DOC.20050428.0004

QA: QA

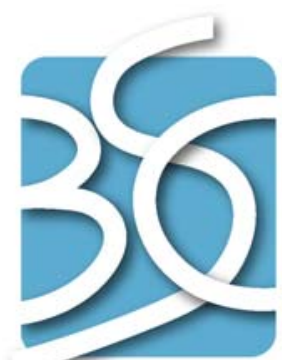

BECHTEL SAIC COMPANYLC
NOTICE OF OPEN CHANGE DOCUMENTS - THIS DOCUMENT IS IMPACTED BY THE LISTED CHANGE DOCUMENT AND CANNOT BE USED WITHOUT IT.

1) ACN-001, DATED 08/12/2005

\title{
Nominal Performance Biosphere Dose Conversion Factor Analysis
}

Prepared for:

U.S. Department of Energy

Office of Civilian Radioactive Waste Management

Office of Repository Development

1551 Hillshire Drive

Las Vegas, Nevada 89134-6321

Prepared by:

Bechtel SAIC Company, LLC

1180 Town Center Drive

Las Vegas, Nevada 89144

Under Contract Number

DE-AC28-01RW12101 


\section{DISCLAIMER}

This report was prepared as an account of work sponsored by an agency of the United States Government. Neither the United States Government nor any agency thereof, nor any of their employees, nor any of their contractors, subcontractors or their employees, makes any warranty, express or implied, or assumes any legal liability or responsibility for the accuracy, completeness, or any third party's use or the results of such use of any information, apparatus, product, or process disclosed, or represents that its use would not infringe privately owned rights. Reference herein to any specific commercial product, process, or service by trade name, trademark, manufacturer, or otherwise, does not necessarily constitute or imply its endorsement, recommendation, or favoring by the United States Government or any agency thereof or its contractors or subcontractors. The views and opinions of authors expressed herein do not necessarily state or reflect those of the United States Government or any agency thereof. 
QA: QA

Nominal Performance Biosphere Dose Conversion Factor Analysis ANL-MGR-MD-000009 REV 04

April 2005 


\begin{tabular}{|l|l|l|}
\hline \multirow{2}{*}{ OCRWM } & Scientific Analysis Signature Page/ Change History & Page iii \\
\cline { 3 - 3 } & & 1. Total Pages: 190 \\
\hline
\end{tabular}

\section{Scientific Analysis Title}

Nominal Performance Biosphere Dose Conversion Factor Analysis

3. DI (including Revision Number)

ANL-MGR-MD-000009 REV 04

\begin{tabular}{|l|l}
\hline 4. Total Appendices & 5. Number of Pages in Each Appendix \\
\hline
\end{tabular}

3

$\mathrm{A}-8$ pages, $\mathrm{B}-8$ pages $+\mathrm{CD}$ ROM, $\mathrm{C}-6$ pages

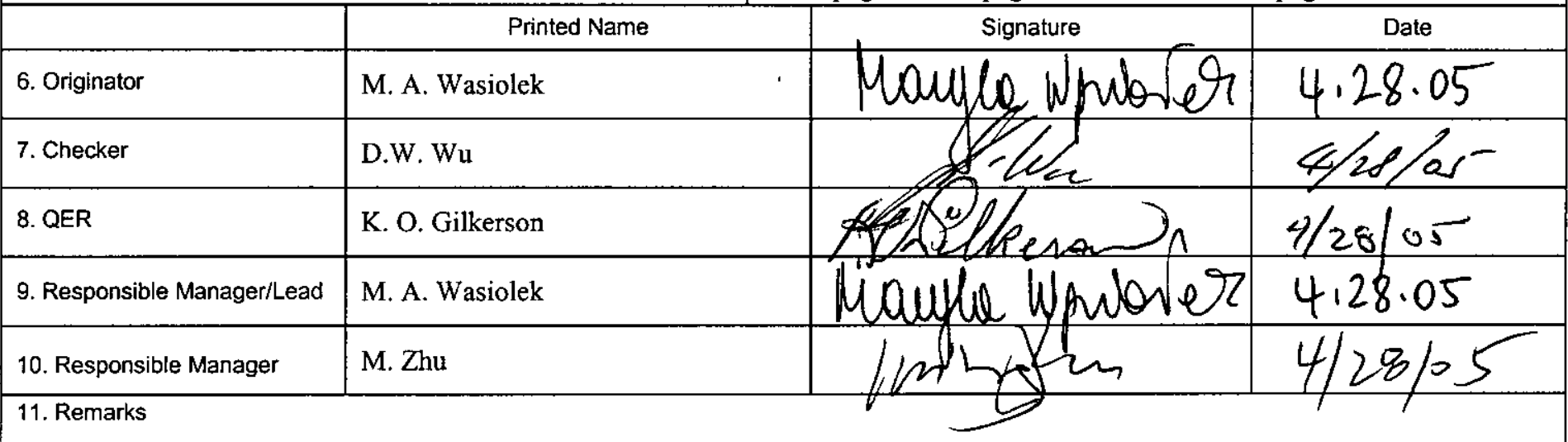

11. Remarks

\section{Change History}

\begin{tabular}{|l|l|}
\hline \multicolumn{1}{|c|}{ 12. Revision No. } & \multicolumn{1}{c|}{ 13. Description of Change } \\
\hline REV 00 ICN 00 & Initial issue \\
\hline REV 01 ICN 00 & $\begin{array}{l}\text { Revision to incorporate the analysis of the climate change effects on the Biosphere Dose Conversion } \\
\text { Factors for nominal performance; add pathway and limited uncertainty analyses; append the list of } \\
\text { radionuclides to include those important for up to 1 million years after the potential repository } \\
\text { closure; remove bounding case; change the document title; and add biosphere model validation } \\
\text { (moved from ANL-MGR-MD-000011 REV 00). Entire AMR has been revised because changes were } \\
\text { extensive. }\end{array}$ \\
\hline REV 02 ICN 00 & Complete revision following development of the new biosphere model. \\
\hline REV 03 ICN 00 & $\begin{array}{l}\text { Revision to incorporate Regulatory Integration Team review comments and to address Condition } \\
\text { Report No. 2222 (implementation of Action No. 2222-001) concerning Latin Hypercube sampling } \\
\text { scheme in GoldSim. The analysis is a complete revision; no change bars were used. }\end{array}$ \\
\hline REV 04 ICN 00 & $\begin{array}{l}\text { Revision to add biosphere dose conversion factors and conversion factors for groundwater protection } \\
\text { standard using updated dosimetry (based on ICRP Publications 60 and 72). }\end{array}$ \\
\hline
\end{tabular}




\section{CONTENTS}

ACRONYMS xiii

1. PURPOSE

2. QUALITY ASSURANCE ........................................................................................ 2-1

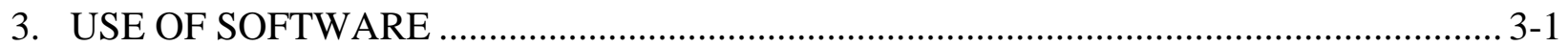

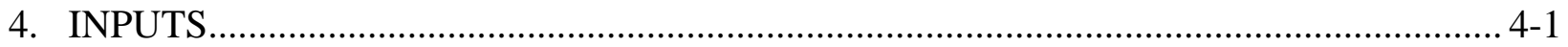

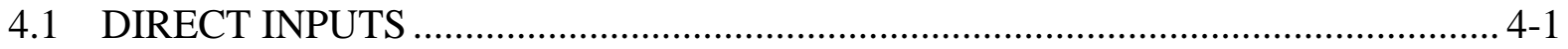

4.1.1 Direct Inputs Used for Calculation of Biosphere Dose Conversion

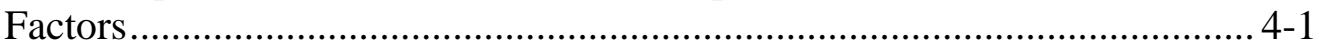

4.1.2 Direct Inputs Used for Calculation of Conversion Factors for Groundwater Protection Standards ............................................................. 4-35

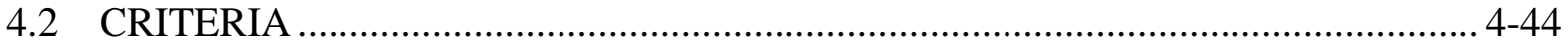

4.3 CODES, STANDARDS, AND REGULATIONS................................................. 4-47

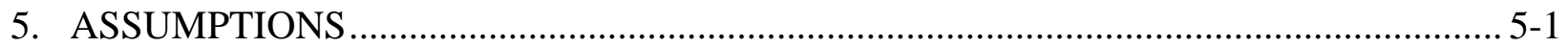

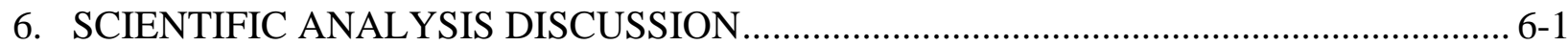

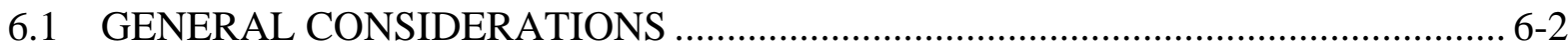

6.1.1 Radionuclides Included in the Analysis....................................................... 6-2

6.1.2 Description of the Groundwater Exposure Scenario ....................................... 6-3

6.1.3 Consideration of Climate Change ......................................................... 6-5

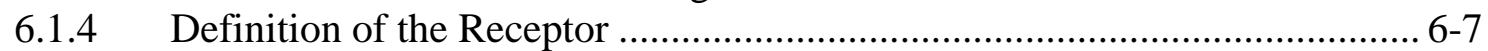

6.1.5 Biosphere Model..................................................................................... 6-7

6.2 DEVELOPMENT OF BIOSPHERE DOSE CONVERSION FACTORS FOR THE GROUNDWATER EXPOSURE SCENARIO................................................ 6-9

6.2.1 Modeling Methods: Treatment of Uncertainty .......................................... 6-10

6.2.2 Modeling Methods: Incorporation of Climate Change............................... 6-10

6.2.3 Modeling Results: Biosphere Dose Conversion Factors .............................. 6-22

6.2.4 TSPA Use of Biosphere Dose Conversion Factors......................................... 6-48

6.2.5 Pathway Analysis................................................................................ 6-49

6.3 DOSE FACTORS FOR GROUNDWATER PROTECTION STANDARDS ............ 6-55

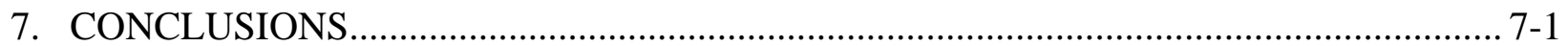

7.1 ANALYSIS SUMMARY …………………….................................................

7.1.1 Incorporation of Uncertainty in Biosphere Dose Conversion Factors........... 7-2

7.1.2 Biosphere Dose Conversion Factors for the Groundwater Exposure Scenario and Their Use in TSPA ............................................................. 7-3

7.1.3 Limitations of Biosphere Modeling......................................................... 7-4

7.1.4 Correlations and Pathway Analysis .............................................................. 7-5

7.1.5 Performance Assessment Calculations for Groundwater Protection

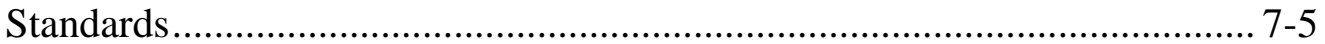

7.2 HOW THE ACCEPTANCE CRITERIA ARE ADDRESSED ................................... 7-5 


\section{CONTENTS (Continued)}

Page

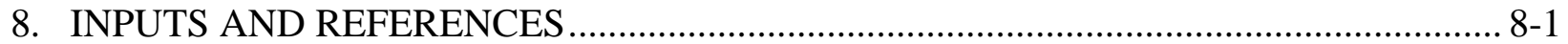

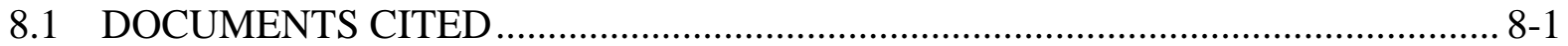

8.2 CODES, STANDARDS, REGULATIONS, AND PROCEDURES ............................. 8-3

8.3 SOURCE DATA, LISTED BY DATA TRACKING NUMBER ……........................ 8-4

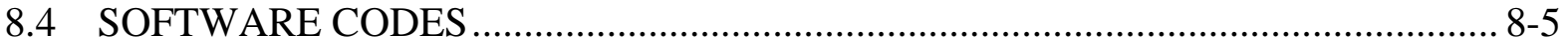

8.5 OUTPUT DATA, LISTED BY DATA TRACKING NUMBER .............................. 8-5

APPENDIX A—LIST AND DESCRIPTION OF GOLDSIM FILES GENERATED IN THIS ANALYSIS ……………………………………………………... A-1

APPENDIX B-DESCRIPTION OF EXCEL FILES GENERATED IN THIS

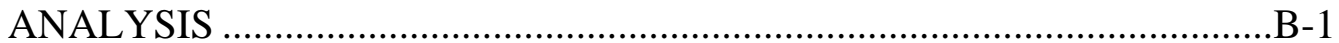

APPENDIX C- VERIFICATION OF ERMYN MODEL FILES FOR GOLDSIM VERSIONS 8.01 SERVICE PACK 4 AND 8.02.300 …................................... 


\section{FIGURES}

1-1. Biosphere Model Documentation and Total System Performance Assessment

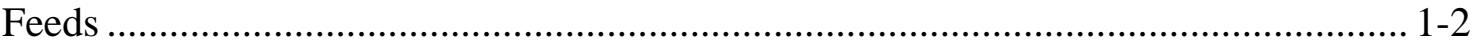

6.1-1. Conceptual Representation of the Biosphere Model for the Groundwater Exposure Scenario 6-4

6.1-2. Relationships Among Submodels for the Groundwater Exposure Scenario

6.2-1. Scaling of the BDCFs

6.2-2. Comparison of BDCFs for the Present-Day Climate Calculated Using ICRP 30 and ICRP 72 Dosimetric Methods

A-1. GoldSim Files for Calculating Groundwater Scenario BDCFs for the PresentDay Climate Generated Using ICRP 72-Based Dosimetric Inputs

A-2. GoldSim Files for Calculating BDCFs for the Upper Bound of the Glacial Transition Climate Generated Using ICRP 72-Based Dosimetric Inputs.

A-3. GoldSim Files for Calculating BDCFs Using Modified Climate-Dependent Input Parameters and ICRP 72-Based Dosimetric Inputs

A-4. GoldSim Files for Calculating BDCFs for the Present-Day Climate Using GoldSim 8.01 Service Pack 4 and ICRP 30-Based Dosimetric Inputs.

A-5. GoldSim Files for Calculating BDCFs for the Upper Bound Glacial Transition Climate Using GoldSim 8.01 Service Pack 4 and ICRP 30-Based Dosimetric Inputs.

A-6. GoldSim Files for Calculating BDCFs Using Modified Climate-Dependent Parameters and GoldSim 8.01 Service Pack 4 and ICRP 30-Based Dosimetric

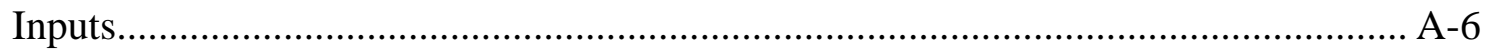

B-1. List of Excel Files Supporting This Analysis ..............................................................

C-1. Model Verification Files Obtained Using GoldSim Versions 7.50.100, 8.01 Service Pack 4, and 8.02.300 C-1 
INTENTIONALLY LEFT BLANK 


\section{TABLES}

Page

1-1. Biosphere-Related Features, Events, and Processes Implicitly Included in TSPA Through the Use of BDCFs for the Groundwater Exposure Scenario.

1-2. Features, Events and Processes Considered in the Development of Conversion Factors for the Groundwater Protection Standards........................................................ 1-5

4.1-1. Summary of Input Parameter Values and Their Uncertainty Distributions.................... 4-2

4.1-2. Primary Radionuclides and Their Decay Products Included in the Biosphere Model.

4.1-3. Dose Coefficients for Inhalation and Ingestion of Radionuclides of Interest Consistent with ICRP 30 Dosimetric Methods

4.1-4. Dose Coefficients for Inhalation and Ingestion of Radionuclides of Interest from ICRP 72.

4.1-5. Dose Coefficients for Exposure to Contaminated Soil for Radionuclides of Interest Consistent with ICRP 30 Dosimetric Methods.

4.1-6. Dose Coefficients for Exposure to Contaminated Soil for Radionuclides of Interest Consistent with ICRP 72 Dosimetric Methods.

4.1-7. ICRP 30-Based Ingestion Dose Coefficients for Beta-Photon Emitting Radionuclides Used in Calculation of Conversion Factors for Groundwater Protection Standards

4.1-8. ICRP 72-Based Ingestion Dose Coefficients for Beta-Photon Emitting Radionuclides Used in Calculation of Conversion Factors for Groundwater Protection Standards

4.2-1. Requirements Applicable to this Analysis .............................................................. 4-44

6.1-1. Receptor Exposure Pathways for the Groundwater Exposure Scenario......................... 6-5

6.1-2. Predicted Future Climates and the Future Climate Analogue Locations....................... 6-6

6.2-1. Mean ICRP 30-Based BDCFs for Climate Extremes and Their Ratios ...................... 6-11

6.2-2. Mean ICRP 72-Based BDCFs for Climate Extremes and Their Ratios ....................... 6-12

6.2-3. Climate-dependent Input Parameters and Their Average Values for the PresentDay Climate and the Upper Bound of the Glacial Transition Climate ........................ 6-13

6.2-4. Correlations (Raw Correlation Coefficients) for ICRP 30-Based BDCFs and Climate-Dependent Input Parameters ...................................................................... 6-14

6.2-5. Correlations (Raw Correlation Coefficients) for ICRP 72-Based BDCFs and Climate-Dependent Input Parameters ....................................................................... 6-16

6.2-6. Dependence of ICRP 30-Based BDCFs for ${ }^{99} \mathrm{Tc},{ }^{129} \mathrm{I}$, and ${ }^{237} \mathrm{~Np}$ on Annual Average Irrigation Rate.

6.2-7. Dependence of ICRP 72-Based BDCFs for ${ }^{99} \mathrm{Tc},{ }^{129} \mathrm{I}$, and ${ }^{237} \mathrm{~Np}$ on Annual Average Irrigation Rate.

6.2-8. Statistics for the ICRP 30-Based BDCFs for the Present-Day Climate, rem/yr per $\mathrm{pCi} / \mathrm{L}$

6.2-9. Statistics for the ICRP 30-Based BDCFs for the Monsoon Climate, rem/yr per $\mathrm{pCi} / \mathrm{L}$

6.2-10. Statistics for the ICRP 30-Based BDCFs for the Glacial Transition Climate, rem/yr per $\mathrm{pCi} / \mathrm{L}$ 


\section{TABLES (Continued)}

Page

6.2-11. Statistics for the ICRP 72-Based BDCFs for the Present-Day Climate, rem/yr per $\mathrm{pCi} / \mathrm{L}$

6.2-12. Statistics for the ICRP 72-Based BDCFs for the Monsoon Climate, rem/yr per

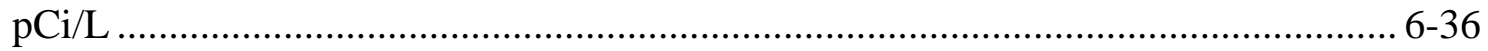

6.2-13. Statistics for the ICRP 72-Based BDCFs for the Glacial Transition Climate, rem/yr per $\mathrm{pCi} / \mathrm{L}$

6.2-14. Correlation Coefficients for the ICRP 30-Based BDCFs for the Present-Day Climate $6-44$

6.2-15. Correlation Coefficients for the ICRP 72-Based BDCFs for the Present-Day Climate...... $6-46$

6.2-16. Calculated Values of Correlation Coefficient and Variable t 6-48

6.2-17. Exposure Pathway Contributions (Percent) for the ICRP 30-Based BDCFs for the Present-Day Climate ..................................................................................... 6-50

6.2-18. Exposure Pathway Contributions (Percent) for the ICRP 30-Based BDCFs for the Upper Bound of Glacial Transition Climate.

6.2-19. Exposure Pathway Contributions (Percent) for the ICRP 72-Based BDCFs for the Present-Day Climate.

6.2-20. Exposure Pathway Contributions (Percent) for the ICRP 72-Based BDCFs for the Upper Bound of Glacial Transition Climate

6.3-1. Limits on Radionuclides in the Representative Volume............................................. 6-55

6.3-2. Primary Radionuclides, Decay Products, and Applicable Groundwater Protection Limits.

6.3-3. Number of Alpha Particles Emitted per One Decay of a Primary Radionuclide Considered in the Gross Alpha Activity Limit of the Groundwater Protection Standards.

6.3-4. Radionuclides and Their Decay Products Included in Calculation of Conversion Factors for Beta-Photon Emitters 6-63

6.3-5. Effective Dose Coefficients ( $\mathrm{Sv/Bq}$ ) Calculated Using ICRP 30-Based Methods for Radionuclides Included in Calculation of Conversion Factors.

6.3-6. Effective Dose Coefficients ( $\mathrm{Sv} / \mathrm{Bq})$ Calculated Using ICRP 72 for Radionuclides Included in Calculation of Conversion Factors.

6.3-7. Conversion Factors (Sv/y per Bq/m3) Developed Using ICRP 30-Based Methods for Calculating Annual Beta-Gamma Dose from Drinking 2 Liters of Water per Day

6.3-8. Conversion Factors (mrem/yr per pCi/L) Developed Using ICRP 30-Based Methods for Calculating Annual Beta-Gamma Dose from Drinking 2 Liters of Water per Day

6.3-9. Conversion Factors $\left(\mathrm{Sv} / \mathrm{y}\right.$ per $\left.\mathrm{Bq} / \mathrm{m}^{3}\right)$ Developed Using ICRP 72 for Calculating Annual Beta-Gamma Dose from Drinking 2 Liters of Water per Day..... 6-75

6.3-10. Conversion Factors (mrem/yr per pCi/L) Developed Using ICRP 72 for Calculating Annual Beta-Gamma Dose from Drinking 2 Liters of Water per Day 6-77

7-1. Output Data Tracking Numbers - ICRP 30 Dosimetric Methods 7-1

7-2. Output Data Tracking Numbers - ICRP 72 Dosimetric Methods $7-1$ 


\section{TABLES (Continued)}

Page

7.1-1. Primary Radionuclides and Decay Products Included in the BDCFs.

C-1. Results of Verification Runs Using GoldSim Versions 7.50.100, 8.01 Service

Pack 4, and 8.02.300 ........................................................................................... 


\section{INTENTIONALLY LEFT BLANK}




\section{ACRONYMS}

AC acceptance criterion

BDCF biosphere dose conversion factor

ERMYN Environmental Radiation Model for Yucca Mountain, Nevada

FEP feature, event, or process

FGR Federal Guidance Report

ICRP International Commission on Radiological Protection

IRT International Review Team

LA license application

NRC U.S. Nuclear Regulatory Commission

QA quality assurance

RMEI reasonably maximally exposed individual

TSPA total system performance assessment

TWP technical work plan

YMRP Yucca Mountain Review Plan 
INTENTIONALLY LEFT BLANK 


\section{PURPOSE}

This analysis report is one of the technical reports containing documentation of the Environmental Radiation Model for Yucca Mountain, Nevada (ERMYN), a biosphere model supporting the Total System Performance Assessment (TSPA) for the license application (LA) for the Yucca Mountain repository. This analysis report describes the development of biosphere dose conversion factors (BDCFs) for the groundwater exposure scenario, and the development of conversion factors for assessing compliance with the groundwater protection standards.

A graphical representation of the documentation hierarchy for the ERMYN is presented in Figure 1-1. This figure shows the interrelationships among the products (i.e., analysis and model reports) developed for biosphere modeling and provides an understanding of how this analysis report contributes to biosphere modeling. This report is one of two reports that develop BDCFs, which are input parameters for the TSPA-LA model. The Biosphere Model Report (BSC 2004 [DIRS 169460]) describes in detail the ERMYN conceptual model and mathematical model. The input parameter reports, shown to the right of the Biosphere Model Report in Figure 1-1, contain detailed description of the model input parameters, their development, and the relationship between the parameters and specific features events and processes (FEPs). This report describes biosphere model calculations and their output, the BDCFs, for the groundwater exposure scenario. This analysis receives direct input from the outputs of the Biosphere Model Report (BSC 2004 [DIRS 169460]) and the five analyses that develop parameter values for the biosphere model (BSC 2005 [DIRS 172827]; BSC 2004 [DIRS 169672]; BSC 2004 [DIRS 169673]; BSC 2004 [DIRS 169458]; BSC 2004 [DIRS 169459]). The results of this report are further analyzed in the Biosphere Dose Conversion Factor Importance and Sensitivity Analysis (Figure 1-1).

The objectives of this analysis are to develop BDCFs for the groundwater exposure scenario for the three climate states (present day, monsoon, and glacial transition) considered in the TSPA-LA, as well as conversion factors for compliance evaluation with the groundwater protection standards. The BDCFs will be used in performance assessment for calculating allpathway annual doses for a given concentration of radionuclides in groundwater. The conversion factors will be used for calculating gross alpha particle activity in groundwater and the annual dose from drinking water for beta- and photon-emitting radionuclides.

This analysis is a revision of the Nominal Performance Biosphere Dose Conversion Factor Analysis (BSC 2004 [DIRS 169674]). The scope of the revision was to calculate BDCFs and conversion factors for compliance evaluation with the groundwater protection standards using updated dosimetric input parameters from, or consistent with, the International Commission on Radiological Protection (ICRP) Publication 72 (ICRP 1996 [DIRS 152446]). The quantities developed previously (BSC 2004 [DIRS 169674]), which reflect the ICRP Publication 30 dosimetric methods (ICRP 30) (ICRP 1979 [DIRS 110386]; ICRP 1980 [DIRS 110351]; ICRP 1981 [DIRS 110352]) were not changed and were retained in this report for comparison purposes.

The analysis was performed in accordance with LP-SIII.9Q-BSC, Scientific Analyses, and the Technical Work Plan for Biosphere Modeling and Expert Support (TWP) (BSC 2005 [DIRS 172782]). 


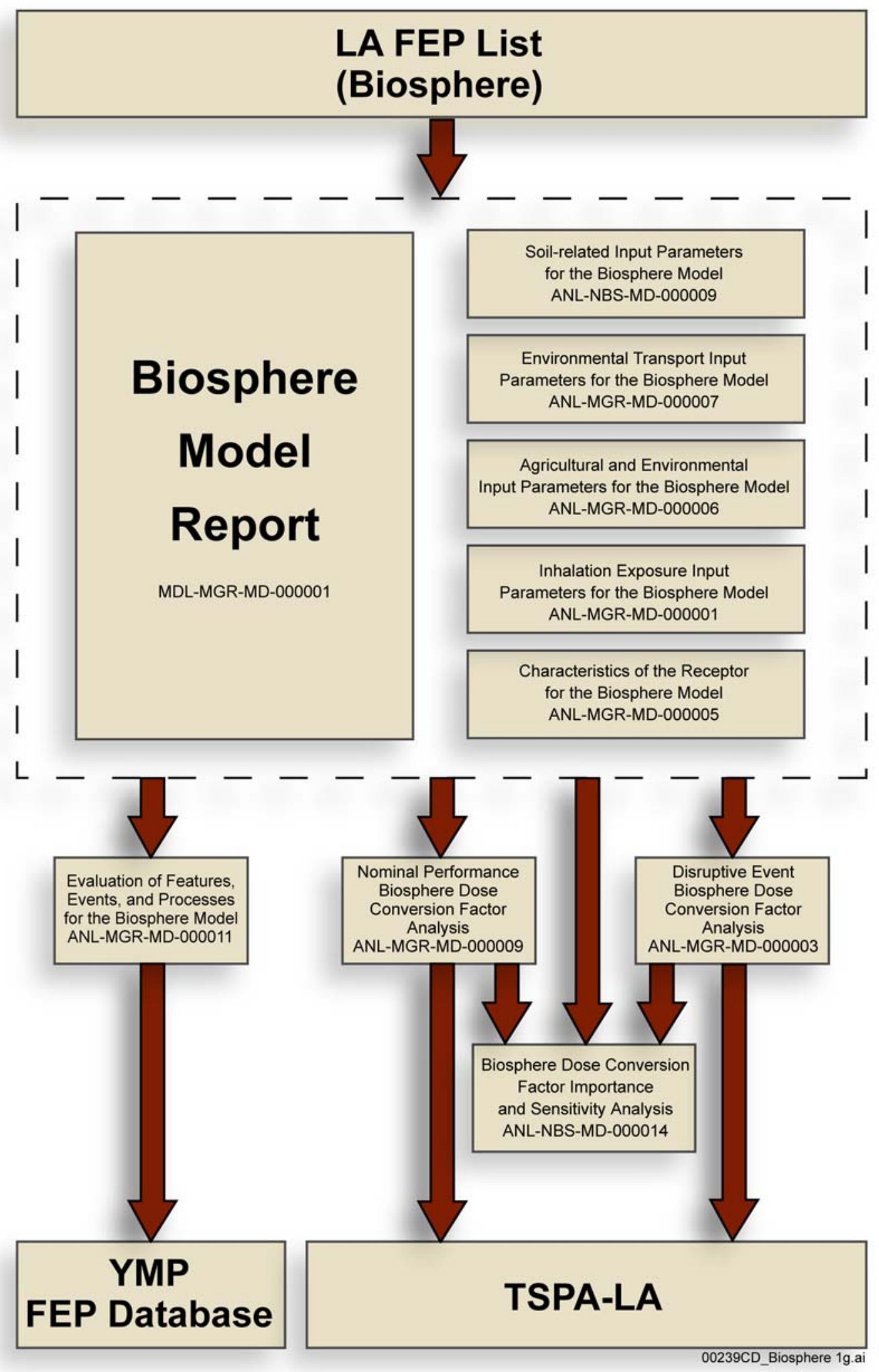

Figure 1-1. Biosphere Model Documentation and Total System Performance Assessment Feeds 
Dosimetric inputs consistent with ICRP Publication 30 are based on the concepts recommended in ICRP Publication 26 (ICRP 1977 [DIRS 101075]) and the dosimetric methods for intakes of radionuclides by workers outlined in ICRP Publication 30 (ICRP 1979 [DIRS 110386]; ICRP 1980 [DIRS 110351]; ICRP 1981 [DIRS 110352]). This approach is consistent with the individual protection standard defined in terms of total effective dose equivalent.

Dosimetric inputs consistent with ICRP Publication 72 are based on the concepts recommended in ICRP Publication 60 (ICRP 1991 [DIRS 101836]) and the updated biokinetic and dosimetric models for intakes of radionuclides by members of the public. ICRP Publication 60 introduced a new dosimetric quantity, the effective dose, which uses an expanded list of tissues and organs and revised values of tissue and organ weighting factors. In addition, biokinetic and dosimetric models were updated to include new information that became available since ICRP Publication 30. A new set of age-dependent dose coefficients for intakes of radionuclides by members of the public was developed (ICRP Publication 30 concerned doses to workers) and documented in a series of reports compiled in ICRP Publication 72 (ICRP 1996 [DIRS 152446]). ICRP Publication 72 is presently the most current compilation of dose coefficients for internal intakes.

For the groundwater exposure scenario, radionuclides enter the biosphere from a well that extracts contaminated groundwater from an aquifer. BDCFs for the groundwater scenario apply to the TSPA-LA modeling cases that consider groundwater release of radionuclides from the repository at Yucca Mountain. These cases include the nominal scenario class, the seismic scenario class, and the igneous intrusion modeling case of the igneous scenario class (BSC 2005 [DIRS 173309], Section 2).

The biosphere model considers features, events, and processes (FEPs) applicable to the Yucca Mountain biosphere (DTN: MO0501SEPFEPLA.001 [DIRS 172601]). Table 1-1 lists FEPs that are included in the biosphere model for the groundwater exposure scenario (BSC 2004 [DIRS 169460], Tables 6.2-1 and 6.3-6). The Biosphere Model Report (BSC 2004 [DIRS 169460]) uses a previous LA FEPs List (DTN: MO0407SEPFEPLA.000 [DIRS 170760]). However, the names, descriptions, and dispositions of the biosphere-related FEPs are the same in both sets.

The BDCFs developed as output of this report, and used as direct inputs to the TSPA, are the results of the biosphere model and use inputs from the supporting parameter reports. The BDCFs, therefore, implicitly include the FEPs that are related to the model and to the model inputs taken from the supporting parameter reports. Consequently, explicit mapping of individual FEPs listed in Table 1-1 to specific sections of this report and BDCFs is not possible. All FEPs considered in the biosphere model and its input parameters are collectively included in BDCFs. Relationships among the biosphere-related FEPs, the biosphere conceptual model, and the exposure scenarios are examined in Section 6.3 of the Biosphere Model Report (BSC 2004 [DIRS 169460]). The disposition of the included FEPs within the mathematical model, and their explicit relationship to the model equations and input parameters is presented in the Biosphere Model Report (BSC 2004 [DIRS 169460], Table 6.7-1). 
Table 1-1. Biosphere-Related Features, Events, and Processes Implicitly Included in TSPA Through the Use of BDCFs for the Groundwater Exposure Scenario

\begin{tabular}{|c|c|}
\hline FEP Name & LA FEP Number \\
\hline Climate change & 1.3.01.00.0A \\
\hline Water table rise affects $\mathrm{SZ}$ & 1.3.07.02.0A \\
\hline Water management activities & 1.4.07.01.0A \\
\hline Wells & 1.4.07.02.0A \\
\hline Chemical characteristics of groundwater in the SZ & 2.2.08.01.0A \\
\hline Soil type & 2.3.02.01.0A \\
\hline Radionuclide accumulation in soils & 2.3.02.02.0A \\
\hline Soil and sediment transport in the biosphere & 2.3.02.03.0A \\
\hline Surface water transport and mixing & 2.3.04.01.0A \\
\hline Precipitation & 2.3.11.01.0A \\
\hline Biosphere characteristics & 2.3.13.01.0A \\
\hline Radionuclide alteration during biosphere transport & 2.3.13.02.0A \\
\hline Human characteristics (physiology, metabolism) & 2.4.01.00.0A \\
\hline Human lifestyle & 2.4.04.01.0A \\
\hline Dwellings & 2.4.07.00.0A \\
\hline Wild and natural land and water use & 2.4.08.00.0A \\
\hline Agricultural land use and irrigation & 2.4.09.01.0B \\
\hline Animal farms and fisheries & 2.4.09.02.0A \\
\hline Urban and industrial land and water use & 2.4.10.00.0A \\
\hline Radioactive decay and ingrowth & 3.1.01.01.0A \\
\hline Atmospheric transport of contaminants & 3.2.10.00.0A \\
\hline Contaminated drinking water, foodstuffs and drugs & 3.3.01.00.0A \\
\hline Plant uptake & 3.3.02.01.0A \\
\hline Animal uptake & 3.3.02.02.0A \\
\hline Fish uptake & 3.3.02.03.0A \\
\hline Contaminated nonfood products and exposure & 3.3.03.01.0A \\
\hline Ingestion & 3.3.04.01.0A \\
\hline Inhalation & 3.3.04.02.0A \\
\hline External exposure & 3.3.04.03.0A \\
\hline Radiation doses & 3.3.05.01.0A \\
\hline Radon and radon daughter exposure & 3.3.08.00.0A \\
\hline
\end{tabular}

Source: DTN: MO0501SEPFEPLA.001 [DIRS 172601].

$\mathrm{SZ}=$ saturated zone

In addition to producing the BDCFs for the groundwater exposure scenario, this analysis develops conversion factor values for evaluating compliance with the groundwater protection standards of 10 CFR 63 [DIRS 173164]. These conversion factors are used by TSPA to calculate gross alpha particle activity in groundwater and the organ and whole-body annual dose 
from beta- and photon-emitting radionuclides resulting from drinking 2 liters of water per day. The FEPs considered in the development of conversion factors are listed in Table 1-2. The disposition of these FEPs in TSPA is through the conversion factors that are inputs for the TSPA-LA.

Table 1-2. Features, Events and Processes Considered in the Development of Conversion Factors for the Groundwater Protection Standards

\begin{tabular}{|l|l|l|}
\hline FEP Number & \multicolumn{1}{|c|}{ FEP Name } & \multicolumn{1}{|c|}{ Description of FEP Disposition } \\
\hline 1.4.07.02.0A & Wells & $\begin{array}{l}\text { A well is the initial source of contaminated groundwater in the } \\
\text { biosphere. }\end{array}$ \\
\hline 2.4.01.00.0A & $\begin{array}{l}\text { Human characteristics } \\
\text { (physiology, metabolism) }\end{array}$ & $\begin{array}{l}\text { Physiology and metabolism of the human receptor were considered } \\
\text { in developing the values of dose coefficient for ingestion, which are } \\
\text { used as input to calculate the values of conversion factors } \\
\text { (Equation 6.3-3). }\end{array}$ \\
\hline $3.1 .01 .01 .0 \mathrm{~A}$ & $\begin{array}{l}\text { Radioactive decay and } \\
\text { ingrowth }\end{array}$ & $\begin{array}{l}\text { Contribution from long-lived and short-lived decay products of } \\
\text { primary radionuclides is included in the values of conversion factors, } \\
\text { through the use of effective dose coefficients for ingestion } \\
\text { (Tables 6.3-5 and 6.3-6) and also through including decay products } \\
\text { in the total number of alpha particles associated with a primary } \\
\text { radionuclide (Table 6.3-3). }\end{array}$ \\
\hline $3.3 .01 .00 .0 \mathrm{~A}$ & $\begin{array}{l}\text { Contaminated drinking } \\
\text { water, foodstuff and drugs }\end{array}$ & $\begin{array}{l}\text { Locally obtained contaminated drinking water causes ingestion dose } \\
\text { (Equations 6.3-2 and 6.3-4) }\end{array}$ \\
\hline $3.3 .04 .01 .0 \mathrm{~A}$ & Ingestion & $\begin{array}{l}\text { Conversion factors for beta-gamma emitters are used in evaluating } \\
\text { exposure of the receptor arising from ingestion of contaminated } \\
\text { water (Equations 6.3-2 to 6.3-4). }\end{array}$ \\
\hline 3.3.05.01.0A & Radiation doses & $\begin{array}{l}\text { Radiation doses arising from ingestion of contaminated water will be } \\
\text { evaluated in TSPA using conversion factors developed in this } \\
\text { analysis. }\end{array}$ \\
\hline
\end{tabular}

Source: DTN: MO0501SEPFEPLA.001 [DIRS 172601]. 


\section{INTENTIONALLY LEFT BLANK}




\section{QUALITY ASSURANCE}

This analysis involved development of data to support performance assessment, as identified in TWP (BSC 2005 [DIRS 172782]), and thus, it was a quality affecting activity in accordance with LP-2.29Q-BSC, Planning for Science Activities. Approved quality assurance procedures identified in TWP (BSC 2005 [DIRS 172782], Section 4) were used to conduct and document the activities described in this report. Specifically, the procedure governing development of this document was LP-SIII.9Q-BSC, Scientific Analyses. Electronic data used in this analysis were controlled in accordance with methods specified in TWP (BSC 2005 [DIRS 172782], Section 8).

The natural barriers and items identified in the Q-List (BSC 2005 [DIRS 171190]) are not pertinent to this analysis and a Safety Category per AP-2.22Q, Classification Analyses and Maintenance of the Q-List, is not applicable. 


\section{INTENTIONALLY LEFT BLANK}




\section{USE OF SOFTWARE}

This analysis was performed using a verified and validated model, the ERMYN model, which is described in the Biosphere Model Report (BSC 2004 [DIRS 169460]). The model files were obtained from the Model Warehouse (DTN: MO0306MWDBGSMF.001 [DIRS 163816]). The model is implemented using the GoldSim Probabilistic Simulation Environment, a graphical, object-oriented computer program for carrying out dynamic, probabilistic simulations (GoldSim Technology Group 2003 [DIRS 166226]).

The analysis was conducted using GoldSim Version 8.02.300 (STN: 10344-8.02-03) (BSC 2004 [DIRS 172588]). The analysis also includes modeling results previously obtained using GoldSim Version 8.01 Service Pack 4 (STN: 10344-8.01SP4-00) (BSC 2004 [DIRS 169695]). Both versions of software were qualified under the Office of Civilian Radioactive Waste Management, Quality Assurance program for use on the Yucca Mountain Project. GoldSim versions 8.01 Service Pack 4 and 8.02.300 were installed by Software Configuration Management on a DELL Precision Workstation 530 computer and run under the Windows 2000 operating system.

The software was appropriate for the application of running ERMYN model, which was implemented using GoldSim software, as described in the Biosphere Model Report (BSC 2004 [DIRS 169460], Section 6.8), and was used within the range of validation in accordance with procedure LP-SI.11Q-BSC, Software Management. The GoldSim files generated in this analysis are shown in Appendix A. The original verification of the model was performed using GoldSim version 7.50.100 (STN: 10344-7.50.100-00) (BSC 2003 [DIRS 161572]). Verification of the model for the use with GoldSim versions 8.01 Service Pack 4 (BSC 2004 [DIRS 169695]) and 8.02.300 (BSC 2004 [DIRS 172588]) is described in Appendix C.

In addition, the commercial off-the-shelf product Microsoft ${ }^{\circledR}$ Excel 2000 (Version 9.0.3821 SR-1) was used for data reduction. Standard functions of Excel were used to calculate values presented in the tables in Section 6. The use of these functions, including formulas or algorithms, inputs, and outputs, is described in Appendix B. 


\section{INTENTIONALLY LEFT BLANK}




\section{INPUTS}

\subsection{DIRECT INPUTS}

\subsubsection{Direct Inputs Used for Calculation of Biosphere Dose Conversion Factors}

The inputs to this analysis are listed in Tables 4.1-1 to 4.1-6. Input parameter values and distributions were generated specifically for biosphere model input and are appropriate for the intended use. The appropriateness for the intended use in this analysis and uncertainty in the input parameters are discussed in the individual analysis reports that document parameter development (BSC 2004 [DIRS 169672]; BSC 2004 [DIRS 169458]; BSC 2004 [DIRS 169673]; BSC 2004 [DIRS 169459]; BSC 2005 [DIRS 172827]).

The parameters were used as input for the ERMYN model for the groundwater exposure scenario (DTN: MO0306MWDBGSMF.001 [DIRS 163816]). Where applicable, Table 4.1-1 includes parameter values and distributions for two climate extremes: the mean present-day climate and one future climate state represented by the upper bound of the glacial transition climate. Direct inputs listed in this section were used for calculation of BDCFs for these two climate states. The BDCFs for the monsoon and glacial transition climates were calculated in this analysis by interpolation between these two extremes.

The half-lives and branching fractions for radionuclides included in the biosphere model are listed in Table 4.1-2. Dose coefficients for inhalation and ingestion consistent with ICRP 30 dosimetric methods (ICRP 30) (ICRP 1979 [DIRS 110386]; ICRP 1980 [DIRS 110351]; ICRP 1981 [DIRS 110352]) are shown in Table 4.1-3. The dose coefficients for inhalation and ingestion from ICRP Publication 72 (ICRP 1996 [DIRS 152446]) are listed in Table 4.1-4. The dose coefficients for exposure to contaminated soil, consistent with ICRP 30 dosimetric methods, are shown in Table 4.1-5. The dose coefficients for exposure to contaminated soil, consistent with the dosimetric methods used in ICRP Publication 72 are listed in Table 4.1-6. The above direct inputs are used in Section 6.2. The BDCF values consistent with ICRP 30 dosimetric methods are presented in this report for comparison purposes.

Additional inputs were taken from the textbook references (sources of established fact data) to help with the statistical analysis of the results. Specifically, Steel and Torrie (1980 [DIRS 150857], pp. 278 to 279) was used as a source of statistical tests of hypotheses and Lide and Frederikse (1997 [DIRS 103178], p. A-105) was used as a source of percentage points for the Student-t distribution.

For brevity, the parameters consistent with the ICRP 30 dosimetric methods are referred to as ICRP 30-based while the parameters consistent with the ICRP 72 dosimetric methods are referred to as ICRP 72-based. 
Table 4.1-1. Summary of Input Parameter Values and Their Uncertainty Distributions

\begin{tabular}{|c|c|c|c|c|c|c|c|c|c|}
\hline \multicolumn{3}{|c|}{ Parameter Name } & $\begin{array}{l}\text { Distribution } \\
\text { Type }\end{array}$ & Units & $\begin{array}{l}\text { Mean, } \\
\text { Mode or } \\
\text { Fixed } \\
\text { Value } \\
\end{array}$ & SD or SE & $\begin{array}{l}\text { Minimum } \\
\text { or Value } \\
\text { for CD }\end{array}$ & $\begin{array}{l}\text { Maximum } \\
\text { or } \\
\text { Percentile } \\
\text { for CD }\end{array}$ & DTN/Reference \\
\hline \multicolumn{10}{|c|}{ SURFACE SOIL SUBMODEL } \\
\hline \multicolumn{3}{|c|}{$\begin{array}{l}\text { Activity concentration of a radionuclide in well } \\
\text { water }\end{array}$} & Fixed & $\mathrm{Bq} / \mathrm{m}^{3}$ & 1 & - & - & - & Source of contamination \\
\hline \multirow{4}{*}{$\begin{array}{l}\text { Annual average } \\
\text { irrigation rate }\end{array}$} & \multicolumn{2}{|c|}{ Present-day climate } & Normal & \multirow{4}{*}{$\mathrm{m} / \mathrm{yr}$} & 0.95 & 0.08 & 0.74 & 1.16 & \multirow{4}{*}{$\begin{array}{l}\text { MO0403SPAAEIBM.002 } \\
\text { [DIRS 169392] }\end{array}$} \\
\hline & \multicolumn{2}{|c|}{$\begin{array}{l}\text { Monsoon climate upper } \\
\text { bound }\end{array}$} & Fixed & & 0.52 & - & - & - & \\
\hline & \multicolumn{2}{|c|}{$\begin{array}{l}\text { Glacial transition climate } \\
\text { lower bound }\end{array}$} & Fixed & & 0.88 & - & - & - & \\
\hline & \multicolumn{2}{|c|}{$\begin{array}{l}\text { Glacial transition climate } \\
\text { upper bound }\end{array}$} & Normal & & 0.50 & 0.04 & 0.40 & 0.60 & \\
\hline \multicolumn{3}{|c|}{ Radionuclide half-life and branching fraction } & Fixed & $\begin{array}{l}\text { See Table } \\
\quad 4.1-2\end{array}$ & $\begin{array}{l}\text { See Table } \\
4.1-2\end{array}$ & - & - & - & $\begin{array}{l}\text { MO0407SPACRBSM.002 } \\
\text { [DIRS 170677] }\end{array}$ \\
\hline \multicolumn{3}{|l|}{ Soil bulk density } & Triangular & $\mathrm{kg} / \mathrm{m}^{3}$ & 1500 & - & 1300 & 1700 & $\begin{array}{l}\text { MO0407SPASRPBM.002 } \\
\text { [DIRS 170755] }\end{array}$ \\
\hline \multicolumn{3}{|c|}{ Surface soil depth (tillage depth) } & Uniform & $\mathrm{m}$ & - & - & 0.05 & 0.30 & $\begin{array}{l}\text { MO0403SPAAEIBM.002 } \\
\text { [DIRS 169392] }\end{array}$ \\
\hline \multicolumn{3}{|c|}{ Surface soil erosion rate } & Triangular & $\mathrm{kg} /\left(\mathrm{m}^{2} \mathrm{yr}\right)$ & 0.19 & - & 0.19 & 1.1 & $\begin{array}{l}\text { MO0407SPASRPBM.002 } \\
\text { [DIRS 170755] }\end{array}$ \\
\hline \multirow{12}{*}{\multicolumn{2}{|c|}{$\begin{array}{l}\text { Soil solid/liquid partition } \\
\text { coefficient }\end{array}$}} & Carbon & \multirow{12}{*}{ Lognormal $^{\mathrm{a}}$} & \multirow{12}{*}{$\mathrm{L} / \mathrm{kg}$} & $1.8 \mathrm{E}+01$ & $6.0 \mathrm{E}+00$ & - & - & \multirow{12}{*}{$\begin{array}{l}\text { MO0407SPASRPBM.002 } \\
\text { [DIRS 170755] }\end{array}$} \\
\hline & & Chlorine & & & $1.4 \mathrm{E}-01$ & $6.0 \mathrm{E}+00$ & - & - & \\
\hline & & Selenium & & & $1.5 \mathrm{E}+02$ & $6.0 \mathrm{E}+00$ & - & - & \\
\hline & & Strontium & & & $2.0 \mathrm{E}+01$ & $5.5 \mathrm{E}+00$ & - & - & \\
\hline & & Technetium & & & $1.4 \mathrm{E}-01$ & $6.0 \mathrm{E}+00$ & - & - & \\
\hline & & Tin & & & $4.5 \mathrm{E}+02$ & $6.0 \mathrm{E}+00$ & - & - & \\
\hline & & lodine & & & $4.5 \mathrm{E}+00$ & $7.4 \mathrm{E}+00$ & - & - & \\
\hline & & Cesium & & & $4.4 \mathrm{E}+03$ & $3.7 \mathrm{E}+00$ & - & - & \\
\hline & & Lead & & & $1.6 \mathrm{E}+04$ & $4.1 \mathrm{E}+00$ & - & - & \\
\hline & & Radium & & & $3.6 \mathrm{E}+04$ & $2.2 \mathrm{E}+01$ & - & - & \\
\hline & & Actinium & & & $1.5 \mathrm{E}+03$ & $6.0 \mathrm{E}+00$ & - & - & \\
\hline & & Thorium & & & $3.0 \mathrm{E}+03$ & $8.2 \mathrm{E}+00$ & - & - & \\
\hline
\end{tabular}


Table 4.1-1. Summary of Input Parameter Values and Their Uncertainty Distributions (Continued)

\begin{tabular}{|c|c|c|c|c|c|c|c|c|}
\hline \multicolumn{2}{|c|}{ Parameter Name } & $\begin{array}{c}\text { Distribution } \\
\text { Type }\end{array}$ & Units & $\begin{array}{l}\text { Mean, } \\
\text { Mode } \\
\text { or Fixed } \\
\text { Value }\end{array}$ & SD or SE & $\begin{array}{l}\text { Minimum } \\
\text { or Value } \\
\text { for CD }\end{array}$ & $\begin{array}{l}\text { Maximum } \\
\quad \text { or } \\
\text { Percentile } \\
\text { for CD }\end{array}$ & DTN/Reference \\
\hline & Protactinium & \multirow{5}{*}{ Lognormal $^{\mathrm{a}}$} & \multirow{5}{*}{$\mathrm{L} / \mathrm{kg}$} & $1.8 \mathrm{E}+03$ & $6.0 \mathrm{E}+00$ & - & - & \\
\hline & Uranium & & & $3.3 E+01$ & $2.5 E+01$ & - & - & \\
\hline & Neptunium & & & $2.5 E+01$ & $3.3 E+00$ & - & - & \\
\hline & Plutonium & & & $1.2 \mathrm{E}+03$ & $3.3 E+00$ & - & - & \\
\hline & Americium & & & $2.0 \mathrm{E}+03$ & $1.3 E+01$ & - & - & \\
\hline \multirow{2}{*}{ Overwatering rate } & $\begin{array}{l}\text { Present-day } \\
\text { climate }\end{array}$ & Cumulative & $\mathrm{m} / \mathrm{yr}$ & - & - & $\begin{array}{l}0.009 \\
0.030 \\
0.045 \\
0.077 \\
0.129 \\
0.233 \\
0.275 \\
\end{array}$ & $\begin{array}{c}0 \% \\
19 \% \\
38 \% \\
57 \% \\
76 \% \\
95 \% \\
100 \% \\
\end{array}$ & \multirow[t]{2}{*}{$\begin{array}{l}\text { MO0403SPAAEIBM.002 } \\
\text { [DIRS 169392] }\end{array}$} \\
\hline & Future climate & Cumulative & $\mathrm{m} / \mathrm{yr}$ & - & - & $\begin{array}{l}0.004 \\
0.020 \\
0.047 \\
0.072 \\
0.104 \\
0.150 \\
0.177 \\
\end{array}$ & $\begin{array}{c}0 \% \\
19 \% \\
38 \% \\
57 \% \\
76 \% \\
95 \% \\
100 \% \\
\end{array}$ & \\
\hline \multicolumn{2}{|l|}{ Volumetric water content } & Uniform & - & - & - & 0.18 & 0.28 & $\begin{array}{l}\text { MO0407SPASRPBM.002 } \\
\text { [DIRS 170755] }\end{array}$ \\
\hline \multicolumn{9}{|c|}{ AIR SUBMODEL } \\
\hline \multicolumn{2}{|c|}{ Mass loading for crops at nominal conditions } & Triangular & $\mathrm{mg} / \mathrm{m}^{3}$ & 0.12 & - & 0.025 & 0.200 & $\begin{array}{l}\text { MO0407SPAINEXI.002 } \\
\text { [DIRS 170597] }\end{array}$ \\
\hline \multirow{4}{*}{$\begin{array}{l}\text { Mass loading for receptor } \\
\text { environments at nominal } \\
\text { conditions }\end{array}$} & Active outdoors & \multirow{4}{*}{ Triangular } & \multirow{4}{*}{$\mathrm{mg} / \mathrm{m}^{3}$} & 5.00 & - & 1.000 & 10.000 & \multirow{4}{*}{$\begin{array}{l}\text { MO0407SPAINEXI.002 } \\
\text { [DIRS 170597] }\end{array}$} \\
\hline & Inactive outdoors & & & 0.06 & - & 0.025 & 0.100 & \\
\hline & Active indoors & & & 0.10 & - & 0.060 & 0.175 & \\
\hline & Asleep indoors & & & 0.03 & - & 0.010 & 0.050 & \\
\hline
\end{tabular}


Table 4.1-1. Summary of Input Parameter Values and Their Uncertainty Distributions (Continued)

\begin{tabular}{|c|c|c|c|c|c|c|c|c|}
\hline \multicolumn{2}{|c|}{ Parameter Name } & $\begin{array}{c}\text { Distribution } \\
\text { Type }\end{array}$ & Units & $\begin{array}{c}\text { Mean, } \\
\text { Mode } \\
\text { or Fixed } \\
\text { Value } \\
\end{array}$ & SD or SE & $\begin{array}{c}\text { Minimum } \\
\text { or Value } \\
\text { for CD }\end{array}$ & $\begin{array}{c}\text { Maximum } \\
\text { or } \\
\text { Percentile } \\
\text { for CD }\end{array}$ & DTN/Reference \\
\hline \multirow{4}{*}{$\begin{array}{l}\text { Enhancement factor at } \\
\text { nominal conditions }\end{array}$} & Active outdoors & Cumulative & - & - & - & $\begin{array}{l}2.2 \\
4.0 \\
6.5\end{array}$ & $\begin{array}{c}0 \% \\
50 \% \\
100 \%\end{array}$ & $\begin{array}{l}\text { MO0407SPASRPBM.002 } \\
\text { [DIRS 170755] }\end{array}$ \\
\hline & Inactive outdoors & \multirow{3}{*}{ Cumulative } & \multirow{3}{*}{-} & \multirow{3}{*}{-} & \multirow{3}{*}{-} & 0.21 & $0 \%$ & \\
\hline & Active indoors & & & & & 0.7 & $50 \%$ & \\
\hline & Asleep indoors & & & & & 1.04 & $100 \%$ & \\
\hline \multicolumn{2}{|c|}{ Evaporative cooler water transfer fraction } & Uniform & - & - & - & 0 & 1 & $\begin{array}{l}\text { MO0406SPAETPBM.002 } \\
\text { [DIRS 170150] }\end{array}$ \\
\hline \multicolumn{2}{|c|}{ Evaporative cooler water use (evaporation) rate } & Lognormal $^{\mathrm{a}}$ & $\mathrm{L} / \mathrm{hr}$ & 17 & 1.7 & - & - & $\begin{array}{l}\text { MO0406SPAETPBM.002 } \\
\text { [DIRS 170150] }\end{array}$ \\
\hline \multicolumn{2}{|c|}{ Evaporative cooler air flow rate } & Cumulative & $\mathrm{m}^{3} / \mathrm{hr}$ & - & - & $\begin{array}{c}1,700 \\
8,300 \\
10,200 \\
\end{array}$ & $\begin{array}{c}0 \% \\
50 \% \\
100 \% \\
\end{array}$ & $\begin{array}{l}\text { MO0406SPAETPBM.002 } \\
\text { [DIRS 170150] }\end{array}$ \\
\hline \multicolumn{2}{|c|}{$\begin{array}{l}\text { Correlation coefficient for evaporative cooler } \\
\text { water use (evaporation) and air flow rates }\end{array}$} & Fixed & - & 0.8 & - & - & - & $\begin{array}{l}\text { MO0406SPAETPBM.002 } \\
\text { [DIRS 170150] }\end{array}$ \\
\hline \multicolumn{2}{|l|}{ Radon release factor } & Fixed & $\begin{array}{c}\left(\mathrm{Bq} / \mathrm{m}^{3}\right) / \\
(\mathrm{Bq} / \mathrm{kg})\end{array}$ & 0.25 & - & - & - & $\begin{array}{l}\text { MO0406SPAETPBM.002 } \\
\text { [DIRS 170150] }\end{array}$ \\
\hline \multirow{2}{*}{ House ventilation rate } & Average & Lognormal $^{b}$ & \multirow{2}{*}{$1 / \mathrm{hr}$} & 1.0 & 1.1 & 0.35 & 2.9 & \multirow{2}{*}{$\begin{array}{l}\text { MO0406SPAETPBM.002 } \\
\text { [DIRS 170150] }\end{array}$} \\
\hline & Evap. cooler on & Uniform & & - & - & 1 & 30 & \\
\hline \multicolumn{2}{|c|}{ Fraction of radon from soil entering the house } & Uniform & - & - & - & 0.1 & 0.25 & $\begin{array}{l}\text { MO0406SPAETPBM.002 } \\
\text { [DIRS 170150] }\end{array}$ \\
\hline \multicolumn{2}{|c|}{$\begin{array}{l}\text { Ratio of Rn- } 222 \text { concentration in air to flux } \\
\text { density from soil }\end{array}$} & Fixed & $\begin{array}{l}\left(\mathrm{Bq} / \mathrm{m}^{3}\right) / \\
\left(\mathrm{Bq} /\left(\mathrm{m}^{2} \mathrm{~s}\right)\right.\end{array}$ & 300 & - & - & - & $\begin{array}{l}\text { MO0406SPAETPBM.002 } \\
\text { [DIRS 170150] }\end{array}$ \\
\hline \multicolumn{2}{|l|}{ Interior wall height } & Cumulative & $\mathrm{m}$ & - & - & $\begin{array}{l}2.1 \\
2.3 \\
2.7 \\
\end{array}$ & $\begin{array}{c}0 \% \\
50 \% \\
100 \% \\
\end{array}$ & $\begin{array}{l}\text { MO0406SPAETPBM.002 } \\
\text { [DIRS 170150] }\end{array}$ \\
\hline \multicolumn{9}{|c|}{ PLANT UPTAKE SUBMODEL } \\
\hline \multirow{5}{*}{$\begin{array}{l}\text { Soil-to-plant transfer factor } \\
\text { for leafy vegetables }\end{array}$} & Chlorine & \multirow{5}{*}{ Lognormal $^{\mathrm{a}}$} & \multirow{5}{*}{$\begin{array}{c}\left(\mathrm{Bq} / \mathrm{kg}_{\text {plant }}\right) / \\
\left(\mathrm{Bq} / \mathrm{kg}_{\text {soil }}\right)\end{array}$} & $6.4 \mathrm{E}+01$ & 2.0 & $1.1 \mathrm{E}+01$ & $3.8 \mathrm{E}+02$ & \multirow{5}{*}{$\begin{array}{l}\text { MO0406SPAETPBM.002 } \\
\text { [DIRS 170150] }\end{array}$} \\
\hline & Selenium & & & 4.6E-02 & 3.8 & $1.4 \mathrm{E}-03$ & $1.4 \mathrm{E}+00$ & \\
\hline & Strontium & & & $1.7 \mathrm{E}+00$ & 2.0 & $2.9 \mathrm{E}-01$ & $1.0 \mathrm{E}+01$ & \\
\hline & Technetium & & & $4.6 \mathrm{E}+01$ & 2.6 & $3.8 \mathrm{E}+00$ & $5.5 \mathrm{E}+02$ & \\
\hline & Tin & & & $3.8 \mathrm{E}-02$ & 2.0 & $6.4 \mathrm{E}-03$ & 2.3E-01 & \\
\hline
\end{tabular}




\begin{tabular}{|c|c|c|c|c|c|c|c|c|}
\hline \multicolumn{2}{|c|}{ Parameter Name } & $\begin{array}{c}\text { Distribution } \\
\text { Type }\end{array}$ & Units & $\begin{array}{c}\text { Mean, } \\
\text { Mode } \\
\text { or Fixed } \\
\text { Value }\end{array}$ & SD or SE & $\begin{array}{l}\text { Minimum } \\
\text { or Value } \\
\text { for } C D\end{array}$ & $\begin{array}{l}\text { Maximum } \\
\text { or } \\
\text { Percentile } \\
\text { for } C D\end{array}$ & DTN/Reference \\
\hline \multirow{11}{*}{$\begin{array}{l}\text { Soil-to-plant transfer factor } \\
\text { for leafy vegetables }\end{array}$} & lodine & \multirow{11}{*}{ Lognormal $^{a}$} & \multirow{11}{*}{$\begin{array}{c}\left(\mathrm{Bq} / \mathrm{kg}_{\text {plant }}\right) / \\
\left(\mathrm{Bq} / \mathrm{kg}_{\text {soil }}\right)\end{array}$} & 2.6E-02 & 9.9 & $7.2 \mathrm{E}-05$ & $9.7 \mathrm{E}+00$ & \multirow{11}{*}{$\begin{array}{l}\text { MO0406SPAETPBM.002 } \\
\text { [DIRS 170150] }\end{array}$} \\
\hline & Cesium & & & $8.5 E-02$ & 2.5 & 7.7E-03 & $9.4 \mathrm{E}-01$ & \\
\hline & Lead & & & $1.5 \mathrm{E}-02$ & 4.6 & $3.0 \mathrm{E}-04$ & 7.7E-01 & \\
\hline & Radium & & & $6.8 \mathrm{E}-02$ & 2.7 & $5.1 \mathrm{E}-03$ & $9.2 \mathrm{E}-01$ & \\
\hline & Actinium & & & 4.3E-03 & 2.0 & $7.2 \mathrm{E}-04$ & $2.6 \mathrm{E}-02$ & \\
\hline & Thorium & & & $4.3 \mathrm{E}-03$ & 2.8 & $3.2 \mathrm{E}-04$ & $5.9 E-02$ & \\
\hline & Protactinium & & & 4.6E-03 & 3.8 & $1.4 \mathrm{E}-04$ & $1.4 \mathrm{E}-01$ & \\
\hline & Uranium & & & 1.1E-02 & 2.0 & $1.8 \mathrm{E}-03$ & $6.6 \mathrm{E}-02$ & \\
\hline & Neptunium & & & $5.9 \mathrm{E}-02$ & 4.4 & 1.3E-03 & $2.6 \mathrm{E}+00$ & \\
\hline & Plutonium & & & $2.9 \mathrm{E}-04$ & 2.0 & $4.9 \mathrm{E}-05$ & $1.7 \mathrm{E}-03$ & \\
\hline & Americium & & & $1.2 \mathrm{E}-03$ & 2.5 & $1.2 \mathrm{E}-04$ & $1.3 \mathrm{E}-02$ & \\
\hline \multirow{16}{*}{$\begin{array}{l}\text { Soil-to-plant transfer factor } \\
\text { for other vegetables }\end{array}$} & Chlorine & \multirow{16}{*}{ Lognormal $^{a}$} & \multirow{16}{*}{$\begin{array}{l}\left(\mathrm{Bq} / \mathrm{kg}_{\text {plant }}\right) / \\
\left(\mathrm{Bq} / \mathrm{kg}_{\text {soil }}\right)\end{array}$} & $6.4 \mathrm{E}+01$ & 2.0 & $1.1 \mathrm{E}+01$ & $3.8 \mathrm{E}+02$ & \multirow{16}{*}{$\begin{array}{l}\text { MO0406SPAETPBM.002 } \\
\text { [DIRS 170150] }\end{array}$} \\
\hline & Selenium & & & 4.6E-02 & 3.8 & $1.4 \mathrm{E}-03$ & $1.4 \mathrm{E}+00$ & \\
\hline & Strontium & & & $7.9 \mathrm{E}-01$ & 2.0 & $1.4 \mathrm{E}-01$ & $4.5 \mathrm{E}+00$ & \\
\hline & Technetium & & & $4.4 \mathrm{E}+00$ & 3.7 & $1.5 \mathrm{E}-01$ & $1.2 \mathrm{E}+02$ & \\
\hline & Tin & & & $1.5 \mathrm{E}-02$ & 3.6 & $5.3 E-04$ & $4.0 \mathrm{E}-01$ & \\
\hline & lodine & & & $3.2 \mathrm{E}-02$ & 4.4 & $7.0 \mathrm{E}-04$ & $1.5 \mathrm{E}+00$ & \\
\hline & Cesium & & & $5.0 \mathrm{E}-02$ & 2.0 & $8.4 \mathrm{E}-03$ & $3.0 \mathrm{E}-01$ & \\
\hline & Lead & & & $9.0 \mathrm{E}-03$ & 3.1 & $5.0 \mathrm{E}-04$ & $1.6 \mathrm{E}-01$ & \\
\hline & Radium & & & 1.2E-02 & 5.3 & 1.6E-04 & 8.6E-01 & \\
\hline & Actinium & & & $1.1 \mathrm{E}-03$ & 4.9 & $1.8 \mathrm{E}-05$ & $6.6 \mathrm{E}-02$ & \\
\hline & Thorium & & & 4.4E-04 & 5.6 & $5.3 E-06$ & $3.6 \mathrm{E}-02$ & \\
\hline & Protactinium & & & 1.1E-03 & 10.0 & $3.0 \mathrm{E}-06$ & 4.3E-01 & \\
\hline & Uranium & & & $6.0 \mathrm{E}-03$ & 2.8 & $4.2 \mathrm{E}-04$ & $8.5 E-02$ & \\
\hline & Neptunium & & & $3.1 \mathrm{E}-02$ & 4.9 & $5.0 \mathrm{E}-04$ & $1.9 \mathrm{E}+00$ & \\
\hline & Plutonium & & & $1.9 \mathrm{E}-04$ & 2.0 & $3.3 E-05$ & $1.1 \mathrm{E}-03$ & \\
\hline & Americium & & & $4.0 \mathrm{E}-04$ & 2.6 & $3.5 \mathrm{E}-05$ & $4.6 \mathrm{E}-03$ & \\
\hline \multirow{4}{*}{$\begin{array}{l}\text { Soil-to-plant transfer factor } \\
\text { for fruit }\end{array}$} & Chlorine & \multirow{4}{*}{ Lognormal $^{a}$} & \multirow{4}{*}{$\begin{array}{c}\left(\mathrm{Bq} / \mathrm{kg}_{\text {plant }}\right) / \\
\left(\mathrm{Bq} / \mathrm{kg}_{\text {soil }}\right)\end{array}$} & $6.4 \mathrm{E}+01$ & 2.0 & $1.1 \mathrm{E}+01$ & $3.8 \mathrm{E}+02$ & \multirow{4}{*}{$\begin{array}{l}\text { MO0406SPAETPBM.002 } \\
\text { [DIRS 170150] }\end{array}$} \\
\hline & Selenium & & & 4.6E-02 & 3.8 & $1.4 \mathrm{E}-03$ & $1.4 \mathrm{E}+00$ & \\
\hline & Strontium & & & $2.9 \mathrm{E}-01$ & 2.3 & $3.6 \mathrm{E}-02$ & $2.4 \mathrm{E}+00$ & \\
\hline & Technetium & & & $4.3 E+00$ & 4.6 & 8.7E-02 & $2.1 \mathrm{E}+02$ & \\
\hline
\end{tabular}




\begin{tabular}{|c|c|c|c|c|c|c|c|c|}
\hline \multicolumn{2}{|c|}{ Parameter Name } & $\begin{array}{c}\text { Distribution } \\
\text { Type }\end{array}$ & Units & $\begin{array}{c}\text { Mean, } \\
\text { Mode } \\
\text { or Fixed } \\
\text { Value }\end{array}$ & SD or SE & $\begin{array}{l}\text { Minimum } \\
\text { or Value } \\
\text { for CD }\end{array}$ & $\begin{array}{l}\text { Maximum } \\
\text { or } \\
\text { Percentile } \\
\text { for CD } \\
\end{array}$ & DTN/Reference \\
\hline \multirow{12}{*}{$\begin{array}{l}\text { Soil-to-plant transfer factor } \\
\text { for fruit }\end{array}$} & Tin & \multirow{12}{*}{ Lognormal $^{a}$} & \multirow{12}{*}{$\begin{array}{l}\left(\mathrm{Bq} / \mathrm{kg}_{\text {plant }}\right) / \\
\left(\mathrm{Bq} / \mathrm{kg}_{\text {soil }}\right)\end{array}$} & $1.5 \mathrm{E}-02$ & 3.6 & $5.3 \mathrm{E}-04$ & 4.0E-01 & \multirow{12}{*}{$\begin{array}{l}\text { MO0406SPAETPBM.002 } \\
\text { [DIRS 170150] }\end{array}$} \\
\hline & lodine & & & $5.7 \mathrm{E}-02$ & 2.8 & $4.1 \mathrm{E}-03$ & $7.9 \mathrm{E}-01$ & \\
\hline & Cesium & & & $5.6 \mathrm{E}-02$ & 2.8 & $3.8 \mathrm{E}-03$ & 8.1E-01 & \\
\hline & Lead & & & $1.2 \mathrm{E}-02$ & 3.3 & $5.8 \mathrm{E}-04$ & $2.6 \mathrm{E}-01$ & \\
\hline & Radium & & & $7.3 \mathrm{E}-03$ & 4.3 & $1.6 \mathrm{E}-04$ & $3.2 \mathrm{E}-01$ & \\
\hline & Actinium & & & $8.5 \mathrm{E}-04$ & 3.4 & 3.7E-05 & $2.0 \mathrm{E}-02$ & \\
\hline & Thorium & & & $2.9 \mathrm{E}-04$ & 4.9 & $4.8 \mathrm{E}-06$ & 1.7E-02 & \\
\hline & Protactinium & & & $1.1 \mathrm{E}-03$ & 10.0 & $3.0 \mathrm{E}-06$ & 4.3E-01 & \\
\hline & Uranium & & & $6.3 \mathrm{E}-03$ & 2.9 & $3.9 \mathrm{E}-04$ & 1.0E-01 & \\
\hline & Neptunium & & & $3.4 \mathrm{E}-02$ & 6.9 & $2.3 \mathrm{E}-04$ & $5.0 \mathrm{E}+00$ & \\
\hline & Plutonium & & & $1.8 \mathrm{E}-04$ & 3.4 & $7.8 \mathrm{E}-06$ & $4.2 \mathrm{E}-03$ & \\
\hline & Americium & & & $5.4 \mathrm{E}-04$ & 2.3 & $6.5 \mathrm{E}-05$ & $4.5 \mathrm{E}-03$ & \\
\hline \multirow{16}{*}{$\begin{array}{l}\text { Soil-to-plant transfer factor } \\
\text { for grain }\end{array}$} & Chlorine & \multirow{16}{*}{ Lognormal $^{a}$} & \multirow{16}{*}{$\begin{array}{l}\left(\mathrm{Bq} / \mathrm{kg}_{\text {plant }}\right) / \\
\left(\mathrm{Bq} / \mathrm{kg}_{\text {soil }}\right)\end{array}$} & $2.4 \mathrm{E}+01$ & 8.4 & $1.0 \mathrm{E}-01$ & $5.8 \mathrm{E}+03$ & \multirow{16}{*}{$\begin{array}{l}\text { MO0406SPAETPBM.002 } \\
\text { [DIRS 170150] }\end{array}$} \\
\hline & Selenium & & & $2.9 \mathrm{E}-02$ & 2.0 & $4.8 \mathrm{E}-03$ & 1.7E-01 & \\
\hline & Strontium & & & 1.7E-01 & 2.0 & $2.8 \mathrm{E}-02$ & $1.0 \mathrm{E}+00$ & \\
\hline & Technetium & & & $1.6 \mathrm{E}+00$ & 4.3 & $3.8 \mathrm{E}-02$ & $6.8 \mathrm{E}+01$ & \\
\hline & Tin & & & $9.2 \mathrm{E}-03$ & 2.0 & $1.5 \mathrm{E}-03$ & $5.5 \mathrm{E}-02$ & \\
\hline & lodine & & & $2.5 \mathrm{E}-02$ & 10.0 & $6.6 \mathrm{E}-05$ & $9.4 \mathrm{E}+00$ & \\
\hline & Cesium & & & $2.0 \mathrm{E}-02$ & 2.2 & $2.7 \mathrm{E}-03$ & $1.6 \mathrm{E}-01$ & \\
\hline & Lead & & & $5.5 \mathrm{E}-03$ & 2.1 & $8.2 \mathrm{E}-04$ & $3.8 \mathrm{E}-02$ & \\
\hline & Radium & & & $3.1 \mathrm{E}-03$ & 4.0 & $8.8 \mathrm{E}-05$ & $1.1 \mathrm{E}-01$ & \\
\hline & Actinium & & & $5.4 \mathrm{E}-04$ & 2.9 & $3.6 \mathrm{E}-05$ & $8.0 \mathrm{E}-03$ & \\
\hline & Thorium & & & $1.7 \mathrm{E}-04$ & 5.2 & $2.4 \mathrm{E}-06$ & $1.2 \mathrm{E}-02$ & \\
\hline & Protactinium & & & $9.5 \mathrm{E}-04$ & 7.2 & $5.9 \mathrm{E}-06$ & $1.5 \mathrm{E}-01$ & \\
\hline & Uranium & & & $1.1 \mathrm{E}-03$ & 3.6 & 4.1E-05 & $3.1 \mathrm{E}-02$ & \\
\hline & Neptunium & & & $4.4 \mathrm{E}-03$ & 6.9 & $3.1 \mathrm{E}-05$ & $6.3 \mathrm{E}-01$ & \\
\hline & Plutonium & & & $1.9 \mathrm{E}-05$ & 4.2 & $4.8 \mathrm{E}-07$ & $7.8 \mathrm{E}-04$ & \\
\hline & Americium & & & $7.5 \mathrm{E}-05$ & 3.2 & $3.8 \mathrm{E}-06$ & $1.5 \mathrm{E}-03$ & \\
\hline \multirow{3}{*}{$\begin{array}{l}\text { Soil-to-plant transfer factor } \\
\text { for forage crops }\end{array}$} & Chlorine & \multirow{3}{*}{ Lognormal $^{a}$} & \multirow{3}{*}{$\begin{array}{l}\left(\mathrm{Bq} / \mathrm{kg}_{\text {plant }}\right) / \\
\left(\mathrm{Bq} / \mathrm{kg}_{\text {soil }}\right)\end{array}$} & $7.5 \mathrm{E}+01$ & 2.0 & $1.3 \mathrm{E}+01$ & $4.5 \mathrm{E}+02$ & \multirow{3}{*}{$\begin{array}{l}\text { MO0406SPAETPBM.002 } \\
\text { [DIRS 170150] }\end{array}$} \\
\hline & Selenium & & & $1.5 \mathrm{E}-01$ & 5.5 & $1.9 \mathrm{E}-03$ & $1.3 \mathrm{E}+01$ & \\
\hline & Strontium & & & $2.1 \mathrm{E}+00$ & 2.1 & $3.2 \mathrm{E}-01$ & $1.3 \mathrm{E}+01$ & \\
\hline
\end{tabular}


Table 4.1-1. Summary of Input Parameter Values and Their Uncertainty Distributions (Continued)

\begin{tabular}{|c|c|c|c|c|c|c|c|c|}
\hline \multicolumn{2}{|c|}{ Parameter Name } & $\begin{array}{l}\text { Distribution } \\
\text { Type }\end{array}$ & Units & $\begin{array}{l}\text { Mean, } \\
\text { Mode } \\
\text { or Fixed } \\
\text { Value }\end{array}$ & SD or SE & $\begin{array}{l}\text { Minimum } \\
\text { or Value } \\
\text { for CD }\end{array}$ & $\begin{array}{l}\text { Maximum } \\
\text { or } \\
\text { Percentile } \\
\text { for } C D\end{array}$ & DTN/Reference \\
\hline \multirow{13}{*}{$\begin{array}{l}\text { Soil-to-plant transfer factor } \\
\text { for forage crops }\end{array}$} & Technetium & \multirow{13}{*}{ Lognormal $^{\mathrm{a}}$} & \multirow{13}{*}{$\begin{array}{l}\left(\mathrm{Bq} / \mathrm{kg}_{\text {plant }}\right) / \\
\left(\mathrm{Bq} / \mathrm{kg}_{\text {soil }}\right)\end{array}$} & $2.7 E+01$ & 2.7 & $2.1 \mathrm{E}+00$ & $3.5 \mathrm{E}+02$ & \multirow{13}{*}{$\begin{array}{l}\text { MO0406SPAETPBM.002 } \\
\text { [DIRS 170150] }\end{array}$} \\
\hline & Tin & & & 1.6E-01 & 5.8 & 1.7E-03 & $1.5 \mathrm{E}+01$ & \\
\hline & lodine & & & $4.0 \mathrm{E}-02$ & 10.0 & $1.1 \mathrm{E}-04$ & $1.5 \mathrm{E}+01$ & \\
\hline & Cesium & & & $1.3 \mathrm{E}-01$ & 3.3 & $6.3 E-03$ & $2.8 \mathrm{E}+00$ & \\
\hline & Lead & & & $1.8 \mathrm{E}-02$ & 7.0 & $1.2 \mathrm{E}-04$ & $2.8 \mathrm{E}+00$ & \\
\hline & Radium & & & $8.2 \mathrm{E}-02$ & 3.0 & $4.9 E-03$ & $1.4 \mathrm{E}+00$ & \\
\hline & Actinium & & & 1.7E-02 & 5.4 & $2.2 \mathrm{E}-04$ & $1.3 E+00$ & \\
\hline & Thorium & & & $1.0 \mathrm{E}-02$ & 4.2 & $2.5 \mathrm{E}-04$ & $3.9 \mathrm{E}-01$ & \\
\hline & Protactinium & & & $1.9 E-02$ & 6.7 & $1.4 \mathrm{E}-04$ & $2.5 E+00$ & \\
\hline & Uranium & & & $1.7 \mathrm{E}-02$ & 6.1 & 1.6E-04 & $1.9 \mathrm{E}+00$ & \\
\hline & Neptunium & & & $5.8 E-02$ & 5.6 & $6.8 \mathrm{E}-04$ & $4.9 \mathrm{E}+00$ & \\
\hline & Plutonium & & & $1.0 \mathrm{E}-03$ & 10.0 & $2.7 E-06$ & $3.9 \mathrm{E}-01$ & \\
\hline & Americium & & & $2.1 \mathrm{E}-03$ & 10.0 & $5.5 \mathrm{E}-06$ & $7.9 \mathrm{E}-01$ & \\
\hline \multicolumn{2}{|c|}{$\begin{array}{l}\text { Correlation coefficient for transfer factors and } \\
\text { solid-liquid partition coefficients }\end{array}$} & Fixed & - & -0.8 & - & - & - & $\begin{array}{l}\text { MO0406SPAETPBM.002 } \\
\text { [DIRS 170150] }\end{array}$ \\
\hline \multirow{2}{*}{ Dry-to-wet weight ratio } & Leafy vegetables & Cumulative & $\mathrm{kg}_{\text {dry }} / \mathrm{kg}_{\text {wet }}$ & - & - & $\begin{array}{l}0.041 \\
0.054 \\
0.060 \\
0.078 \\
0.081 \\
0.084 \\
0.093\end{array}$ & $\begin{array}{c}0 \% \\
17 \% \\
33 \% \\
50 \% \\
67 \% \\
83 \% \\
100 \% \\
\end{array}$ & \multirow[t]{2}{*}{$\begin{array}{l}\text { MO0403SPAAEIBM.002 } \\
\text { [DIRS 169392] }\end{array}$} \\
\hline & Other vegetables & Cumulative & $\mathrm{kg}_{\text {dry }} / \mathrm{kg}_{\text {wet }}$ & - & - & $\begin{array}{c}0.035 \\
0.063 \\
0.078 \\
0.08 \\
0.103 \\
0.122 \\
0.240\end{array}$ & $\begin{array}{c}0 \% \\
17 \% \\
33 \% \\
50 \% \\
67 \% \\
83 \% \\
100 \%\end{array}$ & \\
\hline
\end{tabular}




\begin{tabular}{|c|c|c|c|c|c|c|c|c|c|}
\hline \multicolumn{3}{|c|}{ Parameter Name } & $\begin{array}{l}\text { Distribution } \\
\text { Type }\end{array}$ & Units & $\begin{array}{c}\text { Mean, } \\
\text { Mode } \\
\text { or Fixed } \\
\text { Value } \\
\end{array}$ & SD or SE & $\begin{array}{l}\text { Minimum } \\
\text { or Value } \\
\text { for } C D\end{array}$ & $\begin{array}{l}\text { Maximum } \\
\text { or } \\
\text { Percentile } \\
\text { for CD } \\
\end{array}$ & DTN/Reference \\
\hline \multirow{3}{*}{\multicolumn{2}{|c|}{ Dry-to-wet weight ratio }} & Fruit & Cumulative & $\mathrm{kg}_{\text {dry }} / \mathrm{kg}_{\text {wet }}$ & - & - & $\begin{array}{l}0.062 \\
0.084 \\
0.102 \\
0.155 \\
0.194 \\
\end{array}$ & $\begin{array}{c}0 \% \\
25 \% \\
50 \% \\
75 \% \\
100 \% \\
\end{array}$ & \multirow[t]{3}{*}{$\begin{array}{l}\text { MO0403SPAAEIBM.002 } \\
\text { [DIRS 169392] }\end{array}$} \\
\hline & & Grain & Cumulative & $\mathrm{kg}_{\text {dry }} / \mathrm{kg}_{\text {wet }}$ & - & - & $\begin{array}{l}0.891 \\
0.896 \\
0.906 \\
0.918\end{array}$ & $\begin{array}{c}0 \% \\
33 \% \\
67 \% \\
100 \%\end{array}$ & \\
\hline & & Forage & Cumulative & $\mathrm{kg}_{\text {dry }} / \mathrm{kg}_{\text {wet }}$ & - & - & $\begin{array}{l}0.182 \\
0.227 \\
0.238 \\
\end{array}$ & $\begin{array}{c}0 \% \\
75 \% \\
100 \% \\
\end{array}$ & \\
\hline \multirow{5}{*}{\multicolumn{2}{|c|}{ Translocation factor }} & Leafy vegetables & Fixed & - & 1.0 & - & - & - & \multirow{5}{*}{$\begin{array}{l}\text { MO0406SPAETPBM.002 } \\
\text { [DIRS 170150] }\end{array}$} \\
\hline & & Other vegetables & \multirow{3}{*}{ Cumulative } & \multirow{3}{*}{-} & \multirow{3}{*}{-} & \multirow{3}{*}{-} & 0.05 & $0 \%$ & \\
\hline & & Fruit & & & & & 0.10 & $50 \%$ & \\
\hline & & Grain & & & & & 0.30 & $100 \%$ & \\
\hline & & Forage & Fixed & - & 1.0 & - & - & - & \\
\hline \multirow{5}{*}{\multicolumn{2}{|c|}{$\begin{array}{l}\text { Fraction of overhead } \\
\text { irrigation }\end{array}$}} & Leafy vegetables & \multirow{5}{*}{ Normal } & \multirow{5}{*}{-} & 0.75 & 0.10 & 0.49 & 1.0 & \multirow{5}{*}{$\begin{array}{l}\text { MO0403SPAAEIBM.002 } \\
\text { [DIRS 169392] }\end{array}$} \\
\hline & & Other vegetables & & & 0.75 & 0.10 & 0.49 & 1.0 & \\
\hline & & Fruit & & & 0.50 & 0.10 & 0.24 & 1.0 & \\
\hline & & Grain & & & 0.90 & 0.05 & 0.77 & 1.0 & \\
\hline & & Forage & & & 0.90 & 0.05 & 0.77 & 1.0 & \\
\hline \multicolumn{3}{|c|}{ Weathering half-life } & Cumulative & day & - & - & $\begin{array}{c}5 \\
14 \\
30\end{array}$ & $\begin{array}{c}0 \% \\
50 \% \\
100 \%\end{array}$ & $\begin{array}{l}\text { MO0406SPAETPBM.002 } \\
\text { [DIRS 170150] }\end{array}$ \\
\hline \multirow{5}{*}{$\begin{array}{l}\text { Crop } \\
\text { growing } \\
\text { time }\end{array}$} & \multirow{5}{*}{$\begin{array}{l}\text { Present-day } \\
\text { climate }\end{array}$} & Leafy vegetables & \multirow{5}{*}{ Fixed } & \multirow{5}{*}{ day } & 75 & - & - & - & \multirow{5}{*}{$\begin{array}{l}\text { MO0403SPAAEIBM.002 } \\
\text { [DIRS 169392] }\end{array}$} \\
\hline & & Other vegetables & & & 80 & - & - & - & \\
\hline & & Fruit & & & 160 & - & - & - & \\
\hline & & Grain & & & 200 & - & - & - & \\
\hline & & Forage & & & 75 & - & - & - & \\
\hline
\end{tabular}


Table 4.1-1. Summary of Input Parameter Values and Their Uncertainty Distributions (Continued)

\begin{tabular}{|c|c|c|c|c|c|c|c|c|c|}
\hline \multicolumn{3}{|c|}{ Parameter Name } & $\begin{array}{c}\text { Distribution } \\
\text { Type }\end{array}$ & Units & $\begin{array}{c}\text { Mean, } \\
\text { Mode } \\
\text { or Fixed } \\
\text { Value } \\
\end{array}$ & SD or SE & $\begin{array}{c}\text { Minimum } \\
\text { or Value } \\
\text { for } C D\end{array}$ & $\begin{array}{c}\text { Maximum } \\
\text { or } \\
\text { Percentile } \\
\text { for CD }\end{array}$ & DTN/Reference \\
\hline \multirow{5}{*}{$\begin{array}{l}\text { Crop } \\
\text { growing } \\
\text { time }\end{array}$} & \multirow{5}{*}{$\begin{array}{l}\text { Future } \\
\text { climate }\end{array}$} & Leafy vegetables & \multirow{5}{*}{ Fixed } & \multirow{5}{*}{ day } & 75 & - & - & - & \multirow{5}{*}{$\begin{array}{l}\text { MO0403SPAAEIBM.002 } \\
\text { [DIRS 169392] }\end{array}$} \\
\hline & & Other vegetables & & & 100 & - & - & - & \\
\hline & & Fruit & & & 105 & - & - & - & \\
\hline & & Grain & & & 185 & - & - & - & \\
\hline & & Forage & & & 90 & - & - & - & \\
\hline \multirow{3}{*}{ Crop we } & & Leafy vegetables & Cumulative & $\mathrm{kg} / \mathrm{m}^{2}$ & - & - & $\begin{array}{l}1.08 \\
1.46 \\
1.78 \\
2.01 \\
2.98 \\
3.25 \\
3.83 \\
7.79 \\
7.85 \\
\end{array}$ & $\begin{array}{c}0 \% \\
5 \% \\
20 \% \\
35 \% \\
50 \% \\
65 \% \\
80 \% \\
95 \% \\
100 \% \\
\end{array}$ & \multirow[t]{3}{*}{$\begin{array}{l}\text { MO0403SPAAEIBM.002 } \\
\text { [DIRS 169392] }\end{array}$} \\
\hline & & Other vegetables & Cumulative & $\mathrm{kg} / \mathrm{m}^{2}$ & - & - & $\begin{array}{c}2.8 \\
3.37 \\
3.56 \\
3.64 \\
4.92 \\
5.15 \\
6.61 \\
\end{array}$ & $\begin{array}{c}0 \% \\
5 \% \\
28 \% \\
51 \% \\
72 \% \\
95 \% \\
100 \% \\
\end{array}$ & \\
\hline & & Fruit & Cumulative & $\mathrm{kg} / \mathrm{m}^{2}$ & - & - & $\begin{array}{l}0.73 \\
1.51 \\
2.67 \\
2.92 \\
3.00 \\
3.63 \\
6.89 \\
\end{array}$ & $\begin{array}{c}0 \% \\
5 \% \\
28 \% \\
51 \% \\
72 \% \\
95 \% \\
100 \% \\
\end{array}$ & \\
\hline
\end{tabular}




\begin{tabular}{|c|c|c|c|c|c|c|c|c|}
\hline \multicolumn{2}{|c|}{ Parameter Name } & $\begin{array}{l}\text { Distribution } \\
\text { Type }\end{array}$ & Units & $\begin{array}{l}\text { Mean, } \\
\text { Mode } \\
\text { or Fixed } \\
\text { Value } \\
\end{array}$ & SD or SE & $\begin{array}{l}\text { Minimum } \\
\text { or Value } \\
\text { for CD }\end{array}$ & $\begin{array}{l}\text { Maximum } \\
\text { or } \\
\text { Percentile } \\
\text { for CD }\end{array}$ & DTN/Reference \\
\hline \multirow[t]{2}{*}{ Crop wet yield } & Grain & Cumulative & $\mathrm{kg} / \mathrm{m}^{2}$ & - & - & $\begin{array}{l}0.27 \\
0.28 \\
0.44 \\
0.54 \\
1.10 \\
1.22\end{array}$ & $\begin{array}{c}5 \% \\
35 \% \\
65 \% \\
95 \% \\
100 \%\end{array}$ & [DIRS 169392] \\
\hline & Forage & Cumulative & $\mathrm{kg} / \mathrm{m}^{2}$ & - & - & $\begin{array}{l}0.69 \\
1.02 \\
1.87 \\
5.78 \\
6.28 \\
\end{array}$ & $\begin{array}{c}0 \% \\
5 \% \\
73 \% \\
95 \% \\
100 \% \\
\end{array}$ & \\
\hline \multirow[t]{2}{*}{ Crop dry biomass } & Leafy vegetables & Cumulative & $\mathrm{kg} / \mathrm{m}^{2} \mathrm{c}$ & - & - & $\begin{array}{l}0.10 \\
0.13 \\
0.14 \\
0.15 \\
0.16 \\
0.18 \\
0.30 \\
0.42 \\
0.50 \\
\end{array}$ & $\begin{array}{c}0 \% \\
5 \% \\
20 \% \\
35 \% \\
50 \% \\
65 \% \\
80 \% \\
95 \% \\
100 \% \\
\end{array}$ & $\begin{array}{l}\text { MO0403SPAAEIBM.002 } \\
\text { [DIRS 169392] }\end{array}$ \\
\hline & Other vegetables & Cumulative & $\mathrm{kg} / \mathrm{m}^{2} \mathrm{c}$ & - & - & $\begin{array}{l}0.30 \\
0.40 \\
0.41 \\
0.43 \\
0.44 \\
0.46 \\
0.60 \\
\end{array}$ & $\begin{array}{c}0 \% \\
5 \% \\
28 \% \\
51 \% \\
73 \% \\
95 \% \\
100 \%\end{array}$ & \\
\hline
\end{tabular}




\begin{tabular}{|c|c|c|c|c|c|c|c|c|}
\hline \multicolumn{2}{|c|}{ Parameter Name } & $\begin{array}{l}\text { Distribution } \\
\text { Type }\end{array}$ & Units & $\begin{array}{l}\text { Mean, } \\
\text { Mode } \\
\text { or Fixed } \\
\text { Value } \\
\end{array}$ & SD or SE & $\begin{array}{l}\text { Minimum } \\
\text { or Value } \\
\text { for CD }\end{array}$ & $\begin{array}{l}\text { Maximum } \\
\text { or } \\
\text { Percentile } \\
\text { for CD }\end{array}$ & DTN/Reference \\
\hline \multirow{3}{*}{ Crop dry biomass } & Fruit & Cumulative & $\mathrm{kg} / \mathrm{m}^{2 \mathrm{c}}$ & - & - & $\begin{array}{l}0.10 \\
0.56 \\
0.60 \\
0.65 \\
0.68 \\
1.30\end{array}$ & $\begin{array}{c}0 \% \\
5 \% \\
35 \% \\
65 \% \\
95 \% \\
100 \%\end{array}$ & $\begin{array}{l}\text { MO0403SPAAEIBM.002 } \\
\text { [DIRS 169392] }\end{array}$ \\
\hline & Grain & Cumulative & $\mathrm{kg} / \mathrm{m}^{2} \mathrm{c}$ & - & - & $\begin{array}{l}0.50 \\
0.61 \\
0.74 \\
1.20 \\
1.97 \\
2.20 \\
\end{array}$ & $\begin{array}{c}0 \% \\
5 \% \\
35 \% \\
65 \% \\
95 \% \\
100 \% \\
\end{array}$ & \\
\hline & Forage & Cumulative & $\mathrm{kg} / \mathrm{m}^{2 \mathrm{c}}$ & - & - & $\begin{array}{l}0.10 \\
0.23 \\
0.34 \\
1.38 \\
1.50 \\
\end{array}$ & $\begin{array}{c}0 \% \\
5 \% \\
73 \% \\
95 \% \\
100 \% \\
\end{array}$ & \\
\hline $\begin{array}{l}\text { Daily average irrigation } \\
\text { rate, present-day climate }\end{array}$ & Leafy vegetables & Cumulative & $\mathrm{mm} / \mathrm{day}$ & - & - & $\begin{array}{l}4.00 \\
5.11 \\
5.19 \\
5.21 \\
5.38 \\
5.48 \\
6.00 \\
7.08\end{array}$ & $\begin{array}{c}0 \% \\
5 \% \\
20 \% \\
35 \% \\
50 \% \\
80 \% \\
95 \% \\
100 \%\end{array}$ & $\begin{array}{l}\text { MO0403SPAAEIBM.002 } \\
\text { [DIRS 169392] }\end{array}$ \\
\hline
\end{tabular}




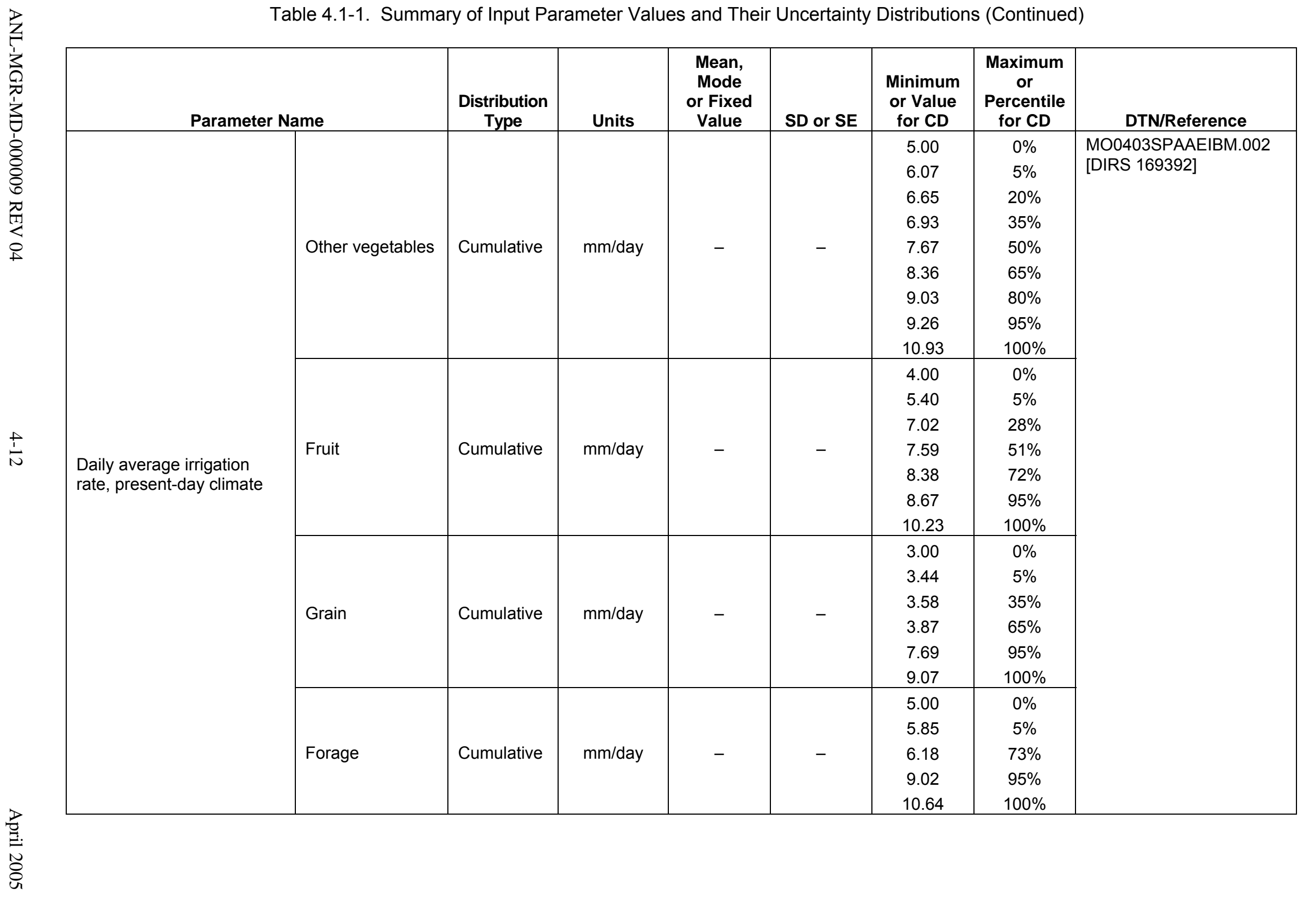




\begin{tabular}{|c|c|c|c|c|c|c|c|c|}
\hline \multicolumn{2}{|c|}{ Parameter Name } & $\begin{array}{c}\text { Distribution } \\
\text { Type }\end{array}$ & Units & $\begin{array}{c}\text { Mean, } \\
\text { Mode } \\
\text { or Fixed } \\
\text { Value }\end{array}$ & SD or SE & $\begin{array}{c}\text { Minimum } \\
\text { or Value } \\
\text { for } C D\end{array}$ & $\begin{array}{c}\text { Maximum } \\
\text { or } \\
\text { Percentile } \\
\text { for CD }\end{array}$ & DTN/Reference \\
\hline \multirow{3}{*}{$\begin{array}{l}\text { Daily average irrigation } \\
\text { rate, future climate }\end{array}$} & Leafy vegetables & Cumulative & $\mathrm{mm} /$ day & - & - & $\begin{array}{l}3.00 \\
3.34 \\
3.51 \\
3.86 \\
3.92 \\
4.02 \\
4.18 \\
4.93 \\
\end{array}$ & $\begin{array}{c}0 \% \\
5 \% \\
20 \% \\
50 \% \\
65 \% \\
80 \% \\
95 \% \\
100 \%\end{array}$ & $\begin{array}{l}\text { MO0403SPAAEIBM.002 } \\
\text { [DIRS 169392] }\end{array}$ \\
\hline & Other vegetables & Cumulative & $\mathrm{mm} /$ day & - & - & $\begin{array}{l}2.00 \\
2.73 \\
3.08 \\
3.48 \\
4.08 \\
4.16 \\
4.43 \\
4.95 \\
5.84\end{array}$ & $\begin{array}{c}0 \% \\
5 \% \\
20 \% \\
35 \% \\
50 \% \\
65 \% \\
80 \% \\
95 \% \\
100 \%\end{array}$ & \\
\hline & Grain & Cumulative & $\mathrm{mm} /$ day & - & - & $\begin{array}{l}2.00 \\
2.51 \\
3.48 \\
4.33 \\
4.38 \\
4.79 \\
5.65 \\
1.00 \\
1.99 \\
3.42 \\
3.93 \\
4.11 \\
4.85\end{array}$ & $\begin{array}{c}0 \% \\
5 \% \\
28 \% \\
51 \% \\
72 \% \\
95 \% \\
100 \% \\
0 \% \\
5 \% \\
35 \% \\
65 \% \\
95 \% \\
100 \%\end{array}$ & \\
\hline
\end{tabular}




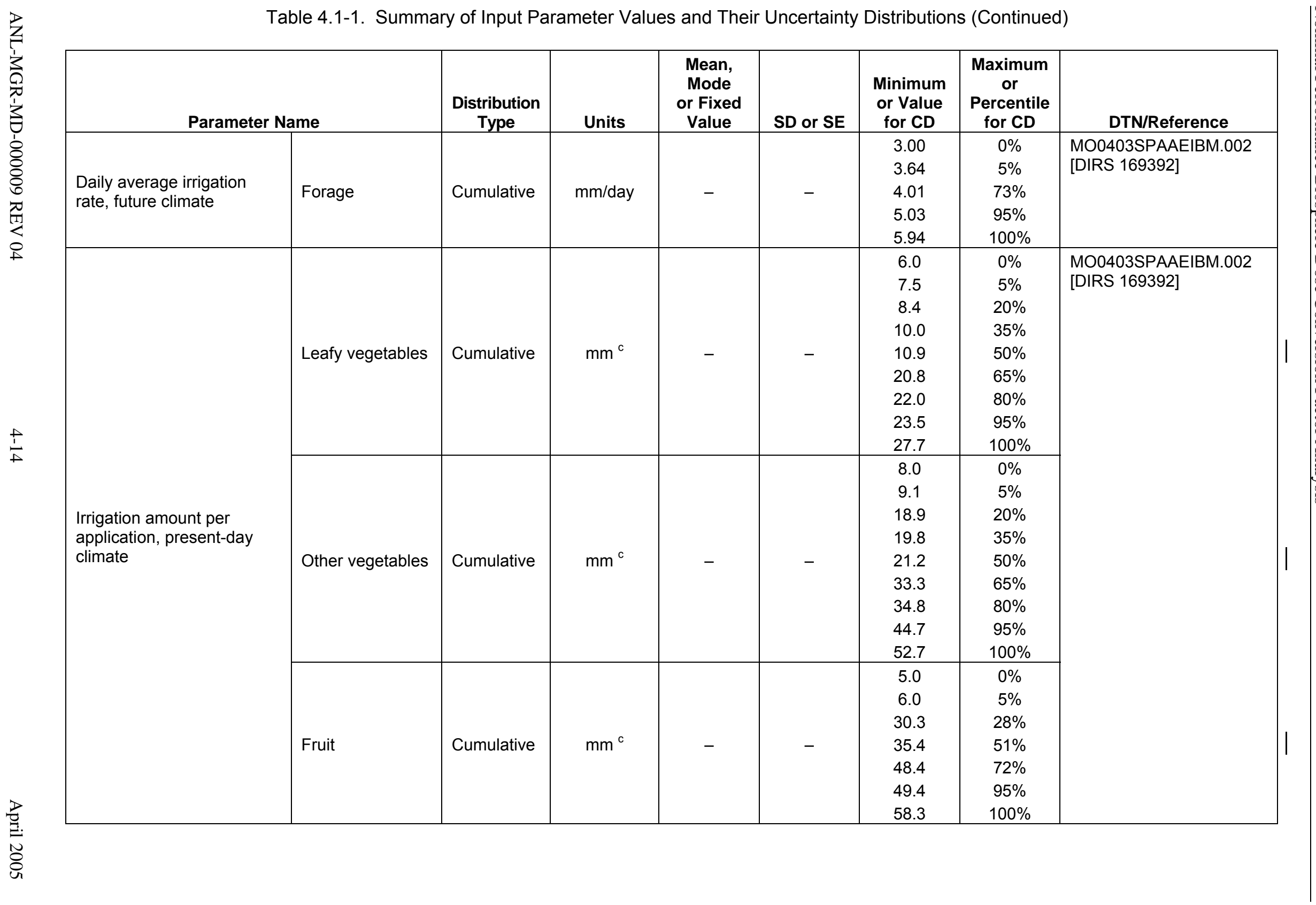


Table 4.1-1. Summary of Input Parameter Values and Their Uncertainty Distributions (Continued)

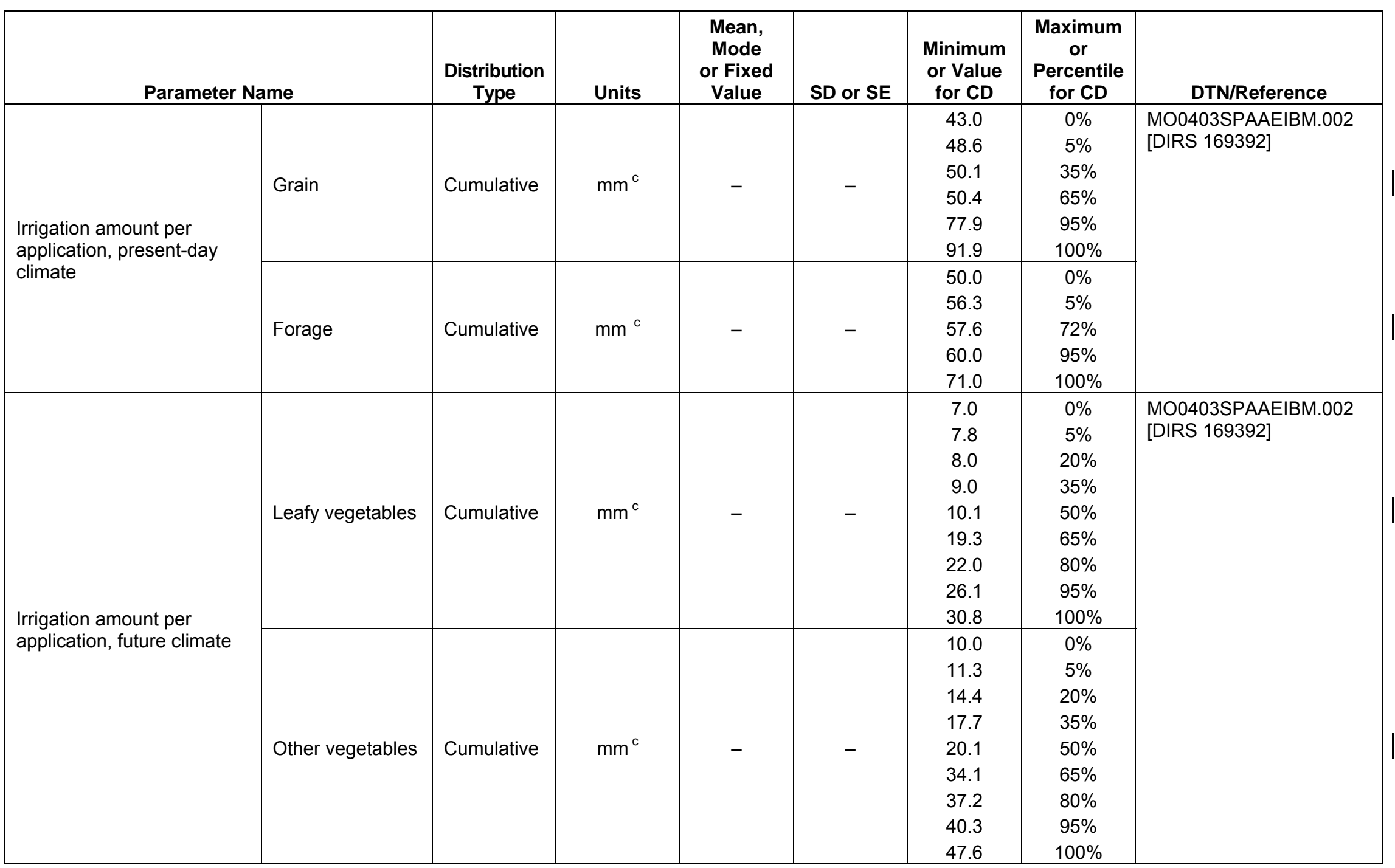




\begin{tabular}{|c|c|c|c|c|c|c|c|c|}
\hline \multicolumn{2}{|c|}{ Parameter Name } & $\begin{array}{l}\text { Distribution } \\
\text { Type }\end{array}$ & Units & $\begin{array}{c}\text { Mean, } \\
\text { Mode } \\
\text { or Fixed } \\
\text { Value }\end{array}$ & SD or SE & $\begin{array}{c}\text { Minimum } \\
\text { or Value } \\
\text { for } C D\end{array}$ & $\begin{array}{l}\text { Maximum } \\
\text { or } \\
\text { Percentile } \\
\text { for CD } \\
\end{array}$ & DTN/Reference \\
\hline \multirow{3}{*}{$\begin{array}{l}\text { Irrigation amount per } \\
\text { application, future climate }\end{array}$} & Fruit & Cumulative & $\mathrm{mm}^{\mathrm{c}}$ & - & - & $\begin{array}{c}7.3 \\
31.4 \\
34.6 \\
43.2 \\
54.4 \\
64.2\end{array}$ & $\begin{array}{c}5 \% \\
28 \% \\
51 \% \\
72 \% \\
95 \% \\
100 \%\end{array}$ & \multirow{3}{*}{$\begin{array}{l}\text { MO0403SPAAEIBM.002 } \\
\text { [DIRS 169392] }\end{array}$} \\
\hline & Grain & Cumulative & $\mathrm{mm}^{\mathrm{c}}$ & - & - & $\begin{array}{l}28.0 \\
32.2 \\
46.2 \\
59.9 \\
66.7 \\
78.7 \\
\end{array}$ & $\begin{array}{c}0 \% \\
5 \% \\
35 \% \\
65 \% \\
95 \% \\
100 \% \\
\end{array}$ & \\
\hline & Forage & Cumulative & $\mathrm{mm}^{\mathrm{c}}$ & - & - & $\begin{array}{l}43.0 \\
48.3 \\
52.5 \\
61.9 \\
73.0 \\
\end{array}$ & $\begin{array}{c}0 \% \\
5 \% \\
73 \% \\
95 \% \\
100 \% \\
\end{array}$ & \\
\hline \multicolumn{2}{|l|}{ Irrigation intensity } & Uniform & $\mathrm{cm} / \mathrm{hr}^{\mathrm{c}}$ & - & - & 1.0 & 7.5 & $\begin{array}{l}\text { MO0403SPAAEIBM.002 } \\
\text { [DIRS 169392] }\end{array}$ \\
\hline \multicolumn{2}{|l|}{ Dry deposition velocity } & Cumulative & $\mathrm{m} / \mathrm{s}$ & - & - & $\begin{array}{l}3 E-4 \\
1 E-3 \\
8 E-3 \\
3 E-2 \\
3 E-1\end{array}$ & $\begin{array}{c}0 \% \\
16 \% \\
50 \% \\
84 \% \\
100 \%\end{array}$ & $\begin{array}{l}\text { MO0406SPAETPBM.002 } \\
\text { [DIRS 170150] }\end{array}$ \\
\hline
\end{tabular}


Table 4.1-1. Summary of Input Parameter Values and Their Uncertainty Distributions (Continued)

\begin{tabular}{|c|c|c|c|c|c|c|c|c|}
\hline \multicolumn{2}{|c|}{ Parameter Name } & \multicolumn{2}{|l|}{$\begin{array}{c}\text { Distribution } \\
\text { Type }\end{array}$} & $\begin{array}{l}\text { Mean, } \\
\text { Mode } \\
\text { or Fixed } \\
\text { Value }\end{array}$ & \multirow{2}{*}{$\frac{\text { SD or SE }}{\text { I O DE L }}$} & \multirow[t]{2}{*}{$\begin{array}{c}\text { Minimum } \\
\text { or Value } \\
\text { for CD }\end{array}$} & \multirow[t]{2}{*}{$\begin{array}{l}\text { Maximum } \\
\text { or } \\
\text { Percentile } \\
\text { for CD }\end{array}$} & \multirow[t]{2}{*}{ DTN/Reference } \\
\hline & & A N I & $A L U P$ & KESUE & & & & \\
\hline \multirow{16}{*}{$\begin{array}{l}\text { Animal product transfer } \\
\text { coefficients for meat }\end{array}$} & Chlorine & \multirow{16}{*}{ Lognormal $^{\mathrm{a}}$} & \multirow{16}{*}{ day $/ \mathrm{kg}$} & $4.6 \mathrm{E}-02$ & 2.0 & 7.7E-03 & $2.7 \mathrm{E}-01$ & \multirow{16}{*}{$\begin{array}{l}\text { MO0406SPAETPBM.002 } \\
\text { [DIRS 170150] }\end{array}$} \\
\hline & Selenium & & & $8.8 \mathrm{E}-02$ & 5.8 & $9.6 \mathrm{E}-04$ & $8.0 \mathrm{E}+00$ & \\
\hline & Strontium & & & $1.4 \mathrm{E}-03$ & 4.4 & $3.1 \mathrm{E}-05$ & $6.2 \mathrm{E}-02$ & \\
\hline & Technetium & & & $1.1 \mathrm{E}-03$ & 7.2 & $6.9 \mathrm{E}-06$ & $1.8 \mathrm{E}-01$ & \\
\hline & Tin & & & $1.9 \mathrm{E}-02$ & 4.6 & $3.8 \mathrm{E}-04$ & 9.9E-01 & \\
\hline & lodine & & & $1.0 \mathrm{E}-02$ & 2.8 & $6.8 \mathrm{E}-04$ & $1.5 \mathrm{E}-01$ & \\
\hline & Cesium & & & $2.4 \mathrm{E}-02$ & 2.6 & $2.1 \mathrm{E}-03$ & $2.7 \mathrm{E}-01$ & \\
\hline & Lead & & & $6.3 E-04$ & 2.6 & $5.4 \mathrm{E}-05$ & $7.5 \mathrm{E}-03$ & \\
\hline & Radium & & & $8.1 \mathrm{E}-04$ & 2.1 & $1.1 \mathrm{E}-04$ & 5.7E-03 & \\
\hline & Actinium & & & $7.9 \mathrm{E}-05$ & 8.2 & $3.5 \mathrm{E}-07$ & $1.8 \mathrm{E}-02$ & \\
\hline & Thorium & & & $1.1 \mathrm{E}-04$ & 10.0 & $2.8 \mathrm{E}-07$ & $4.0 \mathrm{E}-02$ & \\
\hline & Protactinium & & & $6.6 \mathrm{E}-05$ & 10.0 & $1.8 \mathrm{E}-07$ & $2.5 \mathrm{E}-02$ & \\
\hline & Uranium & & & $4.8 \mathrm{E}-04$ & 3.0 & $2.9 \mathrm{E}-05$ & $7.8 \mathrm{E}-03$ & \\
\hline & Neptunium & & & $3.4 \mathrm{E}-04$ & 8.8 & $1.3 \mathrm{E}-06$ & $9.0 \mathrm{E}-02$ & \\
\hline & Plutonium & & & $1.3 \mathrm{E}-05$ & 10.0 & $3.3 E-08$ & 4.7E-03 & \\
\hline & Americium & & & $3.4 \mathrm{E}-05$ & 9.0 & $1.2 \mathrm{E}-07$ & $9.9 \mathrm{E}-03$ & \\
\hline \multirow{12}{*}{$\begin{array}{l}\text { Animal product transfer } \\
\text { coefficients for milk }\end{array}$} & Chlorine & \multirow{12}{*}{ Lognormal $^{a}$} & \multirow{12}{*}{ day/L } & $1.8 \mathrm{E}-02$ & 2.0 & $2.9 \mathrm{E}-03$ & $1.0 \mathrm{E}-01$ & \multirow{12}{*}{$\begin{array}{l}\text { MO0406SPAETPBM.002 } \\
\text { [DIRS 170150] }\end{array}$} \\
\hline & Selenium & & & 5.7E-03 & 2.5 & $5.5 E-04$ & $6.0 \mathrm{E}-02$ & \\
\hline & Strontium & & & 1.7E-03 & 2.0 & $2.8 \mathrm{E}-04$ & $1.0 \mathrm{E}-02$ & \\
\hline & Technetium & & & $2.1 \mathrm{E}-03$ & 6.0 & $2.0 \mathrm{E}-05$ & $2.1 \mathrm{E}-01$ & \\
\hline & Tin & & & $1.1 \mathrm{E}-03$ & 2.0 & $1.8 \mathrm{E}-04$ & $6.3 \mathrm{E}-03$ & \\
\hline & lodine & & & $9.1 \mathrm{E}-03$ & 2.0 & $1.5 \mathrm{E}-03$ & $5.4 \mathrm{E}-02$ & \\
\hline & Cesium & & & 7.7E-03 & 2.0 & $1.3 \mathrm{E}-03$ & $4.6 E-02$ & \\
\hline & Lead & & & 1.7E-04 & 3.0 & $1.0 \mathrm{E}-05$ & $2.9 \mathrm{E}-03$ & \\
\hline & Radium & & & $5.8 \mathrm{E}-04$ & 2.0 & $1.0 \mathrm{E}-04$ & $3.4 \mathrm{E}-03$ & \\
\hline & Actinium & & & 7.6E-06 & 4.1 & $2.0 \mathrm{E}-07$ & $2.9 \mathrm{E}-04$ & \\
\hline & Thorium & & & $4.4 \mathrm{E}-06$ & 2.0 & $7.4 \mathrm{E}-07$ & $2.6 \mathrm{E}-05$ & \\
\hline & Protactinium & & & $4.4 \mathrm{E}-06$ & 2.0 & $7.4 \mathrm{E}-07$ & $2.6 \mathrm{E}-05$ & \\
\hline
\end{tabular}


Table 4.1-1. Summary of Input Parameter Values and Their Uncertainty Distributions (Continued)

\begin{tabular}{|c|c|c|c|c|c|c|c|c|}
\hline \multicolumn{2}{|c|}{ Parameter Name } & $\begin{array}{c}\text { Distribution } \\
\text { Type }\end{array}$ & Units & $\begin{array}{c}\text { Mean, } \\
\text { Mode } \\
\text { or Fixed } \\
\text { Value }\end{array}$ & SD or SE & $\begin{array}{c}\text { Minimum } \\
\text { or Value } \\
\text { for } C D\end{array}$ & $\begin{array}{l}\text { Maximum } \\
\text { or } \\
\text { Percentile } \\
\text { for CD }\end{array}$ & DTN/Reference \\
\hline \multirow{4}{*}{$\begin{array}{l}\text { Animal product transfer } \\
\text { coefficients for milk }\end{array}$} & Uranium & \multirow{4}{*}{ Lognormal $^{\mathrm{a}}$} & \multirow{4}{*}{ day/L } & $4.9 \mathrm{E}-04$ & 2.0 & $8.1 \mathrm{E}-05$ & $2.9 \mathrm{E}-03$ & \multirow{4}{*}{$\begin{array}{l}\text { MO0406SPAETPBM.002 } \\
\text { [DIRS 170150] }\end{array}$} \\
\hline & Neptunium & & & $6.3 \mathrm{E}-06$ & 2.0 & $1.0 \mathrm{E}-06$ & $3.9 \mathrm{E}-05$ & \\
\hline & Plutonium & & & $2.3 \mathrm{E}-07$ & 7.7 & 1.2E-09 & $4.4 \mathrm{E}-05$ & \\
\hline & Americium & & & $1.6 \mathrm{E}-06$ & 4.2 & $3.9 E-08$ & $6.3 \mathrm{E}-05$ & \\
\hline \multirow{16}{*}{$\begin{array}{l}\text { Animal product transfer } \\
\text { coefficients for poultry }\end{array}$} & Chlorine & \multirow{16}{*}{ Lognormal $^{a}$} & \multirow{16}{*}{ day $/ \mathrm{kg}$} & $3.0 \mathrm{E}-02$ & 2.0 & $5.0 \mathrm{E}-03$ & $1.8 \mathrm{E}-01$ & \multirow{16}{*}{$\begin{array}{l}\text { MO0406SPAETPBM.002 } \\
\text { [DIRS 170150] }\end{array}$} \\
\hline & Selenium & & & $5.1 \mathrm{E}+00$ & 3.6 & $1.9 \mathrm{E}-01$ & $1.4 \mathrm{E}+02$ & \\
\hline & Strontium & & & $3.1 \mathrm{E}-02$ & 5.8 & $3.4 \mathrm{E}-04$ & $2.9 \mathrm{E}+00$ & \\
\hline & Technetium & & & $6.3 E-02$ & 10.0 & 1.7E-04 & $2.4 \mathrm{E}+01$ & \\
\hline & Tin & & & $3.5 \mathrm{E}-02$ & 10.0 & $9.4 \mathrm{E}-05$ & $1.3 \mathrm{E}+01$ & \\
\hline & lodine & & & $5.5 \mathrm{E}-02$ & 9.7 & 1.6E-04 & $1.9 \mathrm{E}+01$ & \\
\hline & Cesium & & & $2.6 \mathrm{E}+00$ & 9.8 & $7.2 \mathrm{E}-03$ & $9.3 \mathrm{E}+02$ & \\
\hline & Lead & & & $2.5 \mathrm{E}-02$ & 10.0 & $6.6 \mathrm{E}-05$ & $9.3 \mathrm{E}+00$ & \\
\hline & Radium & & & 1.7E-02 & 10.0 & 4.4E-05 & $6.3 \mathrm{E}+00$ & \\
\hline & Actinium & & & $4.0 \mathrm{E}-03$ & 2.0 & $6.7 \mathrm{E}-04$ & $2.4 \mathrm{E}-02$ & \\
\hline & Thorium & & & $5.9 \mathrm{E}-03$ & 8.0 & 2.7E-05 & $1.3 \mathrm{E}+00$ & \\
\hline & Protactinium & & & $3.0 \mathrm{E}-03$ & 2.0 & $5.1 \mathrm{E}-04$ & $1.8 \mathrm{E}-02$ & \\
\hline & Uranium & & & $2.4 \mathrm{E}-01$ & 10.0 & $6.5 \mathrm{E}-04$ & $9.2 \mathrm{E}+01$ & \\
\hline & Neptunium & & & $3.6 \mathrm{E}-03$ & 2.0 & $6.0 \mathrm{E}-04$ & $2.1 \mathrm{E}-02$ & \\
\hline & Plutonium & & & $1.2 \mathrm{E}-03$ & 10.0 & $3.2 \mathrm{E}-06$ & 4.6E-01 & \\
\hline & Americium & & & $1.8 \mathrm{E}-03$ & 10.0 & $4.8 \mathrm{E}-06$ & $6.7 \mathrm{E}-01$ & \\
\hline \multirow{11}{*}{$\begin{array}{l}\text { Animal product transfer } \\
\text { coefficients for eggs }\end{array}$} & Chlorine & \multirow{11}{*}{ Lognormal $^{a}$} & \multirow{11}{*}{ day $/ \mathrm{kg}$} & $4.4 \mathrm{E}-02$ & 10.0 & 1.2E-04 & $1.7 \mathrm{E}+01$ & \multirow{11}{*}{$\begin{array}{l}\text { MO0406SPAETPBM.002 } \\
\text { [DIRS 170150] }\end{array}$} \\
\hline & Selenium & & & $7.3 \mathrm{E}+00$ & 2.0 & $1.2 \mathrm{E}+00$ & $4.4 \mathrm{E}+01$ & \\
\hline & Strontium & & & 2.7E-01 & 2.0 & $4.5 \mathrm{E}-02$ & 1.6E+00 & \\
\hline & Technetium & & & $2.4 \mathrm{E}+00$ & 2.0 & $4.0 \mathrm{E}-01$ & $1.4 \mathrm{E}+01$ & \\
\hline & Tin & & & 8.7E-02 & 10.0 & 2.3E-04 & $3.3 E+01$ & \\
\hline & lodine & & & $2.6 \mathrm{E}+00$ & 2.0 & $4.4 \mathrm{E}-01$ & $1.6 \mathrm{E}+01$ & \\
\hline & Cesium & & & $3.5 \mathrm{E}-01$ & 5.8 & 3.7E-03 & $3.3 E+01$ & \\
\hline & Lead & & & $5.6 \mathrm{E}-02$ & 10.0 & $1.5 \mathrm{E}-04$ & $2.1 \mathrm{E}+01$ & \\
\hline & Radium & & & $3.9 \mathrm{E}-04$ & 10.0 & $1.0 \mathrm{E}-06$ & $1.5 \mathrm{E}-01$ & \\
\hline & Actinium & & & $2.9 \mathrm{E}-03$ & 2.3 & $3.4 \mathrm{E}-04$ & $2.5 \mathrm{E}-02$ & \\
\hline & Thorium & & & $3.5 \mathrm{E}-03$ & 7.3 & $2.0 \mathrm{E}-05$ & $5.9 \mathrm{E}-01$ & \\
\hline
\end{tabular}


Table 4.1-1. Summary of Input Parameter Values and Their Uncertainty Distributions (Continued)

\begin{tabular}{|c|c|c|c|c|c|c|c|c|}
\hline \multicolumn{2}{|c|}{ Parameter Name } & $\begin{array}{c}\text { Distribution } \\
\text { Type }\end{array}$ & Units & $\begin{array}{l}\text { Mean, } \\
\text { Mode } \\
\text { or Fixed } \\
\text { Value } \\
\end{array}$ & SD or SE & $\begin{array}{c}\text { Minimum } \\
\text { or Value } \\
\text { for CD }\end{array}$ & $\begin{array}{l}\text { Maximum } \\
\text { or } \\
\text { Percentile } \\
\text { for CD }\end{array}$ & DTN/Reference \\
\hline \multirow{5}{*}{$\begin{array}{l}\text { Animal product transfer } \\
\text { coefficients for eggs }\end{array}$} & Protactinium & \multirow{5}{*}{ Lognormal $^{\mathrm{a}}$} & \multirow{5}{*}{ day/kg } & $2.0 \mathrm{E}-03$ & 2.0 & $3.4 \mathrm{E}-04$ & $1.2 \mathrm{E}-02$ & \multirow{5}{*}{$\begin{array}{l}\text { MO0406SPAETPBM.002 } \\
\text { [DIRS 170150] }\end{array}$} \\
\hline & Uranium & & & $6.3 \mathrm{E}-01$ & 2.5 & $6.0 \mathrm{E}-02$ & $6.7 \mathrm{E}+00$ & \\
\hline & Neptunium & & & $3.4 \mathrm{E}-03$ & 2.4 & $3.4 \mathrm{E}-04$ & $3.3 \mathrm{E}-02$ & \\
\hline & Plutonium & & & $1.7 \mathrm{E}-03$ & 7.4 & $9.7 E-06$ & $2.9 \mathrm{E}-01$ & \\
\hline & Americium & & & $4.9 E-03$ & 2.0 & $8.2 \mathrm{E}-04$ & $2.9 \mathrm{E}-02$ & \\
\hline \multirow{4}{*}{$\begin{array}{l}\text { Animal consumption rate } \\
\text { of feed }\end{array}$} & Beef cattle & \multirow{4}{*}{ Uniform } & \multirow{4}{*}{ kg/day } & - & - & 29 & 68 & \multirow{4}{*}{$\begin{array}{l}\text { MO0406SPAETPBM.002 } \\
\text { [DIRS 170150] }\end{array}$} \\
\hline & Dairy cow & & & - & - & 50 & 73 & \\
\hline & Poultry & & & - & - & 0.12 & 0.40 & \\
\hline & Laying hen & & & - & - & 0.12 & 0.40 & \\
\hline \multirow{4}{*}{$\begin{array}{l}\text { Animal consumption rate of } \\
\text { water }\end{array}$} & Beef cattle & Fixed & \multirow{4}{*}{ L/day } & 60 & - & - & - & \multirow{4}{*}{$\begin{array}{l}\text { MO0406SPAETPBM.002 } \\
\text { [DIRS 170150] }\end{array}$} \\
\hline & Dairy cow & Uniform & & - & - & 60 & 100 & \\
\hline & Poultry & Fixed & & 0.5 & - & - & - & \\
\hline & Laying hen & Fixed & & 0.5 & - & - & - & \\
\hline \multirow{4}{*}{$\begin{array}{l}\text { Animal consumption rate of } \\
\text { soil }\end{array}$} & Beef cattle & \multirow{4}{*}{ Uniform } & \multirow{4}{*}{ kg/day } & - & - & 0.4 & 1.0 & \multirow{4}{*}{$\begin{array}{l}\text { MO0406SPAETPBM.002 } \\
\text { [DIRS 170150] }\end{array}$} \\
\hline & Dairy cow & & & - & - & 0.8 & 1.1 & \\
\hline & Poultry & & & - & - & 0.01 & 0.03 & \\
\hline & Laying hen & & & - & - & 0.01 & 0.03 & \\
\hline \multirow{13}{*}{ Bioaccumulation factor } & & & \multicolumn{6}{|c|}{ FISH SUBMODEL } \\
\hline & Carbon & \multirow{12}{*}{ Lognormal $^{a}$} & \multirow{12}{*}{$\mathrm{L} / \mathrm{kg}$} & $4.6 \mathrm{E}+03$ & 3.2 & $2.3 E+02$ & $9.2 \mathrm{E}+04$ & \multirow{12}{*}{$\begin{array}{l}\text { MO0406SPAETPBM.002 } \\
\text { [DIRS 170150] }\end{array}$} \\
\hline & Chlorine & & & $2.2 \mathrm{E}+02$ & 5.6 & $2.6 \mathrm{E}+00$ & $1.9 \mathrm{E}+04$ & \\
\hline & Selenium & & & $2.3 E+02$ & 2.0 & $3.9 E+01$ & $1.4 \mathrm{E}+03$ & \\
\hline & Strontium & & & $4.6 \mathrm{E}+01$ & 2.0 & $7.8 \mathrm{E}+00$ & $2.8 \mathrm{E}+02$ & \\
\hline & Technetium & & & $2.0 \mathrm{E}+01$ & 2.0 & $3.3 E+00$ & $1.2 \mathrm{E}+02$ & \\
\hline & Tin & & & $2.5 E+03$ & 2.0 & $4.2 \mathrm{E}+02$ & $1.5 \mathrm{E}+04$ & \\
\hline & lodine & & & $4.5 \mathrm{E}+01$ & 2.6 & $3.8 \mathrm{E}+00$ & $5.3 \mathrm{E}+02$ & \\
\hline & Cesium & & & $3.5 E+03$ & 2.2 & 4.7E+02 & $2.5 \mathrm{E}+04$ & \\
\hline & Lead & & & $2.9 E+02$ & 2.5 & $2.7 E+01$ & $3.1 \mathrm{E}+03$ & \\
\hline & Radium & & & $6.7 \mathrm{E}+01$ & 2.2 & $9.2 \mathrm{E}+00$ & $5.0 \mathrm{E}+02$ & \\
\hline & Actinium & & & $2.9 E+01$ & 3.0 & $1.7 \mathrm{E}+00$ & $5.0 \mathrm{E}+02$ & \\
\hline & Thorium & & & $1.1 \mathrm{E}+02$ & 2.5 & $1.0 \mathrm{E}+01$ & $1.2 \mathrm{E}+03$ & \\
\hline
\end{tabular}


Table 4.1-1. Summary of Input Parameter Values and Their Uncertainty Distributions (Continued)

\begin{tabular}{|c|c|c|c|c|c|c|c|c|}
\hline \multicolumn{2}{|c|}{ Parameter Name } & $\begin{array}{l}\text { Distribution } \\
\text { Type }\end{array}$ & Units & $\begin{array}{l}\text { Mean, } \\
\text { Mode } \\
\text { or Fixed } \\
\text { Value } \\
\end{array}$ & SD or SE & $\begin{array}{l}\text { Minimum } \\
\text { or Value } \\
\text { for CD }\end{array}$ & $\begin{array}{l}\text { Maximum } \\
\text { or } \\
\text { Percentile } \\
\text { for CD } \\
\end{array}$ & DTN/Reference \\
\hline \multirow{5}{*}{ Bioaccumulation factor } & Protactinium & \multirow{5}{*}{ Lognormal $^{a}$} & \multirow{5}{*}{$\mathrm{L} / \mathrm{kg}$} & $1.2 E+01$ & 2.0 & $2.0 \mathrm{E}+00$ & $7.1 \mathrm{E}+01$ & \multirow{5}{*}{$\begin{array}{l}\text { MO0406SPAETPBM.002 } \\
\text { [DIRS 170150] }\end{array}$} \\
\hline & Uranium & & & $1.4 \mathrm{E}+01$ & 3.0 & $8.4 \mathrm{E}-01$ & $2.3 \mathrm{E}+02$ & \\
\hline & Neptunium & & & $3.0 \mathrm{E}+01$ & 2.9 & $1.9 \mathrm{E}+00$ & 4.7E+02 & \\
\hline & Plutonium & & & 4.1E+01 & 4.7 & $7.9 \mathrm{E}-01$ & $2.2 \mathrm{E}+03$ & \\
\hline & Americium & & & $5.2 \mathrm{E}+01$ & 2.3 & $5.8 \mathrm{E}+00$ & $4.6 \mathrm{E}+02$ & \\
\hline \multirow{2}{*}{$\begin{array}{l}\text { Water concentration } \\
\text { modifying factor, present- } \\
\text { day climate }\end{array}$} & Carbon & Fixed & - & 1.0 & - & - & - & \multirow{4}{*}{$\begin{array}{l}\text { MO0406SPAETPBM.002 } \\
\text { [DIRS 170150] }\end{array}$} \\
\hline & Other elements & Uniform & - & - & - & 2.2 & 6.1 & \\
\hline \multirow{2}{*}{$\begin{array}{l}\text { Water concentration } \\
\text { modifying factor, future } \\
\text { climate }\end{array}$} & Carbon & Fixed & - & 1.0 & - & - & - & \\
\hline & Other elements & Uniform & - & - & - & 1.5 & 3.3 & \\
\hline \multicolumn{9}{|c|}{ SPECIAL CARBON-14 MODEL } \\
\hline \multicolumn{2}{|l|}{ C-14 emission rate } & Fixed & $1 / \mathrm{yr}$ & 22 & - & - & - & $\begin{array}{l}\text { MO0406SPAETPBM.002 } \\
\text { [DIRS 170150] }\end{array}$ \\
\hline \multicolumn{2}{|l|}{ Annual water demand } & Fixed & $\mathrm{m}^{3} / \mathrm{yr}$ & $3,714,450$ & - & - & - & $\begin{array}{l}\text { MO0406SPAETPBM.002 } \\
\text { [DIRS 170150] }\end{array}$ \\
\hline \multirow{2}{*}{$\begin{array}{l}\text { Annual average wind } \\
\text { speed }\end{array}$} & Inhalation & \multirow{2}{*}{ Uniform } & \multirow{2}{*}{$\mathrm{m} / \mathrm{s}$} & - & - & 2.1 & 2.8 & \multirow{2}{*}{$\begin{array}{l}\text { MO0406SPAETPBM.002 } \\
\text { [DIRS 170150] }\end{array}$} \\
\hline & Crops & & & - & - & 1.5 & 2.3 & \\
\hline \multirow{2}{*}{ C-14 mixing height } & Inhalation & \multirow{2}{*}{ Fixed } & \multirow{2}{*}{$\mathrm{m}$} & 2 & - & - & - & \multirow{2}{*}{$\begin{array}{l}\text { MO0406SPAETPBM.002 } \\
\text { [DIRS 170150] }\end{array}$} \\
\hline & Crops & & & 1 & - & - & - & \\
\hline \multicolumn{2}{|c|}{ Fraction of air-derived carbon in plants } & Fixed & - & 0.98 & - & - & - & $\begin{array}{l}\text { MO0406SPAETPBM.002 } \\
\text { [DIRS 170150] }\end{array}$ \\
\hline \multicolumn{2}{|c|}{ Fraction of soil-derived carbon in plants } & Fixed & - & 0.02 & - & - & - & $\begin{array}{l}\text { MO0406SPAETPBM.002 } \\
\text { [DIRS 170150] }\end{array}$ \\
\hline \multirow{5}{*}{$\begin{array}{l}\text { Fraction of stable carbon in } \\
\text { plants }\end{array}$} & Leafy vegetables & \multirow{5}{*}{ Fixed } & \multirow{5}{*}{-} & 0.09 & - & - & - & \multirow{5}{*}{$\begin{array}{l}\text { MO0406SPAETPBM.002 } \\
\text { [DIRS 170150] }\end{array}$} \\
\hline & Other vegetables & & & 0.09 & - & - & - & \\
\hline & Fruit & & & 0.09 & - & - & - & \\
\hline & Grain & & & 0.40 & - & - & - & \\
\hline & Forage & & & 0.09 & - & - & - & \\
\hline \multicolumn{2}{|l|}{ Fraction of stable carbon in soil } & Fixed & - & 0.03 & - & - & - & $\begin{array}{l}\text { MO0406SPAETPBM.002 } \\
\text { [DIRS 170150] }\end{array}$ \\
\hline
\end{tabular}


Table 4.1-1. Summary of Input Parameter Values and Their Uncertainty Distributions (Continued)

\begin{tabular}{|c|c|c|c|c|c|c|c|c|}
\hline \multicolumn{2}{|c|}{ Parameter Name } & $\begin{array}{c}\text { Distribution } \\
\text { Type }\end{array}$ & Units & $\begin{array}{l}\text { Mean, } \\
\text { Mode } \\
\text { or Fixed } \\
\text { Value } \\
\end{array}$ & SD or SE & $\begin{array}{c}\text { Minimum } \\
\text { or Value } \\
\text { for CD }\end{array}$ & $\begin{array}{l}\text { Maximum } \\
\text { or } \\
\text { Percentile } \\
\text { for CD }\end{array}$ & DTN/Reference \\
\hline \multicolumn{2}{|c|}{ Concentration of stable carbon in air } & Fixed & $\mathrm{kg} / \mathrm{m}^{3}$ & $1.8 \mathrm{E}-04$ & - & - & - & $\begin{array}{l}\text { MO0406SPAETPBM.002 } \\
\text { [DIRS 170150] }\end{array}$ \\
\hline \multicolumn{2}{|c|}{ Concentration of stable carbon in water } & Fixed & $\mathrm{kg} / \mathrm{L}$ & $2.0 \mathrm{E}-05$ & - & - & - & $\begin{array}{l}\text { MO0406SPAETPBM.002 } \\
\text { [DIRS 170150] }\end{array}$ \\
\hline \multirow{4}{*}{$\begin{array}{l}\text { Fraction of stable carbon in } \\
\text { animal products }\end{array}$} & Meat & \multirow{4}{*}{ Fixed } & \multirow{4}{*}{-} & 0.24 & - & - & - & \multirow{4}{*}{$\begin{array}{l}\text { MO0406SPAETPBM.002 } \\
\text { [DIRS 170150] }\end{array}$} \\
\hline & Milk & & & 0.07 & - & - & - & \\
\hline & Poultry & & & 0.2 & - & - & - & \\
\hline & Eggs & & & 0.15 & - & - & - & \\
\hline \multicolumn{9}{|c|}{ EXTERNAL EXPOSURE SUBMODEL } \\
\hline \multirow{4}{*}{ Population proportion } & Outdoor workers & \multirow{4}{*}{ Uniform } & \multirow{4}{*}{$\%$} & - & - & 2.9 & 8.1 & \multirow{4}{*}{$\begin{array}{l}\text { MO0407SPACRBSM.002 } \\
\text { [DIRS 170677] }\end{array}$} \\
\hline & Indoor workers & & & - & - & Calculated & Calculated & \\
\hline & Commuters & & & - & - & 33.9 & 44.5 & \\
\hline & Nonworkers & & & - & - & 34.4 & 44.0 & \\
\hline \multirow{5}{*}{$\begin{array}{l}\text { Time spent by outdoor } \\
\text { workers }\end{array}$} & Active outdoors & \multirow{5}{*}{ Lognormal $^{b}$} & \multirow{5}{*}{$\mathrm{hr} /$ day } & 3.1 & 0.2 & 2.6 & 3.7 & \multirow{15}{*}{$\begin{array}{l}\text { MO0407SPACRBSM.002 } \\
\text { [DIRS 170677] }\end{array}$} \\
\hline & Inactive outdoors & & & 4.0 & 0.3 & 3.3 & 4.8 & \\
\hline & Active indoors & & & Calculated & - & - & - & \\
\hline & Asleep indoors & & & 8.3 & 0.1 & 8.0 & 8.6 & \\
\hline & Away & & & 2.0 & 0.4 & 1.2 & 3.3 & \\
\hline \multirow{5}{*}{$\begin{array}{l}\text { Time spent by indoor } \\
\text { workers }\end{array}$} & Active outdoors & \multirow{5}{*}{ Lognormal $^{b}$} & \multirow{5}{*}{$\mathrm{hr} /$ day } & 0.3 & 0.1 & 0.1 & 0.7 & \\
\hline & Inactive outdoors & & & 1.3 & 0.2 & 0.9 & 1.9 & \\
\hline & Active indoors & & & Calculated & - & - & - & \\
\hline & Asleep indoors & & & 8.3 & 0.1 & 8.0 & 8.6 & \\
\hline & Away & & & 2.0 & 0.4 & 1.2 & 3.3 & \\
\hline \multirow{5}{*}{ Time spent by commuters } & Active outdoors & \multirow{5}{*}{ Lognormal $^{b}$} & \multirow{5}{*}{$\mathrm{hr} /$ day } & 0.3 & 0.1 & 0.1 & 0.7 & \\
\hline & Inactive outdoors & & & 1.4 & 0.2 & 1.0 & 2.0 & \\
\hline & Active indoors & & & Calculated & - & - & - & \\
\hline & Asleep indoors & & & 8.3 & 0.1 & 8.0 & 8.6 & \\
\hline & Away & & & 8.0 & 0.5 & 6.8 & 9.4 & \\
\hline
\end{tabular}




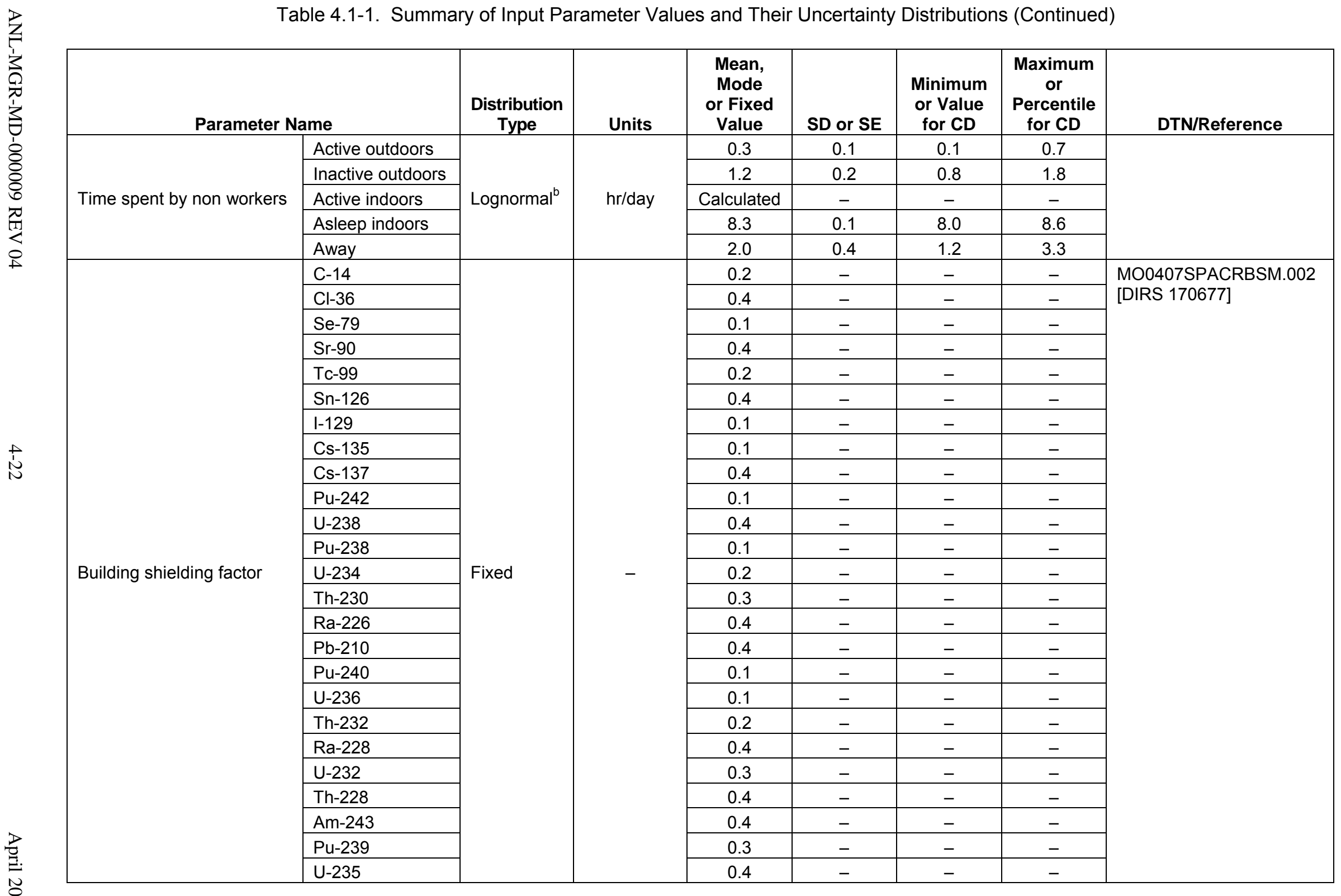


Table 4.1-1. Summary of Input Parameter Values and Their Uncertainty Distributions (Continued)

\begin{tabular}{|c|c|c|c|c|c|c|c|c|}
\hline \multicolumn{2}{|c|}{ Parameter Name } & $\begin{array}{l}\text { Distribution } \\
\text { Type }\end{array}$ & Units & $\begin{array}{l}\text { Mean, } \\
\text { Mode } \\
\text { or Fixed } \\
\text { Value } \\
\end{array}$ & SD or SE & $\begin{array}{l}\text { Minimum } \\
\text { or Value } \\
\text { for CD }\end{array}$ & $\begin{array}{l}\text { Maximum } \\
\quad \text { or } \\
\text { Percentile } \\
\text { for CD } \\
\end{array}$ & DTN/Reference \\
\hline \multirow{6}{*}{ Building shielding factor } & Pa-231 & \multirow{6}{*}{ Fixed } & \multirow{6}{*}{-} & 0.4 & - & - & - & \multirow{6}{*}{$\begin{array}{l}\text { MO0407SPACRBSM.002 } \\
\text { [DIRS 170677] }\end{array}$} \\
\hline & Ac-227 & & & 0.4 & - & - & - & \\
\hline & Am-241 & & & 0.2 & - & - & - & \\
\hline & $\mathrm{Np}-237$ & & & 0.4 & - & - & - & \\
\hline & U-233 & & & 0.4 & - & - & - & \\
\hline & Th-229 & & & 0.4 & - & - & - & \\
\hline \multicolumn{2}{|c|}{$\begin{array}{l}\text { Dose coefficient for exposure to contaminated } \\
\text { soil (infinite depth) }\end{array}$} & Fixed & $\begin{array}{l}(\mathrm{Sv} / \mathrm{yr}) / \\
\left(\mathrm{Bq} / \mathrm{m}^{3}\right)\end{array}$ & $\begin{array}{c}\text { See Tables } \\
4.1-5 \text { and } \\
4.1-6\end{array}$ & - & - & - & $\begin{array}{l}\text { MO0407SPACRBSM.002 } \\
\text { [DIRS 170677] } \\
\text { MO0503SPADCESR.000 } \\
\text { [DIRS 172896] }\end{array}$ \\
\hline \multicolumn{9}{|c|}{ D S UBMODEL } \\
\hline \multirow{5}{*}{ Breathing rate } & Active outdoors & \multirow{5}{*}{ Fixed } & \multirow{5}{*}{$\mathrm{m}^{3} / \mathrm{hr}$} & 1.57 & - & - & - & \multirow{5}{*}{$\begin{array}{l}\text { MO0407SPACRBSM.002 } \\
\text { [DIRS 170677] }\end{array}$} \\
\hline & Inactive outdoors & & & 1.08 & - & - & - & \\
\hline & Active indoors & & & 1.08 & - & - & - & \\
\hline & Asleep indoors & & & 0.39 & - & - & - & \\
\hline & Away & & & $1.08^{d}$ & - & - & - & \\
\hline \multicolumn{2}{|c|}{ Dose conversion factor for inhalation } & Fixed & $\mathrm{Sv} / \mathrm{Bq}$ & $\begin{array}{c}\text { See Tables } \\
4.1-3 \text { and } \\
4.1-4\end{array}$ & - & - & - & $\begin{array}{l}\text { MO0407SPACRBSM.002 } \\
\text { [DIRS 170677] } \\
\text { MO0503SPADCESR.000 } \\
\text { [DIRS 172896] }\end{array}$ \\
\hline \multicolumn{2}{|c|}{ Fraction of houses with evaporative coolers } & Binomial & - & 0.738 & $\begin{array}{l}\text { Batch size } \\
\quad=187\end{array}$ & - & - & $\begin{array}{l}\text { MO0407SPACRBSM.002 } \\
\text { [DIRS 170677] }\end{array}$ \\
\hline \multirow{2}{*}{$\begin{array}{l}\text { Evaporative cooler } \\
\text { use factor }\end{array}$} & Present-day climate & Uniform & - & - & - & 0.32 & 0.46 & \multirow{2}{*}{$\begin{array}{l}\text { MO0407SPACRBSM.002 } \\
\text { [DIRS 170677] }\end{array}$} \\
\hline & Future climate & Uniform & - & - & - & 0.03 & 0.14 & \\
\hline \multirow{2}{*}{$\begin{array}{l}\text { Equilibrium factor for } \\
{ }^{222} \mathrm{Rn} \text { decay products }\end{array}$} & Outdoors & Uniform & - & - & - & 0.5 & 0.7 & \multirow{2}{*}{$\begin{array}{l}\text { MO0406SPAETPBM.002 } \\
\text { [DIRS 170150] }\end{array}$} \\
\hline & Indoors & Uniform & - & - & - & 0.3 & 0.5 & \\
\hline \multicolumn{2}{|c|}{$\begin{array}{l}\text { ICRP 30-based dose conversion factor for } \\
\text { inhalation of }{ }^{222} \mathrm{Rn} \text { decay products }\end{array}$} & Fixed & $\mathrm{Sv} / \mathrm{Bq}$ & 1.33E-8 & - & - & - & $\begin{array}{l}\text { MO0407SPACRBSM.002 } \\
\text { [DIRS 170677] }\end{array}$ \\
\hline \multicolumn{2}{|c|}{$\begin{array}{l}\text { ICRP 72-based dose conversion factor for } \\
\text { inhalation of }{ }^{222} \mathrm{Rn} \text { decay products }\end{array}$} & Fixed & $\mathrm{Sv} / \mathrm{Bq}$ & $6.62 \mathrm{E}-9$ & - & - & - & $\begin{array}{l}\text { MO0503SPADCESR.000 } \\
\text { [DIRS 172896] }\end{array}$ \\
\hline
\end{tabular}


Table 4.1-1. Summary of Input Parameter Values and Their Uncertainty Distributions (Continued)

\begin{tabular}{|c|c|c|c|c|c|c|c|c|}
\hline \multicolumn{2}{|c|}{ Parameter Name } & $\begin{array}{c}\text { Distribution } \\
\text { Type }\end{array}$ & Units & $\begin{array}{l}\text { Mean, } \\
\text { Mode } \\
\text { or Fixed } \\
\text { Value } \\
\end{array}$ & SD or SE & $\begin{array}{l}\text { Minimum } \\
\text { or Value } \\
\text { for CD }\end{array}$ & $\begin{array}{l}\text { Maximum } \\
\quad \text { or } \\
\text { Percentile } \\
\text { for CD } \\
\end{array}$ & DTN/Reference \\
\hline \multicolumn{9}{|c|}{ INGESTION SUBMODEL } \\
\hline \multicolumn{2}{|c|}{ Consumption rate of water } & Fixed & L/day & 2.0 & - & - & - & $\begin{array}{l}\text { MO0407SPACRBSM.002 } \\
\text { [DIRS 170677] }\end{array}$ \\
\hline \multirow{9}{*}{$\begin{array}{l}\text { Consumption rate of } \\
\text { locally produced food }\end{array}$} & Leafy vegetables & \multirow{9}{*}{ Lognormal $^{b}$} & \multirow{9}{*}{$\mathrm{kg} / \mathrm{yr}$} & 3.78 & 0.88 & - & - & \multirow{9}{*}{$\begin{array}{l}\text { MO0407SPACRBSM.002 } \\
\text { [DIRS 170677] }\end{array}$} \\
\hline & Other vegetables & & & 4.73 & 0.67 & - & - & \\
\hline & Fruit & & & 12.68 & 1.36 & - & - & \\
\hline & Grain & & & 0.23 & 0.11 & - & - & \\
\hline & Meat & & & 2.85 & 0.65 & - & - & \\
\hline & Milk & & & 4.66 & 1.68 & - & - & \\
\hline & Poultry & & & 0.42 & 0.13 & - & - & \\
\hline & Eggs & & & 5.30 & 0.83 & - & - & \\
\hline & Fish & & & 0.23 & 0.10 & - & - & \\
\hline \multicolumn{2}{|c|}{ Inadvertent soil ingestion rate } & Cumulative & mg/day & - & - & $\begin{array}{c}50 \\
100 \\
200 \\
\end{array}$ & $\begin{array}{c}0 \% \\
50 \% \\
100 \% \\
\end{array}$ & $\begin{array}{l}\text { MO0407SPACRBSM.002 } \\
\text { [DIRS 170677] }\end{array}$ \\
\hline \multicolumn{2}{|c|}{ Dose conversion factor for ingestion } & Fixed & $\mathrm{Sv} / \mathrm{Bq}$ & $\begin{array}{c}\text { See } \\
\text { Tables } \\
4.1-3 \text { and } \\
4.1-4\end{array}$ & - & - & - & $\begin{array}{l}\text { MO0407SPACRBSM.002 } \\
\text { [DIRS 170677] } \\
\text { MO0503SPADCESR.000 } \\
\text { [DIRS 172896] }\end{array}$ \\
\hline
\end{tabular}

$\mathrm{SD}=$ standard deviation; $\mathrm{SE}=$ standard error; $\mathrm{CD}=$ cumulative distribution

a Lognormal distribution defined using geometric mean and geometric standard deviation

b Lognormal distributions defined using arithmetic mean and arithmetic standard deviation

${ }^{\mathrm{c}}$ The units are not included with the parameter value in GoldSim element because the values are used in an empirical equation. The units are specified in the

element description.

d Breathing rate away (not in contaminated area) has no effect on the results of BDCF calculations. Any value can be used. 
Table 4.1-2. Primary Radionuclides and Their Decay Products Included in the Biosphere Model

\begin{tabular}{|c|c|c|c|}
\hline Primary Radionuclide & $\begin{array}{l}\text { Short-lived Decay } \\
\text { Product }\end{array}$ & Branching Fraction, \% & Half-life \\
\hline Carbon-14 $\left({ }^{14} \mathrm{C}\right)$ & & 100 & $5.730 \mathrm{E}+03 \mathrm{yr}$ \\
\hline Chlorine-36 $\left({ }^{36} \mathrm{Cl}\right)$ & & 100 & $3.01 \mathrm{E}+05 \mathrm{yr}$ \\
\hline Selenium-79 $\left({ }^{79} \mathrm{Se}\right)$ & & 100 & $6.50 \mathrm{E}+04 \mathrm{yr}$ \\
\hline \multirow[t]{2}{*}{ Strontium-90 $\left({ }^{90} \mathrm{Sr}\right)$} & & 100 & $2.912 \mathrm{E}+01 \mathrm{yr}$ \\
\hline & Yttrium-90 $\left({ }^{90} \mathrm{Y}\right)$ & 100 & $6.40 \mathrm{E}+01 \mathrm{hr}$ \\
\hline Technetium-99 $\left({ }^{99} \mathrm{Tc}\right)$ & & 100 & $2.13 \mathrm{E}+05 \mathrm{yr}$ \\
\hline \multirow[t]{3}{*}{ Tin-126 $\left({ }^{126} \mathrm{Sn}\right)$} & & 100 & $1.0 \mathrm{E}+05 \mathrm{yr}$ \\
\hline & Antimony-126m $\left({ }^{126 m} \mathrm{Sb}\right)$ & 100 & $1.90 \mathrm{E}+01 \mathrm{~min}$ \\
\hline & Antimony-126 $\left({ }^{126} \mathrm{Sb}\right)$ & 14 & $1.24 \mathrm{E}+01$ day \\
\hline lodine-129 $\left({ }^{129} \mathrm{I}\right)$ & & 100 & $1.57 \mathrm{E}+07 \mathrm{yr}$ \\
\hline Cesium-135 $\left({ }^{135} \mathrm{Cs}\right)$ & & 100 & $2.3 \mathrm{E}+06 \mathrm{yr}$ \\
\hline \multirow[t]{2}{*}{ Cesium-137 ( $\left.{ }^{137} \mathrm{Cs}\right)$} & & 100 & $3.00 \mathrm{E}+01 \mathrm{yr}$ \\
\hline & Barium-137m $\left({ }^{137 \mathrm{~m}} \mathrm{Ba}\right)$ & 94.60 & $2.552 \mathrm{E}+00 \mathrm{~min}$ \\
\hline \multicolumn{4}{|c|}{ Thorium Series (4n) } \\
\hline Plutonium-240 $\left({ }^{240} \mathrm{Pu}\right)$ & & 100 & $6.537 \mathrm{E}+03 \mathrm{yr}$ \\
\hline Uranium-236 $\left({ }^{236} \mathrm{U}\right)$ & & 100 & $2.3415 \mathrm{E}+07 \mathrm{yr}$ \\
\hline Thorium-232 $\left({ }^{232} \mathrm{Th}\right)$ & & 100 & $1.405 \mathrm{E}+10 \mathrm{yr}$ \\
\hline \multirow[t]{2}{*}{ Radium-228 $\left({ }^{228} \mathrm{Ra}\right)$} & & 100 & $5.75 \mathrm{E}+00 \mathrm{yr}$ \\
\hline & Actinium-228 $\left({ }^{228} \mathrm{Ac}\right)$ & 100 & $6.13 \mathrm{E}+00 \mathrm{hr}$ \\
\hline Uranium-232 $\left({ }^{232} \mathrm{U}\right)$ & & 100 & $7.2 \mathrm{E}+01 \mathrm{yr}$ \\
\hline \multirow[t]{8}{*}{ Thorium-228 $\left({ }^{228} \mathrm{Th}\right)$} & & 100 & $1.9131 \mathrm{E}+00 \mathrm{yr}$ \\
\hline & Radium-224 $\left({ }^{224} \mathrm{Ra}\right)$ & 100 & $3.66 \mathrm{E}+00$ day \\
\hline & Radon-220 $\left({ }^{220} \mathrm{Rn}\right)$ & 100 & $5.56 \mathrm{E}+01 \mathrm{sec}$ \\
\hline & Polonium-216 $\left({ }^{216} \mathrm{Po}\right)$ & 100 & $1.5 \mathrm{E}-01 \mathrm{sec}$ \\
\hline & Lead-212 $\left({ }^{212} \mathrm{~Pb}\right)$ & 100 & $1.064 \mathrm{E}+01 \mathrm{hr}$ \\
\hline & Bismuth-212 $\left({ }^{212} \mathrm{Bi}\right)$ & 100 & $6.055 \mathrm{E}+01 \mathrm{~min}$ \\
\hline & Polonium-212 $\left({ }^{212} \mathrm{Po}\right)$ & 64.07 & $3.05 \mathrm{E}-07 \mathrm{sec}$ \\
\hline & Thallium-208 $\left({ }^{208} \mathrm{TI}\right)$ & 35.93 & $3.07 \mathrm{E}+00 \mathrm{~min}$ \\
\hline \multicolumn{4}{|c|}{ Neptunium Series $(4 n+1)$} \\
\hline Americium-241 $\left({ }^{241} \mathrm{Am}\right)$ & & 100 & $4.322 \mathrm{E}+02 \mathrm{yr}$ \\
\hline \multirow[t]{2}{*}{ Neptunium-237 $\left({ }^{237} \mathrm{~Np}\right)$} & & 100 & $2.14 \mathrm{E}+06 \mathrm{yr}$ \\
\hline & Protactinium-233 $\left({ }^{233} \mathrm{~Pa}\right)$ & 100 & $2.70 \mathrm{E}+01$ day \\
\hline Uranium-233 $\left({ }^{233} \mathrm{U}\right)$ & & 100 & $1.585 \mathrm{E}+05 \mathrm{yr}$ \\
\hline \multirow[t]{9}{*}{ Thorium-229 $\left({ }^{229} \mathrm{Th}\right)$} & & 100 & $7.340 \mathrm{E}+03 \mathrm{yr}$ \\
\hline & Radium-225 $(225 \mathrm{Ra})$ & 100 & $1.48 \mathrm{E}+01$ day \\
\hline & Actinium-225 $\left({ }^{225} \mathrm{Ac}\right)$ & 100 & $1.00 \mathrm{E}+01$ day \\
\hline & Francium-221 $\left({ }^{221} \mathrm{Fr}\right)$ & 100 & $4.8 \mathrm{E}+00 \mathrm{~min}$ \\
\hline & Astatine-217 $\left({ }^{217} \mathrm{At}\right)$ & 100 & $3.23 \mathrm{E}-02 \mathrm{sec}$ \\
\hline & Bismuth-213 $\left({ }^{213} \mathrm{Bi}\right)$ & 100 & $4.565 \mathrm{E}+01 \mathrm{~min}$ \\
\hline & Polonium-213 $\left({ }^{213} \mathrm{Po}\right)$ & 97.84 & $4.2 \mathrm{E}-06 \mathrm{sec}$ \\
\hline & Thallium-209 ( $\left.{ }^{209} \mathrm{TI}\right)$ & 2.16 & $2.20 \mathrm{E}+00 \mathrm{~min}$ \\
\hline & Lead-209 $\left({ }^{209} \mathrm{~Pb}\right)$ & 100 & $3.253 \mathrm{E}+00 \mathrm{hr}$ \\
\hline
\end{tabular}


Table 4.1-2. Primary Radionuclides and Their Decay Products Included in the Biosphere Model (Continued)

\begin{tabular}{|c|c|c|c|}
\hline Primary Radionuclide & $\begin{array}{c}\text { Short-lived Decay } \\
\text { Product }\end{array}$ & Branching Fraction, \% & Half-life \\
\hline \multicolumn{4}{|c|}{ Uranium Series $(4 n+2)$} \\
\hline Plutonium-242 $\left({ }^{242} \mathrm{Pu}\right)$ & & 100 & $3.763 \mathrm{E}+05 \mathrm{yr}$ \\
\hline \multirow[t]{4}{*}{ Uranium-238 $\left({ }^{238} \mathrm{U}\right)$} & & 100 & $4.468 \mathrm{E}+09 \mathrm{yr}$ \\
\hline & Thorium-234 $\left({ }^{234} \mathrm{Th}\right)$ & 100 & $2.410 \mathrm{E}+01$ day \\
\hline & Protactinium-234m $\left({ }^{234 \mathrm{~m}} \mathrm{~Pa}\right)$ & 99.80 & $1.17 \mathrm{E}+00 \mathrm{~min}$ \\
\hline & Protactinium-234 $\left({ }^{234} \mathrm{~Pa}\right)$ & 0.33 & $6.70 \mathrm{E}+00 \mathrm{hr}$ \\
\hline Plutonium-238 $\left({ }^{238} \mathrm{Pu}\right)$ & & 100 & $8.774 \mathrm{E}+01 \mathrm{yr}$ \\
\hline Uranium-234 $\left({ }^{234} U\right)$ & & 100 & $2.445 \mathrm{E}+05 \mathrm{yr}$ \\
\hline Thorium-230 $\left({ }^{230} \mathrm{Th}\right)$ & & 100 & $7.7 \mathrm{E}+04 \mathrm{yr}$ \\
\hline \multirow[t]{8}{*}{ Radium-226 $\left({ }^{226} \mathrm{Ra}\right)$} & & 100 & $1.600 \mathrm{E}+03 \mathrm{yr}$ \\
\hline & Radon-222 $\left({ }^{222} \mathrm{Rn}\right)$ & 100 & $3.8235 \mathrm{E}+00$ day \\
\hline & Polonium-218 $\left({ }^{218} \mathrm{Po}\right)$ & 100 & $3.05 \mathrm{E}+00 \mathrm{~min}$ \\
\hline & Lead-214 $\left({ }^{214} \mathrm{~Pb}\right)$ & 99.98 & $2.68 \mathrm{E}+01 \mathrm{~min}$ \\
\hline & Astatine-218 $\left({ }^{218} \mathrm{At}\right)$ & 0.02 & $2 . E+00 \mathrm{sec}$ \\
\hline & Bismuth-214 $\left({ }^{214} \mathrm{Bi}\right)$ & 100 & $1.99 \mathrm{E}+01 \mathrm{~min}$ \\
\hline & Polonium-214 $\left({ }^{214} \mathrm{Po}\right)$ & 99.98 & $1.643 \mathrm{E}-04 \mathrm{sec}$ \\
\hline & Thallium-210 $\left({ }^{210} \mathrm{TI}\right)$ & 0.02 & $1.3 \mathrm{E}+00 \mathrm{~min}$ \\
\hline \multirow[t]{3}{*}{ Lead-210 $\left({ }^{210} \mathrm{~Pb}\right)$} & & 100 & $2.23 \mathrm{E}+01 \mathrm{yr}$ \\
\hline & Bismuth-210 $\left({ }^{210} \mathrm{Bi}\right)$ & 100 & $5.012 \mathrm{E}+00$ day \\
\hline & Polonium-210 $\left({ }^{210} \mathrm{Po}\right)$ & 100 & $1.3838 \mathrm{E}+02$ day \\
\hline \multicolumn{4}{|c|}{ Actinium Series $(4 n+3)$} \\
\hline \multirow[t]{2}{*}{ Americium-243 $\left({ }^{243} \mathrm{Am}\right)$} & & 100 & $7.380 \mathrm{E}+03 \mathrm{yr}$ \\
\hline & Neptunium-239 $\left({ }^{239} \mathrm{~Np}\right)$ & 100 & $2.355 E+00$ day \\
\hline Plutonium-239 $\left({ }^{239} \mathrm{Pu}\right)$ & & 100 & $2.4065 \mathrm{E}+04 \mathrm{yr}$ \\
\hline \multirow[t]{2}{*}{ Uranium-235 $\left({ }^{235} \mathrm{U}\right)$} & & 100 & $7.038 \mathrm{E}+08 \mathrm{yr}$ \\
\hline & Thorium-231 $\left({ }^{231} \mathrm{Th}\right)$ & 100 & $2.552 \mathrm{E}+01 \mathrm{hr}$ \\
\hline Protactinium-231 $\left({ }^{231} \mathrm{~Pa}\right)$ & & 100 & $3.276 \mathrm{E}+04 \mathrm{yr}$ \\
\hline \multirow[t]{10}{*}{ Actinium-227 $\left({ }^{227} \mathrm{Ac}\right)$} & & 100 & $2.1773 \mathrm{E}+01 \mathrm{yr}$ \\
\hline & Thorium-227 $\left({ }^{227} \mathrm{Th}\right)$ & 98.62 & $1.8718 \mathrm{E}+01$ day \\
\hline & Francium-223 $\left({ }^{223} \mathrm{Fr}\right)$ & 1.38 & $2.18 \mathrm{E}+01 \mathrm{~min}$ \\
\hline & Radium-223 $\left({ }^{223} \mathrm{Ra}\right)$ & 100 & $1.1434 \mathrm{E}+01$ day \\
\hline & Radon-219 $\left({ }^{219} \mathrm{Rn}\right)$ & 100 & $3.96 \mathrm{E}+00 \mathrm{sec}$ \\
\hline & Polonium-215 $\left({ }^{215} \mathrm{Po}\right)$ & 100 & $1.78 \mathrm{E}-03 \mathrm{sec}$ \\
\hline & Lead-211 $\left({ }^{211} \mathrm{~Pb}\right)$ & 100 & $3.61 \mathrm{E}+01 \mathrm{~min}$ \\
\hline & Bismuth-211 $\left({ }^{211} \mathrm{Bi}\right)$ & 100 & $2.14 \mathrm{E}+00 \mathrm{~min}$ \\
\hline & Thallium-207 $\left({ }^{207} \mathrm{TI}\right)$ & 99.72 & $4.77 \mathrm{E}+00 \mathrm{~min}$ \\
\hline & Polonium-211 $\left({ }^{211} \mathrm{Po}\right)$ & 0.28 & $5.16 \mathrm{E}-01 \mathrm{sec}$ \\
\hline
\end{tabular}

Source: DTN: MO0407SPACRBSM.002 [DIRS 170677].

NOTE: Short-lived decay products of primary radionuclides are modeled to be in secular equilibrium with their parents. Only half-lives of primary radionuclides are used as inputs to the biosphere model. 
Table 4.1-3. Dose Coefficients for Inhalation and Ingestion of Radionuclides of Interest Consistent with ICRP 30 Dosimetric Methods

\begin{tabular}{|c|c|c|c|}
\hline \multirow[b]{2}{*}{ Primary Radionuclide } & \multirow{2}{*}{$\begin{array}{c}\text { Short-lived Decay } \\
\text { Product }\end{array}$} & \multicolumn{2}{|c|}{ Dose Coefficients (Sv/Bq) } \\
\hline & & Inhalation & Ingestion \\
\hline Carbon-14 (as $\left.\mathrm{CO}_{2}\right)$ & & $6.36 \mathrm{E}-12$ & $5.64 \mathrm{E}-10$ \\
\hline Chlorine-36 & & $5.93 \mathrm{E}-09$ & $8.18 \mathrm{E}-10$ \\
\hline Selenium-79 & & $2.66 \mathrm{E}-09$ & $2.35 \mathrm{E}-09$ \\
\hline \multirow[t]{2}{*}{ Strontium-90 } & & $6.47 \mathrm{E}-08$ & $3.85 \mathrm{E}-08$ \\
\hline & Yttrium-90 & $2.28 \mathrm{E}-09$ & $2.91 \mathrm{E}-09$ \\
\hline Technetium-99 & & $2.25 \mathrm{E}-09$ & $3.95 E-10$ \\
\hline \multirow[t]{3}{*}{ Tin-126 } & & $2.69 \mathrm{E}-08$ & $5.27 \mathrm{E}-09$ \\
\hline & Antimony-126m & $9.17 \mathrm{E}-12$ & $2.54 \mathrm{E}-11$ \\
\hline & Antimony-126 & 3.17E-09 & $2.89 \mathrm{E}-09$ \\
\hline lodine-129 & & $4.69 \mathrm{E}-08$ & $7.46 \mathrm{E}-08$ \\
\hline Cesium-135 & & $1.23 \mathrm{E}-09$ & $1.91 \mathrm{E}-09$ \\
\hline \multirow[t]{2}{*}{ Cesium-137 } & & 8.63E-09 & $1.35 E-08$ \\
\hline & Barium-137m & - & - \\
\hline \multicolumn{4}{|c|}{ Thorium Series (4n) } \\
\hline Plutonium-240 & & $1.16 \mathrm{E}-04$ & $9.56 \mathrm{E}-07$ \\
\hline Uranium-236 & & $3.39 \mathrm{E}-05$ & $7.26 \mathrm{E}-08$ \\
\hline Thorium-232 & & 4.43E-04 & $7.38 \mathrm{E}-07$ \\
\hline \multirow[t]{2}{*}{ Radium-228 } & & $1.29 \mathrm{E}-06$ & $3.88 \mathrm{E}-07$ \\
\hline & Actinium-228 & 8.33E-08 & $5.85 \mathrm{E}-10$ \\
\hline Uranium-232 & & $1.78 \mathrm{E}-04$ & $3.54 \mathrm{E}-07$ \\
\hline \multirow[t]{8}{*}{ Thorium-228 } & & $9.23 \mathrm{E}-05$ & $1.07 \mathrm{E}-07$ \\
\hline & Radium-224 & $8.53 \mathrm{E}-07$ & $9.89 \mathrm{E}-08$ \\
\hline & Radon-220 & - & - \\
\hline & Polonium-216 & - & - \\
\hline & Lead-212 & $4.56 \mathrm{E}-08$ & $1.23 E-08$ \\
\hline & Bismuth-212 & $5.83 \mathrm{E}-09$ & $2.87 \mathrm{E}-10$ \\
\hline & Polonium-212 & - & - \\
\hline & Thallium-208 & - & - \\
\hline \multicolumn{4}{|c|}{ Neptunium Series $(4 n+1)$} \\
\hline Americium-241 & & $1.20 \mathrm{E}-04$ & $9.84 \mathrm{E}-07$ \\
\hline \multirow[t]{2}{*}{ Neptunium-237 } & & $1.46 \mathrm{E}-04$ & $1.20 \mathrm{E}-06$ \\
\hline & Protactinium-233 & $2.58 \mathrm{E}-09$ & $9.81 \mathrm{E}-10$ \\
\hline Uranium-233 & & $3.66 \mathrm{E}-05$ & $7.81 \mathrm{E}-08$ \\
\hline \multirow[t]{9}{*}{ Thorium-229 } & & $5.80 \mathrm{E}-04$ & $9.54 \mathrm{E}-07$ \\
\hline & Radium-225 & $2.10 \mathrm{E}-06$ & $1.04 \mathrm{E}-07$ \\
\hline & Actinium-225 & $2.92 \mathrm{E}-06$ & $3.00 E-08$ \\
\hline & Francium-221 & - & - \\
\hline & Astatine-217 & - & - \\
\hline & Bismuth-213 & 4.63E-09 & $1.95 \mathrm{E}-10$ \\
\hline & Polonium-213 & - & - \\
\hline & Thallium-209 & - & - \\
\hline & Lead-209 & $2.56 \mathrm{E}-11$ & $5.75 \mathrm{E}-11$ \\
\hline
\end{tabular}


Table 4.1-3. Dose Coefficients for Inhalation and Ingestion of Radionuclides of Interest Consistent with ICRP 30 Dosimetric Methods (Continued)

\begin{tabular}{|c|c|c|c|}
\hline \multirow[b]{2}{*}{ Primary Radionuclide } & \multirow{2}{*}{$\begin{array}{c}\text { Short-lived Decay } \\
\text { Product }\end{array}$} & \multicolumn{2}{|c|}{ Dose Coefficients (Sv/Bq) } \\
\hline & & Inhalation & Ingestion \\
\hline \multicolumn{4}{|c|}{ Uranium Series $(4 n+2)$} \\
\hline Plutonium-242 & & $1.11 \mathrm{E}-04$ & $9.08 \mathrm{E}-07$ \\
\hline \multirow[t]{4}{*}{ Uranium-238 } & & $3.20 \mathrm{E}-05$ & $6.88 \mathrm{E}-08$ \\
\hline & Thorium-234 & $9.47 \mathrm{E}-09$ & $3.69 E-09$ \\
\hline & Protactinium-234m & - & - \\
\hline & Protactinium-234 & $2.20 \mathrm{E}-10$ & $5.84 \mathrm{E}-10$ \\
\hline Plutonium-238 & & 1.06E-04 & $8.65 E-07$ \\
\hline Uranium-234 & & $3.58 \mathrm{E}-05$ & $7.66 \mathrm{E}-08$ \\
\hline Thorium-230 & & $8.80 \mathrm{E}-05$ & $1.48 \mathrm{E}-07$ \\
\hline \multirow[t]{8}{*}{ Radium-226 } & & $2.32 \mathrm{E}-06$ & $3.58 \mathrm{E}-07$ \\
\hline & Radon-222 & - & - \\
\hline & Polonium-218 & - & - \\
\hline & Lead-214 & $2.11 \mathrm{E}-09$ & $1.69 \mathrm{E}-10$ \\
\hline & Astatine-218 & - & - \\
\hline & Bismuth-214 & $1.78 \mathrm{E}-09$ & $7.64 \mathrm{E}-11$ \\
\hline & Polonium-214 & - & - \\
\hline & Thallium-210 & - & - \\
\hline \multirow[t]{3}{*}{ Lead-210 } & & 3.67E-06 & $1.45 \mathrm{E}-06$ \\
\hline & Bismuth-210 & $5.29 \mathrm{E}-08$ & 1.73E-09 \\
\hline & Polonium-210 & $2.54 \mathrm{E}-06$ & $5.14 \mathrm{E}-07$ \\
\hline \multicolumn{4}{|c|}{ Actinium Series $(4 n+3)$} \\
\hline \multirow[t]{2}{*}{ Americium-243 } & & 1.19E-04 & $9.79 \mathrm{E}-07$ \\
\hline & Neptunium-239 & $6.78 \mathrm{E}-10$ & $8.82 \mathrm{E}-10$ \\
\hline Plutonium-239 & & 1.16E-04 & $9.56 \mathrm{E}-07$ \\
\hline \multirow[t]{2}{*}{ Uranium-235 } & & $3.32 \mathrm{E}-05$ & $7.19 \mathrm{E}-08$ \\
\hline & Thorium-231 & $2.37 \mathrm{E}-10$ & $3.65 E-10$ \\
\hline Protactinium-231 & & $3.47 \mathrm{E}-04$ & $2.86 \mathrm{E}-06$ \\
\hline \multirow[t]{10}{*}{ Actinium-227 } & & $1.81 \mathrm{E}-03$ & $3.80 \mathrm{E}-06$ \\
\hline & Thorium-227 & 4.37E-06 & $1.03 \mathrm{E}-08$ \\
\hline & Francium-223 & $1.68 \mathrm{E}-09$ & $2.33 \mathrm{E}-09$ \\
\hline & Radium-223 & $2.12 \mathrm{E}-06$ & $1.78 \mathrm{E}-07$ \\
\hline & Radon-219 & - & - \\
\hline & Polonium-215 $\left({ }^{215} \mathrm{Po}\right)$ & - & - \\
\hline & Lead-211 & $2.35 \mathrm{E}-09$ & $1.42 \mathrm{E}-10$ \\
\hline & Bismuth-211 & - & - \\
\hline & Thallium-207 & - & - \\
\hline & Polonium-211 & - & - \\
\hline
\end{tabular}


Table 4.1-4. Dose Coefficients for Inhalation and Ingestion of Radionuclides of Interest from ICRP 72

\begin{tabular}{|c|c|c|c|}
\hline \multirow[b]{2}{*}{ Primary Radionuclide } & \multirow{2}{*}{$\begin{array}{c}\text { Short-lived Decay } \\
\text { Product }\end{array}$} & \multicolumn{2}{|c|}{ Dose Coefficients (Sv/Bq) } \\
\hline & & Inhalation & Ingestion \\
\hline Carbon-14 (as $\left.\mathrm{CO}_{2}\right)$ & & $6.2 \mathrm{E}-12$ & $5.8 \mathrm{E}-10$ \\
\hline Chlorine-36 & & 7.3E-09 & 9.3E-10 \\
\hline Selenium-79 & & $6.8 \mathrm{E}-09$ & 2.9E-09 \\
\hline \multirow[t]{2}{*}{ Strontium-90 } & & 1.6E-07 & $2.8 \mathrm{E}-08$ \\
\hline & Yttrium-90 & 1.5E-09 & 2.7E-09 \\
\hline Technetium-99 & & 1.3E-08 & $6.4 \mathrm{E}-10$ \\
\hline \multirow[t]{3}{*}{ Tin-126 } & & $2.8 \mathrm{E}-08$ & 4.7E-09 \\
\hline & Antimony-126m & $2.0 \mathrm{E}-11$ & $3.6 \mathrm{E}-11$ \\
\hline & Antimony-126 & $3.2 E-09$ & 2.4E-09 \\
\hline lodine-129 & & $3.6 \mathrm{E}-08$ & 1.1E-07 \\
\hline Cesium-135 & & 8.6E-09 & 2.0E-09 \\
\hline \multirow[t]{2}{*}{ Cesium-137 } & & 3.9E-08 & 1.3E-08 \\
\hline & Barium-137m & - & - \\
\hline \multicolumn{4}{|c|}{ Thorium Series (4n) } \\
\hline Plutonium-240 & & $1.2 \mathrm{E}-04$ & $2.5 \mathrm{E}-07$ \\
\hline Uranium-236 & & 8.7E-06 & 4.7E-08 \\
\hline Thorium-232 & & 1.1E-04 & 2.3E-07 \\
\hline \multirow[t]{2}{*}{ Radium-228 } & & 1.6E-05 & 6.9E-07 \\
\hline & Actinium-228 & $2.5 \mathrm{E}-08$ & 4.3E-10 \\
\hline Uranium-232 & & 3.7E-05 & 3.3E-07 \\
\hline \multirow[t]{8}{*}{ Thorium-228 } & & $4.0 \mathrm{E}-05$ & $7.2 \mathrm{E}-08$ \\
\hline & Radium-224 & $3.4 \mathrm{E}-06$ & $6.5 \mathrm{E}-08$ \\
\hline & Radon-220 & - & - \\
\hline & Polonium-216 & - & - \\
\hline & Lead-212 & $1.9 \mathrm{E}-07$ & 6.0E-09 \\
\hline & Bismuth-212 & $3.1 \mathrm{E}-08$ & $2.6 \mathrm{E}-10$ \\
\hline & Polonium-212 & - & - \\
\hline & Thallium-208 & - & - \\
\hline \multicolumn{4}{|c|}{ Neptunium Series $(4 n+1)$} \\
\hline Americium-241 & & 9.6E-05 & $2.0 \mathrm{E}-07$ \\
\hline \multirow[t]{2}{*}{ Neptunium-237 } & & 5.0E-05 & 1.1E-07 \\
\hline & Protactinium-233 & 3.9E-09 & 8.7E-10 \\
\hline Uranium-233 & & $9.6 \mathrm{E}-06$ & $5.1 \mathrm{E}-08$ \\
\hline \multirow[t]{9}{*}{ Thorium-229 } & & $2.4 \mathrm{E}-04$ & 4.9E-07 \\
\hline & Radium-225 & 7.7E-06 & 9.9E-08 \\
\hline & Actinium-225 & $8.5 E-06$ & $2.4 \mathrm{E}-08$ \\
\hline & Francium-221 & - & - \\
\hline & Astatine-217 & - & - \\
\hline & Bismuth-213 & 3.0E-08 & $2.0 \mathrm{E}-10$ \\
\hline & Polonium-213 & - & - \\
\hline & Thallium-209 & - & - \\
\hline & Lead-209 & $6.1 \mathrm{E}-11$ & 5.7E-11 \\
\hline
\end{tabular}


Table 4.1-4. Dose Coefficients for Inhalation and Ingestion of Radionuclides of Interest from ICRP 72 (Continued)

\begin{tabular}{|c|c|c|c|}
\hline \multirow[b]{2}{*}{ Primary Radionuclide } & \multirow{2}{*}{$\begin{array}{c}\text { Short-lived Decay } \\
\text { Product }\end{array}$} & \multicolumn{2}{|c|}{ Dose Coefficients (Sv/Bq) } \\
\hline & & Inhalation & Ingestion \\
\hline \multicolumn{4}{|c|}{ Uranium Series $(4 n+2)$} \\
\hline Plutonium-242 & & $1.1 \mathrm{E}-04$ & 2.4E-07 \\
\hline \multirow[t]{4}{*}{ Uranium-238 } & & $8.0 \mathrm{E}-06$ & $4.5 \mathrm{E}-08$ \\
\hline & Thorium-234 & 7.7E-09 & $3.4 \mathrm{E}-09$ \\
\hline & Protactinium-234m & - & - \\
\hline & Protactinium-234 & $4.0 \mathrm{E}-10$ & $5.1 \mathrm{E}-10$ \\
\hline Plutonium-238 & & $1.1 \mathrm{E}-04$ & $2.3 \mathrm{E}-07$ \\
\hline Uranium-234 & & 9.4E-06 & $4.9 \mathrm{E}-08$ \\
\hline Thorium-230 & & 1.0E-04 & 2.1E-07 \\
\hline \multirow[t]{8}{*}{ Radium-226 } & & $9.5 \mathrm{E}-06$ & $2.8 \mathrm{E}-07$ \\
\hline & Radon-222 & - & - \\
\hline & Polonium-218 & - & - \\
\hline & Lead-214 & $1.5 \mathrm{E}-08$ & $1.4 \mathrm{E}-10$ \\
\hline & Astatine-218 & - & $-^{a}$ \\
\hline & Bismuth-214 & $1.4 \mathrm{E}-08$ & 1.1E-10 \\
\hline & Polonium-214 & - & - \\
\hline & Thallium-210 & - & - \\
\hline \multirow[t]{3}{*}{ Lead-210 } & & $5.6 \mathrm{E}-06$ & $6.9 \mathrm{E}-07$ \\
\hline & Bismuth-210 & $9.3 \mathrm{E}-08$ & 1.3E-09 \\
\hline & Polonium-210 & $4.3 \mathrm{E}-06$ & $1.2 \mathrm{E}-06$ \\
\hline \multicolumn{4}{|c|}{ Actinium Series $(4 n+3)$} \\
\hline \multirow[t]{2}{*}{ Americium-243 } & & 9.6E-05 & 2.0E-07 \\
\hline & Neptunium-239 & $1.0 \mathrm{E}-09$ & $8.0 \mathrm{E}-10$ \\
\hline Plutonium-239 & & $1.2 \mathrm{E}-04$ & $2.5 \mathrm{E}-07$ \\
\hline \multirow[t]{2}{*}{ Uranium-235 } & & $8.5 \mathrm{E}-06$ & 4.7E-08 \\
\hline & Thorium-231 & $3.3 \mathrm{E}-10$ & $3.4 \mathrm{E}-10$ \\
\hline Protactinium-231 & & 1.4E-04 & 7.1E-07 \\
\hline \multirow[t]{10}{*}{ Actinium-227 } & & $5.5 \mathrm{E}-04$ & $1.1 \mathrm{E}-06$ \\
\hline & Thorium-227 & 1.0E-05 & $8.8 \mathrm{E}-09$ \\
\hline & Francium-223 & $8.9 \mathrm{E}-10$ & $2.4 \mathrm{E}-09$ \\
\hline & Radium-223 & 8.7E-06 & $1.0 \mathrm{E}-07$ \\
\hline & Radon-219 & - & - \\
\hline & Polonium-215 & - & - \\
\hline & Lead-211 & $1.2 \mathrm{E}-08$ & $1.8 \mathrm{E}-10$ \\
\hline & Bismuth-211 & - & - \\
\hline & Thallium-207 & - & - \\
\hline & Polonium-211 & - & - \\
\hline
\end{tabular}


Nominal Performance Biosphere Dose Conversion Factor Analysis

Table 4.1-5. Dose Coefficients for Exposure to Contaminated Soil for Radionuclides of Interest Consistent with ICRP 30 Dosimetric Methods

\begin{tabular}{|c|c|c|}
\hline Primary Radionuclide & Short-lived Decay Product & $\begin{array}{l}\text { Dose Coefficient for Infinite } \\
\left.\text { Depth (Sv/s per } \mathrm{Bq} / \mathrm{m}^{3}\right)\end{array}$ \\
\hline Carbon-14 & & $7.20 \mathrm{E}-23$ \\
\hline Chlorine-36 & & $1.28 \mathrm{E}-20$ \\
\hline Selenium-79 & & 9.96E-23 \\
\hline \multirow[t]{2}{*}{ Strontium-90 } & & 3.77E-21 \\
\hline & Yttrium-90 & $1.28 \mathrm{E}-19$ \\
\hline Technetium-99 & & $6.72 \mathrm{E}-22$ \\
\hline \multirow[t]{3}{*}{ Tin-126 } & & $7.89 \mathrm{E}-19$ \\
\hline & Antimony-126m & $4.98 \mathrm{E}-17$ \\
\hline & Antimony-126 & $9.16 \mathrm{E}-17$ \\
\hline lodine-129 & & $6.93 \mathrm{E}-20$ \\
\hline Cesium-135 & & $2.05 \mathrm{E}-22$ \\
\hline \multirow[t]{2}{*}{ Cesium-137 } & & $4.02 \mathrm{E}-21$ \\
\hline & Barium-137m & $1.93 \mathrm{E}-17$ \\
\hline \multicolumn{3}{|c|}{ Thorium Series (4n) } \\
\hline Plutonium-240 & & $7.85 \mathrm{E}-22$ \\
\hline Uranium-236 & & $1.15 \mathrm{E}-21$ \\
\hline Thorium-232 & & $2.79 \mathrm{E}-21$ \\
\hline \multirow[t]{2}{*}{ Radium-228 } & & $0.00 \mathrm{E}+00$ \\
\hline & Actinium-228 & $3.20 \mathrm{E}-17$ \\
\hline Uranium-232 & & $4.83 \mathrm{E}-21$ \\
\hline \multirow[t]{8}{*}{ Thorium-228 } & & $4.25 \mathrm{E}-20$ \\
\hline & Radium-224 & $2.74 \mathrm{E}-19$ \\
\hline & Radon-220 & $1.23 \mathrm{E}-20$ \\
\hline & Polonium-216 & $5.58 \mathrm{E}-22$ \\
\hline & Lead-212 & 3.77E-18 \\
\hline & Bismuth-212 & $6.27 \mathrm{E}-18$ \\
\hline & Polonium-212 & $0.00 \mathrm{E}+00$ \\
\hline & Thallium-208 & $1.23 \mathrm{E}-16$ \\
\hline \multicolumn{3}{|c|}{ Neptunium Series $(4 n+1)$} \\
\hline Americium-241 & & $2.34 \mathrm{E}-19$ \\
\hline \multirow[t]{2}{*}{ Neptunium-237 } & & 4.17E-19 \\
\hline & Protactinium-233 & $5.46 \mathrm{E}-18$ \\
\hline Uranium-233 & & $7.48 \mathrm{E}-21$ \\
\hline \multirow[t]{9}{*}{ Thorium-229 } & & $1.72 \mathrm{E}-18$ \\
\hline & Radium-225 & $5.90 \mathrm{E}-20$ \\
\hline & Actinium-225 & $3.41 \mathrm{E}-19$ \\
\hline & Francium-221 & $8.22 \mathrm{E}-19$ \\
\hline & Astatine-217 & $9.49 \mathrm{E}-21$ \\
\hline & Bismuth-213 & $4.10 \mathrm{E}-18$ \\
\hline & Polonium-213 & $0.00 \mathrm{E}+00$ \\
\hline & Thallium-209 & $6.92 \mathrm{E}-17$ \\
\hline & Lead-209 & $4.14 \mathrm{E}-21$ \\
\hline
\end{tabular}


Table 4.1-5. Dose Coefficients for Exposure to Contaminated Soil for Radionuclides of Interest Consistent with ICRP 30 Dosimetric Methods (Continued)

\begin{tabular}{|c|c|c|}
\hline Primary Radionuclide & Short-lived Decay Product & $\begin{array}{l}\text { Dose Coefficient for Infinite } \\
\text { Depth }\left(\mathrm{Sv} / \mathrm{s} \text { per } \mathrm{Bq} / \mathrm{m}^{3}\right)\end{array}$ \\
\hline \multicolumn{3}{|c|}{ Uranium Series $(4 n+2)$} \\
\hline Plutonium-242 & & $6.85 \mathrm{E}-22$ \\
\hline \multirow[t]{4}{*}{ Uranium-238 } & & $5.52 \mathrm{E}-22$ \\
\hline & Thorium-234 & $1.29 \mathrm{E}-19$ \\
\hline & Protactinium-234m & $4.80 \mathrm{E}-19$ \\
\hline & Protactinium-234 & $6.18 \mathrm{E}-17$ \\
\hline Plutonium-238 & & $8.10 \mathrm{E}-22$ \\
\hline Uranium-234 & & $2.15 \mathrm{E}-21$ \\
\hline Thorium-230 & & $6.47 \mathrm{E}-21$ \\
\hline \multirow[t]{8}{*}{ Radium-226 } & & $1.70 \mathrm{E}-19$ \\
\hline & Radon-222 & $1.26 \mathrm{E}-20$ \\
\hline & Polonium-218 & $3.02 \mathrm{E}-22$ \\
\hline & Lead-214 & $7.18 \mathrm{E}-18$ \\
\hline & Astatine-218 & $3.13 \mathrm{E}-20$ \\
\hline & Bismuth-214 & $5.25 \mathrm{E}-17$ \\
\hline & Polonium-214 & $2.75 \mathrm{E}-21$ \\
\hline & Thallium-210 & - \\
\hline \multirow[t]{3}{*}{ Lead-210 } & & $1.31 \mathrm{E}-20$ \\
\hline & Bismuth-210 & $1.93 E-20$ \\
\hline & Polonium-210 & $2.80 \mathrm{E}-22$ \\
\hline \multicolumn{3}{|c|}{ Actinium Series $(4 n+3)$} \\
\hline \multirow[t]{2}{*}{ Americium-243 } & & $7.60 \mathrm{E}-19$ \\
\hline & Neptunium-239 & $4.03 E-18$ \\
\hline Plutonium-239 & & $1.58 \mathrm{E}-21$ \\
\hline \multirow[t]{2}{*}{ Uranium-235 } & & $3.86 \mathrm{E}-18$ \\
\hline & Thorium-231 & $1.95 \mathrm{E}-19$ \\
\hline Protactinium-231 & & $1.02 \mathrm{E}-18$ \\
\hline \multirow[t]{10}{*}{ Actinium-227 } & & $2.65 \mathrm{E}-21$ \\
\hline & Thorium-227 & $2.79 \mathrm{E}-18$ \\
\hline & Francium-223 & $1.06 \mathrm{E}-18$ \\
\hline & Radium-223 & $3.23 E-18$ \\
\hline & Radon-219 & $1.65 \mathrm{E}-18$ \\
\hline & Polonium-215 & $5.44 \mathrm{E}-21$ \\
\hline & Lead-211 & $1.64 \mathrm{E}-18$ \\
\hline & Bismuth-211 & 1.37E-18 \\
\hline & Thallium-207 & $1.06 \mathrm{E}-19$ \\
\hline & Polonium-211 & $2.55 \mathrm{E}-19$ \\
\hline \multicolumn{3}{|c|}{ Source: $\quad$ MO0407SPACRBSM.002 [DIRS 170677]. } \\
\hline \multicolumn{3}{|c|}{$\begin{array}{ll}\text { NOTES: } & 1 \mathrm{~Sv}=100 \text { rem } \\
& 1 \mathrm{Ci}=3.7 \times 10^{10} \mathrm{~Bq}\end{array}$} \\
\hline
\end{tabular}


Table 4.1-6. Dose Coefficients for Exposure to Contaminated Soil for Radionuclides of Interest Consistent with ICRP 72 Dosimetric Methods

\begin{tabular}{|c|c|c|}
\hline Primary Radionuclide & Short-lived Decay Product & $\begin{array}{l}\text { Dose Coefficient for Infinite } \\
\left.\text { Depth (Sv/s per } \mathrm{Bq} / \mathrm{m}^{3}\right)\end{array}$ \\
\hline Carbon-14 & & $5.88 \mathrm{E}-23$ \\
\hline Chlorine-36 & & 1.33E-20 \\
\hline Selenium-79 & & 8.19E-23 \\
\hline \multirow[t]{2}{*}{ Strontium-90 } & & $3.46 \mathrm{E}-21$ \\
\hline & Yttrium-90 & $2.15 \mathrm{E}-19$ \\
\hline Technetium-99 & & $5.80 \mathrm{E}-22$ \\
\hline \multirow[t]{3}{*}{ Tin-126 } & & $6.96 \mathrm{E}-19$ \\
\hline & Antimony-126m & 4.67E-17 \\
\hline & Antimony-126 & $8.60 \mathrm{E}-17$ \\
\hline lodine-129 & & $5.11 \mathrm{E}-20$ \\
\hline Cesium-135 & & $1.72 \mathrm{E}-22$ \\
\hline \multirow[t]{2}{*}{ Cesium-137 } & & 4.47E-21 \\
\hline & Barium-137m & $1.81 \mathrm{E}-17$ \\
\hline \multicolumn{3}{|c|}{ Thorium Series $(4 n)$} \\
\hline Plutonium-240 & & $6.02 \mathrm{E}-22$ \\
\hline Uranium-236 & & 9.51E-22 \\
\hline Thorium-232 & & $2.44 \mathrm{E}-21$ \\
\hline \multirow[t]{2}{*}{ Radium-228 } & & $0.00 \mathrm{E}+00$ \\
\hline & Actinium-228 & $3.03 \mathrm{E}-17$ \\
\hline Uranium-232 & & 4.24E-21 \\
\hline \multirow[t]{8}{*}{ Thorium-228 } & & 3.84E-20 \\
\hline & Radium-224 & $2.53 \mathrm{E}-19$ \\
\hline & Radon-220 & 1.15E-20 \\
\hline & Polonium-216 & $5.26 \mathrm{E}-22$ \\
\hline & Lead-212 & $3.46 \mathrm{E}-18$ \\
\hline & Bismuth-212 & $5.96 \mathrm{E}-18$ \\
\hline & Polonium-212 & $0.00 \mathrm{E}+00$ \\
\hline & Thallium-208 & 1.17E-16 \\
\hline \multicolumn{3}{|c|}{ Neptunium Series $(4 n+1)$} \\
\hline Americium-241 & & $1.99 \mathrm{E}-19$ \\
\hline \multirow[t]{2}{*}{ Neptunium-237 } & & $3.72 \mathrm{E}-19$ \\
\hline & Protactinium-233 & $5.04 \mathrm{E}-18$ \\
\hline Uranium-233 & & 6.77E-21 \\
\hline \multirow[t]{9}{*}{ Thorium-229 } & & $1.55 \mathrm{E}-18$ \\
\hline & Radium-225 & 4.61E-20 \\
\hline & Actinium-225 & 3.09E-19 \\
\hline & Francium-221 & 7.56E-19 \\
\hline & Astatine-217 & $8.86 \mathrm{E}-21$ \\
\hline & Bismuth-213 & $3.84 \mathrm{E}-18$ \\
\hline & Polonium-213 & $0.00 \mathrm{E}+00$ \\
\hline & Thallium-209 & $6.56 \mathrm{E}-17$ \\
\hline & Lead-209 & $4.03 E-21$ \\
\hline
\end{tabular}


Table 4.1-6. Dose Coefficients for Exposure to Contaminated Soil for Radionuclides of Interest Consistent with ICRP 72 Dosimetric Methods (Continued)

\begin{tabular}{|c|c|c|}
\hline Primary Radionuclide & Short-lived Decay Product & $\begin{array}{l}\text { Dose Coefficient for Infinite } \\
\text { Depth (Sv/s per Bq/ } \mathrm{m}^{3} \text { ) }\end{array}$ \\
\hline \multicolumn{3}{|c|}{ Uranium Series $(4 n+2)$} \\
\hline Plutonium-242 & & $5.31 \mathrm{E}-22$ \\
\hline \multirow{4}{*}{ Uranium-238 } & & 4.26E-22 \\
\hline & Thorium-234 & 1.14E-19 \\
\hline & Protactinium-234m & $5.28 \mathrm{E}-19$ \\
\hline & Protactinium-234 & $5.83 \mathrm{E}-17$ \\
\hline Plutonium-238 & & $6.24 \mathrm{E}-22$ \\
\hline Uranium-234 & & 1.84E-21 \\
\hline Thorium-230 & & 5.73E-21 \\
\hline \multirow[t]{8}{*}{ Radium-226 } & & 1.56E-19 \\
\hline & Radon-222 & 1.17E-20 \\
\hline & Polonium-218 & $2.85 E-22$ \\
\hline & Lead-214 & $6.65 \mathrm{E}-18$ \\
\hline & Astatine-218 & $2.61 \mathrm{E}-20$ \\
\hline & Bismuth-214 & $5.00 \mathrm{E}-17$ \\
\hline & Polonium-214 & 2.59E-21 \\
\hline & Thallium-210 & - \\
\hline \multirow[t]{3}{*}{ Lead-210 } & & 1.06E-20 \\
\hline & Bismuth-210 & $2.92 E-20$ \\
\hline & Polonium-210 & 2.64E-22 \\
\hline \multicolumn{3}{|c|}{ Actinium Series $(4 n+3)$} \\
\hline \multirow[t]{2}{*}{ Americium-243 } & & $6.65 E-19$ \\
\hline & Neptunium-239 & 3.69E-18 \\
\hline Plutonium-239 & & $1.41 \mathrm{E}-21$ \\
\hline \multirow[t]{2}{*}{ Uranium-235 } & & 3.53E-18 \\
\hline & Thorium-231 & $1.72 \mathrm{E}-19$ \\
\hline Protactinium-231 & & 9.44E-19 \\
\hline \multirow[t]{10}{*}{ Actinium-227 } & & 2.39E-21 \\
\hline & Thorium-227 & $2.57 \mathrm{E}-18$ \\
\hline & Francium-223 & $9.70 \mathrm{E}-19$ \\
\hline & Radium-223 & 2.96E-18 \\
\hline & Radon-219 & $1.53 E-18$ \\
\hline & Polonium-215 & $5.06 \mathrm{E}-21$ \\
\hline & Lead-211 & $1.56 \mathrm{E}-18$ \\
\hline & Bismuth-211 & $1.27 \mathrm{E}-18$ \\
\hline & Thallium-207 & 1.23E-19 \\
\hline & Polonium-211 & $2.40 \mathrm{E}-19$ \\
\hline
\end{tabular}




\subsubsection{Direct Inputs Used for Calculation of Conversion Factors for Groundwater Protection Standards}

Conversion factors for beta- and photon- emitting radionuclides were calculated using the dose coefficients from Federal Guidance Report No. 11 (FGR 11) (DOE 2004 [DIRS 169713]) and from ICRP Publication 72 (ICRP 1999 [DIRS 153114]) for the dosimetric methods consistent with ICRP 30 and ICRP 72, respectively. The values consistent with ICRP 30 dosimetric methods are presented in this report for comparison purposes.

The dose coefficients are considered appropriate for use in this analysis for the following reasons. Dose coefficients tabulated in FGR No. 11 allow calculating committed effective dose equivalent, as defined in 10 CFR 63.2 [DIRS 173164] and are, therefore, appropriate for this analysis. Based on the reliability of the input source, status and purpose of the issuing organizations (U.S. Environmental Protection Agency), and use of the information by the scientific community, the dose coefficients in FGR 11 are considered established fact, consistent with the definition provided in LP-3.15Q-BSC, Managing Technical Product Inputs. Dose coefficients for ingestion were obtained from the on-line interactive database containing FGR 11 dose coefficients, which is available from the Dose and Risk Resources Web Page maintained by the DOE Office of Environmental Policy and Guidance (DOE 2004 [DIRS 169713]). (Note that although the date for this reference appears to be relatively recent, the values included in FGR 11 were developed in the late 1980s. Consistent with the format of the references, the citation uses the year that the data were accessed from the interactive database, rather than the year the data were developed.)

ICRP Publication 72 (ICRP 1996 [DIRS 152446]) contains the most current compilation of dose coefficients for inhalation and ingestion of radionuclides. Based on the reliability of the input source, charter and purpose of the ICRP, and use of the information by the scientific community, the dose coefficients in the publications of the ICRP are considered established fact, consistent with the definition provided in LP-3.15Q-BSC, Managing Technical Product Inputs.

Tables 4.1-7 and 4.1-8 contain organ ingestion dose coefficients for beta- and photon- emitting radionuclides consistent with the ICRP 30 and taken from ICRP 72, respectively. These inputs are used to calculate effective dose coefficients (see Equation 6.3-5) listed in Tables 6.3-5 and 6.3-6, for the dosimetric methods consistent with ICRP 30 and ICRP 72, respectively.

Data from FGR 12 (Eckerman and Ryman 1993 [DIRS 107684]) and the CRC Handbook of Chemistry and Physics (Lide and Frederikse 1997 [DIRS 103178]) regarding fundamental properties of nuclear transformations (such as the type and energy of radiation and the half-life of radionuclides) were also used. These data are considered established fact and are shown in Table 6.3-2. These inputs were used to calculate the number of alpha particles emitted per one decay of a primary radionuclide, as shown in Tables 6.3-2 and 6.3-3.

As before, for brevity, the parameters consistent with the ICRP 30 dosimetric methods are referred to as ICRP 30-based while the parameters consistent with the ICRP 72 dosimetric methods are referred to as ICRP 72-based. 
Table 4.1-7. ICRP 30-Based Ingestion Dose Coefficients for Beta-Photon Emitting Radionuclides Used in Calculation of Conversion Factors for Groundwater Protection Standards

\begin{tabular}{|c|c|c|c|c|c|c|c|c|c|c|c|}
\hline Organ/Tissue & C-14 & $\mathrm{Cl}-36$ & Se-79 & Sr-90 & Y-90 & Tc-99 & Sn-126 & Sb-126m & Sb-126 & I-129 & Cs-135 \\
\hline Adrenal & $5.64 \mathrm{E}-10$ & 7.99E-10 & $9.06 \mathrm{E}-10$ & $1.51 \mathrm{E}-09$ & $1.26 \mathrm{E}-14$ & $6.04 \mathrm{E}-11$ & 8.92E-10 & $2.60 \mathrm{E}-12$ & $1.89 \mathrm{E}-10$ & $1.32 \mathrm{E}-10$ & 1.91E-09 \\
\hline Urinary Bladder Wall & $5.64 \mathrm{E}-10$ & $7.99 \mathrm{E}-10$ & $9.06 \mathrm{E}-10$ & $1.51 \mathrm{E}-09$ & $1.26 \mathrm{E}-14$ & $6.04 \mathrm{E}-11$ & $1.06 \mathrm{E}-09$ & $82 \mathrm{E}-12$ & $8.62 \mathrm{E}-10$ & 1.35E-10 & 1.91E-09 \\
\hline Bone S & $5.64 \mathrm{E}-10$ & 7.99E-10 & $9.06 \mathrm{E}-10$ & 4.19E-07 & 3.67E-13 & $6.04 \mathrm{E}-11$ & 5.06E-09 & $9.53 \mathrm{E}-13$ & $2.27 \mathrm{E}-10$ & $2.17 \mathrm{E}-10$ & $1.91 \mathrm{E}-09$ \\
\hline Brain & $5.64 \mathrm{E}-10$ & $7.99 \mathrm{E}-10$ & $9.06 \mathrm{E}-10$ & $0.00 \mathrm{E}+00$ & $1.26 \mathrm{E}-14$ & $6.04 \mathrm{E}-11$ & $7.28 \mathrm{E}-10$ & $3.21 \mathrm{E}-14$ & 1.06E-11 & $0.00 \mathrm{E}+00$ & $0.00 \mathrm{E}+00$ \\
\hline Breast & $5.64 \mathrm{E}-10$ & $7.99 \mathrm{E}-10$ & $9.06 \mathrm{E}-10$ & $1.51 \mathrm{E}-09$ & $1.27 \mathrm{E}-14$ & $6.04 \mathrm{E}-11$ & $7.96 \mathrm{E}-10$ & $2.06 \mathrm{E}-12$ & $3.53 \mathrm{E}-10$ & $3.31 \mathrm{E}-10$ & $1.91 \mathrm{E}-09$ \\
\hline Esophagus & $5.64 \mathrm{E}-10$ & $7.99 \mathrm{E}-10$ & $9.06 \mathrm{E}-10$ & $1.51 \mathrm{E}-09$ & $1.26 \mathrm{E}-14$ & $6.04 \mathrm{E}-11$ & $5.32 \mathrm{E}-10$ & $4.60 \mathrm{E}-13$ & $3.44 \mathrm{E}-11$ & $3.51 \mathrm{E}-10$ & 1.91E-09 \\
\hline Stomach Wall & $5.64 \mathrm{E}-10$ & 1.11E-09 & $9.70 \mathrm{E}-10$ & 1.75E-09 & 1.07E-09 & 3.39E-09 & 1.60E-09 & $2.64 \mathrm{E}-10$ & 1.14E-09 & $2.19 \mathrm{E}-10$ & 1.99E-09 \\
\hline Small & $5.64 \mathrm{E}-10$ & 7.99E-10 & $9.38 \mathrm{E}-10$ & $1.98 \mathrm{E}-09$ & $2.55 \mathrm{E}-09$ & $1.19 \mathrm{E}-10$ & 4.41E-09 & 7.03E-11 & 3.63E-09 & $1.41 \mathrm{E}-10$ & $1.91 \mathrm{E}-09$ \\
\hline Upper Large Intestine Wall & $5.64 \mathrm{E}-10$ & $7.99 \mathrm{E}-10$ & 1.10E-09 & $5.74 \mathrm{E}-09$ & $1.32 \mathrm{E}-08$ & 4.05E-10 & $1.57 \mathrm{E}-08$ & $2.59 \mathrm{E}-11$ & $8.48 \mathrm{E}-09$ & $1.42 \mathrm{E}-10$ & 1.91E-09 \\
\hline Lower Large Intestine Wall & $5.64 \mathrm{E}-10$ & $7.99 \mathrm{E}-10$ & $1.48 \mathrm{E}-09$ & 1.97E-08 & $3.16 \mathrm{E}-08$ & 1.10E-09 & $4.33 \mathrm{E}-08$ & $7.59 \mathrm{E}-12$ & $1.94 \mathrm{E}-08$ & $1.39 \mathrm{E}-10$ & $1.91 \mathrm{E}-09$ \\
\hline Kidney & $5.64 \mathrm{E}-10$ & $7.99 \mathrm{E}-10$ & $1.25 \mathrm{E}-08$ & $1.51 \mathrm{E}-09$ & $1.26 \mathrm{E}-14$ & $6.04 \mathrm{E}-11$ & $8.19 \mathrm{E}-10$ & $4.53 \mathrm{E}-12$ & $3.93 \mathrm{E}-10$ & $1.34 \mathrm{E}-10$ & $1.91 \mathrm{E}-09$ \\
\hline Liver & $5.64 \mathrm{E}-10$ & $7.99 \mathrm{E}-10$ & $6.48 \mathrm{E}-09$ & $1.51 \mathrm{E}-09$ & $3.65 \mathrm{E}-13$ & $8.23 \mathrm{E}-11$ & $6.85 \mathrm{E}-10$ & $2.77 \mathrm{E}-12$ & $2.99 \mathrm{E}-10$ & $1.38 \mathrm{E}-10$ & $1.91 \mathrm{E}-09$ \\
\hline Lung & $5.64 \mathrm{E}-10$ & $7.99 \mathrm{E}-10$ & $9.06 \mathrm{E}-10$ & $1.51 \mathrm{E}-09$ & $1.26 \mathrm{E}-14$ & $6.04 \mathrm{E}-11$ & $5.99 \mathrm{E}-10$ & $1.88 \mathrm{E}-12$ & $6.85 \mathrm{E}-11$ & 1.65E-10 & $1.91 \mathrm{E}-09$ \\
\hline Muscle & $5.64 \mathrm{E}-10$ & $7.99 \mathrm{E}-10$ & $9.06 \mathrm{E}-10$ & $1.51 \mathrm{E}-09$ & $1.27 \mathrm{E}-14$ & $6.04 \mathrm{E}-11$ & 7.96E-10 & $2.06 \mathrm{E}-12$ & $3.53 \mathrm{E}-10$ & $3.31 \mathrm{E}-10$ & $1.91 \mathrm{E}-09$ \\
\hline Ovaries & $5.64 \mathrm{E}-10$ & $7.99 \mathrm{E}-10$ & $9.06 \mathrm{E}-10$ & $1 \mathrm{E}-09$ & $43 E-14$ & $6.04 \mathrm{E}-11$ & $\mathrm{E}-09$ & 29E-12 & 2.89E-09 & $38 \mathrm{E}-10$ & 1.91E-09 \\
\hline Pancreas & $5.64 \mathrm{E}-10$ & $7.99 \mathrm{E}-10$ & 3.89E-09 & $1.51 \mathrm{E}-09$ & $1.26 \mathrm{E}-14$ & $6.04 \mathrm{E}-11$ & $7.78 \mathrm{E}-10$ & $1.72 \mathrm{E}-11$ & $3.71 \mathrm{E}-10$ & $1.41 \mathrm{E}-10$ & 1.91E-09 \\
\hline Red Marrow & $5.64 \mathrm{E}-10$ & $7.99 \mathrm{E}-10$ & $9.06 \mathrm{E}-10$ & 1.94E-07 & $3.70 \mathrm{E}-13$ & $6.04 \mathrm{E}-11$ & $2.72 \mathrm{E}-09$ & $2.16 \mathrm{E}-12$ & $5.93 \mathrm{E}-10$ & $2.21 \mathrm{E}-10$ & $1.91 \mathrm{E}-09$ \\
\hline Skin & $5.64 \mathrm{E}-10$ & $7.99 \mathrm{E}-10$ & $9.06 \mathrm{E}-10$ & $1.51 \mathrm{E}-09$ & $1.26 \mathrm{E}-14$ & $6.04 \mathrm{E}-11$ & $5.48 \mathrm{E}-10$ & $8.23 \mathrm{E}-13$ & $1.32 \mathrm{E}-10$ & $2.11 \mathrm{E}-10$ & 1.91E-09 \\
\hline Spleen & $5.64 \mathrm{E}-10$ & $7.99 \mathrm{E}-10$ & 4.32E-09 & $1.51 \mathrm{E}-09$ & $1.26 \mathrm{E}-14$ & $6.04 \mathrm{E}-11$ & $7.28 \mathrm{E}-10$ & $9.71 \mathrm{E}-12$ & 3.03E-10 & $1.40 \mathrm{E}-10$ & $1.91 \mathrm{E}-09$ \\
\hline Testes & $5.64 \mathrm{E}-10$ & 7.99E-10 & $9.06 \mathrm{E}-10$ & 1.51E-09 & $1.26 \mathrm{E}-14$ & $6.04 \mathrm{E}-11$ & $6.80 \mathrm{E}-10$ & $2.95 \mathrm{E}-13$ & $2.79 \mathrm{E}-10$ & $1.29 \mathrm{E}-10$ & $1.91 \mathrm{E}-09$ \\
\hline Thymus & $5.64 \mathrm{E}-10$ & $7.99 \mathrm{E}-10$ & $9.06 \mathrm{E}-10$ & $1.51 \mathrm{E}-09$ & $1.26 \mathrm{E}-14$ & $6.04 \mathrm{E}-11$ & $5.32 \mathrm{E}-10$ & $4.60 \mathrm{E}-13$ & $3.44 \mathrm{E}-11$ & $3.51 \mathrm{E}-10$ & $1.91 \mathrm{E}-09$ \\
\hline Thyroid & $5.64 \mathrm{E}-10$ & $7.99 \mathrm{E}-10$ & $9.06 \mathrm{E}-10$ & $1.51 \mathrm{E}-09$ & $1.26 \mathrm{E}-14$ & 1.62E-09 & $5.51 \mathrm{E}-10$ & $1.73 \mathrm{E}-13$ & $1.74 \mathrm{E}-11$ & $2.48 \mathrm{E}-06$ & $1.91 \mathrm{E}-09$ \\
\hline Uterus & $5.64 \mathrm{E}-10$ & $7.99 \mathrm{E}-10$ & $9.06 \mathrm{E}-10$ & $1.51 \mathrm{E}-09$ & $1.26 \mathrm{E}-14$ & $6.04 \mathrm{E}-11$ & 1.27E-09 & $4.60 \mathrm{E}-12$ & $1.18 \mathrm{E}-09$ & $1.38 \mathrm{E}-10$ & 1.91E-09 \\
\hline Whole Body (h_E) & $5.64 \mathrm{E}-10$ & $8.18 \mathrm{E}-10$ & 2.35E-09 & 3.85E-08 & 2.91E-09 & $3.95 \mathrm{E}-10$ & 5.27E-09 & $2.54 \mathrm{E}-11$ & 2.89E-09 & 7.46E-08 & 1.91E-09 \\
\hline
\end{tabular}


Table 4.1-7. ICRP 30-Based Ingestion Dose Coefficients for Beta-Photon Emitting Radionuclides Used in Calculation of Conversion Factors for Groundwater Protection Standards (Continued)

\begin{tabular}{|c|c|c|c|c|c|c|c|c|c|c|c|}
\hline Organ/Tissue & Cs-137 & 3a-137m & Ra-228 & Ac-228 & $\mathrm{Pb}-212$ & Bi-212 & Tl-208 & $\mathrm{Pa}-233$ & Ra-225 & Bi-213 & Tl-209 \\
\hline Adrenal & $1.50 \mathrm{E}-08$ & $0 \mathrm{E}+00$ & $1.58 \mathrm{E}-07$ & $1.80 \mathrm{E}-11$ & $1.66 \mathrm{E}-09$ & $1.27 \mathrm{E}-11$ & $0.00 \mathrm{E}+00$ & $1.25 \mathrm{E}-11$ & 3.37E-08 & 4.86E-12 & $0.00 \mathrm{E}+00$ \\
\hline Urinary Bladder Wall & $1.41 \mathrm{E}-08$ & $0 \mathrm{E}+00$ & $1.57 \mathrm{E}-07$ & 4.09E-11 & $1.71 \mathrm{E}-09$ & $1.29 \mathrm{E}-11$ & $.00 \mathrm{E}+00$ & 7.58E-11 & 3.37E-08 & 4.84E-12 & $.00 \mathrm{E}+00$ \\
\hline Bone Surface & $1.26 \mathrm{E}-08$ & $00 \mathrm{E}+00$ & $5.82 \mathrm{E}-06$ & 3.01E-09 & $1.66 \mathrm{E}-07$ & $9.14 \mathrm{E}-12$ & $0.00 \mathrm{E}+00$ & $1.02 \mathrm{E}-10$ & $1.78 \mathrm{E}-06$ & 4.45E-12 & $.00 \mathrm{E}+00$ \\
\hline Brain & $0.00 \mathrm{E}+00$ & $.00 \mathrm{E}+00$ & $0.00 \mathrm{E}+00$ & $2.54 \mathrm{E}-13$ & $0.00 \mathrm{E}+00$ & $0.00 \mathrm{E}+00$ & $0.00 \mathrm{E}+00$ & $3.41 \mathrm{E}-13$ & $0.00 \mathrm{E}+00$ & $0.00 \mathrm{E}+00$ & $0.00 \mathrm{E}+00$ \\
\hline Breast & $1.24 \mathrm{E}-08$ & $.00 \mathrm{E}+00$ & $1.57 \mathrm{E}-07$ & $2.31 \mathrm{E}-11$ & 1.67E-09 & $1.18 \mathrm{E}-11$ & $0.00 \mathrm{E}+00$ & $2.71 \mathrm{E}-11$ & 3.37E-08 & 4.73E-12 & $.00 \mathrm{E}+00$ \\
\hline Esopha & $1.24 \mathrm{E}-08$ & $.00 \mathrm{E}+00$ & $1.57 \mathrm{E}-07$ & $3.06 \mathrm{E}-12$ & 1.63E-09 & 7.80E-12 & $0.00 \mathrm{E}+00$ & $1.41 \mathrm{E}-12$ & 3.37E-08 & 4.26E-12 & $.00 \mathrm{E}+00$ \\
\hline Stomach Wall & $1.39 \mathrm{E}-08$ & $.00 \mathrm{E}+00$ & $1.57 \mathrm{E}-07$ & $6.21 \mathrm{E}-10$ & $2.93 \mathrm{E}-09$ & 1.60E-09 & $0.00 \mathrm{E}+00$ & $2.92 \mathrm{E}-10$ & $3.38 \mathrm{E}-08$ & 1.31E-09 & $0.00 \mathrm{E}+00$ \\
\hline Small Intestine Wall & $1.44 \mathrm{E}-08$ & $.00 \mathrm{E}+00$ & $1.58 \mathrm{E}-07$ & 1.12E-09 & $5.76 \mathrm{E}-09$ & $1.08 \mathrm{E}-09$ & $0.00 \mathrm{E}+00$ & $8.02 \mathrm{E}-10$ & $3.41 \mathrm{E}-08$ & $7.46 \mathrm{E}-10$ & $0.00 \mathrm{E}+00$ \\
\hline Upper Large Intestine Wall & $1.42 \mathrm{E}-08$ & $0.00 \mathrm{E}+00$ & & & & & $0.00 \mathrm{E}+00$ & & & & $.00 \mathrm{E}+00$ \\
\hline Lower Large Intestine Wall & $1.44 \mathrm{E}-08$ & $0.00 \mathrm{E}+00$ & $1.78 \mathrm{E}-07$ & $1.90 \mathrm{E}-09$ & $1.99 \mathrm{E}-08$ & $1.27 \mathrm{E}-10$ & $0.00 \mathrm{E}+00$ & $1.02 \mathrm{E}-08$ & $6.39 \mathrm{E}-08$ & $1.04 \mathrm{E}-10$ & $0.00 \mathrm{E}+00$ \\
\hline Kidney & 1.37E-08 & $.00 \mathrm{E}+00$ & 1.57E-07 & $3.88 \mathrm{E}-11$ & $1.09 \mathrm{E}-08$ & 1.11E-09 & $0.00 \mathrm{E}+00$ & $3.40 \mathrm{E}-11$ & 3.37E-08 & $6.05 \mathrm{E}-10$ & $0.00 \mathrm{E}+00$ \\
\hline Liver & 1.36E-08 & $.00 \mathrm{E}+00$ & 1.57E-07 & $8.20 \mathrm{E}-10$ & $2.21 \mathrm{E}-08$ & 1.36E-11 & $0.00 \mathrm{E}+00$ & $2.41 \mathrm{E}-11$ & 3.37E-08 & $4.92 \mathrm{E}-12$ & $.00 \mathrm{E}+00$ \\
\hline Lung & 1.27E-08 & $.00 \mathrm{E}+00$ & $1.57 \mathrm{E}-07$ & 7.34E-12 & 1.63E-09 & $9.86 \mathrm{E}-12$ & $0.00 \mathrm{E}+00$ & $3.70 \mathrm{E}-12$ & 3.37E-08 & $4.56 \mathrm{E}-12$ & $0.00 \mathrm{E}+00$ \\
\hline Muscle & $1.24 \mathrm{E}-08$ & $.00 \mathrm{E}+00$ & $1.57 \mathrm{E}-07$ & $2.31 \mathrm{E}-11$ & 1.67E-09 & $1.18 \mathrm{E}-11$ & $0.00 \mathrm{E}+00$ & $2.71 \mathrm{E}-11$ & 3.37E-08 & $4.73 \mathrm{E}-12$ & $0.00 \mathrm{E}+00$ \\
\hline Ovaries & $1.29 \mathrm{E}-08$ & $0.00 \mathrm{E}+00$ & $1.58 \mathrm{E}-07$ & $1.79 \mathrm{E}-10$ & $1.96 \mathrm{E}-09$ & $2.76 \mathrm{E}-11$ & $0.00 \mathrm{E}+00$ & $2.58 \mathrm{E}-10$ & $3.37 \mathrm{E}-08$ & $6.17 \mathrm{E}-12$ & $0.00 \mathrm{E}+00$ \\
\hline Pancreas & tE-08 & $0.00 \mathrm{E}+00$ & $1.57 \mathrm{E}-07$ & $5.05 \mathrm{E}-11$ & $1.69 \mathrm{E}-09$ & $3.18 \mathrm{E}-11$ & $0.00 \mathrm{E}+00$ & 2.93E-11 & $3.37 \mathrm{E}-08$ & $7.69 \mathrm{E}-12$ & $0.00 \mathrm{E}+00$ \\
\hline Red M & $1.32 \mathrm{E}-08$ & $0.00 \mathrm{E}+00$ & $6.53 \mathrm{E}-07$ & $2.75 \mathrm{E}-10$ & $1.51 \mathrm{E}-08$ & 1.29E-11 & $0.00 \mathrm{E}+00$ & $6.89 \mathrm{E}-11$ & $1.68 \mathrm{E}-07$ & $4.89 \mathrm{E}-12$ & $0.00 \mathrm{E}+00$ \\
\hline Skin & 1.04E-08 & $0.00 \mathrm{E}+00$ & 1.57E-07 & $8.84 \mathrm{E}-12$ & 1.64E-09 & $8.86 \mathrm{E}-12$ & $0.00 \mathrm{E}+00$ & $8.58 \mathrm{E}-12$ & 3.37E-08 & 4.38E-12 & $0.00 \mathrm{E}+00$ \\
\hline Spleen & $1.37 \mathrm{E}-08$ & $0.00 \mathrm{E}+00$ & $1.57 \mathrm{E}-07$ & 3.37E-11 & 1.67E-09 & 2.16E-11 & $0.00 \mathrm{E}+00$ & $2.16 \mathrm{E}-11$ & 3.37E-08 & $6.19 \mathrm{E}-12$ & $0.00 \mathrm{E}+00$ \\
\hline Testes & $1.39 \mathrm{E}-08$ & $0.00 \mathrm{E}+00$ & $1.57 \mathrm{E}-07$ & $4.38 \mathrm{E}-11$ & $1.65 \mathrm{E}-09$ & 8.22E-12 & $0.00 \mathrm{E}+00$ & $2.10 \mathrm{E}-11$ & 3.37E-08 & $4.27 \mathrm{E}-12$ & $0.00 \mathrm{E}+00$ \\
\hline Thymus & $1.24 \mathrm{E}-08$ & $0.00 \mathrm{E}+00$ & $1.57 \mathrm{E}-07$ & $3.06 \mathrm{E}-12$ & 1.63E-09 & $7.80 \mathrm{E}-12$ & $0.00 \mathrm{E}+00$ & $1.41 \mathrm{E}-12$ & $3.37 \mathrm{E}-08$ & $4.26 \mathrm{E}-12$ & $0.00 \mathrm{E}+00$ \\
\hline Thyroid & $1.26 \mathrm{E}-08$ & $0.00 \mathrm{E}+00$ & 1.57E-07 & $9.39 \mathrm{E}-13$ & 1.62E-09 & $7.11 \mathrm{E}-12$ & $0.00 \mathrm{E}+00$ & $4.81 \mathrm{E}-13$ & 3.37E-08 & $4.20 \mathrm{E}-12$ & $0.00 \mathrm{E}+00$ \\
\hline Uterus & $1.44 \mathrm{E}-08$ & $0.00 \mathrm{E}+00$ & $1.57 \mathrm{E}-07$ & $8.43 \mathrm{E}-11$ & $1.78 \mathrm{E}-09$ & 2.30E-11 & $0.00 \mathrm{E}+00$ & 1.03E-10 & 3.37E-08 & $5.89 \mathrm{E}-12$ & $0.00 \mathrm{E}+00$ \\
\hline Whole Body (h_E) & $1.35 \mathrm{E}-08$ & $0.00 \mathrm{E}+00$ & 3.88E-07 & $5.85 \mathrm{E}-10$ & 1.23E-08 & 2.87E-10 & $0.00 \mathrm{E}+00$ & $9.81 \mathrm{E}-10$ & 1.04E-07 & $1.95 \mathrm{E}-10$ & $0.00 \mathrm{E}+00$ \\
\hline
\end{tabular}


Table 4.1-7. ICRP 30-Based Ingestion Dose Coefficients for Beta-Photon Emitting Radionuclides Used in Calculation of Conversion Factors for Groundwater Protection Standards (Continued)

\begin{tabular}{|c|c|c|c|c|c|c|c|c|c|c|c|}
\hline Organ/Tissue & $\mathrm{Pb}-209$ & Th-234 & $\mathrm{Pa}-234 \mathrm{~m}$ & $\mathrm{~Pa}-234$ & $\mathrm{~Pb}-214$ & $\mathrm{Bi}-214$ & TI-210 & $\mathrm{Pb}-210$ & $\mathrm{Bi}-210$ & $\mathrm{~Np}-239$ & Th-231 \\
\hline Adrenal & $5.37 \mathrm{E}-13$ & $1.64 \mathrm{E}-12$ & $0.00 \mathrm{E}+00$ & $3.72 \mathrm{E}-11$ & $2.50 \mathrm{E}-11$ & $3.61 \mathrm{E}-12$ & $0.00 \mathrm{E}+00$ & $1.25 \mathrm{E}-07$ & $1.97 \mathrm{E}-11$ & $7.91 \mathrm{E}-12$ & 3.93E-13 \\
\hline Urinary Bladder Wall & $5.37 \mathrm{E}-13$ & $9.15 \mathrm{E}-12$ & $0.00 \mathrm{E}+00$ & $9.06 \mathrm{E}-11$ & $2.47 \mathrm{E}-11$ & $2.25 \mathrm{E}-12$ & $0.00 \mathrm{E}+00$ & $1.25 \mathrm{E}-07$ & $1.97 \mathrm{E}-11$ & $4.74 \mathrm{E}-11$ & 2.89E-12 \\
\hline Bone Surface & $2.09 \mathrm{E}-11$ & $2.08 \mathrm{E}-11$ & $0.00 \mathrm{E}+00$ & $2.74 \mathrm{E}-11$ & 1.10E-09 & $1.51 \mathrm{E}-12$ & $0.00 \mathrm{E}+00$ & $2.16 \mathrm{E}-05$ & $1.97 \mathrm{E}-11$ & $3.59 \mathrm{E}-11$ & 3.17E-12 \\
\hline Brain & $0.00 \mathrm{E}+00$ & $0.00 \mathrm{E}+00$ & $0.00 \mathrm{E}+00$ & $4.48 \mathrm{E}-13$ & $0.00 \mathrm{E}+00$ & $0.00 \mathrm{E}+00$ & $0.00 \mathrm{E}+00$ & $0.00 \mathrm{E}+00$ & $0.00 \mathrm{E}+00$ & $7.93 \mathrm{E}-14$ & $0.00 \mathrm{E}+00$ \\
\hline Breast & $5.37 \mathrm{E}-13$ & $3.57 \mathrm{E}-12$ & $0.00 \mathrm{E}+00$ & $4.99 \mathrm{E}-11$ & $2.42 \mathrm{E}-11$ & $2.55 \mathrm{E}-12$ & $0.00 \mathrm{E}+00$ & $1.25 \mathrm{E}-07$ & $1.97 \mathrm{E}-11$ & $1.72 \mathrm{E}-11$ & 1.44E-12 \\
\hline Esophagus & $5.37 \mathrm{E}-13$ & 4.15E-13 & $0.00 \mathrm{E}+00$ & $6.06 \mathrm{E}-12$ & $2.19 \mathrm{E}-11$ & 1.29E-12 & $0.00 \mathrm{E}+00$ & 1.25E-07 & 1.97E-11 & $8.13 \mathrm{E}-13$ & 3.39E-14 \\
\hline Stomach Wall & $1.88 \mathrm{E}-10$ & $9.95 \mathrm{E}-10$ & $0.00 \mathrm{E}+00$ & $7.80 \mathrm{E}-10$ & $8.75 \mathrm{E}-10$ & $8.65 \mathrm{E}-10$ & $0.00 \mathrm{E}+00$ & 1.25E-07 & 4.65E-10 & $3.46 \mathrm{E}-10$ & 1.95E-10 \\
\hline Small Intestine Wall & $2.24 \mathrm{E}-10$ & $2.55 \mathrm{E}-09$ & $0.00 \mathrm{E}+00$ & $1.48 \mathrm{E}-09$ & $5.62 \mathrm{E}-10$ & $2.32 \mathrm{E}-10$ & $0.00 \mathrm{E}+00$ & $1.25 \mathrm{E}-07$ & 1.06E-09 & $8.76 \mathrm{E}-10$ & $4.54 \mathrm{E}-10$ \\
\hline Upper Large Intestine Wall & $3.50 \mathrm{E}-10$ & $1.47 \mathrm{E}-08$ & $0.00 \mathrm{E}+00$ & 3.13E-09 & $2.49 \mathrm{E}-10$ & $5.86 \mathrm{E}-11$ & $0.00 \mathrm{E}+00$ & $1.26 \mathrm{E}-07$ & 5.77E-09 & 3.85E-09 & $1.90 \mathrm{E}-09$ \\
\hline Lower Large Intestine Wall & $1.73 \mathrm{E}-10$ & 4.30E-08 & $0.00 \mathrm{E}+00$ & $2.46 \mathrm{E}-09$ & $4.89 \mathrm{E}-11$ & $7.31 \mathrm{E}-12$ & $0.00 \mathrm{E}+00$ & $1.30 \mathrm{E}-07$ & $1.54 \mathrm{E}-08$ & $8.72 \mathrm{E}-09$ & $3.42 \mathrm{E}-09$ \\
\hline Kidney & 1.66E-12 & $3.71 \mathrm{E}-12$ & $0.00 \mathrm{E}+00$ & $8.29 \mathrm{E}-11$ & 4.17E-11 & $6.25 \mathrm{E}-11$ & $0.00 \mathrm{E}+00$ & $2.81 \mathrm{E}-06$ & $5.88 \mathrm{E}-09$ & $2.05 \mathrm{E}-11$ & 1.26E-12 \\
\hline Liver & $3.02 \mathrm{E}-12$ & & $0.00 \mathrm{E}+00$ & & & 3.25E-12 & $0.00 \mathrm{E}+00$ & $6.08 \mathrm{E}-06$ & $1.97 \mathrm{E}-11$ & $1.54 \mathrm{E}-11$ & $9.78 \mathrm{E}-13$ \\
\hline Lung & $5.37 \mathrm{E}-13$ & 7.05E-13 & $0.00 \mathrm{E}+00$ & $1.51 \mathrm{E}-11$ & $2.32 \mathrm{E}-11$ & $2.38 \mathrm{E}-12$ & $0.00 \mathrm{E}+00$ & 1.25E-07 & 1.97E-11 & $2.40 \mathrm{E}-12$ & 1.43E-13 \\
\hline Muscle & $5.37 \mathrm{E}-13$ & $3.57 \mathrm{E}-12$ & $0.00 \mathrm{E}+00$ & $4.99 \mathrm{E}-11$ & $2.42 \mathrm{E}-11$ & $2.55 \mathrm{E}-12$ & $0.00 \mathrm{E}+00$ & $1.25 \mathrm{E}-07$ & $1.97 \mathrm{E}-11$ & $1.72 \mathrm{E}-11$ & 1.44E-12 \\
\hline Ovaries & $5.37 \mathrm{E}-13$ & $3.12 \mathrm{E}-11$ & $0.00 \mathrm{E}+00$ & $3.30 \mathrm{E}-10$ & $3.19 \mathrm{E}-11$ & $5.17 \mathrm{E}-12$ & $0.00 \mathrm{E}+00$ & $1.25 \mathrm{E}-07$ & $1.97 \mathrm{E}-11$ & $1.62 \mathrm{E}-10$ & 2.08E-11 \\
\hline Pancreas & $5.37 \mathrm{E}-13$ & $3.49 \mathrm{E}-12$ & $0.00 \mathrm{E}+00$ & $1.06 \mathrm{E}-10$ & $3.86 \mathrm{E}-11$ & $1.58 \mathrm{E}-11$ & $0.00 \mathrm{E}+00$ & 1.25E-07 & $1.97 \mathrm{E}-11$ & 2.16E-11 & $1.72 \mathrm{E}-12$ \\
\hline Red Marrow & 2.19E-12 & $1.84 \mathrm{E}-11$ & $0.00 \mathrm{E}+00$ & $7.86 \mathrm{E}-11$ & $1.12 \mathrm{E}-10$ & $2.51 \mathrm{E}-12$ & $0.00 \mathrm{E}+00$ & $1.48 \mathrm{E}-06$ & $1.97 \mathrm{E}-11$ & 4.66E-11 & $5.30 \mathrm{E}-12$ \\
\hline Skin & $5.37 \mathrm{E}-13$ & $1.31 \mathrm{E}-12$ & $0.00 \mathrm{E}+00$ & $1.87 \mathrm{E}-11$ & $2.24 \mathrm{E}-11$ & 1.49E-12 & $0.00 \mathrm{E}+00$ & 1.25E-07 & 1.97E-11 & $5.04 \mathrm{E}-12$ & $2.88 \mathrm{E}-13$ \\
\hline Spleen & $5.37 \mathrm{E}-13$ & $2.90 \mathrm{E}-12$ & $0.00 \mathrm{E}+00$ & $7.04 \mathrm{E}-11$ & $3.15 \mathrm{E}-11$ & $9.35 \mathrm{E}-12$ & $0.00 \mathrm{E}+00$ & 1.25E-07 & 1.97E-11 & $1.51 \mathrm{E}-11$ & 1.00E-12 \\
\hline Testes & $5.37 \mathrm{E}-13$ & $2.53 \mathrm{E}-12$ & $0.00 \mathrm{E}+00$ & $2.34 \mathrm{E}-11$ & $2.19 \mathrm{E}-11$ & 1.05E-12 & $0.00 \mathrm{E}+00$ & $1.25 \mathrm{E}-07$ & $1.97 \mathrm{E}-11$ & $1.14 \mathrm{E}-11$ & $5.24 \mathrm{E}-13$ \\
\hline Thymus & $5.37 \mathrm{E}-13$ & 4.15E-13 & $0.00 \mathrm{E}+00$ & $6.06 \mathrm{E}-12$ & $2.19 \mathrm{E}-11$ & $1.29 \mathrm{E}-12$ & $0.00 \mathrm{E}+00$ & $1.25 \mathrm{E}-07$ & $1.97 \mathrm{E}-11$ & $8.13 \mathrm{E}-13$ & $3.39 \mathrm{E}-14$ \\
\hline Thyroid & $5.37 \mathrm{E}-13$ & $2.88 \mathrm{E}-13$ & $0.00 \mathrm{E}+00$ & $1.86 \mathrm{E}-12$ & $2.14 \mathrm{E}-11$ & $8.55 \mathrm{E}-13$ & $0.00 \mathrm{E}+00$ & $1.25 \mathrm{E}-07$ & $1.97 \mathrm{E}-11$ & $2.07 \mathrm{E}-13$ & $8.80 \mathrm{E}-15$ \\
\hline Uterus & $5.37 \mathrm{E}-13$ & 1.26E-11 & $0.00 \mathrm{E}+00$ & $1.83 \mathrm{E}-10$ & $3.05 \mathrm{E}-11$ & 4.76E-12 & $0.00 \mathrm{E}+00$ & 1.25E-07 & $1.97 \mathrm{E}-11$ & $6.88 \mathrm{E}-11$ & 4.85E-12 \\
\hline Whole Body (h_E) & $5.75 \mathrm{E}-11$ & 3.69E-09 & $0.00 \mathrm{E}+00$ & $5.84 \mathrm{E}-10$ & 1.69E-10 & $7.64 \mathrm{E}-11$ & $0.00 \mathrm{E}+00$ & 1.45E-06 & 1.73E-09 & $8.82 \mathrm{E}-10$ & $3.65 \mathrm{E}-10$ \\
\hline
\end{tabular}


Table 4.1-7. ICRP 30-Based Ingestion Dose Coefficients for Beta-Photon Emitting Radionuclides Used in Calculation of Conversion Factors for Groundwater Protection Standards (Continued)

\begin{tabular}{|l|c|c|c|c|c|}
\hline \multicolumn{1}{|c|}{ Organ/Tissue } & Ac-227 & Fr-223 & Pb-211 & Bi-211 & Tl-207 \\
\hline Adrenal & $3.89 \mathrm{E}-10$ & $2.32 \mathrm{E}-09$ & $1.91 \mathrm{E}-11$ & $0.00 \mathrm{E}+00$ & $0.00 \mathrm{E}+00$ \\
\hline Urinary Bladder Wall & $5.33 \mathrm{E}-11$ & $2.32 \mathrm{E}-09$ & $1.91 \mathrm{E}-11$ & $0.00 \mathrm{E}+00$ & $0.00 \mathrm{E}+00$ \\
\hline Bone Surface & $6.73 \mathrm{E}-05$ & $2.32 \mathrm{E}-09$ & $1.60 \mathrm{E}-10$ & $0.00 \mathrm{E}+00$ & $0.00 \mathrm{E}+00$ \\
\hline Brain & $1.31 \mathrm{E}-10$ & $5.44 \mathrm{E}-12$ & $0.00 \mathrm{E}+00$ & $0.00 \mathrm{E}+00$ & $0.00 \mathrm{E}+00$ \\
\hline Breast & $1.41 \mathrm{E}-10$ & $2.32 \mathrm{E}-09$ & $1.91 \mathrm{E}-11$ & $0.00 \mathrm{E}+00$ & $0.00 \mathrm{E}+00$ \\
\hline Esophagus & $9.10 \mathrm{E}-11$ & $2.32 \mathrm{E}-09$ & $1.89 \mathrm{E}-11$ & $0.00 \mathrm{E}+00$ & $0.00 \mathrm{E}+00$ \\
\hline Stomach Wall & $1.96 \mathrm{E}-10$ & $2.49 \mathrm{E}-09$ & $1.17 \mathrm{E}-09$ & $0.00 \mathrm{E}+00$ & $0.00 \mathrm{E}+00$ \\
\hline Small Intestine Wall & $2.90 \mathrm{E}-10$ & $2.32 \mathrm{E}-09$ & $5.74 \mathrm{E}-10$ & $0.00 \mathrm{E}+00$ & $0.00 \mathrm{E}+00$ \\
\hline Upper Large Intestine Wall & $1.52 \mathrm{E}-09$ & $2.32 \mathrm{E}-09$ & $2.27 \mathrm{E}-10$ & $0.00 \mathrm{E}+00$ & $0.00 \mathrm{E}+00$ \\
\hline Lower Large Intestine Wall & $7.66 \mathrm{E}-09$ & $2.32 \mathrm{E}-09$ & $4.14 \mathrm{E}-11$ & $0.00 \mathrm{E}+00$ & $0.00 \mathrm{E}+00$ \\
\hline Kidney & $2.95 \mathrm{E}-10$ & $2.32 \mathrm{E}-09$ & $2.73 \mathrm{E}-11$ & $0.00 \mathrm{E}+00$ & $0.00 \mathrm{E}+00$ \\
\hline Liver & $1.54 \mathrm{E}-05$ & $2.32 \mathrm{E}-09$ & $3.68 \mathrm{E}-11$ & $0.00 \mathrm{E}+00$ & $0.00 \mathrm{E}+00$ \\
\hline Lung & $2.20 \mathrm{E}-10$ & $2.32 \mathrm{E}-09$ & $1.90 \mathrm{E}-11$ & $0.00 \mathrm{E}+00$ & $0.00 \mathrm{E}+00$ \\
\hline Muscle & $1.41 \mathrm{E}-10$ & $2.32 \mathrm{E}-09$ & $1.91 \mathrm{E}-11$ & $0.00 \mathrm{E}+00$ & $0.00 \mathrm{E}+00$ \\
\hline Ovaries & $8.31 \mathrm{E}-07$ & $2.32 \mathrm{E}-09$ & $1.97 \mathrm{E}-11$ & $0.00 \mathrm{E}+00$ & $0.00 \mathrm{E}+00$ \\
\hline Pancreas & $3.24 \mathrm{E}-10$ & $2.32 \mathrm{E}-09$ & $2.06 \mathrm{E}-11$ & $0.00 \mathrm{E}+00$ & $0.00 \mathrm{E}+00$ \\
\hline Red Marrow & $5.40 \mathrm{E}-06$ & $2.32 \mathrm{E}-09$ & $3.07 \mathrm{E}-11$ & $0.00 \mathrm{E}+00$ & $0.00 \mathrm{E}+00$ \\
\hline Skin & $8.62 \mathrm{E}-11$ & $2.32 \mathrm{E}-09$ & $1.89 \mathrm{E}-11$ & $0.00 \mathrm{E}+00$ & $0.00 \mathrm{E}+00$ \\
\hline Spleen & $1.07 \mathrm{E}-10$ & $2.32 \mathrm{E}-09$ & $1.98 \mathrm{E}-11$ & $0.00 \mathrm{E}+00$ & $0.00 \mathrm{E}+00$ \\
\hline Testes & $8.31 \mathrm{E}-07$ & $2.32 \mathrm{E}-09$ & $1.89 \mathrm{E}-11$ & $0.00 \mathrm{E}+00$ & $0.00 \mathrm{E}+00$ \\
\hline Thymus & $9.10 \mathrm{E}-11$ & $2.32 \mathrm{E}-09$ & $1.89 \mathrm{E}-11$ & $0.00 \mathrm{E}+00$ & $0.00 \mathrm{E}+00$ \\
\hline Thyroid & $7.55 \mathrm{E}-11$ & $2.32 \mathrm{E}-09$ & $1.88 \mathrm{E}-11$ & $0.00 \mathrm{E}+00$ & $0.00 \mathrm{E}+00$ \\
\hline Uterus & $7.02 \mathrm{E}-11$ & $2.32 \mathrm{E}-09$ & $1.96 \mathrm{E}-11$ & $0.00 \mathrm{E}+00$ & $0.00 \mathrm{E}+00$ \\
\hline Whole Body (h_E) & $3.80 \mathrm{E}-06$ & $2.33 \mathrm{E}-09$ & $1.42 \mathrm{E}-10$ & $0.00 \mathrm{E}+00$ & $0.00 \mathrm{E}+00$ \\
\hline Source: DOE 2004 [DIRS $169713 \mathrm{E}$ & & & & \\
\hline
\end{tabular}


Table 4.1-8. ICRP 72-Based Ingestion Dose Coefficients for Beta-Photon Emitting Radionuclides Used in Calculation of Conversion Factors for Groundwater Protection Standards

\begin{tabular}{|c|c|c|c|c|c|c|c|c|c|c|c|}
\hline Organ/Tissue & C-14 & $\mathrm{Cl}-36$ & Se-79 & Sr-90 & $\mathrm{Y}-90$ & Tc-99 & Sn-126 & Sb-126m & Sb-126 & I-129 & Cs-135 \\
\hline Adrenal & $5.70 \mathrm{E}-10$ & $8.00 \mathrm{E}-10$ & $9.60 \mathrm{E}-10$ & $6.60 \mathrm{E}-10$ & $1.30 \mathrm{E}-14$ & $3.90 \mathrm{E}-11$ & $9.20 \mathrm{E}-10$ & 4.10E-12 & $3.50 \mathrm{E}-10$ & $1.20 \mathrm{E}-10$ & $1.90 \mathrm{E}-09$ \\
\hline Urinary Bladder Wall & $5.70 \mathrm{E}-10$ & $2.10 \mathrm{E}-09$ & 1.10E-09 & 1.50E-09 & $6.10 \mathrm{E}-14$ & $1.60 \mathrm{E}-10$ & 1.10E-09 & 1.60E-12 & 1.00E-09 & 4.30E-10 & 2.20E-09 \\
\hline Bone Surface & $5.70 \mathrm{E}-10$ & $8.00 \mathrm{E}-10$ & $9.60 \mathrm{E}-10$ & 4.10E-07 & $3.70 \mathrm{E}-13$ & $3.90 \mathrm{E}-11$ & $5.40 \mathrm{E}-09$ & 1.20E-12 & 1.10E-09 & $4.00 \mathrm{E}-10$ & $1.90 \mathrm{E}-09$ \\
\hline Brain & $5.70 \mathrm{E}-10$ & $8.00 \mathrm{E}-10$ & $9.60 \mathrm{E}-10$ & $6.60 \mathrm{E}-10$ & 1.30E-14 & $3.90 \mathrm{E}-11$ & $7.00 \mathrm{E}-10$ & $5.50 \mathrm{E}-14$ & $1.20 \mathrm{E}-10$ & 1.40E-10 & $1.90 \mathrm{E}-09$ \\
\hline Breast & $5.70 \mathrm{E}-10$ & $8.00 \mathrm{E}-10$ & $9.60 \mathrm{E}-10$ & $6.60 \mathrm{E}-10$ & $1.30 \mathrm{E}-14$ & $3.90 \mathrm{E}-11$ & $4.60 \mathrm{E}-10$ & $1.00 \mathrm{E}-12$ & $1.20 \mathrm{E}-10$ & 1.10E-10 & 1.90E-09 \\
\hline Esophagus & $5.70 \mathrm{E}-10$ & $8.00 \mathrm{E}-10$ & $9.60 \mathrm{E}-10$ & $6.60 \mathrm{E}-10$ & $1.30 \mathrm{E}-14$ & $3.90 \mathrm{E}-11$ & $5.50 \mathrm{E}-10$ & $7.50 \mathrm{E}-13$ & $1.40 \mathrm{E}-10$ & 1.70E-10 & $1.90 \mathrm{E}-09$ \\
\hline Stomach Wall & $6.30 \mathrm{E}-10$ & 1.10E-09 & $1.00 \mathrm{E}-09$ & $9.00 \mathrm{E}-10$ & 1.10E-09 & $2.20 \mathrm{E}-09$ & $1.60 \mathrm{E}-09$ & $2.60 \mathrm{E}-10$ & $1.20 \mathrm{E}-09$ & $2.00 \mathrm{E}-10$ & $2.00 \mathrm{E}-09$ \\
\hline Small Intestine Wall & $5.70 \mathrm{E}-10$ & 8.10E-10 & $1.00 \mathrm{E}-09$ & 1.10E-09 & 2.60E-09 & $1.80 \mathrm{E}-10$ & 4.30E-09 & $6.40 \mathrm{E}-11$ & $3.10 \mathrm{E}-09$ & $1.30 \mathrm{E}-10$ & $1.90 \mathrm{E}-09$ \\
\hline Upper Large Intestine Wall & $5.80 \mathrm{E}-10$ & $9.00 \mathrm{E}-10$ & 1.40E-09 & $5.80 \mathrm{E}-09$ & $1.40 \mathrm{E}-08$ & 1.40E-09 & $1.60 \mathrm{E}-08$ & $2.40 \mathrm{E}-11$ & 8.00E-09 & $2.20 \mathrm{E}-10$ & 2.20E-09 \\
\hline Lower Large Intestine Wall & $6.00 \mathrm{E}-10$ & 1.10E-09 & 2.30E-09 & $2.20 \mathrm{E}-08$ & $3.10 \mathrm{E}-08$ & $3.90 \mathrm{E}-09$ & $4.40 \mathrm{E}-08$ & $7.50 \mathrm{E}-12$ & $1.80 \mathrm{E}-08$ & 4.30E-10 & $2.60 \mathrm{E}-09$ \\
\hline Colon & $5.90 \mathrm{E}-10$ & $9.80 \mathrm{E}-10$ & $1.80 \mathrm{E}-09$ & $1.30 \mathrm{E}-08$ & $2.10 \mathrm{E}-08$ & $2.50 \mathrm{E}-09$ & $2.80 \mathrm{E}-08$ & $1.70 \mathrm{E}-11$ & $1.20 \mathrm{E}-08$ & $3.10 \mathrm{E}-10$ & $2.40 \mathrm{E}-09$ \\
\hline Kidney & $5.70 \mathrm{E}-10$ & $8.00 \mathrm{E}-10$ & $3.20 \mathrm{E}-08$ & $6.60 \mathrm{E}-10$ & $1.30 \mathrm{E}-14$ & $3.90 \mathrm{E}-11$ & $8.80 \mathrm{E}-10$ & 4.40E-12 & $5.10 \mathrm{E}-10$ & $1.20 \mathrm{E}-10$ & 1.90E-09 \\
\hline Liver & $5.70 \mathrm{E}-10$ & $8.00 \mathrm{E}-10$ & $1.40 \mathrm{E}-08$ & $6.60 \mathrm{E}-10$ & $3.70 \mathrm{E}-13$ & $5.20 \mathrm{E}-11$ & $7.10 \mathrm{E}-10$ & $2.60 \mathrm{E}-12$ & $5.10 \mathrm{E}-10$ & $1.20 \mathrm{E}-10$ & 1.90E-09 \\
\hline Muscle & $5.70 \mathrm{E}-10$ & $8.00 \mathrm{E}-10$ & $9.60 \mathrm{E}-10$ & $6.60 \mathrm{E}-10$ & $1.30 \mathrm{E}-14$ & $3.90 \mathrm{E}-11$ & $8.00 \mathrm{E}-10$ & $2.00 \mathrm{E}-12$ & $4.20 \mathrm{E}-10$ & $3.00 \mathrm{E}-10$ & $1.90 \mathrm{E}-09$ \\
\hline Ovaries & $5.70 \mathrm{E}-10$ & $8.00 \mathrm{E}-10$ & $1.80 \mathrm{E}-09$ & $6.60 \mathrm{E}-10$ & $1.40 \mathrm{E}-14$ & $3.90 \mathrm{E}-11$ & $2.50 \mathrm{E}-09$ & $6.00 \mathrm{E}-12$ & $2.80 \mathrm{E}-09$ & 1.30E-10 & $1.90 \mathrm{E}-09$ \\
\hline Pancreas & $5.70 \mathrm{E}-10$ & $8.00 \mathrm{E}-10$ & $5.00 \mathrm{E}-09$ & $6.60 \mathrm{E}-10$ & 1.30E-14 & $3.90 \mathrm{E}-11$ & $8.50 \mathrm{E}-10$ & $1.50 \mathrm{E}-11$ & $4.80 \mathrm{E}-10$ & $1.20 \mathrm{E}-10$ & $1.90 \mathrm{E}-09$ \\
\hline Red Marrow & $5.70 \mathrm{E}-10$ & $8.00 \mathrm{E}-10$ & $9.60 \mathrm{E}-10$ & $1.80 \mathrm{E}-07$ & $3.70 \mathrm{E}-13$ & $3.90 \mathrm{E}-11$ & $2.80 \mathrm{E}-09$ & $2.20 \mathrm{E}-12$ & $9.00 \mathrm{E}-10$ & $1.40 \mathrm{E}-10$ & $1.90 \mathrm{E}-09$ \\
\hline Extrathoracic Airways & $5.70 \mathrm{E}-10$ & $8.00 \mathrm{E}-10$ & $9.60 \mathrm{E}-10$ & $6.60 \mathrm{E}-10$ & 1.30E-14 & $3.90 \mathrm{E}-11$ & $6.20 \mathrm{E}-10$ & $1.40 \mathrm{E}-13$ & $1.20 \mathrm{E}-10$ & $1.40 \mathrm{E}-10$ & $1.90 \mathrm{E}-09$ \\
\hline Lungs & $5.70 \mathrm{E}-10$ & $8.00 \mathrm{E}-10$ & $9.60 \mathrm{E}-10$ & $6.60 \mathrm{E}-10$ & $1.30 \mathrm{E}-14$ & $3.90 \mathrm{E}-11$ & $6.30 \mathrm{E}-10$ & $1.60 \mathrm{E}-12$ & $1.70 \mathrm{E}-10$ & $1.50 \mathrm{E}-10$ & $1.90 \mathrm{E}-09$ \\
\hline Skin & $5.70 \mathrm{E}-10$ & $8.00 \mathrm{E}-10$ & $9.60 \mathrm{E}-10$ & $6.60 \mathrm{E}-10$ & $1.30 \mathrm{E}-14$ & $3.90 \mathrm{E}-11$ & $5.50 \mathrm{E}-10$ & $8.20 \mathrm{E}-13$ & $2.00 \mathrm{E}-10$ & $1.40 \mathrm{E}-10$ & $1.90 \mathrm{E}-09$ \\
\hline Spleen & $5.70 \mathrm{E}-10$ & $8.00 \mathrm{E}-10$ & $5.60 \mathrm{E}-09$ & $6.60 \mathrm{E}-10$ & $1.30 \mathrm{E}-14$ & $3.90 \mathrm{E}-11$ & $7.10 \mathrm{E}-10$ & $9.40 \mathrm{E}-12$ & $3.70 \mathrm{E}-10$ & $1.20 \mathrm{E}-10$ & 1.90E-09 \\
\hline Testes & $5.70 \mathrm{E}-10$ & $8.00 \mathrm{E}-10$ & $2.90 \mathrm{E}-09$ & $6.60 \mathrm{E}-10$ & 1.30E-14 & $3.90 \mathrm{E}-11$ & $6.60 \mathrm{E}-10$ & $3.90 \mathrm{E}-13$ & $3.60 \mathrm{E}-10$ & $1.20 \mathrm{E}-10$ & $1.90 \mathrm{E}-09$ \\
\hline Thymus & $5.70 \mathrm{E}-10$ & $8.00 \mathrm{E}-10$ & $9.60 \mathrm{E}-10$ & $6.60 \mathrm{E}-10$ & 1.30E-14 & $3.90 \mathrm{E}-11$ & $5.50 \mathrm{E}-10$ & $7.50 \mathrm{E}-13$ & $1.40 \mathrm{E}-10$ & $1.70 \mathrm{E}-10$ & $1.90 \mathrm{E}-09$ \\
\hline Thyroid & $5.70 \mathrm{E}-10$ & $8.00 \mathrm{E}-10$ & $9.60 \mathrm{E}-10$ & $6.60 \mathrm{E}-10$ & $1.30 \mathrm{E}-14$ & $1.00 \mathrm{E}-09$ & $6.20 \mathrm{E}-10$ & $1.40 \mathrm{E}-13$ & $1.20 \mathrm{E}-10$ & $2.10 \mathrm{E}-06$ & 1.90E-09 \\
\hline Uterus & $5.70 \mathrm{E}-10$ & $8.00 \mathrm{E}-10$ & $9.60 \mathrm{E}-10$ & $6.60 \mathrm{E}-10$ & $1.30 \mathrm{E}-14$ & $3.90 \mathrm{E}-11$ & $1.40 \mathrm{E}-09$ & $4.90 \mathrm{E}-12$ & $1.30 \mathrm{E}-09$ & $1.30 \mathrm{E}-10$ & $1.90 \mathrm{E}-09$ \\
\hline Remainder & $5.70 \mathrm{E}-10$ & $8.00 \mathrm{E}-10$ & $1.70 \mathrm{E}-08$ & $6.70 \mathrm{E}-10$ & $5.30 \mathrm{E}-11$ & 4.20E-11 & $8.70 \mathrm{E}-10$ & $3.30 \mathrm{E}-12$ & 4.70E-10 & $2.80 \mathrm{E}-10$ & $1.90 \mathrm{E}-09$ \\
\hline Whole Body (e) & $5.80 \mathrm{E}-10$ & $9.30 \mathrm{E}-10$ & 2.90E-09 & 2.80E-08 & 2.70E-09 & $6.40 \mathrm{E}-10$ & 4.70E-09 & $3.60 \mathrm{E}-11$ & $2.40 \mathrm{E}-09$ & $1.10 \mathrm{E}-07$ & 2.00E-09 \\
\hline
\end{tabular}


Table 4.1-8. ICRP 72-Based Ingestion Dose Coefficients for Beta-Photon Emitting Radionuclides Used in Calculation of Conversion Factors for Groundwater Protection Standards (Continued)

\begin{tabular}{|c|c|c|c|c|c|c|c|c|c|c|c|}
\hline Organ/Tissue & Cs-137 & Ba-137m & Ra-228 & Ac-228 & $\mathrm{Pb}-212$ & $\mathrm{Bi}-212$ & Tl-208 & $\mathrm{Pa}-233$ & Ra-225 & $\mathrm{Bi}-213$ & TI-209 \\
\hline Adrenal & $1.40 \mathrm{E}-08$ & $0.00 \mathrm{E}+00$ & $1.50 \mathrm{E}-07$ & $1.90 \mathrm{E}-11$ & 2.20E-09 & 1.40E-11 & $0.00 \mathrm{E}+00$ & 1.30E-11 & 1.10E-08 & $5.10 \mathrm{E}-12$ & $0.00 \mathrm{E}+00$ \\
\hline Urinary Bladder Wall & $1.40 \mathrm{E}-08$ & $0.00 \mathrm{E}+00$ & $1.50 \mathrm{E}-07$ & 4.10E-11 & 2.40E-09 & $1.80 \mathrm{E}-11$ & $0.00 \mathrm{E}+00$ & $7.50 \mathrm{E}-11$ & 1.10E-08 & $8.20 \mathrm{E}-12$ & $0.00 \mathrm{E}+00$ \\
\hline Bone Surface & $1.40 \mathrm{E}-08$ & $0.00 \mathrm{E}+00$ & $2.20 \mathrm{E}-05$ & $1.50 \mathrm{E}-09$ & $5.20 \mathrm{E}-08$ & $9.70 \mathrm{E}-12$ & $0.00 \mathrm{E}+00$ & $6.60 \mathrm{E}-11$ & $3.60 \mathrm{E}-06$ & $4.60 \mathrm{E}-12$ & $0.00 \mathrm{E}+00$ \\
\hline Brain & $1.20 \mathrm{E}-08$ & $0.00 \mathrm{E}+00$ & $1.50 \mathrm{E}-07$ & $1.60 \mathrm{E}-13$ & 2.10E-09 & $6.90 \mathrm{E}-12$ & $0.00 \mathrm{E}+00$ & $1.50 \mathrm{E}-13$ & 1.10E-08 & $4.30 \mathrm{E}-12$ & $0.00 \mathrm{E}+00$ \\
\hline Breast & 1.10E-08 & $0.00 \mathrm{E}+00$ & 1.50E-07 & 4.30E-12 & 2.20E-09 & $8.80 \mathrm{E}-12$ & $0.00 \mathrm{E}+00$ & $2.00 \mathrm{E}-12$ & 1.10E-08 & $4.50 \mathrm{E}-12$ & $0.00 \mathrm{E}+00$ \\
\hline Esophagus & $1.30 \mathrm{E}-08$ & $0.00 \mathrm{E}+00$ & $1.50 \mathrm{E}-07$ & $3.10 \mathrm{E}-12$ & 2.20E-09 & $8.30 \mathrm{E}-12$ & $0.00 \mathrm{E}+00$ & $1.50 \mathrm{E}-12$ & 1.10E-08 & $4.40 \mathrm{E}-12$ & $0.00 \mathrm{E}+00$ \\
\hline Stomach Wall & 1.30E-08 & $0.00 \mathrm{E}+00$ & 1.50E-07 & $6.10 \mathrm{E}-10$ & 3.40E-09 & 1.60E-09 & $0.00 \mathrm{E}+00$ & $2.90 \mathrm{E}-10$ & 1.10E-08 & 1.30E-09 & $0.00 \mathrm{E}+00$ \\
\hline Small Intestine Wall & 1.40E-08 & $0.00 \mathrm{E}+00$ & $1.50 \mathrm{E}-07$ & $1.00 \mathrm{E}-09$ & $6.00 \mathrm{E}-09$ & 1.10E-09 & $0.00 \mathrm{E}+00$ & $7.70 \mathrm{E}-10$ & $1.20 \mathrm{E}-08$ & $7.40 \mathrm{E}-10$ & $0.00 \mathrm{E}+00$ \\
\hline Upper Large Intestine Wall & 1.40E-08 & $0.00 \mathrm{E}+00$ & 1.70E-07 & $2.40 \mathrm{E}-09$ & 1.80E-08 & $6.70 \mathrm{E}-10$ & $0.00 \mathrm{E}+00$ & 3.70E-09 & $1.80 \mathrm{E}-08$ & $4.40 \mathrm{E}-10$ & $0.00 \mathrm{E}+00$ \\
\hline Lower Large Intestine Wall & 1.70E-08 & $0.00 \mathrm{E}+00$ & $2.20 \mathrm{E}-07$ & $1.90 \mathrm{E}-09$ & $2.00 \mathrm{E}-08$ & $1.30 \mathrm{E}-10$ & $0.00 \mathrm{E}+00$ & $1.00 \mathrm{E}-08$ & 4.70E-08 & $1.00 \mathrm{E}-10$ & $0.00 \mathrm{E}+00$ \\
\hline Colon & $1.50 \mathrm{E}-08$ & $0.00 \mathrm{E}+00$ & $1.90 \mathrm{E}-07$ & $2.20 \mathrm{E}-09$ & $1.90 \mathrm{E}-08$ & $4.30 \mathrm{E}-10$ & $0.00 \mathrm{E}+00$ & $6.50 \mathrm{E}-09$ & $3.00 \mathrm{E}-08$ & $2.90 \mathrm{E}-10$ & $0.00 \mathrm{E}+00$ \\
\hline Kidney & $1.30 \mathrm{E}-08$ & $0.00 \mathrm{E}+00$ & 4.30E-07 & $3.70 \mathrm{E}-11$ & 4.40E-08 & 1.10E-09 & $0.00 \mathrm{E}+00$ & $3.30 \mathrm{E}-11$ & $3.70 \mathrm{E}-08$ & $6.10 \mathrm{E}-10$ & $0.00 \mathrm{E}+00$ \\
\hline Liver & $1.30 \mathrm{E}-08$ & $0.00 \mathrm{E}+00$ & $1.00 \mathrm{E}-06$ & $4.20 \mathrm{E}-10$ & & 1.30E-11 & & 2.10E-11 & & & $.00 \mathrm{E}+00$ \\
\hline Muscle & 1.20E-08 & $0.00 \mathrm{E}+00$ & $1.50 \mathrm{E}-07$ & $2.20 \mathrm{E}-11$ & 2.20E-09 & $1.20 \mathrm{E}-11$ & $0.00 \mathrm{E}+00$ & 2.60E-11 & 1.10E-08 & $4.80 \mathrm{E}-12$ & $0.00 \mathrm{E}+00$ \\
\hline Ovaries & 1.40E-08 & $0.00 \mathrm{E}+00$ & $2.10 \mathrm{E}-07$ & 1.70E-10 & 2.40E-09 & $3.00 \mathrm{E}-11$ & $0.00 \mathrm{E}+00$ & $2.30 \mathrm{E}-10$ & 1.30E-08 & $6.60 \mathrm{E}-12$ & $0.00 \mathrm{E}+00$ \\
\hline Pancreas & $1.40 \mathrm{E}-08$ & $0.00 \mathrm{E}+00$ & $1.50 \mathrm{E}-07$ & $4.60 \mathrm{E}-11$ & 2.20E-09 & $2.90 \mathrm{E}-11$ & $0.00 \mathrm{E}+00$ & $2.70 \mathrm{E}-11$ & 1.10E-08 & $7.30 \mathrm{E}-12$ & $0.00 \mathrm{E}+00$ \\
\hline Red Marrow & $1.30 \mathrm{E}-08$ & $0.00 \mathrm{E}+00$ & $2.30 \mathrm{E}-06$ & $1.50 \mathrm{E}-10$ & $6.70 \mathrm{E}-09$ & 1.30E-11 & $0.00 \mathrm{E}+00$ & $4.60 \mathrm{E}-11$ & $3.50 \mathrm{E}-07$ & $4.90 \mathrm{E}-12$ & $0.00 \mathrm{E}+00$ \\
\hline Extrathoracic Airways & 1.30E-08 & $0.00 \mathrm{E}+00$ & $1.50 \mathrm{E}-07$ & $6.90 \mathrm{E}-13$ & 2.10E-09 & 7.10E-12 & $0.00 \mathrm{E}+00$ & $3.40 \mathrm{E}-13$ & 1.10E-08 & $4.30 \mathrm{E}-12$ & $0.00 \mathrm{E}+00$ \\
\hline Lungs & 1.30E-08 & $0.00 \mathrm{E}+00$ & $1.50 \mathrm{E}-07$ & $6.40 \mathrm{E}-12$ & $2.20 \mathrm{E}-09$ & $9.50 \mathrm{E}-12$ & $0.00 \mathrm{E}+00$ & $3.40 \mathrm{E}-12$ & 1.10E-08 & $4.60 \mathrm{E}-12$ & $0.00 \mathrm{E}+00$ \\
\hline Skin & $1.10 \mathrm{E}-08$ & $0.00 \mathrm{E}+00$ & $1.50 \mathrm{E}-07$ & $8.50 \mathrm{E}-12$ & 2.20E-09 & $8.90 \mathrm{E}-12$ & $0.00 \mathrm{E}+00$ & 8.30E-12 & 1.10E-08 & $4.50 \mathrm{E}-12$ & $0.00 \mathrm{E}+00$ \\
\hline Spleen & 1.30E-08 & $0.00 \mathrm{E}+00$ & 1.50E-07 & $3.10 \mathrm{E}-11$ & 2.20E-09 & $2.10 \mathrm{E}-11$ & $0.00 \mathrm{E}+00$ & 2.10E-11 & 1.10E-08 & $6.20 \mathrm{E}-12$ & $0.00 \mathrm{E}+00$ \\
\hline Testes & $1.20 \mathrm{E}-08$ & $0.00 \mathrm{E}+00$ & $2.10 \mathrm{E}-07$ & $2.70 \mathrm{E}-11$ & 2.20E-09 & $8.30 \mathrm{E}-12$ & $0.00 \mathrm{E}+00$ & 2.10E-11 & $1.30 \mathrm{E}-08$ & $4.40 \mathrm{E}-12$ & $0.00 \mathrm{E}+00$ \\
\hline Thymus & $1.30 \mathrm{E}-08$ & $0.00 \mathrm{E}+00$ & $1.50 \mathrm{E}-07$ & 3.10E-12 & $2.20 \mathrm{E}-09$ & $8.30 \mathrm{E}-12$ & $0.00 \mathrm{E}+00$ & $1.50 \mathrm{E}-12$ & 1.10E-08 & $4.40 \mathrm{E}-12$ & $0.00 \mathrm{E}+00$ \\
\hline Thyroid & $1.30 \mathrm{E}-08$ & $0.00 \mathrm{E}+00$ & $1.50 \mathrm{E}-07$ & $6.90 \mathrm{E}-13$ & 2.10E-09 & 7.10E-12 & $0.00 \mathrm{E}+00$ & $3.40 \mathrm{E}-13$ & 1.10E-08 & $4.30 \mathrm{E}-12$ & $0.00 \mathrm{E}+00$ \\
\hline Uterus & $1.40 \mathrm{E}-08$ & $0.00 \mathrm{E}+00$ & $1.50 \mathrm{E}-07$ & $9.00 \mathrm{E}-11$ & 2.30E-09 & $2.40 \mathrm{E}-11$ & $0.00 \mathrm{E}+00$ & 1.10E-10 & 1.10E-08 & $6.10 \mathrm{E}-12$ & $0.00 \mathrm{E}+00$ \\
\hline Remainder & 1.20E-08 & $0.00 \mathrm{E}+00$ & $1.50 \mathrm{E}-07$ & $4.30 \mathrm{E}-11$ & 2.70E-09 & 4.40E-11 & $0.00 \mathrm{E}+00$ & 4.10E-11 & 1.20E-08 & $2.60 \mathrm{E}-11$ & $0.00 \mathrm{E}+00$ \\
\hline Whole Body (e) & 1.30E-08 & $0.00 \mathrm{E}+00$ & $6.90 \mathrm{E}-07$ & $4.30 \mathrm{E}-10$ & $6.00 \mathrm{E}-09$ & $2.60 \mathrm{E}-10$ & $0.00 \mathrm{E}+00$ & $8.70 \mathrm{E}-10$ & $9.90 \mathrm{E}-08$ & $2.00 \mathrm{E}-10$ & $0.00 E+00$ \\
\hline
\end{tabular}


Table 4.1-8. ICRP 72-Based Ingestion Dose Coefficients for Beta-Photon Emitting Radionuclides Used in Calculation of Conversion Factors for Groundwater Protection Standards (Continued)

\begin{tabular}{|c|c|c|c|c|c|c|c|c|c|c|c|}
\hline Organ/Tissue & Pb-209 & Th-234 & Pa-234m & $\mathrm{Pa}-234$ & $\mathrm{~Pb}-214$ & $\mathrm{Bi}-214$ & TI-210 & $\mathrm{Pb}-210$ & $\mathrm{Bi}-210$ & Np-239 & Th-231 \\
\hline Adrenal & $5.50 \mathrm{E}-13$ & $2.10 \mathrm{E}-12$ & $0.00 \mathrm{E}+00$ & $4.00 \mathrm{E}-11$ & $1.50 \mathrm{E}-11$ & $4.20 \mathrm{E}-12$ & $0.00 \mathrm{E}+00$ & $8.80 \mathrm{E}-08$ & $2.00 \mathrm{E}-11$ & $9.00 \mathrm{E}-12$ & 4.80E-13 \\
\hline Urinary Bladder Wall & $2.50 \mathrm{E}-12$ & $9.80 \mathrm{E}-12$ & $0.00 \mathrm{E}+00$ & $9.00 \mathrm{E}-11$ & $1.80 \mathrm{E}-11$ & $2.50 \mathrm{E}-12$ & $0.00 \mathrm{E}+00$ & $8.90 \mathrm{E}-08$ & $5.80 \mathrm{E}-11$ & $4.60 \mathrm{E}-11$ & $2.90 \mathrm{E}-12$ \\
\hline Bone Surface & 1.40E-11 & $4.70 \mathrm{E}-11$ & $0.00 \mathrm{E}+00$ & $3.20 \mathrm{E}-11$ & $1.90 \mathrm{E}-10$ & 1.70E-12 & $0.00 \mathrm{E}+00$ & $2.30 \mathrm{E}-05$ & $2.00 \mathrm{E}-11$ & $2.50 \mathrm{E}-11$ & $5.80 \mathrm{E}-12$ \\
\hline Brain & $5.50 \mathrm{E}-13$ & $6.50 \mathrm{E}-13$ & $0.00 \mathrm{E}+00$ & $2.80 \mathrm{E}-13$ & $1.00 \mathrm{E}-11$ & $7.10 \mathrm{E}-13$ & $0.00 \mathrm{E}+00$ & $8.80 \mathrm{E}-08$ & $2.00 \mathrm{E}-11$ & $5.30 \mathrm{E}-14$ & $1.80 \mathrm{E}-14$ \\
\hline Breast & $5.50 \mathrm{E}-13$ & $8.80 \mathrm{E}-13$ & $0.00 \mathrm{E}+00$ & $8.70 \mathrm{E}-12$ & $1.10 \mathrm{E}-11$ & $1.70 \mathrm{E}-12$ & $0.00 \mathrm{E}+00$ & $8.80 \mathrm{E}-08$ & $2.00 \mathrm{E}-11$ & 1.10E-12 & $6.00 \mathrm{E}-14$ \\
\hline Esophagus & $5.50 \mathrm{E}-13$ & $8.20 \mathrm{E}-13$ & $0.00 \mathrm{E}+00$ & $6.20 \mathrm{E}-12$ & $1.10 \mathrm{E}-11$ & $1.40 \mathrm{E}-12$ & $0.00 \mathrm{E}+00$ & 8.80E-08 & $2.00 \mathrm{E}-11$ & 8.30E-13 & 4.30E-14 \\
\hline Stomach Wall & $1.90 \mathrm{E}-10$ & $1.00 \mathrm{E}-09$ & $0.00 \mathrm{E}+00$ & $7.60 \mathrm{E}-10$ & $8.60 \mathrm{E}-10$ & $8.60 \mathrm{E}-10$ & $0.00 \mathrm{E}+00$ & $8.80 \mathrm{E}-08$ & $4.70 \mathrm{E}-10$ & $3.40 \mathrm{E}-10$ & $1.90 \mathrm{E}-10$ \\
\hline Small Intestine Wall & $2.20 \mathrm{E}-10$ & $2.50 \mathrm{E}-09$ & $0.00 \mathrm{E}+00$ & 1.30E-09 & $5.50 \mathrm{E}-10$ & $2.30 \mathrm{E}-10$ & $0.00 \mathrm{E}+00$ & $8.80 \mathrm{E}-08$ & 1.10E-09 & $8.50 \mathrm{E}-10$ & $4.40 \mathrm{E}-10$ \\
\hline Upper Large Intestine Wall & $3.50 \mathrm{E}-10$ & $1.50 \mathrm{E}-08$ & $0.00 \mathrm{E}+00$ & $3.00 \mathrm{E}-09$ & $2.40 \mathrm{E}-10$ & $5.70 \mathrm{E}-11$ & $0.00 \mathrm{E}+00$ & $9.10 \mathrm{E}-08$ & $6.00 \mathrm{E}-09$ & 3.90E-09 & 1.90E-09 \\
\hline Lower Large Intestine Wall & $1.70 \mathrm{E}-10$ & 4.30E-08 & $0.00 \mathrm{E}+00$ & $2.40 \mathrm{E}-09$ & $3.80 \mathrm{E}-11$ & 7.30E-12 & $0.00 \mathrm{E}+00$ & $9.80 \mathrm{E}-08$ & 1.60E-08 & 8.70E-09 & 3.40E-09 \\
\hline Colon & $2.70 \mathrm{E}-10$ & $2.70 \mathrm{E}-08$ & $0.00 \mathrm{E}+00$ & $2.80 \mathrm{E}-09$ & $1.50 \mathrm{E}-10$ & $3.60 \mathrm{E}-11$ & $0.00 \mathrm{E}+00$ & $9.40 \mathrm{E}-08$ & $1.00 \mathrm{E}-08$ & $6.00 \mathrm{E}-09$ & 2.50E-09 \\
\hline Kidney & 7.10E-12 & $1.90 \mathrm{E}-11$ & $0.00 \mathrm{E}+00$ & $7.80 \mathrm{E}-11$ & 3.30E-10 & $6.20 \mathrm{E}-11$ & $0.00 \mathrm{E}+00$ & 3.70E-06 & $5.90 \mathrm{E}-09$ & $2.10 \mathrm{E}-11$ & 1.40E-12 \\
\hline Liver & $2.70 \mathrm{E}-12$ & $7.90 \mathrm{E}-12$ & $0.00 \mathrm{E}+00$ & $5.60 \mathrm{E}-11$ & $4.10 \mathrm{E}-11$ & $3.00 \mathrm{E}-12$ & $0.00 \mathrm{E}+00$ & $1.90 \mathrm{E}-06$ & $2.00 \mathrm{E}-11$ & $1.40 \mathrm{E}-11$ & $9.90 \mathrm{E}-13$ \\
\hline Muscle & $5.50 \mathrm{E}-13$ & $3.80 \mathrm{E}-12$ & $0.00 \mathrm{E}+00$ & $4.70 \mathrm{E}-11$ & $1.30 \mathrm{E}-11$ & $2.50 \mathrm{E}-12$ & $0.00 \mathrm{E}+00$ & $8.80 \mathrm{E}-08$ & $2.00 \mathrm{E}-11$ & $1.70 \mathrm{E}-11$ & \\
\hline Ovaries & $5.50 \mathrm{E}-13$ & $3.20 \mathrm{E}-11$ & $0.00 \mathrm{E}+00$ & $3.40 \mathrm{E}-10$ & $2.30 \mathrm{E}-11$ & $6.00 \mathrm{E}-12$ & $0.00 \mathrm{E}+00$ & 8.80E-08 & $2.00 \mathrm{E}-11$ & $1.50 \mathrm{E}-10$ & 1.90E-11 \\
\hline Pancreas & $5.50 \mathrm{E}-13$ & 3.80E-12 & $0.00 \mathrm{E}+00$ & $9.60 \mathrm{E}-11$ & $2.50 \mathrm{E}-11$ & 1.40E-11 & $0.00 \mathrm{E}+00$ & $8.80 \mathrm{E}-08$ & $2.00 \mathrm{E}-11$ & $2.00 \mathrm{E}-11$ & $1.50 \mathrm{E}-12$ \\
\hline Red Marrow & $1.80 \mathrm{E}-12$ & $2.90 \mathrm{E}-11$ & $0.00 \mathrm{E}+00$ & $6.90 \mathrm{E}-11$ & 3.00E-11 & $2.60 \mathrm{E}-12$ & $0.00 \mathrm{E}+00$ & $2.50 \mathrm{E}-06$ & $2.00 \mathrm{E}-11$ & $2.60 \mathrm{E}-11$ & $1.80 \mathrm{E}-12$ \\
\hline Extrathoracic Airways & $5.50 \mathrm{E}-13$ & $6.80 \mathrm{E}-13$ & $0.00 \mathrm{E}+00$ & $1.30 \mathrm{E}-12$ & $1.00 \mathrm{E}-11$ & $8.10 \mathrm{E}-13$ & $0.00 \mathrm{E}+00$ & 8.80E-08 & $2.00 \mathrm{E}-11$ & $1.50 \mathrm{E}-13$ & $2.00 \mathrm{E}-14$ \\
\hline Lungs & $5.50 \mathrm{E}-13$ & $1.00 \mathrm{E}-12$ & $0.00 \mathrm{E}+00$ & $1.30 \mathrm{E}-11$ & $1.20 \mathrm{E}-11$ & $2.10 \mathrm{E}-12$ & $0.00 \mathrm{E}+00$ & $8.80 \mathrm{E}-08$ & $2.00 \mathrm{E}-11$ & $2.10 \mathrm{E}-12$ & $1.20 \mathrm{E}-13$ \\
\hline Skin & $5.50 \mathrm{E}-13$ & 1.70E-12 & $0.00 \mathrm{E}+00$ & $1.80 \mathrm{E}-11$ & 1.10E-11 & $1.50 \mathrm{E}-12$ & $0.00 \mathrm{E}+00$ & 8.80E-08 & $2.00 \mathrm{E}-11$ & $5.00 \mathrm{E}-12$ & $2.90 \mathrm{E}-13$ \\
\hline Spleen & $5.50 \mathrm{E}-13$ & $3.10 \mathrm{E}-12$ & $0.00 \mathrm{E}+00$ & $6.50 \mathrm{E}-11$ & $2.60 \mathrm{E}-11$ & $8.90 \mathrm{E}-12$ & $0.00 \mathrm{E}+00$ & $2.80 \mathrm{E}-06$ & $2.00 \mathrm{E}-11$ & $1.50 \mathrm{E}-11$ & $9.60 \mathrm{E}-13$ \\
\hline Testes & $5.50 \mathrm{E}-13$ & 4.60E-12 & $0.00 \mathrm{E}+00$ & $2.40 \mathrm{E}-11$ & 1.10E-11 & 1.00E-12 & $0.00 \mathrm{E}+00$ & $8.80 \mathrm{E}-08$ & $2.00 \mathrm{E}-11$ & $1.20 \mathrm{E}-11$ & $5.60 \mathrm{E}-13$ \\
\hline Thymus & $5.50 \mathrm{E}-13$ & $8.20 \mathrm{E}-13$ & $0.00 \mathrm{E}+00$ & $6.20 \mathrm{E}-12$ & 1.10E-11 & 1.40E-12 & $0.00 \mathrm{E}+00$ & 8.80E-08 & $2.00 \mathrm{E}-11$ & $8.30 \mathrm{E}-13$ & 4.30E-14 \\
\hline Thyroid & $5.50 \mathrm{E}-13$ & $6.80 \mathrm{E}-13$ & $0.00 \mathrm{E}+00$ & $1.30 \mathrm{E}-12$ & $1.00 \mathrm{E}-11$ & 8.10E-13 & $0.00 \mathrm{E}+00$ & 8.80E-08 & $2.00 \mathrm{E}-11$ & $1.50 \mathrm{E}-13$ & $2.00 \mathrm{E}-14$ \\
\hline Uterus & $5.50 \mathrm{E}-13$ & $1.30 \mathrm{E}-11$ & $0.00 \mathrm{E}+00$ & $1.90 \mathrm{E}-10$ & $2.10 \mathrm{E}-11$ & $5.20 \mathrm{E}-12$ & $0.00 \mathrm{E}+00$ & $8.80 \mathrm{E}-08$ & $2.00 \mathrm{E}-11$ & $7.00 \mathrm{E}-11$ & $4.90 \mathrm{E}-12$ \\
\hline Remainder & $5.30 \mathrm{E}-12$ & $5.70 \mathrm{E}-11$ & $0.00 \mathrm{E}+00$ & $7.20 \mathrm{E}-11$ & $2.70 \mathrm{E}-11$ & $7.80 \mathrm{E}-12$ & $0.00 \mathrm{E}+00$ & $1.40 \mathrm{E}-07$ & $1.00 \mathrm{E}-10$ & $3.30 \mathrm{E}-11$ & $1.00 \mathrm{E}-11$ \\
\hline Whole Body (e) & $5.70 \mathrm{E}-11$ & 3.40E-09 & $0.00 \mathrm{E}+00$ & $5.10 \mathrm{E}-10$ & $1.40 \mathrm{E}-10$ & 1.10E-10 & $0.00 \mathrm{E}+00$ & $6.90 \mathrm{E}-07$ & 1.30E-09 & $8.00 \mathrm{E}-10$ & $3.40 \mathrm{E}-10$ \\
\hline
\end{tabular}


Table 4.1-8. ICRP 72-Based Ingestion Dose Coefficients for Beta-Photon Emitting Radionuclides Used in Calculation of Conversion Factors for Groundwater Protection Standards (Continued)

\begin{tabular}{|c|c|c|c|c|c|}
\hline Organ/Tissue & Ac-227 & Fr-223 & $\mathrm{Pb}-211$ & $\mathrm{Bi}-211$ & Tl-207 \\
\hline Adrenal & $2.50 \mathrm{E}-10$ & 2.30E-09 & 1.70E-11 & $0.00 \mathrm{E}+00$ & $0.00 \mathrm{E}+00$ \\
\hline Urinary Bladder Wall & $4.20 \mathrm{E}-11$ & 2.30E-09 & $1.90 \mathrm{E}-11$ & $0.00 \mathrm{E}+00$ & $0.00 \mathrm{E}+00$ \\
\hline Bone Surface & $3.40 \mathrm{E}-05$ & 2.30E-09 & $2.70 \mathrm{E}-10$ & $0.00 \mathrm{E}+00$ & $0.00 \mathrm{E}+00$ \\
\hline Brain & $5.20 \mathrm{E}-11$ & 2.30E-09 & $1.70 \mathrm{E}-11$ & $0.00 \mathrm{E}+00$ & $0.00 \mathrm{E}+00$ \\
\hline Breast & $5.20 \mathrm{E}-11$ & $2.30 \mathrm{E}-09$ & $1.70 \mathrm{E}-11$ & $0.00 \mathrm{E}+00$ & $0.00 \mathrm{E}+00$ \\
\hline Esophagus & $5.70 \mathrm{E}-11$ & $2.30 \mathrm{E}-09$ & $1.70 \mathrm{E}-11$ & $0.00 \mathrm{E}+00$ & $0.00 \mathrm{E}+00$ \\
\hline Stomach Wall & $1.30 \mathrm{E}-10$ & $2.50 \mathrm{E}-09$ & $1.20 \mathrm{E}-09$ & $0.00 \mathrm{E}+00$ & $0.00 \mathrm{E}+00$ \\
\hline Small Intestine Wall & $2.40 \mathrm{E}-10$ & 2.30E-09 & $4.80 \mathrm{E}-10$ & $0.00 \mathrm{E}+00$ & $0.00 \mathrm{E}+00$ \\
\hline Upper Large Intestine Wall & $1.50 \mathrm{E}-09$ & 2.30E-09 & $2.20 \mathrm{E}-10$ & $0.00 \mathrm{E}+00$ & $0.00 \mathrm{E}+00$ \\
\hline Lower Large Intestine Wall & 7.60E-09 & 2.30E-09 & $3.90 \mathrm{E}-11$ & $0.00 \mathrm{E}+00$ & $0.00 \mathrm{E}+00$ \\
\hline Colon & $4.10 \mathrm{E}-09$ & $2.30 \mathrm{E}-09$ & $1.40 \mathrm{E}-10$ & $0.00 \mathrm{E}+00$ & $0.00 \mathrm{E}+00$ \\
\hline Kidney & $1.70 \mathrm{E}-10$ & $2.30 \mathrm{E}-09$ & $1.70 \mathrm{E}-10$ & $0.00 \mathrm{E}+00$ & $0.00 \mathrm{E}+00$ \\
\hline Liver & 7.70E-06 & $2.30 \mathrm{E}-09$ & $5.30 \mathrm{E}-11$ & $0.00 \mathrm{E}+00$ & $0.00 \mathrm{E}+00$ \\
\hline Muscle & $7.20 \mathrm{E}-11$ & $2.30 \mathrm{E}-09$ & $1.70 \mathrm{E}-11$ & $0.00 \mathrm{E}+00$ & $0.00 \mathrm{E}+00$ \\
\hline Ovaries & $4.20 \mathrm{E}-07$ & $2.30 \mathrm{E}-09$ & $1.80 \mathrm{E}-11$ & $0.00 \mathrm{E}+00$ & $0.00 \mathrm{E}+00$ \\
\hline Pancreas & $1.90 \mathrm{E}-10$ & 2.30E-09 & $1.80 \mathrm{E}-11$ & $0.00 \mathrm{E}+00$ & $0.00 \mathrm{E}+00$ \\
\hline Red Marrow & $2.70 \mathrm{E}-06$ & $2.30 \mathrm{E}-09$ & $3.90 \mathrm{E}-11$ & $0.00 \mathrm{E}+00$ & $0.00 \mathrm{E}+00$ \\
\hline Extrathoracic Airways & $4.20 \mathrm{E}-11$ & 2.30E-09 & $1.70 \mathrm{E}-11$ & $0.00 \mathrm{E}+00$ & $0.00 \mathrm{E}+00$ \\
\hline Lungs & $1.20 \mathrm{E}-10$ & 2.30E-09 & 1.70E-11 & $0.00 \mathrm{E}+00$ & $0.00 \mathrm{E}+00$ \\
\hline Skin & $4.10 \mathrm{E}-11$ & $2.30 \mathrm{E}-09$ & $1.70 \mathrm{E}-11$ & $0.00 \mathrm{E}+00$ & $0.00 \mathrm{E}+00$ \\
\hline Spleen & $6.40 \mathrm{E}-11$ & $2.30 \mathrm{E}-09$ & $1.80 \mathrm{E}-11$ & $0.00 \mathrm{E}+00$ & $0.00 \mathrm{E}+00$ \\
\hline Testes & $4.20 \mathrm{E}-07$ & 2.30E-09 & $1.70 \mathrm{E}-11$ & $0.00 \mathrm{E}+00$ & $0.00 \mathrm{E}+00$ \\
\hline Thymus & $5.70 \mathrm{E}-11$ & 2.30E-09 & 1.70E-11 & $0.00 \mathrm{E}+00$ & $0.00 \mathrm{E}+00$ \\
\hline Thyroid & $4.20 \mathrm{E}-11$ & 2.30E-09 & $1.70 \mathrm{E}-11$ & $0.00 \mathrm{E}+00$ & $0.00 \mathrm{E}+00$ \\
\hline Uterus & $6.40 \mathrm{E}-11$ & 2.30E-09 & $1.80 \mathrm{E}-11$ & $0.00 \mathrm{E}+00$ & $0.00 \mathrm{E}+00$ \\
\hline Remainder & $7.60 \mathrm{E}-11$ & 2.30E-09 & $2.80 \mathrm{E}-11$ & $0.00 \mathrm{E}+00$ & $0.00 \mathrm{E}+00$ \\
\hline Whole Body (e) & 1.10E-06 & $2.40 \mathrm{E}-09$ & $1.80 \mathrm{E}-10$ & $0.00 \mathrm{E}+00$ & $0.00 \mathrm{E}+00$ \\
\hline
\end{tabular}




\subsection{CRITERIA}

Three requirements from the Project Requirements Document (Canori and Leitner 2003 [DIRS 166275], Table 2-3) are applicable to this analysis (Table 4.2-1).

Table 4.2-1. Requirements Applicable to this Analysis

\begin{tabular}{|c|l|c|}
\hline $\begin{array}{c}\text { Requirement } \\
\text { Number }\end{array}$ & \multicolumn{1}{c|}{ Requirement Title } & $\begin{array}{c}\text { Related } \\
\text { Regulation }\end{array}$ \\
\hline PRD-002/T-015 & Requirements for Performance Assessment & 10 CFR 63.114 \\
\hline PRD-002/T-026 & Required Characteristics of the Reference Biosphere & 10 CFR 63.305 \\
\hline PRD-002/T-028 & Required Characteristics of the Reasonably Maximally Exposed Individual & 10 CFR 63.312 \\
\hline
\end{tabular}
Source: Canori and Leitner 2003 [DIRS 166275], Table 2-3.

In addition to the requirements listed in Table 4.2-1, definition of terms in 10 CFR 63.2 and description of concepts in 10 CFR 63.102 [DIRS 173164] that are relevant to biosphere modeling are also applicable to this analysis.

Listed below are NRC (U.S. Nuclear Regulatory Commission) acceptance criteria (AC) from Sections 2.2.1.2.13 (Redistribution of Radionuclides in Soil) and 2.2.1.3.14 (Biosphere Characteristics) of the Yucca Mountain Review Plan, Final Report (NRC 2003 [DIRS 163274]) (YMRP), based on the requirements of 10 CFR 63.114, 63.305, and 63.312 [DIRS 173164], that were considered when modeling biosphere characteristics.

\section{Acceptance Criteria from Section 2.2.1.3.13, Redistribution of Radionuclides in Soil}

\section{Acceptance Criterion 1, System Description and Model Integration Are Adequate.}

(1) Total system performance assessment adequately incorporates important features, physical phenomena and couplings between different models, and uses consistent and appropriate assumptions throughout the abstraction of redistribution of radionuclides in the soil abstraction process;

(2) The total system performance assessment model abstraction identifies and describes aspects of redistribution of radionuclides in soil that are important to repository performance, including the technical bases for these descriptions. For example, the abstraction should include modeling of the deposition of contaminated material in the soil and determination of the depth distribution of the deposited radionuclides;

(3) Relevant site features, events, and processes have been appropriately modeled in the abstraction of redistribution of radionuclides, from surface processes, and sufficient technical bases are provided.

\section{Acceptance Criterion 2, Data Are Sufficient for Model Justification.}

(1) Behavioral, hydrological, and geochemical values used in the license application are adequately justified (e.g., irrigation and precipitation rates, erosion rates, radionuclide 
solubility values, etc.). Adequate descriptions of how the data were used, interpreted, and appropriately synthesized into the parameters are provided; and

(2) Sufficient data (e.g., field, laboratory, and natural analog data) are available to adequately define relevant parameters and conceptual models necessary for developing the abstraction of redistribution of radionuclides in soil in the total system performance assessment.

\section{Acceptance Criterion 3, Data Uncertainty Is Characterized and Propagated Through the Model Abstraction.}

(1) Models use parameter values, assumed ranges, probability distributions, and bounding assumptions that are technically defensible, reasonably account for uncertainties and variabilities, do not result in an under-representation of the risk estimate, and are consistent with the characteristics of the reasonably maximally exposed individual in 10 CFR Part 63;

(2) The technical bases for the parameter values and ranges in the total system performance assessment abstraction are consistent with data from the Yucca Mountain region, e.g., Amargosa Valley survey, studies of surface processes in the Fortymile Wash drainage basin, applicable laboratory testings, natural analogs, or other valid sources of data. For example, soil types, crop types, plow depths, and irrigation rates should be consistent with current farming practices, and data on the airborne particulate concentration should be based on the resuspension of appropriate material in a climate and level of disturbance similar to that which is expected to be found at the location of the reasonably maximally exposed individual, during the compliance time period;

(3) Uncertainty is adequately represented in parameters for conceptual models, process models, and alternative conceptual models considered in developing the total system performance assessment abstraction of redistribution of radionuclides in soil, either through sensitivity analyses, conservative limits, or bounding values supported by data, as necessary. Correlations between input values are appropriately established in the total system performance assessment.

\section{Acceptance Criteria from Section 2.2.1.3.14, Biosphere Characteristics}

\section{Acceptance Criterion 1, System Description and Model Integration Are Adequate.}

(3) Assumptions are consistent between the biosphere characteristics modeling and other abstractions. For example, the U.S. Department of Energy should ensure that the modeling of features, events, and processes, such as climate change, soil types, sorption coefficients, volcanic ash properties, and the physical and chemical properties of radionuclides are consistent with assumptions in other total system performance assessment abstractions. 


\section{Acceptance Criterion 2, Data Are Sufficient for Model Justification.}

(1) The parameter values used in the license application are adequately justified (e.g., behaviors and characteristics of the residents of the Town of Amargosa Valley, Nevada, characteristics of the reference biosphere, etc.) and consistent with the definition of the reasonably maximally exposed individual in 10 CFR Part 63. Adequate descriptions of how the data were used, interpreted, and appropriately synthesized into the parameters are provided; and

(2) Data are sufficient to assess the degree to which features, events, and processes related to biosphere characteristics modeling have been characterized and incorporated in the abstraction. As specified in 10 CFR Part 63, the U.S. Department of Energy should ensure that the modeling of features, events, and processes, such as climate change, soil types, sorption coefficients, volcanic ash properties, and the physical and chemical properties of radionuclides are consistent with assumptions in other total system performance assessment abstractions.

\section{Acceptance Criterion 3, Data Uncertainty Is Characterized and Propagated Through the Model Abstraction.}

(1) Models use parameter values, assumed ranges, probability distributions, and bounding assumptions that are technically defensible, reasonably account for uncertainties and variabilities, do not result in an under-representation of the risk estimate, and are consistent with the definition of the reasonably maximally exposed individual in 10 CFR Part 63;

(2) The technical bases for the parameter values and ranges in the abstraction, such as consumption rates, plant and animal uptake factors, mass-loading factors, and biosphere dose conversion factors, are consistent with site characterization data, and are technically defensible;

(4) Uncertainty is adequately represented in parameter development for conceptual models and process-level models considered in developing the biosphere characteristics modeling, either through sensitivity analyses, conservative limits, or bounding values supported by data, as necessary. Correlations between input values are appropriately established in the total system performance assessment, and the implementation of the abstraction does not inappropriately bias results to a significant degree.

Despite the title of YMRP Section 2.2.1.3.12, Concentrations of Radionuclides in Groundwater, the ACs in that section do not apply to this report on BDCFs for the groundwater exposure scenario and conversion factors for evaluating compliance with the groundwater protection standards because these ACs address only the evaluation of the concentration of radionuclides in the groundwater and not the dose which would result from drinking or being exposed to that water.

Also, despite the title of YMRP Section 2.2.1.4.3, Analysis of Performance that Demonstrates Compliance with the Separate Ground-Water Protection Standards, and some references to 
dose, the ACs in that section do not apply to this report because they address only the evaluation of radionuclide concentrations and locations. Nevertheless, to the extent that these ACs are intended to address compliance with the separate groundwater protection standards, the conversion factors developed in this report are a necessary and accurate component of such a compliance demonstration, as discussed in Sections 4.1.2 and 6.3.

\subsection{CODES, STANDARDS, AND REGULATIONS}

No codes or standards, other than those identified in the Project Requirements Document (Canori and Leitner 2003 [DIRS 166275], Table 2-3) and determined to be applicable, were used in this analysis. 


\section{INTENTIONALLY LEFT BLANK}




\section{ASSUMPTIONS}

No assumptions were used in this analysis. 


\section{INTENTIONALLY LEFT BLANK}




\section{SCIENTIFIC ANALYSIS DISCUSSION}

The objectives of this analysis were to calculate:

1. BDCFs for the groundwater exposure scenario-these BDCFs are used as input to the TSPA-LA model and allow calculating the annual dose to the reasonably maximally exposed individual (RMEI) from a given concentration of radionuclides in groundwater. The dose to the RMEI is used to evaluate compliance with the individual protection standard (10 CFR 63.311 [DIRS 173164]).

2. Conversion factors that are used to calculate gross alpha particle activity in groundwater and the annual dose from beta- and photon-emitting radionuclides resulting from drinking 2 liters of water per day-these conversion factors are used in the TSPA-LA for evaluating compliance with the radionuclide concentration and dose limits of the groundwater protection standards (10 CFR 63.331 [DIRS 173164]).

The above quantities are calculated using two dosimetric approaches: the ICRP 30 and ICRP 72. The first approach is based on the concepts recommended in ICRP Publication 26 (ICRP 1977 [DIRS 101075]) and the dosimetric methods for intakes of radionuclides by workers outlined in ICRP Publication 30 (ICRP 1979 [DIRS 110386]; ICRP 1980 [DIRS 110351]; ICRP 1981 [DIRS 110352]). This approach is consistent with the individual protection standard defined in terms of total effective dose equivalent.

The ICRP 72 approach is based on the concepts recommended in ICRP Publication 60 (ICRP 1991 [DIRS 101836]). ICRP Publication 60 introduced a new dosimetric quantity, the effective dose, which uses an expanded list of tissues and organs and revised values of tissue and organ weighting factors. In addition, biokinetic and dosimetric models were updated to include new information that became available since ICRP Publication 30. A set of age-dependent dose coefficients for intakes of radionuclides by members of the public was developed and documented in a series of reports compiled in ICRP Publication 72 (ICRP 1996 [DIRS 152446]). ICRP Publication 72 is presently the most current compilation of dose coefficients for internal intakes and the approach consistent with the ICRP Publication 72 dosimetric methods is used in this analysis to develop biosphere input to TSPA. The ICRP 30-based values are presented in this report for comparison purposes.

A radionuclide-specific BDCF for the groundwater exposure scenario is numerically equal to the all-pathway annual dose that the RMEI would receive if groundwater containing a unit activity concentration of a given radionuclide were used by the RMEI for irrigation or domestic purposes.

BDCFs for the groundwater exposure scenario apply to the TSPA cases that consider the release of radionuclides to groundwater. These cases include the nominal scenario class, the seismic scenario class, and the igneous intrusion modeling case of the igneous scenario class (BSC 2005 [DIRS 173309], Section 2). The nominal scenario class represents the most likely evolution of the repository system and includes favorable and some potentially adverse future conditions. The igneous and seismic scenario classes consider combinations of FEPs that have a low probability of occurrence but may produce additional potentially adverse future conditions. 
All types of TSPA scenario classes (i.e., the nominal, igneous, and seismic) may result in the release of radionuclides to the groundwater. Assessment of doses for such releases involves BDCFs for the groundwater exposure scenario. BDCFs are developed for a unit radionuclide concentration in groundwater and therefore are independent of the actual processes that resulted in radionuclide release to the groundwater.

Biosphere exposure scenarios should not be confused with TSPA scenario classes. The biosphere exposure scenarios consider radiological consequences of radionuclide releases to the reference biosphere in a given medium, such as the groundwater, irrespective of the cause of contamination in the groundwater.

\subsection{GENERAL CONSIDERATIONS}

\subsubsection{Radionuclides Included in the Analysis}

The radionuclides of interest for the biosphere model depend on the exposure scenario, as discussed in the Biosphere Model Report (BSC 2004 [DIRS 169460], Section 6.1.3). The following twenty-eight radionuclides were identified as important for the TSPA-LA for scenario classes involving radionuclide releases to groundwater: carbon-14 $\left({ }^{14} \mathrm{C}\right)$, chlorine-36 $\left({ }^{36} \mathrm{Cl}\right)$, selenium-79 $\left({ }^{79} \mathrm{Se}\right)$, strontium-90 $\left({ }^{90} \mathrm{Sr}\right)$, technetium-99 $\left({ }^{99} \mathrm{Tc}\right)$, tin-126 $\left({ }^{126} \mathrm{Sn}\right)$, iodine-129 $\left({ }^{129} \mathrm{I}\right)$, cesium-135 ( $\left.{ }^{135} \mathrm{Cs}\right)$, cesium-137 $\left({ }^{137} \mathrm{Cs}\right)$, lead-210 $\left({ }^{210} \mathrm{~Pb}\right)$, radium-226 $\left({ }^{226} \mathrm{Ra}\right)$, actinium-227 $\left({ }^{227} \mathrm{Ac}\right)$, thorium-229 $\left({ }^{229} \mathrm{Th}\right)$, thorium-230 $\left({ }^{230} \mathrm{Th}\right)$, thorium-232 $\left({ }^{232} \mathrm{Th}\right)$, protactinium-231 $\left({ }^{231} \mathrm{~Pa}\right)$, uranium-232 $\left({ }^{232} \mathrm{U}\right)$, uranium-233 $\left({ }^{233} \mathrm{U}\right)$, uranium-234 $\left({ }^{234} \mathrm{U}\right)$, uranium-236 $\left({ }^{236} \mathrm{U}\right)$, uranium-238 $\left({ }^{238} \mathrm{U}\right)$, neptunium-237 $\left({ }^{237} \mathrm{~Np}\right)$, plutonium-238 $\left({ }^{238} \mathrm{Pu}\right)$, plutonium-239 $\left({ }^{239} \mathrm{Pu}\right)$, plutonium-240 $\left({ }^{240} \mathrm{Pu}\right)$, plutonium-242 $\left({ }^{242} \mathrm{Pu}\right)$, americium-241 $\left({ }^{241} \mathrm{Am}\right)$, and americium-243 $\left({ }^{243} \mathrm{Am}\right)$ (BSC 2004 [DIRS 169460], Section 6.1.3). These radionuclides are referred to in this analysis as the primary radionuclides. The list includes radionuclides that are of potential importance during both the first 20,000 years and the period of up to 1,000,000 years (BSC 2004 [DIRS 169460], Section 6.1.3).

The biosphere model accounts for the decay products of the primary radionuclides. The short-lived decay products (half-lives less than 180 days) are considered to be in secular equilibrium with the parent radionuclide and their contributions to the BDCFs are included in the BDCF for the long-lived radionuclide (either as a primary radionuclide or its long-lived decay product) (BSC 2004 [DIRS 169460], Section 6.3.5). These decay products for radionuclides of interest are listed in Table 4.1-2. The biosphere model also accounts for the decay and ingrowth of the long-lived decay products in the soil, and adds BDCF contributions of the long-lived decay products to that of the parent primary radionuclide (BSC 2004 [DIRS 169460], Section 6.4.1.2). Two decay products of the primary radionuclides, ${ }^{228} \mathrm{Th}$ and ${ }^{228} \mathrm{Ra}$, have halflives greater than 180 days and are not automatically included in the BDCFs of the parent when the biosphere model is executed. For biosphere modeling, ${ }^{228} \mathrm{Th}$ and ${ }^{228} \mathrm{Ra}$ are treated like primary radionuclides. After BDCFs are calculated for these radionuclides, their BDCFs are added to the BDCF of the parent primary radionuclide. In the case of ${ }^{232} \mathrm{Th}$, the BDCF includes the contribution from ${ }^{228} \mathrm{Ra},{ }^{228} \mathrm{Th}$, and their short-lived decay products. The BDCF for ${ }^{232} \mathrm{U}$ includes the contribution from ${ }^{228} \mathrm{Th}$ and its short-lived decay products. 


\subsubsection{Description of the Groundwater Exposure Scenario}

A detailed description of the groundwater exposure scenario, including the associated conceptual and mathematical models, is presented in the Biosphere Model Report (BSC 2004 [DIRS 169460], Sections 6.3.1 and 6.4). A brief summary of the main concepts and the modeling approach for the groundwater exposure scenario is presented in this section.

Radionuclide release to the reference biosphere, environmental transport, and exposure of the receptor are shown schematically in Figure 6.1-1. The source of radionuclides in the biosphere is contaminated groundwater extracted from a well, which is the interface between the geosphere and biosphere. The biosphere model does not consider water treatment between extraction and use, and therefore, radionuclide concentrations in the well water are the same as those in the groundwater. This and other biosphere analyses frequently use the term groundwater, rather than well water, when referring to the water used in the reference biosphere.

Radionuclide transport in the reference biosphere is initiated when contaminated groundwater is used for agricultural and domestic purposes. Five modes of groundwater use are considered in the model: human consumption, domestic use, crop irrigation, animal consumption, and fish farming.

After radionuclides enter the reference biosphere, migration through the biosphere occurs due to a number of transport processes that lead to radionuclide contamination and accumulation in environmental media (e.g., soil, air, flora, and fauna). The following environmental transport processes are explicitly included in the biosphere model:

- Radionuclide accumulation in soil as a result of long-term irrigation with contaminated water

- Resuspension of contaminated soil

- Radionuclide deposition on crop surfaces by dry processes (resuspension of contaminated soil and subsequent adhesion of soil particles on crop surfaces)

- Radionuclide deposition on crop surfaces by wet processes resulting from using contaminated irrigation water

- Translocation of contaminants from the deposition site to the edible tissues of vegetation

- Post-deposition retention by crops (including weathering processes)

- Radionuclide uptake by crops through the roots

- Release of gaseous radionuclides $\left({ }^{222} \mathrm{Rn},{ }^{14} \mathrm{CO}_{2}\right)$ from the soil

- Absorption of ${ }^{14} \mathrm{CO}_{2}$ by crops from the atmosphere

- Radionuclide uptake by animals through consumption of contaminated feed, water, and soil, followed by transfer to animal products 


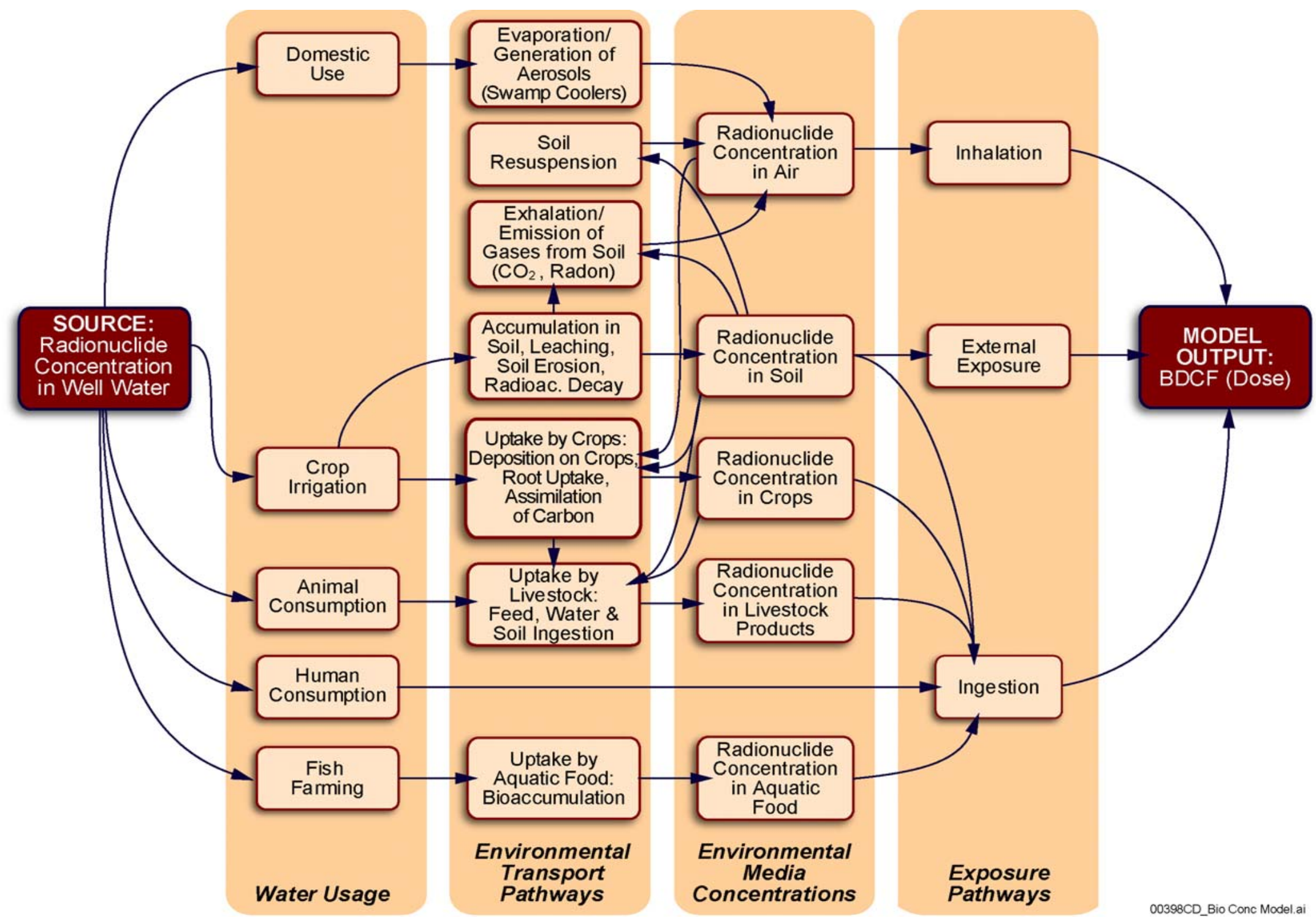


- Radionuclide transfer from water to air via evaporative coolers

- Radionuclide transfer from water to fish (aquatic food).

Exposure of humans to radionuclides in the environment arises when people come in direct (inhalation and ingestion) or indirect (external exposure) contact with contaminated environmental media. Table 6.1-1 provides a summary of human exposure pathways considered in the biosphere model, as well as environmental media and typical activities that may potentially lead to radiation exposure. Other activities and the associated exposures were evaluated for inclusion in the biosphere model (BSC 2004 [DIRS 169460]) but were not included because of low consequences. Such an approach did not underestimate the risk to the RMEI.

Table 6.1-1. Receptor Exposure Pathways for the Groundwater Exposure Scenario

\begin{tabular}{|c|c|c|c|}
\hline $\begin{array}{l}\text { Environmental } \\
\text { Medium }\end{array}$ & $\begin{array}{l}\text { Exposure } \\
\text { Mode }\end{array}$ & Exposure Pathways & Examples of Typical Activities \\
\hline Water & Ingestion & Water intake. & $\begin{array}{l}\text { Drinking water and water-based } \\
\text { beverages and water used in food } \\
\text { preparation. }\end{array}$ \\
\hline \multirow[t]{2}{*}{ Soil } & Ingestion & Inadvertent soil ingestion. & $\begin{array}{l}\text { Recreational activities, occupational } \\
\text { activities, gardening, and } \\
\text { consumption of fresh fruit and } \\
\text { vegetables. }\end{array}$ \\
\hline & External & External radiation exposure. & $\begin{array}{l}\text { Activities on or near contaminated } \\
\text { soils. }\end{array}$ \\
\hline Air & Inhalation & $\begin{array}{l}\text { Breathing resuspended particles, gases } \\
\left({ }^{222} \mathrm{Rn} \text { and progeny, plus }{ }^{14} \mathrm{CO}_{2}\right) \text {, and } \\
\text { aerosols from evaporative coolers. }\end{array}$ & $\begin{array}{l}\text { Any outdoor activities, including soil- } \\
\text { disturbing activities related to work } \\
\text { and recreation. Domestic activities, } \\
\text { including sleeping. }\end{array}$ \\
\hline Plants & Ingestion & $\begin{array}{l}\text { Consumption of locally produced crops: } \\
\text { leafy vegetables, other vegetables, fruit, and } \\
\text { grain. }\end{array}$ & Eating contaminated crop foodstuffs. \\
\hline Animals & Ingestion & $\begin{array}{l}\text { Consumption of locally produced animal } \\
\text { products: meat, poultry, milk, and eggs. }\end{array}$ & $\begin{array}{l}\text { Eating contaminated animal product } \\
\text { foodstuffs. }\end{array}$ \\
\hline Fish & Ingestion & $\begin{array}{l}\text { Consumption of locally produced freshwater } \\
\text { fish. }\end{array}$ & Eating contaminated fish. \\
\hline
\end{tabular}

Source: BSC 2004 [DIRS 169460], Table 6.3-1.

\subsubsection{Consideration of Climate Change}

Climate refers to the meteorological conditions that characteristically prevail in a particular region. The climate model for the Yucca Mountain region was formulated using paleoclimate and paleoenvironmental reconstructions based on microfossil evaluations in Owens Lake, California, and cores and calcite isotope records from Devils Hole, Death Valley National Park, Nevada. The sequence and duration of past climate periods are identified and applied to the Yucca Mountain site, which has a similar climate setting.

The climate at Yucca Mountain is expected to change in the future. Although forecasting long-term (e.g., for the next 10,000 years) climate change is speculative, the future climate at Yucca Mountain was forecasted based on the study of past climates (BSC 2004 [DIRS 170002], Section 1). Temperature and precipitation records from present-day meteorological stations at 
colder and wetter sites were selected to represent future climate states (BSC 2004 [DIRS 170002], Section 6.6.1). The forecasted future climate states, durations, and future climate analogue locations are summarized in Table 6.1-2.

Table 6.1-2. Predicted Future Climates and the Future Climate Analogue Locations

\begin{tabular}{|c|c|c|}
\hline Climate State & Duration & Representative Meteorological Stations \\
\hline Present-day interglacial climate & 400 to 600 years & Yucca Mountain region \\
\hline Monsoon climate & 900 to 1,400 years & $\begin{array}{l}\text { Average lower bound: } \\
\text { Yucca Mountain region } \\
\text { Average upper bound: } \\
\text { Nogales, Arizona } \\
\text { Hobbs, New Mexico }\end{array}$ \\
\hline Glacial transition climate & 8,000 to 8,700 years & $\begin{array}{l}\text { Average lower bound: } \\
\text { Beowawe, Nevada } \\
\text { Delta, Utah } \\
\text { Average upper bound: } \\
\text { Spokane, Washington } \\
\text { Rosalia, Washington } \\
\text { St. John, Washington }\end{array}$ \\
\hline
\end{tabular}

Source: BSC 2004 [DIRS 170002], Table 6-1.

For modeling climate change for TSPA, the climate shifts in a series of step changes between three different climate states in the first 10,000 years: present-day climate, monsoon climate (about twice the precipitation of the present-day climate), and glacial transition climate (colder than monsoon but similar precipitation) (BSC 2003 [DIRS 166296], p. 79). Within the TSPALA model, these shifts require coordinating the coupled submodels because they must all simultaneously change to the appropriate climate state. To support climate change modeling for TSPA, BDCFs are developed for the three climate states (Section 6.2.2).

A proportionality function was developed based on BDCFs for the present-day (interglacial) climate and BDCFs for the average upper bound of the glacial transition climate. These climates represent the two extreme conditions, with respect to temperature and precipitation, forecasted to occur in the Yucca Mountain region during the 10,000-year postclosure period. To determine the dependence of BDCFs on climate, the range of each climate-dependent biosphere model input value was calculated. The range for each parameter spanned from the value for the present-day climate to the value for the upper bound of the glacial transition climate. Model simulations were run using these parameter ranges as input. Correlation coefficients were calculated for the climate-dependent model-input parameters and the model output (i.e., the BDCFs), as described in Section 6.2.2. Correlation coefficients were highest for the long-term average annual irrigation rate (Tables 6.2-4 and 6.2-5). The BDCFs for a given radionuclide appear to be proportional to the value of the annual average irrigation rate. Using this proportionality, BDCFs for the monsoon climate and the glacial transition climate for each radionuclide were calculated by interpolation between the values (extremes) for the present-day climate and the upper bound of the glacial transition climate (Section 6.2.2). 


\subsubsection{Definition of the Receptor}

The regulations for licensing the repository include an individual protection standard for the performance of the repository. This standard is expressed as the annual dose limit to a hypothetical person called the RMEI (10 CFR 63.311 [DIRS 173164]). Analysis of annual dose includes potential pathways of radionuclide transport and exposure (10 CFR 63.311 [DIRS 173164]). Changes in the reference biosphere, other than climate changes, are not included.

According to 10 CFR 63.312 [DIRS 173164] the RMEI is a hypothetical receptor that:

- Lives above the highest concentration of radionuclides in the plume of contamination

- Has a diet and lifestyle representative of people who now reside in the Amargosa Valley based on surveys of the people residing in the Amargosa Valley that determine current diets and lifestyles, and then use the mean values of these factors in the assessments conducted for 10 CFR 63.311 and 10 CFR 63.321

- Uses well water with average concentrations of radionuclides based on an annual water demand of 3,000 acre-feet

- Drinks 2 liters of water per day from wells drilled into the groundwater from a point above the highest concentration of radionuclides in the plume of contamination

- Is an adult who is metabolically and physiologically consistent with present knowledge of adults.

To meet the requirements of 10 CFR 63.312(b) [DIRS 173164], the dietary and lifestyle characteristics of the RMEI were determined based on surveys of people living in the Amargosa Valley combined with national information on behavior patterns. Characteristics of the RMEI were developed in a separate analysis (BSC 2005 [DIRS 172827]).

\subsubsection{Biosphere Model}

This analysis was performed using a verified and validated model, ERMYN, which is described in the Biosphere Model Report (BSC 2004 [DIRS 169460]). The model files were obtained from the Model Warehouse (DTN: MO0306MWDBGSMF.001 [DIRS 163816]). These model files were created using GoldSim version 7.50.100. Since then, updated versions of GoldSim were baselined. Two of these versions: version 8.01 Service Pack 4 (BSC 2004 [DIRS 169695]) and 8.02.300 (BSC 2004 [DIRS 172588]) were used in this analysis. Verification of the model files for GoldSim versions 8.01 Service Pack 4 and 8.02.300 is presented in Appendix C.

The ERMYN model (BSC 2004 [DIRS 169460]), which this analysis uses to calculate BDCFs, models biosphere processes for radionuclides released from the repository to the biosphere, the environmental transport of these radionuclides, and human exposure. The Biosphere Model Report (BSC 2004 [DIRS 169460]), which describes the ERMYN model: 
1. Describes the biosphere model objectives, reference biosphere, human receptor, exposure scenarios, environmental transport pathways, and human exposure pathways (BSC 2004 [DIRS 169460], Section 6.1).

2. Develops the conceptual model based on site-specific FEPs, the reference biosphere and human receptor, and a number of other approximations (BSC 2004 [DIRS 169460], Sections 6.2 and 6.3).

3. Describes the mathematical model and submodels based on the conceptual model and other published biosphere models (BSC 2004 [DIRS 169460], Section 6.4).

4. Summarizes model input parameters and uncertainty distributions (BSC 2004 [DIRS 169460], Section 6.6).

5. Identifies model improvements compared with the previous biosphere model (BSC 2004 [DIRS 169460], Section 6.7).

6. Constructs the ERMYN implementation tool based on the mathematical model using GoldSim stochastic simulation software (BSC 2004 [DIRS 169460], Section 6.8).

7. Verifies the ERMYN implementation tool in GoldSim (BSC 2004 [DIRS 169460], Section 6.10).

8. Validates the ERMYN model by comparing the conceptual and mathematical models and by comparing the numerical results with results from other published biosphere models (BSC 2004 [DIRS 169460], Section 7).

The ERMYN model was designed to perform an environmental radiation dose assessment and can calculate radionuclide-specific dose or provide a radionuclide-specific BDCF for a given receptor. Use of the ERMYN model in performance assessment is limited to the calculation of BDCFs. Input parameters for the biosphere model are developed and documented in a series of five model parameter reports:

- Agricultural and Environmental Parameters for the Biosphere Model (BSC 2004 [DIRS 169673]).

- Characteristics of the Receptor for the Biosphere Model (BSC 2005 [DIRS 172827]).

- Environmental Transport Input Parameters for the Biosphere Model (BSC 2004 [DIRS 169672]).

- Inhalation Exposure Input Parameters for the Biosphere Model (BSC 2004 [DIRS 169458]).

- Soil-Related Input Parameters for the Biosphere Model (BSC 2004 [DIRS 169459]).

The architecture of the biosphere model for the groundwater exposure scenario, including the submodels, is shown in Figure 6.1-2. The submodels address radionuclide transport to, and 
accumulation in, specific environmental media (e.g., soil, air, plants, animals, and fish) and the inhalation, ingestion, and external exposure pathways.

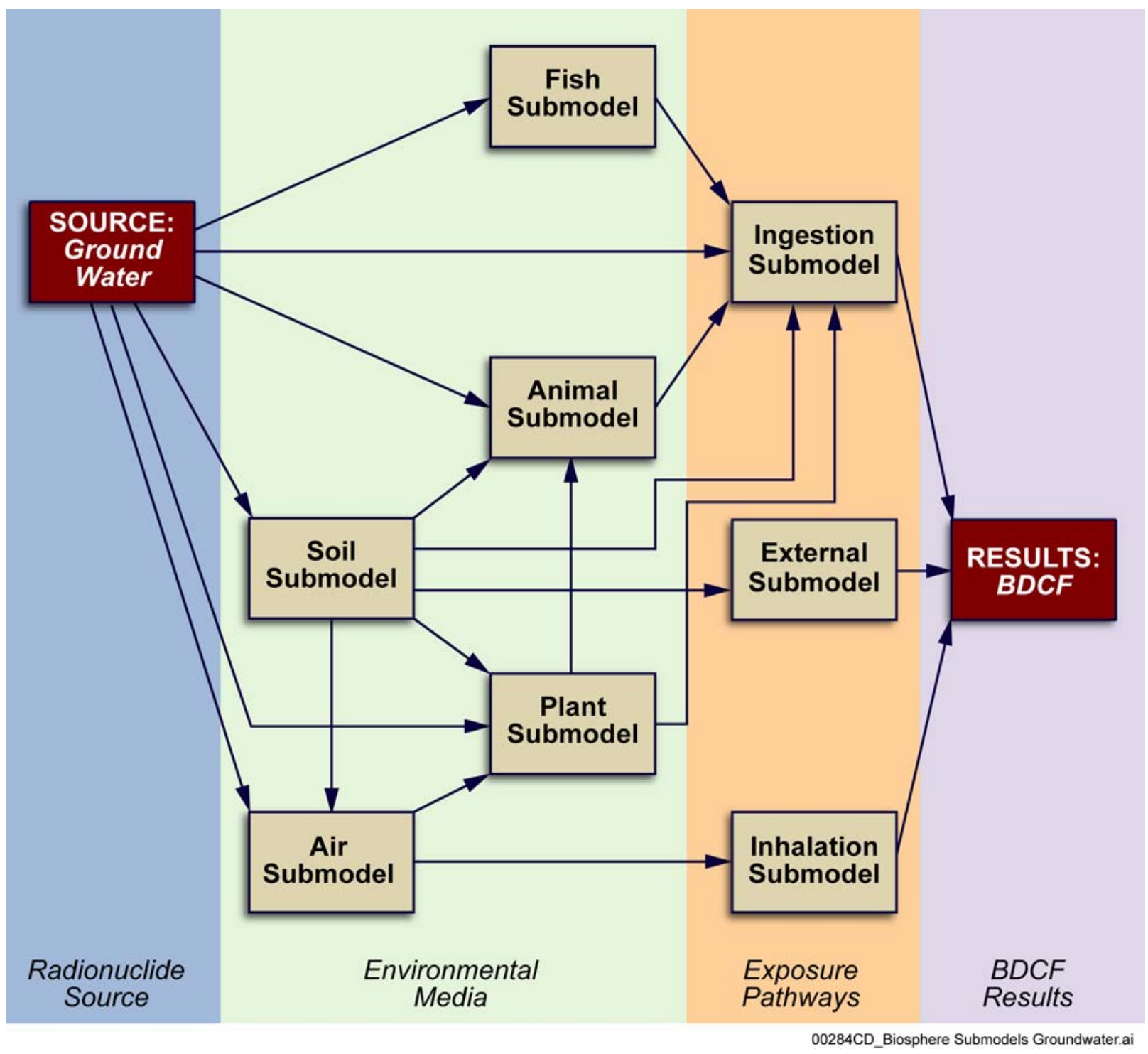

Figure 6.1-2. Relationships Among Submodels for the Groundwater Exposure Scenario

\subsection{DEVELOPMENT OF BIOSPHERE DOSE CONVERSION FACTORS FOR THE GROUNDWATER EXPOSURE SCENARIO}

The BDCFs for the groundwater exposure scenario were calculated, using probabilistic analysis, in a series of simulations for each of the 28 primary radionuclides and 2 long-lived decay products, ${ }^{228}$ Th and ${ }^{228} \mathrm{Ra}$ (Section 6.1.1). Each simulation resulted in 1,000 model realizations. A model realization is one of the possible model outcomes obtained as a result of a single round of sampling of the model input parameters. This section describes the format and the summary of the results of biosphere modeling for the groundwater exposure scenario, as well as their use in the TSPA model. 


\subsubsection{Modeling Methods: Treatment of Uncertainty}

The probabilistic approach was chosen to develop BDCFs. This approach allows statistical sampling of parameter values defined by their probability distribution functions. This method, called Monte Carlo analysis, provides a quantitative evaluation of the parameter uncertainties and their impacts on the modeling outcome. Uncertainty in the model outcome is represented by the probability distribution functions of the BDCFs. Input parameter values were sampled using the Latin Hypercube sampling method for consistency with the sampling technique to be used in TSPA (BSC 2005 [DIRS 173309], Table 2.10-2). With Latin Hypercube sampling, the probability distribution is divided into intervals of equal probability. The code then randomly samples a value within each interval, which results in a more even and consistent sampling compared with the conventional Monte Carlo random sampling scheme. The value of the random seed in GoldSim was set to 1.

\subsubsection{Modeling Methods: Incorporation of Climate Change}

The biosphere model was constructed for a reference biosphere with an arid or semi-arid climate. The climate during the compliance period is projected to change from the present-day (interglacial) climate, through a monsoon climate, to a glacial transition climate. Although the monsoon climate generally is wetter, and the glacial transition climate is wetter and cooler than the present-day climate, the radionuclide environmental transport pathways and the human exposure pathways are expected to remain essentially unchanged. The conceptual and mathematical structure of the ERMYN model was designed such that it is appropriate for the entire 10,000-year postclosure period. Climate change is addressed through model input parameters, which use different values depending on the climate.

Detailed model input was developed for the anticipated extremes of climate, the present-day climate and the upper bound of the glacial transition climate, which is forecasted to start in about 2,000 years and last through the compliance period (BSC 2004 [DIRS 170002]). For the upper bound of the glacial transition climate, climate-dependent model input parameters were based on future climate analogue sites represented by meteorological conditions in east central Washington state. Although these are extremes in climate predictions, they remain arid to semiarid. During the compliance period, the climate conditions are expected to be variable and may fall between the two extremes for which input data were developed. The approach adopted, to provide the TSPA-LA with BDCFs representing possible future climate variation between the two extremes, is provided below.

BDCFs were first calculated for two climates (the present-day climate and one future climate state, i.e., the upper bound of the glacial transition climate) representing the upper and lower bounds of the expected range of the temperature and precipitation over the 10,000-year postclosure period. The mean values of the BDCFs for these two climate states, and the ratio of the two, are shown in Tables 6.2-1 and 6.2-2 for the ICRP 30 and ICRP 72 dosimetric approaches, respectively. In the next step, the BDCFs for the monsoon and the glacial transition climates were calculated as described in this section. 
Table 6.2-1. Mean ICRP 30-Based BDCFs for Climate Extremes and Their Ratios

\begin{tabular}{|l|c|c|c|}
\hline \multirow{2}{*}{ Radionuclide } & \multicolumn{2}{|c|}{ Mean BDCF, rem/yr per pCi/L } & \multirow{2}{*}{$\begin{array}{c}\text { BDCF Ratio (Glacial } \\
\text { Transition to Present- } \\
\text { Day Climate) }\end{array}$} \\
\cline { 2 - 3 } & Present-Day Climate & $\begin{array}{c}\text { Glacial Transition } \\
\text { Climate- Upper Bound }\end{array}$ & 0.94 \\
\hline C-14 & $9.10 \mathrm{E}-06$ & $8.52 \mathrm{E}-06$ & 0.75 \\
\hline Cl-36 & $2.07 \mathrm{E}-05$ & $1.55 \mathrm{E}-05$ & 0.64 \\
\hline Sr-90 & $6.19 \mathrm{E}-05$ & $3.97 \mathrm{E}-05$ & 0.87 \\
\hline Tc-99 & $1.64 \mathrm{E}-04$ & $1.43 \mathrm{E}-04$ & 0.85 \\
\hline Sn-126 & $2.26 \mathrm{E}-06$ & $1.93 \mathrm{E}-06$ & 0.55 \\
\hline I-129 & $6.33 \mathrm{E}-03$ & $3.51 \mathrm{E}-03$ & 0.86 \\
\hline Cs-135 & $3.36 \mathrm{E}-04$ & $2.91 \mathrm{E}-04$ & 0.61 \\
\hline Cs-137 & $5.57 \mathrm{E}-05$ & $3.39 \mathrm{E}-05$ & 0.59 \\
\hline Pb-210 & $4.82 \mathrm{E}-04$ & $2.85 \mathrm{E}-04$ & 0.83 \\
\hline Ra-226 & $9.04 \mathrm{E}-03$ & $7.48 \mathrm{E}-03$ & 0.59 \\
\hline Ac-227 & $9.69 \mathrm{E}-02$ & $5.71 \mathrm{E}-02$ & 0.56 \\
\hline Th-229 & $3.11 \mathrm{E}-02$ & $1.75 \mathrm{E}-02$ & 0.54 \\
\hline Th-230 & $3.41 \mathrm{E}-02$ & $1.84 \mathrm{E}-02$ & 0.59 \\
\hline Th-232 & $2.93 \mathrm{E}-02$ & $1.72 \mathrm{E}-02$ & 0.55 \\
\hline Pa-231 & $4.45 \mathrm{E}-02$ & $2.44 \mathrm{E}-02$ & 0.57 \\
\hline U-232 & $1.01 \mathrm{E}-01$ & $5.76 \mathrm{E}-02$ & 0.56 \\
\hline U-233 & $5.82 \mathrm{E}-03$ & $3.26 \mathrm{E}-03$ & 0.57 \\
\hline U-234 & $1.86 \mathrm{E}-03$ & $1.06 \mathrm{E}-03$ & 0.57 \\
\hline U-236 & $1.28 \mathrm{E}-03$ & $7.27 \mathrm{E}-04$ & 0.57 \\
\hline U-238 & $1.14 \mathrm{E}-03$ & $6.43 \mathrm{E}-04$ & 0.57 \\
\hline Np-237 & $1.13 \mathrm{E}-03$ & $6.44 \mathrm{E}-04$ & 0.73 \\
\hline Pu-238 & $6.98 \mathrm{E}-03$ & $5.13 \mathrm{E}-03$ & 0.72 \\
\hline Pu-239 & $4.69 \mathrm{E}-03$ & $3.38 \mathrm{E}-03$ & 0.64 \\
\hline Pu-240 & $9.25 \mathrm{E}-03$ & $5.96 \mathrm{E}-03$ & 0.64 \\
\hline Pu-242 & $9.01 \mathrm{E}-03$ & $5.83 \mathrm{E}-03$ & \\
\hline Am-241 & $8.91 \mathrm{E}-03$ & $5.73 \mathrm{E}-03$ & \\
\hline Am-243 & $6.83 \mathrm{E}-03$ & $4.65 \mathrm{E}-03$ & 0.64 \\
\hline Soun & $6.14 \mathrm{E}-03$ & \\
\hline
\end{tabular}

Source: MO0407SPAINEXI.002 [DIRS 170597]; MO0407SPASRPBM.002 [DIRS 170755];

MO0403SPAAEIBM.002 [DIRS 169392]; MO0407SPACRBSM.002 [DIRS 170677];

MO0406SPAETPBM.002 [DIRS 170150]; MO0306MWDBGSMF.001 [DIRS 163816].

NOTE: The mean BDCF values and their ratios were calculated using Excel (file name $=G W B D C F$

Realizations_MC and FC_Rev 4.xls, worksheet name = Comparison_Climates; Appendix B). 
Table 6.2-2. Mean ICRP 72-Based BDCFs for Climate Extremes and Their Ratios

\begin{tabular}{|c|c|c|c|}
\hline \multirow[b]{2}{*}{ Radionuclide } & \multicolumn{2}{|c|}{ Mean BDCF, rem/yr per pCi/L } & \multirow{2}{*}{$\begin{array}{c}\text { BDCF Ratio (Glacial } \\
\text { Transition to Present- } \\
\text { Day Climate) }\end{array}$} \\
\hline & Present-Day Climate & $\begin{array}{l}\text { Glacial Transition } \\
\text { Climate- Upper Bound }\end{array}$ & \\
\hline C-14 & $9.35 \mathrm{E}-06$ & $8.76 \mathrm{E}-06$ & 0.94 \\
\hline $\mathrm{Cl}-36$ & $2.35 \mathrm{E}-05$ & 1.76E-05 & 0.75 \\
\hline Se-79 & $7.65 \mathrm{E}-05$ & 4.90E-05 & 0.64 \\
\hline Sr-90 & $1.24 \mathrm{E}-04$ & $1.07 \mathrm{E}-04$ & 0.86 \\
\hline Tc-99 & $3.74 \mathrm{E}-06$ & $3.14 \mathrm{E}-06$ & 0.84 \\
\hline Sn-126 & 5.92E-03 & $3.28 \mathrm{E}-03$ & 0.55 \\
\hline $\mathrm{I}-129$ & 4.95E-04 & 4.29E-04 & 0.87 \\
\hline Cs-135 & 5.87E-05 & $3.57 E-05$ & 0.61 \\
\hline Cs-137 & 4.59E-04 & $2.72 \mathrm{E}-04$ & 0.59 \\
\hline $\mathrm{Pb}-210$ & 8.74E-03 & $7.21 \mathrm{E}-03$ & 0.83 \\
\hline Ra-226 & $5.32 \mathrm{E}-02$ & $3.12 E-02$ & 0.59 \\
\hline Ac-227 & $9.65 \mathrm{E}-03$ & $5.39 \mathrm{E}-03$ & 0.56 \\
\hline Th-229 & 1.60E-02 & 8.81E-03 & 0.55 \\
\hline Th-230 & $1.92 \mathrm{E}-02$ & 1.11E-02 & 0.58 \\
\hline Th-232 & $2.38 \mathrm{E}-02$ & 1.38E-02 & 0.58 \\
\hline Pa-231 & $3.33 \mathrm{E}-02$ & $1.86 \mathrm{E}-02$ & 0.56 \\
\hline U-232 & $3.21 \mathrm{E}-03$ & 2.17E-03 & 0.67 \\
\hline$U-233$ & $7.24 \mathrm{E}-04$ & 4.49E-04 & 0.62 \\
\hline U-234 & $4.68 \mathrm{E}-04$ & $3.02 E-04$ & 0.65 \\
\hline U-236 & $3.96 \mathrm{E}-04$ & $2.58 \mathrm{E}-04$ & 0.65 \\
\hline U-238 & $4.26 \mathrm{E}-04$ & 2.79E-04 & 0.65 \\
\hline $\mathrm{Np}-237$ & $1.45 \mathrm{E}-03$ & 8.64E-04 & 0.59 \\
\hline Pu-238 & $2.59 \mathrm{E}-03$ & 1.43E-03 & 0.55 \\
\hline Pu-239 & $6.83 \mathrm{E}-03$ & $3.74 \mathrm{E}-03$ & 0.55 \\
\hline Pu-240 & 6.60E-03 & 3.61E-03 & 0.55 \\
\hline $\mathrm{Pu}-242$ & $6.38 \mathrm{E}-03$ & $3.51 \mathrm{E}-03$ & 0.55 \\
\hline Am-241 & 3.47E-03 & 1.90E-03 & 0.55 \\
\hline Am-243 & $5.70 \mathrm{E}-03$ & $3.11 \mathrm{E}-03$ & 0.55 \\
\hline
\end{tabular}

Source: MO0407SPAINEXI.002 [DIRS 170597]; MO0407SPASRPBM.002 [DIRS 170755]; MO0403SPAAEIBM.002 [DIRS 169392]; MO0407SPACRBSM.002 [DIRS 170677]; MO0406SPAETPBM.002 [DIRS 170150]; MO0306MWDBGSMF.001 [DIRS 163816]; MO0503SPADCESR.000 [DIRS 172896]

NOTE: The mean BDCF values and their ratios were calculated using Excel (file name $=G W B D C F$ Realizations_MC and FC_Rev 4.xIs, worksheet name = Comparison_Climates; Appendix B).

Regardless of the dosimetric approach, the BDCF values for the upper bound of the glacial transition climate are consistently lower than the corresponding values for the present-day climate. The differences between the BDCFs are within a factor of two. The ratios of the BDCFs for the glacial transition and the present-day climates, depending on the dosimetry system used, ranged from 0.84 to 0.85 for ${ }^{99} \mathrm{Tc}$; and 0.86 to 0.87 for ${ }^{129} \mathrm{I}$; for actinides, less than 0.73 for the ICRP 30 dosimetry and less than 0.67 for the ICRP 72 dosimetry; and 0.94 for ${ }^{14} \mathrm{C}$ regardless of the dosimetric approach. Inspection of the BDCF distributions for the present-day 
climate (see Tables 6.2-8 and 6.2-11) indicates that the BDCF variability, as measured by the 95th- to the 5th-percentile point, is at least a factor of about 4.2 for ${ }^{14} \mathrm{C}, 3.3$ for ${ }^{99} \mathrm{Tc}, 2.1$ for ${ }^{129} \mathrm{I}$, and 2.7 for ${ }^{237} \mathrm{~Np}$. Variability in the BDCF values due to normal parametric uncertainty dominates the change in expected mean BDCF values caused by extreme climate change. It was therefore appropriate to use interpolation between the two extreme climate states, for which detailed information was available, to generate BDCFs for the expected distribution of climates in the future.

To evaluate this approach, the influence of climate change on the BDCF values was investigated. For the input parameters that are affected by climate change (Table 6.2-3), uniform distributions of parameter values were constructed ranging between the parameter averages for the extreme climates (i.e., the present-day and the upper bound of the glacial transition climates). The uniform distribution was selected because it is the simplest distribution that captures the range of parameter values.

Table 6.2-3. Climate-dependent Input Parameters and Their Average Values for the Present-Day Climate and the Upper Bound of the Glacial Transition Climate

\begin{tabular}{|l|c|c|}
\hline \multicolumn{1}{|c|}{ Parameter } & Present-Day Climate & $\begin{array}{c}\text { Glacial Transition Climate } \\
\text { (Upper Bound) }\end{array}$ \\
\hline Growing time, other vegetables & 80 days & 100 days \\
\hline Growing time, fruits & 160 days & 105 days \\
\hline Growing time, grains & 200 days & 185 days \\
\hline Growing time, cattle forage & 75 days & 90 days \\
\hline Irrigation application, leafy vegetables & $14.7 \mathrm{~mm}$ & $14.6 \mathrm{~mm}$ \\
\hline Irrigation application, other vegetables & $26.0 \mathrm{~mm}$ & $25.0 \mathrm{~mm}$ \\
\hline Irrigation application, fruits & $33.9 \mathrm{~mm}$ & $34.2 \mathrm{~mm}$ \\
\hline Irrigation application, grains & $56.7 \mathrm{~mm}$ & $51.3 \mathrm{~mm}$ \\
\hline Irrigation application, cattle forage & $57.8 \mathrm{~mm}$ & $53.5 \mathrm{~mm}$ \\
\hline Average annual irrigation rate & $0.95 \mathrm{~m} / \mathrm{yr}$ & $0.50 \mathrm{~m} / \mathrm{yr}$ \\
\hline Daily average irrigation rate, leafy vegetables & $5.41 \mathrm{~mm} /$ day & $3.81 \mathrm{~mm} /$ day \\
\hline Daily average irrigation rate, other vegetables & $7.71 \mathrm{~mm} /$ day & $3.84 \mathrm{~mm} /$ day \\
\hline Daily average irrigation rate, fruits & $7.41 \mathrm{~mm} /$ day & $3.90 \mathrm{~mm} /$ day \\
\hline Daily average irrigation rate, grains & $4.64 \mathrm{~mm} /$ day & $3.36 \mathrm{~mm} /$ day \\
\hline Daily average irrigation rate, cattle forage & $6.55 \mathrm{~mm} /$ day & $4.14 \mathrm{~mm} /$ day \\
\hline Overwatering rate & $0.079 \mathrm{~m} / \mathrm{yr}$ & $0.067 \mathrm{~m} / \mathrm{yr}$ \\
\hline Water concentration modifying factor (for fisheries) & $4.15^{\mathrm{a}}$ & $2.4^{\text {a }}$ \\
\hline Evaporative cooler use factor & 0.39 & 0.085 \\
\hline
\end{tabular}

Source: MO0403SPAAEIBM.002 [DIRS 169392]; MO0407SPACRBSM.002 [DIRS 170677]; MO0406SPAETPBM.002 [DIRS 170150].

${ }^{a}$ All radionuclides except $\mathrm{C}-14$, for which 1 was used.

The model was then executed using these distributions for the climate-dependent parameters with the other input parameter distributions and values left unchanged. BDCF values for 1,000 model realizations were calculated for each primary radionuclide (Section 6.1.1). The influence of climate-dependent input parameters on the model output was determined by calculating correlation coefficients for the BDCFs and climate-dependent input parameters (Tables 6.2-4 and 6.2-5, for the ICRP 30- and ICRP 72-based approach, respectively). 
Table 6.2-4. Correlations (Raw Correlation Coefficients) for ICRP 30-Based BDCFs and Climate- I Dependent Input Parameters

\begin{tabular}{|c|c|c|c|c|c|c|c|c|c|}
\hline \multirow[b]{2}{*}{ 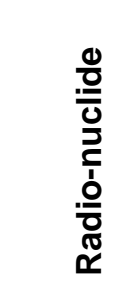 } & \multicolumn{4}{|c|}{ Growing time } & \multicolumn{5}{|c|}{ Irrigation Application } \\
\hline & 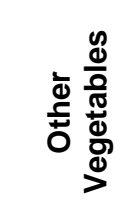 & $\begin{array}{l}\frac{n}{5} \\
\frac{5}{4}\end{array}$ & 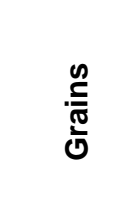 & 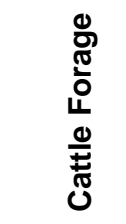 & 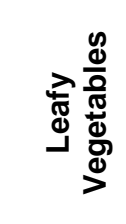 & 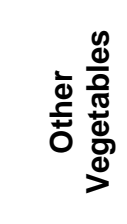 & 量 & $\begin{array}{l}\stackrel{n}{\bar{\sigma}} \\
\frac{\pi}{0}\end{array}$ & 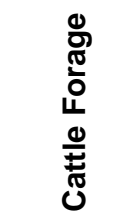 \\
\hline C-14 & -0.0022 & -0.0041 & -0.0026 & -0.0711 & -0.0233 & 0.0076 & 0.0015 & -0.0575 & -0.0174 \\
\hline Cl-36 & -0.0182 & 0.0181 & 0.0080 & 0.0161 & 0.0079 & -0.0081 & -0.0191 & -0.0007 & -0.0683 \\
\hline Se-79 & 0.0388 & -0.0142 & -0.0192 & 0.0312 & -0.0161 & -0.0432 & -0.0191 & -0.0407 & -0.0036 \\
\hline Sr-90 & 0.0330 & 0.0071 & 0.0070 & -0.0185 & 0.0593 & -0.0058 & 0.0756 & 0.0171 & -0.0124 \\
\hline Tc-99 & 0.0426 & 0.0801 & -0.0374 & -0.0059 & 0.0125 & 0.0261 & 0.0077 & 0.0348 & -0.0329 \\
\hline Sn-126 & 0.0257 & 0.0294 & 0.0451 & 0.0185 & 0.0688 & -0.0210 & -0.0192 & -0.0413 & 0.0296 \\
\hline $\mathrm{I}-129$ & 0.0260 & 0.0216 & -0.0249 & 0.0061 & -0.0407 & 0.0575 & 0.0093 & 0.0003 & -0.0358 \\
\hline Cs-135 & 0.0263 & 0.0184 & 0.0412 & -0.0236 & -0.0292 & 0.0534 & 0.0129 & 0.0037 & -0.0460 \\
\hline Cs-137 & 0.0417 & 0.0046 & 0.0607 & -0.0189 & 0.0172 & 0.0425 & 0.0236 & 0.0182 & -0.0545 \\
\hline $\mathrm{Pb}-210$ & 0.0306 & 0.0109 & -0.0351 & 0.0050 & 0.0266 & 0.0119 & 0.0234 & -0.0630 & -0.0154 \\
\hline Ra-226 & -0.0050 & 0.0488 & 0.0117 & 0.0165 & 0.0157 & 0.0276 & 0.0248 & 0.0057 & 0.0448 \\
\hline Ac-227 & -0.0057 & -0.0294 & 0.0223 & -0.0370 & 0.0338 & -0.0305 & -0.0242 & 0.0126 & -0.0223 \\
\hline Th-229 & 0.0164 & 0.0428 & 0.0240 & -0.0186 & -0.0015 & -0.0220 & 0.0098 & 0.0010 & -0.0331 \\
\hline Th-230 & 0.0141 & 0.0390 & 0.0151 & 0.0115 & -0.0012 & -0.0075 & 0.0167 & 0.0085 & 0.0298 \\
\hline Th-232 & 0.0208 & 0.0430 & 0.0248 & -0.0183 & 0.0052 & -0.0217 & 0.0114 & -0.0019 & -0.0289 \\
\hline $\mathrm{Pa}-231$ & 0.0334 & 0.0653 & 0.0341 & -0.0206 & -0.0049 & -0.0205 & 0.0270 & -0.0304 & -0.0069 \\
\hline U-232 & -0.0370 & -0.0225 & -0.0127 & -0.0506 & 0.0121 & 0.0019 & -0.0125 & 0.0040 & -0.0153 \\
\hline U-233 & 0.0029 & 0.0225 & -0.0504 & -0.0212 & -0.0236 & 0.0417 & 0.0116 & 0.0063 & -0.0404 \\
\hline U-234 & -0.0106 & 0.0212 & -0.0486 & -0.0251 & -0.0183 & 0.0360 & 0.0090 & 0.0081 & -0.0365 \\
\hline U-236 & -0.0207 & 0.0165 & -0.0455 & -0.0255 & -0.0127 & 0.0321 & 0.0057 & 0.0044 & -0.0377 \\
\hline U-238 & -0.0198 & 0.0168 & -0.0462 & -0.0257 & -0.0132 & 0.0334 & 0.0068 & 0.0045 & -0.0376 \\
\hline Np-237 & 0.0053 & 0.0353 & 0.0155 & 0.0030 & 0.0283 & -0.0004 & -0.0132 & 0.0140 & -0.0243 \\
\hline Pu-238 & 0.0223 & -0.0109 & 0.0178 & -0.0191 & 0.0260 & -0.0037 & 0.0040 & 0.0050 & -0.0251 \\
\hline Pu-239 & 0.0487 & 0.0369 & 0.0056 & -0.0193 & 0.0148 & -0.0073 & 0.0089 & -0.0268 & -0.0139 \\
\hline $\mathrm{Pu}-240$ & 0.0475 & 0.0370 & 0.0072 & -0.0187 & 0.0161 & -0.0076 & 0.0091 & -0.0257 & -0.0142 \\
\hline Pu-242 & 0.0491 & 0.0369 & 0.0050 & -0.0196 & 0.0143 & -0.0072 & 0.0088 & -0.0273 & -0.0139 \\
\hline Am-241 & 0.0264 & 0.0253 & 0.0462 & -0.0277 & 0.0102 & -0.0253 & 0.0385 & -0.0155 & -0.0251 \\
\hline Am-243 & 0.0231 & 0.0447 & 0.0328 & -0.0348 & -0.0013 & -0.0146 & 0.0335 & -0.0375 & -0.0185 \\
\hline
\end{tabular}


Table 6.2-4. Correlations (Raw Correlation Coefficients) for ICRP 30-Based BDCFs and ClimateDependent Input Parameters (Continued)

\begin{tabular}{|c|c|c|c|c|c|c|c|c|c|}
\hline \multirow[b]{2}{*}{ 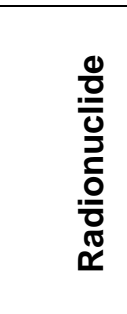 } & \multirow[b]{2}{*}{ 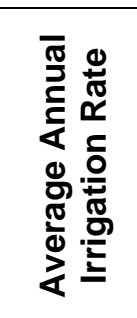 } & \multicolumn{5}{|c|}{ Daily Average Irrigation Rate } & \multirow[b]{2}{*}{ 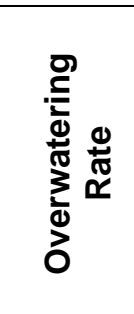 } & \multirow[b]{2}{*}{ 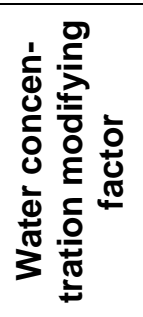 } & \multirow{2}{*}{ 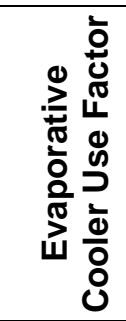 } \\
\hline & & 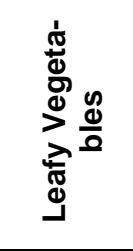 & 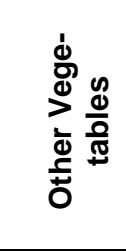 & $\stackrel{\frac{n}{5}}{\frac{5}{2}}$ & $\begin{array}{l}\mathscr{0} \\
\stackrel{\frac{\pi}{\pi}}{0} \\
0\end{array}$ & 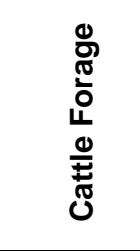 & & & \\
\hline C-14 & -0.0020 & -0.0128 & 0.0669 & 0.0239 & 0.0157 & 0.0090 & 0.0001 & 0.0000 & 0.0170 \\
\hline $\mathrm{Cl}-36$ & 0.1035 & -0.0419 & 0.0726 & 0.0166 & -0.0056 & 0.0579 & -0.0382 & -0.0155 & 0.0196 \\
\hline Se-79 & 0.0276 & 0.0243 & 0.0410 & 0.0015 & -0.0417 & 0.0056 & 0.0123 & -0.0007 & -0.0116 \\
\hline Sr-90 & 0.2395 & 0.0419 & 0.0089 & 0.0737 & -0.0191 & 0.0288 & -0.0245 & 0.0317 & -0.0047 \\
\hline Tc-99 & 0.1254 & 0.0801 & -0.0022 & -0.0069 & -0.0029 & 0.0260 & -0.0379 & 0.0274 & 0.0202 \\
\hline Sn-126 & 0.2457 & -0.0004 & 0.0167 & 0.0053 & 0.0044 & -0.0063 & -0.0042 & -0.0080 & 0.0270 \\
\hline $\mathrm{I}-129$ & 0.0945 & 0.0928 & 0.0219 & -0.0111 & 0.0165 & 0.0534 & 0.0267 & -0.0239 & -0.0131 \\
\hline Cs-135 & 0.0862 & 0.0123 & -0.0229 & 0.0096 & -0.0223 & -0.0302 & -0.0057 & 0.1090 & -0.0231 \\
\hline Cs-137 & 0.1721 & -0.0059 & -0.0183 & 0.0363 & -0.0254 & -0.0448 & -0.0126 & 0.1119 & 0.0033 \\
\hline $\mathrm{Pb}-210$ & 0.0547 & 0.0323 & -0.0008 & -0.0555 & -0.0120 & 0.0066 & 0.0589 & 0.1311 & 0.0242 \\
\hline Ra-226 & 0.3346 & -0.0041 & 0.0366 & 0.0055 & -0.0098 & 0.0029 & -0.0201 & 0.0203 & -0.0982 \\
\hline Ac-227 & 0.0912 & 0.0231 & 0.1079 & 0.0207 & -0.0028 & -0.0205 & -0.0016 & 0.0231 & 0.3807 \\
\hline Th-229 & 0.2393 & 0.0198 & 0.0760 & 0.0113 & 0.0224 & 0.0140 & -0.0099 & -0.0034 & 0.0214 \\
\hline Th-230 & 0.1995 & 0.0099 & 0.0174 & -0.0371 & 0.0001 & 0.0254 & -0.0367 & -0.0044 & -0.0578 \\
\hline Th-232 & 0.2724 & 0.0214 & 0.0584 & 0.0002 & 0.0207 & 0.0168 & -0.0147 & -0.0057 & 0.0019 \\
\hline $\mathrm{Pa}-231$ & 0.2221 & 0.0194 & 0.0554 & -0.0067 & 0.0157 & 0.0051 & -0.0366 & -0.0027 & 0.0022 \\
\hline U-232 & 0.1138 & 0.0079 & 0.0621 & -0.0005 & -0.0319 & 0.0088 & -0.0183 & 0.0489 & 0.2468 \\
\hline U-233 & 0.0831 & 0.0130 & -0.0106 & 0.0108 & -0.0471 & 0.0474 & 0.0055 & 0.0166 & 0.0343 \\
\hline U-234 & 0.0834 & 0.0111 & 0.0000 & 0.0166 & -0.0478 & 0.0447 & 0.0018 & 0.0254 & 0.0645 \\
\hline $\mathrm{U}-236$ & 0.0760 & 0.0124 & 0.0077 & 0.0216 & -0.0481 & 0.0391 & 0.0033 & 0.0283 & 0.0726 \\
\hline U-238 & 0.0791 & 0.0114 & 0.0050 & 0.0215 & -0.0484 & 0.0397 & 0.0023 & 0.0282 & 0.0695 \\
\hline Np-237 & 0.1729 & -0.0064 & 0.0775 & 0.0067 & -0.0226 & 0.0103 & -0.0183 & -0.0059 & 0.1338 \\
\hline Pu-238 & 0.1305 & -0.0226 & 0.1179 & 0.0334 & -0.0017 & -0.0090 & 0.0256 & 0.0469 & 0.1783 \\
\hline $\mathrm{Pu}-239$ & 0.2282 & 0.0112 & 0.0988 & 0.0185 & 0.0097 & -0.0099 & 0.0018 & 0.0140 & 0.0533 \\
\hline Pu-240 & 0.2302 & 0.0095 & 0.1017 & 0.0207 & 0.0105 & -0.0090 & 0.0029 & 0.0140 & 0.0555 \\
\hline Pu-242 & 0.2273 & 0.0119 & 0.0977 & 0.0177 & 0.0094 & -0.0102 & 0.0014 & 0.0139 & 0.0526 \\
\hline Am-241 & 0.2136 & 0.0306 & 0.1021 & 0.0463 & -0.0134 & 0.0040 & -0.0228 & 0.0353 & 0.1053 \\
\hline Am-243 & 0.2117 & 0.0411 & 0.0738 & 0.0227 & 0.0029 & -0.0030 & -0.0356 & 0.0204 & 0.0371 \\
\hline
\end{tabular}

Source: MO0407SPAINEXI.002 [DIRS 170597]; MO0407SPASRPBM.002 [DIRS 170755]; MO0403SPAAEIBM.002 [DIRS 169392]; MO0407SPACRBSM.002 [DIRS 170677]; MO0406SPAETPBM.002 [DIRS 170150]; MO0306MWDBGSMF.001 [DIRS 163816].

NOTE: Values were calculated using Excel (Correlations for climate dependent parameters_Rev 3.xIs; Appendix B). Correlation coefficients in shaded cells differ significantly from zero at the $99 \%$ confidence level. 
Table 6.2-5. Correlations (Raw Correlation Coefficients) for ICRP 72-Based BDCFs and ClimateDependent Input Parameters

\begin{tabular}{|c|c|c|c|c|c|c|c|c|c|}
\hline \multirow[b]{2}{*}{ 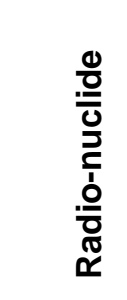 } & \multicolumn{4}{|c|}{ Growing time } & \multicolumn{5}{|c|}{ Irrigation Application } \\
\hline & 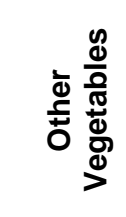 & $\begin{array}{l}\stackrel{0}{5} \\
\frac{5}{4}\end{array}$ & 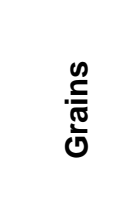 & 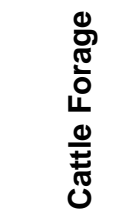 & 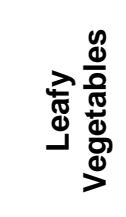 & 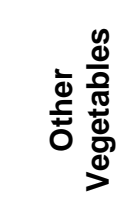 & 点 & $\begin{array}{l}\stackrel{n}{\bar{\sigma}} \\
\frac{\pi}{0}\end{array}$ & 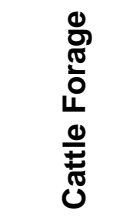 \\
\hline C-14 & -0.0022 & -0.0041 & -0.0026 & -0.0711 & -0.0233 & 0.0076 & 0.0015 & -0.0575 & -0.0174 \\
\hline $\mathrm{Cl}-36$ & -0.0182 & 0.0181 & 0.0080 & 0.0161 & 0.0079 & -0.0081 & -0.0191 & -0.0007 & -0.0683 \\
\hline Se-79 & 0.0387 & -0.0141 & -0.0191 & 0.0312 & -0.0161 & -0.0432 & -0.0191 & -0.0406 & -0.0036 \\
\hline Sr-90 & 0.0330 & 0.0052 & 0.0082 & -0.0206 & 0.0611 & -0.0068 & 0.0740 & 0.0172 & -0.0138 \\
\hline Tc-99 & 0.0417 & 0.0790 & -0.0368 & -0.0072 & 0.0129 & 0.0256 & 0.0065 & 0.0349 & -0.0330 \\
\hline Sn-126 & 0.0257 & 0.0294 & 0.0451 & 0.0186 & 0.0689 & -0.0210 & -0.0192 & -0.0414 & 0.0296 \\
\hline $\mathrm{I}-129$ & 0.0262 & 0.0216 & -0.0247 & 0.0061 & -0.0405 & 0.0574 & 0.0094 & 0.0003 & -0.0359 \\
\hline Cs-135 & 0.0263 & 0.0187 & 0.0414 & -0.0238 & -0.0291 & 0.0532 & 0.0129 & 0.0035 & -0.0460 \\
\hline Cs-137 & 0.0415 & 0.0050 & 0.0609 & -0.0188 & 0.0166 & 0.0431 & 0.0234 & 0.0183 & -0.0541 \\
\hline $\mathrm{Pb}-210$ & 0.0305 & 0.0108 & -0.0350 & 0.0047 & 0.0268 & 0.0117 & 0.0233 & -0.0630 & -0.0155 \\
\hline Ra-226 & -0.0059 & 0.0498 & 0.0108 & 0.0126 & 0.0171 & 0.0239 & 0.0243 & 0.0020 & 0.0415 \\
\hline Ac-227 & -0.0055 & -0.0296 & 0.0221 & -0.0371 & 0.0341 & -0.0307 & -0.0240 & 0.0125 & -0.0228 \\
\hline Th-229 & 0.0171 & 0.0434 & 0.0248 & -0.0186 & -0.0001 & -0.0223 & 0.0107 & 0.0009 & -0.0335 \\
\hline Th-230 & 0.0161 & 0.0408 & 0.0167 & 0.0069 & -0.0010 & -0.0106 & 0.0154 & 0.0055 & 0.0215 \\
\hline Th-232 & 0.0251 & 0.0436 & 0.0262 & -0.0185 & 0.0159 & -0.0207 & 0.0128 & -0.0042 & -0.0254 \\
\hline $\mathrm{Pa}-231$ & 0.0336 & 0.0652 & 0.0345 & -0.0209 & -0.0044 & -0.0206 & 0.0268 & -0.0311 & -0.0064 \\
\hline U-232 & -0.0350 & -0.0168 & -0.0153 & -0.0508 & 0.0087 & 0.0114 & 0.0016 & 0.0069 & -0.0175 \\
\hline U-233 & 0.0085 & 0.0244 & -0.0499 & -0.0196 & -0.0266 & 0.0453 & 0.0139 & 0.0081 & -0.0407 \\
\hline U-234 & -0.0030 & 0.0256 & -0.0482 & -0.0239 & -0.0241 & 0.0424 & 0.0136 & 0.0131 & -0.0364 \\
\hline U-236 & -0.0222 & 0.0183 & -0.0426 & -0.0246 & -0.0146 & 0.0367 & 0.0086 & 0.0083 & -0.0387 \\
\hline $\mathrm{U}-238$ & -0.0187 & 0.0188 & -0.0450 & -0.0253 & -0.0162 & 0.0402 & 0.0121 & 0.0081 & -0.0383 \\
\hline Np-237 & 0.0090 & 0.0340 & 0.0089 & 0.0014 & 0.0270 & -0.0115 & -0.0180 & 0.0117 & -0.0147 \\
\hline Pu-238 & 0.0198 & -0.0086 & 0.0246 & -0.0268 & 0.0368 & -0.0247 & -0.0022 & -0.0036 & -0.0340 \\
\hline Pu-239 & 0.0443 & 0.0405 & 0.0070 & -0.0190 & 0.0143 & -0.0122 & 0.0070 & -0.0296 & -0.0161 \\
\hline Pu-240 & 0.0432 & 0.0406 & 0.0087 & -0.0185 & 0.0158 & -0.0129 & 0.0070 & -0.0287 & -0.0165 \\
\hline Pu-242 & 0.0448 & 0.0403 & 0.0063 & -0.0193 & 0.0137 & -0.0118 & 0.0070 & -0.0299 & -0.0160 \\
\hline Am-241 & 0.0224 & 0.0217 & 0.0435 & -0.0287 & 0.0092 & -0.0310 & 0.0357 & -0.0190 & -0.0279 \\
\hline Am-243 & 0.0208 & 0.0437 & 0.0315 & -0.0349 & -0.0023 & -0.0176 & 0.0322 & -0.0395 & -0.0199 \\
\hline
\end{tabular}


Table 6.2-5. Correlations (Raw Correlation Coefficients) for ICRP 72-Based BDCFs and ClimateDependent Input Parameters (Continued)

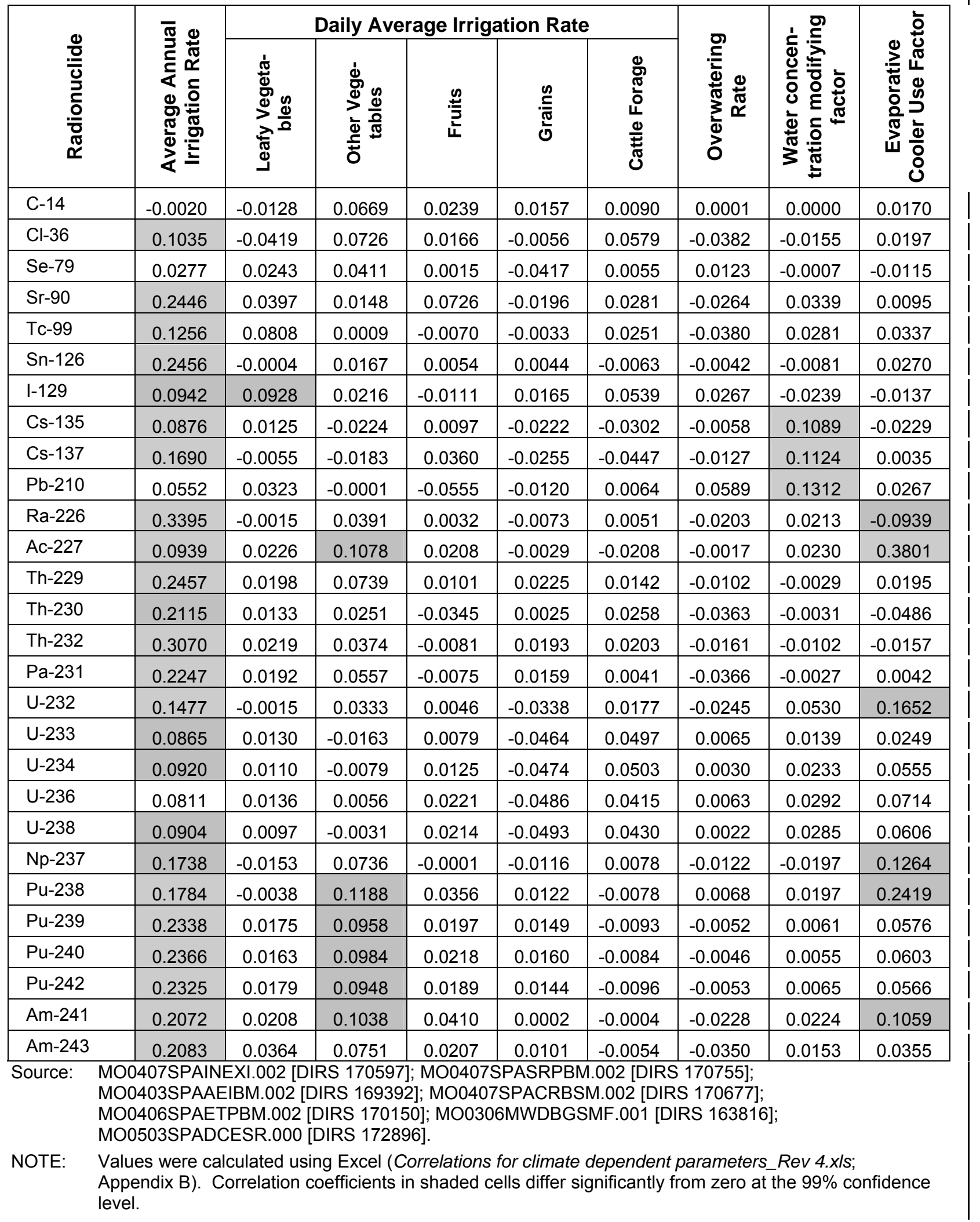


The shaded cells contain correlation coefficients with values that differ from zero at the 99 percent confidence level (see Section 6.2.3 for discussion of the statistical test to determine values of correlation coefficients that represent true correlation at a given confidence level). The calculations were carried out using Excel (Correlations for climate dependent parameters_Rev 3.xls and Correlations for climate dependent parameters_Rev 4.xls; Appendix B).

The parameter for which correlation coefficients are consistently among the highest is the average annual irrigation rate (Tables 6.2-4 and 6.2-5). For a few radionuclides, the BDCFs are also correlated with the daily irrigation rates for leafy and other vegetables, water concentration modifying factor, and evaporative cooler use factor. The relationships between the average annual irrigation rate and the BDCFs for ${ }^{99} \mathrm{Tc}$, ${ }^{129} \mathrm{I}$, and ${ }^{237} \mathrm{~Np}$ are shown in Tables 6.2-6 and 6.2-7, for the ICRP 30- and ICRP 72-based dosimetric methods, respectively. If the raw individual realization data are plotted, the stochastic variability among BDCFs from the multiple random inputs results in plots that show little discernable trend. To generate graphs illustrating the trend, averaging over realizations to minimize the impact of other variables was required. The results of model realizations were sorted by annual irrigation rate, and the irrigation rate and the corresponding BDCFs were averaged in blocks of 100 values. Graphical representations for selected radionuclides are shown in Tables 6.2-6 and 6.2-7 for the ICRP 30- and ICRP 72-based methods, respectively. The graphs were generated in Excel (Dependence of BDCFs on Irrigation Rate_Rev 3.xls and Dependence of BDCFs on Irrigation Rate_Rev 4.xls; Appendix B).

BDCFs for 5 radionuclides also show a positive correlation with the evaporative cooler use factor, and BDCFs for 3 radionuclides show a positive correlation with the water concentration modifying factor. There is a positive correlation between the average annual irrigation rate, the evaporative cooler use factor, and the fishpond water concentration modifying factor because all these parameters have higher values in hotter climates. Therefore, the annual average irrigation rate was considered a surrogate parameter to represent the dependence of the BDCFs on the evaporative cooler use factor and the water concentration modifying factor.

The correlation between the average annual irrigation rate and the BDCF for ${ }^{14} \mathrm{C}$ is negative. This is because the BDCF for ${ }^{14} \mathrm{C}$ depends on the concentration of ${ }^{14} \mathrm{C}$ in the air, which is proportional to the square root of the irrigated area; which in turn, is inversely proportional to the amount of average annual irrigation (i.e., for a given volume of water, a greater area can be irrigated if the irrigation rate is lower). In spite of this, the mean BDCF for ${ }^{14} \mathrm{C}$ for the upper bound of the glacial transition climate is less than the mean BDCF for the present-day climate (Table 6.2-1) due to the combined influence of other climate-dependent parameters, which reduce the $\mathrm{BDCF}$ value for ${ }^{14} \mathrm{C}$ for the upper bound of the glacial transition climate. 
Table 6.2-6. Dependence of ICRP 30-Based BDCFs for ${ }^{99} \mathrm{Tc},{ }^{129} \mathrm{I}$, and ${ }^{237} \mathrm{~Np}$ on Annual Average | Irrigation Rate

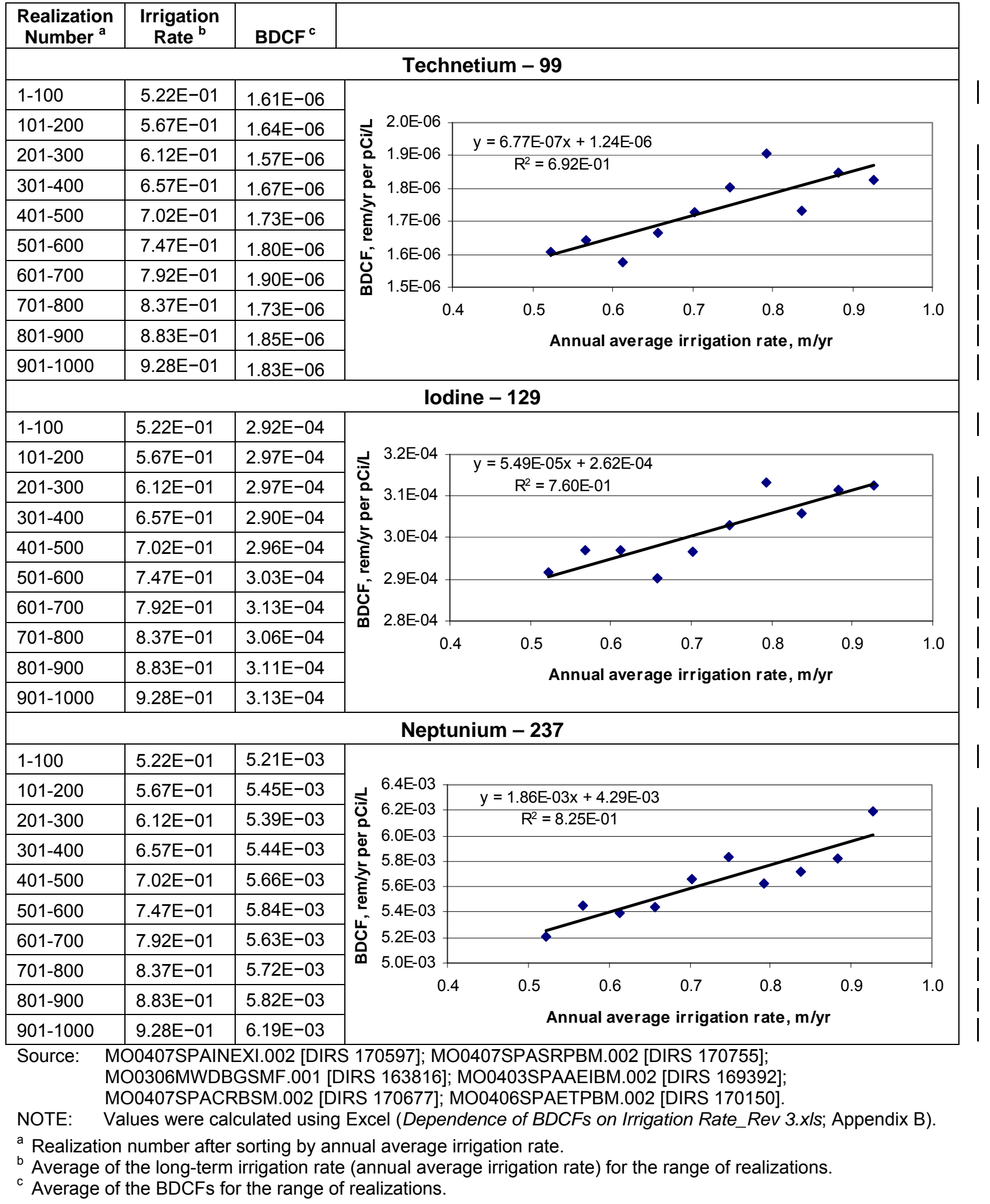


Table 6.2-7. Dependence of ICRP 72-Based BDCFs for ${ }^{99} \mathrm{Tc},{ }^{129} \mathrm{I}$, and ${ }^{237} \mathrm{~Np}$ on Annual Average Irrigation Rate

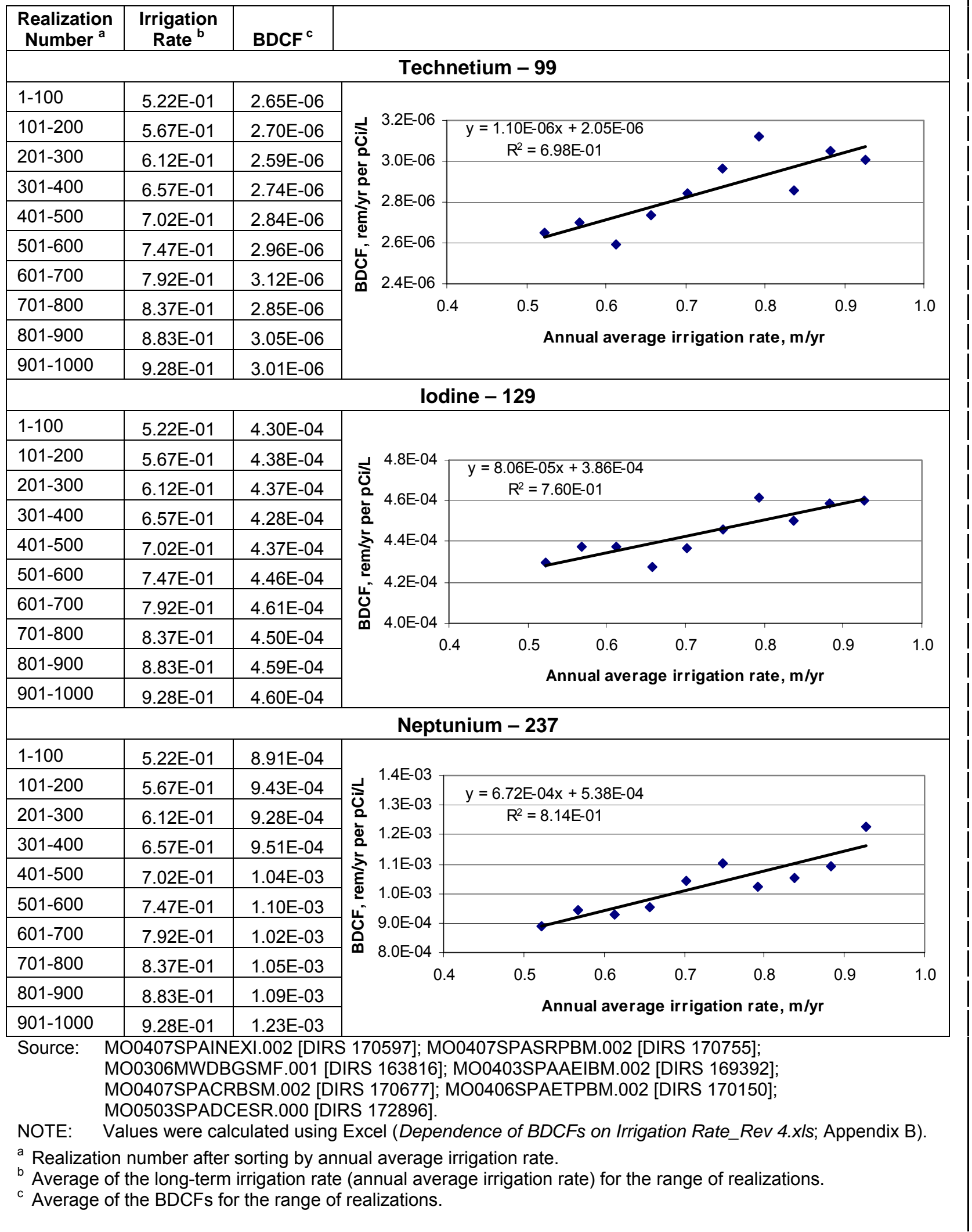


Given that the BDCFs can be shown to increase linearly with the value of a climate-dependent parameter, the annual average irrigation rate (Tables 6.2-6 and 6.2-7), the BDCFs for the average upper bound of the monsoon and the average lower bound of the glacial transition climates were calculated by interpolation between the BDCF values for the present-day and the upper bound of the glacial transition climates. The strength of the observed dependencies shows that BDCFs can be scaled with the average annual irrigation rate. Based on the future climate analogue sites (DTN: MO0403SPAAEIBM.002 [DIRS 169392]), values of the average annual irrigation rates are:

- $0.95 \mathrm{~m} / \mathrm{yr}$ for the present-day climate and the lower bound of the monsoon climate

- $0.88 \mathrm{~m} / \mathrm{yr}$ for the lower bound of the glacial transition climate (based on Delta, Utah)

- $0.52 \mathrm{~m} / \mathrm{yr}$ for the upper bound of the monsoon climate (based on Nogales, Arizona)

- $0.50 \mathrm{~m} / \mathrm{yr}$ for the upper bound of the glacial transition climate (based on Spokane and other locations in east central Washington).

Because differences between the irrigation rates for the upper bound of the monsoon ( $0.52 \mathrm{~m} / \mathrm{yr})$ and the upper bound of the glacial transition climates $(0.50 \mathrm{~m} / \mathrm{yr})$ are small, one value of $0.50 \mathrm{~m} / \mathrm{yr}$ is used. The relationship between the average annual irrigation rates and the BDCFs for the climate states under consideration is shown in Figure 6.2-1.

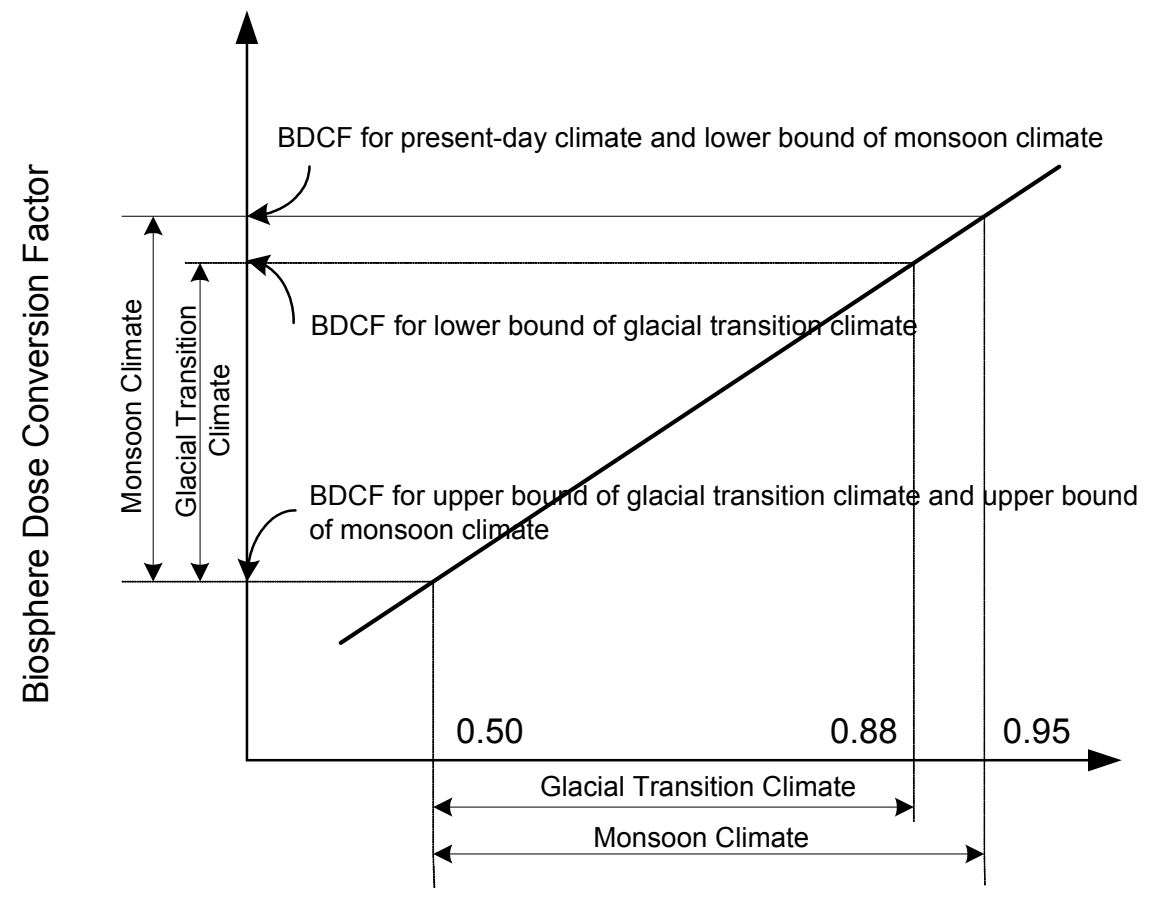

Annual average irrigation rate $(\mathrm{m} / \mathrm{yr})$

Figure 6.2-1. Scaling of the BDCFs 
BDCF values for the lower bound of the glacial transition climate can be calculated as:

$$
B D C F_{L B G T}=B D C F_{U B G T}+\frac{0.88-0.50}{0.95-0.50}\left(B D C F_{I C}-B D C F_{U B G T}\right)
$$

where

$$
\begin{aligned}
B D C F_{L B G T}= & \text { BDCF for the lower bound of glacial transition climate } \\
B D C F_{U B G T}= & \text { BDCF for the upper bound of glacial transition climate } \\
B D C F_{I C}= & \text { BDCF for the present-day climate (interglacial) } \\
0.88-0.50 & \text { Scaling factor equal to the difference in average annual irrigation } \\
0.95-0.50= & \begin{array}{l}
\text { rates between the lower and upper bounds of the glacial transition } \\
\text { climate divided by the difference in average annual irrigation rates } \\
\text { between the present-day climate and the upper bound of the glacial } \\
\text { transition climate. }
\end{array}
\end{aligned}
$$

BDCF values for individual realizations for the monsoon and the glacial transition climates were then calculated by randomly sampling over the range of BDCF values for these climates. For the monsoon climate, the sampling was over the entire range between the two extreme BDCF values for an individual realization (i.e., the values for the present-day climate and the upper bound of the glacial transition climate), and was calculated as

$$
B D C F_{M C}=B D C F_{U B G T}+\left(B D C F_{I C}-B D C F_{U B G T}\right) R A N D
$$

where

$$
\begin{array}{ll}
B D C F_{M C} & =\text { BDCF for the monsoon climate } \\
R A N D & =\text { random number greater than or equal to } 0 \text { and less than } 1
\end{array}
$$

For the glacial transition climate, the sampling was between the value for the lower and upper bounds of the glacial transition climate and was calculated as

$$
B D C F_{G T C}=B D C F_{U B G T}+\frac{0.88-0.50}{0.95-0.50}\left(B D C F_{I C}-B D C F_{U B G T}\right) R A N D
$$

where

$$
B D C F_{G T C} \quad=\quad \text { BDCF for the glacial transition climate }
$$

These calculations were performed using Excel (GW BDCF Realizations_MC and FC_Rev 4.xls; | Appendix B).

\subsubsection{Modeling Results: Biosphere Dose Conversion Factors}

BDCFs for the present-day climate, monsoon climate, and glacial transition climate were calculated as described in the previous section. The BDCFs for a specific climate state consist of a set of 1,000 row vectors, with vector elements representing BDCFs for different radionuclides and a row (vector) number corresponding to a model realization. The full sets of BDCF vectors for the three climate states are given in the Excel files GW BDCF Realizations_MC and FC_Rev 
4.xls for the ICRP 30- and ICRP 72-based dosimetry (Appendix B). Summary statistics for the BDCFs consistent with the ICRP 30 dosimetry are presented in Tables 6.2-8 to 6.2-10 for the present-day climate, monsoon climate, and glacial transition climate, respectively. The statistics include the mean, standard deviation (STD), minimum, maximum, and percentiles of cumulative distribution in increments of 5 percent. The corresponding BDCFs for the ICRP 72 dosimetry are listed in Tables 6.2-11 to 6.2-13. The values listed in Tables 6.2-8 to 6.2-13 are presented with three significant digits although only two significant digits are significant. This is to avoid round-off errors in the TSPA calculation of the annual dose. The BDCFs can be considered as intermediate results and, as such, should be given with an additional significant digit.

In all of the tables containing the results, the values for ${ }^{232} \mathrm{Th}$ include contributions from ${ }^{228} \mathrm{Ra}$ and ${ }^{228} \mathrm{Th}$ and their short-lived decay products. Similarly, values for ${ }^{232} \mathrm{U}$ include contributions from ${ }^{228} \mathrm{Th}$ and its short-lived decay products. In addition, the combined value of BDCF for ${ }^{226} \mathrm{Ra}$ and ${ }^{210} \mathrm{~Pb}$ is provided, in case the concentration of ${ }^{210} \mathrm{~Pb}$ in groundwater is not calculated in the TSPA-LA model. The combined ${ }^{226} \mathrm{Ra}-{ }^{210} \mathrm{~Pb}$ value was calculated for the activity concentration of ${ }^{210} \mathrm{~Pb}$ in groundwater equal to that of ${ }^{226} \mathrm{Ra}$, i.e., for the condition of radioactive equilibrium between these two radionuclides.

The mean BDCF values for the present-day climate calculated using the ICRP 30 and ICRP 72 dosimetries are compared in Figure 6.2-2. Generally, the BDCFs for the actinides are lower when the ICRP 72 dosimetric methods are used. For the radionuclides having a lower atomic mass, BDCFs can be either higher or lower, depending on the dosimetry. Notably, the BDCFs for ${ }^{99}$ Tc and ${ }^{129}$ I are over 60 percent and 40 percent higher, respectively, using ICRP 72 methods. 
Table 6.2-8. Statistics for the ICRP 30-Based BDCFs for the Present-Day Climate, rem/yr per pCi/L

\begin{tabular}{|c|c|c|c|c|c|c|c|c|c|c|c|}
\hline & C-14 & $\mathrm{Cl}-36$ & Se-79 & Sr-90 & Tc-99 & Sn-126 & I-129 & Cs-135 & Cs-137 & $\mathrm{Pb}-210$ & Ra-226 \\
\hline Mean & $9.10 \mathrm{E}-06$ & $2.07 \mathrm{E}-05$ & $6.19 \mathrm{E}-05$ & $1.64 \mathrm{E}-04$ & $2.26 \mathrm{E}-06$ & $6.33 \mathrm{E}-03$ & $3.36 \mathrm{E}-04$ & $5.57 \mathrm{E}-05$ & $4.82 \mathrm{E}-04$ & $9.04 \mathrm{E}-03$ & $9.69 \mathrm{E}-02$ \\
\hline STD & $6.67 \mathrm{E}-06$ & $4.23 \mathrm{E}-05$ & $1.05 \mathrm{E}-04$ & 2.57E-05 & $2.38 \mathrm{E}-06$ & $3.94 \mathrm{E}-03$ & $1.14 \mathrm{E}-04$ & $4.01 \mathrm{E}-05$ & $2.28 \mathrm{E}-04$ & $3.54 \mathrm{E}-03$ & 4.77E-02 \\
\hline Minimum & $4.15 \mathrm{E}-06$ & $3.43 \mathrm{E}-06$ & $9.71 \mathrm{E}-06$ & 1.23E-04 & $1.18 \mathrm{E}-06$ & $2.03 \mathrm{E}-04$ & $2.21 \mathrm{E}-04$ & $1.15 \mathrm{E}-05$ & $1.52 \mathrm{E}-04$ & $5.80 \mathrm{E}-03$ & $2.48 \mathrm{E}-03$ \\
\hline $5 \%$ & $4.92 \mathrm{E}-06$ & $5.21 \mathrm{E}-06$ & $1.50 \mathrm{E}-05$ & $1.35 \mathrm{E}-04$ & $1.31 \mathrm{E}-06$ & $1.12 \mathrm{E}-03$ & $2.43 \mathrm{E}-04$ & $1.90 \mathrm{E}-05$ & $2.33 \mathrm{E}-04$ & $6.26 \mathrm{E}-03$ & $3.89 \mathrm{E}-02$ \\
\hline $10 \%$ & $5.20 \mathrm{E}-06$ & $5.78 \mathrm{E}-06$ & $1.77 \mathrm{E}-05$ & $1.39 \mathrm{E}-04$ & $1.36 \mathrm{E}-06$ & $1.85 \mathrm{E}-03$ & $2.51 \mathrm{E}-04$ & 2.22E-05 & $2.59 \mathrm{E}-04$ & $6.45 \mathrm{E}-03$ & 4.69E-02 \\
\hline $15 \%$ & $5.47 \mathrm{E}-06$ & $6.52 \mathrm{E}-06$ & $1.98 \mathrm{E}-05$ & $1.43 \mathrm{E}-04$ & $1.42 \mathrm{E}-06$ & $2.57 \mathrm{E}-03$ & $2.58 \mathrm{E}-04$ & $2.53 \mathrm{E}-05$ & $2.84 \mathrm{E}-04$ & $6.61 \mathrm{E}-03$ & $5.26 \mathrm{E}-02$ \\
\hline $20 \%$ & $5.66 \mathrm{E}-06$ & 7.07E-06 & $2.18 \mathrm{E}-05$ & $1.45 \mathrm{E}-04$ & $1.46 \mathrm{E}-06$ & $3.15 \mathrm{E}-03$ & $2.64 \mathrm{E}-04$ & $2.82 \mathrm{E}-05$ & $3.01 \mathrm{E}-04$ & $6.76 \mathrm{E}-03$ & $5.79 \mathrm{E}-02$ \\
\hline $25 \%$ & $5.90 \mathrm{E}-06$ & $7.73 \mathrm{E}-06$ & $2.40 \mathrm{E}-05$ & $1.48 \mathrm{E}-04$ & $1.51 \mathrm{E}-06$ & $3.60 \mathrm{E}-03$ & $2.70 \mathrm{E}-04$ & $3.10 \mathrm{E}-05$ & $3.26 \mathrm{E}-04$ & $6.94 \mathrm{E}-03$ & $6.25 \mathrm{E}-02$ \\
\hline $30 \%$ & $6.09 \mathrm{E}-06$ & 8.65E-06 & $2.65 \mathrm{E}-05$ & $1.50 \mathrm{E}-04$ & $1.55 \mathrm{E}-06$ & 3.97E-03 & $2.78 \mathrm{E}-04$ & 3.35E-05 & 3.47E-04 & $7.11 \mathrm{E}-03$ & $6.75 \mathrm{E}-02$ \\
\hline $35 \%$ & $6.34 \mathrm{E}-06$ & $9.51 \mathrm{E}-06$ & $2.82 \mathrm{E}-05$ & $1.52 \mathrm{E}-04$ & $1.61 \mathrm{E}-06$ & $4.25 \mathrm{E}-03$ & $2.84 \mathrm{E}-04$ & 3.62E-05 & 3.64E-04 & 7.25E-03 & $7.20 \mathrm{E}-02$ \\
\hline $40 \%$ & $6.54 \mathrm{E}-06$ & $1.05 \mathrm{E}-05$ & $3.05 \mathrm{E}-05$ & $1.54 \mathrm{E}-04$ & 1.67E-06 & $4.56 \mathrm{E}-03$ & $2.91 \mathrm{E}-04$ & $3.91 \mathrm{E}-05$ & $3.85 \mathrm{E}-04$ & $7.46 \mathrm{E}-03$ & 7.73E-02 \\
\hline $45 \%$ & $6.83 \mathrm{E}-06$ & 1.16E-05 & $3.29 \mathrm{E}-05$ & $1.56 \mathrm{E}-04$ & $1.72 \mathrm{E}-06$ & $5.08 \mathrm{E}-03$ & $2.99 \mathrm{E}-04$ & 4.17E-05 & 4.03E-04 & $7.69 \mathrm{E}-03$ & $8.20 \mathrm{E}-02$ \\
\hline $50 \%$ & $7.04 \mathrm{E}-06$ & $1.28 \mathrm{E}-05$ & $3.57 \mathrm{E}-05$ & $1.59 \mathrm{E}-04$ & $1.78 \mathrm{E}-06$ & $5.54 \mathrm{E}-03$ & $3.09 \mathrm{E}-04$ & $4.53 \mathrm{E}-05$ & 4.27E-04 & $7.94 \mathrm{E}-03$ & $8.81 \mathrm{E}-02$ \\
\hline $55 \%$ & $7.36 \mathrm{E}-06$ & $1.42 \mathrm{E}-05$ & $3.84 \mathrm{E}-05$ & 1.62E-04 & $1.85 \mathrm{E}-06$ & $5.98 \mathrm{E}-03$ & $3.16 \mathrm{E}-04$ & $4.85 \mathrm{E}-05$ & $4.51 \mathrm{E}-04$ & $8.20 \mathrm{E}-03$ & $9.33 \mathrm{E}-02$ \\
\hline $60 \%$ & $7.78 \mathrm{E}-06$ & $1.56 \mathrm{E}-05$ & $4.25 \mathrm{E}-05$ & $1.64 \mathrm{E}-04$ & $1.93 \mathrm{E}-06$ & $6.47 \mathrm{E}-03$ & $3.29 \mathrm{E}-04$ & $5.17 \mathrm{E}-05$ & $4.74 \mathrm{E}-04$ & 8.47E-03 & $9.93 \mathrm{E}-02$ \\
\hline $65 \%$ & $8.35 \mathrm{E}-06$ & $1.76 \mathrm{E}-05$ & $4.75 \mathrm{E}-05$ & $1.66 \mathrm{E}-04$ & $2.03 E-06$ & $6.96 \mathrm{E}-03$ & $3.39 \mathrm{E}-04$ & $5.57 \mathrm{E}-05$ & $5.09 \mathrm{E}-04$ & $8.83 \mathrm{E}-03$ & $1.06 \mathrm{E}-01$ \\
\hline $70 \%$ & $8.84 \mathrm{E}-06$ & $1.93 \mathrm{E}-05$ & $5.33 \mathrm{E}-05$ & $1.71 \mathrm{E}-04$ & $2.14 \mathrm{E}-06$ & $7.75 \mathrm{E}-03$ & $3.50 \mathrm{E}-04$ & $6.09 \mathrm{E}-05$ & $5.38 \mathrm{E}-04$ & $9.19 \mathrm{E}-03$ & $1.14 \mathrm{E}-01$ \\
\hline $75 \%$ & $9.55 \mathrm{E}-06$ & 2.22E-05 & $6.15 \mathrm{E}-05$ & $1.75 \mathrm{E}-04$ & $2.30 \mathrm{E}-06$ & $8.53 \mathrm{E}-03$ & $3.64 \mathrm{E}-04$ & $6.60 \mathrm{E}-05$ & $5.73 \mathrm{E}-04$ & $9.66 \mathrm{E}-03$ & $1.21 \mathrm{E}-01$ \\
\hline $80 \%$ & $1.06 \mathrm{E}-05$ & $2.53 \mathrm{E}-05$ & $7.22 \mathrm{E}-05$ & $1.79 \mathrm{E}-04$ & $2.49 \mathrm{E}-06$ & $9.35 \mathrm{E}-03$ & $3.83 \mathrm{E}-04$ & $7.35 \mathrm{E}-05$ & $6.21 \mathrm{E}-04$ & $1.04 \mathrm{E}-02$ & $1.32 \mathrm{E}-01$ \\
\hline $85 \%$ & $1.19 \mathrm{E}-05$ & $2.91 \mathrm{E}-05$ & $9.01 \mathrm{E}-05$ & $1.86 \mathrm{E}-04$ & $2.85 \mathrm{E}-06$ & $1.03 \mathrm{E}-02$ & 4.07E-04 & $8.74 \mathrm{E}-05$ & $6.76 \mathrm{E}-04$ & 1.12E-02 & $1.44 \mathrm{E}-01$ \\
\hline $90 \%$ & $1.40 \mathrm{E}-05$ & $3.71 \mathrm{E}-05$ & $1.19 \mathrm{E}-04$ & $1.95 \mathrm{E}-04$ & $3.46 \mathrm{E}-06$ & $1.16 \mathrm{E}-02$ & $4.50 \mathrm{E}-04$ & $1.04 \mathrm{E}-04$ & $7.51 \mathrm{E}-04$ & $1.26 \mathrm{E}-02$ & $1.63 \mathrm{E}-01$ \\
\hline $95 \%$ & $2.05 \mathrm{E}-05$ & $5.33 \mathrm{E}-05$ & 1.67E-04 & 2.13E-04 & $4.41 \mathrm{E}-06$ & $1.42 \mathrm{E}-02$ & $5.18 \mathrm{E}-04$ & $1.32 \mathrm{E}-04$ & $9.28 \mathrm{E}-04$ & $1.60 \mathrm{E}-02$ & $1.84 \mathrm{E}-01$ \\
\hline Maximum & $9.35 \mathrm{E}-05$ & $8.85 \mathrm{E}-04$ & $1.89 \mathrm{E}-03$ & $3.78 \mathrm{E}-04$ & $6.19 \mathrm{E}-05$ & $2.03 E-02$ & $1.98 \mathrm{E}-03$ & 4.77E-04 & $1.69 \mathrm{E}-03$ & $3.66 \mathrm{E}-02$ & 4.27E-01 \\
\hline
\end{tabular}




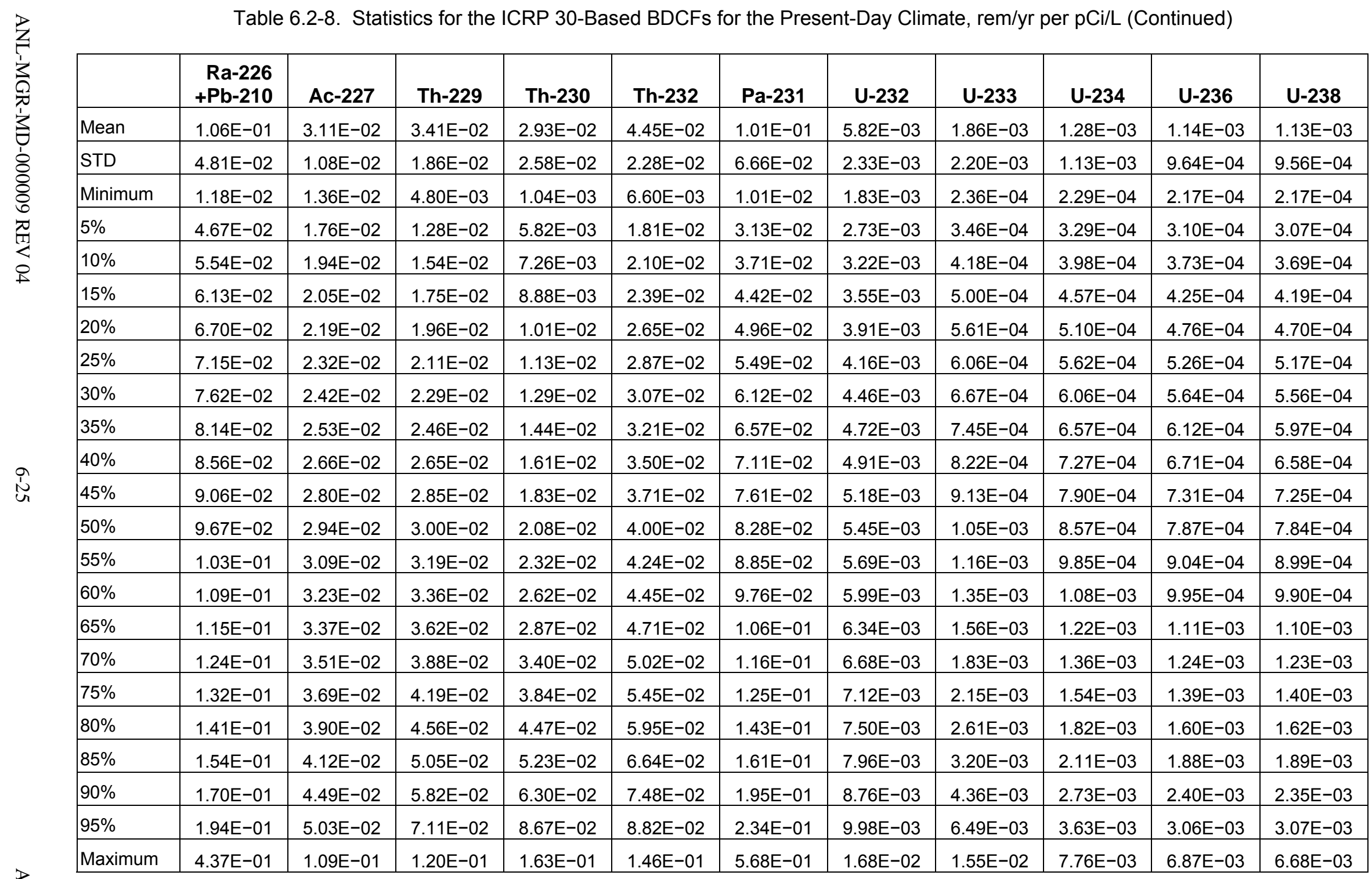


Table 6.2-8. $\quad$ Statistics for the ICRP 30-Based BDCFs for the Present-Day Climate, rem/yr per pCi/L I (Continued)

\begin{tabular}{|c|c|c|c|c|c|c|c|}
\hline & Np-237 & Pu-238 & Pu-239 & $\mathrm{Pu}-240$ & $\mathrm{Pu}-242$ & Am-241 & Am-243 \\
\hline Mean & $6.98 \mathrm{E}-03$ & $4.69 \mathrm{E}-03$ & $9.25 \mathrm{E}-03$ & $9.01 \mathrm{E}-03$ & $8.91 \mathrm{E}-03$ & $6.83 \mathrm{E}-03$ & $9.57 \mathrm{E}-03$ \\
\hline STD & $2.50 \mathrm{E}-03$ & $1.14 \mathrm{E}-03$ & $3.75 \mathrm{E}-03$ & $3.52 \mathrm{E}-03$ & $3.67 \mathrm{E}-03$ & $2.06 \mathrm{E}-03$ & $4.23 E-03$ \\
\hline Minimum & $3.85 \mathrm{E}-03$ & $2.90 \mathrm{E}-03$ & $3.73 \mathrm{E}-03$ & $3.73 \mathrm{E}-03$ & $3.56 \mathrm{E}-03$ & $2.97 \mathrm{E}-03$ & $2.96 \mathrm{E}-03$ \\
\hline $5 \%$ & 4.45E-03 & 3.37E-03 & $5.09 \mathrm{E}-03$ & $5.04 \mathrm{E}-03$ & $4.86 \mathrm{E}-03$ & $4.29 \mathrm{E}-03$ & $4.75 \mathrm{E}-03$ \\
\hline $10 \%$ & $4.77 \mathrm{E}-03$ & $3.57 \mathrm{E}-03$ & $5.54 \mathrm{E}-03$ & $5.47 \mathrm{E}-03$ & $5.30 \mathrm{E}-03$ & $4.71 \mathrm{E}-03$ & $5.35 \mathrm{E}-03$ \\
\hline $15 \%$ & $4.99 \mathrm{E}-03$ & $3.68 \mathrm{E}-03$ & $5.96 \mathrm{E}-03$ & $5.87 \mathrm{E}-03$ & $5.70 \mathrm{E}-03$ & $5.02 \mathrm{E}-03$ & $5.81 \mathrm{E}-03$ \\
\hline $20 \%$ & $5.19 \mathrm{E}-03$ & $3.81 \mathrm{E}-03$ & $6.28 \mathrm{E}-03$ & $6.21 \mathrm{E}-03$ & $6.01 \mathrm{E}-03$ & $5.21 \mathrm{E}-03$ & $6.25 \mathrm{E}-03$ \\
\hline $25 \%$ & $5.37 \mathrm{E}-03$ & $3.91 \mathrm{E}-03$ & $6.60 \mathrm{E}-03$ & $6.53 \mathrm{E}-03$ & $6.32 \mathrm{E}-03$ & $5.40 \mathrm{E}-03$ & $6.71 \mathrm{E}-03$ \\
\hline $30 \%$ & $5.56 \mathrm{E}-03$ & 4.04E-03 & $6.85 \mathrm{E}-03$ & $6.77 \mathrm{E}-03$ & $6.55 \mathrm{E}-03$ & $5.58 \mathrm{E}-03$ & $7.02 \mathrm{E}-03$ \\
\hline $35 \%$ & $5.75 \mathrm{E}-03$ & $4.13 \mathrm{E}-03$ & $7.18 \mathrm{E}-03$ & $7.08 \mathrm{E}-03$ & $6.87 \mathrm{E}-03$ & $5.86 \mathrm{E}-03$ & $7.35 \mathrm{E}-03$ \\
\hline $40 \%$ & $5.91 \mathrm{E}-03$ & $4.26 \mathrm{E}-03$ & $7.60 \mathrm{E}-03$ & $7.51 \mathrm{E}-03$ & $7.28 \mathrm{E}-03$ & $6.05 \mathrm{E}-03$ & $7.71 \mathrm{E}-03$ \\
\hline $45 \%$ & $6.10 \mathrm{E}-03$ & 4.35E-03 & $7.96 \mathrm{E}-03$ & $7.79 \mathrm{E}-03$ & $7.64 \mathrm{E}-03$ & $6.26 \mathrm{E}-03$ & $8.17 \mathrm{E}-03$ \\
\hline $50 \%$ & 6.37E-03 & $4.45 \mathrm{E}-03$ & 8.35E-03 & $8.20 \mathrm{E}-03$ & 8.02E-03 & $6.45 \mathrm{E}-03$ & $8.62 E-03$ \\
\hline $55 \%$ & $6.57 \mathrm{E}-03$ & $4.56 \mathrm{E}-03$ & $8.72 \mathrm{E}-03$ & $8.57 \mathrm{E}-03$ & $8.41 \mathrm{E}-03$ & $6.62 \mathrm{E}-03$ & $9.11 \mathrm{E}-03$ \\
\hline $60 \%$ & $6.82 \mathrm{E}-03$ & $4.70 \mathrm{E}-03$ & $9.14 \mathrm{E}-03$ & $8.94 \mathrm{E}-03$ & 8.77E-03 & $6.87 \mathrm{E}-03$ & $9.48 \mathrm{E}-03$ \\
\hline $65 \%$ & $7.06 \mathrm{E}-03$ & $4.82 \mathrm{E}-03$ & $9.64 \mathrm{E}-03$ & $9.37 \mathrm{E}-03$ & $9.28 \mathrm{E}-03$ & $7.09 \mathrm{E}-03$ & $1.01 \mathrm{E}-02$ \\
\hline $70 \%$ & $7.44 \mathrm{E}-03$ & $4.99 \mathrm{E}-03$ & $1.02 \mathrm{E}-02$ & $9.99 \mathrm{E}-03$ & $9.84 \mathrm{E}-03$ & $7.42 \mathrm{E}-03$ & 1.07E-02 \\
\hline $75 \%$ & $7.89 \mathrm{E}-03$ & $5.19 \mathrm{E}-03$ & $1.09 \mathrm{E}-02$ & 1.06E-02 & 1.05E-02 & $7.75 \mathrm{E}-03$ & $1.14 \mathrm{E}-02$ \\
\hline $80 \%$ & $8.30 \mathrm{E}-03$ & $5.42 \mathrm{E}-03$ & $1.16 \mathrm{E}-02$ & $1.13 \mathrm{E}-02$ & $1.13 \mathrm{E}-02$ & $8.14 \mathrm{E}-03$ & $1.24 \mathrm{E}-02$ \\
\hline $85 \%$ & $8.82 \mathrm{E}-03$ & $5.68 \mathrm{E}-03$ & $1.28 \mathrm{E}-02$ & $1.23 \mathrm{E}-02$ & $1.23 \mathrm{E}-02$ & $8.68 \mathrm{E}-03$ & $1.36 \mathrm{E}-02$ \\
\hline $90 \%$ & $9.89 \mathrm{E}-03$ & $6.12 \mathrm{E}-03$ & 1.42E-02 & $1.38 \mathrm{E}-02$ & $1.38 \mathrm{E}-02$ & $9.38 \mathrm{E}-03$ & $1.49 \mathrm{E}-02$ \\
\hline $95 \%$ & 1.19E-02 & $6.82 \mathrm{E}-03$ & $1.68 \mathrm{E}-02$ & $1.62 \mathrm{E}-02$ & 1.62E-02 & $1.08 \mathrm{E}-02$ & 1.77E-02 \\
\hline Maximum & $2.49 \mathrm{E}-02$ & $1.41 \mathrm{E}-02$ & $2.76 \mathrm{E}-02$ & $2.66 \mathrm{E}-02$ & $2.67 \mathrm{E}-02$ & $1.71 \mathrm{E}-02$ & $3.68 \mathrm{E}-02$ \\
\hline Source: & \multicolumn{7}{|c|}{$\begin{array}{l}\text { MO0407SPAINEXI.002 [DIRS 170597]; MO0407SPASRPBM.002 [DIRS 170755]; } \\
\text { MO0306MWDBGSMF.001 [DIRS 163816]; MO0403SPAAEIBM.002 [DIRS 169392]; } \\
\text { MO0407SPACRBSM.002 [DIRS 170677];, MO0406SPAETPBM.002 [DIRS 170150]. }\end{array}$} \\
\hline NOTE: & \multicolumn{7}{|c|}{$\begin{array}{l}\text { See Excel file GW BDCF Realizations_MC and FC_Rev 4.xIs in Appendix B for details of } \\
\text { calculations. } \\
\text { indard deviation. }\end{array}$} \\
\hline
\end{tabular}


Table 6.2-9. Statistics for the ICRP 30-Based BDCFs for the Monsoon Climate, rem/yr per pCi/L

\begin{tabular}{|c|c|c|c|c|c|c|c|c|c|c|c|}
\hline & C-14 & $\mathrm{Cl}-36$ & Se-79 & Sr-90 & Tc-99 & Sn-126 & I-129 & Cs-135 & Cs-137 & $\mathrm{Pb}-210$ & Ra-226 \\
\hline Mean & $8.80 \mathrm{E}-06$ & $1.83 \mathrm{E}-05$ & $5.07 \mathrm{E}-05$ & 1.53E-04 & $2.10 \mathrm{E}-06$ & $4.96 \mathrm{E}-03$ & $3.13 \mathrm{E}-04$ & 4.44E-05 & 3.83E-04 & $8.26 \mathrm{E}-03$ & $7.65 E-02$ \\
\hline STD & $6.67 \mathrm{E}-06$ & $4.04 \mathrm{E}-05$ & $7.82 \mathrm{E}-05$ & $2.22 \mathrm{E}-05$ & $2.14 \mathrm{E}-06$ & 3.23E-03 & $9.42 \mathrm{E}-05$ & $3.16 \mathrm{E}-05$ & $1.87 \mathrm{E}-04$ & $2.82 \mathrm{E}-03$ & $3.99 \mathrm{E}-02$ \\
\hline Minimum & $3.79 \mathrm{E}-06$ & 3.13E-06 & $8.91 \mathrm{E}-06$ & $1.21 \mathrm{E}-04$ & 1.15E-06 & $1.54 \mathrm{E}-04$ & $2.19 \mathrm{E}-04$ & $9.72 \mathrm{E}-06$ & 1.16E-04 & $5.60 \mathrm{E}-03$ & 2.23E-03 \\
\hline $5 \%$ & 4.65E-06 & 4.64E-06 & 1.35E-05 & $1.30 \mathrm{E}-04$ & 1.27E-06 & $8.91 \mathrm{E}-04$ & $2.35 \mathrm{E}-04$ & $1.55 \mathrm{E}-05$ & $1.81 \mathrm{E}-04$ & $6.03 \mathrm{E}-03$ & $2.83 \mathrm{E}-02$ \\
\hline $10 \%$ & $4.88 \mathrm{E}-06$ & $5.18 \mathrm{E}-06$ & $1.50 \mathrm{E}-05$ & 1.33E-04 & $1.30 \mathrm{E}-06$ & $1.40 \mathrm{E}-03$ & $2.42 \mathrm{E}-04$ & $1.78 \mathrm{E}-05$ & $2.02 \mathrm{E}-04$ & $6.22 \mathrm{E}-03$ & $3.52 \mathrm{E}-02$ \\
\hline $15 \%$ & $5.18 \mathrm{E}-06$ & $5.60 \mathrm{E}-06$ & $1.71 \mathrm{E}-05$ & $1.35 \mathrm{E}-04$ & $1.34 \mathrm{E}-06$ & $1.99 \mathrm{E}-03$ & $2.48 \mathrm{E}-04$ & $2.03 E-05$ & $2.22 \mathrm{E}-04$ & $6.32 \mathrm{E}-03$ & $3.98 \mathrm{E}-02$ \\
\hline $20 \%$ & 5.37E-06 & $6.19 \mathrm{E}-06$ & $1.85 \mathrm{E}-05$ & 1.37E-04 & $1.38 \mathrm{E}-06$ & $2.42 \mathrm{E}-03$ & $2.53 \mathrm{E}-04$ & $2.23 \mathrm{E}-05$ & $2.36 \mathrm{E}-04$ & $6.46 \mathrm{E}-03$ & $4.50 \mathrm{E}-02$ \\
\hline $25 \%$ & $5.59 \mathrm{E}-06$ & $6.62 \mathrm{E}-06$ & $2.01 \mathrm{E}-05$ & $1.39 \mathrm{E}-04$ & $1.42 \mathrm{E}-06$ & $2.66 \mathrm{E}-03$ & $2.58 \mathrm{E}-04$ & $2.48 \mathrm{E}-05$ & $2.53 \mathrm{E}-04$ & $6.57 \mathrm{E}-03$ & $4.84 \mathrm{E}-02$ \\
\hline $30 \%$ & $5.81 \mathrm{E}-06$ & $7.21 \mathrm{E}-06$ & $2.19 \mathrm{E}-05$ & $1.41 \mathrm{E}-04$ & $1.46 \mathrm{E}-06$ & $2.98 \mathrm{E}-03$ & 2.63E-04 & $2.74 \mathrm{E}-05$ & $2.71 \mathrm{E}-04$ & $6.68 \mathrm{E}-03$ & $5.22 \mathrm{E}-02$ \\
\hline $35 \%$ & $6.01 \mathrm{E}-06$ & $8.12 \mathrm{E}-06$ & $2.36 \mathrm{E}-05$ & $1.43 \mathrm{E}-04$ & $1.50 \mathrm{E}-06$ & $3.26 \mathrm{E}-03$ & $2.70 \mathrm{E}-04$ & $2.95 \mathrm{E}-05$ & 2.87E-04 & $6.85 \mathrm{E}-03$ & $5.68 \mathrm{E}-02$ \\
\hline $40 \%$ & $6.28 \mathrm{E}-06$ & $8.92 \mathrm{E}-06$ & $2.53 \mathrm{E}-05$ & 1.45E-04 & $1.54 \mathrm{E}-06$ & $3.55 \mathrm{E}-03$ & $2.76 \mathrm{E}-04$ & $3.18 \mathrm{E}-05$ & $3.04 \mathrm{E}-04$ & 7.03E-03 & $6.08 \mathrm{E}-02$ \\
\hline $45 \%$ & $6.53 \mathrm{E}-06$ & $9.76 \mathrm{E}-06$ & $2.70 \mathrm{E}-05$ & $1.47 \mathrm{E}-04$ & $1.60 \mathrm{E}-06$ & $3.91 \mathrm{E}-03$ & $2.82 \mathrm{E}-04$ & $3.38 \mathrm{E}-05$ & $3.21 \mathrm{E}-04$ & $7.18 \mathrm{E}-03$ & $6.43 \mathrm{E}-02$ \\
\hline $50 \%$ & $6.77 \mathrm{E}-06$ & $1.06 \mathrm{E}-05$ & $2.90 \mathrm{E}-05$ & $1.49 \mathrm{E}-04$ & $1.65 \mathrm{E}-06$ & 4.22E-03 & $2.90 \mathrm{E}-04$ & $3.64 \mathrm{E}-05$ & $3.40 \mathrm{E}-04$ & $7.34 \mathrm{E}-03$ & $6.92 \mathrm{E}-02$ \\
\hline $55 \%$ & 7.03E-06 & 1.16E-05 & 3.15E-05 & $1.50 \mathrm{E}-04$ & $1.71 \mathrm{E}-06$ & $4.69 \mathrm{E}-03$ & $2.95 \mathrm{E}-04$ & $3.87 \mathrm{E}-05$ & $3.59 \mathrm{E}-04$ & $7.54 \mathrm{E}-03$ & $7.27 \mathrm{E}-02$ \\
\hline $60 \%$ & $7.51 \mathrm{E}-06$ & $1.30 \mathrm{E}-05$ & $3.47 \mathrm{E}-05$ & $1.53 \mathrm{E}-04$ & $1.78 \mathrm{E}-06$ & $4.99 \mathrm{E}-03$ & $3.04 \mathrm{E}-04$ & 4.14E-05 & $3.81 \mathrm{E}-04$ & 7.79E-03 & $7.82 \mathrm{E}-02$ \\
\hline $65 \%$ & 8.03E-06 & 1.47E-05 & $3.92 \mathrm{E}-05$ & $1.55 \mathrm{E}-04$ & $1.86 \mathrm{E}-06$ & $5.44 \mathrm{E}-03$ & $3.14 \mathrm{E}-04$ & $4.46 \mathrm{E}-05$ & 4.06E-04 & $8.08 \mathrm{E}-03$ & $8.27 \mathrm{E}-02$ \\
\hline $70 \%$ & 8.56E-06 & 1.66E-05 & $4.45 \mathrm{E}-05$ & $1.58 \mathrm{E}-04$ & $1.99 \mathrm{E}-06$ & $6.03 \mathrm{E}-03$ & $3.23 \mathrm{E}-04$ & 4.94E-05 & $4.25 \mathrm{E}-04$ & 8.43E-03 & $9.00 \mathrm{E}-02$ \\
\hline $75 \%$ & $9.26 \mathrm{E}-06$ & $1.87 \mathrm{E}-05$ & $5.08 \mathrm{E}-05$ & $1.61 \mathrm{E}-04$ & $2.11 \mathrm{E}-06$ & $6.64 \mathrm{E}-03$ & $3.38 \mathrm{E}-04$ & $5.32 \mathrm{E}-05$ & $4.57 \mathrm{E}-04$ & $8.78 \mathrm{E}-03$ & $9.65 \mathrm{E}-02$ \\
\hline $80 \%$ & $1.04 \mathrm{E}-05$ & $2.21 \mathrm{E}-05$ & $6.04 \mathrm{E}-05$ & $1.65 \mathrm{E}-04$ & $2.30 \mathrm{E}-06$ & $7.47 \mathrm{E}-03$ & $3.53 \mathrm{E}-04$ & $5.80 \mathrm{E}-05$ & 4.97E-04 & $9.25 \mathrm{E}-03$ & $1.07 \mathrm{E}-01$ \\
\hline $85 \%$ & 1.16E-05 & $2.59 \mathrm{E}-05$ & $7.48 \mathrm{E}-05$ & 1.70E-04 & $2.56 \mathrm{E}-06$ & $8.18 \mathrm{E}-03$ & 3.74E-04 & $6.65 \mathrm{E}-05$ & 5.43E-04 & $1.01 \mathrm{E}-02$ & $1.14 \mathrm{E}-01$ \\
\hline $90 \%$ & 1.37E-05 & $3.32 \mathrm{E}-05$ & $9.45 \mathrm{E}-05$ & $1.78 \mathrm{E}-04$ & $3.17 \mathrm{E}-06$ & $9.06 \mathrm{E}-03$ & $4.05 \mathrm{E}-04$ & $8.14 \mathrm{E}-05$ & $6.18 \mathrm{E}-04$ & 1.12E-02 & $1.27 \mathrm{E}-01$ \\
\hline $95 \%$ & $2.03 \mathrm{E}-05$ & 4.94E-05 & 1.33E-04 & $1.93 \mathrm{E}-04$ & $4.08 \mathrm{E}-06$ & $1.11 \mathrm{E}-02$ & $4.68 \mathrm{E}-04$ & $1.00 \mathrm{E}-04$ & 7.13E-04 & $1.41 \mathrm{E}-02$ & $1.51 \mathrm{E}-01$ \\
\hline Maximum & 9.33E-05 & 8.37E-04 & $1.05 \mathrm{E}-03$ & $3.74 \mathrm{E}-04$ & $5.40 \mathrm{E}-05$ & $2.00 \mathrm{E}-02$ & $1.58 \mathrm{E}-03$ & $4.21 \mathrm{E}-04$ & $1.62 \mathrm{E}-03$ & $2.84 \mathrm{E}-02$ & $3.96 \mathrm{E}-01$ \\
\hline
\end{tabular}


Table 6.2-9. Statistics for the ICRP 30-Based BDCFs for the Monsoon Climate, rem/yr per pCi/L (Continued)

\begin{tabular}{|c|c|c|c|c|c|c|c|c|c|c|c|}
\hline & $\begin{array}{r}\mathrm{Ra}-226 \\
+\mathrm{Pb}-210 \\
\end{array}$ & Ac-227 & Th-229 & Th-230 & Th-232 & Pa-231 & U-232 & U-233 & U-234 & U-236 & U-238 \\
\hline Mean & $18 \mathrm{E}-02$ & $2.45 \mathrm{E}-02$ & $2.64 \mathrm{E}-02$ & $2.32 \mathrm{E}-02$ & $3.45 \mathrm{E}-02$ & $7.95 \mathrm{E}-02$ & $4.60 \mathrm{E}-03$ & $1.46 \mathrm{E}-03$ & $9.93 E-04$ & $.79 E-04$ & $8.80 \mathrm{E}-04$ \\
\hline STD & $1 \mathrm{E}-02$ & $8.34 \mathrm{E}-03$ & $1.50 \mathrm{E}-02$ & $2.08 \mathrm{E}-02$ & $1.88 \mathrm{E}-02$ & $5.35 \mathrm{E}-02$ & $1.82 \mathrm{E}-03$ & 1.75E-03 & $8.80 \mathrm{E}-04$ & $7.45 \mathrm{E}-04$ & $7.36 \mathrm{E}-04$ \\
\hline Minin & 1E-02 & 1.27E-02 & $4.48 \mathrm{E}-03$ & 7.47E-04 & $6.02 \mathrm{E}-03$ & $9.79 \mathrm{E}-03$ & $1.80 \mathrm{E}-03$ & $2.34 \mathrm{E}-04$ & $2.25 \mathrm{E}-04$ & 2.11E-04 & $2.11 \mathrm{E}-04$ \\
\hline $5 \%$ & $58 \mathrm{E}-02$ & $1.50 \mathrm{E}-02$ & $9.40 \mathrm{E}-03$ & $4.39 \mathrm{E}-03$ & 1.30E-02 & $2.50 \mathrm{E}-02$ & $2.34 \mathrm{E}-03$ & $2.98 \mathrm{E}-04$ & $2.89 \mathrm{E}-04$ & $.67 \mathrm{E}-04$ & 2.64E-04 \\
\hline $10 \%$ & $30 \mathrm{E}-02$ & $1.59 \mathrm{E}-02$ & 1.15E-02 & $5.65 \mathrm{E}-03$ & $1.58 \mathrm{E}-02$ & $2.97 \mathrm{E}-02$ & $2.59 \mathrm{E}-03$ & $3.48 \mathrm{E}-04$ & $3.17 \mathrm{E}-04$ & $3.01 \mathrm{E}-04$ & $3.03 \mathrm{E}-04$ \\
\hline $15 \%$ & $7 \mathrm{E}-02$ & $1.69 \mathrm{E}-02$ & $1.34 \mathrm{E}-02$ & $6.76 \mathrm{E}-03$ & $1.80 \mathrm{E}-02$ & $3.39 \mathrm{E}-02$ & $2.82 \mathrm{E}-03$ & $3.86 \mathrm{E}-04$ & 3.57E-04 & 3.37E-04 & $3.36 \mathrm{E}-04$ \\
\hline $20 \%$ & $32 \mathrm{E}-02$ & 1.75E-02 & 1.46E-02 & $7.93 \mathrm{E}-03$ & $1.97 \mathrm{E}-02$ & $3.79 \mathrm{E}-02$ & 3.06E-03 & 4.32E-04 & $3.91 \mathrm{E}-04$ & 3.66E-04 & $3.61 \mathrm{E}-04$ \\
\hline $25 \%$ & $64 \mathrm{E}-02$ & 1.83E-02 & 1.61E-02 & $9.09 \mathrm{E}-03$ & 2.17E-02 & 4.23E-02 & $3.29 \mathrm{E}-03$ & 4.79E-04 & 4.32E-04 & $4.01 \mathrm{E}-04$ & $3.94 \mathrm{E}-04$ \\
\hline $30 \%$ & 04E-02 & $1.92 \mathrm{E}-02$ & 1.73E-02 & 1.03E-02 & 2.33E-02 & 4.65E-02 & $3.48 \mathrm{E}-03$ & $5.15 \mathrm{E}-04$ & $4.75 \mathrm{E}-04$ & 4.37E-04 & 4.36E-04 \\
\hline $35 \%$ & $46 \mathrm{E}-02$ & $2.00 \mathrm{E}-02$ & $1.85 \mathrm{E}-02$ & 1.13E-02 & $2.50 \mathrm{E}-02$ & $5.12 \mathrm{E}-02$ & $3.70 \mathrm{E}-03$ & $5.66 \mathrm{E}-04$ & $5.16 \mathrm{E}-04$ & $4.79 \mathrm{E}-04$ & 4.79E-04 \\
\hline $40 \%$ & $92 \mathrm{E}-02$ & $2.07 \mathrm{E}-02$ & $2.00 \mathrm{E}-02$ & $1.30 \mathrm{E}-02$ & $2.64 \mathrm{E}-02$ & $5.58 \mathrm{E}-02$ & $3.89 \mathrm{E}-03$ & $6.31 \mathrm{E}-04$ & $5.71 \mathrm{E}-04$ & $5.25 \mathrm{E}-04$ & $5.13 \mathrm{E}-04$ \\
\hline $45 \%$ & $23 \mathrm{E}-02$ & $2.17 \mathrm{E}-02$ & 2.12E-02 & $1.44 \mathrm{E}-02$ & $2.80 \mathrm{E}-02$ & $5.95 \mathrm{E}-02$ & 4.07E-03 & $7.05 \mathrm{E}-04$ & $6.09 \mathrm{E}-04$ & $5.61 \mathrm{E}-04$ & $5.69 \mathrm{E}-04$ \\
\hline $50 \%$ & $71 \mathrm{E}-02$ & $2.26 \mathrm{E}-02$ & $2.30 \mathrm{E}-02$ & 1.63E-02 & $2.96 \mathrm{E}-02$ & $6.39 \mathrm{E}-02$ & $4.27 \mathrm{E}-03$ & 7.96E-04 & $6.83 \mathrm{E}-04$ & $6.20 \mathrm{E}-04$ & $6.21 \mathrm{E}-04$ \\
\hline $55 \%$ & $11 \mathrm{E}-02$ & $2.37 \mathrm{E}-02$ & $2.46 \mathrm{E}-02$ & 1.87E-02 & $3.15 \mathrm{E}-02$ & $7.02 \mathrm{E}-02$ & $4.48 \mathrm{E}-03$ & $9.14 \mathrm{E}-04$ & $7.51 \mathrm{E}-04$ & $6.88 \mathrm{E}-04$ & $6.82 \mathrm{E}-04$ \\
\hline $60 \%$ & 8.65E-02 & $2.48 \mathrm{E}-02$ & 2.63E-02 & $2.03 \mathrm{E}-02$ & $3.46 \mathrm{E}-02$ & $7.56 \mathrm{E}-02$ & $4.71 \mathrm{E}-03$ & $1.05 \mathrm{E}-03$ & $8.14 \mathrm{E}-04$ & $7.65 \mathrm{E}-04$ & 7.57E-04 \\
\hline $65 \%$ & $9.18 \mathrm{E}-02$ & $2.58 \mathrm{E}-02$ & $2.84 \mathrm{E}-02$ & $2.33 \mathrm{E}-02$ & $3.74 \mathrm{E}-02$ & $8.16 \mathrm{E}-02$ & 4.94E-03 & $1.23 \mathrm{E}-03$ & $9.43 \mathrm{E}-04$ & $8.59 \mathrm{E}-04$ & $8.49 \mathrm{E}-04$ \\
\hline $70 \%$ & $9.80 \mathrm{E}-02$ & $2.70 \mathrm{E}-02$ & 3.05E-02 & $2.69 \mathrm{E}-02$ & $4.01 \mathrm{E}-02$ & $9.23 \mathrm{E}-02$ & $5.18 \mathrm{E}-03$ & $1.40 \mathrm{E}-03$ & $1.07 \mathrm{E}-03$ & $9.62 \mathrm{E}-04$ & $9.35 E-04$ \\
\hline $75 \%$ & $1.06 \mathrm{E}-01$ & $2.90 \mathrm{E}-02$ & $3.26 \mathrm{E}-02$ & 3.03E-02 & $4.29 \mathrm{E}-02$ & $1.02 \mathrm{E}-01$ & $5.46 \mathrm{E}-03$ & $1.68 \mathrm{E}-03$ & $1.23 \mathrm{E}-03$ & 1.10E-03 & $1.04 \mathrm{E}-03$ \\
\hline $80 \%$ & 1.15E-01 & $3.06 \mathrm{E}-02$ & $3.56 \mathrm{E}-02$ & $3.52 \mathrm{E}-02$ & 4.69E-02 & 1.13E-01 & $5.85 \mathrm{E}-03$ & $1.97 \mathrm{E}-03$ & $1.41 \mathrm{E}-03$ & $1.24 \mathrm{E}-03$ & $1.24 \mathrm{E}-03$ \\
\hline $85 \%$ & $1.23 \mathrm{E}-01$ & $3.27 \mathrm{E}-02$ & $3.88 \mathrm{E}-02$ & 4.15E-02 & $5.23 \mathrm{E}-02$ & $1.26 \mathrm{E}-01$ & $6.44 \mathrm{E}-03$ & $2.45 \mathrm{E}-03$ & $1.71 \mathrm{E}-03$ & $1.45 \mathrm{E}-03$ & $1.46 \mathrm{E}-03$ \\
\hline $90 \%$ & 1.37E-01 & $3.50 \mathrm{E}-02$ & $4.40 \mathrm{E}-02$ & $4.88 \mathrm{E}-02$ & $5.77 \mathrm{E}-02$ & $1.48 \mathrm{E}-01$ & $6.99 \mathrm{E}-03$ & $3.27 E-03$ & $2.09 \mathrm{E}-03$ & $1.74 \mathrm{E}-03$ & $1.83 E-03$ \\
\hline $95 \%$ & $1.59 \mathrm{E}-01$ & $3.93 \mathrm{E}-02$ & $5.69 \mathrm{E}-02$ & $6.56 \mathrm{E}-02$ & $6.95 \mathrm{E}-02$ & $1.88 \mathrm{E}-01$ & $7.99 \mathrm{E}-03$ & $4.93 \mathrm{E}-03$ & $2.87 \mathrm{E}-03$ & $2.43 \mathrm{E}-03$ & $2.40 \mathrm{E}-03$ \\
\hline Maximum & $4.06 \mathrm{E}-01$ & $9.57 \mathrm{E}-02$ & $9.68 \mathrm{E}-02$ & $1.41 \mathrm{E}-01$ & $1.28 \mathrm{E}-01$ & $5.05 \mathrm{E}-01$ & $1.44 \mathrm{E}-02$ & 1.33E-02 & 7.41E-03 & $6.27 \mathrm{E}-03$ & $4.74 \mathrm{E}-03$ \\
\hline
\end{tabular}


Table 6.2-9. Statistics for the ICRP 30-Based BDCFs for the Monsoon Climate, rem/yr per pCi/L I (Continued)

\begin{tabular}{|c|c|c|c|c|c|c|c|}
\hline & Np-237 & Pu-238 & Pu-239 & $\mathrm{Pu}-240$ & Pu-242 & Am-241 & Am-243 \\
\hline Mean & $6.05 \mathrm{E}-03$ & $4.07 \mathrm{E}-03$ & $7.57 \mathrm{E}-03$ & $7.41 \mathrm{E}-03$ & $7.31 \mathrm{E}-03$ & $5.74 \mathrm{E}-03$ & $7.88 \mathrm{E}-03$ \\
\hline STD & $2.02 \mathrm{E}-03$ & $9.48 \mathrm{E}-04$ & $3.00 \mathrm{E}-03$ & $2.89 \mathrm{E}-03$ & $3.03 E-03$ & $1.69 \mathrm{E}-03$ & $3.41 \mathrm{E}-03$ \\
\hline Minimum & $3.57 \mathrm{E}-03$ & $2.62 \mathrm{E}-03$ & $3.25 \mathrm{E}-03$ & $3.15 \mathrm{E}-03$ & $3.19 \mathrm{E}-03$ & $2.84 \mathrm{E}-03$ & $2.94 \mathrm{E}-03$ \\
\hline $5 \%$ & 4.07E-03 & $3.01 \mathrm{E}-03$ & 4.32E-03 & $4.33 \mathrm{E}-03$ & $4.11 \mathrm{E}-03$ & $3.75 \mathrm{E}-03$ & $4.08 \mathrm{E}-03$ \\
\hline $10 \%$ & $4.28 \mathrm{E}-03$ & $3.14 \mathrm{E}-03$ & $4.71 \mathrm{E}-03$ & $4.65 \mathrm{E}-03$ & $4.46 \mathrm{E}-03$ & $4.04 \mathrm{E}-03$ & $4.55 \mathrm{E}-03$ \\
\hline $15 \%$ & $4.41 \mathrm{E}-03$ & $3.22 \mathrm{E}-03$ & $4.94 \mathrm{E}-03$ & $4.91 \mathrm{E}-03$ & $4.68 \mathrm{E}-03$ & $4.24 \mathrm{E}-03$ & 4.97E-03 \\
\hline $20 \%$ & $4.62 \mathrm{E}-03$ & $3.33 \mathrm{E}-03$ & $5.21 \mathrm{E}-03$ & $5.15 \mathrm{E}-03$ & $4.94 \mathrm{E}-03$ & $4.40 \mathrm{E}-03$ & $5.23 E-03$ \\
\hline $25 \%$ & 4.77E-03 & $3.40 \mathrm{E}-03$ & $5.46 \mathrm{E}-03$ & $5.33 \mathrm{E}-03$ & $5.17 \mathrm{E}-03$ & 4.57E-03 & $5.48 \mathrm{E}-03$ \\
\hline $30 \%$ & $4.90 \mathrm{E}-03$ & $3.48 \mathrm{E}-03$ & $5.71 \mathrm{E}-03$ & $5.63 \mathrm{E}-03$ & $5.42 \mathrm{E}-03$ & 4.73E-03 & $5.80 \mathrm{E}-03$ \\
\hline $35 \%$ & $5.03 \mathrm{E}-03$ & $3.57 \mathrm{E}-03$ & $5.96 \mathrm{E}-03$ & $5.88 \mathrm{E}-03$ & $5.74 \mathrm{E}-03$ & $4.90 \mathrm{E}-03$ & $6.12 \mathrm{E}-03$ \\
\hline $40 \%$ & $5.16 \mathrm{E}-03$ & $3.67 \mathrm{E}-03$ & $6.19 \mathrm{E}-03$ & $6.14 \mathrm{E}-03$ & $6.05 \mathrm{E}-03$ & $5.05 \mathrm{E}-03$ & $6.38 \mathrm{E}-03$ \\
\hline $45 \%$ & $5.30 \mathrm{E}-03$ & $3.75 \mathrm{E}-03$ & $6.44 \mathrm{E}-03$ & $6.40 \mathrm{E}-03$ & $6.33 \mathrm{E}-03$ & $5.21 \mathrm{E}-03$ & $6.73 \mathrm{E}-03$ \\
\hline $50 \%$ & $5.45 \mathrm{E}-03$ & $3.84 \mathrm{E}-03$ & $6.75 \mathrm{E}-03$ & $6.74 \mathrm{E}-03$ & $6.59 \mathrm{E}-03$ & $5.37 \mathrm{E}-03$ & $6.99 \mathrm{E}-03$ \\
\hline $55 \%$ & $5.66 \mathrm{E}-03$ & $3.97 \mathrm{E}-03$ & $7.10 \mathrm{E}-03$ & $7.00 \mathrm{E}-03$ & $6.88 \mathrm{E}-03$ & $5.52 \mathrm{E}-03$ & $7.29 \mathrm{E}-03$ \\
\hline $60 \%$ & $5.84 \mathrm{E}-03$ & 4.07E-03 & $7.48 \mathrm{E}-03$ & $7.26 \mathrm{E}-03$ & $7.20 \mathrm{E}-03$ & $5.77 \mathrm{E}-03$ & $7.78 \mathrm{E}-03$ \\
\hline $65 \%$ & $6.10 \mathrm{E}-03$ & $4.19 \mathrm{E}-03$ & $7.83 \mathrm{E}-03$ & $7.60 \mathrm{E}-03$ & $7.48 \mathrm{E}-03$ & $6.03 E-03$ & $8.21 \mathrm{E}-03$ \\
\hline $70 \%$ & $6.39 \mathrm{E}-03$ & 4.33E-03 & $8.20 \mathrm{E}-03$ & $7.99 \mathrm{E}-03$ & $7.93 \mathrm{E}-03$ & $6.28 \mathrm{E}-03$ & $8.75 \mathrm{E}-03$ \\
\hline $75 \%$ & $6.70 \mathrm{E}-03$ & 4.45E-03 & $8.75 \mathrm{E}-03$ & $8.55 \mathrm{E}-03$ & $8.50 \mathrm{E}-03$ & $6.50 \mathrm{E}-03$ & $9.44 \mathrm{E}-03$ \\
\hline $80 \%$ & $7.06 \mathrm{E}-03$ & $4.65 \mathrm{E}-03$ & $9.43 \mathrm{E}-03$ & $9.10 \mathrm{E}-03$ & $9.13 \mathrm{E}-03$ & $6.82 \mathrm{E}-03$ & $1.01 \mathrm{E}-02$ \\
\hline $85 \%$ & $7.64 \mathrm{E}-03$ & 4.92E-03 & $1.05 \mathrm{E}-02$ & $1.01 \mathrm{E}-02$ & $9.94 \mathrm{E}-03$ & $7.26 \mathrm{E}-03$ & 1.10E-02 \\
\hline $90 \%$ & $8.44 \mathrm{E}-03$ & $5.27 \mathrm{E}-03$ & 1.15E-02 & 1.13E-02 & $1.11 \mathrm{E}-02$ & $7.81 \mathrm{E}-03$ & $1.21 \mathrm{E}-02$ \\
\hline $95 \%$ & $1.01 \mathrm{E}-02$ & $5.91 \mathrm{E}-03$ & 1.37E-02 & 1.33E-02 & 1.33E-02 & $8.98 \mathrm{E}-03$ & $1.49 \mathrm{E}-02$ \\
\hline Maximum & $1.85 \mathrm{E}-02$ & $1.02 \mathrm{E}-02$ & $2.45 \mathrm{E}-02$ & $2.30 \mathrm{E}-02$ & $2.63 E-02$ & $1.67 \mathrm{E}-02$ & $2.73 \mathrm{E}-02$ \\
\hline Source: $\quad N$ & \multicolumn{7}{|c|}{$\begin{array}{l}\text { MO0407SPAINEXI.002 [DIRS 170597]; MO0407SPASRPBM.002 [DIRS 170755]; } \\
\text { MO0306MWDBGSMF.001 [DIRS 163816]; MO0403SPAAEIBM.002 [DIRS 169392]; } \\
\text { MO0407SPACRBSM.002 [DIRS 170677]; MO0406SPAETPBM.002 [DIRS 170150]. }\end{array}$} \\
\hline $\mathrm{TE}: \quad \mathrm{S}$ & \multicolumn{7}{|c|}{$\begin{array}{l}\text { See Excel file GW BDCF Realizations_MC and FC_Rev 4.xIs in Appendix B for details of } \\
\text { calculations. }\end{array}$} \\
\hline
\end{tabular}


Table 6.2-10. Statistics for the ICRP 30-Based BDCFs for the Glacial Transition Climate, rem/yr per pCi/L

\begin{tabular}{|c|c|c|c|c|c|c|c|c|c|c|c|}
\hline & C-14 & $\mathrm{Cl}-36$ & Se-79 & Sr-90 & Tc-99 & Sn-126 & I-129 & Cs-135 & Cs-137 & $\mathrm{Pb}-210$ & Ra-226 \\
\hline Mean & 8.77E-06 & 1.77E-05 & 4.97E-05 & $1.52 \mathrm{E}-04$ & $2.07 \mathrm{E}-06$ & $4.71 \mathrm{E}-03$ & $3.10 \mathrm{E}-04$ & 4.33E-05 & $3.66 \mathrm{E}-04$ & $8.18 \mathrm{E}-03$ & 7.37E-02 \\
\hline STD & $6.67 \mathrm{E}-06$ & $3.79 \mathrm{E}-05$ & $8.73 \mathrm{E}-05$ & $2.09 \mathrm{E}-05$ & $2.13 \mathrm{E}-06$ & $3.02 \mathrm{E}-03$ & $9.10 \mathrm{E}-05$ & $3.14 \mathrm{E}-05$ & $1.76 \mathrm{E}-04$ & $2.85 \mathrm{E}-03$ & 3.83E-02 \\
\hline Minimum & $3.78 \mathrm{E}-06$ & $3.19 \mathrm{E}-06$ & $8.85 \mathrm{E}-06$ & $1.22 \mathrm{E}-04$ & $1.16 \mathrm{E}-06$ & $1.53 \mathrm{E}-04$ & 2.17E-04 & $1.03 \mathrm{E}-05$ & $1.22 \mathrm{E}-04$ & $5.68 \mathrm{E}-03$ & $2.21 \mathrm{E}-03$ \\
\hline $5 \%$ & 4.61E-06 & $4.48 \mathrm{E}-06$ & $1.32 \mathrm{E}-05$ & $1.29 \mathrm{E}-04$ & $1.26 \mathrm{E}-06$ & $8.26 \mathrm{E}-04$ & $2.33 \mathrm{E}-04$ & $1.53 \mathrm{E}-05$ & $1.73 \mathrm{E}-04$ & $6.04 \mathrm{E}-03$ & 2.73E-02 \\
\hline $10 \%$ & $4.89 \mathrm{E}-06$ & $5.05 \mathrm{E}-06$ & $1.53 \mathrm{E}-05$ & $1.32 \mathrm{E}-04$ & $1.30 \mathrm{E}-06$ & $1.43 \mathrm{E}-03$ & $2.41 \mathrm{E}-04$ & $1.79 \mathrm{E}-05$ & $1.94 \mathrm{E}-04$ & $6.17 \mathrm{E}-03$ & $3.38 \mathrm{E}-02$ \\
\hline $15 \%$ & $5.13 \mathrm{E}-06$ & $5.54 \mathrm{E}-06$ & 1.67E-05 & $1.35 \mathrm{E}-04$ & $1.34 \mathrm{E}-06$ & $1.90 \mathrm{E}-03$ & 2.47E-04 & $2.02 \mathrm{E}-05$ & $2.14 \mathrm{E}-04$ & $6.30 \mathrm{E}-03$ & $3.90 \mathrm{E}-02$ \\
\hline $20 \%$ & $5.33 \mathrm{E}-06$ & $6.09 \mathrm{E}-06$ & $1.82 \mathrm{E}-05$ & $1.37 \mathrm{E}-04$ & $1.37 \mathrm{E}-06$ & $2.34 \mathrm{E}-03$ & $2.52 \mathrm{E}-04$ & $2.23 \mathrm{E}-05$ & $2.31 \mathrm{E}-04$ & $6.42 \mathrm{E}-03$ & $4.24 \mathrm{E}-02$ \\
\hline $25 \%$ & $5.56 \mathrm{E}-06$ & $6.53 \mathrm{E}-06$ & $1.94 \mathrm{E}-05$ & $1.38 \mathrm{E}-04$ & $1.41 \mathrm{E}-06$ & $2.62 \mathrm{E}-03$ & $2.57 \mathrm{E}-04$ & $2.45 \mathrm{E}-05$ & $2.47 \mathrm{E}-04$ & $6.54 \mathrm{E}-03$ & $4.71 \mathrm{E}-02$ \\
\hline $30 \%$ & $5.77 \mathrm{E}-06$ & 7.16E-06 & $2.13 \mathrm{E}-05$ & $1.40 \mathrm{E}-04$ & $1.45 \mathrm{E}-06$ & $2.84 \mathrm{E}-03$ & 2.62E-04 & $2.59 \mathrm{E}-05$ & 2.61E-04 & $6.65 \mathrm{E}-03$ & $5.04 \mathrm{E}-02$ \\
\hline $35 \%$ & $5.98 \mathrm{E}-06$ & 7.95E-06 & $2.26 \mathrm{E}-05$ & 1.42E-04 & $1.49 \mathrm{E}-06$ & $3.11 \mathrm{E}-03$ & 2.67E-04 & $2.85 \mathrm{E}-05$ & $2.73 \mathrm{E}-04$ & $6.78 \mathrm{E}-03$ & $5.48 \mathrm{E}-02$ \\
\hline $40 \%$ & $6.19 \mathrm{E}-06$ & $8.60 \mathrm{E}-06$ & $2.46 \mathrm{E}-05$ & $1.44 \mathrm{E}-04$ & 1.53E-06 & 3.47E-03 & $2.72 \mathrm{E}-04$ & $3.08 \mathrm{E}-05$ & $2.91 \mathrm{E}-04$ & $6.92 \mathrm{E}-03$ & $5.88 \mathrm{E}-02$ \\
\hline $45 \%$ & $6.47 \mathrm{E}-06$ & $9.55 \mathrm{E}-06$ & $2.63 \mathrm{E}-05$ & $1.46 \mathrm{E}-04$ & $1.58 \mathrm{E}-06$ & $3.75 \mathrm{E}-03$ & $2.78 \mathrm{E}-04$ & $3.28 \mathrm{E}-05$ & 3.07E-04 & $7.12 \mathrm{E}-03$ & $6.28 \mathrm{E}-02$ \\
\hline $50 \%$ & $6.70 \mathrm{E}-06$ & $1.06 \mathrm{E}-05$ & $2.85 \mathrm{E}-05$ & $1.48 \mathrm{E}-04$ & 1.63E-06 & $4.06 \mathrm{E}-03$ & $2.85 \mathrm{E}-04$ & $3.51 \mathrm{E}-05$ & $3.26 \mathrm{E}-04$ & 7.27E-03 & $6.70 \mathrm{E}-02$ \\
\hline $55 \%$ & $6.99 \mathrm{E}-06$ & 1.16E-05 & $3.12 \mathrm{E}-05$ & $1.50 \mathrm{E}-04$ & $1.69 \mathrm{E}-06$ & $4.38 \mathrm{E}-03$ & $2.94 \mathrm{E}-04$ & $3.87 E-05$ & $3.41 \mathrm{E}-04$ & $7.49 \mathrm{E}-03$ & 7.07E-02 \\
\hline $60 \%$ & $7.46 \mathrm{E}-06$ & $1.26 \mathrm{E}-05$ & $3.36 \mathrm{E}-05$ & $1.52 \mathrm{E}-04$ & $1.75 \mathrm{E}-06$ & $4.79 \mathrm{E}-03$ & $3.01 \mathrm{E}-04$ & 4.07E-05 & $3.60 \mathrm{E}-04$ & 7.70E-03 & $7.45 \mathrm{E}-02$ \\
\hline $65 \%$ & $8.04 \mathrm{E}-06$ & $1.42 \mathrm{E}-05$ & $3.68 \mathrm{E}-05$ & $1.54 \mathrm{E}-04$ & $1.82 \mathrm{E}-06$ & $5.19 \mathrm{E}-03$ & $3.08 \mathrm{E}-04$ & $4.32 \mathrm{E}-05$ & $3.85 \mathrm{E}-04$ & $7.98 \mathrm{E}-03$ & $8.08 \mathrm{E}-02$ \\
\hline $70 \%$ & $8.50 \mathrm{E}-06$ & 1.62E-05 & 4.27E-05 & $1.57 \mathrm{E}-04$ & $1.95 \mathrm{E}-06$ & $5.63 \mathrm{E}-03$ & $3.21 \mathrm{E}-04$ & 4.63E-05 & $4.08 \mathrm{E}-04$ & 8.27E-03 & $8.58 \mathrm{E}-02$ \\
\hline $75 \%$ & $9.25 \mathrm{E}-06$ & $1.82 \mathrm{E}-05$ & $4.80 \mathrm{E}-05$ & $1.59 \mathrm{E}-04$ & $2.07 \mathrm{E}-06$ & $6.27 \mathrm{E}-03$ & $3.35 \mathrm{E}-04$ & $4.99 E-05$ & $4.39 \mathrm{E}-04$ & 8.67E-03 & $9.25 \mathrm{E}-02$ \\
\hline $80 \%$ & $1.03 \mathrm{E}-05$ & $2.14 \mathrm{E}-05$ & $5.60 \mathrm{E}-05$ & $1.63 \mathrm{E}-04$ & $2.25 \mathrm{E}-06$ & $6.95 \mathrm{E}-03$ & $3.48 \mathrm{E}-04$ & $5.63 E-05$ & $4.71 \mathrm{E}-04$ & $9.10 \mathrm{E}-03$ & $1.00 \mathrm{E}-01$ \\
\hline $85 \%$ & 1.15E-05 & $2.51 \mathrm{E}-05$ & 7.07E-05 & 1.69E-04 & $2.56 \mathrm{E}-06$ & 7.77E-03 & $3.69 \mathrm{E}-04$ & $6.48 \mathrm{E}-05$ & $5.13 \mathrm{E}-04$ & $9.84 \mathrm{E}-03$ & 1.07E-01 \\
\hline $90 \%$ & 1.37E-05 & $3.22 \mathrm{E}-05$ & $9.23 \mathrm{E}-05$ & 1.76E-04 & $3.13 \mathrm{E}-06$ & $8.86 \mathrm{E}-03$ & $3.98 \mathrm{E}-04$ & $7.95 \mathrm{E}-05$ & $5.80 \mathrm{E}-04$ & $1.09 \mathrm{E}-02$ & $1.25 \mathrm{E}-01$ \\
\hline $95 \%$ & $2.03 \mathrm{E}-05$ & $4.47 \mathrm{E}-05$ & $1.40 \mathrm{E}-04$ & $1.91 \mathrm{E}-04$ & $3.93 E-06$ & $1.07 \mathrm{E}-02$ & $4.57 \mathrm{E}-04$ & $9.82 \mathrm{E}-05$ & $6.99 \mathrm{E}-04$ & $1.35 \mathrm{E}-02$ & 1.44E-01 \\
\hline Maximum & $9.32 \mathrm{E}-05$ & 7.32E-04 & $1.73 \mathrm{E}-03$ & $3.47 \mathrm{E}-04$ & $5.44 \mathrm{E}-05$ & $1.85 \mathrm{E}-02$ & $1.61 \mathrm{E}-03$ & $3.29 \mathrm{E}-04$ & $1.49 \mathrm{E}-03$ & $3.08 \mathrm{E}-02$ & $3.71 \mathrm{E}-01$ \\
\hline
\end{tabular}


Table 6.2-10. Statistics for the ICRP 30-Based BDCFs for the Glacial Transition Climate, rem/yr per pCi/L (Continued)

\begin{tabular}{|c|c|c|c|c|c|c|c|c|c|c|c|}
\hline & $\begin{array}{r}\mathrm{Ra}-226 \\
+\mathrm{Pb}-210 \\
\end{array}$ & Ac-227 & Th-229 & Th-230 & Th-232 & $\mathrm{Pa}-231$ & U-232 & U-233 & U-234 & U-236 & U-238 \\
\hline Mean & $8.19 \mathrm{E}-02$ & $2.33 E-02$ & $2.53 \mathrm{E}-02$ & $2.23 \mathrm{E}-02$ & $3.30 \mathrm{E}-02$ & $7.58 \mathrm{E}-02$ & $4.38 \mathrm{E}-03$ & $1.40 \mathrm{E}-03$ & 9.57E-04 & $8.50 \mathrm{E}-04$ & $8.50 \mathrm{E}-04$ \\
\hline STD & $3.86 \mathrm{E}-02$ & $7.30 \mathrm{E}-03$ & $1.45 \mathrm{E}-02$ & $1.99 \mathrm{E}-02$ & 1.77E-02 & $5.04 \mathrm{E}-02$ & $1.68 \mathrm{E}-03$ & $1.70 \mathrm{E}-03$ & $8.52 \mathrm{E}-04$ & $7.22 \mathrm{E}-04$ & $7.11 \mathrm{E}-04$ \\
\hline Minimum & $1.04 \mathrm{E}-02$ & $1.27 \mathrm{E}-02$ & $4.50 \mathrm{E}-03$ & $8.89 \mathrm{E}-04$ & $5.63 \mathrm{E}-03$ & $9.74 \mathrm{E}-03$ & $1.70 \mathrm{E}-03$ & 2.27E-04 & $2.23 \mathrm{E}-04$ & $2.14 \mathrm{E}-04$ & $2.11 \mathrm{E}-04$ \\
\hline $5 \%$ & $3.51 \mathrm{E}-02$ & $1.48 \mathrm{E}-02$ & $9.60 \mathrm{E}-03$ & $4.25 \mathrm{E}-03$ & $1.29 \mathrm{E}-02$ & $2.38 \mathrm{E}-02$ & $2.28 \mathrm{E}-03$ & $2.84 \mathrm{E}-04$ & $2.84 \mathrm{E}-04$ & $2.64 \mathrm{E}-04$ & $2.66 \mathrm{E}-04$ \\
\hline $10 \%$ & 4.15E-02 & $1.57 \mathrm{E}-02$ & 1.14E-02 & $5.57 \mathrm{E}-03$ & $1.54 \mathrm{E}-02$ & $2.92 \mathrm{E}-02$ & $2.59 \mathrm{E}-03$ & $3.35 \mathrm{E}-04$ & 3.17E-04 & $2.93 \mathrm{E}-04$ & $2.96 \mathrm{E}-04$ \\
\hline $15 \%$ & 4.67E-02 & $1.65 \mathrm{E}-02$ & $1.28 \mathrm{E}-02$ & $6.49 \mathrm{E}-03$ & $1.73 \mathrm{E}-02$ & 3.33E-02 & $2.81 \mathrm{E}-03$ & $3.74 \mathrm{E}-04$ & $3.53 E-04$ & $3.23 \mathrm{E}-04$ & $3.24 \mathrm{E}-04$ \\
\hline $20 \%$ & $5.11 \mathrm{E}-02$ & $1.72 \mathrm{E}-02$ & $1.42 \mathrm{E}-02$ & $7.56 \mathrm{E}-03$ & $1.91 \mathrm{E}-02$ & 3.77E-02 & $3.00 \mathrm{E}-03$ & $4.21 \mathrm{E}-04$ & $3.82 \mathrm{E}-04$ & $3.51 \mathrm{E}-04$ & $3.53 E-04$ \\
\hline $25 \%$ & $5.49 \mathrm{E}-02$ & $1.79 \mathrm{E}-02$ & $1.55 \mathrm{E}-02$ & 8.62E-03 & $2.05 \mathrm{E}-02$ & 4.16E-02 & 3.17E-03 & $4.58 \mathrm{E}-04$ & 4.19E-04 & 3.87E-04 & $3.82 \mathrm{E}-04$ \\
\hline $30 \%$ & $5.86 \mathrm{E}-02$ & $1.85 \mathrm{E}-02$ & 1.67E-02 & $9.73 \mathrm{E}-03$ & $2.19 \mathrm{E}-02$ & 4.47E-02 & $3.36 \mathrm{E}-03$ & $5.03 \mathrm{E}-04$ & $4.51 \mathrm{E}-04$ & 4.19E-04 & 4.14E-04 \\
\hline $35 \%$ & $6.26 \mathrm{E}-02$ & $1.93 \mathrm{E}-02$ & $1.79 \mathrm{E}-02$ & $1.09 \mathrm{E}-02$ & $2.36 \mathrm{E}-02$ & $4.94 \mathrm{E}-02$ & $3.53 E-03$ & $5.51 \mathrm{E}-04$ & $4.89 \mathrm{E}-04$ & $4.52 \mathrm{E}-04$ & $4.49 \mathrm{E}-04$ \\
\hline $40 \%$ & $6.65 \mathrm{E}-02$ & $2.01 \mathrm{E}-02$ & $1.91 \mathrm{E}-02$ & $1.23 \mathrm{E}-02$ & $2.54 \mathrm{E}-02$ & $5.27 \mathrm{E}-02$ & $3.71 \mathrm{E}-03$ & $6.07 \mathrm{E}-04$ & $5.29 \mathrm{E}-04$ & 4.87E-04 & $4.89 \mathrm{E}-04$ \\
\hline $45 \%$ & $7.06 \mathrm{E}-02$ & $2.10 \mathrm{E}-02$ & $2.02 \mathrm{E}-02$ & 1.37E-02 & $2.71 \mathrm{E}-02$ & $5.64 \mathrm{E}-02$ & $3.88 \mathrm{E}-03$ & $6.83 \mathrm{E}-04$ & $5.85 \mathrm{E}-04$ & $5.35 \mathrm{E}-04$ & $5.44 \mathrm{E}-04$ \\
\hline $50 \%$ & $7.51 \mathrm{E}-02$ & $2.17 \mathrm{E}-02$ & $2.17 \mathrm{E}-02$ & $1.53 \mathrm{E}-02$ & 2.86E-02 & $6.09 \mathrm{E}-02$ & 4.06E-03 & 7.73E-04 & $6.48 \mathrm{E}-04$ & $5.93 \mathrm{E}-04$ & $5.99 E-04$ \\
\hline $55 \%$ & $7.85 \mathrm{E}-02$ & $2.25 \mathrm{E}-02$ & $2.31 \mathrm{E}-02$ & 1.76E-02 & $3.08 \mathrm{E}-02$ & $6.73 \mathrm{E}-02$ & $4.23 \mathrm{E}-03$ & $8.80 \mathrm{E}-04$ & 7.13E-04 & $6.54 \mathrm{E}-04$ & $6.57 \mathrm{E}-04$ \\
\hline $60 \%$ & $8.30 \mathrm{E}-02$ & $2.33 \mathrm{E}-02$ & $2.48 \mathrm{E}-02$ & $1.99 \mathrm{E}-02$ & $3.30 \mathrm{E}-02$ & $7.25 \mathrm{E}-02$ & 4.47E-03 & $1.01 \mathrm{E}-03$ & 7.95E-04 & $7.28 \mathrm{E}-04$ & 7.36E-04 \\
\hline $65 \%$ & 8.94E-02 & $2.44 \mathrm{E}-02$ & $2.65 \mathrm{E}-02$ & $2.27 \mathrm{E}-02$ & $3.50 \mathrm{E}-02$ & $7.90 \mathrm{E}-02$ & $4.70 \mathrm{E}-03$ & 1.16E-03 & $8.94 \mathrm{E}-04$ & $8.22 \mathrm{E}-04$ & $8.29 \mathrm{E}-04$ \\
\hline $70 \%$ & $9.50 \mathrm{E}-02$ & $2.56 \mathrm{E}-02$ & $2.88 \mathrm{E}-02$ & $2.56 \mathrm{E}-02$ & $3.73 E-02$ & $8.58 \mathrm{E}-02$ & $4.91 \mathrm{E}-03$ & $1.36 \mathrm{E}-03$ & $1.02 \mathrm{E}-03$ & $9.19 \mathrm{E}-04$ & $9.20 \mathrm{E}-04$ \\
\hline $75 \%$ & $1.01 \mathrm{E}-01$ & $2.70 \mathrm{E}-02$ & $3.08 \mathrm{E}-02$ & $2.96 \mathrm{E}-02$ & $4.05 \mathrm{E}-02$ & $9.39 \mathrm{E}-02$ & $5.15 \mathrm{E}-03$ & $1.57 \mathrm{E}-03$ & $1.19 \mathrm{E}-03$ & $1.05 \mathrm{E}-03$ & $1.07 \mathrm{E}-03$ \\
\hline $80 \%$ & $1.09 \mathrm{E}-01$ & $2.86 \mathrm{E}-02$ & $3.39 \mathrm{E}-02$ & $3.31 \mathrm{E}-02$ & $4.40 \mathrm{E}-02$ & $1.06 \mathrm{E}-01$ & $5.50 \mathrm{E}-03$ & $1.95 \mathrm{E}-03$ & 1.37E-03 & $1.19 \mathrm{E}-03$ & $1.18 \mathrm{E}-03$ \\
\hline $85 \%$ & 1.17E-01 & $3.05 \mathrm{E}-02$ & $3.77 \mathrm{E}-02$ & $3.93 \mathrm{E}-02$ & $4.91 \mathrm{E}-02$ & $1.21 \mathrm{E}-01$ & $5.93 \mathrm{E}-03$ & $2.40 \mathrm{E}-03$ & $1.63 \mathrm{E}-03$ & $1.44 \mathrm{E}-03$ & $1.41 \mathrm{E}-03$ \\
\hline $90 \%$ & 1.33E-01 & $3.33 \mathrm{E}-02$ & $4.25 \mathrm{E}-02$ & $4.79 \mathrm{E}-02$ & $5.78 \mathrm{E}-02$ & $1.42 \mathrm{E}-01$ & $6.56 \mathrm{E}-03$ & $3.26 \mathrm{E}-03$ & $1.98 \mathrm{E}-03$ & $1.73 \mathrm{E}-03$ & 1.76E-03 \\
\hline $95 \%$ & $1.53 \mathrm{E}-01$ & $3.71 \mathrm{E}-02$ & $5.70 \mathrm{E}-02$ & $6.39 \mathrm{E}-02$ & $6.63 \mathrm{E}-02$ & $1.81 \mathrm{E}-01$ & $7.54 \mathrm{E}-03$ & 4.62E-03 & $2.65 \mathrm{E}-03$ & $2.31 \mathrm{E}-03$ & $2.29 \mathrm{E}-03$ \\
\hline Maximum & $3.80 \mathrm{E}-01$ & 7.01E-02 & $9.81 \mathrm{E}-02$ & $1.18 \mathrm{E}-01$ & $1.12 \mathrm{E}-01$ & $3.91 \mathrm{E}-01$ & 1.37E-02 & 1.33E-02 & $6.27 \mathrm{E}-03$ & $5.36 \mathrm{E}-03$ & $5.02 E-03$ \\
\hline
\end{tabular}


Table 6.2-10. Statistics for the ICRP 30-Based BDCFs for the Glacial Transition Climate, rem/yr per | $\mathrm{pCi} / \mathrm{L}$ (Continued)

\begin{tabular}{|c|c|c|c|c|c|c|c|}
\hline & Np-237 & Pu-238 & Pu-239 & Pu-240 & Pu-242 & Am-241 & Am-243 \\
\hline Mean & $5.92 \mathrm{E}-03$ & $3.92 \mathrm{E}-03$ & 7.36E-03 & $7.20 \mathrm{E}-03$ & & $5.60 \mathrm{E}-03$ & 7.60E-03 \\
\hline STD & $2.05 \mathrm{E}-03$ & 8.86E-04 & $2.90 \mathrm{E}-03$ & $2.73 E-03$ & $2.78 \mathrm{E}-03$ & $1.62 \mathrm{E}-03$ & $3.14 \mathrm{E}-03$ \\
\hline Minimum & & & & $3.17 \mathrm{E}-03$ & & & $2.90 \mathrm{E}-03$ \\
\hline $5 \%$ & $4.00 \mathrm{E}-03$ & $2.98 \mathrm{E}-03$ & $4.27 \mathrm{E}-03$ & $4.24 \mathrm{E}-03$ & 4.06E-03 & $3.70 \mathrm{E}-03$ & $4.02 \mathrm{E}-03$ \\
\hline $10 \%$ & & & & & & & \\
\hline $15 \%$ & $4.35 \mathrm{E}-03$ & $3.19 \mathrm{E}-03$ & $4.79 \mathrm{E}-03$ & $4.82 \mathrm{E}-03$ & 4.62E-03 & $4.18 \mathrm{E}-03$ & $4.79 \mathrm{E}-03$ \\
\hline $20 \%$ & $4.49 \mathrm{E}-03$ & $3.28 \mathrm{E}-03$ & $5.05 \mathrm{E}-03$ & $5.05 \mathrm{E}-03$ & $4.86 \mathrm{E}-03$ & $4.34 \mathrm{E}-03$ & $5.08 \mathrm{E}-03$ \\
\hline $25 \%$ & $4.66 \mathrm{E}-03$ & $3.35 \mathrm{E}-03$ & $5.30 \mathrm{E}-03$ & $5.30 \mathrm{E}-03$ & $5.15 \mathrm{E}-03$ & $4.49 \mathrm{E}-03$ & $5.34 \mathrm{E}-03$ \\
\hline $30 \%$ & $4.77 \mathrm{E}-0$ & $3.43 \mathrm{E}-03$ & $5.55 \mathrm{E}-03$ & $54 \mathrm{E}-03$ & $5.36 \mathrm{E}-03$ & 4.67E-03 & $5.69 \mathrm{E}-03$ \\
\hline $35 \%$ & $4.94 \mathrm{E}-03$ & $3.49 \mathrm{E}-03$ & $5.82 \mathrm{E}-03$ & $5.75 \mathrm{E}-03$ & $5.60 \mathrm{E}-03$ & $4.81 \mathrm{E}-03$ & $5.98 \mathrm{E}-03$ \\
\hline $40 \%$ & $5.07 \mathrm{E}-03$ & $3.58 \mathrm{E}-03$ & $6.10 \mathrm{E}-03$ & $5.97 \mathrm{E}-03$ & $5.84 \mathrm{E}-03$ & $4.95 \mathrm{E}-03$ & $6.26 \mathrm{E}-03$ \\
\hline $45 \%$ & $5.20 \mathrm{E}-03$ & 3.63E-03 & $6.34 \mathrm{E}-03$ & $6.21 \mathrm{E}-03$ & $6.10 \mathrm{E}-03$ & $5.10 \mathrm{E}-03$ & $6.53 E-03$ \\
\hline $50 \%$ & $5.38 \mathrm{E}-03$ & $3.71 \mathrm{E}-03$ & $6.59 \mathrm{E}-03$ & $6.47 \mathrm{E}-03$ & $6.41 \mathrm{E}-03$ & $5.25 \mathrm{E}-03$ & $6.79 \mathrm{E}-03$ \\
\hline $55 \%$ & $5.56 \mathrm{E}-03$ & $3.79 \mathrm{E}-03$ & $6.89 \mathrm{E}-03$ & $6.77 \mathrm{E}-03$ & $6.67 \mathrm{E}-03$ & $5.38 \mathrm{E}-03$ & $7.20 \mathrm{E}-03$ \\
\hline $60 \%$ & $5.71 \mathrm{E}-03$ & $3.89 \mathrm{E}-03$ & $7.18 \mathrm{E}-03$ & $7.11 \mathrm{E}-03$ & $6.99 \mathrm{E}-03$ & $5.53 \mathrm{E}-03$ & 7.63E-03 \\
\hline $65 \%$ & $5.90 \mathrm{E}-03$ & $4.00 \mathrm{E}-03$ & $7.63 \mathrm{E}-03$ & $7.48 \mathrm{E}-03$ & $7.40 \mathrm{E}-03$ & $5.75 \mathrm{E}-03$ & $8.05 \mathrm{E}-03$ \\
\hline $70 \%$ & $6.15 \mathrm{E}-03$ & $4.12 \mathrm{E}-03$ & $8.06 \mathrm{E}-03$ & 7.84E-03 & $7.83 E-03$ & $5.96 \mathrm{E}-03$ & $8.52 E-03$ \\
\hline $75 \%$ & $6.55 \mathrm{E}-03$ & $4.28 \mathrm{E}-03$ & $8.49 \mathrm{E}-03$ & $8.38 \mathrm{E}-03$ & $8.32 \mathrm{E}-03$ & $6.26 \mathrm{E}-03$ & $9.05 E-03$ \\
\hline $80 \%$ & $6.92 \mathrm{E}-03$ & $4.44 \mathrm{E}-03$ & $9.16 \mathrm{E}-03$ & $8.91 \mathrm{E}-03$ & $8.80 \mathrm{E}-03$ & $6.67 \mathrm{E}-03$ & $9.76 \mathrm{E}-03$ \\
\hline $85 \%$ & $7.34 \mathrm{E}-03$ & $4.65 \mathrm{E}-03$ & $1.01 \mathrm{E}-02$ & $9.82 \mathrm{E}-03$ & $9.56 \mathrm{E}-03$ & $7.06 \mathrm{E}-03$ & $1.06 \mathrm{E}-02$ \\
\hline $90 \%$ & $8.17 \mathrm{E}-03$ & $4.99 \mathrm{E}-03$ & 1.13E-02 & $1.09 \mathrm{E}-02$ & $1.08 \mathrm{E}-02$ & $7.69 \mathrm{E}-03$ & 1.17E-02 \\
\hline $95 \%$ & $9.89 \mathrm{E}-03$ & $5.55 \mathrm{E}-03$ & $1.31 \mathrm{E}-02$ & $1.25 \mathrm{E}-02$ & $1.28 \mathrm{E}-02$ & $8.79 \mathrm{E}-03$ & 1.36E-02 \\
\hline Maximum & $2.34 \mathrm{E}-02$ & $1.25 \mathrm{E}-02$ & $2.36 \mathrm{E}-02$ & $2.40 \mathrm{E}-02$ & $2.05 \mathrm{E}-02$ & $1.52 \mathrm{E}-02$ & $3.10 \mathrm{E}-02$ \\
\hline
\end{tabular}

Source: MO0407SPAINEXI.002 [DIRS 170597]; MO0407SPASRPBM.002 [DIRS 170755]; MO0306MWDBGSMF.001 [DIRS 163816]; MO0403SPAAEIBM.002 [DIRS 169392]; MO0407SPACRBSM.002 [DIRS 170677]; MO0406SPAETPBM.002 [DIRS 170150].

NOTE: See Excel file GW BDCF Realizations_MC and FC_Rev 4.xls in Appendix B for details of calculations.

STD = standard deviation . 
Table 6.2-11. Statistics for the ICRP 72-Based BDCFs for the Present-Day Climate, rem/yr per pCi/L

\begin{tabular}{|c|c|c|c|c|c|c|c|c|c|c|c|}
\hline & C-14 & $\mathrm{Cl}-36$ & Se-79 & Sr-90 & Tc-99 & Sn-126 & I-129 & Cs-135 & Cs-137 & $\mathrm{Pb}-210$ & $\mathrm{Ra}-226$ \\
\hline Mean & $9.35 \mathrm{E}-06$ & 2.35E-05 & 7.65E-05 & 1.24E-04 & $3.74 \mathrm{E}-06$ & $5.92 \mathrm{E}-03$ & 4.95E-04 & 5.87E-05 & 4.59E-04 & 8.74E-03 & 5.32E-02 \\
\hline STD & $6.86 \mathrm{E}-06$ & 4.81E-05 & 1.30E-04 & $1.93 \mathrm{E}-05$ & 3.87E-06 & $3.69 \mathrm{E}-03$ & 1.67E-04 & 4.20E-05 & 2.19E-04 & $3.40 \mathrm{E}-03$ & $2.56 \mathrm{E}-02$ \\
\hline Minimum & 4.27E-06 & 3.91E-06 & 1.20E-05 & 9.17E-05 & $1.94 \mathrm{E}-06$ & $1.88 \mathrm{E}-04$ & 3.26E-04 & 1.23E-05 & 1.44E-04 & 5.59E-03 & 1.66E-03 \\
\hline $5 \%$ & 5.06E-06 & 5.93E-06 & 1.85E-05 & 1.02E-04 & 2.18E-06 & 1.05E-03 & $3.58 \mathrm{E}-04$ & 2.01E-05 & 2.21E-04 & 6.06E-03 & $2.19 \mathrm{E}-02$ \\
\hline $10 \%$ & 5.35E-06 & $6.58 \mathrm{E}-06$ & 2.18E-05 & 1.04E-04 & $2.28 \mathrm{E}-06$ & 1.73E-03 & $3.69 \mathrm{E}-04$ & 2.34E-05 & 2.46E-04 & $6.25 \mathrm{E}-03$ & $2.63 \mathrm{E}-02$ \\
\hline $15 \%$ & $5.62 \mathrm{E}-06$ & $7.42 \mathrm{E}-06$ & $2.45 \mathrm{E}-05$ & 1.07E-04 & 2.36E-06 & $2.40 \mathrm{E}-03$ & $3.81 \mathrm{E}-04$ & $2.68 \mathrm{E}-05$ & 2.69E-04 & $6.39 \mathrm{E}-03$ & $2.95 \mathrm{E}-02$ \\
\hline $20 \%$ & $5.82 \mathrm{E}-06$ & 8.04E-06 & 2.71E-05 & 1.09E-04 & 2.43E-06 & 2.95E-03 & 3.89E-04 & 2.98E-05 & 2.87E-04 & 6.54E-03 & $3.24 \mathrm{E}-02$ \\
\hline $25 \%$ & 6.07E-06 & 8.80E-06 & 2.97E-05 & 1.11E-04 & $2.51 \mathrm{E}-06$ & 3.37E-03 & 3.98E-04 & $3.28 \mathrm{E}-05$ & 3.10E-04 & 6.69E-03 & $3.48 \mathrm{E}-02$ \\
\hline $30 \%$ & 6.26E-06 & $9.84 \mathrm{E}-06$ & $3.29 \mathrm{E}-05$ & 1.13E-04 & 2.60E-06 & $3.72 \mathrm{E}-03$ & $4.09 \mathrm{E}-04$ & $3.54 \mathrm{E}-05$ & 3.30E-04 & $6.88 \mathrm{E}-03$ & $3.73 \mathrm{E}-02$ \\
\hline $35 \%$ & $6.52 \mathrm{E}-06$ & $1.08 \mathrm{E}-05$ & $3.49 \mathrm{E}-05$ & 1.14E-04 & 2.69E-06 & $3.98 \mathrm{E}-03$ & $4.18 \mathrm{E}-04$ & $3.84 \mathrm{E}-05$ & $3.46 \mathrm{E}-04$ & 7.01E-03 & $4.00 \mathrm{E}-02$ \\
\hline $40 \%$ & $6.73 \mathrm{E}-06$ & 1.20E-05 & 3.77E-05 & 1.16E-04 & 2.77E-06 & 4.27E-03 & 4.28E-04 & 4.13E-05 & 3.67E-04 & 7.23E-03 & $4.28 \mathrm{E}-02$ \\
\hline $45 \%$ & 7.02E-06 & 1.32E-05 & 4.07E-05 & 1.18E-04 & 2.87E-06 & 4.76E-03 & 4.39E-04 & $4.40 \mathrm{E}-05$ & 3.83E-04 & 7.46E-03 & 4.54E-02 \\
\hline $50 \%$ & 7.24E-06 & $1.45 \mathrm{E}-05$ & $4.42 \mathrm{E}-05$ & $1.20 \mathrm{E}-04$ & 2.96E-06 & $5.19 \mathrm{E}-03$ & $4.54 \mathrm{E}-04$ & 4.79E-05 & $4.05 \mathrm{E}-04$ & 7.69E-03 & 4.85E-02 \\
\hline $55 \%$ & 7.57E-06 & 1.62E-05 & 4.75E-05 & 1.22E-04 & $3.06 \mathrm{E}-06$ & $5.60 \mathrm{E}-03$ & 4.65E-04 & 5.12E-05 & 4.29E-04 & 7.93E-03 & $5.11 \mathrm{E}-02$ \\
\hline $60 \%$ & 8.00E-06 & 1.78E-05 & 5.25E-05 & 1.23E-04 & 3.19E-06 & 6.06E-03 & 4.85E-04 & 5.45E-05 & 4.52E-04 & 8.19E-03 & $5.48 \mathrm{E}-02$ \\
\hline $65 \%$ & 8.59E-06 & 2.00E-05 & 5.87E-05 & $1.25 \mathrm{E}-04$ & 3.36E-06 & $6.52 \mathrm{E}-03$ & 4.99E-04 & 5.86E-05 & 4.84E-04 & 8.54E-03 & $5.86 \mathrm{E}-02$ \\
\hline $70 \%$ & $9.10 \mathrm{E}-06$ & $2.20 \mathrm{E}-05$ & $6.59 \mathrm{E}-05$ & $1.29 \mathrm{E}-04$ & $3.56 \mathrm{E}-06$ & 7.26E-03 & $5.16 \mathrm{E}-04$ & $6.41 \mathrm{E}-05$ & 5.11E-04 & 8.88E-03 & $6.23 \mathrm{E}-02$ \\
\hline $75 \%$ & 9.82E-06 & $2.52 \mathrm{E}-05$ & 7.60E-05 & 1.32E-04 & $3.80 \mathrm{E}-06$ & 7.99E-03 & 5.36E-04 & 6.99E-05 & $5.45 \mathrm{E}-04$ & 9.33E-03 & 6.69E-02 \\
\hline $80 \%$ & $1.09 \mathrm{E}-05$ & $2.88 \mathrm{E}-05$ & 8.91E-05 & 1.35E-04 & 4.13E-06 & 8.76E-03 & $5.63 \mathrm{E}-04$ & $7.74 \mathrm{E}-05$ & $5.89 \mathrm{E}-04$ & $9.99 \mathrm{E}-03$ & 7.21E-02 \\
\hline $85 \%$ & 1.23E-05 & 3.31E-05 & $1.11 \mathrm{E}-04$ & $1.40 \mathrm{E}-04$ & 4.67E-06 & 9.67E-03 & $5.98 \mathrm{E}-04$ & $9.21 \mathrm{E}-05$ & $6.46 \mathrm{E}-04$ & 1.08E-02 & 7.93E-02 \\
\hline $90 \%$ & 1.44E-05 & 4.22E-05 & 1.47E-04 & 1.46E-04 & 5.64E-06 & 1.09E-02 & 6.62E-04 & $1.09 \mathrm{E}-04$ & 7.19E-04 & 1.22E-02 & 8.84E-02 \\
\hline $95 \%$ & 2.11E-05 & 6.07E-05 & 2.06E-04 & 1.61E-04 & 7.27E-06 & 1.33E-02 & 7.62E-04 & 1.39E-04 & 8.87E-04 & $1.55 \mathrm{E}-02$ & 9.97E-02 \\
\hline Maximum & 9.61E-05 & 1.01E-03 & 2.33E-03 & 2.84E-04 & $1.00 \mathrm{E}-04$ & 1.90E-02 & 2.91E-03 & $5.00 \mathrm{E}-04$ & 1.62E-03 & 3.53E-02 & $2.21 \mathrm{E}-01$ \\
\hline
\end{tabular}




\begin{tabular}{|c|c|c|c|c|c|c|c|c|c|c|c|}
\hline & $\begin{array}{r}\mathrm{Ra}-226 \\
+\mathrm{Pb}-210\end{array}$ & Ac-227 & Th-229 & Th-230 & Th-232 & Pa-231 & $\mathrm{U}-232$ & U-233 & U-234 & U-236 & U-238 \\
\hline Mean & 6.19E-02 & $9.65 \mathrm{E}-03$ & $1.60 \mathrm{E}-02$ & 1.92E-02 & 2.38E-02 & 3.33E-02 & $3.21 \mathrm{E}-03$ & 7.24E-04 & 4.68E-04 & 3.96E-04 & 4.26E-04 \\
\hline STD & 2.60E-02 & 3.38E-03 & 8.37E-03 & $1.52 \mathrm{E}-02$ & 1.07E-02 & $2.21 \mathrm{E}-02$ & $1.02 \mathrm{E}-03$ & 8.30E-04 & $3.60 \mathrm{E}-04$ & 2.63E-04 & 2.91E-04 \\
\hline Minimum & 1.03E-02 & 4.18E-03 & $2.58 \mathrm{E}-03$ & 1.13E-03 & 4.72E-03 & 2.62E-03 & 1.46E-03 & 1.49E-04 & 1.42E-04 & 1.36E-04 & 1.41E-04 \\
\hline $5 \%$ & 2.91E-02 & $5.42 \mathrm{E}-03$ & $6.40 \mathrm{E}-03$ & 4.63E-03 & 1.07E-02 & $1.01 \mathrm{E}-02$ & $1.86 \mathrm{E}-03$ & $1.88 \mathrm{E}-04$ & $1.78 \mathrm{E}-04$ & 1.69E-04 & 1.74E-04 \\
\hline $10 \%$ & 3.43E-02 & $6.00 \mathrm{E}-03$ & 7.47E-03 & 5.86E-03 & 1.25E-02 & $1.20 \mathrm{E}-02$ & 2.04E-03 & $2.10 \mathrm{E}-04$ & $1.95 \mathrm{E}-04$ & 1.84E-04 & 1.87E-04 \\
\hline $15 \%$ & 3.77E-02 & $6.35 \mathrm{E}-03$ & $8.53 \mathrm{E}-03$ & $6.87 \mathrm{E}-03$ & $1.41 \mathrm{E}-02$ & $1.45 \mathrm{E}-02$ & $2.21 \mathrm{E}-03$ & 2.35E-04 & 2.15E-04 & 2.01E-04 & 2.07E-04 \\
\hline $20 \%$ & 4.07E-02 & $6.77 \mathrm{E}-03$ & $9.45 \mathrm{E}-03$ & 7.71E-03 & 1.52E-02 & 1.63E-02 & 2.36E-03 & $2.50 \mathrm{E}-04$ & $2.28 \mathrm{E}-04$ & 2.13E-04 & 2.20E-04 \\
\hline $25 \%$ & 4.32E-02 & $7.18 \mathrm{E}-03$ & $1.02 \mathrm{E}-02$ & $8.50 \mathrm{E}-03$ & $1.61 \mathrm{E}-02$ & $1.81 \mathrm{E}-02$ & $2.45 \mathrm{E}-03$ & 2.64E-04 & 2.42E-04 & 2.26E-04 & $2.29 \mathrm{E}-04$ \\
\hline $30 \%$ & 4.57E-02 & $7.50 \mathrm{E}-03$ & 1.10E-02 & $9.41 \mathrm{E}-03$ & 1.73E-02 & 2.01E-02 & 2.57E-03 & 2.83E-04 & 2.53E-04 & $2.35 \mathrm{E}-04$ & 2.40E-04 \\
\hline $35 \%$ & 4.84E-02 & 7.87E-03 & 1.17E-02 & 1.04E-02 & $1.81 \mathrm{E}-02$ & 2.16E-02 & $2.70 \mathrm{E}-03$ & 3.07E-04 & $2.68 \mathrm{E}-04$ & $2.48 \mathrm{E}-04$ & 2.53E-04 \\
\hline $40 \%$ & $5.10 \mathrm{E}-02$ & $8.25 \mathrm{E}-03$ & $1.26 \mathrm{E}-02$ & 1.16E-02 & $1.92 \mathrm{E}-02$ & $2.35 \mathrm{E}-02$ & $2.83 \mathrm{E}-03$ & 3.32E-04 & 2.92E-04 & 2.66E-04 & 2.75E-04 \\
\hline $45 \%$ & 5.39E-02 & $8.70 \mathrm{E}-03$ & $1.34 \mathrm{E}-02$ & $1.30 \mathrm{E}-02$ & $2.04 \mathrm{E}-02$ & $2.50 \mathrm{E}-02$ & $2.93 \mathrm{E}-03$ & $3.63 \mathrm{E}-04$ & 3.11E-04 & $2.84 \mathrm{E}-04$ & 2.93E-04 \\
\hline $50 \%$ & $5.65 \mathrm{E}-02$ & $9.14 \mathrm{E}-03$ & $1.43 \mathrm{E}-02$ & 1.44E-02 & 2.13E-02 & $2.73 \mathrm{E}-02$ & $3.06 \mathrm{E}-03$ & $4.14 \mathrm{E}-04$ & 3.37E-04 & 3.01E-04 & 3.21E-04 \\
\hline $55 \%$ & $6.00 \mathrm{E}-02$ & $9.61 \mathrm{E}-03$ & $1.50 \mathrm{E}-02$ & $1.58 \mathrm{E}-02$ & $2.29 \mathrm{E}-02$ & $2.93 \mathrm{E}-02$ & $3.17 \mathrm{E}-03$ & $4.58 \mathrm{E}-04$ & 3.63E-04 & $3.29 \mathrm{E}-04$ & $3.51 \mathrm{E}-04$ \\
\hline $60 \%$ & $6.38 \mathrm{E}-02$ & $1.00 \mathrm{E}-02$ & $1.58 \mathrm{E}-02$ & $1.74 \mathrm{E}-02$ & 2.39E-02 & 3.23E-02 & $3.32 \mathrm{E}-03$ & $5.23 \mathrm{E}-04$ & 3.96E-04 & 3.54E-04 & 3.83E-04 \\
\hline $65 \%$ & $6.76 \mathrm{E}-02$ & $1.05 \mathrm{E}-02$ & 1.69E-02 & 1.96E-02 & $2.57 \mathrm{E}-02$ & $3.51 \mathrm{E}-02$ & $3.44 \mathrm{E}-03$ & $5.95 \mathrm{E}-04$ & 4.42E-04 & 3.85E-04 & 4.20E-04 \\
\hline $70 \%$ & $7.18 \mathrm{E}-02$ & $1.09 \mathrm{E}-02$ & $1.81 \mathrm{E}-02$ & 2.21E-02 & $2.74 \mathrm{E}-02$ & $3.81 \mathrm{E}-02$ & $3.59 \mathrm{E}-03$ & 6.96E-04 & 4.92E-04 & 4.23E-04 & 4.71E-04 \\
\hline $75 \%$ & 7.62E-02 & 1.15E-02 & $1.95 \mathrm{E}-02$ & $2.48 \mathrm{E}-02$ & $2.90 \mathrm{E}-02$ & 4.14E-02 & 3.77E-03 & $8.11 \mathrm{E}-04$ & $5.49 \mathrm{E}-04$ & 4.70E-04 & $5.18 \mathrm{E}-04$ \\
\hline $80 \%$ & 8.18E-02 & $1.21 \mathrm{E}-02$ & 2.12E-02 & 2.86E-02 & 3.14E-02 & 4.76E-02 & 3.97E-03 & $9.79 \mathrm{E}-04$ & $6.47 \mathrm{E}-04$ & 5.33E-04 & 5.97E-04 \\
\hline $85 \%$ & $8.87 \mathrm{E}-02$ & $1.28 \mathrm{E}-02$ & $2.36 \mathrm{E}-02$ & 3.25E-02 & 3.53E-02 & 5.37E-02 & 4.24E-03 & $1.20 \mathrm{E}-03$ & 7.38E-04 & $6.06 \mathrm{E}-04$ & $6.86 \mathrm{E}-04$ \\
\hline $90 \%$ & $9.72 \mathrm{E}-02$ & $1.40 \mathrm{E}-02$ & 2.67E-02 & 3.90E-02 & 3.87E-02 & $6.45 \mathrm{E}-02$ & $4.50 \mathrm{E}-03$ & $1.62 \mathrm{E}-03$ & $9.38 \mathrm{E}-04$ & 7.44E-04 & $8.12 \mathrm{E}-04$ \\
\hline $95 \%$ & 1.10E-01 & $1.57 \mathrm{E}-02$ & $3.24 \mathrm{E}-02$ & 5.31E-02 & 4.49E-02 & 7.70E-02 & $5.17 \mathrm{E}-03$ & $2.41 \mathrm{E}-03$ & 1.19E-03 & $9.13 \mathrm{E}-04$ & 1.02E-03 \\
\hline Maximum & 2.31E-01 & $3.40 \mathrm{E}-02$ & $5.46 \mathrm{E}-02$ & 9.04E-02 & 6.71E-02 & 1.87E-01 & 7.47E-03 & 6.11E-03 & $2.58 \mathrm{E}-03$ & 1.92E-03 & 1.97E-03 \\
\hline
\end{tabular}


Table 6.2-11. Statistics for the ICRP 72-Based BDCFs for the Present-Day Climate, rem/yr per pCi/L (Continued)

\begin{tabular}{|c|c|c|c|c|c|c|c|}
\hline & Np-237 & Pu-238 & Pu-239 & $\mathrm{Pu}-240$ & $\mathrm{Pu}-242$ & Am-241 & Am-243 \\
\hline Mean & $1.45 \mathrm{E}-03$ & $2.59 \mathrm{E}-03$ & 6.83E-03 & 6.60E-03 & 6.38E-03 & $3.47 \mathrm{E}-03$ & $5.70 \mathrm{E}-03$ \\
\hline STD & $8.80 \mathrm{E}-04$ & $9.21 \mathrm{E}-04$ & 3.69E-03 & $3.45 \mathrm{E}-03$ & $3.47 \mathrm{E}-03$ & $1.58 \mathrm{E}-03$ & $3.33 \mathrm{E}-03$ \\
\hline Minimum & $3.80 \mathrm{E}-04$ & $9.21 \mathrm{E}-04$ & 1.63E-03 & $1.61 \mathrm{E}-03$ & $1.54 \mathrm{E}-03$ & 7.01E-04 & 7.05E-04 \\
\hline $5 \%$ & 5.72E-04 & 1.43E-03 & 2.79E-03 & $2.78 \mathrm{E}-03$ & 2.61E-03 & $1.52 \mathrm{E}-03$ & 1.91E-03 \\
\hline $10 \%$ & $6.81 \mathrm{E}-04$ & $1.59 \mathrm{E}-03$ & 3.27E-03 & $3.21 \mathrm{E}-03$ & 3.04E-03 & $1.84 \mathrm{E}-03$ & $2.38 \mathrm{E}-03$ \\
\hline $15 \%$ & $7.60 \mathrm{E}-04$ & 1.71E-03 & 3.62E-03 & 3.57E-03 & 3.37E-03 & $2.04 \mathrm{E}-03$ & $2.68 \mathrm{E}-03$ \\
\hline $20 \%$ & $8.33 E-04$ & 1.83E-03 & $3.93 \mathrm{E}-03$ & 3.87E-03 & $3.66 \mathrm{E}-03$ & $2.22 \mathrm{E}-03$ & $3.08 \mathrm{E}-03$ \\
\hline $25 \%$ & 8.79E-04 & $1.93 \mathrm{E}-03$ & 4.24E-03 & 4.18E-03 & $3.94 \mathrm{E}-03$ & 2.37E-03 & 3.45E-03 \\
\hline $30 \%$ & 9.53E-04 & 2.03E-03 & 4.52E-03 & 4.43E-03 & 4.23E-03 & $2.56 \mathrm{E}-03$ & 3.67E-03 \\
\hline $35 \%$ & 1.00E-03 & $2.13 E-03$ & 4.84E-03 & 4.77E-03 & 4.53E-03 & $2.71 \mathrm{E}-03$ & 3.99E-03 \\
\hline $40 \%$ & 1.08E-03 & $2.22 \mathrm{E}-03$ & $5.17 \mathrm{E}-03$ & $5.06 \mathrm{E}-03$ & $4.81 \mathrm{E}-03$ & $2.86 \mathrm{E}-03$ & $4.23 \mathrm{E}-03$ \\
\hline $45 \%$ & 1.14E-03 & $2.32 \mathrm{E}-03$ & 5.57E-03 & $5.43 E-03$ & $5.18 \mathrm{E}-03$ & $3.03 E-03$ & $4.51 \mathrm{E}-03$ \\
\hline $50 \%$ & $1.21 \mathrm{E}-03$ & $2.42 \mathrm{E}-03$ & $5.92 \mathrm{E}-03$ & $5.75 \mathrm{E}-03$ & $5.54 \mathrm{E}-03$ & 3.19E-03 & 4.92E-03 \\
\hline $55 \%$ & 1.30E-03 & $2.52 \mathrm{E}-03$ & $6.26 \mathrm{E}-03$ & $6.10 \mathrm{E}-03$ & $5.86 \mathrm{E}-03$ & $3.33 \mathrm{E}-03$ & $5.31 \mathrm{E}-03$ \\
\hline $60 \%$ & $1.38 \mathrm{E}-03$ & $2.65 \mathrm{E}-03$ & $6.62 \mathrm{E}-03$ & $6.45 \mathrm{E}-03$ & $6.19 \mathrm{E}-03$ & $3.53 \mathrm{E}-03$ & $5.65 \mathrm{E}-03$ \\
\hline $65 \%$ & $1.48 \mathrm{E}-03$ & $2.76 \mathrm{E}-03$ & $7.13 \mathrm{E}-03$ & $6.89 \mathrm{E}-03$ & $6.65 \mathrm{E}-03$ & 3.67E-03 & $6.09 \mathrm{E}-03$ \\
\hline $70 \%$ & 1.59E-03 & $2.89 \mathrm{E}-03$ & $7.78 \mathrm{E}-03$ & $7.53 \mathrm{E}-03$ & $7.24 \mathrm{E}-03$ & $3.86 \mathrm{E}-03$ & $6.59 \mathrm{E}-03$ \\
\hline $75 \%$ & 1.75E-03 & 3.06E-03 & $8.41 \mathrm{E}-03$ & $8.08 \mathrm{E}-03$ & $7.86 \mathrm{E}-03$ & 4.17E-03 & 7.16E-03 \\
\hline $80 \%$ & $1.92 \mathrm{E}-03$ & 3.23E-03 & $9.09 \mathrm{E}-03$ & 8.73E-03 & $8.52 \mathrm{E}-03$ & $4.48 \mathrm{E}-03$ & 7.95E-03 \\
\hline $85 \%$ & $2.08 \mathrm{E}-03$ & $3.46 \mathrm{E}-03$ & 1.02E-02 & $9.66 \mathrm{E}-03$ & 9.54E-03 & 4.84E-03 & $8.89 \mathrm{E}-03$ \\
\hline $90 \%$ & $2.45 \mathrm{E}-03$ & 3.81E-03 & 1.17E-02 & 1.10E-02 & 1.09E-02 & $5.43 \mathrm{E}-03$ & 9.94E-03 \\
\hline $95 \%$ & $3.21 \mathrm{E}-03$ & 4.39E-03 & 1.43E-02 & 1.37E-02 & 1.34E-02 & 6.67E-03 & 1.19E-02 \\
\hline Maximum & $6.52 \mathrm{E}-03$ & 7.55E-03 & $2.49 \mathrm{E}-02$ & 2.30E-02 & $2.36 \mathrm{E}-02$ & 1.19E-02 & $2.68 \mathrm{E}-02$ \\
\hline \multicolumn{8}{|l|}{ Source: } \\
\hline NOTE: & $\begin{array}{l}\text { See Excel file } \\
\text { calculations. }\end{array}$ & $W B D C F R$ & lizations_M & and $F C \_R E$ & & ndix B for & \\
\hline
\end{tabular}


Table 6.2-12. Statistics for the ICRP 72-Based BDCFs for the Monsoon Climate, rem/yr per pCi/L

\begin{tabular}{|c|c|c|c|c|c|c|c|c|c|c|c|}
\hline & C-14 & $\mathrm{Cl}-36$ & Se-79 & Sr-90 & Tc-99 & Sn-126 & I-129 & Cs-135 & Cs-137 & $\mathrm{Pb}-210$ & Ra-226 \\
\hline Mean & 9.07E-06 & 2.06E-05 & $6.31 \mathrm{E}-05$ & 1.15E-04 & $3.45 \mathrm{E}-06$ & 4.64E-03 & 4.62E-04 & 4.76E-05 & $3.68 \mathrm{E}-04$ & $8.00 \mathrm{E}-03$ & 4.26E-02 \\
\hline STD & $6.85 \mathrm{E}-06$ & $4.24 \mathrm{E}-05$ & $1.01 \mathrm{E}-04$ & 1.67E-05 & $3.42 \mathrm{E}-06$ & $3.01 \mathrm{E}-03$ & $1.38 \mathrm{E}-04$ & $3.38 \mathrm{E}-05$ & $1.84 \mathrm{E}-04$ & 2.85E-03 & 2.17E-02 \\
\hline Minimum & $3.98 \mathrm{E}-06$ & $3.59 \mathrm{E}-06$ & $1.09 \mathrm{E}-05$ & $8.93 \mathrm{E}-05$ & 1.87E-06 & 1.49E-04 & $3.19 \mathrm{E}-04$ & $9.96 \mathrm{E}-06$ & $1.20 \mathrm{E}-04$ & $5.43 \mathrm{E}-03$ & $1.52 \mathrm{E}-03$ \\
\hline $5 \%$ & 4.77E-06 & 5.18E-06 & 1.64E-05 & $9.74 \mathrm{E}-05$ & 2.07E-06 & 8.32E-04 & $3.45 \mathrm{E}-04$ & 1.67E-05 & 1.71E-04 & $5.86 \mathrm{E}-03$ & 1.61E-02 \\
\hline $10 \%$ & 5.06E-06 & 5.89E-06 & $1.89 \mathrm{E}-05$ & 9.97E-05 & 2.16E-06 & $1.39 \mathrm{E}-03$ & 3.57E-04 & 1.95E-05 & 1.92E-04 & $6.01 \mathrm{E}-03$ & 2.01E-02 \\
\hline $15 \%$ & $5.35 \mathrm{E}-06$ & $6.52 \mathrm{E}-06$ & 2.08E-05 & $1.01 \mathrm{E}-04$ & $2.23 \mathrm{E}-06$ & $1.84 \mathrm{E}-03$ & $3.64 \mathrm{E}-04$ & $2.21 \mathrm{E}-05$ & 2.12E-04 & $6.12 \mathrm{E}-03$ & 2.25E-02 \\
\hline $20 \%$ & $5.58 \mathrm{E}-06$ & 7.07E-06 & $2.28 \mathrm{E}-05$ & $1.02 \mathrm{E}-04$ & $2.28 \mathrm{E}-06$ & $2.27 \mathrm{E}-03$ & 3.73E-04 & $2.40 \mathrm{E}-05$ & 2.27E-04 & $6.24 \mathrm{E}-03$ & 2.45E-02 \\
\hline $25 \%$ & $5.79 \mathrm{E}-06$ & 7.63E-06 & 2.53E-05 & $1.04 \mathrm{E}-04$ & 2.35E-06 & $2.59 \mathrm{E}-03$ & $3.82 \mathrm{E}-04$ & 2.63E-05 & $2.41 \mathrm{E}-04$ & 6.37E-03 & 2.65E-02 \\
\hline $30 \%$ & $6.00 \mathrm{E}-06$ & $8.46 \mathrm{E}-06$ & $2.75 \mathrm{E}-05$ & $1.06 \mathrm{E}-04$ & $2.41 \mathrm{E}-06$ & $2.80 \mathrm{E}-03$ & $3.90 \mathrm{E}-04$ & $2.82 \mathrm{E}-05$ & $2.54 \mathrm{E}-04$ & $6.50 \mathrm{E}-03$ & 2.92E-02 \\
\hline $35 \%$ & $6.20 \mathrm{E}-06$ & $9.15 \mathrm{E}-06$ & 2.93E-05 & 1.07E-04 & $2.48 \mathrm{E}-06$ & $3.02 \mathrm{E}-03$ & $3.98 \mathrm{E}-04$ & 3.05E-05 & $2.72 \mathrm{E}-04$ & $6.60 \mathrm{E}-03$ & 3.07E-02 \\
\hline $40 \%$ & $6.51 \mathrm{E}-06$ & $1.01 \mathrm{E}-05$ & $3.18 \mathrm{E}-05$ & $1.09 \mathrm{E}-04$ & 2.57E-06 & 3.36E-03 & 4.07E-04 & $3.29 \mathrm{E}-05$ & $2.93 \mathrm{E}-04$ & $6.78 \mathrm{E}-03$ & 3.33E-02 \\
\hline $45 \%$ & $6.77 \mathrm{E}-06$ & $1.09 \mathrm{E}-05$ & $3.36 \mathrm{E}-05$ & 1.10E-04 & 2.66E-06 & $3.58 \mathrm{E}-03$ & 4.15E-04 & $3.56 \mathrm{E}-05$ & $3.10 \mathrm{E}-04$ & $6.93 \mathrm{E}-03$ & $3.58 \mathrm{E}-02$ \\
\hline $50 \%$ & $7.00 \mathrm{E}-06$ & $1.20 \mathrm{E}-05$ & $3.68 \mathrm{E}-05$ & $1.11 \mathrm{E}-04$ & 2.73E-06 & 3.93E-03 & 4.27E-04 & 3.82E-05 & 3.28E-04 & 7.09E-03 & $3.85 \mathrm{E}-02$ \\
\hline $55 \%$ & $7.29 \mathrm{E}-06$ & $1.35 \mathrm{E}-05$ & 4.01E-05 & 1.13E-04 & $2.82 \mathrm{E}-06$ & 4.22E-03 & 4.40E-04 & 4.13E-05 & 3.45E-04 & 7.34E-03 & 4.16E-02 \\
\hline $60 \%$ & 7.73E-06 & $1.52 \mathrm{E}-05$ & 4.37E-05 & 1.15E-04 & 2.92E-06 & $4.68 \mathrm{E}-03$ & 4.53E-04 & 4.48E-05 & 3.63E-04 & 7.54E-03 & $4.39 \mathrm{E}-02$ \\
\hline $65 \%$ & $8.28 \mathrm{E}-06$ & 1.69E-05 & 4.95E-05 & 1.17E-04 & $3.07 \mathrm{E}-06$ & $5.09 \mathrm{E}-03$ & 4.65E-04 & 4.81E-05 & $3.82 \mathrm{E}-04$ & $7.78 \mathrm{E}-03$ & 4.63E-02 \\
\hline $70 \%$ & $8.80 \mathrm{E}-06$ & $1.86 \mathrm{E}-05$ & $5.55 \mathrm{E}-05$ & 1.19E-04 & $3.27 \mathrm{E}-06$ & $5.58 \mathrm{E}-03$ & $4.80 \mathrm{E}-04$ & $5.31 \mathrm{E}-05$ & $4.12 \mathrm{E}-04$ & 8.09E-03 & $5.05 \mathrm{E}-02$ \\
\hline $75 \%$ & $9.49 \mathrm{E}-06$ & $2.10 \mathrm{E}-05$ & $6.27 \mathrm{E}-05$ & $1.23 \mathrm{E}-04$ & $3.48 \mathrm{E}-06$ & $6.20 \mathrm{E}-03$ & $5.03 \mathrm{E}-04$ & $5.65 \mathrm{E}-05$ & 4.41E-04 & 8.41E-03 & $5.36 \mathrm{E}-02$ \\
\hline $80 \%$ & $1.06 \mathrm{E}-05$ & $2.52 \mathrm{E}-05$ & 7.13E-05 & $1.25 \mathrm{E}-04$ & 3.81E-06 & 6.91E-03 & 5.23E-04 & $6.25 \mathrm{E}-05$ & 4.79E-04 & 8.86E-03 & $5.83 E-02$ \\
\hline $85 \%$ & 1.20E-05 & $2.88 \mathrm{E}-05$ & 9.17E-05 & 1.30E-04 & 4.27E-06 & 7.73E-03 & $5.52 \mathrm{E}-04$ & 7.22E-05 & $5.18 \mathrm{E}-04$ & $9.59 \mathrm{E}-03$ & 6.34E-02 \\
\hline $90 \%$ & 1.42E-05 & 3.73E-05 & $1.20 \mathrm{E}-04$ & 1.35E-04 & 5.22E-06 & 8.85E-03 & $6.00 \mathrm{E}-04$ & 8.92E-05 & 5.95E-04 & $1.09 \mathrm{E}-02$ & 7.12E-02 \\
\hline $95 \%$ & 2.07E-05 & 5.41E-05 & 1.76E-04 & 1.45E-04 & 6.57E-06 & 1.05E-02 & $6.81 \mathrm{E}-04$ & $1.11 \mathrm{E}-04$ & 7.22E-04 & 1.32E-02 & 8.46E-02 \\
\hline Maximum & $9.58 \mathrm{E}-05$ & $8.42 \mathrm{E}-04$ & $1.59 \mathrm{E}-03$ & $2.57 \mathrm{E}-04$ & $8.50 \mathrm{E}-05$ & 1.66E-02 & $2.35 \mathrm{E}-03$ & $3.90 \mathrm{E}-04$ & $1.53 \mathrm{E}-03$ & 3.10E-02 & 1.73E-01 \\
\hline
\end{tabular}


Table 6.2-12. Statistics for the ICRP 72-Based BDCFs for the Monsoon Climate, rem/yr per pCi/L (Continued)

\begin{tabular}{|c|c|c|c|c|c|c|c|c|c|c|c|}
\hline & $\begin{array}{r}\mathrm{Ra}-226 \\
+\mathrm{Pb}-210 \\
\end{array}$ & Ac-227 & Th-229 & Th-230 & Th-232 & Pa-231 & U-232 & U-233 & U-234 & U-236 & U-238 \\
\hline Mean & $5.06 \mathrm{E}-02$ & 7.56E-03 & 1.25E-02 & $1.53 \mathrm{E}-02$ & 1.90E-02 & 2.62E-02 & 2.70E-03 & 5.94E-04 & $3.88 \mathrm{E}-04$ & $3.29 \mathrm{E}-04$ & $3.55 \mathrm{E}-04$ \\
\hline STD & 22E-02 & $2.54 \mathrm{E}-03$ & 6.82E-03 & 1.26E-02 & 8.89E-03 & 1.76E-02 & 8.27E-04 & $6.71 \mathrm{E}-04$ & 2.91E-04 & 2.09E-04 & 2.33E-04 \\
\hline Minimum & 8.84E-03 & $3.79 \mathrm{E}-03$ & $2.28 \mathrm{E}-03$ & $8.68 \mathrm{E}-04$ & $3.82 \mathrm{E}-03$ & $2.48 \mathrm{E}-03$ & $1.44 \mathrm{E}-03$ & $1.48 \mathrm{E}-04$ & 1.41E-04 & 1.35E-04 & $1.39 \mathrm{E}-04$ \\
\hline $5 \%$ & $2.36 \mathrm{E}-02$ & $4.61 \mathrm{E}-03$ & 4.86E-03 & $3.58 \mathrm{E}-03$ & 8.38E-03 & 7.54E-03 & 1.67E-03 & 1.72E-04 & $1.62 \mathrm{E}-04$ & $1.55 \mathrm{E}-04$ & $1.58 \mathrm{E}-04$ \\
\hline $10 \%$ & $2.75 \mathrm{E}-02$ & 4.97E-03 & $5.81 \mathrm{E}-03$ & $4.58 \mathrm{E}-03$ & 9.94E-03 & $9.69 \mathrm{E}-03$ & $1.80 \mathrm{E}-03$ & $1.84 \mathrm{E}-04$ & 1.73E-04 & 1.64E-04 & 1.69E-04 \\
\hline $15 \%$ & $2.99 \mathrm{E}-02$ & $5.23 \mathrm{E}-03$ & $6.55 \mathrm{E}-03$ & $5.30 \mathrm{E}-03$ & 1.09E-02 & $1.09 \mathrm{E}-02$ & $1.91 \mathrm{E}-03$ & 2.01E-04 & 1.87E-04 & 1.75E-04 & $1.80 \mathrm{E}-04$ \\
\hline $20 \%$ & 3.19E-02 & $5.48 \mathrm{E}-03$ & 7.07E-03 & $5.85 \mathrm{E}-03$ & 1.16E-02 & $1.25 \mathrm{E}-02$ & $2.01 \mathrm{E}-03$ & 2.12E-04 & 1.96E-04 & 1.85E-04 & $1.89 \mathrm{E}-04$ \\
\hline $25 \%$ & $3.41 \mathrm{E}-02$ & $5.68 \mathrm{E}-03$ & 7.75E-03 & $6.53 \mathrm{E}-03$ & 1.26E-02 & $1.39 \mathrm{E}-02$ & 2.10E-03 & 2.27E-04 & 2.06E-04 & 1.92E-04 & 1.99E-04 \\
\hline $30 \%$ & 3.67E-02 & $5.88 \mathrm{E}-03$ & 8.31E-03 & $7.26 \mathrm{E}-03$ & $1.36 \mathrm{E}-02$ & $1.53 \mathrm{E}-02$ & $2.20 \mathrm{E}-03$ & $2.43 \mathrm{E}-04$ & $2.18 \mathrm{E}-04$ & 2.03E-04 & 2.09E-04 \\
\hline $35 \%$ & $3.88 \mathrm{E}-02$ & $6.18 \mathrm{E}-03$ & $8.90 \mathrm{E}-03$ & $8.20 \mathrm{E}-03$ & $1.45 \mathrm{E}-02$ & 1.67E-02 & $2.28 \mathrm{E}-03$ & $2.57 \mathrm{E}-04$ & 2.31E-04 & 2.13E-04 & 2.21E-04 \\
\hline $40 \%$ & 4.05E-02 & $6.51 \mathrm{E}-03$ & $9.50 \mathrm{E}-03$ & $9.16 \mathrm{E}-03$ & $1.52 \mathrm{E}-02$ & $1.81 \mathrm{E}-02$ & $2.35 \mathrm{E}-03$ & $2.85 \mathrm{E}-04$ & $2.44 \mathrm{E}-04$ & 2.23E-04 & 2.34E-04 \\
\hline $45 \%$ & 4.34E-02 & $6.73 \mathrm{E}-03$ & $1.01 \mathrm{E}-02$ & $1.00 \mathrm{E}-02$ & $1.59 \mathrm{E}-02$ & $1.94 \mathrm{E}-02$ & $2.44 \mathrm{E}-03$ & $3.08 \mathrm{E}-04$ & $2.60 \mathrm{E}-04$ & $2.38 \mathrm{E}-04$ & $2.48 \mathrm{E}-04$ \\
\hline $50 \%$ & 4.65E-02 & $7.05 \mathrm{E}-03$ & $1.08 \mathrm{E}-02$ & 1.12E-02 & $1.71 \mathrm{E}-02$ & $2.10 \mathrm{E}-02$ & $2.53 \mathrm{E}-03$ & $3.43 \mathrm{E}-04$ & 2.77E-04 & 2.50E-04 & $2.65 \mathrm{E}-04$ \\
\hline $55 \%$ & 4.89E-02 & 7.31E-03 & 1.17E-02 & $1.24 \mathrm{E}-02$ & $1.79 \mathrm{E}-02$ & 2.32E-02 & $2.64 \mathrm{E}-03$ & $3.72 \mathrm{E}-04$ & 2.97E-04 & 2.69E-04 & $2.86 \mathrm{E}-04$ \\
\hline $60 \%$ & $5.17 \mathrm{E}-02$ & 7.70E-03 & $1.26 \mathrm{E}-02$ & 1.36E-02 & $1.90 \mathrm{E}-02$ & $2.52 \mathrm{E}-02$ & 2.76E-03 & $4.20 \mathrm{E}-04$ & $3.25 \mathrm{E}-04$ & 2.90E-04 & 3.13E-04 \\
\hline $65 \%$ & $5.43 \mathrm{E}-02$ & 8.06E-03 & $1.34 \mathrm{E}-02$ & 1.57E-02 & 2.02E-02 & 2.77E-02 & $2.85 \mathrm{E}-03$ & 4.90E-04 & 3.64E-04 & 3.17E-04 & 3.49E-04 \\
\hline $70 \%$ & $5.85 \mathrm{E}-02$ & $8.41 \mathrm{E}-03$ & 1.43E-02 & 1.76E-02 & $2.16 \mathrm{E}-02$ & 3.02E-02 & 2.96E-03 & $5.65 \mathrm{E}-04$ & 4.03E-04 & 3.47E-04 & $3.88 \mathrm{E}-04$ \\
\hline $75 \%$ & $6.19 \mathrm{E}-02$ & $8.80 \mathrm{E}-03$ & $1.52 \mathrm{E}-02$ & $1.98 \mathrm{E}-02$ & $2.31 \mathrm{E}-02$ & 3.35E-02 & $3.10 \mathrm{E}-03$ & $6.51 \mathrm{E}-04$ & $4.50 \mathrm{E}-04$ & 3.91E-04 & $4.28 \mathrm{E}-04$ \\
\hline $80 \%$ & $6.67 \mathrm{E}-02$ & $9.38 \mathrm{E}-03$ & 1.66E-02 & $2.25 \mathrm{E}-02$ & $2.52 \mathrm{E}-02$ & $3.73 \mathrm{E}-02$ & $3.30 \mathrm{E}-03$ & 7.83E-04 & $5.19 \mathrm{E}-04$ & 4.38E-04 & 4.89E-04 \\
\hline $85 \%$ & 7.25E-02 & 9.96E-03 & 1.89E-02 & 2.65E-02 & 2.81E-02 & 4.24E-02 & 3.57E-03 & 9.44E-04 & $6.08 \mathrm{E}-04$ & $5.10 \mathrm{E}-04$ & $5.63 \mathrm{E}-04$ \\
\hline $90 \%$ & $8.11 \mathrm{E}-02$ & $1.08 \mathrm{E}-02$ & $2.15 \mathrm{E}-02$ & $3.18 \mathrm{E}-02$ & $3.12 \mathrm{E}-02$ & $5.04 \mathrm{E}-02$ & $3.77 \mathrm{E}-03$ & $1.31 \mathrm{E}-03$ & 7.58E-04 & $5.94 \mathrm{E}-04$ & 6.66E-04 \\
\hline $95 \%$ & $9.33 \mathrm{E}-02$ & $1.21 \mathrm{E}-02$ & $2.55 \mathrm{E}-02$ & 4.17E-02 & 3.62E-02 & $6.22 \mathrm{E}-02$ & $4.25 \mathrm{E}-03$ & 1.93E-03 & $1.01 \mathrm{E}-03$ & 7.96E-04 & $8.68 \mathrm{E}-04$ \\
\hline Maximum & 1.81E-01 & $2.28 \mathrm{E}-02$ & $5.12 \mathrm{E}-02$ & 8.73E-02 & 5.99E-02 & 1.32E-01 & $6.77 \mathrm{E}-03$ & $5.51 \mathrm{E}-03$ & $2.39 \mathrm{E}-03$ & 1.67E-03 & $1.80 \mathrm{E}-03$ \\
\hline
\end{tabular}


Table 6.2-12. Statistics for the ICRP 72-Based BDCFs for the Monsoon Climate, rem/yr per pCi/L (Continued)

\begin{tabular}{|c|c|c|c|c|c|c|c|}
\hline & Np-237 & Pu-238 & Pu-239 & Pu-240 & Pu-242 & Am-241 & Am-243 \\
\hline Mean & 1.17E-03 & 2.02E-03 & 5.33E-03 & $5.15 \mathrm{E}-03$ & 4.99E-03 & $2.70 \mathrm{E}-03$ & 4.42E-03 \\
\hline STD & 7.30E-04 & 7.34E-04 & 3.01E-03 & 2.82E-03 & 2.84E-03 & $1.25 \mathrm{E}-03$ & 2.60E-03 \\
\hline Minimum & $3.71 \mathrm{E}-04$ & 8.38E-04 & $1.21 \mathrm{E}-03$ & $1.20 \mathrm{E}-03$ & 1.14E-03 & $6.52 \mathrm{E}-04$ & $6.56 \mathrm{E}-04$ \\
\hline $5 \%$ & 4.74E-04 & 1.13E-03 & $2.08 \mathrm{E}-03$ & 2.06E-03 & $1.95 \mathrm{E}-03$ & $1.21 \mathrm{E}-03$ & $1.48 \mathrm{E}-03$ \\
\hline $10 \%$ & $5.45 \mathrm{E}-04$ & $1.25 \mathrm{E}-03$ & $2.45 \mathrm{E}-03$ & $2.41 \mathrm{E}-03$ & 2.30E-03 & 1.39E-03 & $1.85 \mathrm{E}-03$ \\
\hline $15 \%$ & 6.10E-04 & 1.33E-03 & 2.69E-03 & 2.66E-03 & $2.52 \mathrm{E}-03$ & $1.58 \mathrm{E}-03$ & $2.12 \mathrm{E}-03$ \\
\hline $20 \%$ & $6.58 \mathrm{E}-04$ & 1.40E-03 & 2.99E-03 & 2.95E-03 & 2.79E-03 & $1.68 \mathrm{E}-03$ & $2.34 \mathrm{E}-03$ \\
\hline $25 \%$ & 7.04E-04 & $1.48 \mathrm{E}-03$ & 3.24E-03 & 3.19E-03 & 3.03E-03 & $1.81 \mathrm{E}-03$ & $2.54 \mathrm{E}-03$ \\
\hline $30 \%$ & 7.53E-04 & $1.56 \mathrm{E}-03$ & $3.48 \mathrm{E}-03$ & 3.40E-03 & 3.25E-03 & $1.95 \mathrm{E}-03$ & $2.74 \mathrm{E}-03$ \\
\hline $35 \%$ & 7.93E-04 & 1.63E-03 & $3.76 \mathrm{E}-03$ & 3.66E-03 & $3.49 \mathrm{E}-03$ & 2.05E-03 & $2.99 \mathrm{E}-03$ \\
\hline $40 \%$ & 8.49E-04 & 1.71E-03 & 3.94E-03 & 3.86E-03 & 3.68E-03 & 2.18E-03 & $3.25 \mathrm{E}-03$ \\
\hline $45 \%$ & 9.01E-04 & 1.79E-03 & 4.17E-03 & 4.07E-03 & 3.89E-03 & 2.30E-03 & $3.49 \mathrm{E}-03$ \\
\hline $50 \%$ & 9.47E-04 & 1.88E-03 & 4.47E-03 & 4.36E-03 & 4.19E-03 & 2.42E-03 & $3.72 \mathrm{E}-03$ \\
\hline $55 \%$ & 1.02E-03 & 1.95E-03 & 4.75E-03 & 4.62E-03 & 4.44E-03 & 2.57E-03 & $4.01 \mathrm{E}-03$ \\
\hline $60 \%$ & 1.12E-03 & 2.06E-03 & 5.30E-03 & 5.13E-03 & 4.95E-03 & 2.71E-03 & 4.37E-03 \\
\hline $65 \%$ & $1.18 \mathrm{E}-03$ & $2.14 \mathrm{E}-03$ & $5.63 \mathrm{E}-03$ & $5.49 \mathrm{E}-03$ & $5.28 \mathrm{E}-03$ & 2.89E-03 & 4.79E-03 \\
\hline $70 \%$ & 1.27E-03 & $2.28 \mathrm{E}-03$ & 6.09E-03 & $5.90 \mathrm{E}-03$ & $5.68 \mathrm{E}-03$ & 3.09E-03 & 5.17E-03 \\
\hline $75 \%$ & 1.37E-03 & $2.40 \mathrm{E}-03$ & $6.49 \mathrm{E}-03$ & 6.30E-03 & 6.03E-03 & 3.33E-03 & $5.61 \mathrm{E}-03$ \\
\hline $80 \%$ & $1.53 \mathrm{E}-03$ & $2.56 \mathrm{E}-03$ & 7.29E-03 & 6.86E-03 & $6.80 \mathrm{E}-03$ & 3.51E-03 & $6.11 \mathrm{E}-03$ \\
\hline $85 \%$ & $1.74 \mathrm{E}-03$ & 2.72E-03 & 8.17E-03 & 7.79E-03 & 7.66E-03 & 3.77E-03 & $6.91 \mathrm{E}-03$ \\
\hline $90 \%$ & 1.97E-03 & 2.94E-03 & $9.46 \mathrm{E}-03$ & 8.98E-03 & 8.86E-03 & 4.20E-03 & 7.96E-03 \\
\hline $95 \%$ & $2.58 \mathrm{E}-03$ & 3.43E-03 & 1.11E-02 & 1.06E-02 & 1.04E-02 & $5.21 \mathrm{E}-03$ & $9.45 \mathrm{E}-03$ \\
\hline Maximum & $6.35 \mathrm{E}-03$ & $5.64 \mathrm{E}-03$ & $2.32 \mathrm{E}-02$ & $2.15 \mathrm{E}-02$ & $2.19 \mathrm{E}-02$ & $8.44 \mathrm{E}-03$ & $1.89 \mathrm{E}-02$ \\
\hline \multicolumn{8}{|c|}{$\begin{array}{ll}\text { Source: } & \text { MO0407SPAINEXI.002 [DIRS 170597]; MO0407SPASRPBM.002 [DIRS 170755]; } \\
& \text { MO0306MWDBGSMF.001 [DIRS 163816]; MO0403SPAAEIBM.002 [DIRS 169392]; } \\
& \text { MO0407SPACRBSM.002 [DIRS 170677]; MO0406SPAETPBM.002 [DIRS 170150]; } \\
& \text { MO0503SPADCESR.000 [DIRS 172896]. }\end{array}$} \\
\hline NOTE: & ee Excel file & ס זחס אחי & alizations_M & & & ndix $B$ for $d$ & \\
\hline
\end{tabular}


Table 6.2-13. Statistics for the ICRP 72-Based BDCFs for the Glacial Transition Climate, rem/yr per pCi/L

\begin{tabular}{|c|c|c|c|c|c|c|c|c|c|c|c|}
\hline & C-14 & $\mathrm{Cl}-36$ & Se-79 & Sr-90 & Tc-99 & Sn-126 & I-129 & Cs-135 & Cs-137 & $\mathrm{Pb}-210$ & Ra-226 \\
\hline Mean & 9.02E-06 & $2.04 \mathrm{E}-05$ & 6.16E-05 & 1.14E-04 & $3.41 \mathrm{E}-06$ & $4.49 \mathrm{E}-03$ & 4.58E-04 & 4.63E-05 & $3.58 \mathrm{E}-04$ & 7.87E-03 & 4.10E-02 \\
\hline STD & $6.85 \mathrm{E}-06$ & 4.51E-05 & 1.06E-04 & $1.58 \mathrm{E}-05$ & $3.51 \mathrm{E}-06$ & 2.92E-03 & 1.33E-04 & $3.41 \mathrm{E}-05$ & $1.83 \mathrm{E}-04$ & $2.57 \mathrm{E}-03$ & 2.09E-02 \\
\hline Minimum & $4.00 \mathrm{E}-06$ & 3.61E-06 & 1.07E-05 & 8.97E-05 & 1.87E-06 & $1.41 \mathrm{E}-04$ & 3.22E-04 & 1.02E-05 & 1.13E-04 & $5.40 \mathrm{E}-03$ & $1.55 \mathrm{E}-03$ \\
\hline $5 \%$ & 4.76E-06 & $5.23 \mathrm{E}-06$ & 1.63E-05 & $9.67 \mathrm{E}-05$ & 2.07E-06 & 7.73E-04 & $3.45 \mathrm{E}-04$ & $1.58 \mathrm{E}-05$ & 1.63E-04 & $5.80 \mathrm{E}-03$ & $1.61 \mathrm{E}-02$ \\
\hline $10 \%$ & $5.04 \mathrm{E}-06$ & 5.81E-06 & 1.87E-05 & $9.90 \mathrm{E}-05$ & $2.14 \mathrm{E}-06$ & $1.28 \mathrm{E}-03$ & $3.54 \mathrm{E}-04$ & $1.89 \mathrm{E}-05$ & $1.84 \mathrm{E}-04$ & $5.95 \mathrm{E}-03$ & 1.96E-02 \\
\hline $15 \%$ & $5.28 \mathrm{E}-06$ & $6.38 \mathrm{E}-06$ & 2.07E-05 & 1.01E-04 & 2.22E-06 & 1.80E-03 & 3.63E-04 & 2.16E-05 & 2.01E-04 & $6.08 \mathrm{E}-03$ & $2.21 \mathrm{E}-02$ \\
\hline $20 \%$ & $5.50 \mathrm{E}-06$ & $6.87 \mathrm{E}-06$ & 2.27E-05 & $1.02 \mathrm{E}-04$ & 2.27E-06 & 2.17E-03 & 3.71E-04 & 2.37E-05 & 2.21E-04 & $6.19 \mathrm{E}-03$ & $2.44 \mathrm{E}-02$ \\
\hline $25 \%$ & 5.73E-06 & 7.42E-06 & $2.45 \mathrm{E}-05$ & $1.04 \mathrm{E}-04$ & 2.33E-06 & $2.50 \mathrm{E}-03$ & $3.79 \mathrm{E}-04$ & 2.59E-05 & 2.36E-04 & $6.32 \mathrm{E}-03$ & $2.61 \mathrm{E}-02$ \\
\hline $30 \%$ & 5.94E-06 & 8.14E-06 & 2.65E-05 & 1.05E-04 & 2.40E-06 & $2.75 \mathrm{E}-03$ & 3.87E-04 & 2.77E-05 & 2.54E-04 & $6.45 \mathrm{E}-03$ & $2.82 \mathrm{E}-02$ \\
\hline $35 \%$ & $6.19 \mathrm{E}-06$ & 8.86E-06 & 2.86E-05 & 1.06E-04 & 2.46E-06 & $3.01 \mathrm{E}-03$ & 3.96E-04 & 2.97E-05 & 2.70E-04 & $6.59 \mathrm{E}-03$ & 3.00E-02 \\
\hline $40 \%$ & $6.40 \mathrm{E}-06$ & 9.91E-06 & $3.10 \mathrm{E}-05$ & $1.08 \mathrm{E}-04$ & $2.53 \mathrm{E}-06$ & $3.27 \mathrm{E}-03$ & 4.04E-04 & 3.24E-05 & 2.84E-04 & 6.77E-03 & $3.20 \mathrm{E}-02$ \\
\hline $45 \%$ & $6.66 \mathrm{E}-06$ & 1.07E-05 & 3.34E-05 & $1.09 \mathrm{E}-04$ & 2.61E-06 & $3.53 \mathrm{E}-03$ & 4.13E-04 & $3.51 \mathrm{E}-05$ & 2.96E-04 & $6.91 \mathrm{E}-03$ & $3.40 \mathrm{E}-02$ \\
\hline $50 \%$ & $6.95 \mathrm{E}-06$ & $1.20 \mathrm{E}-05$ & $3.57 \mathrm{E}-05$ & 1.11E-04 & $2.69 \mathrm{E}-06$ & $3.78 \mathrm{E}-03$ & 4.24E-04 & $3.75 \mathrm{E}-05$ & $3.10 \mathrm{E}-04$ & 7.07E-03 & 3.67E-02 \\
\hline $55 \%$ & 7.18E-06 & 1.30E-05 & 3.92E-05 & 1.13E-04 & $2.78 \mathrm{E}-06$ & 4.05E-03 & 4.36E-04 & $4.00 \mathrm{E}-05$ & $3.28 \mathrm{E}-04$ & 7.27E-03 & $3.88 \mathrm{E}-02$ \\
\hline $60 \%$ & 7.67E-06 & 1.47E-05 & 4.34E-05 & 1.14E-04 & 2.88E-06 & 4.47E-03 & 4.47E-04 & 4.26E-05 & $3.50 \mathrm{E}-04$ & $7.50 \mathrm{E}-03$ & 4.16E-02 \\
\hline $65 \%$ & 8.27E-06 & 1.66E-05 & 4.79E-05 & 1.16E-04 & 3.07E-06 & 4.84E-03 & 4.60E-04 & 4.53E-05 & 3.75E-04 & 7.73E-03 & 4.45E-02 \\
\hline $70 \%$ & 8.80E-06 & $1.87 \mathrm{E}-05$ & 5.35E-05 & $1.18 \mathrm{E}-04$ & 3.25E-06 & $5.39 \mathrm{E}-03$ & 4.75E-04 & 4.97E-05 & 4.00E-04 & 8.03E-03 & 4.73E-02 \\
\hline $75 \%$ & $9.46 \mathrm{E}-06$ & $2.08 \mathrm{E}-05$ & $6.15 \mathrm{E}-05$ & $1.20 \mathrm{E}-04$ & 3.46E-06 & $5.99 \mathrm{E}-03$ & $4.90 \mathrm{E}-04$ & $5.47 \mathrm{E}-05$ & $4.30 \mathrm{E}-04$ & 8.39E-03 & $5.09 \mathrm{E}-02$ \\
\hline $80 \%$ & 1.06E-05 & 2.36E-05 & $6.93 \mathrm{E}-05$ & $1.24 \mathrm{E}-04$ & 3.76E-06 & $6.56 \mathrm{E}-03$ & $5.15 \mathrm{E}-04$ & $6.18 \mathrm{E}-05$ & 4.64E-04 & 8.80E-03 & $5.63 E-02$ \\
\hline $85 \%$ & $1.20 \mathrm{E}-05$ & 2.89E-05 & 8.69E-05 & 1.27E-04 & 4.21E-06 & 7.38E-03 & $5.50 \mathrm{E}-04$ & 7.03E-05 & 5.04E-04 & $9.51 \mathrm{E}-03$ & $6.13 \mathrm{E}-02$ \\
\hline $90 \%$ & $1.41 \mathrm{E}-05$ & 3.69E-05 & 1.13E-04 & 1.33E-04 & 4.97E-06 & 8.52E-03 & $5.88 \mathrm{E}-04$ & 8.35E-05 & $5.64 \mathrm{E}-04$ & $1.06 \mathrm{E}-02$ & $6.97 \mathrm{E}-02$ \\
\hline $95 \%$ & $2.08 \mathrm{E}-05$ & $5.18 \mathrm{E}-05$ & $1.68 \mathrm{E}-04$ & $1.43 \mathrm{E}-04$ & $6.52 \mathrm{E}-06$ & 1.03E-02 & $6.74 \mathrm{E}-04$ & $1.09 \mathrm{E}-04$ & $7.24 \mathrm{E}-04$ & $1.28 \mathrm{E}-02$ & $7.99 \mathrm{E}-02$ \\
\hline Maximum & $9.60 \mathrm{E}-05$ & $9.49 \mathrm{E}-04$ & $2.02 \mathrm{E}-03$ & 2.42E-04 & 8.96E-05 & 1.72E-02 & 2.32E-03 & $4.68 \mathrm{E}-04$ & 1.47E-03 & $2.57 \mathrm{E}-02$ & $1.94 \mathrm{E}-01$ \\
\hline
\end{tabular}


Table 6.2-13. Statistics for the ICRP 72-Based BDCFs for the Glacial Transition Climate, rem/yr per pCi/L (Continued)

\begin{tabular}{|c|c|c|c|c|c|c|c|c|c|c|c|}
\hline & $\begin{array}{r}\mathrm{Ra}-226 \\
+\mathrm{Pb}-210 \\
\end{array}$ & Ac-227 & Th-229 & Th-230 & Th-232 & $\mathrm{Pa}-231$ & U-232 & U-233 & U-234 & U-236 & U-238 \\
\hline Mean & 4.89E-02 & 7.32E-03 & $1.20 \mathrm{E}-02$ & $1.47 \mathrm{E}-02$ & 1.83E-02 & 2.52E-02 & 2.64E-03 & 5.73E-04 & 3.77E-04 & $3.21 \mathrm{E}-04$ & $3.46 \mathrm{E}-04$ \\
\hline STD & 2.13E-02 & 2.49E-03 & 6.63E-03 & 1.18E-02 & 8.48E-03 & 1.72E-02 & 8.11E-04 & 6.40E-04 & 2.80E-04 & 2.06E-04 & 2.27E-04 \\
\hline Minimum & 8.78E-03 & $3.70 \mathrm{E}-03$ & $2.41 \mathrm{E}-03$ & 1.03E-03 & 4.18E-03 & $2.45 \mathrm{E}-03$ & $1.41 \mathrm{E}-03$ & $1.48 \mathrm{E}-04$ & $1.41 \mathrm{E}-04$ & 1.35E-04 & 1.39E-04 \\
\hline $5 \%$ & 2.31E-02 & 4.49E-03 & 4.64E-03 & $3.50 \mathrm{E}-03$ & 7.99E-03 & 7.67E-03 & $1.68 \mathrm{E}-03$ & 1.70E-04 & $1.61 \mathrm{E}-04$ & 1.53E-04 & $1.58 \mathrm{E}-04$ \\
\hline $10 \%$ & $2.68 \mathrm{E}-02$ & $4.85 \mathrm{E}-03$ & $5.70 \mathrm{E}-03$ & $4.48 \mathrm{E}-03$ & $9.74 \mathrm{E}-03$ & $9.25 \mathrm{E}-03$ & 1.77E-03 & 1.84E-04 & $1.72 \mathrm{E}-04$ & 1.63E-04 & 1.67E-04 \\
\hline $15 \%$ & $2.95 \mathrm{E}-02$ & $5.14 \mathrm{E}-03$ & 6.31E-03 & $5.02 \mathrm{E}-03$ & 1.07E-02 & 1.06E-02 & 1.87E-03 & $1.96 \mathrm{E}-04$ & $1.82 \mathrm{E}-04$ & 1.72E-04 & 1.76E-04 \\
\hline $20 \%$ & $3.20 \mathrm{E}-02$ & 5.35E-03 & $6.89 \mathrm{E}-03$ & $5.81 \mathrm{E}-03$ & 1.16E-02 & $1.22 \mathrm{E}-02$ & $1.96 \mathrm{E}-03$ & 2.08E-04 & 1.93E-04 & $1.81 \mathrm{E}-04$ & 1.86E-04 \\
\hline $25 \%$ & $3.39 \mathrm{E}-02$ & $5.54 \mathrm{E}-03$ & 7.53E-03 & $6.40 \mathrm{E}-03$ & 1.23E-02 & 1.36E-02 & $2.06 \mathrm{E}-03$ & 2.24E-04 & 2.03E-04 & $1.90 \mathrm{E}-04$ & 1.96E-04 \\
\hline $30 \%$ & $3.56 \mathrm{E}-02$ & $5.75 \mathrm{E}-03$ & 8.06E-03 & 7.07E-03 & 1.30E-02 & $1.50 \mathrm{E}-02$ & 2.16E-03 & 2.39E-04 & 2.14E-04 & 1.99E-04 & 2.05E-04 \\
\hline $35 \%$ & $3.75 \mathrm{E}-02$ & $6.04 \mathrm{E}-03$ & 8.69E-03 & 7.89E-03 & $1.36 \mathrm{E}-02$ & 1.63E-02 & $2.24 \mathrm{E}-03$ & $2.54 \mathrm{E}-04$ & 2.26E-04 & 2.10E-04 & 2.16E-04 \\
\hline $40 \%$ & 3.96E-02 & $6.33 \mathrm{E}-03$ & $9.17 \mathrm{E}-03$ & 8.89E-03 & $1.46 \mathrm{E}-02$ & 1.75E-02 & $2.30 \mathrm{E}-03$ & 2.74E-04 & 2.39E-04 & 2.20E-04 & $2.29 \mathrm{E}-04$ \\
\hline $45 \%$ & 4.18E-02 & $6.53 \mathrm{E}-03$ & $9.84 \mathrm{E}-03$ & $9.77 \mathrm{E}-03$ & $1.54 \mathrm{E}-02$ & $1.88 \mathrm{E}-02$ & 2.38E-03 & $3.00 \mathrm{E}-04$ & $2.52 \mathrm{E}-04$ & 2.31E-04 & 2.43E-04 \\
\hline $50 \%$ & 4.43E-02 & $6.75 \mathrm{E}-03$ & 1.04E-02 & 1.07E-02 & 1.62E-02 & 2.02E-02 & $2.48 \mathrm{E}-03$ & 3.34E-04 & 2.73E-04 & $2.46 \mathrm{E}-04$ & 2.62E-04 \\
\hline $55 \%$ & 4.64E-02 & $7.00 \mathrm{E}-03$ & 1.10E-02 & 1.18E-02 & $1.73 \mathrm{E}-02$ & 2.22E-02 & $2.59 \mathrm{E}-03$ & $3.71 \mathrm{E}-04$ & $2.94 \mathrm{E}-04$ & 2.64E-04 & 2.85E-04 \\
\hline $60 \%$ & 4.90E-02 & 7.32E-03 & 1.18E-02 & 1.34E-02 & 1.82E-02 & 2.36E-02 & 2.68E-03 & 4.09E-04 & 3.17E-04 & 2.84E-04 & 3.04E-04 \\
\hline $65 \%$ & $5.28 \mathrm{E}-02$ & $7.65 \mathrm{E}-03$ & $1.28 \mathrm{E}-02$ & $1.49 \mathrm{E}-02$ & $1.97 \mathrm{E}-02$ & $2.58 \mathrm{E}-02$ & $2.79 \mathrm{E}-03$ & 4.76E-04 & $3.54 \mathrm{E}-04$ & 3.11E-04 & 3.39E-04 \\
\hline $70 \%$ & $5.60 \mathrm{E}-02$ & 8.01E-03 & $1.38 \mathrm{E}-02$ & 1.70E-02 & 2.10E-02 & $2.87 \mathrm{E}-02$ & $2.88 \mathrm{E}-03$ & $5.50 \mathrm{E}-04$ & $3.82 \mathrm{E}-04$ & $3.40 \mathrm{E}-04$ & 3.74E-04 \\
\hline $75 \%$ & $5.95 \mathrm{E}-02$ & 8.46E-03 & 1.46E-02 & 1.91E-02 & 2.29E-02 & 3.18E-02 & 3.00E-03 & $6.23 \mathrm{E}-04$ & 4.38E-04 & 3.74E-04 & 4.15E-04 \\
\hline $80 \%$ & $6.50 \mathrm{E}-02$ & $9.05 \mathrm{E}-03$ & $1.59 \mathrm{E}-02$ & 2.14E-02 & $2.45 \mathrm{E}-02$ & 3.53E-02 & 3.17E-03 & 7.46E-04 & 5.06E-04 & 4.13E-04 & 4.64E-04 \\
\hline $85 \%$ & $6.94 \mathrm{E}-02$ & $9.66 \mathrm{E}-03$ & 1.79E-02 & $2.53 \mathrm{E}-02$ & 2.64E-02 & 4.01E-02 & $3.38 \mathrm{E}-03$ & $9.70 \mathrm{E}-04$ & $6.04 \mathrm{E}-04$ & 4.79E-04 & $5.48 \mathrm{E}-04$ \\
\hline $90 \%$ & 7.79E-02 & $1.03 \mathrm{E}-02$ & 2.02E-02 & $3.13 \mathrm{E}-02$ & 2.96E-02 & 4.72E-02 & 3.67E-03 & $1.28 \mathrm{E}-03$ & 7.29E-04 & $5.83 E-04$ & 6.46E-04 \\
\hline $95 \%$ & 8.87E-02 & $1.18 \mathrm{E}-02$ & 2.49E-02 & 4.02E-02 & $3.49 \mathrm{E}-02$ & $6.04 \mathrm{E}-02$ & 4.10E-03 & $1.79 \mathrm{E}-03$ & 9.33E-04 & 7.31E-04 & 7.99E-04 \\
\hline Maximum & 2.03E-01 & $3.08 \mathrm{E}-02$ & 4.72E-02 & 7.07E-02 & $5.91 \mathrm{E}-02$ & $1.48 \mathrm{E}-01$ & 6.81E-03 & 5.16E-03 & 2.05E-03 & $1.54 \mathrm{E}-03$ & 1.58E-03 \\
\hline
\end{tabular}


Table 6.2-13. Statistics for the ICRP 72-Based BDCFs for the Glacial Transition Climate, rem/yr per $\mathrm{pCi} / \mathrm{L}$ (Continued)

\begin{tabular}{|c|c|c|c|c|c|c|c|}
\hline & Np-237 & Pu-238 & Pu-239 & Pu-240 & Pu-242 & Am-241 & Am-243 \\
\hline Mean & 1.13E-03 & 1.95E-03 & $5.13 \mathrm{E}-03$ & 4.95E-03 & $4.80 \mathrm{E}-03$ & $2.61 \mathrm{E}-03$ & $4.28 \mathrm{E}-03$ \\
\hline STD & $7.00 \mathrm{E}-04$ & 7.32E-04 & $2.92 \mathrm{E}-03$ & $2.75 \mathrm{E}-03$ & $2.74 \mathrm{E}-03$ & $1.28 \mathrm{E}-03$ & $2.65 \mathrm{E}-03$ \\
\hline Minimum & 3.61E-04 & 8.07E-04 & $1.20 \mathrm{E}-03$ & 1.19E-03 & 1.13E-03 & $6.05 \mathrm{E}-04$ & $6.09 \mathrm{E}-04$ \\
\hline $5 \%$ & 4.71E-04 & 1.11E-03 & $2.08 \mathrm{E}-03$ & $2.06 \mathrm{E}-03$ & 1.95E-03 & 1.19E-03 & $1.42 \mathrm{E}-03$ \\
\hline $10 \%$ & $5.41 \mathrm{E}-04$ & $1.21 \mathrm{E}-03$ & $2.41 \mathrm{E}-03$ & $2.38 \mathrm{E}-03$ & $2.25 \mathrm{E}-03$ & 1.36E-03 & 1.76E-03 \\
\hline $15 \%$ & $5.91 \mathrm{E}-04$ & $1.29 \mathrm{E}-03$ & $2.66 \mathrm{E}-03$ & $2.62 \mathrm{E}-03$ & $2.49 \mathrm{E}-03$ & $1.52 \mathrm{E}-03$ & $2.08 \mathrm{E}-03$ \\
\hline $20 \%$ & $6.39 \mathrm{E}-04$ & 1.37E-03 & 2.92E-03 & $2.88 \mathrm{E}-03$ & $2.73 E-03$ & 1.63E-03 & $2.29 \mathrm{E}-03$ \\
\hline $25 \%$ & $6.82 \mathrm{E}-04$ & $1.45 \mathrm{E}-03$ & $3.16 \mathrm{E}-03$ & $3.09 \mathrm{E}-03$ & $2.95 \mathrm{E}-03$ & $1.74 \mathrm{E}-03$ & $2.45 \mathrm{E}-03$ \\
\hline $30 \%$ & 7.24E-04 & $1.51 \mathrm{E}-03$ & 3.40E-03 & 3.31E-03 & 3.17E-03 & 1.86E-03 & 2.67E-03 \\
\hline $35 \%$ & $7.68 \mathrm{E}-04$ & $1.58 \mathrm{E}-03$ & 3.57E-03 & $3.52 \mathrm{E}-03$ & 3.34E-03 & 1.96E-03 & 2.87E-03 \\
\hline $40 \%$ & $8.15 \mathrm{E}-04$ & 1.65E-03 & $3.80 \mathrm{E}-03$ & $3.71 \mathrm{E}-03$ & $3.55 \mathrm{E}-03$ & $2.05 \mathrm{E}-03$ & $3.09 \mathrm{E}-03$ \\
\hline $45 \%$ & 8.64E-04 & $1.72 \mathrm{E}-03$ & $4.05 \mathrm{E}-03$ & 3.96E-03 & $3.78 \mathrm{E}-03$ & $2.20 \mathrm{E}-03$ & 3.36E-03 \\
\hline $50 \%$ & $9.21 \mathrm{E}-04$ & $1.78 \mathrm{E}-03$ & $4.28 \mathrm{E}-03$ & 4.19E-03 & 4.02E-03 & 2.31E-03 & $3.63 \mathrm{E}-03$ \\
\hline $55 \%$ & 9.82E-04 & 1.85E-03 & 4.65E-03 & 4.51E-03 & 4.34E-03 & $2.45 \mathrm{E}-03$ & $3.90 \mathrm{E}-03$ \\
\hline $60 \%$ & $1.04 \mathrm{E}-03$ & $1.96 \mathrm{E}-03$ & $5.01 \mathrm{E}-03$ & 4.83E-03 & 4.66E-03 & $2.61 \mathrm{E}-03$ & 4.16E-03 \\
\hline $65 \%$ & 1.13E-03 & 2.07E-03 & $5.30 \mathrm{E}-03$ & $5.16 \mathrm{E}-03$ & 4.97E-03 & $2.76 \mathrm{E}-03$ & $4.52 \mathrm{E}-03$ \\
\hline $70 \%$ & $1.22 \mathrm{E}-03$ & 2.17E-03 & $5.75 \mathrm{E}-03$ & 5.53E-03 & $5.40 \mathrm{E}-03$ & $2.91 \mathrm{E}-03$ & 4.87E-03 \\
\hline $75 \%$ & 1.33E-03 & $2.29 \mathrm{E}-03$ & $6.24 \mathrm{E}-03$ & $6.01 \mathrm{E}-03$ & $5.84 \mathrm{E}-03$ & $3.12 \mathrm{E}-03$ & $5.38 \mathrm{E}-03$ \\
\hline $80 \%$ & 1.47E-03 & $2.44 \mathrm{E}-03$ & $6.84 \mathrm{E}-03$ & $6.62 \mathrm{E}-03$ & $6.37 \mathrm{E}-03$ & 3.37E-03 & $5.94 \mathrm{E}-03$ \\
\hline $85 \%$ & $1.70 \mathrm{E}-03$ & $2.58 \mathrm{E}-03$ & 7.66E-03 & 7.34E-03 & $7.20 \mathrm{E}-03$ & 3.73E-03 & $6.70 \mathrm{E}-03$ \\
\hline $90 \%$ & 1.93E-03 & $2.86 \mathrm{E}-03$ & $8.71 \mathrm{E}-03$ & $8.25 \mathrm{E}-03$ & $8.14 \mathrm{E}-03$ & $4.26 \mathrm{E}-03$ & $7.55 \mathrm{E}-03$ \\
\hline $95 \%$ & $2.58 \mathrm{E}-03$ & 3.38E-03 & $1.09 \mathrm{E}-02$ & 1.05E-02 & 1.03E-02 & $5.02 \mathrm{E}-03$ & $9.29 \mathrm{E}-03$ \\
\hline Maximum & $5.54 \mathrm{E}-03$ & $6.86 \mathrm{E}-03$ & $2.17 \mathrm{E}-02$ & $2.01 \mathrm{E}-02$ & 2.05E-02 & $1.03 \mathrm{E}-02$ & 2.10E-02 \\
\hline
\end{tabular}

Source: MO0407SPAINEXI.002 [DIRS 170597]; MO0407SPASRPBM.002 [DIRS 170755]; MO0306MWDBGSMF.001 [DIRS 163816]; MO0403SPAAEIBM.002 [DIRS 169392]; MO0407SPACRBSM.002 [DIRS 170677]; MO0406SPAETPBM.002 [DIRS 170150]; MO0503SPADCESR.000 [DIRS 172896].

NOTE: See Excel file GW BDCF Realizations_MC and FC_Rev 4.xIs in Appendix B for details of calculations.

STD = standard deviation . 


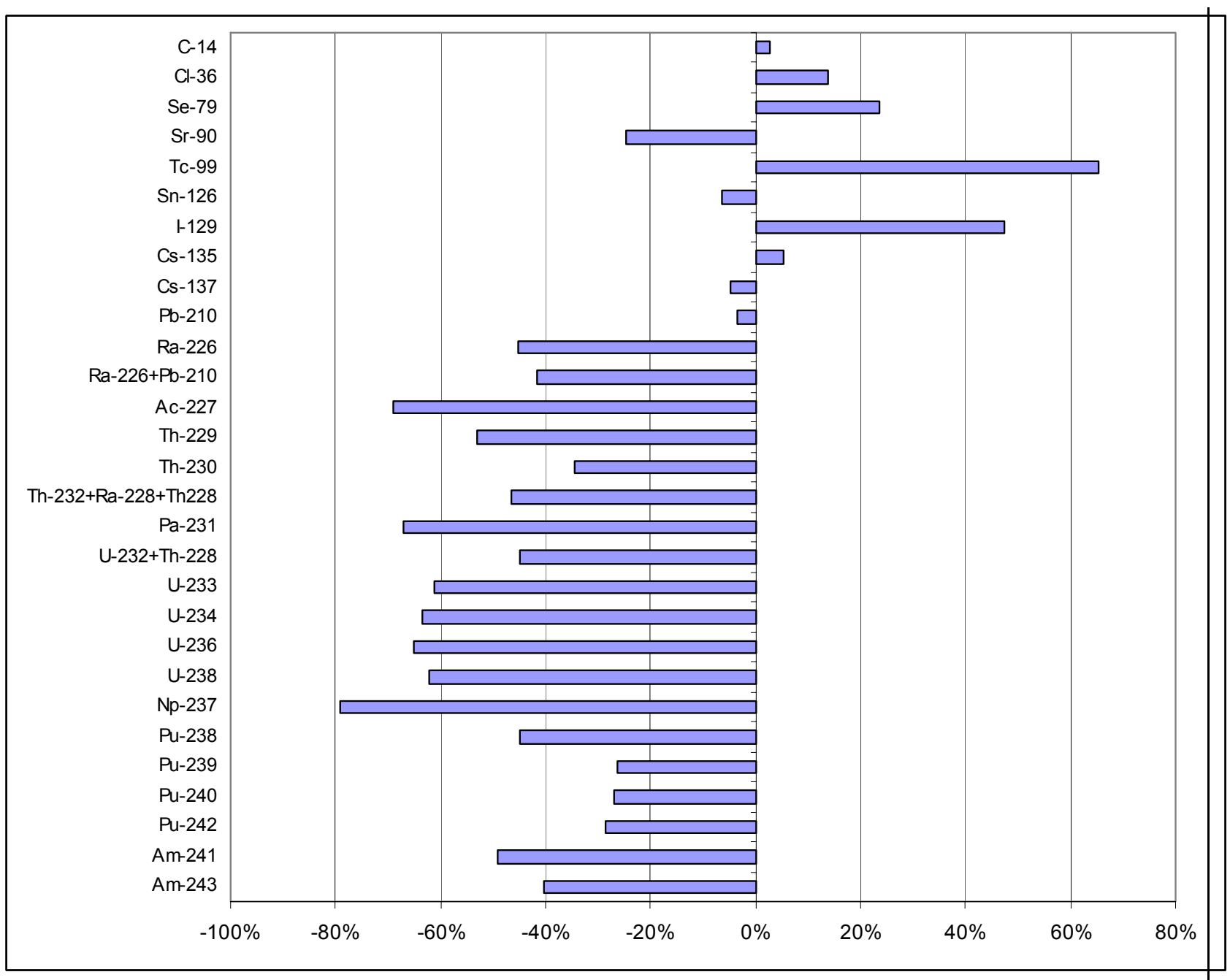

NOTE: The figure shows percent difference relative to the BDCF calculated methods consistent with ICRP 30.

Source: The values were calculated in Excel file GW BDCF Realizations_MC and FC_Rev 4.xls. See Appendix B for details of calculations.

Figure 6.2-2. Comparison of BDCFs for the Present-Day Climate Calculated Using ICRP 30 and ICRP 72 Dosimetric Methods

Some of the BDCFs for the groundwater scenario are correlated (Tables 6.2-14 and 6.2-15). To show correlations between the BDCFs, correlation coefficients for present-day climate BDCFs were calculated for selected radionuclides. These calculations were done using Excel (Correlations for Groundwater BDCFs MC_Rev 3.xls and Correlations for Groundwater BDCFs MC_Rev 4.xls; Appendix B). Certain correlation coefficients in Tables 6.2-14 and 6.2-15 are shown as zero. The lack of correlation was determined by performing a statistical test on the correlation coefficients. The null hypothesis was that the (true) population correlation coefficient is zero. If the calculated value of the correlation coefficient for the sampling distribution is $r$, values of $t$ can be calculated as 


$$
t=\frac{r}{\sqrt{\frac{\left(1-r^{2}\right)}{n-2}}}
$$

where $n$ is the number of data points in the sampling distribution (i.e., 1,000). The $t$-values then can be compared with Student's $t$-values for $n-2$ degrees of freedom (Steel and Torrie 1980 [DIRS 150857], pp. 278 to 279). Values of $t$ calculated for different values of $r$ are listed in Table 6.2-16. The null hypothesis that the population correlation coefficient is equal to zero (no correlation) can be rejected at the 99 percent confidence level if the value of $t$ is less than 2.576 (Lide and Frederikse 1997 [DIRS 103178], p. A-105) because the one-tail area under the probability distribution function for $t$ is equal to 0.995 for $t=2.576$. This corresponds to the value of $r$ equal to 0.0813 . The distribution of $t$ approaches a normal distribution when the degrees of freedom are large, which is the case here. Thus, for correlation coefficients less than 0.0813, the value is set to zero in Tables 6.2-14 and 6.2-15.

The correlations between the BDCFs for different radionuclides are generally the highest for the actinides and ${ }^{226} \mathrm{Ra}$. These radionuclides have similar exposure pathways, similar energies and types of radiation, and their BDCFs depend strongly on radionuclide concentration in surface soil. This latter dependence causes also higher correlations with the actinides for radionuclides that have a significant BDCF contribution from external exposure pathway $\left({ }^{126} \mathrm{Sn}\right.$ and $\left.{ }^{137} \mathrm{Cs}\right)$.

Correlations between the BDCFs are reflected in the TSPA through sampling of the row vectors, as described in Section 6.2.4, rather than through the correlation matrix. 
Table 6.2-14. Correlation Coefficients for the ICRP 30-Based BDCFs for the Present-Day Climate

\begin{tabular}{|c|c|c|c|c|c|c|c|c|c|c|c|c|c|c|}
\hline & C-14 & $\mathrm{Cl}-36$ & Se-79 & Sr-90 & Tc-99 & Sn-126 & I-129 & Cs-135 & Cs-137 & $\mathrm{Pb}-210$ & Ra-226 & Ac-227 & Th-229 & Th-230 \\
\hline C-14 & 1.000 & & & & & & & & & & & & & \\
\hline $\mathrm{Cl}-36$ & 0 & 1.000 & & & & & & & & & & & & \\
\hline Se-79 & 0 & 0 & 1.000 & & & & & & & & & & & \\
\hline Sr-90 & 0 & 0.155 & 0.096 & 1.000 & & & & & & & & & & \\
\hline \begin{tabular}{|l|} 
Tc-99 \\
\end{tabular} & 0 & 0.092 & 0 & 0.284 & 1.000 & & & & & & & & & \\
\hline Sn-126 & 0 & 0.094 & 0 & 0.134 & 0 & 1.000 & & & & & & & & \\
\hline I-129 & 0 & 0.144 & 0.100 & 0.225 & 0.121 & 0 & 1.000 & & & & & & & \\
\hline Cs-135 & 0 & 0 & 0 & 0 & 0 & 0.165 & 0 & 1.000 & & & & & & \\
\hline Cs-137 & 0 & 0 & 0 & 0.201 & 0 & 0 & 0 & 0.718 & 1.000 & & & & & \\
\hline $\mathrm{Pb}-210$ & 0 & 0 & 0 & 0.093 & 0 & 0 & 0 & 0.153 & 0.175 & 1.000 & & & & \\
\hline Ra-226 & 0 & 0 & 0 & 0.160 & 0 & 0.444 & 0.118 & 0.187 & 0.146 & 0 & 1.000 & & & \\
\hline Ac-227 & 0 & 0 & 0 & 0.118 & 0 & 0 & 0 & 0 & 0.135 & 0 & 0 & 1.000 & & \\
\hline Th-229 & 0 & 0 & 0 & 0.132 & 0 & 0.377 & 0 & 0.222 & 0.131 & 0 & 0.415 & 0.350 & 1.000 & \\
\hline Th-230 & 0 & 0 & 0 & 0 & 0 & 0.490 & 0.094 & 0.229 & 0 & 0 & 0.653 & 0 & 0.609 & 1.000 \\
\hline Th-232 & 0 & 0 & 0 & 0.129 & 0 & 0.443 & 0.085 & 0.240 & 0.117 & 0 & 0.483 & 0.274 & 0.980 & 0.723 \\
\hline $\mathrm{Pa}-231$ & 0 & 0 & 0 & 0.126 & 0 & 0.406 & 0 & 0.178 & 0 & 0 & 0.405 & 0.188 & 0.801 & 0.514 \\
\hline U-232 & 0 & 0 & 0 & 0.163 & 0 & 0 & 0 & 0 & 0.130 & 0 & 0.145 & 0.713 & 0.390 & 0 \\
\hline U-233 & 0 & 0 & 0 & 0 & 0 & 0.264 & 0 & 0.090 & 0 & 0 & 0.233 & 0.104 & 0.510 & 0.400 \\
\hline U-234 & 0 & 0 & 0 & 0 & 0 & 0.235 & 0 & 0 & 0 & 0 & 0.245 & 0.202 & 0.476 & 0.334 \\
\hline U-236 & 0 & 0 & 0 & 0 & 0 & 0.212 & 0 & 0 & 0 & 0 & 0.219 & 0.232 & 0.472 & 0.270 \\
\hline U-238 & 0 & 0 & 0 & 0 & 0 & 0.215 & 0 & 0 & 0 & 0 & 0.221 & 0.220 & 0.462 & 0.272 \\
\hline Np-237 & 0 & 0.160 & 0.116 & 0.176 & 0.175 & 0.277 & 0.130 & 0.150 & 03 & 0 & 0.193 & 0.377 & 0.469 & 0.288 \\
\hline Pu-238 & 0 & 0 & 0 & 0.200 & 0 & 0.015 & 0 & 0.121 & 0.267 & 0 & 0.164 & 0.687 & 0.457 & 0 \\
\hline Pu-239 & 0 & 0 & 0 & 0.134 & 0 & 0.386 & 0 & 0.208 & 0.100 & 0 & 0.391 & 0.317 & 0.831 & 0.497 \\
\hline \begin{tabular}{|l|}
$\mathrm{Pu}-240$ \\
\end{tabular} & 0 & 0 & 0 & 0.143 & 0 & 0.375 & 0 & 0.206 & 0.114 & 0 & 0.389 & 0.337 & 0.832 & 0.473 \\
\hline Pu-242 & 0 & 0 & 0 & 0.130 & 0 & 0.390 & 0 & 0.208 & 0.095 & 0 & 0.391 & 0.309 & 0.831 & 0.506 \\
\hline Am-241 & 0 & 0 & 0 & 0.237 & 0 & 0.234 & 0.087 & 0.151 & 0.231 & 0 & 0.300 & 0.542 & 0.688 & 0.215 \\
\hline Am-243 & 0 & 0 & 0 & 0.139 & 0 & 0.371 & 0 & 0.185 & 0.117 & 0 & 0.376 & 0.309 & 0.731 & 0.461 \\
\hline
\end{tabular}


Table 6.2-14. Correlations Coefficients for the ICRP 30-Based BDCFs for the Present-Day Climate (Continued)

\begin{tabular}{|c|c|c|c|c|c|c|c|c|c|c|c|c|c|c|}
\hline & Th-232 & Pa-231 & U-232 & U-233 & U-234 & U-236 & U-238 & Np-237 & Pu-238 & Pu-239 & Pu-240 & Pu-242 & Am-241 & Am-243 \\
\hline \multicolumn{15}{|l|}{ C-14 } \\
\hline \multicolumn{15}{|l|}{$\mathrm{Cl}-36$} \\
\hline \multicolumn{15}{|l|}{ Se-79 } \\
\hline \multicolumn{15}{|l|}{ Sr-90 } \\
\hline \multicolumn{15}{|l|}{ Tc-99 } \\
\hline \multicolumn{15}{|l|}{ Sn-126 } \\
\hline \multicolumn{15}{|l|}{ I-129 } \\
\hline \multicolumn{15}{|l|}{ Cs-135 } \\
\hline \multicolumn{15}{|l|}{ Cs-137 } \\
\hline \multicolumn{15}{|l|}{ Pb-210 } \\
\hline \multicolumn{15}{|l|}{ Ra-226 } \\
\hline \multicolumn{15}{|l|}{ Ac-227 } \\
\hline \multicolumn{15}{|l|}{ Th-229 } \\
\hline \multicolumn{15}{|l|}{ Th-230 } \\
\hline Th-232 & 1.000 & & & & & & & & & & & & & \\
\hline $\mathrm{Pa}-231$ & 0.776 & 1.000 & & & & & & & & & & & & \\
\hline U-232 & 0.327 & 0.272 & 1.000 & & & & & & & & & & & \\
\hline U-233 & 0.510 & 0.459 & 0.508 & 1.000 & & & & & & & & & & \\
\hline U-234 & 0.460 & 0.444 & 0.642 & 0.972 & 1.000 & & & & & & & & & \\
\hline U-236 & 0.443 & 0.443 & 0.682 & 0.949 & 0.993 & 1.000 & & & & & & & & \\
\hline U-238 & 0.436 & 0.435 & 0.678 & 0.950 & 0.994 & 1.000 & 1.000 & & & & & & & \\
\hline Np-237 & 0.450 & 0.453 & 0.353 & 0.307 & 0.311 & 0.310 & 0.305 & 1.000 & & & & & & \\
\hline Pu-238 & 0.369 & 0.335 & 0.552 & 0.132 & 0.211 & 0.247 & 0.235 & 0.326 & 1.000 & & & & & \\
\hline Pu-239 & 0.797 & 0.809 & 0.342 & 0.455 & 0.441 & 0.442 & 0.433 & 0.499 & 0.574 & 1.000 & & & & \\
\hline Pu-240 & 0.792 & 0.805 & 0.359 & 0.447 & 0.439 & 0.443 & 0.433 & 0.499 & 0.605 & 0.999 & 1.000 & & & \\
\hline Pu-242 & 0.798 & 0.810 & 0.336 & 0.458 & 0.442 & 0.442 & 0.432 & 0.500 & 0.562 & 1.000 & 0.998 & 1.000 & & \\
\hline $\mathrm{Am}-241$ & 0.615 & 0.633 & 0.485 & 0.314 & 0.352 & 0.378 & 0.366 & 0.459 & 0.607 & 0.669 & 0.685 & 0.662 & 1.000 & \\
\hline
\end{tabular}


Table 6.2-15. Correlation Coefficients for the ICRP 72-Based BDCFs for the Present-Day Climate

\begin{tabular}{|c|c|c|c|c|c|c|c|c|c|c|c|c|c|c|}
\hline & C-14 & $\mathrm{Cl}-36$ & Se-79 & Sr-90 & TC-99 & Sn-126 & |-129 & Cs-135 & Cs-137 & $\mathrm{Pb}-210$ & Ra-226 & Ac-227 & Th-229 & Th-230 \\
\hline C-14 & 1.000 & & & & & & & & & & & & & \\
\hline $\mathrm{Cl}-36$ & 0 & 1.000 & & & & & & & & & & & & \\
\hline Se-79 & 0 & 0 & 1.0000 & & & & & & & & & & & \\
\hline Sr-90 & 0 & 0.156 & 0.097 & 1.000 & & & & & & & & & & \\
\hline Tc-99 & 0 & 0.092 & 0 & 0.284 & 1.000 & & & & & & & & & \\
\hline Sn-126 & 0 & 0.094 & 0 & 0.136 & 0 & 1.000 & & & & & & & & \\
\hline $\mathrm{I}-129$ & 0 & 0.144 & 0.100 & 0.226 & 0.121 & 0 & 1.000 & & & & & & & \\
\hline Cs-135 & 0 & 0 & 0 & 0 & 0 & 0.166 & 0 & 1.000 & & & & & & \\
\hline Cs-137 & 0 & 0 & 0 & 0.204 & 0 & 0 & 0 & 0.721 & 1.000 & & & & & \\
\hline $\mathrm{Pb}-210$ & 0 & 0 & 0 & 0.094 & 0 & 0 & 0 & 0.153 & 0.175 & 1.000 & & & & \\
\hline Ra-226 & 0 & 0 & 0 & 0.167 & 0 & 0.455 & 0.117 & 0.199 & 0.148 & 0 & 1.000 & & & \\
\hline Ac-227 & 0 & 0 & 0 & 0.154 & 0 & 0 & 0 & 0 & 0.137 & 0 & 0.082 & 1.000 & & \\
\hline Th-229 & 0 & 0 & 0 & 0.149 & 0 & 0.385 & 0 & 0.231 & 0.134 & 0 & 0.441 & 0.344 & 1.000 & \\
\hline Th-230 & 0 & 0 & 0 & 0 & 0 & 0.499 & 0.094 & 0.244 & 0 & 0 & 0.650 & 0 & 0.696 & 1.000 \\
\hline Th-232 & 0 & 0 & 0 & 0.149 & 0 & 0.493 & 0.108 & 0.253 & 0.116 & 0 & 0.555 & 0.202 & 0.924 & 0.841 \\
\hline $\mathrm{Pa}-231$ & 0 & 0 & 0 & 0.133 & 0 & 0.408 & 0 & 0.183 & 0 & 0 & 0.427 & 0.193 & 0.799 & 0.578 \\
\hline U-232 & 0 & 0 & 0 & 0.236 & 0 & 0.083 & 0.087 & 0 & 0.155 & 0 & 0.167 & 0.518 & 0.318 & 0.071 \\
\hline U-233 & 0 & 0 & 0 & 0 & 0 & 0.273 & 0 & 0.096 & 0 & 0 & 0.245 & 0 & 0.504 & 0.460 \\
\hline U-234 & 0 & 0 & 0 & 0 & 0 & 0.252 & 0 & 0.086 & 0 & 0 & 0.269 & 0.159 & 0.464 & 0.413 \\
\hline $\mathrm{U}-236$ & 0 & 0 & 0 & 0.082 & 0 & 0.213 & 0 & 0 & 0 & 0 & 0.229 & 0.217 & 0.454 & 0.306 \\
\hline U-238 & 0 & 0 & 0 & 0.081 & 0 & 0.221 & 0 & 0 & 0 & 0 & 0.234 & 0.176 & 0.421 & 0.307 \\
\hline $\mathrm{Np}-237$ & 0 & 0.131 & 0.109 & 0.153 & 0.184 & 0.268 & 0.102 & 0.115 & 0 & 0 & 0.197 & 0.354 & 0.451 & 0.310 \\
\hline Pu-238 & 0 & 0 & 0 & 0.235 & 0 & 0 & 0 & 0.097 & 0.258 & 0 & 0.199 & 0.855 & 0.563 & 0.086 \\
\hline Pu-239 & 0 & 0 & 0 & 0.133 & 0 & 0.394 & 0 & 0.196 & 0.083 & 0 & 0.402 & 0.325 & 0.846 & 0.566 \\
\hline Pu-240 & 0 & 0 & 0 & 0.142 & 0 & 0.384 & 0 & 0.193 & 0.096 & 0 & 0.401 & 0.347 & 0.848 & 0.545 \\
\hline Pu-242 & 0 & 0 & 0 & 0.129 & 0 & 0.397 & 0 & 0.197 & 0 & 0 & 0.402 & 0.317 & 0.844 & 0.574 \\
\hline $\mathrm{Am}-241$ & 0 & 0 & 0 & 0.221 & 0 & 0.224 & 0 & 0.131 & 0.208 & 0 & 0.302 & 0.551 & 0.692 & 0.275 \\
\hline Am-243 & 0 & 0 & 0 & 0.132 & 0 & 0.364 & 0 & 0.176 & 0.105 & 0 & 0.384 & 0.310 & 0.734 & 0.514 \\
\hline
\end{tabular}


Table 6.2-15. Correlation Coefficients for the ICRP 72-Based BDCFs for the Present-Day Climate (Continued)

\begin{tabular}{|c|c|c|c|c|c|c|c|c|c|c|c|c|c|c|}
\hline & Th-232 & Pa-231 & U-232 & U-233 & U-234 & U-236 & U-238 & Np-237 & Pu-238 & Pu-239 & Pu-240 & Pu-242 & Am-241 & Am-243 \\
\hline \multicolumn{15}{|l|}{ C-14 } \\
\hline \multicolumn{15}{|l|}{$\mathrm{Cl}-36$} \\
\hline \multicolumn{15}{|l|}{ Se-79 } \\
\hline \multicolumn{15}{|l|}{ Sr-90 } \\
\hline \multicolumn{15}{|l|}{ Tc-99 } \\
\hline \multicolumn{15}{|l|}{ Sn-126 } \\
\hline \multicolumn{15}{|l|}{ I-129 } \\
\hline \multicolumn{15}{|l|}{ Cs-135 } \\
\hline \multicolumn{15}{|l|}{ Cs-137 } \\
\hline \multicolumn{15}{|l|}{$\mathrm{Pb}-210$} \\
\hline \multicolumn{15}{|l|}{ Ra-226 } \\
\hline \multicolumn{15}{|l|}{ Ac-227 } \\
\hline \multicolumn{15}{|l|}{ Th-229 } \\
\hline \multicolumn{15}{|l|}{ Th-230 } \\
\hline Th-232 & 1.000 & & & & & & & & & & & & & \\
\hline Pa-231 & 0.710 & 1.000 & & & & & & & & & & & & \\
\hline U-232 & 0.235 & 0.232 & 1.000 & & & & & & & & & & & \\
\hline U-233 & 0.483 & 0.449 & 0.490 & 1.000 & & & & & & & & & & \\
\hline $\mathrm{U}-234$ & 0.432 & 0.433 & 0.624 & 0.971 & 1.000 & & & & & & & & & \\
\hline U-236 & 0.381 & 0.430 & 0.705 & 0.922 & 0.976 & 1.000 & & & & & & & & \\
\hline U-238 & 0.369 & 0.403 & 0.705 & 0.924 & 0.980 & 0.995 & 1.000 & & & & & & & \\
\hline Np-237 & 0.395 & 0.446 & 0.270 & 0.292 & 0.296 & 0.292 & 0.274 & 1.000 & & & & & & \\
\hline Pu-238 & 0.362 & 0.431 & 0.542 & 0.148 & 0.229 & 0.305 & 0.257 & 0.394 & 1.000 & & & & & \\
\hline $\mathrm{Pu}-239$ & 0.731 & 0.827 & 0.278 & 0.459 & 0.440 & 0.442 & 0.408 & 0.498 & 0.588 & 1.000 & & & & \\
\hline Pu-240 & 0.725 & 0.826 & 0.294 & 0.451 & 0.436 & 0.444 & 0.409 & 0.498 & 0.617 & 0.999 & 1.000 & & & \\
\hline Pu-242 & 0.733 & 0.827 & 0.272 & 0.462 & 0.441 & 0.441 & 0.407 & 0.497 & 0.577 & 1.000 & 0.998 & 1.000 & & \\
\hline $\mathrm{Am}-241$ & 0.523 & 0.641 & 0.399 & 0.294 & 0.324 & 0.373 & 0.331 & 0.444 & 0.742 & 0.688 & 0.706 & 0.680 & 1.000 & \\
\hline $\mathrm{Am}-243$ & 0.646 & 0.733 & 0.242 & 0.411 & 0.394 & 0.396 & 0.366 & 0.453 & 0.479 & 0.736 & 0.737 & 0.735 & 0.893 & 1.000 \\
\hline
\end{tabular}

Source: MO0407SPAINEXI.002 [DIRS 170597]; MO0407SPASRPBM.002 [DIRS 170755]; MO0306MWDBGSMF.001 [DIRS 163816]; MO0403SPAAEIBM.002 [DIRS 169392]; MO0407SPACRBSM.002 [DIRS 170677]; MO0406SPAETPBM.002 [DIRS 170150], MO0503SPADCESR.000 [DIRS 172896]

NOTE: See Excel file Correlations for Groundwater BDCFs MC_Rev 4.xls in Appendix B for details of calculations. 
Table 6.2-16. Calculated Values of Correlation Coefficient and Variable $t$

\begin{tabular}{|c|c|}
\hline Calculated Correlation Coefficient, $r$ & $\mathbf{t}$ \\
\hline 0.0000 & 0.000 \\
\hline 0.0100 & 0.316 \\
\hline 0.0200 & 0.632 \\
\hline 0.0300 & 0.948 \\
\hline 0.0400 & 1.265 \\
\hline 0.0500 & 1.582 \\
\hline 0.0600 & 1.899 \\
\hline 0.0610 & 1.931 \\
\hline 0.0620 & 1.962 \\
\hline 0.0630 & 1.994 \\
\hline 0.0700 & 2.217 \\
\hline 0.0780 & 2.472 \\
\hline 0.0790 & 2.504 \\
\hline 0.0800 & 2.535 \\
\hline 0.0810 & 2.567 \\
\hline 0.0812 & 2.574 \\
\hline 0.0813 & 2.577 \\
\hline 0.0820 & 2.599 \\
\hline 0.1000 & 3.175 \\
\hline 0.1200 & 3.819 \\
\hline 0.1400 & 4.467 \\
\hline 0.1600 & 5.121 \\
\hline 0.1800 & 5.781 \\
\hline 0.2000 & 6.449 \\
\hline
\end{tabular}

NOTE: $\quad$ See Excel file Correlations for Groundwater BDCFs MC_Rev 3.xIs or Correlations for Groundwater BDCFs MC_Rev 4.XIs in Appendix B for details of calculations.

\subsubsection{TSPA Use of Biosphere Dose Conversion Factors}

The assessment of annual doses will be carried out in the TSPA-LA model, which uses BDCFs as input parameters. The TSPA-LA model calculates annual fluxes of individual radionuclides at a specified distance from the repository, which, when divided by the annual water demand, yield radionuclide concentrations in the groundwater used by the receptor. The total annual dose is the sum of the annual doses from the 28 radionuclides tracked in the TSPA-LA model. The total annual dose is calculated (BSC 2004 [DIRS 169460], Section 6.4.10.4) as

$$
D_{\text {total }}(t)=\sum_{i} B D C F_{i} \times C w_{i}(t)
$$

where

$$
\begin{aligned}
D_{\text {total }}(t)= & \text { time-dependent total annual dose to a defined receptor resulting from the release of } \\
& \text { radionuclides from the repository; includes contributions from all radionuclides } \\
& \text { considered in the TSPA-LA (mrem/yr) } \\
B D C F_{i}= & \text { biosphere dose conversion factor for radionuclide } i(\mathrm{mrem} / \mathrm{yr} \text { per pCi/L) }
\end{aligned}
$$




$$
C w_{i}(t) \quad=\text { time-dependent activity concentration of radionuclide } i \text { in the groundwater }(\mathrm{pCi} / \mathrm{L}) \text {. }
$$

Equation 6.2-5 is based on a linear relationship between groundwater concentrations and dose. The BDCFs are calculated for constant activity concentration of radionuclides in the soil, which occurs after the equilibrium concentration of radionuclides in soil has been reached. Equilibrium conditions are reached if irrigation is sustained for a sufficiently long period of time, which varies among radionuclides from years to thousands of years, depending on the effective removal rate of the radionuclide from soil.

Some radionuclides, such as isotopes of thorium, plutonium, and americium, have low removal rates from soils. These radionuclides build up slowly in the soil and it may take on the order of thousands of years to reach equilibrium concentrations. In the event that equilibrium conditions for a radionuclide have not been reached and groundwater concentrations are increasing, this approximation will result in overestimating the dose for that radionuclide (BSC 2004 [DIRS 169460], Section 6.3.1.4). If concentrations in groundwater are decreasing, the dose may be underestimated (BSC 2004 [DIRS 169460], Section 6.3.1.4). However, it is unlikely that groundwater concentrations will decrease until long after the period of 20,000 years used for the TSPA-LA. The annual dose for a point in time, $t$, is calculated using the activity concentration in water at time $t, C w(t)$. The product of the radionuclide concentration and the BDCF represents the dose that would prevail if the radionuclide concentration in water, $C w(t)$, persisted prior to time, $t$, long enough for this radionuclide to reach equilibrium in the soil.

\subsubsection{Pathway Analysis}

Pathway analysis was conducted to determine the relative importance of individual exposure pathways in terms of contributions to BDCFs for various radionuclides. The biosphere model explicitly addresses 15 exposure pathways, and percent contributions to the BDCF were calculated using the mean values of the BDCFs. The results for the present-day climate BDCFs calculated using methods consistent with ICRP 30 are presented in Table 6.2-17. These calculations were performed using Excel (GW BDCF Pathway Analysis MC_Rev 3.xls; Appendix B).

Pathway contributions differ among radionuclides. Inhalation of particulate matter tends to dominate doses for actinides (e.g., isotopes of thorium, uranium, plutonium, and americium). Inhalation of radioactive aerosols generated by evaporative coolers also is an important inhalation exposure pathway for these radionuclides. Ingestion of water is a consistently high contributor to dose. Other pathways are only important for a few radionuclides. For instance, external exposure is a dominant pathway for ${ }^{126} \mathrm{Sn}$ and ${ }^{137} \mathrm{Cs}$, inhalation of radon decay products for ${ }^{226} \mathrm{Ra}$ and ${ }^{230} \mathrm{Th}$, and fish consumption for ${ }^{14} \mathrm{C}$, isotopes of cesium, and ${ }^{210} \mathrm{~Pb}$. Ingestion pathways generally are more important for radionuclides with atomic numbers less than about 88 .

For the future climate, the importance of the evaporative cooler pathway is greatly reduced, and for most radionuclides, the inhalation of particulate matter and the consumption of water are dominant pathways (Table 6.2-18). These calculations were performed using Excel (GW BDCF | Pathway Analysis FC_Rev 3.xls; Appendix B). 
Table 6.2-17. Exposure Pathway Contributions (Percent) for the ICRP 30-Based BDCFs for the Present-Day Climate

\begin{tabular}{|c|c|c|c|c|c|c|c|c|c|c|c|c|c|c|c|}
\hline \multirow[b]{2}{*}{$\begin{array}{l}\text { Radio- } \\
\text { nuclide }\end{array}$} & \multirow[b]{2}{*}{$\begin{array}{c}\text { External } \\
\text { exposure }\end{array}$} & \multicolumn{3}{|c|}{ Inhalation } & \multicolumn{11}{|c|}{ Ingestion } \\
\hline & & $\begin{array}{c}\text { Particul. } \\
\text { Matter }\end{array}$ & $\begin{array}{l}\text { Evap. } \\
\text { Cooler }\end{array}$ & Radon & Water & $\begin{array}{l}\text { Leafy } \\
\text { Veget. }\end{array}$ & $\begin{array}{l}\text { Other } \\
\text { Veget. }\end{array}$ & Fruit & Grain & Meat & Milk & Poultry & Eggs & Fish & Soil \\
\hline C-14 & $\begin{array}{l}0.0 \\
\end{array}$ & 0.0 & 0.0 & 0.0 & 16.8 & 2.8 & 5.0 & 13.0 & 0.6 & 7.0 & 3.3 & 0.6 & 5.5 & 45.3 & 0.0 \\
\hline $\mathrm{Cl}-36$ & 0.1 & 0.0 & 0.2 & 0.0 & 10.7 & 3.2 & 4.9 & 16.5 & 2.6 & 22.7 & 18.5 & 0.0 & 9.0 & 11.4 & 0.0 \\
\hline Se-79 & 0.0 & 0.1 & 0.0 & 0.0 & 10.3 & 0.7 & 0.5 & 1.6 & 0.1 & 55.1 & 3.2 & 2.0 & 21.9 & 3.8 & 0.6 \\
\hline Sr-90 & 0.5 & 0.1 & 0.3 & 0.0 & 68.2 & 6.6 & 3.7 & 6.2 & 0.6 & 3.0 & 3.7 & 0.0 & 1.6 & 5.1 & 0.4 \\
\hline Tc-99 & 0.0 & 0.0 & 0.7 & 0.0 & 47.2 & 12.0 & 2.1 & 7.7 & 0.5 & 4.3 & 13.5 & 0.2 & 10.0 & 1.6 & 0.0 \\
\hline Sn-126 & 98.2 & 0.0 & 0.0 & 0.0 & 0.2 & 0.0 & 0.0 & 0.0 & 0.0 & 0.4 & 0.0 & 0.0 & 0.1 & 1.0 & 0.0 \\
\hline I-129 & 0.1 & 0.1 & 0.1 & 0.0 & 59.9 & 2.6 & 0.7 & 3.0 & 0.4 & 5.0 & 7.5 & 0.2 & 14.2 & 5.4 & 0.8 \\
\hline Cs-135 & 0.0 & 0.1 & 0.0 & 0.0 & 9.3 & 1.4 & 0.9 & 4.2 & 0.2 & 10.3 & 5.8 & 7.3 & 5.5 & 54.0 & 1.1 \\
\hline Cs-137 & 43.0 & 0.0 & 0.0 & 0.0 & 7.6 & 0.4 & 0.1 & 0.5 & 0.0 & 1.7 & 1.0 & 0.9 & 0.7 & 44.1 & 0.1 \\
\hline Pb-210 & 0.0 & 0.2 & 0.5 & 0.0 & 58.8 & 2.6 & 0.6 & 2.3 & 0.3 & 0.3 & 0.2 & 0.1 & 1.4 & 32.4 & 0.4 \\
\hline Ra-226 & 6.8 & 0.3 & 0.0 & 89.2 & 1.0 & 0.3 & 0.2 & 0.6 & 0.0 & 0.1 & 0.1 & 0.0 & 0.7 & 0.1 & 0.6 \\
\hline Ac-227 & 0.3 & 16.8 & 42.6 & 0.0 & 34.7 & 1.4 & 0.3 & 1.2 & 0.1 & 0.1 & 0.0 & 0.0 & 0.0 & 2.2 & 0.2 \\
\hline Th-229 & 3.0 & 71.9 & 12.5 & 0.0 & 8.6 & 0.5 & 0.1 & 0.4 & 0.0 & 0.1 & 0.0 & 0.0 & 0.0 & 1.8 & 0.9 \\
\hline Th-230 & 5.7 & 13.6 & 2.2 & 74.5 & 1.4 & 0.3 & 0.1 & 0.6 & 0.0 & 0.1 & 0.1 & 0.0 & 0.6 & 0.3 & 0.6 \\
\hline Th-232 & 25.0 & 53.9 & 8.8 & 0.0 & 8.2 & 0.7 & 0.2 & 0.6 & 0.1 & 0.1 & 0.1 & 0.0 & 0.0 & 1.5 & 0.9 \\
\hline Pa-231 & 1.3 & 84.7 & 2.5 & 0.0 & 7.7 & 0.6 & 0.2 & 0.8 & 0.1 & 0.1 & 0.0 & 0.0 & 0.0 & 0.2 & 1.8 \\
\hline U-232 & 12.3 & 20.6 & 33.9 & 0.0 & 26.6 & 1.1 & 0.3 & 0.9 & 0.1 & 0.1 & 0.1 & 0.2 & 0.8 & 2.7 & 0.3 \\
\hline U-233 & 1.3 & 68.3 & 14.3 & 0.0 & 11.4 & 0.6 & 0.2 & 0.6 & 0.1 & 0.1 & 0.1 & 0.2 & 1.6 & 0.3 & 0.9 \\
\hline U-234 & 0.4 & 52.3 & 20.4 & 4.9 & 16.2 & 0.8 & 0.2 & 0.7 & 0.1 & 0.1 & 0.1 & 0.3 & 2.2 & 0.5 & 0.7 \\
\hline U-236 & 0.0 & 54.9 & 21.7 & 0.0 & 17.3 & 0.8 & 0.2 & 0.8 & 0.1 & 0.1 & 0.2 & 0.4 & 2.3 & 0.5 & 0.8 \\
\hline U-238 & 3.7 & 52.1 & 20.6 & 0.0 & 17.3 & 0.8 & 0.2 & 0.8 & 0.1 & 0.1 & 0.2 & 0.4 & 2.4 & 0.5 & 0.8 \\
\hline Np-237 & 2.6 & 22.5 & 15.2 & 0.0 & 46.5 & 2.6 & 1.0 & 4.3 & 0.2 & 0.9 & 0.0 & 0.0 & 0.0 & 3.0 & 1.2 \\
\hline Pu-238 & 0.0 & 20.6 & 16.4 & 0.0 & 49.8 & 2.1 & 0.5 & 1.7 & 0.2 & 0.0 & 0.0 & 0.0 & 0.0 & 7.3 & 1.1 \\
\hline Pu-239 & 0.0 & 52.5 & 9.1 & 0.0 & 27.9 & 1.5 & 0.3 & 1.3 & 0.2 & 0.0 & 0.0 & 0.0 & 0.1 & 4.1 & 2.9 \\
\hline Pu-240 & 0.0 & 51.5 & 9.4 & 0.0 & 28.7 & 1.5 & 0.3 & 1.3 & 0.2 & 0.0 & 0.0 & 0.0 & 0.1 & 4.2 & 2.8 \\
\hline Pu-242 & 0.0 & 53.1 & 9.1 & 0.0 & 27.5 & 1.5 & 0.3 & 1.3 & 0.2 & 0.0 & 0.0 & 0.0 & 0.1 & 4.1 & 2.9 \\
\hline Am-241 & 0.1 & 38.2 & 12.8 & 0.0 & 39.0 & 1.8 & 0.4 & 1.6 & 0.2 & 0.1 & 0.0 & 0.0 & 0.0 & 3.7 & 2.1 \\
\hline Am-243 & 5.6 & 49.0 & 9.1 & 0.0 & 27.7 & 1.5 & 0.3 & 1.3 & 0.1 & 0.1 & 0.0 & 0.0 & 0.0 & 2.6 & 2.7 \\
\hline
\end{tabular}
[DIRS 169392]; MO0407SPACRBSM.002 [DIRS 170677]; MO0406SPAETPBM.002 [DIRS 170150].

NOTES: See Excel file GW BDCF Pathway Analysis MC_Rev 3.xls in Appendix B for details of calculations.

Particul. $=$ Particulate; Evap. $=$ Evaporative; Veget. $=$ Vegetables 
Table 6.2-18. Exposure Pathway Contributions (Percent) for the ICRP 30-Based BDCFs for the Upper Bound of Glacial Transition Climate

\begin{tabular}{|c|c|c|c|c|c|c|c|c|c|c|c|c|c|c|c|}
\hline \multirow[b]{2}{*}{$\begin{array}{l}\text { Radio- } \\
\text { nuclide }\end{array}$} & \multirow[b]{2}{*}{$\begin{array}{c}\text { External } \\
\text { exposure }\end{array}$} & \multicolumn{3}{|c|}{ Inhalation } & \multicolumn{11}{|c|}{ Ingestion } \\
\hline & & $\begin{array}{c}\text { Particul. } \\
\text { Matter }\end{array}$ & $\begin{array}{l}\text { Evap. } \\
\text { Cooler }\end{array}$ & Radon & Water & $\begin{array}{l}\text { Leafy } \\
\text { Veget. }\end{array}$ & $\begin{array}{l}\text { Other } \\
\text { Veget. }\end{array}$ & Fruit & Grain & Meat & Milk & Poultry & Eggs & Fish & Soil \\
\hline $\mathrm{C}-14$ & 0.0 & 0.0 & 0.0 & 0.0 & 17.9 & 2.9 & 3.7 & 10.1 & 0.7 & 6.4 & 3.1 & 0.7 & 6.2 & 48.4 & 0.0 \\
\hline $\mathrm{Cl}-36$ & 0.1 & 0.0 & 0.1 & 0.0 & 14.3 & 3.2 & 4.8 & 16.2 & 2.5 & 22.1 & 17.9 & 0.0 & 10.0 & 8.8 & 0.0 \\
\hline Se-79 & 0.0 & 0.1 & 0.0 & 0.0 & 16.0 & 0.7 & 0.5 & 1.5 & 0.1 & 51.8 & 3.0 & 1.8 & 20.4 & 3.4 & 0.6 \\
\hline Sr-90 & 0.3 & 0.1 & 0.1 & 0.0 & 78.4 & 4.8 & 2.4 & 3.9 & 0.4 & 2.1 & 2.6 & 0.0 & 1.2 & 3.4 & 0.2 \\
\hline Tc-99 & 0.0 & 0.0 & 0.2 & 0.0 & 55.3 & 10.5 & 1.8 & 6.2 & 0.4 & 3.7 & 11.6 & 0.2 & 9.0 & 1.1 & 0.0 \\
\hline Sn-126 & 98.0 & 0.0 & 0.0 & 0.0 & 0.4 & 0.0 & 0.0 & 0.0 & 0.0 & 0.4 & 0.0 & 0.0 & 0.1 & 1.0 & 0.0 \\
\hline $\mathrm{I}-129$ & 0.1 & 0.1 & 0.0 & 0.0 & 69.3 & 2.1 & 0.5 & 1.9 & 0.3 & 3.9 & 5.8 & 0.2 & 11.6 & 3.6 & 0.6 \\
\hline Cs-135 & 0.0 & 0.1 & 0.0 & 0.0 & 15.2 & 1.3 & 0.8 & 3.7 & 0.2 & 9.4 & 5.3 & 6.6 & 5.0 & 51.4 & 1.0 \\
\hline Cs-137 & 38.4 & 0.0 & 0.0 & 0.0 & 12.8 & 0.4 & 0.1 & 0.5 & 0.0 & 1.8 & 1.0 & 0.9 & 0.7 & 43.3 & 0.1 \\
\hline $\mathrm{Pb}-210$ & 0.0 & 0.1 & 0.1 & 0.0 & 71.0 & 2.2 & 0.4 & 1.4 & 0.2 & 0.2 & 0.1 & 0.0 & 1.2 & 22.6 & 0.3 \\
\hline Ra-226 & 6.1 & 0.3 & 0.0 & 89.5 & 1.7 & 0.3 & 0.1 & 0.5 & 0.0 & 0.1 & 0.1 & 0.0 & 0.6 & 0.1 & 0.5 \\
\hline Ac-227 & 0.3 & 15.8 & 16.4 & 0.0 & 61.6 & 1.8 & 0.3 & 1.0 & 0.2 & 0.1 & 0.0 & 0.0 & 0.0 & 2.3 & 0.2 \\
\hline Th-229 & 3.0 & 71.8 & 5.0 & 0.0 & 16.0 & 0.6 & 0.1 & 0.4 & 0.1 & 0.1 & 0.0 & 0.0 & 0.1 & 2.0 & 0.9 \\
\hline Th-230 & 5.3 & 12.4 & 0.8 & 76.6 & 2.3 & 0.3 & 0.1 & 0.5 & 0.0 & 0.1 & 0.1 & 0.0 & 0.5 & 0.3 & 0.6 \\
\hline Th-232 & 24.5 & 52.8 & 3.5 & 0.0 & 14.9 & 0.8 & 0.2 & 0.5 & 0.1 & 0.1 & 0.1 & 0.0 & 0.0 & 1.6 & 0.9 \\
\hline $\mathrm{Pa}-231$ & 1.3 & 80.6 & 1.0 & 0.0 & 13.4 & 0.7 & 0.2 & 0.7 & 0.1 & 0.1 & 0.0 & 0.0 & 0.0 & 0.2 & 1.7 \\
\hline U-232 & 12.1 & 20.2 & 13.2 & 0.0 & 47.4 & 1.4 & 0.2 & 0.9 & 0.1 & 0.1 & 0.1 & 0.2 & 1.0 & 2.7 & 0.3 \\
\hline U-233 & 1.4 & 68.3 & 5.5 & 0.0 & 20.0 & 0.7 & 0.1 & 0.5 & 0.1 & 0.1 & 0.1 & 0.3 & 1.7 & 0.3 & 0.9 \\
\hline U-234 & 0.4 & 51.7 & 7.8 & 5.6 & 28.5 & 0.9 & 0.2 & 0.7 & 0.1 & 0.1 & 0.2 & 0.4 & 2.3 & 0.5 & 0.7 \\
\hline U-236 & 0.0 & 54.6 & 8.3 & 0.0 & 30.5 & 1.0 & 0.2 & 0.7 & 0.1 & 0.1 & 0.2 & 0.4 & 2.5 & 0.5 & 0.8 \\
\hline U-238 & 3.7 & 51.5 & 7.9 & 0.0 & 30.4 & 1.0 & 0.2 & 0.7 & 0.1 & 0.1 & 0.2 & 0.4 & 2.5 & 0.5 & 0.8 \\
\hline Np-237 & 2.2 & 18.8 & 4.5 & 0.0 & 63.3 & 2.4 & 0.8 & 3.5 & 0.2 & 0.8 & 0.0 & 0.0 & 0.0 & 2.4 & 1.0 \\
\hline Pu-238 & 0.0 & 15.2 & 5.0 & 0.0 & 69.1 & 2.1 & 0.3 & 1.2 & 0.2 & 0.0 & 0.0 & 0.0 & 0.0 & 5.9 & 0.8 \\
\hline Pu-239 & 0.0 & 44.3 & 3.1 & 0.0 & 43.3 & 1.6 & 0.3 & 1.0 & 0.2 & 0.0 & 0.0 & 0.0 & 0.1 & 3.7 & 2.5 \\
\hline Pu-240 & 0.0 & 43.2 & 3.1 & 0.0 & 44.3 & 1.6 & 0.3 & 1.0 & 0.2 & 0.0 & 0.0 & 0.0 & 0.1 & 3.8 & 2.4 \\
\hline Pu-242 & 0.0 & 44.9 & 3.1 & 0.0 & 42.8 & 1.5 & 0.3 & 1.0 & 0.2 & 0.0 & 0.0 & 0.0 & 0.1 & 3.6 & 2.5 \\
\hline Am-241 & 0.1 & 30.2 & 4.1 & 0.0 & 57.2 & 1.8 & 0.3 & 1.2 & 0.2 & 0.1 & 0.0 & 0.0 & 0.0 & 3.1 & 1.7 \\
\hline Am-243 & 4.7 & 41.3 & 3.1 & 0.0 & 43.2 & 1.5 & 0.3 & 1.0 & 0.2 & 0.1 & 0.0 & 0.0 & 0.0 & 2.3 & 2.3 \\
\hline Source: & $\begin{array}{l}\text { MO0407SP } \\
\text { [DIRS } 1693\end{array}$ & $\begin{array}{l}\text { AINEXI.00 } \\
92] ; \text { MO04 }\end{array}$ & $\begin{array}{l}2 \text { [DIRS } 1 \\
07 \text { SPACF }\end{array}$ & $\begin{array}{l}\text { 70597]; N } \\
\text { BSM.002 }\end{array}$ & $\begin{array}{l}\text { D407SF } \\
\text { DIRS } 17\end{array}$ & $\begin{array}{l}\text { ASRPBM } \\
\text { 677]; MC }\end{array}$ & $\begin{array}{l}002 \text { [DIR } \\
0406 \text { SPA }\end{array}$ & $\begin{array}{l}170755 \\
\text { TPBM. }\end{array}$ & $\begin{array}{l}\text { MO0306 } \\
2 \text { [DIRS }\end{array}$ & $\begin{array}{l}\text { WDBGS } \\
70150] .\end{array}$ & F.001 & RS 1638 & ]; $\mathrm{MOO}$ & 3SPAA & M.002 \\
\hline
\end{tabular}


The results for the present-day climate BDCFs calculated using methods consistent with ICRP 72 are presented in Table 6.2-19. These calculations were performed using Excel (GW BDCF Pathway Analysis MC_Rev 4.xls; Appendix B).

Analogous to the case of ICRP 30-based dosimetry, pathway contributions differ among radionuclides for the BDCFs calculated using ICRP 72 dosimetric methods. Inhalation of particulate matter dominates doses for actinides to an even greater degree than for the ICRP 30based dosimetry. Inhalation of radioactive aerosols generated by evaporative coolers also is an important inhalation exposure pathway for these radionuclides. Ingestion of water is a consistently high contributor to dose. Generally, inhalation pathway tends to be more important for the actinides for the BDCFs calculated using ICRP 72 dosimetry, compared with the older dosimetry. This is in part due to the decreased absorption of these radionuclides from the alimentary tract and the resulting overall decrease in the ingestion pathway contribution. Pathways other than inhalation of particulate matter and evaporative cooler aerosols and well as ingestion of water are only important for a few radionuclides. For instance, external exposure is a dominant pathway for ${ }^{126} \mathrm{Sn}$ and ${ }^{137} \mathrm{Cs}$, inhalation of radon decay products for ${ }^{226} \mathrm{Ra}$ and ${ }^{230} \mathrm{Th}$, and fish consumption for ${ }^{14} \mathrm{C}$, isotopes of cesium, and ${ }^{210} \mathrm{~Pb}$. Ingestion pathways generally are more important for radionuclides with atomic numbers less than that of radium.

For the future climate, the importance of the evaporative cooler pathway is greatly reduced, and for most radionuclides, the inhalation of particulate matter and the consumption of water are dominant pathways (Table 6.2-20). These calculations were performed using Excel (GW BDCF Pathway Analysis FC_Rev 4.xls; Appendix B). 
Table 6.2-19. Exposure Pathway Contributions (Percent) for the ICRP 72-Based BDCFs for the Present-Day Climate

\begin{tabular}{|c|c|c|c|c|c|c|c|c|c|c|c|c|c|c|c|}
\hline \multirow[b]{2}{*}{$\begin{array}{l}\text { Radio- } \\
\text { nuclide }\end{array}$} & \multirow[b]{2}{*}{$\begin{array}{l}\text { External } \\
\text { exposure }\end{array}$} & \multicolumn{3}{|c|}{ Inhalation } & \multicolumn{11}{|c|}{ Ingestion } \\
\hline & & $\begin{array}{c}\text { Particul. } \\
\text { Matter }\end{array}$ & $\begin{array}{l}\text { Evap. } \\
\text { Cooler }\end{array}$ & Radon & Water & $\begin{array}{l}\text { Leafy } \\
\text { Veget. }\end{array}$ & $\begin{array}{l}\text { Other } \\
\text { Veget. }\end{array}$ & Fruit & Grain & Meat & Milk & Poultry & Eggs & Fish & Soil \\
\hline C-14 & 0.0 & 0.0 & 0.0 & 0.0 & 16.8 & 2.8 & 5.0 & 13.0 & 0.6 & 7.0 & 3.3 & 0.6 & 5.5 & 45.3 & 0.0 \\
\hline $\mathrm{Cl}-36$ & 0.1 & 0.0 & 0.2 & 0.0 & 10.7 & 3.2 & 4.9 & 16.5 & 2.6 & 22.7 & 18.5 & 0.0 & 9.0 & 11.4 & 0.0 \\
\hline Se-79 & 0.0 & 0.2 & 0.1 & 0.0 & 10.2 & 0.7 & 0.5 & 1.6 & 0.1 & 55.0 & 3.2 & 2.0 & 21.9 & 3.8 & 0.6 \\
\hline Sr-90 & 1.1 & 0.3 & 1.0 & 0.0 & 67.2 & 6.5 & 3.6 & 6.1 & 0.6 & 3.0 & 3.7 & 0.0 & 1.6 & 5.0 & 0.4 \\
\hline Tc-99 & 0.0 & 0.1 & 2.5 & 0.0 & 46.3 & 11.8 & 2.1 & 7.6 & 0.5 & 4.3 & 13.3 & 0.2 & 9.8 & 1.5 & 0.0 \\
\hline Sn-126 & 98.3 & 0.0 & 0.0 & 0.0 & 0.2 & 0.0 & 0.0 & 0.0 & 0.0 & 0.3 & 0.0 & 0.0 & 0.1 & 1.0 & 0.0 \\
\hline $\mid-129$ & 0.1 & 0.0 & 0.1 & 0.0 & 60.0 & 2.6 & 0.7 & 3.0 & 0.4 & 5.0 & 7.5 & 0.2 & 14.2 & 5.4 & 0.8 \\
\hline Cs-135 & 0.0 & 0.7 & 0.1 & 0.0 & 9.2 & 1.4 & 0.9 & 4.2 & 0.2 & 10.2 & 5.7 & 7.2 & 5.5 & 53.6 & 1.1 \\
\hline Cs-137 & 42.3 & 0.0 & 0.1 & 0.0 & 7.7 & 0.4 & 0.1 & 0.5 & 0.0 & 1.7 & 1.0 & 0.9 & 0.7 & 44.6 & 0.1 \\
\hline $\mathrm{Pb}-210$ & 0.0 & 0.3 & 0.8 & 0.0 & 58.5 & 2.5 & 0.6 & 2.3 & 0.2 & 0.3 & 0.2 & 0.1 & 1.4 & 32.2 & 0.4 \\
\hline Ra-226 & 11.7 & 1.4 & 0.1 & 80.8 & 1.4 & 0.5 & 0.3 & 1.0 & 0.1 & 0.1 & 0.1 & 0.0 & 1.2 & 0.2 & 1.0 \\
\hline Ac-227 & 0.9 & 16.9 & 42.9 & 0.0 & 33.9 & 1.4 & 0.3 & 1.1 & 0.1 & 0.1 & 0.0 & 0.0 & 0.0 & 2.2 & 0.2 \\
\hline Th-229 & 6.0 & 67.2 & 11.7 & 0.0 & 10.4 & 0.6 & 0.1 & 0.5 & 0.1 & 0.1 & 0.0 & 0.0 & 0.1 & 2.2 & 1.1 \\
\hline Th-230 & 8.3 & 24.0 & 3.8 & 56.6 & 3.0 & 0.5 & 0.2 & 0.8 & 0.1 & 0.1 & 0.1 & 0.0 & 0.9 & 0.6 & 1.0 \\
\hline Th-232 & 44.2 & 31.7 & 5.2 & 0.0 & 12.1 & 1.3 & 0.6 & 1.1 & 0.1 & 0.2 & 0.3 & 0.0 & 0.0 & 1.8 & 1.3 \\
\hline Pa-231 & 3.7 & 84.3 & 3.1 & 0.0 & 5.8 & 0.5 & 0.2 & 0.6 & 0.1 & 0.1 & 0.0 & 0.0 & 0.0 & 0.1 & 1.6 \\
\hline U-232 & 21.2 & 11.2 & 18.3 & 0.0 & 39.8 & 1.7 & 0.4 & 1.4 & 0.2 & 0.2 & 0.2 & 0.3 & 1.4 & 3.4 & 0.4 \\
\hline U-233 & 3.2 & 60.5 & 9.7 & 0.0 & 19.1 & 1.0 & 0.3 & 1.0 & 0.1 & 0.1 & 0.2 & 0.4 & 2.6 & 0.6 & 1.4 \\
\hline U-234 & 1.0 & 39.2 & 14.6 & 6.6 & 28.3 & 1.4 & 0.4 & 1.3 & 0.1 & 0.2 & 0.3 & 0.6 & 3.8 & 0.9 & 1.3 \\
\hline U-236 & 0.0 & 40.5 & 16.0 & 0.0 & 32.1 & 1.5 & 0.4 & 1.4 & 0.2 & 0.2 & 0.3 & 0.7 & 4.4 & 1.0 & 1.4 \\
\hline U-238 & 10.2 & 34.6 & 13.7 & 0.0 & 30.7 & 1.5 & 0.4 & 1.4 & 0.1 & 0.2 & 0.3 & 0.6 & 4.2 & 0.9 & 1.4 \\
\hline Np-237 & 11.5 & 36.9 & 25.1 & 0.0 & 20.6 & 1.1 & 0.4 & 1.9 & 0.1 & 0.4 & 0.0 & 0.0 & 0.0 & 1.3 & 0.5 \\
\hline Pu-238 & 0.0 & 38.7 & 30.9 & 0.0 & 24.0 & 1.0 & 0.2 & 0.8 & 0.1 & 0.0 & 0.0 & 0.0 & 0.0 & 3.5 & 0.5 \\
\hline Pu-239 & 0.0 & 73.6 & 12.8 & 0.0 & 9.9 & 0.5 & 0.1 & 0.5 & 0.1 & 0.0 & 0.0 & 0.0 & 0.0 & 1.5 & 1.0 \\
\hline Pu-240 & 0.0 & 72.7 & 13.2 & 0.0 & 10.2 & 0.5 & 0.1 & 0.5 & 0.1 & 0.0 & 0.0 & 0.0 & 0.0 & 1.5 & 1.0 \\
\hline Pu-242 & 0.0 & 73.5 & 12.6 & 0.0 & 10.2 & 0.6 & 0.1 & 0.5 & 0.1 & 0.0 & 0.0 & 0.0 & 0.0 & 1.5 & 1.1 \\
\hline Am-241 & 0.2 & 60.1 & 20.1 & 0.0 & 15.6 & 0.7 & 0.2 & 0.6 & 0.1 & 0.0 & 0.0 & 0.0 & 0.0 & 1.5 & 0.8 \\
\hline Am-243 & \begin{tabular}{|l|}
8.6 \\
\end{tabular} & 66.6 & 12.3 & 0.0 & 9.5 & 0.5 & 0.1 & 0.4 & 0.1 & 0.0 & 0.0 & 0.0 & 0.0 & 0.9 & 0.9 \\
\hline Source: & \multicolumn{15}{|c|}{$\begin{array}{l}\text { MO0407SPAINEXI.002 [DIRS 170597]; MO0407SPASRPBM.002 [DIRS 170755]; MO0306MWDBGSMF.001 [DIRS 163816]; MO0403SPAAEIBM.002 } \\
\text { [DIRS 169392]; MO0407SPACRBSM.002 [DIRS 170677]; MO0406SPAETPBM.002 [DIRS 170150]; MO0503SPADCESR.000 [DIRS 172896]. }\end{array}$} \\
\hline NOTES: & $\begin{array}{l}\text { See Excel fil } \\
\text { Particul. = P }\end{array}$ & file GW BD & CF Path & $\begin{array}{l}\text { Analys } \\
\text { aporativ }\end{array}$ & $\begin{array}{l}S M C \_R \\
\text {; Veget. }\end{array}$ & $\begin{array}{l}\text { 4.xls in } \\
\text { Vegeta }\end{array}$ & pendix & for det & of calc & tions. & & & & & \\
\hline
\end{tabular}


Table 6.2-20. Exposure Pathway Contributions (Percent) for the ICRP 72-Based BDCFs for the Upper Bound of Glacial Transition Climate

\begin{tabular}{|c|c|c|c|c|c|c|c|c|c|c|c|c|c|c|c|}
\hline \multirow[b]{2}{*}{$\begin{array}{l}\text { Radio- } \\
\text { nuclide }\end{array}$} & \multirow[b]{2}{*}{$\begin{array}{l}\text { External } \\
\text { exposure }\end{array}$} & \multicolumn{3}{|c|}{ Inhalation } & \multicolumn{11}{|c|}{ Ingestion } \\
\hline & & \begin{tabular}{|c|} 
Particul. \\
Matter
\end{tabular} & $\begin{array}{l}\text { Evap. } \\
\text { Cooler }\end{array}$ & Radon & Water & $\begin{array}{l}\text { Leafy } \\
\text { Veget. }\end{array}$ & $\begin{array}{l}\text { Other } \\
\text { Veget. }\end{array}$ & Fruit & Grain & Meat & Milk & Poultry & Eggs & Fish & Soil \\
\hline C-14 & 0.0 & 0.0 & 0.0 & 0.0 & 17.9 & 2.9 & 3.7 & 10.1 & 0.7 & 6.4 & 3.1 & 0.7 & 6.2 & 48.4 & 0.0 \\
\hline $\mathrm{Cl}-36$ & 0.1 & 0.0 & 0.1 & 0.0 & 14.3 & 3.2 & 4.8 & 16.2 & 2.5 & 22.1 & 17.9 & 0.0 & 10.0 & 8.8 & 0.0 \\
\hline Se-79 & 0.0 & 0.2 & 0.0 & 0.0 & 16.0 & 0.7 & 0.5 & 1.5 & 0.1 & 51.8 & 3.0 & 1.8 & 20.3 & 3.4 & 0.6 \\
\hline Sr-90 & 0.7 & 0.2 & 0.2 & 0.0 & 77.9 & 4.8 & 2.4 & 3.9 & 0.4 & 2.1 & 2.6 & 0.0 & 1.2 & 3.4 & 0.2 \\
\hline Tc-99 & 0.0 & 0.1 & 0.7 & 0.0 & 55.0 & 10.4 & 1.8 & 6.2 & 0.4 & 3.7 & 11.5 & 0.2 & 9.0 & 1.0 & 0.0 \\
\hline Sn-126 & 98.1 & 0.0 & 0.0 & 0.0 & 0.4 & 0.0 & 0.0 & 0.0 & 0.0 & 0.4 & 0.0 & 0.0 & 0.1 & 1.0 & 0.0 \\
\hline I-129 & 0.0 & 0.0 & 0.0 & 0.0 & 69.4 & 2.1 & 0.5 & 1.9 & 0.3 & 3.9 & 5.8 & 0.2 & 11.6 & 3.6 & 0.6 \\
\hline Cs-135 & 0.0 & 0.6 & 0.0 & 0.0 & 15.1 & 1.3 & 0.8 & 3.7 & 0.2 & 9.4 & 5.2 & 6.6 & 5.0 & 51.1 & 1.0 \\
\hline Cs-137 & 37.7 & 0.0 & 0.0 & 0.0 & 12.9 & 0.4 & 0.1 & 0.5 & 0.0 & 1.8 & 1.0 & 1.0 & 0.7 & 43.7 & 0.1 \\
\hline $\mathrm{Pb}-210$ & 0.0 & 0.2 & 0.2 & 0.0 & 70.9 & 2.1 & 0.4 & 1.4 & 0.2 & 0.2 & 0.1 & 0.0 & 1.2 & 22.6 & 0.3 \\
\hline Ra-226 & 10.6 & 1.2 & 0.0 & 81.5 & 2.4 & 0.5 & 0.2 & 0.9 & 0.1 & 0.1 & 0.1 & 0.0 & 1.1 & 0.2 & 0.9 \\
\hline Ac-227 & 0.8 & 16.0 & 16.7 & 0.0 & 60.6 & 1.8 & 0.3 & 1.0 & 0.2 & 0.1 & 0.0 & 0.0 & 0.0 & 2.3 & 0.2 \\
\hline Th-229 & 5.9 & 65.8 & 4.6 & 0.0 & 18.8 & 0.7 & 0.1 & 0.5 & 0.1 & 0.1 & 0.0 & 0.0 & 0.1 & 2.3 & 1.1 \\
\hline Th-230 & 7.7 & 22.4 & 1.4 & 59.2 & 5.1 & 0.5 & 0.2 & 0.8 & 0.1 & 0.1 & 0.1 & 0.0 & 0.8 & 0.6 & 1.0 \\
\hline Th-232 & 41.1 & 29.5 & 1.9 & 0.0 & 20.8 & 1.4 & 0.5 & 1.0 & 0.1 & 0.2 & 0.3 & 0.0 & 0.0 & 1.8 & 1.2 \\
\hline Pa-231 & 3.6 & 81.8 & 1.2 & 0.0 & 10.3 & 0.6 & 0.2 & 0.6 & 0.1 & 0.1 & 0.0 & 0.0 & 0.0 & 0.1 & 1.5 \\
\hline U-232 & 17.3 & 9.2 & 5.9 & 0.0 & 59.1 & 1.8 & 0.3 & 1.1 & 0.2 & 0.2 & 0.2 & 0.3 & 1.4 & 2.9 & 0.4 \\
\hline U-233 & 3.0 & 55.6 & 3.4 & 0.0 & 30.7 & 1.1 & 0.2 & 0.8 & 0.1 & 0.1 & 0.2 & 0.4 & 2.5 & 0.5 & 1.3 \\
\hline U-234 & 0.9 & 34.2 & 4.9 & 6.6 & 43.8 & 1.5 & 0.3 & 1.1 & 0.2 & 0.2 & 0.3 & 0.6 & 3.6 & 0.8 & 1.1 \\
\hline U-236 & 0.0 & 34.9 & 5.3 & 0.0 & 49.2 & 1.6 & 0.3 & 1.2 & 0.2 & 0.2 & 0.3 & 0.6 & 4.1 & 0.9 & 1.2 \\
\hline U-238 & 8.8 & 29.8 & 4.5 & 0.0 & 46.9 & 1.5 & 0.3 & 1.1 & 0.2 & 0.2 & 0.3 & 0.6 & 3.9 & 0.8 & 1.2 \\
\hline $\mathrm{Np}-237$ & 11.9 & 38.2 & 9.2 & 0.0 & 34.7 & 1.3 & 0.4 & 1.9 & 0.1 & 0.4 & 0.0 & 0.0 & 0.0 & 1.3 & 0.6 \\
\hline Pu-238 & 0.0 & 37.5 & 12.2 & 0.0 & 43.6 & 1.3 & 0.2 & 0.8 & 0.1 & 0.0 & 0.0 & 0.0 & 0.0 & 3.7 & 0.5 \\
\hline Pu-239 & 0.0 & 73.0 & 5.1 & 0.0 & 18.1 & 0.7 & 0.1 & 0.4 & 0.1 & 0.0 & 0.0 & 0.0 & 0.0 & 1.5 & 1.0 \\
\hline Pu-240 & 0.0 & 72.1 & 5.3 & 0.0 & 18.7 & 0.7 & 0.1 & 0.4 & 0.1 & 0.0 & 0.0 & 0.0 & 0.0 & 1.6 & 1.0 \\
\hline Pu-242 & 0.0 & 72.6 & 5.0 & 0.0 & 18.5 & 0.7 & 0.1 & 0.4 & 0.1 & 0.0 & 0.0 & 0.0 & 0.0 & 1.6 & 1.1 \\
\hline Am-241 & 0.2 & 59.1 & 8.0 & 0.0 & 28.5 & 0.9 & 0.2 & 0.6 & 0.1 & 0.0 & 0.0 & 0.0 & 0.0 & 1.5 & 0.8 \\
\hline Am-243 & 8.5 & 66.0 & 4.9 & 0.0 & 17.5 & 0.6 & 0.1 & 0.4 & 0.1 & 0.0 & 0.0 & 0.0 & 0.0 & 0.9 & 0.9 \\
\hline Source: & $\begin{array}{l}\text { MO0407SP } \\
\text { [DIRS } 1693\end{array}$ & $\begin{array}{l}\text { AINEXI.00 } \\
92] ; \text { MO04 }\end{array}$ & 2 [DIRS & $\begin{array}{l}70597] ; M \\
\text { BSM.002 }\end{array}$ & 0407SF & $\begin{array}{l}\text { ASRPBM } \\
\text { 0677]; MC }\end{array}$ & 02 [DIR & $\begin{array}{l}170755 \\
\text { TPBM. }\end{array}$ & $\begin{array}{l}\text { MO0306 } \\
2 \text { [DIRS }\end{array}$ & $\begin{array}{l}\text { WDBG } \\
\text { 70150]; }\end{array}$ & $\begin{array}{l}\text { IF.001 } \\
\text { O0503 }\end{array}$ & $\begin{array}{l}\text { IRS } 1638 \\
\text { ADCESR }\end{array}$ & $\begin{array}{l}\text { 6]; MO0 } \\
00 \text { [DIR }\end{array}$ & $\begin{array}{l}3 \text { SPAA } \\
17289\end{array}$ & M.002 \\
\hline NOTES: & See Excel & IIe GVV B & r Patm & ay Analys & $M C \_R E$ & $\begin{aligned} & 4 . x I s \text { in } \\
= & \text { Vegetab }\end{aligned}$ & pendix & For det & of calc & tions. & & & & & \\
\hline
\end{tabular}




\subsection{DOSE FACTORS FOR GROUNDWATER PROTECTION STANDARDS}

The groundwater protection standards (10 CFR 63.331 [DIRS 173164]) prohibit releasing radionuclides from the repository to the accessible environment in excess of the values in Table 6.3-1 in 3,000-acre feet of water (representative volume) (10 CFR 63.332 [DIRS 173164]).

Table 6.3-1. Limits on Radionuclides in the Representative Volume

\begin{tabular}{|l|l|l|}
\hline \multicolumn{1}{|c|}{ Radionuclide or type of radiation emitted } & \multicolumn{1}{c|}{ Limit } & $\begin{array}{c}\text { Is natural } \\
\text { background } \\
\text { included? }\end{array}$ \\
\hline Combined radium-226 and radium-228 & 5 picocuries per liter & Yes \\
\hline $\begin{array}{l}\text { Gross alpha activity (including radium-226 but } \\
\text { excluding radon and uranium) }\end{array}$ & 15 picocuries per liter & Yes \\
\hline $\begin{array}{l}\text { Combined beta and photon emitting } \\
\text { radionuclides }\end{array}$ & $\begin{array}{l}0.04 \text { mSv (4 mrem) per year to the whole body } \\
\text { or any organ, based on drinking 2 liters of water } \\
\text { per day from the representative volume }\end{array}$ & No \\
\hline
\end{tabular}

Source: 10 CFR 63.331 [DIRS 173164].

Gross alpha particle activity means the total radioactivity due to alpha particle emission as inferred from measurements on a dry sample (40 CFR 141.2 [DIRS 173245]). For this analysis, evaluation of gross alpha activity for consideration in TSPA is based on calculation, rather than measurement, of total alpha emissions from the primary radionuclides and their decay products (Section 6.1.1). The calculation of radionuclide concentrations in water is based on the representative volume, which contains 3,000 acre-feet of water (about 3,714,450,000 liters) (10 CFR 63.332 [DIRS 173164]).

Table 6.3-2 lists primary radionuclides (Section 6.1.1), short-lived decay products, radionuclide emissions, and applicable limits from the groundwater protection standards. 
Table 6.3-2. Primary Radionuclides, Decay Products, and Applicable Groundwater Protection Limits

\begin{tabular}{|c|c|c|c|c|c|c|c|c|c|}
\hline \multirow{2}{*}{\multicolumn{2}{|c|}{$\begin{array}{c}\text { Primary Radionuclide and } \\
\text { Mode of Decay }\end{array}$}} & \multirow{2}{*}{\multicolumn{2}{|c|}{$\begin{array}{c}\text { Short-lived Decay } \\
\text { Product and Mode of } \\
\text { Decay }\end{array}$}} & \multirow{3}{*}{$\begin{array}{l}\mathrm{BF} \\
1\end{array}$} & \multicolumn{3}{|c|}{$\begin{array}{l}\text { Type and Energy of } \\
\text { Radiation } \\
\text { (MeV per nuclear } \\
\text { transformation) }\end{array}$} & \multirow[b]{2}{*}{ Half-life } & \multirow[b]{2}{*}{ Applicable Limit } \\
\hline & & & & & \multirow{2}{*}{\begin{tabular}{l|} 
Alpha \\
-
\end{tabular}} & \multirow{2}{*}{\begin{tabular}{|l|} 
Electron \\
0.049 \\
\end{tabular}} & \multirow{2}{*}{\begin{tabular}{|l|} 
Photon \\
-
\end{tabular}} & & \\
\hline Carbon-14 & $\beta-$ & & & & & & & $5730 \mathrm{yr}$ & $0.04 \mathrm{mSv}(4 \mathrm{mrem})$ per year; $\beta, \gamma$ \\
\hline Chlorine-36 & $E C \beta+\beta-$ & & & 1 & - & 0.274 & $<$ & $3.01 \mathrm{E}+05 \mathrm{yr}$ & $0.04 \mathrm{mSv}$ (4 mrem) per year; $\beta, \gamma$ \\
\hline Selenium-79 & $\beta-$ & & & 1 & - & 0.056 & - & $6.50 \mathrm{E}+04 \mathrm{yr}$ & $0.04 \mathrm{mSv}(4 \mathrm{mrem})$ per year; $\beta, \gamma$ \\
\hline Strontium-90 & $\beta-$ & & & 1 & - & 0.196 & - & $29.12 \mathrm{yr}$ & $0.04 \mathrm{mSv}$ (4 mrem) per year; $\beta, \gamma$ \\
\hline & & Yttrium-90 & $\beta-$ & 1 & - & 0.935 & $<$ & $64.0 \mathrm{hr}$ & $0.04 \mathrm{mSv}$ (4 mrem) per year; $\beta, \gamma$ \\
\hline Technetium-99 & $\beta-$ & & & 1 & - & 0.101 & - & $2.13 \mathrm{E}+05 \mathrm{yr}$ & $0.04 \mathrm{mSv}$ (4 mrem) per year; $\beta, \gamma$ \\
\hline Tin-126 & $\beta-$ & & & 1 & - & 0.172 & 0.057 & $1.0 \mathrm{E}+05 \mathrm{yr}$ & $0.04 \mathrm{mSv}(4 \mathrm{mrem})$ per year; $\beta, \gamma$ \\
\hline & & Antimony-126m & IT $\beta-$ & 1 & - & 0.591 & 1.548 & $19.0 \mathrm{~min}$ & $0.04 \mathrm{mSv}$ (4 mrem) per year; $\beta, \gamma$ \\
\hline & & Antimony-126 & $\beta-$ & 0.14 & - & 0.283 & 2.834 & 12.4 day & $0.04 \mathrm{mSv}$ (4 mrem) per year; $\beta, \gamma$ \\
\hline lodine-129 & $\beta-$ & & & 1 & - & 0.064 & 0.025 & $1.57 \mathrm{E}+07 \mathrm{yr}$ & $0.04 \mathrm{mSv}(4 \mathrm{mrem})$ per year; $\beta, \gamma$ \\
\hline Cesium-135 & $\beta-$ & & & 1 & - & 0.067 & - & $2.3 \mathrm{E}+06 \mathrm{yr}$ & $0.04 \mathrm{mSv}(4 \mathrm{mrem})$ per year; $\beta, \gamma$ \\
\hline Cesium-137 & $\beta-$ & & & 1 & - & 0.187 & - & $30.0 \mathrm{yr}$ & $0.04 \mathrm{mSv}(4 \mathrm{mrem})$ per year; $\beta, \gamma$ \\
\hline & & Barium-137m & IT & 0.946 & - & 0.065 & 0.597 & $2.552 \mathrm{~min}$ & $0.04 \mathrm{mSv}$ (4 mrem) per year; $\beta, \gamma$ \\
\hline \multicolumn{10}{|c|}{ Thorium Series (4n) } \\
\hline Plutonium-240 & $\mathrm{SF} \alpha$ & & & 1 & 5.156 & 0.011 & 0.002 & $6537 \mathrm{yr}$ & $15 \mathrm{pCi} / \mathrm{L} ; \alpha$ \\
\hline Uranium-236 & $\alpha$ & & & 1 & 4.505 & 0.011 & 0.002 & $2.3415 \mathrm{E}+07 \mathrm{yr}$ & excluded \\
\hline Thorium-232 & $\alpha$ & & & 1 & 3.996 & 0.012 & 0.001 & $1.405 \mathrm{E}+10 \mathrm{yr}$ & $15 \mathrm{pCi} / \mathrm{L} ; \alpha$ \\
\hline Radium-228 & $\beta-$ & & & 1 & - & 0.017 & $<$ & $5.75 \mathrm{yr}$ & $\begin{array}{l}5 \mathrm{pCi} / \mathrm{L} \text { of Ra-226 + Ra-228 } \\
0.04 \mathrm{mSv}(4 \mathrm{mrem}) \text { per year; } \beta, \gamma\end{array}$ \\
\hline & & Actinium-228 & $\beta-$ & 1 & - & 0.475 & 0.971 & $6.13 \mathrm{hr}$ & $0.04 \mathrm{mSv}(4 \mathrm{mrem})$ per year; $\beta, \gamma$ \\
\hline Uranium-232 & $\alpha$ & & & 1 & 5.302 & 0.017 & 0.002 & $72 \mathrm{yr}$ & excluded \\
\hline \multirow[t]{4}{*}{ Thorium-228 } & $\alpha$ & & & 1 & 5.400 & 0.021 & 0.003 & $1.9131 \mathrm{yr}$ & $15 \mathrm{pCi} / \mathrm{L} ; \alpha$ \\
\hline & & Radium-224 & $\alpha$ & 1 & 5.674 & 0.002 & 0.010 & 3.66 day & $15 \mathrm{pCi} / \mathrm{L} ; \alpha$ \\
\hline & & Radon-220 & $\alpha$ & 1 & 6.288 & $<$ & $<$ & $55.6 \mathrm{sec}$ & excluded \\
\hline & & Polonium-216 & $\alpha$ & 1 & 6.779 & $<$ & $<$ & $0.15 \mathrm{sec}$ & $15 \mathrm{pCi} / \mathrm{L} ; \alpha$ \\
\hline
\end{tabular}


Table 6.3-2. Primary Radionuclides, Decay Products, and Applicable Groundwater Protection Limits (Continued)

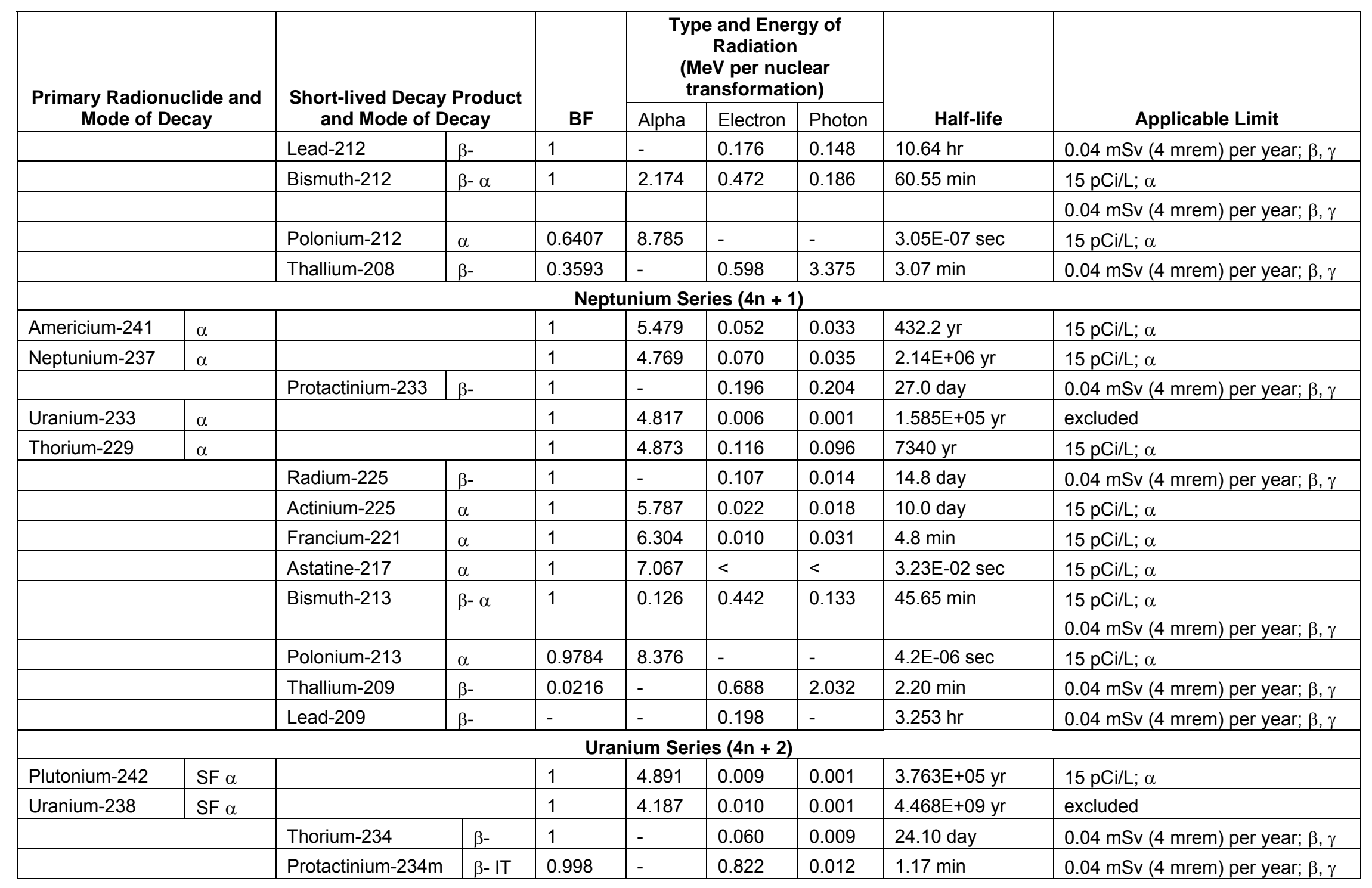


Table 6.3-2. Primary Radionuclides, Decay Products, and Applicable Groundwater Protection Limits (Continued)

\begin{tabular}{|c|c|c|c|c|c|c|c|c|c|}
\hline \multirow{2}{*}{\multicolumn{2}{|c|}{$\begin{array}{l}\text { Primary Radionuclide and } \\
\text { Mode of Decay }\end{array}$}} & \multirow{2}{*}{\multicolumn{2}{|c|}{$\begin{array}{l}\text { Short-lived Decay Product } \\
\text { and Mode of Decay }\end{array}$}} & \multirow{3}{*}{$\begin{array}{c}\text { BF } \\
0.0033\end{array}$} & \multicolumn{3}{|c|}{$\begin{array}{l}\text { Type and Energy of } \\
\text { Radiation } \\
\text { (MeV per nuclear } \\
\text { transformation) }\end{array}$} & \multirow[b]{2}{*}{ Half-life } & \multirow[b]{2}{*}{ Applicable Limit } \\
\hline & & & & & \multirow{2}{*}{\begin{tabular}{|l|} 
Alpha \\
-
\end{tabular}} & \multirow{2}{*}{\begin{tabular}{|l|} 
Electron \\
0.494 \\
\end{tabular}} & \multirow{2}{*}{$\begin{array}{l}\text { Photon } \\
1.919 \\
\end{array}$} & & \\
\hline & & Protactinium-234 & $\beta-$ & & & & & $6.70 \mathrm{hr}$ & $0.04 \mathrm{mSv}$ (4 mrem) per year; $\beta, \gamma$ \\
\hline Plutonium-238 & $\mathrm{SF} \alpha$ & & & 1 & 5.487 & 0.011 & 0.002 & $87.74 \mathrm{yr}$ & $15 \mathrm{pCi} / \mathrm{L} ; \alpha$ \\
\hline Uranium-234 & $\alpha$ & & & 1 & 4.758 & 0.013 & 0.002 & $2.445 \mathrm{E}+05 \mathrm{yr}$ & excluded \\
\hline Thorium-230 & $\alpha$ & & & 1 & 4.671 & 0.015 & 0.002 & $7.7 \mathrm{E}+04 \mathrm{yr}$ & $15 \mathrm{pCi} / \mathrm{L} ; \alpha$ \\
\hline Radium-226 & $\alpha$ & & & 1 & 4.774 & 0.004 & 0.007 & $1600 \mathrm{yr}$ & $\begin{array}{l}5 \mathrm{pCi} / \mathrm{L} \text { of Ra-226 + Ra-228 } \\
15 \mathrm{pCi} / \mathrm{L} ; \alpha\end{array}$ \\
\hline & & Radon-222 & $\alpha$ & 1 & 5.489 & $<$ & $<$ & 3.8235 day & excluded \\
\hline & & Polonium-218 & $\alpha$ & 1 & 6.001 & $<$ & $<$ & $3.05 \mathrm{~min}$ & $15 \mathrm{pCi} / \mathrm{L} ; \alpha$ \\
\hline & & Lead-214 & $\beta-$ & 0.9998 & - & 0.293 & 0.250 & $26.8 \mathrm{~min}$ & $0.04 \mathrm{mSv}$ (4 mrem) per year; $\beta, \gamma$ \\
\hline & & Astatine-218 & $\alpha$ & 0.0002 & 6.697 & 0.040 & 0.007 & $2 \mathrm{sec}$ & $15 \mathrm{pCi} / \mathrm{L} ; \alpha$ \\
\hline & & Bismuth-214 & $\beta-$ & 1 & - & 0.659 & 1.508 & $19.9 \mathrm{~min}$ & $\begin{array}{l}15 \mathrm{pCi} / \mathrm{L} ; \alpha \\
0.04 \mathrm{mSv}(4 \mathrm{mrem}) \text { per year; } \beta, \gamma\end{array}$ \\
\hline & & Polonium-214 & $\alpha$ & 0.9998 & 7.687 & $<$ & $<$ & 1.643E-04 sec & $15 \mathrm{pCi} / \mathrm{L} ; \alpha$ \\
\hline & & Thallium-210 & $\beta-$ & 0.0002 & - & & & $1.3 \mathrm{~min}$ & $0.04 \mathrm{mSv}$ (4 mrem) per year; $\beta, \gamma$ \\
\hline Lead-210 & $\beta-$ & & & 1 & - & 0.038 & 0.005 & $22.3 \mathrm{yr}$ & $0.04 \mathrm{mSv}$ (4 mrem) per year; $\beta, \gamma$ \\
\hline & & Bismuth-210 & $\beta-$ & 1 & - & 0.389 & - & 5.012 day & $0.04 \mathrm{mSv}$ (4 mrem) per year; $\beta, \gamma$ \\
\hline & & Polonium-210 & $\alpha$ & 1 & 5.297 & $<$ & $<$ & 138.38 day & $15 \mathrm{pCi} / \mathrm{L} ; \alpha$ \\
\hline & & & & Act & ium Se & $(4 n+3)$ & & & \\
\hline Americium-243 & $\alpha$ & & & 1 & 5.270 & 0.022 & 0.056 & $7380 \mathrm{yr}$ & $15 \mathrm{pCi} / \mathrm{L} ; \alpha$ \\
\hline & & Neptunium-239 & $\beta-$ & 1 & - & 0.260 & 0.173 & 2.355 day & $0.04 \mathrm{mSv}$ (4 mrem) per year; $\beta, \gamma$ \\
\hline Plutonium-239 & $\alpha$ & & & 1 & 5.148 & 0.007 & $<$ & $2.4065 \mathrm{E}+04 \mathrm{yr}$ & $15 \mathrm{pCi} / \mathrm{L} ; \alpha$ \\
\hline Uranium-235 & $\alpha$ & & & 1 & 4.396 & 0.049 & 0.156 & $7.038 \mathrm{E}+08 \mathrm{yr}$ & excluded \\
\hline & & Thorium-231 & $\beta-$ & 1 & - & 0.165 & 0.026 & $25.52 \mathrm{hr}$ & $0.04 \mathrm{mSv}(4 \mathrm{mrem})$ per year; $\beta, \gamma$ \\
\hline Protactinium-231 & $\alpha$ & & & 1 & 4.969 & 0.065 & 0.048 & $3.276 \mathrm{E}+04 \mathrm{yr}$ & $15 \mathrm{pCi} / \mathrm{L} ; \alpha$ \\
\hline Actinium-227 & $\beta-\alpha$ & & & 1 & 0.068 & 0.016 & $<$ & $21.773 \mathrm{yr}$ & $\begin{array}{l}15 \mathrm{pCi} / \mathrm{L} ; \alpha \\
0.04 \mathrm{mSv}(4 \mathrm{mrem}) \text { per year; } \beta, \gamma\end{array}$ \\
\hline
\end{tabular}


Table 6.3-2. Primary Radionuclides, Decay Products, and Applicable Groundwater Protection Limits (Continued)

\begin{tabular}{|c|c|c|c|c|c|c|c|c|}
\hline \multirow{3}{*}{$\begin{array}{c}\text { Primary Radionuclide and } \\
\text { Mode of Decay }\end{array}$} & \multirow{2}{*}{\multicolumn{2}{|c|}{$\begin{array}{c}\text { Short-lived Decay Product } \\
\text { and Mode of Decay }\end{array}$}} & \multirow[b]{2}{*}{ BF } & \multicolumn{3}{|c|}{$\begin{array}{l}\text { Type and Energy of } \\
\text { Radiation } \\
\text { (MeV per nuclear } \\
\text { transformation) }\end{array}$} & \multirow[b]{2}{*}{ Half-life } & \multirow[b]{2}{*}{ Applicable Limit } \\
\hline & & & & Alpha & Electron & Photon & & \\
\hline & Thorium-227 & $\alpha$ & 0.9862 & 5.884 & 0.053 & 0.110 & 18.718 day & $15 \mathrm{pCi} / \mathrm{L} ; \alpha$ \\
\hline & Francium-223 & $\beta-$ & 0.0138 & - & 0.400 & 0.059 & $21.8 \mathrm{~min}$ & $0.04 \mathrm{mSv}$ (4 mrem) per year; $\beta, \gamma$ \\
\hline & Radium-223 & $\alpha$ & 1 & 5.667 & 0.076 & 0.134 & 11.434 day & $15 \mathrm{pCi} / L ; \alpha$ \\
\hline & Radon-219 & $\alpha$ & 1 & 6.757 & 0.006 & 0.056 & $3.96 \mathrm{sec}$ & excluded \\
\hline & Polonium-215 & $\alpha$ & 1 & 7.386 & $<$ & $<$ & $1.78 \mathrm{E}-3 \mathrm{sec}$ & $15 \mathrm{pCi} / \mathrm{L} ; \alpha$ \\
\hline & Lead-211 & $\beta-$ & 1 & - & 0.456 & 0.051 & $36.1 \mathrm{~min}$ & $0.04 \mathrm{mSv}(4 \mathrm{mrem})$ per year; $\beta, \gamma$ \\
\hline & Bismuth-211 & $\alpha \beta-$ & 1 & 6.550 & 0.010 & 0.047 & $2.14 \mathrm{~min}$ & $15 \mathrm{pCi} / \mathrm{L} ; \alpha$ \\
\hline & Thallium-207 & $\beta-$ & 0.9972 & - & 0.493 & 0.002 & $4.77 \mathrm{~min}$ & $0.04 \mathrm{mSv}(4 \mathrm{mrem})$ per year; $\beta, \gamma$ \\
\hline & Polonium-211 & $\alpha$ & 0.0028 & 7.442 & $<$ & 0.008 & $0.516 \mathrm{sec}$ & $15 \mathrm{pCi} / \mathrm{L} ; \alpha$ \\
\hline
\end{tabular}


The combined activity concentration of ${ }^{226} \mathrm{Ra}$ and ${ }^{228} \mathrm{Ra}$ in the groundwater is calculated based on the annual mass flux of these radionuclides and the representative volume. The natural background concentrations of ${ }^{226} \mathrm{Ra}$ and ${ }^{228} \mathrm{Ra}$ in groundwater must be included with the calculated activity (10 CFR 63.331 [DIRS 173164]) for comparison to the limit of 5 pCi/L.

For determining alpha activity concentration (including ${ }^{226}$ Ra but excluding radon and uranium) for comparison with the limit for gross alpha activity of $15 \mathrm{pCi} / \mathrm{L}$, the activity concentration of primary radionuclides in groundwater is calculated based on the annual mass flux of these radionuclides and the representative volume. Alpha particle activity is calculated as the total of alpha emissions from all primary radionuclides and decay products included in the model (Section 6.1.1). Consistent with the approach used in the biosphere model (BSC 2004 [DIRS 169460], Section 6.3.1.4), short-lived decay products of a primary radionuclide are modeled to be in secular equilibrium with the primary radionuclide. After the activity concentration of a primary radionuclide in the groundwater is calculated, the value is multiplied by the number of alpha particles included in the decay chain to calculate the total number of alpha particles associated with the decay of the primary radionuclide. The number of alpha particles is shown in Table 6.3-3. For example, if the calculated activity concentration of ${ }^{229} \mathrm{Th}$ in groundwater is $2 \mathrm{pCi} / \mathrm{L}$, the alpha activity associated with the decay of ${ }^{229} \mathrm{Th}$ is $2 \mathrm{pCi} / \mathrm{L} \times 5$ alpha particles per decay $=10 \mathrm{pCi} / \mathrm{L}$. The natural background concentrations of alpha emitters in groundwater (including ${ }^{226} \mathrm{Ra}$ but excluding radon and uranium) (10 CFR 63.331 [DIRS 173164]) must be included in the calculation of alpha activity concentration.

Table 6.3-3. $\quad$ Number of Alpha Particles Emitted per One Decay of a Primary Radionuclide Considered in the Gross Alpha Activity Limit of the Groundwater Protection Standards

\begin{tabular}{|c|c|c|c|c|}
\hline \multicolumn{2}{|c|}{ Primary Radionuclide } & \multicolumn{2}{|c|}{ Short-lived Decay Products } & $\begin{array}{c}\text { Number of Alpha } \\
\text { Particles }\end{array}$ \\
\hline \multicolumn{5}{|c|}{ Thorium Series $(4 n)$} \\
\hline Plutonium-240 & $100 \%^{a}$ & & & 1 \\
\hline Uranium-236 $^{\mathrm{b}}$ & $(100 \%)$ & & & $0^{\mathrm{b}}$ \\
\hline Thorium-232 ${ }^{\mathrm{C}}$ & $100 \%$ & & & 1 \\
\hline Uranium-232 ${ }^{\mathrm{b}, \mathrm{d}}$ & $(100 \%)$ & & & $0^{\mathrm{b}}$ \\
\hline Thorium-228 ${ }^{\mathrm{c}, \mathrm{d}}$ & $100 \%$ & & & $4^{b}$ \\
\hline & & Radium-224 & $100 \%$ & \\
\hline & & Radon-220 ${ }^{\mathrm{b}}$ & $(100 \%)$ & \\
\hline & & Polonium-216 & $100 \%$ & \\
\hline & & Bismuth-212 & $35.93 \%$ & \\
\hline & & Polonium-212 & $64.07 \%$ & \\
\hline \multicolumn{5}{|c|}{ Neptunium Series $(4 n+1)$} \\
\hline Americium-241 & $100 \%$ & & & 1 \\
\hline Neptunium-237 & $100 \%$ & & & 1 \\
\hline Uranium-233 ${ }^{\mathrm{b}}$ & $(100 \%)$ & & & $0^{\mathrm{b}}$ \\
\hline \multirow[t]{6}{*}{ Thorium-229 } & $100 \%$ & & & 5 \\
\hline & & Actinium-225 & $100 \%$ & \\
\hline & & Francium-221 & $100 \%$ & \\
\hline & & Astatine-217 & $100 \%$ & \\
\hline & & Bismuth-213 & $2.16 \%$ & \\
\hline & & Polonium-213 & $97.84 \%$ & \\
\hline
\end{tabular}


Table 6.3-3. N Number of Alpha Particles Emitted per One Decay of a Primary Radionuclide Considered in the Gross Alpha Activity Limit of the Groundwater Protection Standards (Continued)

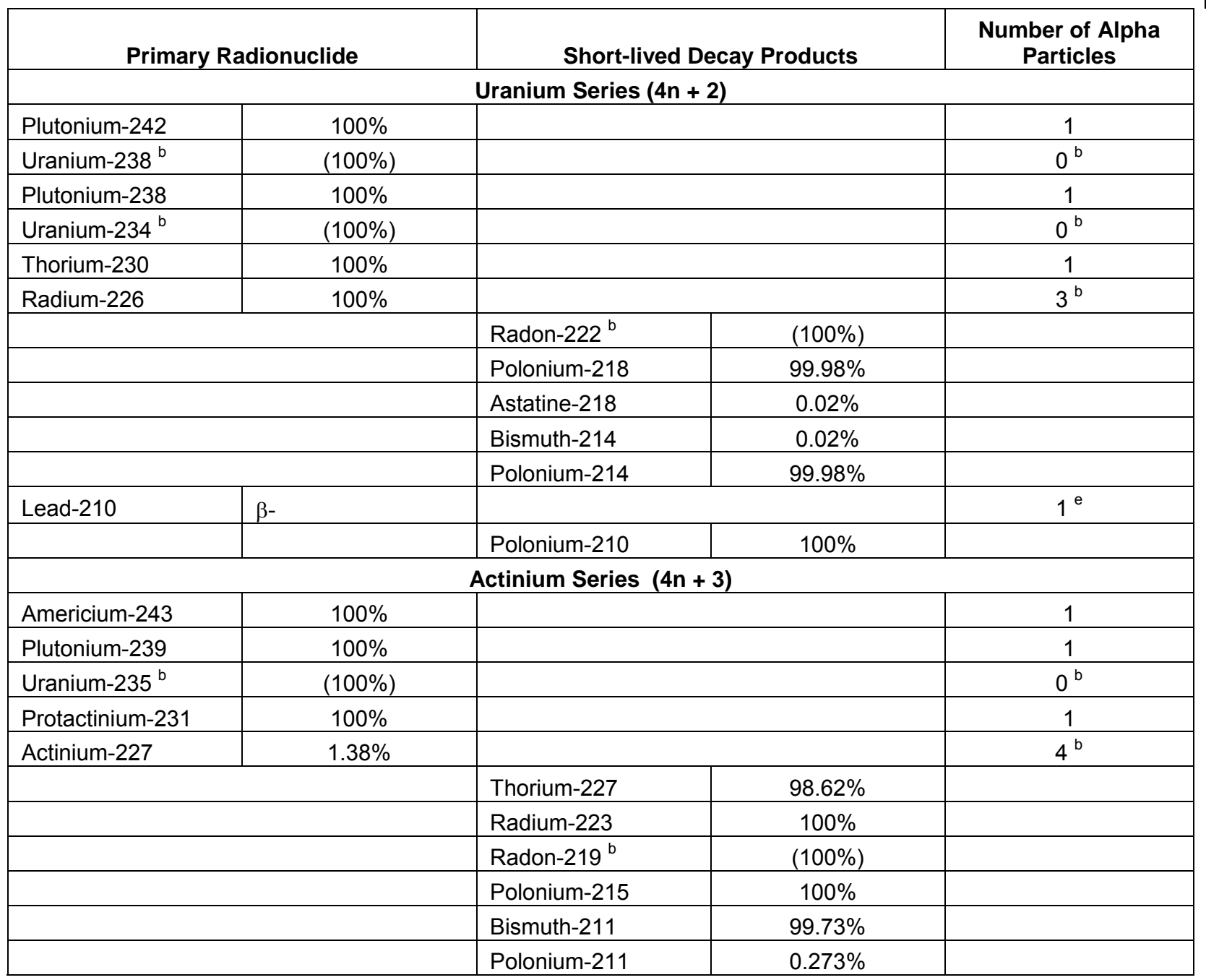

${ }^{\text {a }}$ Percent alpha particles emitted per disintegration.

${ }^{\mathrm{b}}$ Isotopes of radon and uranium have been excluded, per 10 CFR 63.331 [DIRS 173164].

${ }^{c}{ }^{232} \mathrm{Th}$ is accompanied in the groundwater by its relatively long-lived decay products, ${ }^{228} \mathrm{Ra}$ and ${ }^{228} \mathrm{Th}$ (and their short-lived decay products), which are not being tracked in TSPA. If radioactive equilibrium between ${ }^{232} \mathrm{Th},{ }^{228} \mathrm{Ra}$ and ${ }^{228} \mathrm{Th}$ is assumed, the number of alpha particles for ${ }^{228} \mathrm{Th}$ should be added to that for ${ }^{232} \mathrm{Th}$ for a total of 5 . ${ }^{228} \mathrm{Ra}$ and its decay product ${ }^{228} \mathrm{Ac}$ are beta-emitters so they do not contribute to the alpha particle number.)

d ${ }^{232} \mathrm{U}$ is accompanied in the groundwater by its relatively long-lived decay product, ${ }^{228} \mathrm{Th}$ (and its short-lived decay products). If radioactive equilibrium between ${ }^{232} \mathrm{U}$ and ${ }^{228} \mathrm{Th}$ is assumed, the number of alpha particles for ${ }^{228} \mathrm{Th}$ should be attributed to that for ${ }^{232} U$ for a total of 4 .

e If concentration of ${ }^{210} \mathrm{~Pb}$ in groundwater is not calculated, it can be assumed that ${ }^{210} \mathrm{~Pb}$ is in radioactive equilibrium with ${ }^{226} \mathrm{Ra}$. If this is the case, the number of alpha particles for ${ }^{210} \mathrm{~Pb}$ should be added to that for ${ }^{226} \mathrm{Ra}$ for a total of 4 . 
The alpha particle activity concentration in water is calculated as

$$
C_{\alpha}=\sum_{i} C w_{i} N_{\alpha, i}
$$

where

$$
\begin{aligned}
C_{\alpha}= & \text { total alpha particle activity concentration in groundwater }\left(\mathrm{Bq} / \mathrm{m}^{3} \text { or } \mathrm{pCi} / \mathrm{L}\right) \\
C w_{i}= & \text { activity concentration of a primary radionuclide } i \text { in groundwater }\left(\mathrm{Bq} / \mathrm{m}^{3}\right. \text { or } \\
& \text { pCi/L) } \\
N_{\alpha, i}= & \begin{array}{l}
\text { number of alpha particles attributed to one decay of a primary radionuclide } i \\
\text { (from Table } 6.3-3)
\end{array}
\end{aligned}
$$

The annual dose limit for beta- and photon-emitting radionuclides is $0.04 \mathrm{mSv}$ (4 mrem) per year, based on the consumption of 2 liters of water per day (10 CFR 63.331 [DIRS 173164]). This limit applies to radionuclides other than alpha emitters. Alpha emitters are covered under the gross alpha limit of the groundwater protection standards. If a radionuclide decays with emissions of alpha and beta radiation, this analysis considers the radionuclide for both gross alpha and annual dose. This is the case for several radionuclides (Table 6.3-2). Such an approach is conservative and ensures that all types of radiation emitted from a radionuclide are considered.

Dose contributions for beta-photon emitters were calculated using dose coefficients from FGR 11 (DOE 2004 [DIRS 169713]) and ICRP Publication 72 (ICRP 1999 [DIRS 153114]). These dose coefficients include contributions from all emissions for each radionuclide. For example, if the fraction of decays of a radionuclide that primarily is a beta emitter undergoes an alpha decay, the dose from alpha particles is included in the dose coefficient. Radionuclides such as ${ }^{212} \mathrm{Bi}$ that have large fractions of alpha and beta decays are double counted (i.e., they are included in the gross alpha component and the dose component to ensure that all radionuclide emissions are counted). Radionuclides classified as alpha-beta emitters (Eckerman and Ryman 1993 [DIRS 107684], Table A.1), with a large fraction of alpha emission (about 99 percent or more; e.g., ${ }^{218} \mathrm{Po}, 99.98$ percent $\alpha ;{ }^{211} \mathrm{Bi}, 99.73$ percent $\alpha$ ), are not included in calculating beta-photon dose; primary radionuclides that were included are listed in Table 6.3-4. 
Table 6.3-4. Radionuclides and Their Decay Products Included in Calculation of Conversion Factors for Beta-Photon Emitters

\begin{tabular}{|c|c|c|c|}
\hline \multicolumn{2}{|c|}{ Primary Radionuclide and Mode of Decay } & \multicolumn{2}{|c|}{ Short-lived Decay Product and Mode of Decay } \\
\hline Carbon-14 & $\beta-$ & & \\
\hline Chlorine-36 & $E C, \beta+, \beta-$ & & \\
\hline Selenium-79 & $\beta-$ & & \\
\hline Strontium-90 & $\beta-$ & Yttrium-90 & $\beta-$ \\
\hline Technetium-99 & $\beta-$ & & \\
\hline \multirow[t]{2}{*}{ Tin-126 } & \multirow[t]{2}{*}{$\beta-$} & Antimony-126m & IT, $\beta$ - \\
\hline & & Antimony-126 & $\beta-$ \\
\hline lodine-129 & $\beta-$ & & \\
\hline Cesium-135 & $\beta-$ & & \\
\hline Cesium-137 & $\beta-$ & Barium-137m & IT \\
\hline \multicolumn{4}{|c|}{ Thorium Series (4n) } \\
\hline Radium-228 ${ }^{a}$ & $\beta-$ & Actinium-228 & $\beta-$ \\
\hline \multirow[t]{3}{*}{ Thorium-228 ${ }^{a}$} & \multirow[t]{3}{*}{$\alpha$} & Lead-212 & $\beta-$ \\
\hline & & Bismuth-212 & $\beta-\alpha$ \\
\hline & & Thallium-208 & $\beta-$ \\
\hline \multicolumn{4}{|c|}{ Neptunium Series $(4 n+1)$} \\
\hline Neptunium-237 & $\alpha$ & Protactinium-233 & $\beta-$ \\
\hline \multirow[t]{4}{*}{ Thorium-229 } & \multirow[t]{4}{*}{$\alpha$} & Radium-225 & $\beta-$ \\
\hline & & Bismuth-213 & $\beta-\alpha$ \\
\hline & & Thallium-209 & $\beta-$ \\
\hline & & Lead-209 & $\beta-$ \\
\hline \multicolumn{4}{|c|}{ Uranium Series $(4 n+2)$} \\
\hline \multirow[t]{3}{*}{ Uranium-238 } & \multirow[t]{3}{*}{ SF, $\alpha$} & Thorium-234 & $\beta-$ \\
\hline & & Protactinium-234m & $\beta-$ IT \\
\hline & & Protactinium-234 & $\beta-$ \\
\hline \multirow[t]{3}{*}{ Radium-226 } & \multirow[t]{3}{*}{$\alpha$} & Lead-214 & $\beta-$ \\
\hline & & Bismuth-214 & $\beta-$ \\
\hline & & Thallium-210 & $\beta-$ \\
\hline Lead-210 & $\beta-$ & Bismuth-210 & $\beta-$ \\
\hline \multicolumn{4}{|c|}{ Actinium Series $(4 n+3)$} \\
\hline Americium-243 & $\alpha$ & Neptunium-239 & $\beta-$ \\
\hline \multirow[t]{3}{*}{ Actinium-227 } & \multirow[t]{3}{*}{$\beta-\alpha$} & Francium-223 & $\beta-$ \\
\hline & & Lead-211 & $\beta-$ \\
\hline & & Thallium-207 & $\beta-$ \\
\hline
\end{tabular}

${ }^{\text {a }}{ }^{228} \mathrm{Ra}$ and ${ }^{228} \mathrm{Th}$ are not primary radionuclides (their concentration in groundwater are not calculated in TSPA) but they are treated as primary radionuclides in the biosphere model, as described in Section 6.1.1.

$\mathrm{EC}=$ electron capture, IT = isomeric transformation, $\mathrm{SF}=$ spontaneous fission. 
The annual dose from drinking 2 liters of water per day for a given concentration of a primary beta-photon-emitting radionuclide in groundwater is calculated as

$$
\begin{aligned}
& D_{i}(S v / y r)=C w_{i}\left(B q / m^{3}\right) C F_{i}\left(\frac{S v / y r}{B q / m^{3}}\right) \\
& \text { or } \\
& D_{i}(\mathrm{mrem} / \mathrm{yr})=C w_{i}(p C i / L) C F_{i}\left(\frac{\mathrm{mrem} / \mathrm{yr}}{p C i / L}\right)
\end{aligned}
$$

where

$$
\begin{aligned}
& D_{i}=\quad \begin{array}{l}
\text { annual dose (committed effective dose equivalent or committed organ dose } \\
\text { equivalent) from intake of radionuclide } i \text { by ingestion resulting from daily } \\
\text { consumption of } 2 \text { liters of water ( } \mathrm{Sv} / \mathrm{yr} \text { or mrem/yr, depending on the set of } \\
\text { units used) }
\end{array} \\
& C w_{i}=\quad \begin{array}{l}
\text { activity concentration of radionuclide } i \text { in groundwater }\left(\mathrm{Bq} / \mathrm{m}^{3} \text { or } \mathrm{pCi} / \mathrm{L}\right. \text {, } \\
\text { depending on the set of units used) }
\end{array} \\
& C F_{i}=\quad \begin{array}{l}
\text { conversion factor for calculating beta-photon dose from radionuclide } i(\mathrm{~Sv} / \mathrm{yr} \\
\text { per } \mathrm{Bq} / \mathrm{m}^{3} \text { or mrem } / \mathrm{yr} \text { per pCi/L, depending on the set of units used). }
\end{array}
\end{aligned}
$$

The conversion factor, $C F$, is numerically equal to the annual dose resulting from daily consumption of 2 liters of water containing a unit activity concentration of a given primary radionuclide and associated short-lived decay products. Conversion factors are calculated as

$$
\begin{aligned}
& C F_{i}\left(\frac{S v / y r}{B q / m^{3}}\right)=2 \frac{L}{d} \times 365.25 \frac{d}{y r} \times 10^{-3} \frac{m^{3}}{L} \times E D C F_{i}\left(\frac{S v}{B q}\right) \\
& \text { or } \\
& C F_{i}\left(\frac{m r e m / y r}{p C i / L}\right)=2 \frac{L}{d} \times 365.25 \frac{d}{y r} \times \frac{3.7 \times 10^{-2} B q}{p C i} \times E D C F_{i}\left(\frac{S v}{B q}\right) \times \frac{100 \mathrm{rem}}{S v} \times \frac{1000 \mathrm{mrem}}{\mathrm{rem}}
\end{aligned}
$$

where

$$
E D C F_{i}=\text { effective dose coefficient for ingestion of primary radionuclide } i(\mathrm{~Sv} / \mathrm{Bq})
$$


The total dose from ingesting beta-gamma emitters in the groundwater is calculated as

$$
D=\sum_{i} C w_{i} C F_{i}
$$

The effective dose coefficient for the ingestion of a primary radionuclide includes contributions from dose coefficients for the short-lived decay products. The effective dose coefficient is calculated as the weighted sum of the organ or whole body dose coefficients for a primary radionuclide and its short-lived decay products, with the weights corresponding to the branching fractions:

$$
E D C F_{i}=D C F_{i}+\sum_{s} D C F_{s, i} \times B F_{s, i}
$$

where

$$
\begin{aligned}
& D C F_{i}= \text { dose coefficient for primary radionuclide } i(\mathrm{~Sv} / \mathrm{Bq}) \text {, equal to zero if a primary } \\
& \text { radionuclide is not a beta-photon emitter } \\
& D C F_{s, i}= \begin{array}{l}
\text { dose coefficient for short-lived beta-photon emitting radionuclide } s \text { in a decay } \\
\text { chain of a primary radionuclide } i(\mathrm{~Sv} / \mathrm{Bq})
\end{array} \\
& B F_{s, i}=\begin{array}{l}
\text { branching fraction for short-lived beta-photon emitting radionuclide } s \text { in a decay } \\
\text { chain of a primary radionuclide } i \text { (dimensionless) }
\end{array}
\end{aligned}
$$

In this case, the effective dose coefficients account only for contributions from beta- and photonemitting radionuclides. Alpha emitters with a fraction of alpha decays greater than 99 percent are not included. Effective dose coefficients for calculating annual beta-photon dose resulting from consumption of 2 liters of water per day are summarized in Tables 6.3-5 and 6.3-6, for the ICRP 30-based and ICRP 72 methodologies, respectively. The calculations were carried out in Excel (see files Conversion Factors for Groundwater Protection Standard_Rev 3.xls and Conversion Factors for Groundwater Protection Standard_Rev 4.xls in Appendix B for details).

The effective dose coefficient for ${ }^{232} \mathrm{Th}$ includes contributions from ${ }^{228} \mathrm{Ra}$ and ${ }^{228} \mathrm{Th}$ and their short-lived decay products. ${ }^{232} \mathrm{Th}$ is accompanied in the groundwater by its relatively long -lived decay products, ${ }^{228} \mathrm{Ra}$ and ${ }^{228} \mathrm{Th}$ (and their short-lived decay products), which are not primary radionuclides (Section 6.1.1). The effective dose coefficient for ${ }^{232} \mathrm{Th}$ is calculated for the conditions of radioactive equilibrium between ${ }^{232} \mathrm{Th},{ }^{228} \mathrm{Ra}$, and ${ }^{228} \mathrm{Th} .{ }^{232} \mathrm{Th}$ itself is an alpha emitter, so it does not contribute to the value of its effective dose coefficient.

Similarly, ${ }^{232} \mathrm{U}$ is accompanied in the groundwater by its relatively long-lived decay product, ${ }^{228} \mathrm{Th}$ (and its short-lived decay products). The effective dose coefficient attributable to ${ }^{232} \mathrm{U}$ is calculated by including the contribution from ${ }^{228} \mathrm{Th}$ and its short-lived decay products under the conditions of radioactive equilibrium between ${ }^{232} \mathrm{U}$ and ${ }^{228} \mathrm{Th} .{ }^{232} \mathrm{U}$ is an alpha emitter, so by itself does not contribute to the value of its effective dose coefficient.

Tables 6.3-5 and 6.3-6 include the value of the effective dose coefficient for ${ }^{226} \mathrm{Ra}$ (and its short-lived decay products), ${ }^{210} \mathrm{~Pb}$ (and its short-lived decay products) and, separately, the value 
for ${ }^{226} \mathrm{Ra}$ that includes a contribution from ${ }^{210} \mathrm{~Pb}$. The latter value was developed so that the contribution of this relatively long-lived radionuclide to the beta-photon dose is accounted for, in case its concentration in groundwater is not calculated in TSPA. ${ }^{210} \mathrm{~Pb}$ was screened out from the TSPA-LA calculations for the period of up to 20,000 years (BSC 2004 [DIRS 169460], Section 6.1.3).

Tables 6.3-7 and 6.3-8 list conversion factors for calculating the beta-photon doses expressed in the units of $\mathrm{Sv} / \mathrm{yr}$ per $\mathrm{Bq} / \mathrm{m}^{3}$ and mrem/yr per $\mathrm{pCi} / \mathrm{L}$, respectively, for the ICRP 30-based dosimetry. The corresponding set of conversion factor calculated using ICRP 72 dosimetry is presented in Tables 6.3-9 and 6.3-10. The conversion factors were calculated using Equation 6.3-3.

For the individual organs, the difference between the ICRP 30- and ICRP 72-based sets of conversion factors results only from the differences in the ingestion dose coefficients for these organs. The whole body conversion factors are different between the two sets also because the ICRP 72 considers additional organs (colon, extrathoracic pathways, and reminder) and because the organ weighting factors are different. 


\begin{tabular}{|c|c|c|c|c|c|c|c|c|c|c|}
\hline $\begin{array}{l}\text { Effectiv } \\
\text { Conver }\end{array}$ & actors & & & & & & & & & \\
\hline Organ or Tissue & C-14 & $\mathrm{Cl}-36$ & Se-79 & Sr-90 & Tc-99 & Sn-126 & I-129 & Cs-135 & Cs-137 & Th-232 \\
\hline Urinary bladder wall & $5.64 \mathrm{E}-10$ & $7.99 \mathrm{E}-10$ & $9.06 \mathrm{E}-10$ & $1.51 \mathrm{E}-09$ & $6.04 \mathrm{E}-11$ & $1.18 \mathrm{E}-09$ & 1.35E-10 & 1.91E-09 & $1.41 \mathrm{E}-08$ & $1.59 \mathrm{E}-07$ \\
\hline Bone surface & $5.64 \mathrm{E}-10$ & $7.99 \mathrm{E}-10$ & $9.06 \mathrm{E}-10$ & 4.19E-07 & $6.04 \mathrm{E}-11$ & $5.09 \mathrm{E}-09$ & $2.17 \mathrm{E}-10$ & $1.91 \mathrm{E}-09$ & $1.26 \mathrm{E}-08$ & $5.99 \mathrm{E}-06$ \\
\hline Brain & $5.64 \mathrm{E}-10$ & $7.99 \mathrm{E}-10$ & $9.06 \mathrm{E}-10$ & $1.26 \mathrm{E}-14$ & $6.04 \mathrm{E}-11$ & $7.30 \mathrm{E}-10$ & $0.00 \mathrm{E}+00$ & $0.00 \mathrm{E}+00$ & $0.00 \mathrm{E}+00$ & $2.54 \mathrm{E}-13$ \\
\hline Stomach wall & $5.64 \mathrm{E}-10$ & 1.11E-09 & $9.70 \mathrm{E}-10$ & $2.82 \mathrm{E}-09$ & $3.39 \mathrm{E}-09$ & $2.02 \mathrm{E}-09$ & $2.19 \mathrm{E}-10$ & $1.99 \mathrm{E}-09$ & $1.39 \mathrm{E}-08$ & $1.62 \mathrm{E}-07$ \\
\hline Small intestine wall & $5.64 \mathrm{E}-10$ & $7.99 \mathrm{E}-10$ & $9.38 \mathrm{E}-10$ & $4.53 \mathrm{E}-09$ & $1.19 \mathrm{E}-10$ & $4.99 \mathrm{E}-09$ & $1.41 \mathrm{E}-10$ & $1.91 \mathrm{E}-09$ & $1.44 \mathrm{E}-08$ & $1.66 \mathrm{E}-07$ \\
\hline Upper large intestine wall & $5.64 \mathrm{E}-10$ & $7.99 \mathrm{E}-10$ & $1.10 \mathrm{E}-09$ & $1.89 \mathrm{E}-08$ & $4.05 \mathrm{E}-10$ & $1.69 \mathrm{E}-08$ & $1.42 \mathrm{E}-10$ & $1.91 \mathrm{E}-09$ & $1.42 \mathrm{E}-08$ & $1.83 \mathrm{E}-07$ \\
\hline Lower large intestine wall & $5.64 \mathrm{E}-10$ & $7.99 \mathrm{E}-10$ & $1.48 \mathrm{E}-09$ & $5.13 \mathrm{E}-08$ & $1.10 \mathrm{E}-09$ & $4.60 \mathrm{E}-08$ & $1.39 \mathrm{E}-10$ & $1.91 \mathrm{E}-09$ & $1.44 \mathrm{E}-08$ & $2.00 \mathrm{E}-07$ \\
\hline Kidney & $5.64 \mathrm{E}-10$ & 7.99E-10 & $1.25 \mathrm{E}-08$ & $1.51 \mathrm{E}-09$ & $6.04 \mathrm{E}-11$ & $8.79 \mathrm{E}-10$ & $1.34 \mathrm{E}-10$ & $1.91 \mathrm{E}-09$ & 1.37E-08 & 1.69E-07 \\
\hline Liver & $5.64 \mathrm{E}-10$ & $7.99 \mathrm{E}-10$ & $6.48 \mathrm{E}-09$ & $1.51 \mathrm{E}-09$ & $8.23 \mathrm{E}-11$ & 7.30E-10 & $1.38 \mathrm{E}-10$ & $1.91 \mathrm{E}-09$ & $1.36 \mathrm{E}-08$ & $1.80 \mathrm{E}-07$ \\
\hline Pancreas & $5.64 \mathrm{E}-10$ & 7.99E-10 & 3.89E-09 & $1.51 \mathrm{E}-09$ & $6.04 \mathrm{E}-11$ & 8.47E-10 & $1.41 \mathrm{E}-10$ & $1.91 \mathrm{E}-09$ & $1.34 \mathrm{E}-08$ & $1.59 \mathrm{E}-07$ \\
\hline Red bone marrow & $5.64 \mathrm{E}-10$ & $7.99 \mathrm{E}-10$ & $9.06 \mathrm{E}-10$ & $1.94 \mathrm{E}-07$ & $6.04 \mathrm{E}-11$ & 2.81E-09 & $2.21 \mathrm{E}-10$ & $1.91 \mathrm{E}-09$ & $1.32 \mathrm{E}-08$ & $6.68 \mathrm{E}-07$ \\
\hline Skin & $5.64 \mathrm{E}-10$ & $7.99 \mathrm{E}-10$ & $9.06 \mathrm{E}-10$ & $1.51 \mathrm{E}-09$ & $6.04 \mathrm{E}-11$ & $5.67 \mathrm{E}-10$ & $2.11 \mathrm{E}-10$ & $1.91 \mathrm{E}-09$ & 1.04E-08 & $1.59 \mathrm{E}-07$ \\
\hline Spleen & $5.64 \mathrm{E}-10$ & $7.99 \mathrm{E}-10$ & 4.32E-09 & $1.51 \mathrm{E}-09$ & $6.04 \mathrm{E}-11$ & $7.80 \mathrm{E}-10$ & 1.40E-10 & $1.91 \mathrm{E}-09$ & 1.37E-08 & 1.59E-07 \\
\hline Testes & $5.64 \mathrm{E}-10$ & $7.99 \mathrm{E}-10$ & $9.06 \mathrm{E}-10$ & $1.51 \mathrm{E}-09$ & $6.04 \mathrm{E}-11$ & $7.19 \mathrm{E}-10$ & $1.29 \mathrm{E}-10$ & $1.91 \mathrm{E}-09$ & $1.39 \mathrm{E}-08$ & $1.59 \mathrm{E}-07$ \\
\hline Thymus & $5.64 \mathrm{E}-10$ & $7.99 \mathrm{E}-10$ & $9.06 \mathrm{E}-10$ & $1.51 \mathrm{E}-09$ & $6.04 \mathrm{E}-11$ & $5.37 \mathrm{E}-10$ & $3.51 \mathrm{E}-10$ & $1.91 \mathrm{E}-09$ & $1.24 \mathrm{E}-08$ & $1.59 \mathrm{E}-07$ \\
\hline Thyroid & $5.64 \mathrm{E}-10$ & 7.99E-10 & $9.06 \mathrm{E}-10$ & $1.51 \mathrm{E}-09$ & 1.62E-09 & $5.54 \mathrm{E}-10$ & $2.48 \mathrm{E}-06$ & $1.91 \mathrm{E}-09$ & 1.26E-08 & 1.59E-07 \\
\hline Uterus & $5.64 \mathrm{E}-10$ & $7.99 \mathrm{E}-10$ & $9.06 \mathrm{E}-10$ & $1.51 \mathrm{E}-09$ & $6.04 \mathrm{E}-11$ & $1.44 \mathrm{E}-09$ & $1.38 \mathrm{E}-10$ & $1.91 \mathrm{E}-09$ & $1.44 \mathrm{E}-08$ & $1.59 \mathrm{E}-07$ \\
\hline Whole body (h_E) & $5.64 \mathrm{E}-10$ & $8.18 \mathrm{E}-10$ & 2.35E-09 & $4.14 \mathrm{E}-08$ & 3.95E-10 & $5.70 \mathrm{E}-09$ & $7.46 \mathrm{E}-08$ & $1.91 \mathrm{E}-09$ & 1.35E-08 & $4.01 \mathrm{E}-07$ \\
\hline
\end{tabular}




\begin{tabular}{|c|c|c|c|c|c|c|c|c|c|}
\hline $\begin{array}{l}\text { Effectiv } \\
\text { Conver }\end{array}$ & Factors $(C$ & $\begin{array}{l}\text { nts (Sv } \\
\text { tinued) }\end{array}$ & & & & & & & \\
\hline Organ or Tissue & $\mathrm{U}-232$ & Np-237 & Th-229 & U-238 & Ra-226 & $\mathrm{Pb}-210$ & $\begin{array}{c}\mathrm{Ra}-226+ \\
\mathrm{Pb}-210\end{array}$ & Am-243 & Ac-227 \\
\hline Adrenal & 1.67E-09 & $1.25 \mathrm{E}-11$ & 3.37E-08 & $1.76 \mathrm{E}-12$ & $2.86 \mathrm{E}-11$ & $1.25 \mathrm{E}-07$ & $1.25 \mathrm{E}-07$ & $7.91 \mathrm{E}-12$ & $4.40 \mathrm{E}-10$ \\
\hline Urinary bladder wall & $1.72 \mathrm{E}-09$ & $7.58 \mathrm{E}-11$ & 3.37E-08 & $9.45 \mathrm{E}-12$ & $2.69 \mathrm{E}-11$ & $1.25 \mathrm{E}-07$ & $1.25 \mathrm{E}-07$ & 4.74E-11 & $1.04 \mathrm{E}-10$ \\
\hline Bone surface & $1.66 \mathrm{E}-07$ & $1.02 \mathrm{E}-10$ & $1.78 \mathrm{E}-06$ & $2.09 \mathrm{E}-11$ & 1.10E-09 & $2.16 \mathrm{E}-05$ & $2.16 \mathrm{E}-05$ & $3.59 \mathrm{E}-11$ & $6.73 E-05$ \\
\hline Esophagus & 1.64E-09 & $1.41 \mathrm{E}-12$ & $3.37 \mathrm{E}-08$ & $4.35 \mathrm{E}-13$ & $2.32 \mathrm{E}-11$ & $1.25 \mathrm{E}-07$ & $1.25 \mathrm{E}-07$ & 8.13E-13 & $1.42 \mathrm{E}-10$ \\
\hline Stomach wall & 4.53E-09 & $2.92 \mathrm{E}-10$ & $3.53 \mathrm{E}-08$ & $9.98 \mathrm{E}-10$ & 1.74E-09 & $1.25 \mathrm{E}-07$ & $1.27 \mathrm{E}-07$ & $3.46 \mathrm{E}-10$ & $1.40 \mathrm{E}-09$ \\
\hline Small intestine wall & $6.84 \mathrm{E}-09$ & $8.02 \mathrm{E}-10$ & $3.51 \mathrm{E}-08$ & $2.55 \mathrm{E}-09$ & $7.94 \mathrm{E}-10$ & $1.26 \mathrm{E}-07$ & $1.27 \mathrm{E}-07$ & $8.76 \mathrm{E}-10$ & $8.96 \mathrm{E}-10$ \\
\hline Upper large intestine wall & $1.74 \mathrm{E}-08$ & $3.62 \mathrm{E}-09$ & $3.99 \mathrm{E}-08$ & $1.47 \mathrm{E}-08$ & $3.08 \mathrm{E}-10$ & $1.32 \mathrm{E}-07$ & $1.32 \mathrm{E}-07$ & $3.85 \mathrm{E}-09$ & $1.78 \mathrm{E}-09$ \\
\hline Lower large intestine wall & $2.00 \mathrm{E}-08$ & $1.02 \mathrm{E}-08$ & $6.42 \mathrm{E}-08$ & $4.30 \mathrm{E}-08$ & $5.62 \mathrm{E}-11$ & $1.45 \mathrm{E}-07$ & $1.45 \mathrm{E}-07$ & $8.72 \mathrm{E}-09$ & 7.73E-09 \\
\hline Kidney & $1.20 \mathrm{E}-08$ & $3.40 \mathrm{E}-11$ & $3.43 \mathrm{E}-08$ & $3.98 \mathrm{E}-12$ & $1.04 \mathrm{E}-10$ & $2.82 \mathrm{E}-06$ & $2.82 \mathrm{E}-06$ & $2.05 \mathrm{E}-11$ & $3.54 \mathrm{E}-10$ \\
\hline Pancreas & $1.72 \mathrm{E}-09$ & $2.93 \mathrm{E}-11$ & 3.37E-08 & $3.84 \mathrm{E}-12$ & $5.44 \mathrm{E}-11$ & $1.25 \mathrm{E}-07$ & $1.25 \mathrm{E}-07$ & $2.16 \mathrm{E}-11$ & $3.77 \mathrm{E}-10$ \\
\hline Red bone marrow & $1.51 \mathrm{E}-08$ & $6.89 \mathrm{E}-11$ & $1.68 \mathrm{E}-07$ & $1.87 \mathrm{E}-11$ & $1.14 \mathrm{E}-10$ & $1.48 \mathrm{E}-06$ & $1.48 \mathrm{E}-06$ & $4.66 \mathrm{E}-11$ & $5.40 \mathrm{E}-06$ \\
\hline Skin & 1.65E-09 & $8.58 \mathrm{E}-12$ & $3.37 \mathrm{E}-08$ & $1.37 \mathrm{E}-12$ & $2.39 \mathrm{E}-11$ & $1.25 \mathrm{E}-07$ & $1.25 \mathrm{E}-07$ & $5.04 \mathrm{E}-12$ & $1.37 \mathrm{E}-10$ \\
\hline Spleen & $1.69 \mathrm{E}-09$ & $2.16 \mathrm{E}-11$ & 3.37E-08 & $3.13 \mathrm{E}-12$ & $4.08 \mathrm{E}-11$ & $1.25 \mathrm{E}-07$ & $1.25 \mathrm{E}-07$ & $1.51 \mathrm{E}-11$ & $1.59 \mathrm{E}-10$ \\
\hline Testes & $1.66 \mathrm{E}-09$ & $2.10 \mathrm{E}-11$ & $3.37 \mathrm{E}-08$ & $2.61 \mathrm{E}-12$ & $2.29 \mathrm{E}-11$ & $1.25 \mathrm{E}-07$ & $1.25 \mathrm{E}-07$ & $1.14 \mathrm{E}-11$ & $8.31 \mathrm{E}-07$ \\
\hline Thymus & 1.64E-09 & $1.41 \mathrm{E}-12$ & 3.37E-08 & 4.35E-13 & $2.32 \mathrm{E}-11$ & $1.25 \mathrm{E}-07$ & $1.25 \mathrm{E}-07$ & $8.13 \mathrm{E}-13$ & $1.42 \mathrm{E}-10$ \\
\hline Thyroid & 1.63E-09 & $4.81 \mathrm{E}-13$ & $3.37 \mathrm{E}-08$ & $2.94 \mathrm{E}-13$ & $2.23 \mathrm{E}-11$ & $1.25 \mathrm{E}-07$ & $1.25 \mathrm{E}-07$ & $2.07 \mathrm{E}-13$ & $1.26 \mathrm{E}-10$ \\
\hline Uterus & $1.80 \mathrm{E}-09$ & $1.03 \mathrm{E}-10$ & $3.37 \mathrm{E}-08$ & $1.32 \mathrm{E}-11$ & $3.53 \mathrm{E}-11$ & $1.25 \mathrm{E}-07$ & $1.25 \mathrm{E}-07$ & $6.88 \mathrm{E}-11$ & $1.22 \mathrm{E}-10$ \\
\hline Whole body ( $\mathrm{h}$ EE) & $1.26 \mathrm{E}-08$ & $9.81 \mathrm{E}-10$ & $1.04 \mathrm{E}-07$ & $3.69 \mathrm{E}-09$ & $2.45 \mathrm{E}-10$ & $1.45 \mathrm{E}-06$ & $1.45 \mathrm{E}-06$ & $8.82 \mathrm{E}-10$ & $3.80 \mathrm{E}-06$ \\
\hline
\end{tabular}


Table 6.3-6. Effective Dose Coefficients (Sv/Bq) Calculated Using ICRP 72 for Radionuclides Included in Calculation of Conversion Factors

\begin{tabular}{|c|c|c|c|c|c|c|c|c|c|c|}
\hline Organ or Tissue & C-14 & $\mathrm{Cl}-36$ & Se-79 & Sr-90 & TC-99 & Sn-126 & I-129 & Cs-135 & Cs-137 & Th-232 \\
\hline Adrenal & $5.70 \mathrm{E}-10$ & 8.00E-10 & $9.60 \mathrm{E}-10$ & $6.60 \mathrm{E}-10$ & $3.90 \mathrm{E}-11$ & $9.73 E-10$ & $1.20 \mathrm{E}-10$ & 1.90E-09 & $1.40 \mathrm{E}-08$ & $1.52 \mathrm{E}-07$ \\
\hline Urinary bladder wall & $5.70 \mathrm{E}-10$ & $2.10 \mathrm{E}-09$ & 1.10E-09 & 1.50E-09 & $1.60 \mathrm{E}-10$ & 1.24E-09 & 4.30E-10 & 2.20E-09 & 1.40E-08 & $1.52 \mathrm{E}-07$ \\
\hline Bone surface & $5.70 \mathrm{E}-10$ & $8.00 \mathrm{E}-10$ & $9.60 \mathrm{E}-10$ & 4.10E-07 & $3.90 \mathrm{E}-11$ & $5.56 \mathrm{E}-09$ & $4.00 \mathrm{E}-10$ & 1.90E-09 & $1.40 \mathrm{E}-08$ & $2.21 \mathrm{E}-05$ \\
\hline Brain & $5.70 \mathrm{E}-10$ & 8.00E-10 & $9.60 \mathrm{E}-10$ & $6.60 \mathrm{E}-10$ & $3.90 \mathrm{E}-11$ & 7.17E-10 & $1.40 \mathrm{E}-10$ & 1.90E-09 & $1.20 \mathrm{E}-08$ & $1.52 \mathrm{E}-07$ \\
\hline Breast & $5.70 \mathrm{E}-10$ & 8.00E-10 & $9.60 \mathrm{E}-10$ & $6.60 \mathrm{E}-10$ & $3.90 \mathrm{E}-11$ & $4.78 \mathrm{E}-10$ & $1.10 \mathrm{E}-10$ & 1.90E-09 & 1.10E-08 & $1.52 \mathrm{E}-07$ \\
\hline Esophagus & $5.70 \mathrm{E}-10$ & $8.00 \mathrm{E}-10$ & $9.60 \mathrm{E}-10$ & $6.60 \mathrm{E}-10$ & $3.90 \mathrm{E}-11$ & $5.70 \mathrm{E}-10$ & 1.70E-10 & 1.90E-09 & $1.30 \mathrm{E}-08$ & $1.52 \mathrm{E}-07$ \\
\hline Stomach wall & $6.30 \mathrm{E}-10$ & 1.10E-09 & 1.00E-09 & 2.00E-09 & 2.20E-09 & 2.03E-09 & $2.00 \mathrm{E}-10$ & 2.00E-09 & 1.30E-08 & $1.56 \mathrm{E}-07$ \\
\hline Small intestine wall & $5.70 \mathrm{E}-10$ & $8.10 \mathrm{E}-10$ & 1.00E-09 & 3.70E-09 & $1.80 \mathrm{E}-10$ & 4.80E-09 & $1.30 \mathrm{E}-10$ & 1.90E-09 & $1.40 \mathrm{E}-08$ & $1.58 \mathrm{E}-07$ \\
\hline Upper large intestine wall & $5.80 \mathrm{E}-10$ & $9.00 \mathrm{E}-10$ & 1.40E-09 & $1.98 \mathrm{E}-08$ & 1.40E-09 & $1.71 \mathrm{E}-08$ & $2.20 \mathrm{E}-10$ & $2.20 \mathrm{E}-09$ & $1.40 \mathrm{E}-08$ & $1.91 \mathrm{E}-07$ \\
\hline Lower large intestine wall & $6.00 \mathrm{E}-10$ & 1.10E-09 & 2.30E-09 & $5.30 \mathrm{E}-08$ & 3.90E-09 & 4.65E-08 & $4.30 \mathrm{E}-10$ & 2.60E-09 & $1.70 \mathrm{E}-08$ & 2.42E-07 \\
\hline Colon & $5.90 \mathrm{E}-10$ & $9.80 \mathrm{E}-10$ & $1.80 \mathrm{E}-09$ & $3.40 \mathrm{E}-08$ & $2.50 \mathrm{E}-09$ & 2.97E-08 & $3.10 \mathrm{E}-10$ & $2.40 \mathrm{E}-09$ & $1.50 \mathrm{E}-08$ & 2.12E-07 \\
\hline Kidney & $5.70 \mathrm{E}-10$ & 8.00E-10 & $3.20 \mathrm{E}-08$ & $6.60 \mathrm{E}-10$ & $3.90 \mathrm{E}-11$ & $9.56 \mathrm{E}-10$ & $1.20 \mathrm{E}-10$ & 1.90E-09 & $1.30 \mathrm{E}-08$ & 4.75E-07 \\
\hline Liver & $5.70 \mathrm{E}-10$ & 8.00E-10 & 1.40E-08 & $6.60 \mathrm{E}-10$ & $5.20 \mathrm{E}-11$ & $7.84 \mathrm{E}-10$ & $1.20 \mathrm{E}-10$ & 1.90E-09 & $1.30 \mathrm{E}-08$ & $1.01 \mathrm{E}-06$ \\
\hline Muscle & $5.70 \mathrm{E}-10$ & $8.00 \mathrm{E}-10$ & $9.60 \mathrm{E}-10$ & $6.60 \mathrm{E}-10$ & $3.90 \mathrm{E}-11$ & $8.61 \mathrm{E}-10$ & $3.00 \mathrm{E}-10$ & 1.90E-09 & $1.20 \mathrm{E}-08$ & $1.52 \mathrm{E}-07$ \\
\hline Ovaries & $5.70 \mathrm{E}-10$ & $8.00 \mathrm{E}-10$ & 1.80E-09 & $6.60 \mathrm{E}-10$ & $3.90 \mathrm{E}-11$ & 2.90E-09 & $1.30 \mathrm{E}-10$ & 1.90E-09 & $1.40 \mathrm{E}-08$ & 2.13E-07 \\
\hline Pancreas & $5.70 \mathrm{E}-10$ & $8.00 \mathrm{E}-10$ & $5.00 \mathrm{E}-09$ & $6.60 \mathrm{E}-10$ & $3.90 \mathrm{E}-11$ & $9.32 \mathrm{E}-10$ & $1.20 \mathrm{E}-10$ & $1.90 \mathrm{E}-09$ & $1.40 \mathrm{E}-08$ & $1.52 \mathrm{E}-07$ \\
\hline Red bone marrow & $5.70 \mathrm{E}-10$ & $8.00 \mathrm{E}-10$ & $9.60 \mathrm{E}-10$ & $1.80 \mathrm{E}-07$ & $3.90 \mathrm{E}-11$ & 2.93E-09 & $1.40 \mathrm{E}-10$ & 1.90E-09 & $1.30 \mathrm{E}-08$ & $2.31 \mathrm{E}-06$ \\
\hline Extrathoracic airways & $5.70 \mathrm{E}-10$ & $8.00 \mathrm{E}-10$ & $9.60 \mathrm{E}-10$ & $6.60 \mathrm{E}-10$ & $3.90 \mathrm{E}-11$ & $6.37 \mathrm{E}-10$ & 1.40E-10 & $1.90 \mathrm{E}-09$ & $1.30 \mathrm{E}-08$ & $1.52 \mathrm{E}-07$ \\
\hline Lungs & $5.70 \mathrm{E}-10$ & $8.00 \mathrm{E}-10$ & $9.60 \mathrm{E}-10$ & $6.60 \mathrm{E}-10$ & $3.90 \mathrm{E}-11$ & $6.55 \mathrm{E}-10$ & $1.50 \mathrm{E}-10$ & 1.90E-09 & $1.30 \mathrm{E}-08$ & $1.52 \mathrm{E}-07$ \\
\hline Skin & $5.70 \mathrm{E}-10$ & 8.00E-10 & $9.60 \mathrm{E}-10$ & $6.60 \mathrm{E}-10$ & $3.90 \mathrm{E}-11$ & $5.79 \mathrm{E}-10$ & 1.40E-10 & 1.90E-09 & 1.10E-08 & $1.52 \mathrm{E}-07$ \\
\hline Spleen & $5.70 \mathrm{E}-10$ & 8.00E-10 & $5.60 \mathrm{E}-09$ & $6.60 \mathrm{E}-10$ & $3.90 \mathrm{E}-11$ & $7.71 \mathrm{E}-10$ & $1.20 \mathrm{E}-10$ & $1.90 \mathrm{E}-09$ & $1.30 \mathrm{E}-08$ & $1.52 \mathrm{E}-07$ \\
\hline Testes & $5.70 \mathrm{E}-10$ & 8.00E-10 & 2.90E-09 & $6.60 \mathrm{E}-10$ & $3.90 \mathrm{E}-11$ & $7.11 \mathrm{E}-10$ & $1.20 \mathrm{E}-10$ & $1.90 \mathrm{E}-09$ & $1.20 \mathrm{E}-08$ & 2.12E-07 \\
\hline Thymus & $5.70 \mathrm{E}-10$ & 8.00E-10 & $9.60 \mathrm{E}-10$ & $6.60 \mathrm{E}-10$ & $3.90 \mathrm{E}-11$ & $5.70 \mathrm{E}-10$ & 1.70E-10 & 1.90E-09 & 1.30E-08 & $1.52 \mathrm{E}-07$ \\
\hline Thyroid & $5.70 \mathrm{E}-10$ & 8.00E-10 & $9.60 \mathrm{E}-10$ & $6.60 \mathrm{E}-10$ & $1.00 \mathrm{E}-09$ & 6.37E-10 & $2.10 \mathrm{E}-06$ & 1.90E-09 & 1.30E-08 & $1.52 \mathrm{E}-07$ \\
\hline Uterus & $5.70 \mathrm{E}-10$ & 8.00E-10 & $9.60 \mathrm{E}-10$ & $6.60 \mathrm{E}-10$ & $3.90 \mathrm{E}-11$ & 1.59E-09 & $1.30 \mathrm{E}-10$ & 1.90E-09 & 1.40E-08 & $1.52 \mathrm{E}-07$ \\
\hline Remainder & $5.70 \mathrm{E}-10$ & $8.00 \mathrm{E}-10$ & 1.70E-08 & $7.23 \mathrm{E}-10$ & $4.20 \mathrm{E}-11$ & $9.39 \mathrm{E}-10$ & $2.80 \mathrm{E}-10$ & $1.90 \mathrm{E}-09$ & $1.20 \mathrm{E}-08$ & $1.53 \mathrm{E}-07$ \\
\hline Whole body (e) & $5.80 \mathrm{E}-10$ & $9.30 \mathrm{E}-10$ & $2.90 \mathrm{E}-09$ & $3.07 \mathrm{E}-08$ & $6.40 \mathrm{E}-10$ & $5.07 \mathrm{E}-09$ & 1.10E-07 & $2.00 \mathrm{E}-09$ & $1.30 \mathrm{E}-08$ & 6.97E-07 \\
\hline
\end{tabular}




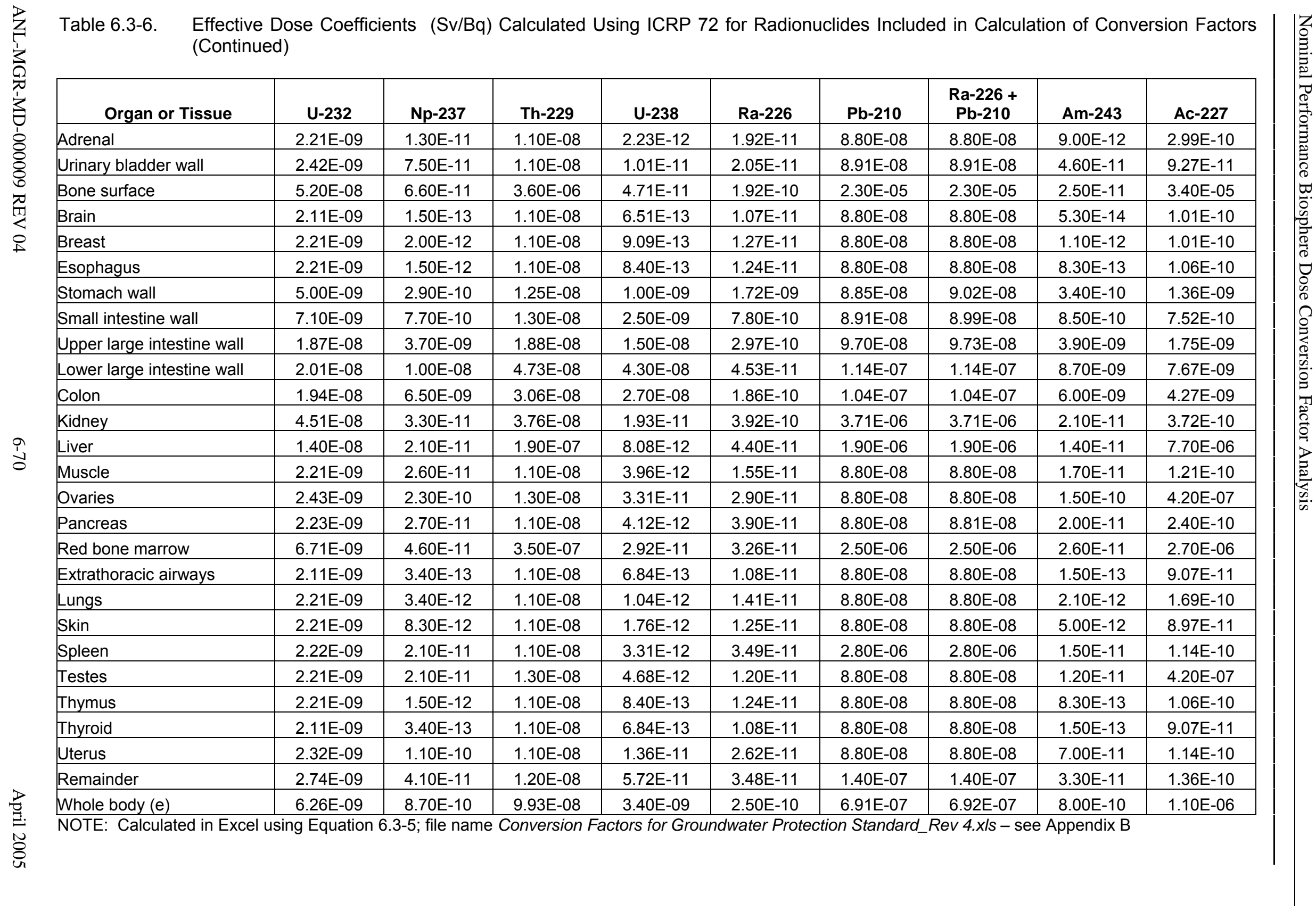


Table 6.3-7. Conversion Factors (Sv/y per Bq/m3) Developed Using ICRP 30-Based Methods for Calculating Annual Beta-Gamma Dose from Drinking 2 Liters of Water per Day

\begin{tabular}{|c|c|c|c|c|c|c|c|c|c|c|}
\hline Organ or Tissue & C-14 & $\mathrm{Cl}-36$ & Se-79 & Sr-90 & TC-99 & Sn-126 & I-129 & Cs-135 & Cs-137 & $\begin{array}{c}\text { Th-232/Ra- } \\
228\end{array}$ \\
\hline Adrenal & $4.12 \mathrm{E}-10$ & $5.84 \mathrm{E}-10$ & $6.62 \mathrm{E}-10$ & 1.10E-09 & $4.41 \mathrm{E}-11$ & $6.73 \mathrm{E}-10$ & $9.64 \mathrm{E}-11$ & 1.40E-09 & $1.10 \mathrm{E}-08$ & $1.17 \mathrm{E}-07$ \\
\hline Urinary bladder wall & $4.12 \mathrm{E}-10$ & $5.84 \mathrm{E}-10$ & $6.62 \mathrm{E}-10$ & 1.10E-09 & $4.41 \mathrm{E}-11$ & $8.64 \mathrm{E}-10$ & $9.86 \mathrm{E}-11$ & $1.40 \mathrm{E}-09$ & $1.03 \mathrm{E}-08$ & $1.16 \mathrm{E}-07$ \\
\hline Bone surface & 4.12E-10 & $5.84 \mathrm{E}-10$ & $6.62 \mathrm{E}-10$ & 3.06E-07 & $4.41 \mathrm{E}-11$ & 3.72E-09 & $1.59 \mathrm{E}-10$ & $1.40 \mathrm{E}-09$ & $9.20 \mathrm{E}-09$ & 4.37E-06 \\
\hline Brain & 4.12E-10 & $5.84 \mathrm{E}-10$ & $6.62 \mathrm{E}-10$ & $9.20 \mathrm{E}-15$ & $4.41 \mathrm{E}-11$ & $5.33 \mathrm{E}-10$ & $0.00 \mathrm{E}+00$ & $0.00 \mathrm{E}+00$ & $0.00 \mathrm{E}+00$ & $1.86 \mathrm{E}-13$ \\
\hline Breast & $4.12 \mathrm{E}-10$ & $5.84 \mathrm{E}-10$ & $6.62 \mathrm{E}-10$ & $1.10 \mathrm{E}-09$ & $4.41 \mathrm{E}-11$ & $6.19 \mathrm{E}-10$ & $2.42 \mathrm{E}-10$ & $1.40 \mathrm{E}-09$ & 9.06E-09 & $1.16 \mathrm{E}-07$ \\
\hline Esophagus & $4.12 \mathrm{E}-10$ & $5.84 \mathrm{E}-10$ & $6.62 \mathrm{E}-10$ & 1.10E-09 & $4.41 \mathrm{E}-11$ & $3.92 \mathrm{E}-10$ & $2.56 \mathrm{E}-10$ & 1.40E-09 & $9.06 \mathrm{E}-09$ & $1.16 \mathrm{E}-07$ \\
\hline Stomach wall & 4.12E-10 & $8.11 \mathrm{E}-10$ & $7.09 \mathrm{E}-10$ & 2.06E-09 & $2.48 \mathrm{E}-09$ & $1.48 \mathrm{E}-09$ & $1.60 \mathrm{E}-10$ & $1.45 \mathrm{E}-09$ & $1.02 \mathrm{E}-08$ & $1.18 \mathrm{E}-07$ \\
\hline Small intestine wall & $4.12 \mathrm{E}-10$ & $5.84 \mathrm{E}-10$ & $6.85 \mathrm{E}-10$ & 3.31E-09 & $8.69 \mathrm{E}-11$ & $3.64 \mathrm{E}-09$ & $1.03 \mathrm{E}-10$ & $1.40 \mathrm{E}-09$ & $1.05 \mathrm{E}-08$ & $1.21 \mathrm{E}-07$ \\
\hline Upper large intestine wall & $4.12 \mathrm{E}-10$ & $5.84 \mathrm{E}-10$ & $8.04 \mathrm{E}-10$ & $1.38 \mathrm{E}-08$ & $2.96 \mathrm{E}-10$ & $1.24 \mathrm{E}-08$ & $1.04 \mathrm{E}-10$ & 1.40E-09 & $1.04 \mathrm{E}-08$ & $1.34 \mathrm{E}-07$ \\
\hline Lower large intestine wall & 4.12E-10 & $5.84 \mathrm{E}-10$ & $1.08 \mathrm{E}-09$ & $3.75 \mathrm{E}-08$ & $8.04 \mathrm{E}-10$ & $3.36 \mathrm{E}-08$ & $1.02 \mathrm{E}-10$ & $1.40 \mathrm{E}-09$ & $1.05 \mathrm{E}-08$ & $1.46 \mathrm{E}-07$ \\
\hline Kidney & $4.12 \mathrm{E}-10$ & $5.84 \mathrm{E}-10$ & $9.13 \mathrm{E}-09$ & 1.10E-09 & $4.41 \mathrm{E}-11$ & $6.42 \mathrm{E}-10$ & $9.79 \mathrm{E}-11$ & 1.40E-09 & $1.00 \mathrm{E}-08$ & 1.23E-07 \\
\hline Liver & $4.12 \mathrm{E}-10$ & $5.84 \mathrm{E}-10$ & 4.73E-09 & $1.10 \mathrm{E}-09$ & $6.01 \mathrm{E}-11$ & $5.33 \mathrm{E}-10$ & $1.01 \mathrm{E}-10$ & $1.40 \mathrm{E}-09$ & 9.93E-09 & $1.31 \mathrm{E}-07$ \\
\hline Lung & 4.12E-10 & $5.84 \mathrm{E}-10$ & $6.62 \mathrm{E}-10$ & 1.10E-09 & $4.41 \mathrm{E}-11$ & $4.46 \mathrm{E}-10$ & $1.21 \mathrm{E}-10$ & $1.40 \mathrm{E}-09$ & $9.28 \mathrm{E}-09$ & $1.16 \mathrm{E}-07$ \\
\hline Muscle & $4.12 \mathrm{E}-10$ & $5.84 \mathrm{E}-10$ & $6.62 \mathrm{E}-10$ & 1.10E-09 & $4.41 \mathrm{E}-11$ & $6.19 \mathrm{E}-10$ & $2.42 \mathrm{E}-10$ & 1.40E-09 & $9.06 \mathrm{E}-09$ & $1.16 \mathrm{E}-07$ \\
\hline Ovaries & 4.12E-10 & $5.84 \mathrm{E}-10$ & $6.62 \mathrm{E}-10$ & 1.10E-09 & $4.41 \mathrm{E}-11$ & 2.06E-09 & $1.01 \mathrm{E}-10$ & $1.40 \mathrm{E}-09$ & $9.42 \mathrm{E}-09$ & 1.17E-07 \\
\hline Pancreas & 4.12E-10 & $5.84 \mathrm{E}-10$ & 2.84E-09 & $1.10 \mathrm{E}-09$ & $4.41 \mathrm{E}-11$ & $6.19 \mathrm{E}-10$ & $1.03 \mathrm{E}-10$ & $1.40 \mathrm{E}-09$ & $9.79 \mathrm{E}-09$ & 1.16E-07 \\
\hline Red bone marrow & 4.12E-10 & $5.84 \mathrm{E}-10$ & $6.62 \mathrm{E}-10$ & $1.42 \mathrm{E}-07$ & $4.41 \mathrm{E}-11$ & $2.05 \mathrm{E}-09$ & $1.61 \mathrm{E}-10$ & $1.40 \mathrm{E}-09$ & $9.64 \mathrm{E}-09$ & $4.88 \mathrm{E}-07$ \\
\hline Skin & $4.12 \mathrm{E}-10$ & $5.84 \mathrm{E}-10$ & $6.62 \mathrm{E}-10$ & $1.10 \mathrm{E}-09$ & $4.41 \mathrm{E}-11$ & 4.14E-10 & $1.54 \mathrm{E}-10$ & $1.40 \mathrm{E}-09$ & $7.60 \mathrm{E}-09$ & 1.16E-07 \\
\hline Spleen & 4.12E-10 & $5.84 \mathrm{E}-10$ & $3.16 \mathrm{E}-09$ & $1.10 \mathrm{E}-09$ & $4.41 \mathrm{E}-11$ & $5.70 \mathrm{E}-10$ & $1.02 \mathrm{E}-10$ & $1.40 \mathrm{E}-09$ & $1.00 \mathrm{E}-08$ & 1.16E-07 \\
\hline Testes & $4.12 \mathrm{E}-10$ & $5.84 \mathrm{E}-10$ & $6.62 \mathrm{E}-10$ & $1.10 \mathrm{E}-09$ & $4.41 \mathrm{E}-11$ & $5.25 \mathrm{E}-10$ & $9.42 \mathrm{E}-11$ & $1.40 \mathrm{E}-09$ & $1.02 \mathrm{E}-08$ & $1.16 \mathrm{E}-07$ \\
\hline Thymus & 4.12E-10 & $5.84 \mathrm{E}-10$ & $6.62 \mathrm{E}-10$ & 1.10E-09 & $4.41 \mathrm{E}-11$ & $3.92 \mathrm{E}-10$ & $2.56 \mathrm{E}-10$ & $1.40 \mathrm{E}-09$ & $9.06 \mathrm{E}-09$ & $1.16 \mathrm{E}-07$ \\
\hline Thyroid & $4.12 \mathrm{E}-10$ & $5.84 \mathrm{E}-10$ & $6.62 \mathrm{E}-10$ & 1.10E-09 & $1.18 \mathrm{E}-09$ & $4.04 \mathrm{E}-10$ & $1.81 \mathrm{E}-06$ & 1.40E-09 & $9.20 \mathrm{E}-09$ & $1.16 \mathrm{E}-07$ \\
\hline Uterus & 4.12E-10 & $5.84 \mathrm{E}-10$ & $6.62 \mathrm{E}-10$ & $1.10 \mathrm{E}-09$ & $4.41 \mathrm{E}-11$ & $1.05 \mathrm{E}-09$ & $1.01 \mathrm{E}-10$ & $1.40 \mathrm{E}-09$ & $1.05 \mathrm{E}-08$ & $1.16 \mathrm{E}-07$ \\
\hline Whole body (h_E) & $4.12 \mathrm{E}-10$ & $5.98 \mathrm{E}-10$ & $1.72 \mathrm{E}-09$ & $3.03 E-08$ & $2.89 \mathrm{E}-10$ & $4.16 \mathrm{E}-09$ & $5.45 \mathrm{E}-08$ & $1.40 \mathrm{E}-09$ & $9.86 \mathrm{E}-09$ & $2.93 \mathrm{E}-07$ \\
\hline
\end{tabular}




\begin{tabular}{|c|c|c|c|c|c|c|c|c|c|}
\hline Organ or Tissue & U-232 & Np-237 & Th-229 & U-238 & Ra-226 & $\mathrm{Pb}-210$ & $\begin{array}{c}\text { Ra-226+Pb- } \\
210\end{array}$ & Am-243 & Ac-227 \\
\hline Adrenal & $1.22 \mathrm{E}-09$ & $9.13 \mathrm{E}-12$ & $2.46 \mathrm{E}-08$ & $1.29 \mathrm{E}-12$ & $2.09 \mathrm{E}-11$ & $9.13 \mathrm{E}-08$ & $9.13 \mathrm{E}-08$ & $5.78 \mathrm{E}-12$ & $3.22 \mathrm{E}-10$ \\
\hline Urinary bladder wall & $1.26 \mathrm{E}-09$ & $5.54 \mathrm{E}-11$ & $2.46 \mathrm{E}-08$ & $6.90 \mathrm{E}-12$ & $1.97 \mathrm{E}-11$ & $9.13 \mathrm{E}-08$ & $9.13 \mathrm{E}-08$ & $3.46 \mathrm{E}-11$ & $7.63 \mathrm{E}-11$ \\
\hline Bone surface & $1.21 \mathrm{E}-07$ & $7.45 \mathrm{E}-11$ & $1.30 \mathrm{E}-06$ & $1.53 \mathrm{E}-11$ & $8.04 \mathrm{E}-10$ & $1.58 \mathrm{E}-05$ & $1.58 \mathrm{E}-05$ & $2.62 \mathrm{E}-11$ & $4.92 \mathrm{E}-05$ \\
\hline Esophagus & $1.20 \mathrm{E}-09$ & $1.03 \mathrm{E}-12$ & $2.46 \mathrm{E}-08$ & $3.18 \mathrm{E}-13$ & $1.69 \mathrm{E}-11$ & $9.13 \mathrm{E}-08$ & $9.13 \mathrm{E}-08$ & $5.94 \mathrm{E}-13$ & $1.04 \mathrm{E}-10$ \\
\hline Stomach wall & 3.31E-09 & $2.13 \mathrm{E}-10$ & $2.58 \mathrm{E}-08$ & $7.29 \mathrm{E}-10$ & 1.27E-09 & 9.17E-08 & $9.29 \mathrm{E}-08$ & $2.53 \mathrm{E}-10$ & $1.02 \mathrm{E}-09$ \\
\hline Small intestine wall & $5.00 \mathrm{E}-09$ & $5.86 \mathrm{E}-10$ & $2.56 \mathrm{E}-08$ & $1.87 \mathrm{E}-09$ & $5.80 \mathrm{E}-10$ & $9.21 \mathrm{E}-08$ & $9.27 \mathrm{E}-08$ & $6.40 \mathrm{E}-10$ & $6.55 \mathrm{E}-10$ \\
\hline Upper large intestine wall & $1.27 \mathrm{E}-08$ & $2.64 \mathrm{E}-09$ & $2.91 \mathrm{E}-08$ & $1.07 \mathrm{E}-08$ & $2.25 \mathrm{E}-10$ & $9.63 \mathrm{E}-08$ & $9.65 \mathrm{E}-08$ & $2.81 \mathrm{E}-09$ & 1.30E-09 \\
\hline Lower large intestine wall & $1.46 \mathrm{E}-08$ & $7.45 \mathrm{E}-09$ & $4.69 \mathrm{E}-08$ & $3.14 \mathrm{E}-08$ & $4.11 \mathrm{E}-11$ & $1.06 \mathrm{E}-07$ & $1.06 \mathrm{E}-07$ & 6.37E-09 & $5.65 \mathrm{E}-09$ \\
\hline Kidney & 8.77E-09 & $2.48 \mathrm{E}-11$ & $2.51 \mathrm{E}-08$ & $2.91 \mathrm{E}-12$ & $7.61 \mathrm{E}-11$ & $2.06 \mathrm{E}-06$ & $2.06 \mathrm{E}-06$ & $1.50 \mathrm{E}-11$ & $2.59 \mathrm{E}-10$ \\
\hline Pancreas & $1.26 \mathrm{E}-09$ & $2.14 \mathrm{E}-11$ & $2.46 \mathrm{E}-08$ & $2.80 \mathrm{E}-12$ & $3.97 \mathrm{E}-11$ & $9.13 \mathrm{E}-08$ & $9.14 \mathrm{E}-08$ & $1.58 \mathrm{E}-11$ & $2.75 \mathrm{E}-10$ \\
\hline Red bone marrow & 1.10E-08 & $5.03 E-11$ & 1.23E-07 & $1.36 \mathrm{E}-11$ & $8.36 \mathrm{E}-11$ & $1.08 \mathrm{E}-06$ & $1.08 \mathrm{E}-06$ & $3.40 \mathrm{E}-11$ & $3.94 \mathrm{E}-06$ \\
\hline Skin & $1.20 \mathrm{E}-09$ & $6.27 \mathrm{E}-12$ & $2.46 \mathrm{E}-08$ & $1.00 \mathrm{E}-12$ & $1.74 \mathrm{E}-11$ & $9.13 \mathrm{E}-08$ & $9.13 \mathrm{E}-08$ & $3.68 \mathrm{E}-12$ & $1.00 \mathrm{E}-10$ \\
\hline Spleen & $1.24 \mathrm{E}-09$ & $1.58 \mathrm{E}-11$ & $2.46 \mathrm{E}-08$ & $2.29 \mathrm{E}-12$ & $2.98 \mathrm{E}-11$ & $9.13 \mathrm{E}-08$ & $9.14 \mathrm{E}-08$ & $1.10 \mathrm{E}-11$ & 1.16E-10 \\
\hline Testes & $1.21 \mathrm{E}-09$ & $1.53 \mathrm{E}-11$ & $2.46 \mathrm{E}-08$ & $1.90 \mathrm{E}-12$ & $1.68 \mathrm{E}-11$ & $9.13 \mathrm{E}-08$ & $9.13 E-08$ & $8.33 E-12$ & $6.07 \mathrm{E}-07$ \\
\hline Thymus & $1.20 \mathrm{E}-09$ & $1.03 \mathrm{E}-12$ & $2.46 \mathrm{E}-08$ & $3.18 \mathrm{E}-13$ & $1.69 \mathrm{E}-11$ & $9.13 \mathrm{E}-08$ & $9.13 E-08$ & $5.94 \mathrm{E}-13$ & $1.04 \mathrm{E}-10$ \\
\hline Thyroid & 1.19E-09 & $3.51 \mathrm{E}-13$ & $2.46 \mathrm{E}-08$ & $2.15 \mathrm{E}-13$ & $1.63 \mathrm{E}-11$ & 9.13E-08 & $9.13 E-08$ & $1.51 \mathrm{E}-13$ & $9.23 \mathrm{E}-11$ \\
\hline Uterus & 1.32E-09 & $7.52 \mathrm{E}-11$ & $2.46 \mathrm{E}-08$ & $9.65 \mathrm{E}-12$ & $2.58 \mathrm{E}-11$ & $9.13 \mathrm{E}-08$ & $9.14 \mathrm{E}-08$ & $5.03 \mathrm{E}-11$ & $8.90 \mathrm{E}-11$ \\
\hline Whole body (h_E) & $9.19 \mathrm{E}-09$ & $7.17 \mathrm{E}-10$ & 7.62E-08 & $2.70 \mathrm{E}-09$ & $1.79 \mathrm{E}-10$ & $1.06 \mathrm{E}-06$ & $1.06 \mathrm{E}-06$ & $6.44 \mathrm{E}-10$ & $2.78 \mathrm{E}-06$ \\
\hline
\end{tabular}




\begin{tabular}{|c|c|c|c|c|c|c|c|c|c|c|}
\hline Organ or Tissue & C-14 & $\mathrm{Cl}-36$ & Se-79 & Sr-90 & TC-99 & Sn-126 & I-129 & Cs-135 & Cs-137 & $\begin{array}{c}\text { Th-232/Ra- } \\
228\end{array}$ \\
\hline Adrenal & $1.52 \mathrm{E}-03$ & 2.16E-03 & $2.45 \mathrm{E}-03$ & $4.08 \mathrm{E}-03$ & 1.63E-04 & $2.49 \mathrm{E}-03$ & $3.57 \mathrm{E}-04$ & $5.16 \mathrm{E}-03$ & $4.05 \mathrm{E}-02$ & $4.32 \mathrm{E}-01$ \\
\hline Urinary bladder & $1.52 \mathrm{E}-03$ & $2.16 \mathrm{E}-03$ & $2.45 \mathrm{E}-03$ & $4.08 \mathrm{E}-03$ & $1.63 \mathrm{E}-04$ & $3.20 \mathrm{E}-03$ & $3.65 \mathrm{E}-04$ & $5.16 \mathrm{E}-03$ & $3.81 \mathrm{E}-02$ & $4.29 \mathrm{E}-01$ \\
\hline Bone surface & $1.52 \mathrm{E}-03$ & 2.16E-03 & $2.45 \mathrm{E}-03$ & $1.13 \mathrm{E}+00$ & $1.63 \mathrm{E}-04$ & $1.38 \mathrm{E}-02$ & $5.87 \mathrm{E}-04$ & $5.16 \mathrm{E}-03$ & $3.41 \mathrm{E}-02$ & $1.62 \mathrm{E}+01$ \\
\hline Esophagus & $1.52 \mathrm{E}-03$ & $2.16 \mathrm{E}-03$ & $2.45 \mathrm{E}-03$ & $4.08 \mathrm{E}-03$ & $1.63 \mathrm{E}-04$ & $1.45 \mathrm{E}-03$ & $9.49 \mathrm{E}-04$ & $5.16 \mathrm{E}-03$ & $3.35 \mathrm{E}-02$ & $4.29 \mathrm{E}-01$ \\
\hline Stomach wall & $1.52 \mathrm{E}-03$ & $3.00 \mathrm{E}-03$ & $2.62 \mathrm{E}-03$ & $7.62 \mathrm{E}-03$ & $9.16 \mathrm{E}-03$ & $5.47 \mathrm{E}-03$ & $5.92 \mathrm{E}-04$ & $5.38 \mathrm{E}-03$ & $3.76 \mathrm{E}-02$ & $4.38 \mathrm{E}-01$ \\
\hline Small intestine wall & $1.52 \mathrm{E}-03$ & $2.16 \mathrm{E}-03$ & $2.54 \mathrm{E}-03$ & $1.22 \mathrm{E}-02$ & $3.22 \mathrm{E}-04$ & $1.35 \mathrm{E}-02$ & $3.81 \mathrm{E}-04$ & $5.16 \mathrm{E}-03$ & $3.89 \mathrm{E}-02$ & $4.49 \mathrm{E}-01$ \\
\hline Upper large intestine wall & $1.52 \mathrm{E}-03$ & $2.16 \mathrm{E}-03$ & 2.97E-03 & $5.12 \mathrm{E}-02$ & $1.09 \mathrm{E}-03$ & $4.57 \mathrm{E}-02$ & $3.84 \mathrm{E}-04$ & $5.16 \mathrm{E}-03$ & $3.84 \mathrm{E}-02$ & 4.94E-01 \\
\hline Lower large intestine wall & $1.52 \mathrm{E}-03$ & $2.16 \mathrm{E}-03$ & $4.00 \mathrm{E}-03$ & $1.39 \mathrm{E}-01$ & $2.97 \mathrm{E}-03$ & $1.24 \mathrm{E}-01$ & $3.76 \mathrm{E}-04$ & $5.16 \mathrm{E}-03$ & $3.89 \mathrm{E}-02$ & $5.40 \mathrm{E}-01$ \\
\hline Kidney & $1.52 \mathrm{E}-03$ & $2.16 \mathrm{E}-03$ & $3.38 \mathrm{E}-02$ & $4.08 \mathrm{E}-03$ & $1.63 \mathrm{E}-04$ & $2.37 \mathrm{E}-03$ & $3.62 \mathrm{E}-04$ & $5.16 \mathrm{E}-03$ & $3.70 \mathrm{E}-02$ & 4.57E-01 \\
\hline Pancreas & $1.52 \mathrm{E}-03$ & $2.16 \mathrm{E}-03$ & $1.05 \mathrm{E}-02$ & $4.08 \mathrm{E}-03$ & 1.63E-04 & $2.29 \mathrm{E}-03$ & $3.81 \mathrm{E}-04$ & $5.16 \mathrm{E}-03$ & $3.62 \mathrm{E}-02$ & $4.29 \mathrm{E}-01$ \\
\hline Red bone marrow & $1.52 \mathrm{E}-03$ & $2.16 \mathrm{E}-03$ & $2.45 \mathrm{E}-03$ & $5.24 \mathrm{E}-01$ & 1.63E-04 & $7.58 \mathrm{E}-03$ & 5.97E-04 & $5.16 \mathrm{E}-03$ & $3.57 \mathrm{E}-02$ & $1.81 \mathrm{E}+00$ \\
\hline Skin & $1.52 \mathrm{E}-03$ & 2.16E-03 & $2.45 \mathrm{E}-03$ & $4.08 \mathrm{E}-03$ & $1.63 \mathrm{E}-04$ & $1.53 \mathrm{E}-03$ & $5.70 \mathrm{E}-04$ & $5.16 \mathrm{E}-03$ & $2.81 \mathrm{E}-02$ & $4.29 \mathrm{E}-01$ \\
\hline Spleen & $1.52 \mathrm{E}-03$ & $2.16 \mathrm{E}-03$ & 1.17E-02 & $4.08 \mathrm{E}-03$ & 1.63E-04 & $2.11 \mathrm{E}-03$ & $3.78 \mathrm{E}-04$ & $5.16 \mathrm{E}-03$ & $3.70 \mathrm{E}-02$ & $4.29 \mathrm{E}-01$ \\
\hline Testes & $1.52 \mathrm{E}-03$ & $2.16 \mathrm{E}-03$ & $2.45 \mathrm{E}-03$ & $4.08 \mathrm{E}-03$ & 1.63E-04 & $1.94 \mathrm{E}-03$ & $3.49 \mathrm{E}-04$ & $5.16 \mathrm{E}-03$ & $3.76 \mathrm{E}-02$ & $4.29 \mathrm{E}-01$ \\
\hline Thymus & $1.52 \mathrm{E}-03$ & $2.16 \mathrm{E}-03$ & $2.45 \mathrm{E}-03$ & $4.08 \mathrm{E}-03$ & 1.63E-04 & $1.45 \mathrm{E}-03$ & $9.49 \mathrm{E}-04$ & $5.16 \mathrm{E}-03$ & $3.35 \mathrm{E}-02$ & $4.29 \mathrm{E}-01$ \\
\hline Thyroid & $1.52 \mathrm{E}-03$ & $2.16 \mathrm{E}-03$ & $2.45 \mathrm{E}-03$ & $4.08 \mathrm{E}-03$ & $4.38 \mathrm{E}-03$ & $1.50 \mathrm{E}-03$ & $6.70 \mathrm{E}+00$ & $5.16 \mathrm{E}-03$ & $3.41 \mathrm{E}-02$ & 4.29E-01 \\
\hline Uterus & $1.52 \mathrm{E}-03$ & $2.16 \mathrm{E}-03$ & $2.45 \mathrm{E}-03$ & $4.08 \mathrm{E}-03$ & 1.63E-04 & $3.89 \mathrm{E}-03$ & 3.73E-04 & $5.16 \mathrm{E}-03$ & $3.89 \mathrm{E}-02$ & $4.29 \mathrm{E}-01$ \\
\hline Whole body (h_E) & $1.52 \mathrm{E}-03$ & $2.21 \mathrm{E}-03$ & $6.35 \mathrm{E}-03$ & $1.12 \mathrm{E}-01$ & $1.07 \mathrm{E}-03$ & $1.54 \mathrm{E}-02$ & $2.02 \mathrm{E}-01$ & $5.16 \mathrm{E}-03$ & $3.65 \mathrm{E}-02$ & $1.08 \mathrm{E}+00$ \\
\hline
\end{tabular}




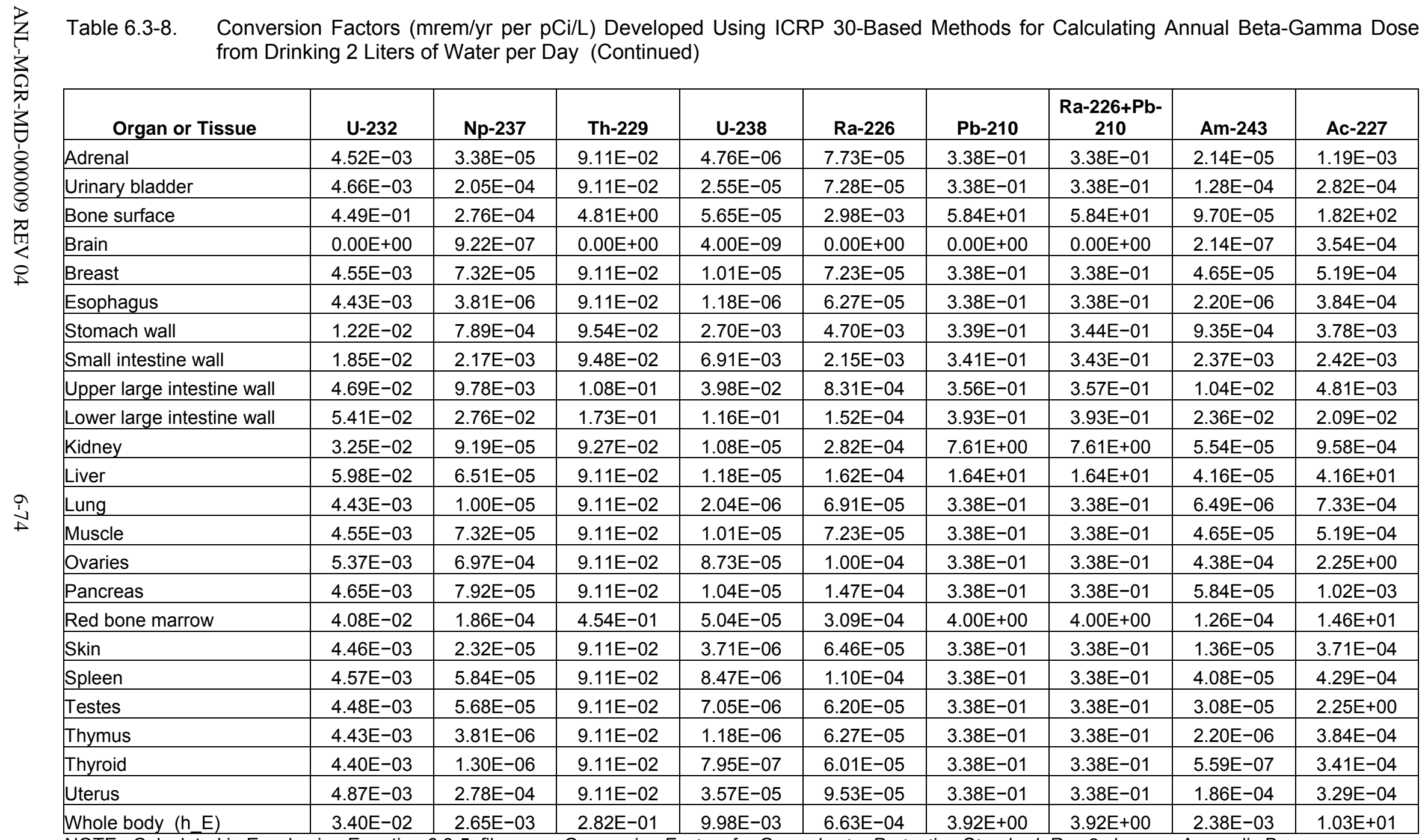




\begin{tabular}{|c|c|c|c|c|c|c|c|c|c|c|}
\hline Table 6.3-9. & Day & 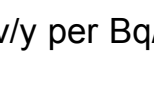 & 3) Deve & Using & 121 & Uutan & ual BE & mima & trom Dr & Liters \\
\hline Organ or Tissue & C-14 & $\mathrm{Cl}-36$ & Se-79 & Sr-90 & Tc-99 & Sn-126 & I-129 & Cs-135 & Cs-137 & $\begin{array}{c}\text { Th-232/Ra- } \\
228\end{array}$ \\
\hline Adrenal & $4.16 \mathrm{E}-10$ & $5.84 \mathrm{E}-10$ & $7.01 \mathrm{E}-10$ & $4.82 \mathrm{E}-10$ & $2.85 \mathrm{E}-11$ & $7.11 \mathrm{E}-10$ & 8.77E-11 & 1.39E-09 & $1.02 \mathrm{E}-08$ & $1.11 \mathrm{E}-07$ \\
\hline Urinary bladder wall & $4.16 \mathrm{E}-10$ & 1.53E-09 & 8.04E-10 & 1.10E-09 & 1.17E-10 & $9.07 \mathrm{E}-10$ & 3.14E-10 & 1.61E-09 & $1.02 \mathrm{E}-08$ & 1.11E-07 \\
\hline Bone surface & 4.16E-10 & $5.84 \mathrm{E}-10$ & $7.01 \mathrm{E}-10$ & $3.00 \mathrm{E}-07$ & $2.85 \mathrm{E}-11$ & 4.06E-09 & $2.92 \mathrm{E}-10$ & 1.39E-09 & $1.02 E-08$ & $1.61 \mathrm{E}-05$ \\
\hline Brain & 4.16E-10 & $5.84 \mathrm{E}-10$ & 7.01E-10 & 4.82E-10 & 2.85E-11 & $5.24 \mathrm{E}-10$ & 1.02E-10 & 1.39E-09 & 8.77E-09 & $1.11 \mathrm{E}-07$ \\
\hline Breast & $4.16 \mathrm{E}-10$ & $5.84 \mathrm{E}-10$ & $7.01 \mathrm{E}-10$ & $4.82 \mathrm{E}-10$ & $2.85 \mathrm{E}-11$ & $3.49 \mathrm{E}-10$ & $8.04 \mathrm{E}-11$ & 1.39E-09 & 8.04E-09 & 1.11E-07 \\
\hline Esophagus & 4.16E-10 & $5.84 \mathrm{E}-10$ & $7.01 \mathrm{E}-10$ & $4.82 \mathrm{E}-10$ & $2.85 \mathrm{E}-11$ & 4.17E-10 & $1.24 \mathrm{E}-10$ & 1.39E-09 & 9.50E-09 & $1.11 \mathrm{E}-07$ \\
\hline Stomach wall & $4.60 \mathrm{E}-10$ & $8.04 \mathrm{E}-10$ & $7.31 \mathrm{E}-10$ & $1.46 \mathrm{E}-09$ & $1.61 \mathrm{E}-09$ & $1.48 \mathrm{E}-09$ & $1.46 \mathrm{E}-10$ & $1.46 \mathrm{E}-09$ & 9.50E-09 & 1.14E-07 \\
\hline Small intestine wall & $4.16 \mathrm{E}-10$ & $5.92 \mathrm{E}-10$ & $7.31 \mathrm{E}-10$ & 2.70E-09 & $1.31 \mathrm{E}-10$ & $3.50 \mathrm{E}-09$ & $9.50 \mathrm{E}-11$ & 1.39E-09 & $1.02 \mathrm{E}-08$ & 1.15E-07 \\
\hline Upper large intestine wall & $4.24 \mathrm{E}-10$ & $6.57 \mathrm{E}-10$ & 1.02E-09 & $1.45 \mathrm{E}-08$ & 1.02E-09 & $1.25 \mathrm{E}-08$ & $1.61 \mathrm{E}-10$ & 1.61E-09 & $1.02 \mathrm{E}-08$ & $1.40 \mathrm{E}-07$ \\
\hline Lower large intestine wall & $4.38 \mathrm{E}-10$ & 8.04E-10 & $1.68 \mathrm{E}-09$ & 3.87E-08 & 2.85E-09 & $3.40 \mathrm{E}-08$ & $3.14 \mathrm{E}-10$ & $1.90 \mathrm{E}-09$ & $1.24 \mathrm{E}-08$ & 1.77E-07 \\
\hline Colon & $4.31 \mathrm{E}-10$ & $7.16 \mathrm{E}-10$ & 1.31E-09 & $2.48 \mathrm{E}-08$ & 1.83E-09 & 2.17E-08 & $2.26 \mathrm{E}-10$ & 1.75E-09 & 1.10E-08 & $1.55 \mathrm{E}-07$ \\
\hline Kidney & $4.16 \mathrm{E}-10$ & $5.84 \mathrm{E}-10$ & 2.34E-08 & 4.82E-10 & $2.85 \mathrm{E}-11$ & $6.98 \mathrm{E}-10$ & 8.77E-11 & 1.39E-09 & $9.50 \mathrm{E}-09$ & 3.47E-07 \\
\hline Liver & $4.16 \mathrm{E}-10$ & $5.84 \mathrm{E}-10$ & 1.02E-08 & $4.82 \mathrm{E}-10$ & $3.80 \mathrm{E}-11$ & $5.73 \mathrm{E}-10$ & 8.77E-11 & 1.39E-09 & $9.50 \mathrm{E}-09$ & $7.41 \mathrm{E}-07$ \\
\hline Muscle & 4.16E-10 & $5.84 \mathrm{E}-10$ & 7.01E-10 & $4.82 \mathrm{E}-10$ & $2.85 \mathrm{E}-11$ & $6.29 \mathrm{E}-10$ & $2.19 \mathrm{E}-10$ & $1.39 \mathrm{E}-09$ & 8.77E-09 & $1.11 \mathrm{E}-07$ \\
\hline Ovaries & $4.16 \mathrm{E}-10$ & $5.84 \mathrm{E}-10$ & 1.31E-09 & 4.82E-10 & 2.85E-11 & 2.12E-09 & $9.50 \mathrm{E}-11$ & 1.39E-09 & $1.02 \mathrm{E}-08$ & $1.55 \mathrm{E}-07$ \\
\hline Pancreas & $4.16 \mathrm{E}-10$ & $5.84 \mathrm{E}-10$ & $3.65 \mathrm{E}-09$ & $4.82 \mathrm{E}-10$ & $2.85 \mathrm{E}-11$ & $6.81 \mathrm{E}-10$ & $8.77 \mathrm{E}-11$ & 1.39E-09 & $1.02 \mathrm{E}-08$ & $1.11 \mathrm{E}-07$ \\
\hline Red bone marrow & $4.16 \mathrm{E}-10$ & $5.84 \mathrm{E}-10$ & 7.01E-10 & $1.31 \mathrm{E}-07$ & $2.85 \mathrm{E}-11$ & $2.14 \mathrm{E}-09$ & 1.02E-10 & 1.39E-09 & $9.50 \mathrm{E}-09$ & $1.69 \mathrm{E}-06$ \\
\hline Extrathoracic airways & $4.16 \mathrm{E}-10$ & $5.84 \mathrm{E}-10$ & 7.01E-10 & $4.82 \mathrm{E}-10$ & 2.85E-11 & 4.65E-10 & $1.02 \mathrm{E}-10$ & 1.39E-09 & $9.50 \mathrm{E}-09$ & 1.11E-07 \\
\hline Lungs & $4.16 \mathrm{E}-10$ & $5.84 \mathrm{E}-10$ & $7.01 \mathrm{E}-10$ & $4.82 \mathrm{E}-10$ & $2.85 \mathrm{E}-11$ & $4.79 \mathrm{E}-10$ & 1.10E-10 & 1.39E-09 & $9.50 \mathrm{E}-09$ & $1.11 \mathrm{E}-07$ \\
\hline Skin & $4.16 \mathrm{E}-10$ & $5.84 \mathrm{E}-10$ & $7.01 \mathrm{E}-10$ & $4.82 \mathrm{E}-10$ & $2.85 \mathrm{E}-11$ & $4.23 E-10$ & $1.02 \mathrm{E}-10$ & 1.39E-09 & 8.04E-09 & $1.11 \mathrm{E}-07$ \\
\hline Spleen & $4.16 \mathrm{E}-10$ & $5.84 \mathrm{E}-10$ & 4.09E-09 & $4.82 \mathrm{E}-10$ & 2.85E-11 & $5.63 \mathrm{E}-10$ & 8.77E-11 & 1.39E-09 & $9.50 \mathrm{E}-09$ & 1.11E-07 \\
\hline Testes & $4.16 \mathrm{E}-10$ & $5.84 \mathrm{E}-10$ & 2.12E-09 & $4.82 \mathrm{E}-10$ & $2.85 \mathrm{E}-11$ & $5.19 \mathrm{E}-10$ & $8.77 \mathrm{E}-11$ & 1.39E-09 & 8.77E-09 & $1.55 \mathrm{E}-07$ \\
\hline Thymus & 4.16E-10 & $5.84 \mathrm{E}-10$ & $7.01 \mathrm{E}-10$ & $4.82 \mathrm{E}-10$ & $2.85 \mathrm{E}-11$ & 4.17E-10 & $1.24 \mathrm{E}-10$ & 1.39E-09 & 9.50E-09 & $1.11 \mathrm{E}-07$ \\
\hline Thyroid & 4.16E-10 & $5.84 \mathrm{E}-10$ & $7.01 \mathrm{E}-10$ & $4.82 \mathrm{E}-10$ & $7.31 \mathrm{E}-10$ & $4.65 \mathrm{E}-10$ & 1.53E-06 & 1.39E-09 & $9.50 \mathrm{E}-09$ & 1.11E-07 \\
\hline Uterus & $4.16 \mathrm{E}-10$ & $5.84 \mathrm{E}-10$ & 7.01E-10 & $4.82 \mathrm{E}-10$ & $2.85 \mathrm{E}-11$ & 1.16E-09 & $9.50 \mathrm{E}-11$ & 1.39E-09 & $1.02 \mathrm{E}-08$ & 1.11E-07 \\
\hline Remainder & $4.16 \mathrm{E}-10$ & $5.84 \mathrm{E}-10$ & 1.24E-08 & $5.28 \mathrm{E}-10$ & 3.07E-11 & $6.86 \mathrm{E}-10$ & $2.05 \mathrm{E}-10$ & 1.39E-09 & $8.77 \mathrm{E}-09$ & 1.12E-07 \\
\hline Whole body (e) & $4.24 \mathrm{E}-10$ & $6.79 \mathrm{E}-10$ & 2.12E-09 & 2.24E-08 & $4.68 \mathrm{E}-10$ & 3.71E-09 & $8.04 \mathrm{E}-08$ & 1.46E-09 & $9.50 \mathrm{E}-09$ & $5.09 \mathrm{E}-07$ \\
\hline
\end{tabular}









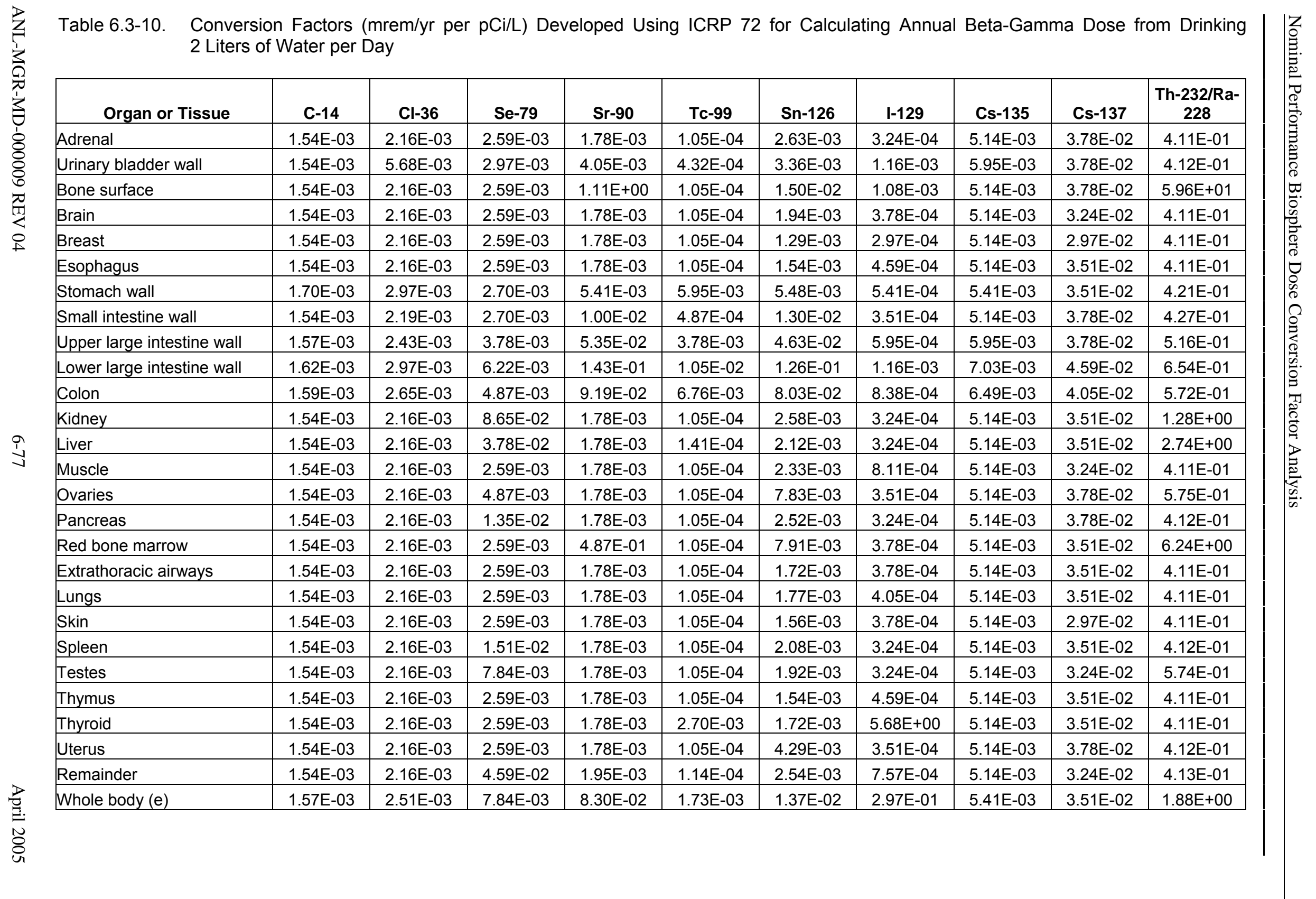




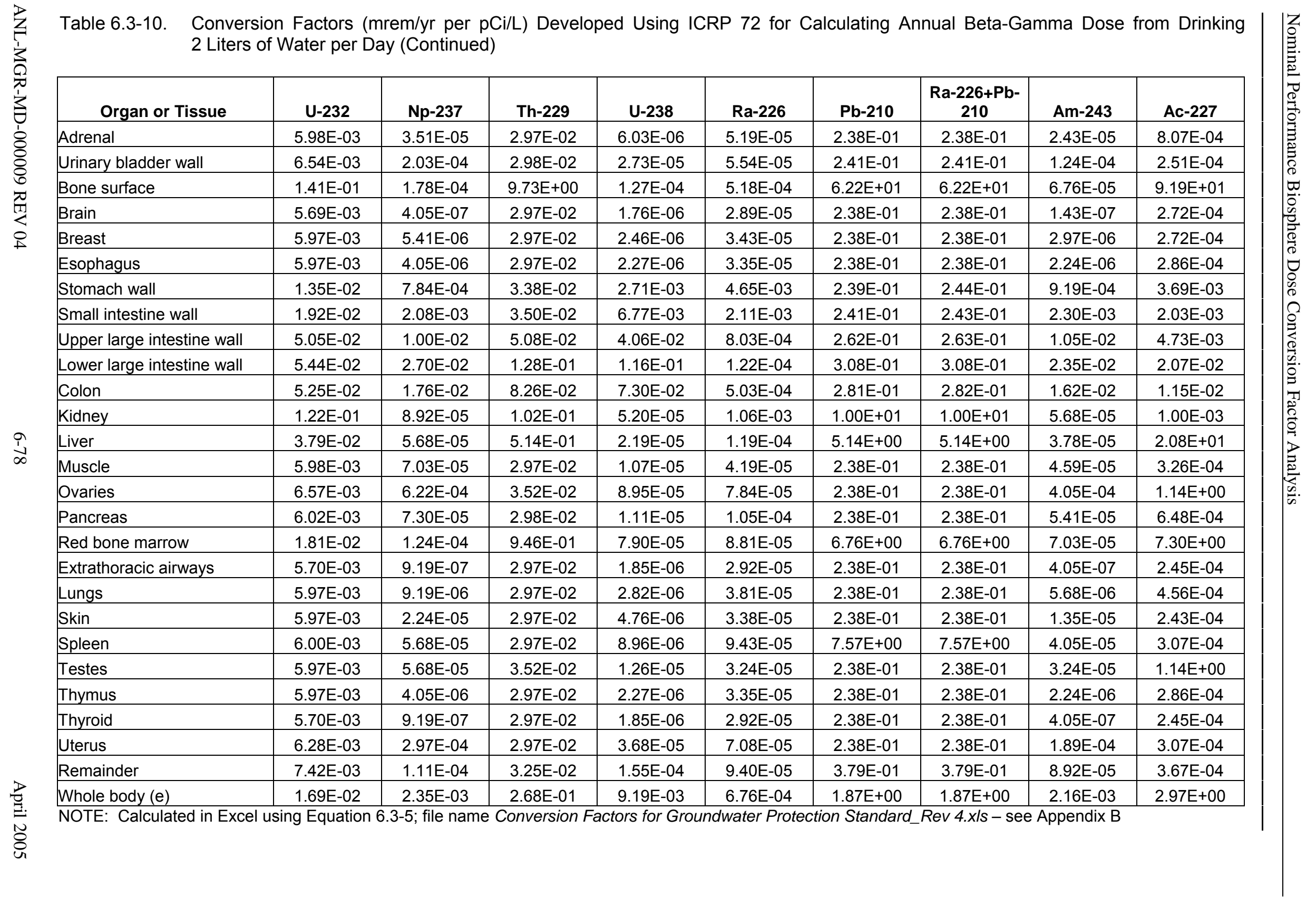




\section{CONCLUSIONS}

This section summarizes the derivation of BDCFs for the groundwater exposure scenario and the methods for calculating values for evaluation of compliance with the groundwater protection standards. The output of this analysis is included in several data sets, as shown in Tables 7-1 and 7-2 for the analysis using ICRP 30 and ICRP 72 dosimetric methods, respectively.

Table 7-1. Output Data Tracking Numbers - ICRP 30 Dosimetric Methods

\begin{tabular}{|l|l|l|}
\hline \multicolumn{1}{|c|}{ DTN } & \multicolumn{1}{|c|}{ DTN Title } & \multicolumn{1}{c|}{ DTN Description and Comments } \\
\hline M00402SPABPHER.000 & $\begin{array}{l}\text { Conversion Factors for Beta- } \\
\text { Photon Emitting Radionuclides } \\
\text { for Groundwater Protection } \\
\text { Standard }\end{array}$ & $\begin{array}{l}\text { Conversion factors for organs or whole body for } \\
\text { calculating beta-photon dose from drinking 2 liters } \\
\text { of water per day calculated using ICRP 30-based } \\
\text { methods. }\end{array}$ \\
\hline M00407MWDBDCFG.000 & $\begin{array}{l}\text { Biosphere Dose Conversion } \\
\text { Factors for the Groundwater } \\
\text { Exposure Scenario }\end{array}$ & $\begin{array}{l}\text { BDCFs for the groundwater exposure scenario } \\
\text { developed using ICRP 30-based methods. }\end{array}$ \\
\hline M00407SPACFCAC.000 & $\begin{array}{l}\text { Conversion Factors for } \\
\text { Calculating Alpha Activity } \\
\text { Concentration in Groundwater }\end{array}$ & $\begin{array}{l}\text { Multipliers to be used with activity concentration } \\
\text { of a primary radionuclide in groundwater to obtain } \\
\text { alpha activity concentration associated with this } \\
\text { radionuclide. }\end{array}$ \\
\hline M0407MWDGSBMF.000 & $\begin{array}{l}\text { GoldSim Biosphere Model Files } \\
\text { for Calculating Biosphere Dose } \\
\text { Conversion Factors for } \\
\text { Groundwater Exposure Scenario }\end{array}$ & $\begin{array}{l}\text { GoldSim files used for calculation of BDCFs for } \\
\text { the groundwater exposure scenario using ICRP } \\
\text { 30-based dosimetric methods. }\end{array}$ \\
\hline
\end{tabular}

Table 7-2. Output Data Tracking Numbers - ICRP 72 Dosimetric Methods

\begin{tabular}{|l|l|l|}
\hline \multicolumn{1}{|c|}{ DTN } & \multicolumn{1}{|c|}{ DTN Title } & \multicolumn{1}{c|}{ DTN Description and Comments } \\
\hline M00501SPAGWPRO.000 & $\begin{array}{l}\text { Conversion Factors for Beta- } \\
\text { Photon Emitting Radionuclides } \\
\text { for Groundwater Protection } \\
\text { Standard Calculated Using ICRP } \\
\text { 72 Dose Coefficients }\end{array}$ & $\begin{array}{l}\text { Conversion factors for organs or whole body for } \\
\text { calculating beta-photon dose from drinking 2 liters } \\
\text { of water per day calculated using ICRP 72 } \\
\text { methods. }\end{array}$ \\
\hline M00501MWDBDCFS.000 & $\begin{array}{l}\text { Biosphere Dose Conversion } \\
\text { Factors for the Groundwater } \\
\text { Exposure Scenario Calculated } \\
\text { Using ICRP 72 Dose Coefficients }\end{array}$ & $\begin{array}{l}\text { BDCFs for the groundwater exposure scenario } \\
\text { developed using ICRP 72 methods. }\end{array}$ \\
\hline M00407SPACFCAC.000 & $\begin{array}{l}\text { Conversion Factors for } \\
\text { Calculating Alpha Activity } \\
\text { Concentration in Groundwater }\end{array}$ & $\begin{array}{l}\text { Multipliers to be used with activity concentration } \\
\text { of a primary radionuclide in groundwater to obtain } \\
\text { alpha activity concentration associated with this } \\
\text { radionuclide. }\end{array}$ \\
\hline MO0503MWDGSBMG.000 & $\begin{array}{l}\text { GoldSim Biosphere Model Files } \\
\text { Used for Calculation of } \\
\text { Groundwater Scenario BDCFs } \\
\text { Based on ICRP 72 Dosimetric } \\
\text { Methods }\end{array}$ & $\begin{array}{l}\text { GoldSim files used for calculation of BDCFs for } \\
\text { the groundwater exposure scenario using ICRP } \\
\text { 72 dosimetric methods. }\end{array}$ \\
\hline
\end{tabular}

The values of the BDCFs were developed using the ERMYN biosphere model and remain valid within the application and validation limits of the model (BSC 2004 [DIRS 169460], Section 8.2). Specifically, the BDCFs were developed for a specific assessment context, 
described in BSC (2004 [DIRS 169460]), the reference biosphere, and the receptor. If used for I other situations, the BDCFs may not apply, as further discussed in Section 7.1.3.

It is recommended that the values developed using the updated dosimetric methods (consistent with ICRP 72) be used in the TSPA model (Table 7-2). This includes DTN: MO0501MWDBDCFS.000 containing BDCFs for the groundwater exposure scenario and DTN: MO0501SPAGWPRO.000 containing conversion factors for organs or whole body for calculating beta-photon dose from drinking 2 liters of water per day. Conversion factors for calculating alpha activity concentration in groundwater (DTN: MO0407SPACFCAC.000) do not depend on the choice of dosimetric methods.

The basis for recommending the use of ICRP 72-based BDCFs is to provide a more accurate estimate of annual dose using a more current scientific understanding. This recommendation is supported by conclusions reached by an International Atomic Energy Agency International Review Team (IRT), which reviewed the biosphere modeling done by the U.S. Department of Energy for the Yucca Mountain Site Characterization Project in 2001 (IAEA 2001 [DIRS 155188]). In their review the IRT stated that ICRP Publication 72 was the preferred basis for the international approach to dosimetric modeling and noted that "even if the [dosimetric] data are fixed in regulatory compliance calculations, it is important to test the implications of applying more modern dosimetric data such as presented in ICRP Publications 72 and 74 . The IRT observes that ICRP 72 data are adopted in the IAEA Basic Safety Standards (IAEA 1996 [DIRS 156655]) and have legal force in EU [European Union] Member States through implementation of the EURATOM Directive 96/29” (IAEA 2001 [DIRS 155188], p. 43).

\subsection{ANALYSIS SUMMARY}

\subsubsection{Incorporation of Uncertainty in Biosphere Dose Conversion Factors}

The BDCFs were calculated in a series of biosphere model realizations using a probabilistic approach that allows statistical sampling of parameter values defined by their probability distribution functions. Such an approach provides a quantitative evaluation of the parameter uncertainties and their impacts on the modeling outcome, the BDCFs. Uncertainty in the model outcome is represented by the probability distribution functions of the BDCFs. The BDCFs were developed for three climate states: the present-day climate, the monsoon climate, and the glacial transition climate (Section 6.2.2). BDCFs for each climate are in the format of 1,000 row vectors. Rows represent individual model realizations, while the vector elements correspond to the BDCFs for individual radionuclides of interest for a given model realization. The BDCFs were calculated for 28 radionuclides, so each row vector has 28 elements. The full set of BDCFs consists of 84,000 values (3 climate states $\times 28$ radionuclides $\times 1,000$ model realizations) (DTN: MO0501MWDBDCFS.000 or DTN: MO0407MWDBDCFG.000, depending on the dosimetric method used). A vector can be regarded as a one-dimensional array containing the results of a single realization of the biosphere model for the primary radionuclides. 


\subsubsection{Biosphere Dose Conversion Factors for the Groundwater Exposure Scenario and Their Use in TSPA}

Some BDCFs (radionuclide specific) include contributions from decay products (Section 6.1.1). The primary radionuclides and the decay products that were included in the BDCF with the primary radionuclides are presented in Table 7.1-1.

Table 7.1-1. Primary Radionuclides and Decay Products Included in the BDCFs

\begin{tabular}{|l|l|}
\hline Primary Radionuclide & \multicolumn{1}{|c|}{ Decay Products Included in BDCF } \\
\hline C-14 & \\
\hline Cl-36 & \\
\hline Se-79 & Y-90 \\
\hline Sr-90 & \\
\hline Tc-99 & \\
\hline Sn-126 & \\
\hline I-129 & Ba-137m \\
\hline Cs-135 & Bi-210, Po-210 \\
\hline Cs-137 & Rn-222, Po-218, Pb-214, At-218, Bi-214, Po-214, TI-210 \\
\hline Pb-210 & Th-227, Fr-223, Ra-223, Rn-219, Po-215, Pb-211, Bi-211, TI-207, Po-211 \\
\hline Ra-226 & Ra-225, Ac-225, Fr-221, At-223, Bi-213, Po-213, TI-209, Pb-209 \\
\hline Ac-227 & \\
\hline Th-229 & Ra-228, Ac-228, Th-228, Ra-224, Rn-220, Po-216, Pb-212, Bi-212, Po-212, TI-208 \\
\hline Th-230 & \\
\hline Th-232 & Th-228, Ra-224, Rn-220, Po-216, Pb-212, Bi-212, Po-212, Tl-208 \\
\hline Pa-231 & \\
\hline U-232 & \\
\hline U-233 & \\
\hline U-234 & \\
\hline U-236 & Th-234, Pa-234m, Pa-234 \\
\hline U-238 & Pa-233 \\
\hline Np-237 & \\
\hline Pu-238 & \\
\hline Pu-239 & \\
\hline Pu-240 & \\
\hline Pu-242 & \\
\hline Am-241 & \\
\hline Am-243 & \\
\hline & \\
\hline
\end{tabular}

The total annual dose is the sum of the annual doses from individual radionuclides. The total annual dose is calculated in TSPA as

$$
D_{\text {total }}(t)=\sum_{i} B D C F_{i} \times C w_{i}(t)
$$

where 
$D_{\text {total }}(t)=$ time-dependent total annual dose to a defined receptor resulting from the release of radionuclides from the repository; includes contributions from all radionuclides considered in the TSPA-LA (Sv/yr)

$B D C F_{i}=$ climate-specific biosphere dose conversion factor for radionuclide $i$ (Sv/yr per $\mathrm{Bq} / \mathrm{m}^{3}$ )

$C w_{i}(t) \quad=$ time-dependent activity concentration of radionuclide $i$ the groundwater $\left(\mathrm{Bq} / \mathrm{m}^{3}\right)$.

Equation 7-1 uses a linear relationship between radionuclide concentrations in groundwater and the resulting doses. Calculations of the total dose for a given point in time should use the set of BDCFs corresponding to the climate at that time (DTN: MO0501MWDBDCFS.000). The BDCF values in DTN: MO0501MWDBDCFS.000 are given with three significant digits although only two significant digits are significant. This is to avoid round-off errors in the TSPA calculation of the annual dose.

As shown in Table 7.1-1, BDCFs for ${ }^{226} \mathrm{Ra}$ and ${ }^{210} \mathrm{~Pb}$ are calculated separately, although ${ }^{210} \mathrm{~Pb}$ is a decay product of ${ }^{226} \mathrm{Ra}$. If the activity concentration of ${ }^{210} \mathrm{~Pb}$ in the groundwater is not calculated by the TSPA-LA model, the BDCF for ${ }^{210} \mathrm{~Pb}$ should be added to that of ${ }^{226} \mathrm{Ra}$ to account for that part of the uranium decay chain. This summation is based on the condition of radioactive equilibrium between ${ }^{226} \mathrm{Ra},{ }^{210} \mathrm{~Pb}$, and their short-lived decay products.

\subsubsection{Limitations of Biosphere Modeling}

The ERMYN model applies to the specific environments identified in 10 CFR 63.305 [DIRS 173164]. It uses certain approximations and simplifications. Therefore, the ERMYN model only applies within a certain assessment context (BSC 2004 [DIRS 169460], Section 6.1). The radionuclide sources for the biosphere model are specific to the groundwater exposure scenario. The ERMYN model focuses on the radionuclides that were screened for the TSPA-LA (BSC 2004 [DIRS 169460], Section 6.1.3). The model applies to assessing chronic radiation doses and is valid for all values of input parameters reasonably expected to occur in the arid to semi-arid region surrounding Yucca Mountain.

For the contaminated groundwater scenario, the ERMYN model applies to an agricultural situation with long-term irrigation and soil contamination at long-term equilibrium conditions. If soils are not at equilibrium concentrations of radionuclides, the ERMYN model might overestimate the radiation dose. The biosphere model applies to an arid or semi-arid climate, and it is valid only for limited groundwater discharge to the surface and limited surface water transport, as long as the radionuclide concentration in the surface water is the same as in the groundwater and the reference biosphere is not greatly altered. For example, if permanent surface waters such as rivers or lakes are present, the environment would be sufficiently different to change the reference biosphere, and other pathways would have to be added for the ERMYN model to remain valid. 


\subsubsection{Correlations and Pathway Analysis}

Correlation coefficients for the groundwater exposure scenario BDCF for individual radionuclides are listed in Tables 6.2-14 and 6.2-15 for the ICRP 30- and ICRP 72-based values, respectively. Correlation coefficients generally are the highest for the isotopes of actinides.

Results of pathway analysis for the ICRP 30-based BDCFs are presented in Tables 6.2-17 and 6.2-18 for the present-day climate and the upper bound of the glacial transition climate, respectively. The analogous results for the ICRP 72-based BDCFs are presented in Tables 6.2-19 and 6.2-20 for the present-day climate and the upper bound of the glacial transition climate, respectively. For both climates, inhalation of particulate matter tends to dominate doses for actinides (e.g., isotopes of thorium, uranium, plutonium, and americium). Inhalation of radioactive aerosols generated by evaporative coolers also is an important inhalation exposure pathway for these radionuclides, especially for the present-day climate. Contribution from the inhalation pathway for the actinides is greater for the ICRP 72-based BDCFs than for the ICRP 30-based BDCFs.

For the upper bound of the glacial transition climate, the importance of the evaporative cooler pathway is greatly reduced. Ingestion of water is a consistently high contributor to dose for all radionuclides and for both climates. Other pathways are only important for a few radionuclides. For instance, external exposure is a dominant pathway for ${ }^{126} \mathrm{Sn}$ and ${ }^{137} \mathrm{Cs}$, inhalation of radon decay products for ${ }^{226} \mathrm{Ra}$ and ${ }^{230} \mathrm{Th}$, and fish consumption for ${ }^{14} \mathrm{C}$, isotopes of cesium, and ${ }^{210} \mathrm{~Pb}$. Consumption pathways generally are more important for radionuclides with atomic numbers less than that of radium.

\subsubsection{Performance Assessment Calculations for Groundwater Protection Standards}

For calculating alpha activity concentration in groundwater and the annual dose from beta- and photon-emitting radionuclides for evaluation of compliance with the groundwater protection standards, the methods and the conversion factor values described in Section 6.3 should be used using the values of conversion factors developed in this analysis. Natural background activity concentrations must be added to calculated values for comparison with the combined ${ }^{226} \mathrm{Ra}$ and

${ }^{228} \mathrm{Ra}$ and the gross alpha activity limits. The alpha particle activity concentration in the groundwater should be calculated using Equation 6.3-1 and the number of alpha particles from Table 6.3-3 (DTN: MO0407SPACFCAC.000 for either dosimetric method). The beta-gamma dose should be calculated using Equation 6.3-4 and conversion factors from Table 6.3-9 or 6.3-10 for the ICRP 72-based methods (DTN: MO0501SPAGWPRO.000).

\subsection{HOW THE ACCEPTANCE CRITERIA ARE ADDRESSED}

The primary function of this analysis was to calculate BDCFs for the groundwater exposure scenario. As noted before, this analysis integrates the model (BSC 2004 [DIRS 169460]) and the model input parameters (BSC 2005 [DIRS 172827]; BSC 2004 [DIRS 169672]; BSC 2004 [DIRS 169673]; BSC 2004 [DIRS 169458]; BSC 2004 [DIRS 169459]) to produce the output of the biosphere model. All ACs addressed by the model and input parameters reports are implicitly included in this analysis and the modeling results. 
In addition, the ACs identified as applicable to this analysis in Section 4.2, that are related to the model abstraction were addressed. The biosphere modeling does not utilize the model abstraction step, but rather, the BDCFs are calculated as the model output. In this sense, the BDCFs, which are the input to the TSPA-LA model, serve as a collapsed model abstraction. The uncertainty in the model and its input parameters is propagated onto and reflected in the BDCFs through the development of the BDCF vectors representing individual model realizations (see Section 7.1.2), consistent with ACs 2.2.1.3.13.3, 2.2.1.3.13.4, and 2.2.1.3.14.3 (see Table 4.2-2).

The specific ACs were addressed as follows:

\section{Section 2.2.1.3.13.3: Redistribution of Radionuclides in Soils}

AC 1 (1): As discussed in Sections 1 and 7.1.2, because the BDCFs are used directly in the TSPA, the TSPA adequately incorporates the results of human exposures to groundwater.

AC 1(2): By including the BDCFs for the groundwater exposure scenario, the TSPA identifies and describes an aspect of radionuclide redistribution in soil that is important to repository performance. See, Section 7.1.2 and 7.1.3. The technical bases for the groundwater exposure scenario are adequately described in Section 6.1.2.

AC 1 (3): Relevant site FEPs (Section 1), including climate change (Section 6.1.3), receptor characteristics (Section 6.1.4), and the biosphere model (Section 6.1.5) have been appropriately modeled in the BDCFs. Sufficient technical bases are provided for the BDCFs.

AC 2 (1): The BDCF development process described in Section 6 shows that the BDCFs used in the license application are justified. Adequate descriptions of how data was used to develop BDCFs are presented in Section 6.2, which includes a pathway analysis (Section 6.2.5), climate change (Section 6.2.2), and uncertainty (Section 6.2.1).

AC 2 (2): As shown in Sections 4.1.1 and 4.1.2, sufficient data are available to adequately define the parameters for the BDCFs.

AC 3 (1): The calculations described in Section 6.2.2 for the incorporation of climate change, the resulting BDCFs presented in Section 6.2.3, and the development of dose factors described in Section 6.3 show that the parameter values are technically defensible, reasonably account for uncertainties, and do not result in an under-representation of the risk.

AC 3 (2): The model input data related to agricultural parameters (Section 4.1.1) are consistent with the current farming practices. Data on the airborne particulate concentration is based on the resuspension of appropriate material in a climate and level of disturbance, which is expected to be found at the location of the RMEI.

AC 3 (3): Sections 6.2.1 and 7.1.1 shows that uncertainty was adequately represented in the BDCFs by a probability distribution which was generated from a thousand realizations using a Monte Carlo technique. 


\section{Section 2.2.1.3.14.3: Biosphere Characteristics}

AC 1 (3): Section 6.1.3 shows how the impact of climate change on the BDCFs is consistent with the three climate states used in other TSPA abstractions.

AC 2 (1): The BDCF development process described in Section 6 shows that the BDCFs used in the license application are justified. Section 6.1.4 shows that the analysis is consistent with the definition of the RMEI. Adequate descriptions of how data was used to develop BDCFs are presented in Section 6.2, which includes a pathway analysis (Section 6.2.5), climate change (Section 6.2.2), and uncertainty (Section 6.2.1).

AC 2 (2): As shown in Sections 4.1.1 and 4.1.2, sufficient data are available to adequately define | the degree to which the BDCFs incorporate FEPs and are consistent with the present knowledge of the conditions in the region surrounding Yucca Mountain.

AC 3 (1): Sections 4.1.1 and 4.1.2 show that the parameter values are technically defensible, | reasonably account for uncertainties, do not result in an under-representation of the risk, and are consistent with the definition of the RMEI.

AC 3 (2): Sections 4.1.1 and 4.1.2 shows that the technical bases for the parameter values and ranges used to generate the BDCFs are consistent with site characterization data and technically defensible.

AC 3 (4): Sections 6.2.1 and 7.1.1 show that uncertainty is adequately represented in parameter development through a Monte Carlo analysis. 


\section{INTENTIONALLY LEFT BLANK}




\section{INPUTS AND REFERENCES}

\subsection{DOCUMENTS CITED}

BSC (Bechtel SAIC Company) 2003. Total System Performance Assessment-

166296

License Application Methods and Approach. TDR-WIS-PA-000006 REV 00 ICN 01. Las Vegas, Nevada: Bechtel SAIC Company. ACC: DOC.20031215.0001.

BSC 2004. Agricultural and Environmental Input Parameters for the Biosphere Model. ANL-MGR-MD-000006, Rev. 02. Las Vegas, Nevada: Bechtel SAIC Company. ACC: DOC.20040915.0007.

BSC 2004. Biosphere Model Report. MDL-MGR-MD-000001, Rev. 01. Las Vegas, Nevada: Bechtel SAIC Company. ACC: DOC.20041108.0005.

BSC 2004. Environmental Transport Input Parameters for the Biosphere Model. ANL-MGR-MD-000007, Rev. 02. Las Vegas, Nevada: Bechtel SAIC Company. ACC: DOC.20040913.0003.

BSC 2004. Future Climate Analysis. ANL-NBS-GS-000008, Rev. 01. Las Vegas, Nevada: Bechtel SAIC Company. ACC: DOC.20040908.0005.

BSC 2004. Inhalation Exposure Input Parameters for the Biosphere Model. ANLMGR-MD-000001, Rev. 03. Las Vegas, Nevada: Bechtel SAIC Company. ACC: DOC.20040913.0001.

BSC 2004. Nominal Performance Biosphere Dose Conversion Factor Analysis. ANL-MGR-MD-000009 REV 03. Las Vegas, Nevada: Bechtel SAIC Company. ACC: DOC.20040909.0003.

BSC 2004. Soil-Related Input Parameters for the Biosphere Model. ANL-NBSMD-000009, Rev. 02. Las Vegas, Nevada: Bechtel SAIC Company. ACC: DOC.20040913.0002.

BSC 2005. Characteristics of the Receptor for the Biosphere Model. ANL-MGR172827 MD-000005 REV 04. Las Vegas, Nevada: Bechtel SAIC Company. ACC: DOC.20050405.0005.

BSC 2005. Q-List. 000-30R-MGR0-00500-000-001. Las Vegas, Nevada: Bechtel SAIC Company. ACC: ENG.20050217.0010.

BSC 2005. Technical Work Plan for Biosphere Modeling and Expert Support. TWP-NBS-MD-000004 REV 04. Las Vegas, Nevada: Bechtel SAIC Company. ACC: DOC.20050131.0012. 
BSC 2005. Technical Work Plan For: TSPA-LA FY 05-06 Activities. TWP-MGRPA-000031 REV 00. Las Vegas, Nevada: Bechtel SAIC Company. ACC: DOC.20050401.0006.

Canori, G.F. and Leitner, M.M. 2003. Project Requirements Document. TER166275 MGR-MD-000001 REV 02. Las Vegas, Nevada: Bechtel SAIC Company. ACC: DOC.20031222.0006.

[DOE (U.S. Department of Energy)] 2004. "FGR 11 Table 2.2. Ingestion Intakes 169713 (for various radionuclides)." [Oak Ridge, Tennessee: U.S. Department of Energy, Oak Ridge National Laboratory]. Accessed March 11, 2004. ACC:

MOL.20040504.0294. http://www.ornl.gov/cgibin/cgiwrap?user=wlj\&script=fgrx.pl

Eckerman, K.F. and Ryman, J.C. 1993. External Exposure to Radionuclides in Air, Water, and Soil, Exposure-to-Dose Coefficients for General Application, Based on the 1987 Federal Radiation Protection Guidance. EPA 402-R-93-081. Federal Guidance Report No. 12. Washington, D.C.: U.S. Environmental Protection Agency, Office of Radiation and Indoor Air. TIC: 225472.

GoldSim Technology Group. 2003. User's Guide, GoldSim Probabilistic Simulation Environment. Version 8.01. Redmond, Washington: Golder Associates. TIC: 255170.

ICRP (International Commission on Radiological Protection) 1977. Recommendations of the International Commission on Radiological Protection. Volume 1, No. 3 of Annals of the ICRP. ICRP Publication 26. Reprinted 1982. New York, New York: Pergamon Press. TIC: 221568.

ICRP 1979. Limits for Intakes of Radionuclides by Workers. Volume 2, No. 3/4 of 110386 Annals of the ICRP. Sowby, F.D., ed. ICRP Publication 30 Part 1. New York, New York: Pergamon Press. TIC: 4939.

ICRP 1980. Limits for Intakes of Radionuclides by Workers. Volume 4, No. 3/4 of Annals of the ICRP. Sowby, F.D., ed. ICRP Publication 30 Part 2. Reprinted 1990. Elmsford, New York: Pergamon Press. TIC: 4941.

ICRP 1981. Limits for Intakes of Radionuclides by Workers. Volume 6, No. 2/3 of 166226 Annals of the ICRP. Sowby, F.D., ed. ICRP Publication 30 Part 3, Including Addendum to Parts 1 and 2. New York, New York: Pergamon Press. TIC: 4943.

ICRP 1991. "1990 Recommendations of the International Commission on 101836 Radiological Protection." Volume 21, No. 1-3 of Annals of the ICRP. ICRP Publication 60. New York, New York: Pergamon Press. TIC: 235864. 
ICRP 1996. Age-Dependent Doses to Members of the Public from Intake of

Radionuclides: Part 5 Compilation of Ingestion and Inhalation Dose Coefficients. Volume 26, No. 1 of Annals of the ICRP. Smith, H., ed. ICRP Publication 72. New York, New York: Pergamon Press. TIC: 235870.

IAEA (International Atomic Energy Agency) 1996. International Basic Safety Standards for Protection Against Ionizing Radiation and for the Safety of Radiation Sources. Safety Series No. 115. Vienna, Austria: International Atomic Energy Agency. TIC: 250383.

ICRP 1999. The ICRP Database of Dose Coefficients: Workers and Members of the Public. Version 1.0. [New York, New York]: Pergamon. TIC: 248811.

IAEA (International Atomic Energy Agency) 2001. An International Peer Review 155188 of the Biosphere Modelling Programme of the US Department of Energy's Yucca Mountain Site Characterization Project, Report of the IAEA International Review Team. Vienna, Austria: International Atomic Energy Agency. TIC: 250092.

Lide, D.R. and Frederikse, H.P.R., eds. 1997. CRC Handbook of Chemistry and Physics. 78th Edition. Boca Raton, Florida: CRC Press. TIC: 243741.

NRC (U.S. Nuclear Regulatory Commission) 2003. Yucca Mountain Review Plan, 163274 Final Report. NUREG-1804, Rev. 2. Washington, D.C.: U.S. Nuclear Regulatory Commission, Office of Nuclear Material Safety and Safeguards. TIC: 254568.

Steel, R.G.D. and Torrie, J.H. 1980. Principles and Procedures of Statistics: A Biometrical Approach. 2nd Edition. New York, New York: McGraw Hill. TIC: 6322.

\subsection{CODES, STANDARDS, REGULATIONS, AND PROCEDURES}

10 CFR 63. 2004 Energy: Disposal of High-Level Radioactive Wastes in a

40 CFR 141. 2004 Protection of Environment: National Primary Drinking Water Regulations. ACC: MOL.20050331.0079.

AP-2.22Q, Rev. 1, ICN 1. Classification Analyses and Maintenance of the Q-List. Washington, D.C.: U.S. Department of Energy, Office of Civilian Radioactive Waste Management. ACC: DOC.20040714.0002.

LP-2.29Q-BSC, Rev. 0, ICN 0. Planning for Science Activities. Washington, D.C.: U.S. Department of Energy, Office of Civilian Radioactive Waste Management. ACC: DOC.20050114.0001. 
LP-3.15Q-BSC, Rev. 0, ICN 0. Managing Technical Product Inputs. Washington, D.C.: U.S. Department of Energy, Office of Civilian Radioactive Waste Management. Acc: DOC.20050113.0002.

LP-SIII.9Q-BSC, Rev. 0, ICN 0. Scientific Analyses. Washington, D.C.: U.S. Department of Energy, Office of Civilian Radioactive Waste Management.

ACC: DOC.20050203.0010.

LP-SI.11Q-BSC Rev. 0, ICN 1. Software Management. Washington, D.C.: U.S. Department of Energy, Office of Civilian Radioactive Waste Management.

ACC: DOC.20041005.0008.

\subsection{SOURCE DATA, LISTED BY DATA TRACKING NUMBER}

MO0306MWDBGSMF.001. Biosphere Goldsim Model Files. Submittal date:

163816 06/13/2003.

MO0403SPAAEIBM.002. Agricultural and Environmental Input Parameters for 169392 the Biosphere Model. Submittal date: 03/22/2004.

MO0406SPAETPBM.002. Environmental Transport Input Parameters for the 170150 Biosphere Model. Submittal date: 06/24/2004.

MO0407SPACRBSM.002. Characteristics of the Receptor for the Biosphere Model. Submittal date: 07/19/2004.

MO0407SEPFEPLA.000. LA FEP List. Submittal date: 07/20/2004.

MO0407SPAINEXI.002. Inhalation Exposure Input Parameters for the Biosphere Model. Submittal date: 07/12/2004.

MO0407SPASRPBM.002. Soil Related Parameters for the Biosphere Model.

MO0501SEPFEPLA.001. LA FEP List and Screening. Submittal date: 01/17/2005.

MO0503SPADCESR.000. Dose Coefficients for Internal and External Exposure 172896 to Selected Radionuclides Consistent with ICRP 72 Dosimetric Methods.

Submittal date: 03/01/2005. 


\subsection{SOFTWARE CODES}

BSC 2003. Software Code: GoldSim. V7.50.100. PC. 10344-7.50.100-00.

BSC 2004. Software Code: GoldSim. V8.01 Service Pack 4. PC, Windows 169695 2000. 10344-8.01SP4-00.

BSC 2004. Software Code: GoldSim. V.8.02.300. PC, Windows 2000. 10344-

172588 8.02-03.

\subsection{OUTPUT DATA, LISTED BY DATA TRACKING NUMBER}

MO0402SPABPHER.000 Conversion Factors for Beta-Photon Emitting Radionuclides for Groundwater Protection Standard

MO0407MWDBDCFG.000 Biosphere Dose Conversion Factors for the Groundwater Exposure Scenario

MO0407SPACFCAC.000 Conversion Factors for Calculating Alpha Activity Concentration in Groundwater

MO0407MWDGSBMF.000 GoldSim Biosphere Model Files for Calculating Biosphere Dose Conversion Factors for Groundwater Exposure Scenario

MO0501SPAGWPRO.000 Conversion Factors for Beta-Photon Emitting Radionuclides for Groundwater Protection Standard Calculated Using ICRP 72 Dose Coefficients

MO0501MWDBDCFS.000 Biosphere Dose Conversion Factors for the Groundwater Exposure Scenario Calculated Using ICRP 72 Dose Coefficients

MO0503MWDGSBMG.000 GoldSim Biosphere Model Files Used for Calculation of Groundwater Scenario BDCFs Based on ICRP 72 Dosimetric Methods 


\section{INTENTIONALLY LEFT BLANK}


APPENDIX A

LIST AND DESCRIPTION OF GOLDSIM FILES GENERATED IN THIS ANALYSIS 


\section{A. LIST AND DESCRIPTION OF GOLDSIM FILES GENERATED IN THIS ANALYSIS}

This appendix contains the list and description of GoldSim biosphere model files generated in this and in the previous revision of the analysis. The files are included in DTN: MO0503MWDGSBMG.000 and MO0407MWDGSBMF.000, respectively. The analysis was conducted using GoldSim Version 8.02.300 (STN: 10344-8.02-03) (BSC 2004 [DIRS 172588]) and GoldSim 8.01 Service Pack 4 (STN: 10344-8.01SP4-00) (BSC 2004 [DIRS 169695]).

Figure A-1 shows the list of GoldSim files for the present-day climate (file names ERMYN_GW_M_<radionuclide symbol>_ICRP72) generated using GoldSim 8.02.300 and ICRP 72-based dosimetric inputs. These files are included in DTN: MO0503MWDGSBMG.000.

\begin{tabular}{|c|c|c|c|c|}
\hline \multirow{4}{*}{ Present-day climate } & Name $A$ & Size & Type & Modified \\
\hline & B]ERMYN_GW_M_AC227D_ICRP72.gsm & $11,469 \mathrm{~KB}$ & GoldSim Model & $1 / 11 / 20052: 55 \mathrm{PN}$ \\
\hline & (3)ERMYN_GW_M_Am241_ICRP72.gsm & $11,469 \mathrm{~KB}$ & GoldSim Model & $1 / 11 / 20053: 25 \mathrm{PN}$ \\
\hline & B]ERMYN_GW_M_Am243D_ICRP72.gsm & $11,469 \mathrm{~KB}$ & GoldSim Model & $1 / 11 / 20054: 00 \mathrm{PV}$ \\
\hline \multirow{27}{*}{$\begin{array}{l} \pm \text { This folder is Online. } \\
\text { Select an item to view its description. }\end{array}$} & B]ERMYN_GW_M_C14_ICRP72.gsm & $11,469 \mathrm{~KB}$ & GoldSim Model & $1 / 11 / 20052: 13 \mathrm{PV}$ \\
\hline & BEERYN_GW_M_Cl36_ICRP72.gsm & $11,469 \mathrm{~KB}$ & GoldSim Model & $1 / 11 / 20052: 15 \mathrm{PN}$ \\
\hline & 8ERMYN_GW_M_Cs135_ICRP72.gsm & $11,469 \mathrm{~KB}$ & GoldSim Model & $1 / 11 / 20052: 27 \mathrm{PV}$ \\
\hline & B]ERMYN_GW_M_Cs137D_ICRP72.gsm & $11,469 \mathrm{~KB}$ & GoldSim Model & $1 / 11 / 20052: 28 \mathrm{PV}$ \\
\hline & BERMYN_GW_M_I129_ICRP72.gsm & $11,469 \mathrm{~KB}$ & GoldSim Model & $1 / 11 / 20052: 25 \mathrm{PV}$ \\
\hline & B]ERMYN_GW_M_Np237D_ICRP72.gsm & $11,469 \mathrm{~KB}$ & GoldSim Model & $1 / 11 / 20053: 17 \mathrm{PV}$ \\
\hline & BERMYN_GW_M_Pa231_ICRP72.gsm & $11,469 \mathrm{~KB}$ & GoldSim Model & $1 / 11 / 20053: 03 \mathrm{PN}$ \\
\hline & (2EERYYN_GW_M_Pb210D_ICRP72.gsm & $11,469 \mathrm{~KB}$ & GoldSim Model & $1 / 11 / 20052: 30 \mathrm{PV}$ \\
\hline & BEEMYN_GW_M_PU238_ICRP72.gsm & $11,469 \mathrm{~KB}$ & GoldSim Model & $1 / 11 / 20053: 19 \mathrm{PV}$ \\
\hline & BERMYN_GW_M_PU239_ICRP72.gsm & $11,469 \mathrm{~KB}$ & GoldSim Model & $1 / 11 / 20053: 21 \mathrm{PV}$ \\
\hline & 8EERMYN_GW_M_Pu240_ICRP72.gsm & $11,469 \mathrm{~KB}$ & GoldSim Model & $1 / 11 / 20053: 22 \mathrm{PV}$ \\
\hline & 38ERMYN_GW_M_Pu242_ICRP72.gsm & $11,469 \mathrm{~KB}$ & GoldSim Model & $1 / 11 / 20053: 24 \mathrm{PV}$ \\
\hline & (3)ERMYN_GW_M_Ra226D_ICRP72.gsm & $11,469 \mathrm{~KB}$ & GoldSim Model & $1 / 11 / 20052: 50 \mathrm{PV}$ \\
\hline & BEEMYN_GW_M_Ra228D_ICRP72.gsm & $11,469 \mathrm{~KB}$ & GoldSim Model & $1 / 11 / 20052: 53 \mathrm{PV}$ \\
\hline & BERMYN_GW_M_Se79_ICRP72.gsm & $11,469 \mathrm{~KB}$ & GoldSim Model & $1 / 11 / 20052: 17 \mathrm{PV}$ \\
\hline & BERMYN_GW_M_Sn126D_ICRP72.gsm & $11,469 \mathrm{~KB}$ & GoldSim Model & $1 / 11 / 20052: 23 \mathrm{PV}$ \\
\hline & B]ERMYN_GW_M_Sr90D_ICRP72.gsm & $11,469 \mathrm{~KB}$ & GoldSim Model & $1 / 11 / 20052: 19 \mathrm{PV}$ \\
\hline & B]ERMYN_GW_M_Tc99_ICRP72.gsm & $11,469 \mathrm{~KB}$ & GoldSim Model & $1 / 11 / 20052: 21 \mathrm{PV}$ \\
\hline & BERMYN_GW_M_Th228D_ICRP72.gsm & $11,469 \mathrm{~KB}$ & GoldSim Model & $1 / 11 / 20052: 56 \mathrm{PV}$ \\
\hline & BEERYN_GW_M_Th229D_ICRP72.gsm & $11,469 \mathrm{~KB}$ & GoldSim Model & $1 / 11 / 20052: 58 \mathrm{PN}$ \\
\hline & EERMYN_GW_M_Th230_ICRP72.gsm & $11,469 \mathrm{~KB}$ & GoldSim Model & $1 / 11 / 20052: 59 \mathrm{PN}$ \\
\hline & BEERMYN_GW_M_Th232_ICRP72.gsm & $11,469 \mathrm{~KB}$ & GoldSim Model & $1 / 11 / 20053: 01 \mathrm{PV}$ \\
\hline & BEERMYN_GW_M_U232_ICRP72.gsm & $11,469 \mathrm{~KB}$ & GoldSim Model & $1 / 11 / 20053: 04 \mathrm{PV}$ \\
\hline & BEERMYN_GW_M_U233_ICRP72.gsm & $11,469 \mathrm{~KB}$ & GoldSim Model & $1 / 11 / 20053: 06 \mathrm{PV}$ \\
\hline & BEERMYN_GW_M_U234_ICRP72.gsm & $11,469 \mathrm{~KB}$ & GoldSim Model & $1 / 11 / 20053: 08 \mathrm{PV}$ \\
\hline & BERMYN_GW_M_U236_ICRP72.gsm & $11,469 \mathrm{~KB}$ & GoldSim Model & $1 / 11 / 20053: 11 \mathrm{PV}$ \\
\hline & BERMYN_GW_M_U238D_ICRP72.gsm & $11,469 \mathrm{~KB}$ & GoldSim Model & $1 / 11 / 20053: 13 \mathrm{PV}$ \\
\hline
\end{tabular}

Figure A-1. GoldSim Files for Calculating Groundwater Scenario BDCFs for the Present-Day Climate Generated Using ICRP 72-Based Dosimetric Inputs 
Figure A-2 shows the list of GoldSim files for the upper bound of the glacial transition climate (file names ERMYN_GW_F_<radionuclide symbol>_ICRP72) generated using GoldSim 8.02.300 and ICRP 72-based dosimetric inputs. These files are included in DTN: MO0503MWDGSBMG.000.

\begin{tabular}{|c|c|c|c|c|}
\hline \multirow[t]{2}{*}{$7 \quad 2 \quad 7$} & Name $A$ & \multirow{2}{*}{$\begin{array}{r}\text { Size } \\
11,470 \mathrm{~KB}\end{array}$} & \multirow{2}{*}{$\begin{array}{l}\text { Type } \\
\text { GoldSim Model }\end{array}$} & \multirow{2}{*}{$\begin{array}{l}\text { Modified } \\
1 / 12 / 20059: 34 \mathrm{AM}\end{array}$} \\
\hline & BERMYN_GW_F_AC227D_ICRP72.gsm & & & \\
\hline 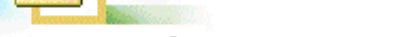 & BERMYN_GW_F_Am241_ICRP72.gsm & $11,470 \mathrm{~KB}$ & GoldSim Model & $1 / 12 / 200510: 12 \mathrm{AM}$ \\
\hline Future Climate & BEEMYN_GW_F_Am243D_ICRP72.gsm & $11,470 \mathrm{~KB}$ & GoldSim Model & $1 / 12 / 200510: 14 \mathrm{AM}$ \\
\hline \multirow{3}{*}{ \pm This folder is Online. } & B]ERMYN_GW_F_C14_ICRP72.gsm & $11,470 \mathrm{~KB}$ & GoldSim Model & $1 / 12 / 20058: 51 \mathrm{AM}$ \\
\hline & B]ERMYN_GW_F_Cl36_ICRP72.gsm & $11,470 \mathrm{~KB}$ & GoldSim Model & 1/12/2005 9:07 AM \\
\hline & (B)ERMYN_GW_F_Cs135_ICRP72.gsm & $11,470 \mathrm{~KB}$ & GoldSim Model & 1/12/2005 9:23 AM \\
\hline Select an item to view its description. & 38ERYN_GW_F_Cs137D_ICRP72.gsm & $11,470 \mathrm{~KB}$ & GoldSim Model & 1/12/2005 9:24 AM \\
\hline See also: & 3 ERMYN_GW_F_I129_ICRP72.gsm & $11,470 \mathrm{~KB}$ & GoldSim Model & $1 / 12 / 20059: 21 \mathrm{AM}$ \\
\hline My Documents & 3 ERMYN_GW_F_Np237D_ICRP72.gsm & $11,470 \mathrm{~KB}$ & GoldSim Model & $1 / 12 / 200510: 00 \mathrm{AM}$ \\
\hline My Network Places & BERMYN_GW_F_Pa231_ICRP72.gsm & $11,470 \mathrm{~KB}$ & GoldSim Model & $1 / 12 / 20059: 48 \mathrm{AM}$ \\
\hline \multirow[t]{20}{*}{ My Computer } & 18 ERMYN_GW_F_Pb210D_ICRP72.gsm & $11,470 \mathrm{~KB}$ & GoldSim Model & $1 / 12 / 20059: 26 \mathrm{AM}$ \\
\hline & 3 ERMYN_GW_F_PU238_ICRP72.gsm & $11,470 \mathrm{~KB}$ & GoldSim Model & $1 / 12 / 200510: 02 \mathrm{AM}$ \\
\hline & BERMYN_GW_F_Pu239_ICRP72.gsm & $11,470 \mathrm{~KB}$ & GoldSim Model & $1 / 12 / 200510: 06 \mathrm{AM}$ \\
\hline & BERMYN_GW_F_Pu240_ICRP72.gsm & $11,470 \mathrm{~KB}$ & GoldSim Model & 1/12/2005 10:08 AM \\
\hline & 38EMYN_GW_F_PU242_ICRP72.gsm & $11,470 \mathrm{~KB}$ & GoldSim Model & $1 / 12 / 200510: 10 \mathrm{AM}$ \\
\hline & $B$ ERMYN_GW_F_Ra226D_ICRP72.gsm & $11,470 \mathrm{~KB}$ & GoldSim Model & $1 / 12 / 20059: 28 \mathrm{AM}$ \\
\hline & BERMYN_GW_F_Ra228D_ICRP72.gsm & $11,470 \mathrm{~KB}$ & GoldSim Model & $1 / 12 / 20059: 31 \mathrm{AM}$ \\
\hline & B]ERMYN_GW_F_Se79_ICRP72.gsm & $11,470 \mathrm{~KB}$ & GoldSim Model & $1 / 12 / 20059: 09$ AM \\
\hline & BEERYN_GW_F_Sn126D_ICRP72.gsm & $11,470 \mathrm{~KB}$ & GoldSim Model & $1 / 12 / 20059: 19$ AM \\
\hline & 8ERMYN_GW_F_Sr90D_ICRP72.gsm & $11,470 \mathrm{~KB}$ & GoldSim Model & $1 / 12 / 20059: 11 \mathrm{AM}$ \\
\hline & BEERMYN_GW_F_Tc99_ICRP72.gsm & $11,470 \mathrm{~KB}$ & GoldSim Model & $1 / 12 / 20059: 13 \mathrm{AM}$ \\
\hline & BERMYN_GW_F_Th228D_ICRP72.gsm & $11,470 \mathrm{~KB}$ & GoldSim Model & $1 / 12 / 20059: 37 \mathrm{AM}$ \\
\hline & BERMYN_GW_F_Th229D_ICRP72.gsm & $11,470 \mathrm{~KB}$ & GoldSim Model & $1 / 12 / 20059: 42 \mathrm{AM}$ \\
\hline & 83ERMYN_GW_F_Th230_ICRP72.gsm & $11,470 \mathrm{~KB}$ & GoldSim Model & $1 / 12 / 20059: 45 \mathrm{AM}$ \\
\hline & BEEMYN_GW_F_Th232_ICRP72.gsm & $11,470 \mathrm{~KB}$ & GoldSim Model & $1 / 12 / 20059: 46 \mathrm{AM}$ \\
\hline & BEEMYN_GW_F_U232_ICRP72.gsm & $11,470 \mathrm{~KB}$ & GoldSim Model & $1 / 12 / 20059: 50 \mathrm{AM}$ \\
\hline & 8 ERMYN_GW_F_U233_ICRP72.gsm & $11,470 \mathrm{~KB}$ & GoldSim Model & $1 / 12 / 20059: 52 \mathrm{AM}$ \\
\hline & (3)ERMYN_GW_F_U234_ICRP72.gsm & $11,470 \mathrm{~KB}$ & GoldSim Model & 1/12/2005 9:54 AM \\
\hline & (3)ERMYN_GW_F_U236_ICRP72.gsm & $11,470 \mathrm{~KB}$ & GoldSim Model & $1 / 12 / 20059: 56 \mathrm{AM}$ \\
\hline & BEERMYN_GW_F_U238D_ICRP72.gsm & $11,470 \mathrm{~KB}$ & GoldSim Model & $1 / 12 / 20059: 58 \mathrm{AM}$ \\
\hline
\end{tabular}

Figure A-2. GoldSim Files for Calculating BDCFs for the Upper Bound of the Glacial Transition Climate Generated Using ICRP 72-Based Dosimetric Inputs 
Figure A-3 shows the list of GoldSim files with modified climate-dependent input parameters (file names ERMYN_GW_Climates_<radionuclide symbol>_ICRP72) generated using GoldSim 8.02.300 and ICRP 72-based dosimetric inputs. These files are included in DTN: MO0503MWDGSBMG.000.

\begin{tabular}{|c|c|c|c|c|}
\hline \multirow[t]{4}{*}{$7, \quad 2$} & Name $\Lambda$ & \multirow{2}{*}{$\begin{array}{r}\text { Size } \\
11,550 \mathrm{~KB}\end{array}$} & \multirow{2}{*}{$\begin{array}{l}\text { Type } \\
\text { GoldSim Model }\end{array}$} & \multirow{2}{*}{$\begin{array}{l}\text { Modified } \\
1 / 21 / 20058: 30 \mathrm{AM}\end{array}$} \\
\hline & BERMYN_GW_Climates_AC227D_ICRP72.gsm & & & \\
\hline & 3]ERMYN_GW_Climates_Am241_ICRP72.gsm & $11,550 \mathrm{~KB}$ & GoldSim Model & $1 / 21 / 200510: 40 \mathrm{AM}$ \\
\hline & 13 ERMYN_GW_Climates_Am243D_ICRP72.gsm & $11,550 \mathrm{~KB}$ & GoldSim Model & $1 / 21 / 200510: 42 \mathrm{AM}$ \\
\hline \multirow{3}{*}{$\oplus$ This folder is Online. } & B]ERMYN_GW_Climates_C14_ICRP72.gsm & $11,550 \mathrm{~KB}$ & GoldSim Model & 1/21/2005 8:02 AM \\
\hline & (3)ERMYN_GW_Climates_Cl36_ICRP72.gsm & $11,550 \mathrm{~KB}$ & GoldSim Model & 1/21/2005 8:07 AM \\
\hline & (3)ERMYN_GW_Climates_Cs135_ICRP72.gsm & $11,550 \mathrm{~KB}$ & GoldSim Model & 1/21/2005 8:22 AM \\
\hline \multirow[t]{4}{*}{ Select an item to view its description. } & 18ERMYN_GW_Climates_Cs137D_ICRP72.gsm & $11,550 \mathrm{~KB}$ & GoldSim Model & $1 / 21 / 20058: 23$ AM \\
\hline & 18 ERMYN_GW_Climates_I129_ICRP72.gsm & $11,550 \mathrm{~KB}$ & GoldSim Model & 1/21/2005 8:18 AM \\
\hline & (3)ERMYN_GW_Climates_Np237D_ICRP72.gsm & $11,550 \mathrm{~KB}$ & GoldSim Model & $1 / 21 / 20059: 27 \mathrm{AM}$ \\
\hline & (3)ERMYN_GW_Climates_Pa231_ICRP72.gsm & $11,550 \mathrm{~KB}$ & GoldSim Model & $1 / 21 / 20058: 39 \mathrm{AM}$ \\
\hline \multirow{20}{*}{ My Computer } & 18 ERMYN_GW_Climates_Pb210D_ICRP72.gsm & $11,550 \mathrm{~KB}$ & GoldSim Model & $1 / 21 / 20058: 25$ AM \\
\hline & B]ERMYN_GW_Climates_Pu238_ICRP72.gsm & $11,550 \mathrm{~KB}$ & GoldSim Model & $1 / 21 / 20059: 40 \mathrm{AM}$ \\
\hline & (3)ERMYN_GW_Climates_PU239_ICRP72.gsm & $11,550 \mathrm{~KB}$ & GoldSim Model & $1 / 21 / 200510: 33 \mathrm{AM}$ \\
\hline & 13 ERMYN_GW_Climates_PU240_ICRP72.gsm & $11,550 \mathrm{~KB}$ & GoldSim Model & $1 / 21 / 200510: 36 \mathrm{AM}$ \\
\hline & 13 ERMYN_GW_Climates_PU242_ICRP72.gsm & $11,550 \mathrm{~KB}$ & GoldSim Model & $1 / 21 / 200510: 37 \mathrm{AM}$ \\
\hline & 19]ERMYN_GW_Climates_Ra226D_ICRP72.gsm & $11,550 \mathrm{~KB}$ & GoldSim Model & $1 / 21 / 20058: 27 \mathrm{AM}$ \\
\hline & 13]ERMYN_GW_Climates_Ra228D_ICRP72.gsm & $11,550 \mathrm{~KB}$ & GoldSim Model & $1 / 21 / 20058: 28 \mathrm{AM}$ \\
\hline & BERMYN_GW_Climates_Se79_ICRP72.gsm & $11,550 \mathrm{~KB}$ & GoldSim Model & $1 / 21 / 20058: 08 \mathrm{AM}$ \\
\hline & B]ERMYN_GW_Climates_Sn126D_ICRP72.gsm & $11,550 \mathrm{~KB}$ & GoldSim Model & $1 / 21 / 20058: 16 \mathrm{AM}$ \\
\hline & BERMYN_GW_Climates_Sr90D_ICRP72.gsm & $11,550 \mathrm{~KB}$ & GoldSim Model & $1 / 21 / 20058: 10 \mathrm{AM}$ \\
\hline & B]ERMYN_GW_Climates_Tc99_ICRP72.gsm & $11,550 \mathrm{~KB}$ & GoldSim Model & $1 / 21 / 20058: 12 \mathrm{AM}$ \\
\hline & B]ERMYN_GW_Climates_Th228D_ICRP72.gsm & $11,550 \mathrm{~KB}$ & GoldSim Model & $1 / 21 / 20058: 33$ AM \\
\hline & 3]ERMYN_GW_Climates_Th229D_ICRP72.gsm & $11,550 \mathrm{~KB}$ & GoldSim Model & $1 / 21 / 20058: 34 \mathrm{AM}$ \\
\hline & BERMYN_GW_Climates_Th230_ICRP72.gsm & $11,550 \mathrm{~KB}$ & GoldSim Model & $1 / 21 / 20058: 36 \mathrm{AM}$ \\
\hline & BERMYN_GW_Climates_Th232_ICRP72.gsm & $11,550 \mathrm{~KB}$ & GoldSim Model & 1/21/2005 8:37 AM \\
\hline & BERMYN_GW_Climates_U232_ICRP72.gsm & $11,550 \mathrm{~KB}$ & GoldSim Model & $1 / 21 / 20058: 41 \mathrm{AM}$ \\
\hline & BERMYN_GW_Climates_U233_ICRP72.gsm & $11,550 \mathrm{~KB}$ & GoldSim Model & $1 / 21 / 20058: 42 \mathrm{AM}$ \\
\hline & BERMYN_GW_Climates_U234_ICRP72.gsm & $11,550 \mathrm{~KB}$ & GoldSim Model & $1 / 21 / 20059: 19$ AM \\
\hline & B]ERMYN_GW_Climates_U236_ICRP72.gsm & $11,550 \mathrm{~KB}$ & GoldSim Model & $1 / 21 / 20059: 21 \mathrm{AM}$ \\
\hline & B]ERMYN_GW_Climates_U238D_ICRP72.gsm & $11,550 \mathrm{~KB}$ & GoldSim Model & $1 / 21 / 20059: 23 \mathrm{AM}$ \\
\hline
\end{tabular}

Figure A-3. GoldSim Files for Calculating BDCFs Using Modified Climate-Dependent Input Parameters and ICRP 72-Based Dosimetric Inputs 
Figure A-4 shows the list of GoldSim files for the present-day climate (file names ERMYN_GW_MC8_<radionuclide symbol>) generated using GoldSim 8.01 Service Pack 4 with dosimetric inputs based on ICRP 30 methods. These files are included in DTN: MO0407MWDGSBMF.000.

\begin{tabular}{|c|c|c|c|c|}
\hline \multirow{5}{*}{$\begin{array}{l}\text { 801SP } 4 \text { Runs for } \\
\text { Modern Climate }\end{array}$} & Name $A$ & Size & Type & Modified \\
\hline & BERMYN_GW_MC8_Ac227D.gsm & $11,460 \mathrm{~KB}$ & GoldSim Model & $6 / 16 / 20047: 35 \mathrm{AM}$ \\
\hline & YERMYN_GW_MC8_Am241.gsm & $11,460 \mathrm{~KB}$ & GoldSim Model & $6 / 16 / 20047: 41 \mathrm{AM}$ \\
\hline & BERMYN_GW_MC8_Am243D.gsm & $11,460 \mathrm{~KB}$ & GoldSim Model & $6 / 16 / 20047: 44 \mathrm{AM}$ \\
\hline & BERMYN_GW_MC8_C14.gSm & $11,460 \mathrm{~KB}$ & GoldSim Model & $6 / 16 / 20047: 48 \mathrm{AM}$ \\
\hline \multirow{3}{*}{ \pm This folder is Online. } & BERMYN_GW_MC8_Cl36.gsm & $11,460 \mathrm{~KB}$ & GoldSim Model & $6 / 16 / 20047: 51 \mathrm{AM}$ \\
\hline & BERMYN_GW_MC8_Cs135.gsm & $11,460 \mathrm{~KB}$ & GoldSim Model & 6/16/2004 8:48 AM \\
\hline & 13ERMYN_GW_MC8_Cs137D.gsm & $11,460 \mathrm{~KB}$ & GoldSim Model & 6/16/2004 9:06 AM \\
\hline \multirow{23}{*}{$\begin{array}{l}\text { Select an item to view its description. } \\
\text { See also: } \\
\text { My Documents } \\
\text { My Network Places } \\
\text { My Computer }\end{array}$} & BERMYN_GW_MC8_I129.gsm & $11,460 \mathrm{~KB}$ & GoldSim Model & 6/16/20049:11 AM \\
\hline & BERMYN_GW_MC8_Np237D.gsm & $11,460 \mathrm{~KB}$ & GoldSim Model & 6/16/2004 9:14 AM \\
\hline & BERMYN_GW_MC8_Pa231.gsm & $11,460 \mathrm{~KB}$ & GoldSim Model & $6 / 16 / 20049: 16 \mathrm{AM}$ \\
\hline & BERMYN_GW_MC8_Pb210D.gsm & $11,460 \mathrm{~KB}$ & GoldSim Model & 6/16/2004 9:18 AM \\
\hline & BERMYN_GW_MC8_Pu238.gsm & $11,460 \mathrm{~KB}$ & GoldSim Model & 6/16/2004 9:20 AM \\
\hline & |ZERMYN_GW_MC8_Pu239.gsm & $11,460 \mathrm{~KB}$ & GoldSim Model & 6/16/2004 9:22 AM \\
\hline & BERMYN_GW_MC8_Pu240.gsm & $11,460 \mathrm{~KB}$ & GoldSim Model & 6/16/2004 9:24 AM \\
\hline & BERMYN_GW_MC8_Pu242.gsm & $11,460 \mathrm{~KB}$ & GoldSim Model & $6 / 16 / 20049: 28 \mathrm{AM}$ \\
\hline & BERMYN_GW_MC8_Ra226D.gsm & $11,460 \mathrm{~KB}$ & GoldSim Model & $6 / 16 / 20049: 30 \mathrm{AM}$ \\
\hline & BERMYN_GW_MC8_Ra228D.gsm & $11,460 \mathrm{~KB}$ & GoldSim Model & 6/16/2004 9:33 AM \\
\hline & QERMYN_GW_MC8_Se79.gsm & $11,460 \mathrm{~KB}$ & GoldSim Model & 6/16/2004 9:36 AM \\
\hline & BERMYN_GW_MC8_Sn126D.gsm & $11,460 \mathrm{~KB}$ & GoldSim Model & 6/16/2004 9:38 AM \\
\hline & QERMYN_GW_MC8_Sr90D.gsm & $11,460 \mathrm{~KB}$ & GoldSim Model & 6/16/2004 9:41 AM \\
\hline & YERMYN_GW_MC8_Tc99.gsm & $11,460 \mathrm{~KB}$ & GoldSim Model & 6/16/2004 9:43 AM \\
\hline & (3ERMYN_GW_MC8_Th228D.gsm & $11,460 \mathrm{~KB}$ & GoldSim Model & $6 / 16 / 200410: 10 \mathrm{AM}$ \\
\hline & BeRMYN_GW_MC8_Th229D.gsm & $11,460 \mathrm{~KB}$ & GoldSim Model & $6 / 16 / 200410: 20 \mathrm{AM}$ \\
\hline & (3ERMYN_GW_MC8_Th230.gsm & $11,460 \mathrm{~KB}$ & GoldSim Model & $6 / 16 / 2004$ 10:26 AM \\
\hline & 18ERMYN_GW_MC8_Th232.gsm & $11,460 \mathrm{~KB}$ & GoldSim Model & 6/16/2004 10:54 AM \\
\hline & 13ERMYN_GW_MC8_U232.gsm & $11,460 \mathrm{~KB}$ & GoldSim Model & $6 / 16 / 200410: 59 \mathrm{AM}$ \\
\hline & 8]ERMYN_GW_MC8_U233.gsm & $11,460 \mathrm{~KB}$ & GoldSim Model & 6/16/2004 11:01 AM \\
\hline & BERMYN_GW_MC8_U234.gsm & $11,460 \mathrm{~KB}$ & GoldSim Model & $6 / 16 / 200411: 03 \mathrm{AM}$ \\
\hline & 18ERMYN_GW_MC8_U236.gsm & $11,460 \mathrm{~KB}$ & GoldSim Model & 6/16/2004 11:04 AM \\
\hline & BERMYN_GW_MC8_U238D.gsm & $11,460 \mathrm{~KB}$ & GoldSim Model & $6 / 16 / 200411: 06 \mathrm{AM}$ \\
\hline & & & $335 \mathrm{MB}$ & 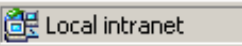 \\
\hline
\end{tabular}

Figure A-4. GoldSim Files for Calculating BDCFs for the Present-Day Climate Using GoldSim 8.01 Service Pack 4 and ICRP 30-Based Dosimetric Inputs 
Figure A-5 shows the list of GoldSim files for the upper bound of the glacial transition climate (file names ERMYN_GW_FC8_<radionuclide symbol>) generated using GoldSim 8.01 Service Pack 4 with dosimetric inputs based on ICRP 30 methods. These files are included in DTN: MO0407MWDGSBMF.000.

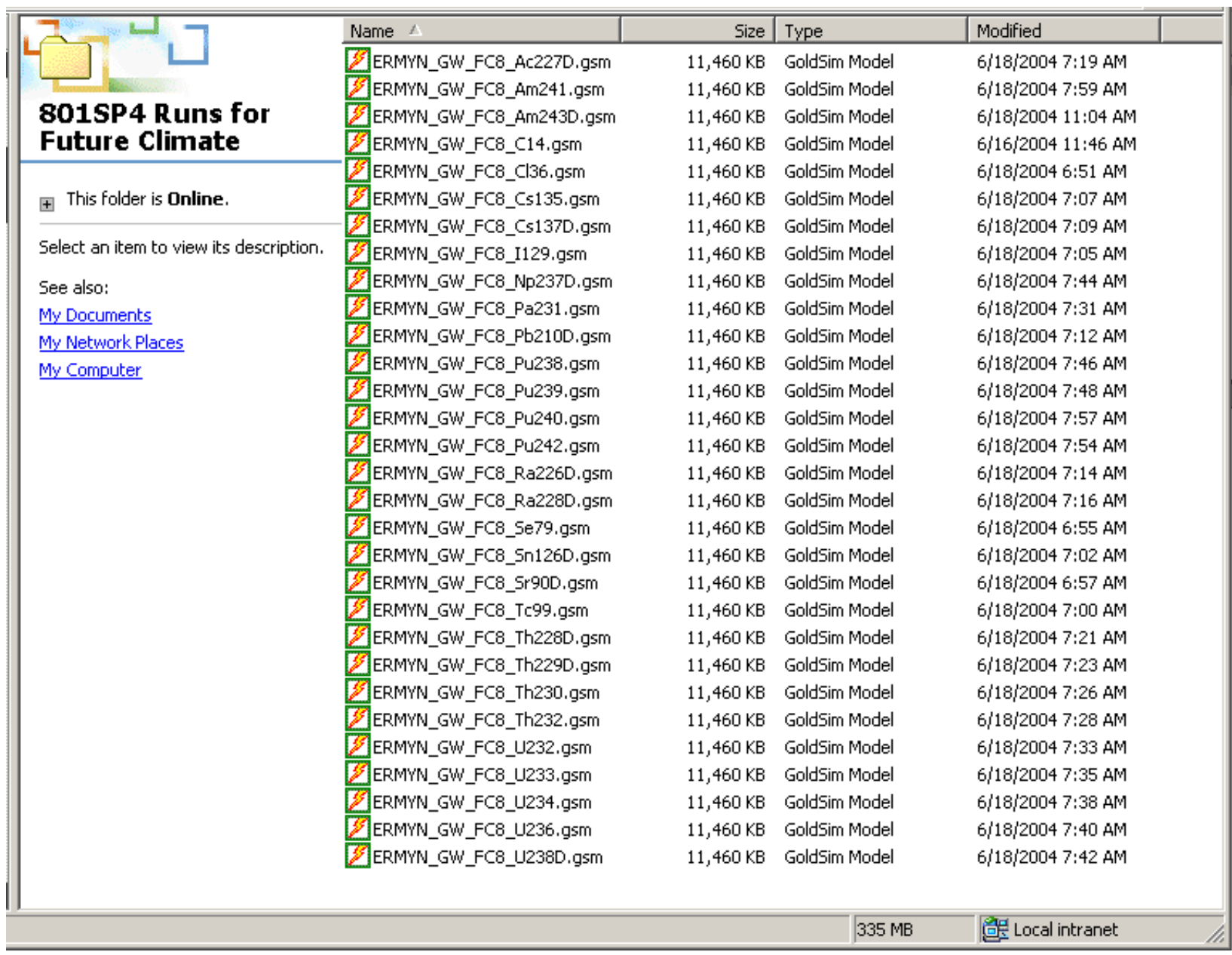

Figure A-5. GoldSim Files for Calculating BDCFs for the Upper Bound Glacial Transition Climate Using GoldSim 8.01 Service Pack 4 and ICRP 30-Based Dosimetric Inputs 
Figure A-6 shows the list of the GoldSim files with modified climate-dependent input parameters (file names ERMYN_GW_Climates8_<radionuclide symbol>), as described in Section 6.2.2 and in Appendix B. The files were generated using GoldSim 8.01 Service Pack 4 with dosimetric inputs based on ICRP 30 methods. These files are included in DTN: MO0407MWDGSBMF.000.

\begin{tabular}{|c|c|c|c|c|}
\hline \multirow{5}{*}{$\begin{array}{l}\overbrace{\text { Boisp4 Runs for }} \\
\text { Climates }\end{array}$} & Name $A$ & Size & Type & Modified \\
\hline & BERMYN_GW_Climates8_Ac227D.gsm & $11,540 \mathrm{~KB}$ & GoldSim Model & $7 / 18 / 200411: 39 \mathrm{AM}$ \\
\hline & BgERYN_GW_Climates8_Am241.gsm & $11,540 \mathrm{~KB}$ & GoldSim Model & $7 / 18 / 200411: 42 \mathrm{AM}$ \\
\hline & B]ERMYN_GW_Climates8_Am243D.gsm & $11,540 \mathrm{~KB}$ & GoldSim Model & $7 / 18 / 200411: 47 \mathrm{AM}$ \\
\hline & (3)ERMYN_GW_Climates8_C14.gsm & $11,540 \mathrm{~KB}$ & GoldSim Model & $7 / 18 / 200411: 49 \mathrm{AM}$ \\
\hline \multirow{27}{*}{$\begin{array}{l}\text { Select an item to view its description. } \\
\text { See also: } \\
\text { My Documents } \\
\text { My Network Places } \\
\text { My Computer }\end{array}$} & [3]ERMYN_GW_Climates8_Cl36.gsm & $11,540 \mathrm{~KB}$ & GoldSim Model & $7 / 18 / 200411: 51 \mathrm{AM}$ \\
\hline & 8)ERMYN_GW_Climates8_Cs135.gsm & $11,540 \mathrm{~KB}$ & GoldSim Model & $7 / 18 / 200411: 52 \mathrm{AM}$ \\
\hline & BEEMYN_GW_Climates8_Cs137D.gsm & $11,540 \mathrm{~KB}$ & GoldSim Model & $7 / 18 / 200411: 59 \mathrm{AM}$ \\
\hline & B]ERMYN_GW_Climates8_I129.gsm & $11,540 \mathrm{~KB}$ & GoldSim Model & $7 / 18 / 200412: 01 \mathrm{PM}$ \\
\hline & B]ERMYN_GW_Climates8_Np237D.gsm & $11,540 \mathrm{~KB}$ & GoldSim Model & $7 / 18 / 200412: 03 \mathrm{PM}$ \\
\hline & 8E ERMYN_GW_Climates8_Pa231.gsm & $11,540 \mathrm{~KB}$ & GoldSim Model & $7 / 18 / 200412: 04 \mathrm{PM}$ \\
\hline & 8 ERMYN_GW_Climates8_Pb210D.gsm & $11,540 \mathrm{~KB}$ & GoldSim Model & $7 / 18 / 200412: 06 \mathrm{PM}$ \\
\hline & 18 ERMYN_GW_Climates8_Pu238.gsm & $11,540 \mathrm{~KB}$ & GoldSim Model & $7 / 18 / 200412: 08 \mathrm{PM}$ \\
\hline & BERMYN_GW_Climates8_Pu239.gsm & $11,540 \mathrm{~KB}$ & GoldSim Model & $7 / 18 / 200412: 10 \mathrm{PM}$ \\
\hline & 38ERYN_GW_Climates8_Pu240.gsm & $11,540 \mathrm{~KB}$ & GoldSim Model & $7 / 18 / 200412: 12 \mathrm{PM}$ \\
\hline & 3 ERMYN_GW_Climates8_Pu242.gsm & $11,540 \mathrm{~KB}$ & GoldSim Model & $7 / 18 / 200412: 14 \mathrm{PM}$ \\
\hline & (8)ERMYN_GW_Climates8_Ra226D.gsm & $11,540 \mathrm{~KB}$ & GoldSim Model & $7 / 18 / 200412: 16 \mathrm{PM}$ \\
\hline & (3)ERMYN_GW_Climates8_Ra228D.gsm & $11,540 \mathrm{~KB}$ & GoldSim Model & $7 / 18 / 200412: 18 \mathrm{PM}$ \\
\hline & B]ERMYN_GW_Climates8_Se79.gsm & $11,540 \mathrm{~KB}$ & GoldSim Model & $7 / 18 / 200412: 20 \mathrm{PM}$ \\
\hline & BEERYN_GW_Climates8_Sn126D.gsm & $11,540 \mathrm{~KB}$ & GoldSim Model & $7 / 18 / 200412: 22 \mathrm{PM}$ \\
\hline & BgERYN_GW_Climates8_Sr90D.gsm & $11,540 \mathrm{~KB}$ & GoldSim Model & $7 / 18 / 200412: 23 \mathrm{PM}$ \\
\hline & EEEMYN_GW_Climates8_Tc99.gsm & $11,540 \mathrm{~KB}$ & GoldSim Model & $7 / 18 / 200412: 25 \mathrm{PM}$ \\
\hline & BgERYN_GW_Climates8_Th228D.gsm & $11,540 \mathrm{~KB}$ & GoldSim Model & $7 / 18 / 200412: 27 \mathrm{PM}$ \\
\hline & BEERYN_GW_Climates8_Th229D.gsm & $11,540 \mathrm{~KB}$ & GoldSim Model & $7 / 18 / 200412: 30 \mathrm{PM}$ \\
\hline & BgERYN_GW_Climates8_Th230.gsm & $11,540 \mathrm{~KB}$ & GoldSim Model & $7 / 18 / 200412: 31 \mathrm{PM}$ \\
\hline & BEEMYN_GW_Climates8_Th232.gsm & $11,540 \mathrm{~KB}$ & GoldSim Model & $7 / 18 / 200412: 33 \mathrm{PM}$ \\
\hline & BgERYN_GW_Climates8_U232.gsm & $11,540 \mathrm{~KB}$ & GoldSim Model & $7 / 18 / 200412: 36 \mathrm{PM}$ \\
\hline & BERMYN_GW_Climates8_U233.gsm & $11,540 \mathrm{~KB}$ & GoldSim Model & $7 / 18 / 200412: 38 \mathrm{PM}$ \\
\hline & B]ERMYN_GW_Climates8_U234.gsm & $11,540 \mathrm{~KB}$ & GoldSim Model & $7 / 18 / 200412: 39 \mathrm{PM}$ \\
\hline & BERMYN_GW_Climates8_U236.gsm & $11,540 \mathrm{~KB}$ & GoldSim Model & $7 / 18 / 200412: 41 \mathrm{PM}$ \\
\hline & BgEMYN_GW_Climates8_U238D.gsm & $11,540 \mathrm{~KB}$ & GoldSim Model & $7 / 18 / 200412: 43 \mathrm{PM}$ \\
\hline & & & $338 \mathrm{MB}$ & 番总 Local intranet \\
\hline
\end{tabular}

Figure A-6. GoldSim Files for Calculating BDCFs Using Modified Climate-Dependent Parameters and GoldSim 8.01 Service Pack 4 and ICRP 30-Based Dosimetric Inputs 


\section{APPENDIX B}

\section{DESCRIPTION OF EXCEL FILES GENERATED IN THIS ANALYSIS}




\section{B. LIST AND DESCRIPTION OF EXCEL FILES GENERATED IN THIS ANALYSIS}

This appendix contains descriptions of the Excel files that were used for calculations in this analysis. For the files that contain the stochastic results of the GoldSim runs, the values were copied from the RESULTS container of the appropriate GoldSim files and pasted into the worksheets. The files can be found on the CD ROM included as an electronic attachment to this document.

\section{B.1 LIST OF EXCEL FILES}

Figure B-1 shows the list of the Excel files supporting this analysis. The description of the files follows in Section B.2.

\begin{tabular}{|c|c|c|c|c|}
\hline \multirow{4}{*}{ 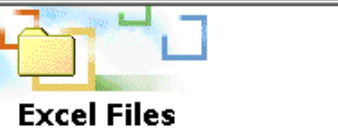 } & Name $A$ & Size & Type & Modified \\
\hline & 2] Conversion Factors for Groundwater Protection Standard_Rev 3.xls & $118 \mathrm{~KB}$ & Microsoft Excel Worksheet & $3 / 18 / 200511: 40 \mathrm{AM}$ \\
\hline & (2] Conversion Factors for Groundwater Protection Standard_Rev 4.xls & $116 \mathrm{~KB}$ & Microsoft Excel Worksheet & $1 / 12 / 20055: 53 \mathrm{PM}$ \\
\hline & (1] Correlations for climate dependent parameters_Rev $3 . x \mathrm{~s}$ & $1,005 \mathrm{~KB}$ & Microsoft Excel Worksheet & $3 / 18 / 20051: 36 \mathrm{PM}$ \\
\hline \multirow{3}{*}{$\oplus$ This folder is Online. } & 20 Correlations for climate dependent parameters_Rev 4.xls & $991 \mathrm{~KB}$ & Microsoft Excel Worksheet & $3 / 18 / 20051: 35 \mathrm{PM}$ \\
\hline & Correlations for Groundwater BDCFs MC_Rev 3.xls & $871 \mathrm{~KB}$ & Microsoft Excel Worksheet & $2 / 28 / 20056: 38 \mathrm{PM}$ \\
\hline & (20) Correlations for Groundwater BDCFs MC_Rev 4.xls & $810 \mathrm{~KB}$ & Microsoft Excel Worksheet & $3 / 18 / 200511: 40 \mathrm{AM}$ \\
\hline Select an item to view its description. & (2) Dependence of BDCFs on Irrigation Rate_Rev 3.xls & $166 \mathrm{~KB}$ & Microsoft Excel Worksheet & $4 / 7 / 20058: 50 \mathrm{AM}$ \\
\hline See also: & (1)Dependence of BDCFs on Irrigation Rate_Rev 4.xls & $166 \mathrm{~KB}$ & Microsoft Excel Worksheet & 4/7/2005 8:50 AM \\
\hline My Documents & (2] GW BDCF Realizations_MC and FC_Rev 4.xls & $12,322 \mathrm{~KB}$ & Microsoft Excel Worksheet & $3 / 4 / 20053: 16 \mathrm{PM}$ \\
\hline My Network Places & X1 GW BDCFs Pathway Analysis FC_Rev 3.xls & $10,020 \mathrm{~KB}$ & Microsoft Excel Worksheet & $3 / 4 / 20054: 24 \mathrm{PM}$ \\
\hline \multirow{3}{*}{ My Computer } & 1] GW BDCFs Pathway Analysis FC_Rev 4.xls & $10,644 \mathrm{~KB}$ & Microsoft Excel Worksheet & $3 / 16 / 200512: 53 \mathrm{PM}$ \\
\hline & (1] GW BDCFs Pathway Analysis MC_Rev 3.xls & $10,014 \mathrm{~KB}$ & Microsoft Excel Worksheet & $3 / 4 / 20054: 24 \mathrm{PM}$ \\
\hline & (1) GW BDCFs Pathway Analysis MC_Rev 4.xls & $10,646 \mathrm{~KB}$ & Microsoft Excel Worksheet & 3/16/2005 12:53 PM \\
\hline
\end{tabular}

Figure B-1. List of Excel Files Supporting This Analysis

\section{B.2 DESCRIPTION OF EXCEL FILES}

GW BDCF Realizations_MC and FC_Rev 4.xls-This Excel file contains six worksheets. The worksheets BDCF Calculations FGR11 and BDCF Calculations ICRP72 contain the results of 1,000 biosphere model realizations for the groundwater exposure scenario from files shown in Figures A-1, A-2 for the ICRP 72 dosimetry and Figures A-4 and A-5 for the ICRP 30-based dosimetry (FGR 11). The worksheets also contain the calculated values of BDCFs for the monsoon and the glacial transition climates. The BDCFs are arranged by radionuclide in columns B to AH for the present-day climate (average interglacial) and in columns AJ to BQ for the average upper bound of the glacial transition climate. The results of individual model realizations are in rows 31 to 1,030. The values from the present-day climate and the upper bound of the glacial transition climate were copied from the Final_BDCF_Dist element of the Results container of the GoldSim files for individual radionuclides and pasted into the worksheet.

Columns BS to $\mathrm{CZ}$ and rows 31 to 1,030 contain calculated BDCF values for the monsoon climate. Each BDCF value was calculated as

$$
B D C F_{M C, i j}=B D C F_{U B G T, i, j}+\left(B D C F_{I C, i, j}-B D C F_{U B G T, i, j}\right) R A N D(0,1) \quad(E q . \text { B-1 })
$$

where 


$\begin{array}{ll}B D C F_{M C, i, j} & =\begin{array}{l}\text { BDCF for model realization } i \text { and radionuclide } j \text { for monsoon } \\ \text { climate }\end{array} \\ B D C F_{U B G T, i, j}= & \begin{array}{l}\text { BDCF for model realization } i \text { and radionuclide } j \text { for upper bound of } \\ \text { glacial transition climate }\end{array} \\ B D C F_{I C, i, j} & =\begin{array}{l}\text { BDCF for model realization } i \text { and radionuclide } j \text { for present-day } \\ \text { (interglacial) climate }\end{array} \\ R A N D(0,1) & =\end{array}$

Columns DB to EI and rows 31 to 1,030 contain calculated BDCF values for the glacial | transition climate. Each BDCF value was calculated as

$$
B D C F_{G T C, i j}=B D C F_{U B G T, i, j}+\left(B D C F_{I C, i, j}-B D C F_{U B G T, i, j}\right) \frac{0.88-0.50}{0.95-0.50} R A N D(0,1)
$$

where

$$
\begin{array}{ll}
\mathrm{BDCF}_{G T C, i, j}= & \begin{array}{l}
\text { BDCF for model realization } i \text { and radionuclide } j \text { for glacial transition } \\
\text { climate }
\end{array} \\
B D C F_{U B G T, i, j}= & \begin{array}{l}
\text { BDCF for model realization } i \text { and radionuclide } j \text { for upper bound of } \\
\text { glacial transition climate }
\end{array} \\
\frac{0.88-0.50}{0.95-0.50}= & \begin{array}{l}
\text { scaling factor, which is a fraction of the BDCF interval that is being } \\
\text { sampled; scaling factor is calculated using the values of average } \\
\text { annual irrigation rate for the present-day interglacial climate, and } \\
\text { lower and upper bounds of the glacial transition climate, as } \\
\text { explained in Section } 6.2 .2
\end{array} \\
\operatorname{RAND}(0,1)= & \text { random number between } 0 \text { and } 1 .
\end{array}
$$

For all climates, the BDCF values for ${ }^{232}$ Th and its decay products were calculated by adding the values for ${ }^{232} \mathrm{Th},{ }^{228} \mathrm{Ra}$, and ${ }^{228} \mathrm{Th}$. The BDCF values for ${ }^{232} \mathrm{U}$ and its decay products were calculated by adding the values for ${ }^{232} \mathrm{U}$ and ${ }^{228} \mathrm{Th}$.

Row 5 contains the means of the values in rows 31 to 1,030 of the corresponding columns, calculated using the Excel AVERAGE function for the specified cell range.

Row 6 contains standard deviations of the values in rows 31 to 1,030 of the corresponding columns, calculated using the Excel STDEV function for the specified cell range.

Row 7 contains the minima of the values in rows 31 to 1,030 of the corresponding columns, calculated using the Excel MIN function for the specified cell range.

Row 27 contains the maxima of the values in rows 31 to 1,030 of the corresponding columns, calculated using the Excel MAX function for the specified cell range.

Rows 8 to 26 contain the percentiles, in increments of 5, of the values in rows 31 to 1,030 of the corresponding columns, calculated using the Excel PERCENTILE function for the specified cell range. 
The worksheets, BDCF Values ICRP72 and BDCF Values FGR11, contain BDCF values copied from the worksheets, BDCF Calculations ICRP72 and BDCF Calculations FGR11, respectively. This was done to stabilize the results of random sampling executed in the first and second worksheets to provide final BDCF results for the TSPA model.

The statistics from rows 5 to 27 of the worksheets BDCF Values ICRP72 and BDCF Values FGR11 were copied and pasted into the worksheets, Comparison_Climates and Comparison_Dosimetries to compare the effect of climate change and dosimetry change on the BDCF values. The analysis was conducted using mean BDCF values.

The worksheet Comparison_Climates contains the BDCF statistics for the present-day and upper bound of the glacial transition climates (rows 6 to 28 and 34 to 56, respectively) and for the ICRP 30- and ICRP 72-based dosimetric parameters (columns A to AH and AJ to BQ for the ICRP 72 and ICRP 30 values, respectively). Percent difference between these BDCF relative to the present-day climate values is calculated in rows 61 to 83 . The results for the means BDCFs are summarized in rows 95 to 127.

Likewise, a comparison of BDCFs developed using ICRP 30 and ICRP 72-based dosimetric input parameters is presented in worksheet Comparison_Dosimetries. This worksheet contains the statistics for the ICRP 72-based BDCFs (rows 6 to 28) and ICRP 30-based BDCFs (rows 34 to 56) and for the present-day (columns $\mathrm{A}$ to $\mathrm{AH}$ ) and the upper bound of the glacial transition climates (columns AJ to BQ). Percent difference between these BDCF relative to the ICRP 30based values is calculated in rows 61 to 83 . The results for the means BDCFs are summarized in rows 97 to 125 .

Correlations for climate dependent parameters_Rev 3.xls and Correlations for climate dependent parameters_Rev 4.xls-These Excel file contain calculations of correlation coefficients for the climate-dependent parameters and the BDCFs, which were calculated by replacing the values of climate-dependent parameters with uniform distributions between the values for the extreme climates (i.e., for the present-day climate and the upper bound of the glacial transition climate). (..._Rev 3.xls file contains the calculations for the ICRP 30-based BDCFs; ..._Rev 4.xls file contains the calculations for the ICRP 72-based BDCFs. The workbook consists of two worksheets: Summary and Raw Correlations Calculation . The Raw Correlations Calculation worksheet contains the results of sampling of individual climate-dependent model input parameters (there are 18 such parameters). The results are in columns $\mathrm{C}$ to $\mathrm{T}$ and rows 39 to 1,038. Columns $\mathrm{V}$ to BA and rows 39 to 1,038 contain BDCF values for individual radionuclides and model realizations. The BDCFs were generated using modified distributions for climate-dependent parameters, as described in Section 6.2.2. In rows 4 to 33, and in columns from $\mathrm{C}$ to $\mathrm{T}$, the values of correlation coefficients for the climatedependent model input parameters and the BDCFs are shown, calculated using the Excel CORREL function.

Worksheet Summary contains the correlation coefficients for the primary radionuclides copied from the worksheet Raw Correlations Calculation. They appear in columns $\mathrm{C}$ to $\mathrm{T}$ and in rows 8 to 35 of the worksheet. 
Dependence of BDCFs on Irrigation Rate_Rev 3.xls and Dependence of BDCFs on Irrigation Rate_Rev 4.xls-These Excel files are used to generate graphs showing the linear dependence of BDCFs on annual average irrigation rate, for the ICRP 30- and ICRP 72-based BDCFs, respectively. First, the columns containing the sampling results for annual average irrigation rate and the BDCFs for ${ }^{14} \mathrm{C},{ }^{99} \mathrm{Tc},{ }^{129} \mathrm{I}$, and ${ }^{237} \mathrm{~Np}$ were copied from files Correlations for Climate Dependent Parameters_Rev 3.xls and Correlations for Climate Dependent Parameters_Rev 4.xls. The values are in columns $\mathrm{C}$ to $\mathrm{G}$ of the worksheet. The values then were sorted by average annual irrigation rate. The irrigation rates and the BDCFs were then averaged in groups of 100 (columns $\mathrm{K}$ to $\mathrm{O}$, rows 8 to 17). The average values were used to produce four graphs, one for each of the four radionuclides.

Correlations for Groundwater BDCFs MC_Rev 3.xls and Correlations for Groundwater BDCFs MC_Rev 4.xls-These Excel files contain calculations of the correlation coefficients for groundwater exposure scenario BDCFs for different radionuclides for the present-day climate, for the ICRP 30- and ICRP 72-based BDCFs, respectively. The BDCF values were copied from the file GW BDCF Realizations_MC and FC_Rev 4.xls.

The BDCFs for radionuclides of interest are in columns B to AE. Rows 1,012 to 1,039 and columns B to AC contain the 28 by 28 table of rank correlation coefficients for the BDCFs for 28 primary radionuclides, calculated using the Excel CORREL function for the specified range of cells containing ranks for the BDCFs.

The rows beneath row 1,040 contain supplementary calculations of the Student's $t$ values for the range of correlation coefficient values.

GW BDCFs Pathway Analysis MC_Rev 3.xls, GW BDCFs Pathway Analysis FC_Rev 3.xls, GW BDCFs Pathway Analysis MC_Rev 4.xls and GW BDCFs Pathway Analysis FC_Rev 4.xls - These Excel files contain calculations of pathway contributions to BDCFs for the present-day climate and upper bound of the glacial transition climate, for the ICRP 30- and ICRP 72-based BDCFs, respectively. The files contain worksheets for individual radionuclides (28 primary radionuclides, ${ }^{228} \mathrm{Ra}$ and ${ }^{228} \mathrm{Th}$, as well as the worksheets that include contribution of long-lived decay products for selected radionuclides) and a summary worksheet. The first worksheet (Pathway Summary) contains the summary of the mean pathway BDCFs, the following worksheets contain the pathway BDCFs from individual realizations for radionuclides of interest, as well as their mean values. The pathway BDCFs for 1,000 realizations are in rows 10 to 1,009 of each worksheet for an individual radionuclide. The values were copied from the Pathway_BDCF_Dist element of the Results container of the GoldSim files for individual radionuclides and pasted into the worksheets for the corresponding radionuclides (files shown in Figures A-1 and A-2 for ICRP 72-based values and in Figures A-4 and A-5 for ICRP 30-based values). Row 6 contains the mean values of BDCFs from individual model realizations (rows 10 to 1,009) calculated using the Excel AVERAGE function.

The Pathway Summary worksheet contains the summary of the mean values of pathway BDCFs for the individual radionuclides copied from the radionuclide worksheets as well as calculation of the fractional and percent contributions of the individual pathways to BDCFs. 
Conversion Factors for Groundwater Protection Standard_Rev 3.xls and Conversion Factors for Groundwater Protection Standard_Rev 4.xls-These Excel files contains calculations of conversion factors for calculating beta-photon annual dose resulting from drinking 2 liters of water per day using ICRP 30- and ICRP 72-based dose coefficients, respectively. The calculation of annual dose from beta-photon emitting radionuclides is conducted for evaluation of compliance with the groundwater protection standards. The calculations are performed for 18 radionuclides of importance during the compliance period. For the ICRP 30-based calculations, column B to CO, rows 6 to 29 of the set Organ Conversion Factors contain calculation of the effective dose coefficients for ingestion of radionuclides. The effective dose coefficients are summarized in rows 35 to 58. The effective dose coefficients are calculated by adding the dose coefficients for beta-photon emitting radionuclides weighted by their branching fractions using equation 6.3-5. Rows 64 to 87 and 93 to 116 contain calculations of the conversion factors in units of $\mathrm{Sv} / \mathrm{yr}$ per $\mathrm{Bq} / \mathrm{m}^{3}$ or in $\mathrm{mrem} / \mathrm{yr}$ per $\mathrm{pCi} / \mathrm{L}$, respectively. The values are calculated using Equation 6.3-3.

The corresponding calculations using ICRP 72 dose coefficients use three more organs (extrathoracic pathways, colon, and remainder) than those for ICRP 30-based values. As a result, there are more rows in the file where the ICRP 72-based values are calculated, i.e., in Conversion Factors for Groundwater Protection Standard_Rev 4.xls. 


\section{INTENTIONALLY LEFT BLANK}


APPENDIX C

VERIFICATION OF ERMYN MODEL FILES FOR GOLDSIM VERSIONS

8.01 SERVICE PACK 4 AND 8.02.300 


\section{VERIFICATION OF ERMYN MODEL FILES FOR GOLDSIM VERSIONS 8.01 SERVICE PACK 4 AND 8.02.300}

The ERMYN model for the groundwater exposure scenario (DTN: MO0306MWDBGSMF.001) was constructed and verified for GoldSim version 7.50.100. This analysis was conducted using Version 8.02.300 of the software (STN: 10344-8.02-03, Windows 2000) (BSC 2004 [DIRS 172588]) qualified under the Office of Civilian Radioactive Waste Management, Quality Assurance program for use on the Yucca Mountain Project. It also includes the previously developed BDCF, which were calculated using GoldSim 8.01 Service Pack 4 (BSC 2004 [DIRS 169695]).

Verification was conducted for three radionuclides: ${ }^{14} \mathrm{C},{ }^{226} \mathrm{Ra}$, and ${ }^{239} \mathrm{Pu}$ because these three radionuclides are representative of all environmental transport and exposure pathways included in the model for the groundwater exposure scenario. To verify the applicability of GoldSim versions 8.01 Service Pack 4 and 8.02.300 as software supporting the biosphere model, the original model verification files were opened in GoldSim 8.01 Service Pack 4 and 8.02.300, and the verification calculations were repeated.

Figure C-1 shows the list of the original verification files as well as the model files generated in this analysis when the original files were run using GoldSim 8.01 Service Pack 4 and GoldSim 8.02.300. The files that are labeled with *_GS801SP4.gsm correspond to runs performed using GoldSim Version 8.01 Service Pack 4, the files that are labeled with *_GS802300.gsm correspond to runs performed using GoldSim Version 8.02.300, whereas, those without the GoldSim version designation are runs performed using GoldSim Version 7.50.100. Table C-1 shows the results from the original verification files and their re-runs in GoldSim 8.01 Service Pack 4 and 8.02.300. The results are identical, so the model can be executed using GoldSim 8.01 Service Pack 4 as well as GoldSim 8.02.300. The files shown in Figure C-1 are included in DTN: MO0503MWDGSBMG.000.

\begin{tabular}{|c|c|c|c|c|}
\hline $7, \quad 2=$ & Name $\triangle$ & Size & Type & Modified \\
\hline & BERMYN_GW_C14verf.gsm & $3,931 \mathrm{~KB}$ & GoldSim Model & $6 / 23 / 20032: 29 \mathrm{PM}$ \\
\hline \multirow[b]{2}{*}{ GoldSim Verification } & (8EMYN_GW_C14verf_GS8015P4.gsm & $3,526 \mathrm{~KB}$ & GoldSim Model & 7/20/2004 11:15 AM \\
\hline & BERMYN_GW_Pu239verf.gsm & $3,931 \mathrm{~KB}$ & GoldSim Model & $6 / 23 / 20032: 29 \mathrm{PM}$ \\
\hline \multirow{3}{*}{ \pm This folder is Online. } & BERMYN_GW_PU239verf_GS8015P4.gsm & $3,526 \mathrm{~KB}$ & GoldSim Model & $7 / 20 / 200411: 16 \mathrm{AM}$ \\
\hline & BERMYN_GW_Ra226verf.gsm & $3,931 \mathrm{~KB}$ & GoldSim Model & $6 / 23 / 20032: 29 \mathrm{PM}$ \\
\hline & BERMYN_GW_Ra226verf_GS8015P4.gsm & $3,526 \mathrm{~KB}$ & GoldSim Model & $7 / 20 / 200411: 18 \mathrm{AM}$ \\
\hline Select an item to view its description. & BEEMYN_GW_C14verf_GS802300.gsm & $3,538 \mathrm{~KB}$ & GoldSim Model & $3 / 4 / 20053: 43 \mathrm{PM}$ \\
\hline See also: & (3ERMYN_GW_PU239verf_GS802300.gsm & $3,538 \mathrm{~KB}$ & GoldSim Model & $3 / 4 / 20053: 44 \mathrm{PM}$ \\
\hline My Documents & [8ERMYN_GW_Ra226verf_GS802300.gsm & $3,538 \mathrm{~KB}$ & GoldSim Model & $3 / 4 / 20053: 45 \mathrm{PM}$ \\
\hline \multicolumn{5}{|l|}{$\overline{\text { My Network Places }}$} \\
\hline \multicolumn{5}{|l|}{ My Computer } \\
\hline & & & $32.2 \mathrm{MB}$ & Local intranet \\
\hline
\end{tabular}

Figure C-1. Model Verification Files Obtained Using GoldSim Versions 7.50.100, 8.01 Service Pack 4, and 8.02.300 
Nominal Performance Biosphere Dose Conversion Factor Analysis

Table C-1. Results of Verification Runs Using GoldSim Versions 7.50.100, 8.01 Service Pack 4, and 8.02.300

\begin{tabular}{|c|c|c|c|}
\hline \multirow[b]{2}{*}{ Radionuclide } & \multirow[b]{2}{*}{ Pathway } & \multicolumn{2}{|c|}{ BDCF (rem/yr per pCi/L) } \\
\hline & & GoldSim 7.50.100 & GoldSim 8.01 Service Pack 4 and 8.02.300 \\
\hline \multirow[t]{17}{*}{ C-14 } & All pathways & 7.14602E-06 & $7.14602 \mathrm{E}-06$ \\
\hline & External & $3.26180 \mathrm{E}-13$ & $3.26180 \mathrm{E}-13$ \\
\hline & Inhalation & 1.63130E-09 & 1.63130E-09 \\
\hline & Evaporative Cooler & $3.40450 \mathrm{E}-11$ & $3.40450 \mathrm{E}-11$ \\
\hline & Radon & $0.00000 \mathrm{E}+00$ & $0.00000 \mathrm{E}+00$ \\
\hline & Water & $1.52440 \mathrm{E}-06$ & $1.52440 \mathrm{E}-06$ \\
\hline & Leafy Vegetables & $2.52710 \mathrm{E}-07$ & $2.52710 \mathrm{E}-07$ \\
\hline & Other Vegetables & $4.42120 \mathrm{E}-07$ & $4.42120 \mathrm{E}-07$ \\
\hline & Fruit & $1.15850 \mathrm{E}-06$ & $1.15850 \mathrm{E}-06$ \\
\hline & Grain & $5.87210 \mathrm{E}-08$ & $5.87210 \mathrm{E}-08$ \\
\hline & Meat & $6.31780 \mathrm{E}-07$ & $6.31780 \mathrm{E}-07$ \\
\hline & Milk & $3.01670 \mathrm{E}-07$ & $3.01670 \mathrm{E}-07$ \\
\hline & Poultry & $5.41440 \mathrm{E}-08$ & $5.41440 \mathrm{E}-08$ \\
\hline & Eggs & $5.12430 \mathrm{E}-07$ & $5.12430 \mathrm{E}-07$ \\
\hline & Fish & $2.20780 \mathrm{E}-06$ & $2.20780 \mathrm{E}-06$ \\
\hline & Soil Ingestion & $8.67940 \mathrm{E}-12$ & $8.67940 \mathrm{E}-12$ \\
\hline & File name & ERMYN_GW_C14verf.gsm & $\begin{array}{l}\text { ERMYN_GW_C14verf_GS801SP4.gsm } \\
\text { ERMYN_GW_C14verf_GS802300.gsm }\end{array}$ \\
\hline \multirow[t]{17}{*}{ Pu-239 } & All pathways & $7.63326 \mathrm{E}-03$ & $\begin{array}{c}7.63326 \mathrm{E}-03 \\
\end{array}$ \\
\hline & External & 1.38220E-07 & $1.38220 \mathrm{E}-07$ \\
\hline & Inhalation & $3.92780 \mathrm{E}-03$ & $3.92780 \mathrm{E}-03$ \\
\hline & Evaporative Cooler & $6.20940 \mathrm{E}-04$ & $6.20940 \mathrm{E}-04$ \\
\hline & Radon & $0.00000 \mathrm{E}+00$ & $0.00000 \mathrm{E}+00$ \\
\hline & Water & $2.58390 \mathrm{E}-03$ & $2.58390 \mathrm{E}-03$ \\
\hline & Leafy Vegetables & $7.55490 \mathrm{E}-05$ & $7.55490 \mathrm{E}-05$ \\
\hline & Other Vegetables & $1.52470 \mathrm{E}-05$ & $1.52470 \mathrm{E}-05$ \\
\hline & Fruit & $4.95180 \mathrm{E}-05$ & 4.95180E-05 \\
\hline & Grain & $5.87800 \mathrm{E}-06$ & $5.87800 \mathrm{E}-06$ \\
\hline & Meat & $2.57590 \mathrm{E}-07$ & $2.57590 \mathrm{E}-07$ \\
\hline & Milk & $9.85630 \mathrm{E}-09$ & $9.85630 \mathrm{E}-09$ \\
\hline & Poultry & $6.33900 \mathrm{E}-08$ & $6.33900 \mathrm{E}-08$ \\
\hline & Eggs & $1.13320 \mathrm{E}-06$ & 1.13320E-06 \\
\hline & Fish & $1.38430 \mathrm{E}-04$ & $1.38430 \mathrm{E}-04$ \\
\hline & Soil Ingestion & $2.14330 \mathrm{E}-04$ & $2.14330 \mathrm{E}-04$ \\
\hline & File name & ERMYN_GW_Pu239verf.gsm & $\begin{array}{l}\text { ERMYN_GW_Pu239verf_GS801SP4.gsm } \\
\text { ERMYN_GW_Pu239verf_GS802300.gsm }\end{array}$ \\
\hline \multirow[t]{7}{*}{ Ra-226 } & All pathways & $7.45638 \mathrm{E}-02$ & $7.45638 \mathrm{E}-02$ \\
\hline & External & $5.64900 \mathrm{E}-03$ & $5.64900 \mathrm{E}-03$ \\
\hline & Inhalation & $2.44180 \mathrm{E}-04$ & $2.44180 \mathrm{E}-04$ \\
\hline & Evaporative Cooler & $1.24400 \mathrm{E}-05$ & $1.24400 \mathrm{E}-05$ \\
\hline & Radon & $6.66370 \mathrm{E}-02$ & $6.66370 \mathrm{E}-02$ \\
\hline & Water & $9.68280 \mathrm{E}-04$ & $9.68280 \mathrm{E}-04$ \\
\hline & Leafy Vegetables & $1.10450 \mathrm{E}-04$ & $1.10450 \mathrm{E}-04$ \\
\hline
\end{tabular}


Table C-1. Results of Verification Runs Using GoldSim Versions 7.50.100, 8.01 Service Pack 4, and 8.02.300 (Continued)

\begin{tabular}{|c|c|c|c|}
\hline \multirow[b]{2}{*}{ Radionuclide } & \multirow[b]{2}{*}{ Pathway } & \multicolumn{2}{|c|}{ BDCF (rem/yr per pCi/L) } \\
\hline & & GoldSim 7.50.100 & GoldSim 8.01 Service Pack 4 and 8.02 .300 \\
\hline \multirow[t]{10}{*}{ Ra-226 (cont.) } & Other Vegetables & $6.20040 \mathrm{E}-05$ & $6.20040 \mathrm{E}-05$ \\
\hline & Fruit & $2.28720 \mathrm{E}-04$ & $2.28720 \mathrm{E}-04$ \\
\hline & Grain & 1.54740E-05 & $1.54740 \mathrm{E}-05$ \\
\hline & Meat & $2.58540 \mathrm{E}-05$ & $2.58540 \mathrm{E}-05$ \\
\hline & Milk & $2.39060 \mathrm{E}-05$ & $2.39060 \mathrm{E}-05$ \\
\hline & Poultry & $2.55110 \mathrm{E}-06$ & $2.55110 \mathrm{E}-06$ \\
\hline & Eggs & $6.36060 \mathrm{E}-05$ & $6.36060 \mathrm{E}-05$ \\
\hline & Fish & 8.47680E-05 & 8.47680E-05 \\
\hline & Soil Ingestion & 4.35430E-04 & $4.35430 \mathrm{E}-04$ \\
\hline & File name & ERMYN_GW_Ra226verf.gsm & $\begin{array}{l}\text { ERMYN_GW_Ra226verf_GS801SP4.gsm } \\
\text { ERMYN_GW_Ra226verf_GS802300.gsm }\end{array}$ \\
\hline
\end{tabular}




\section{INTENTIONALLY LEFT BLANK}

